\title{
Supporting Information Formamide-Catalyzed Nucleophilic Substitutions: Mechanistic Insight and Rationalization of Catalytic Activity
}

Corinna Kohlmeyer, ${ }^{1}$ André Schäfer, ${ }^{2, *}$ Peter H. Huy, ${ }^{3,4 *}$ Gerhard Hilt ${ }^{1, *}$

1. Oldenburg University, Institute of Chemistry, Carl-von-Ossietzky-Str. 9-11, 26111 Oldenburg, Germany; gerhard.hilt@uni-oldenburg.de. 2. Saarland University, Institute of Inorganic Chemistry, 66123 Saarbruecken, Germany; andre.schaefer@uni-saarland.de. 3.

Saarland University, Institute of Organic Chemistry, 66123 Saarbruecken, Germany. 4. Rostock University, Institute for Chemistry, Albert-Einstein-Straße 3a, 18059 Rostock, Germany; peter.huy@uni-rostock.de.

\author{
Homepages \\ https://www.uni-saarland.de/fakultaet-nt/schaefer/ \\ Peterhuylab.de \\ https://uol.de/oc-hilt
}

Twitter

@orgmet_Schaefer

@Peterhuylab 


\section{Content}

1 ROBUSTNESS SCREEN AND SENSITIVITY ASSESSMENT ............................... 1

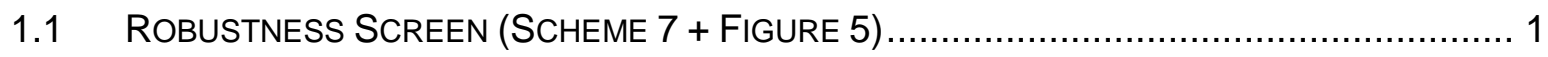

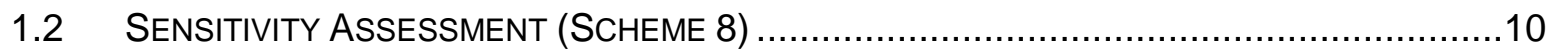

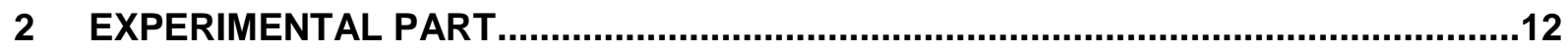

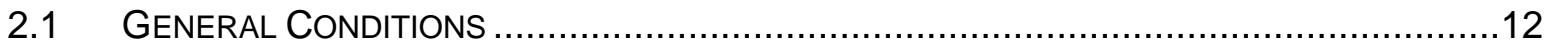

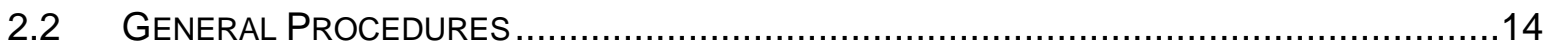

2.2.1 General Procedure 1: Formamide catalyzed Synthesis of 4-tert-Butylbenzyl Chloride

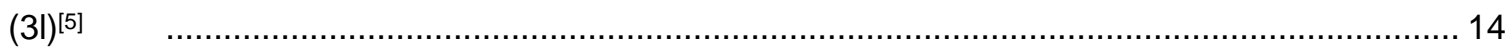

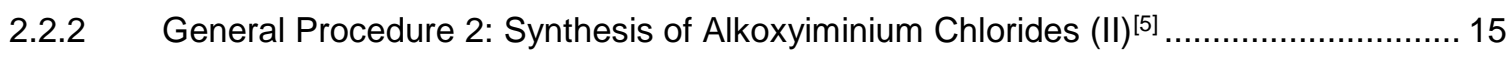

2.2.3 General Procedure 3: Synthesis of Alkoxyiminium Hexafluorophosphates ................... 15

2.2.4 General Procedure 4: Appel type Synthesis of 4-tert-Butylbenzyl Chloride ${ }^{[5]} \ldots \ldots \ldots \ldots . . . . .16$

2.2.5 General Procedure 5: Synthesis of 4-tert-Butylbenzyl Chloride with Thionylchloride ... 17

2.2.6 General Procedure 6: Synthesis of Formamides 5 via PIDA-catalyzed amidation ....... 18

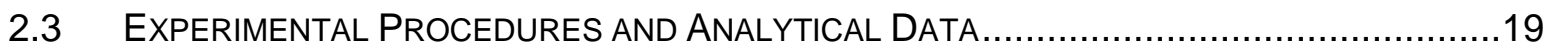

2.3.1 Synthesis of Alkoxyiminium Chlorides II (Scheme 3 A) ............................................ 19

2.3.2 Synthesis of Alkoxyiminium Hexafluorophosphates III (Scheme 3 B) ........................... 23

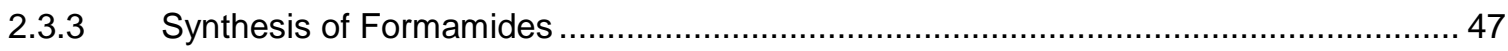

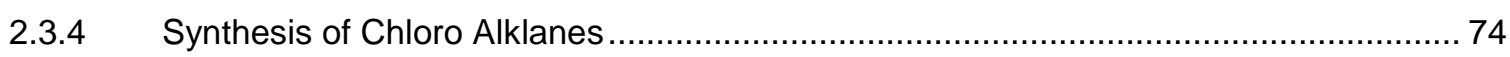

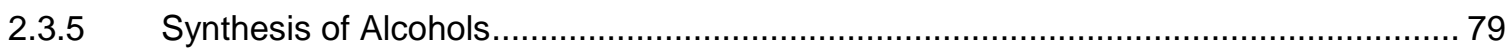

2.3.6 Analytical Data of the Additives (16) and Internal Standard for the Robustness Screen..

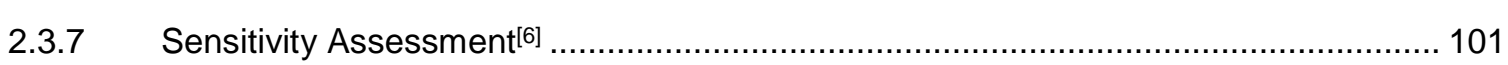

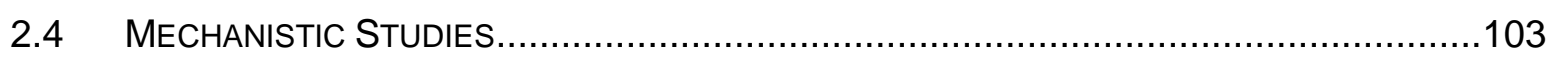

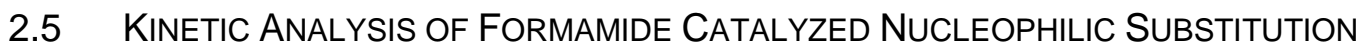

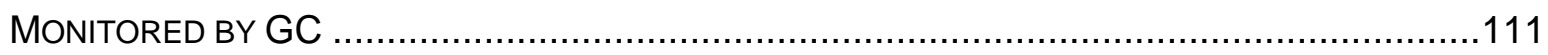

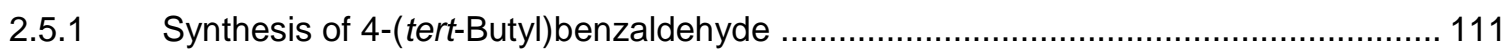

2.5.2 Synthesis of 4-(tert-Butyl)phenyl)methanol (1I) ................................................... 112

2.5.3 General Procedure for the Kinetic Analysis of Formamide Catalyzed Nucleophilic

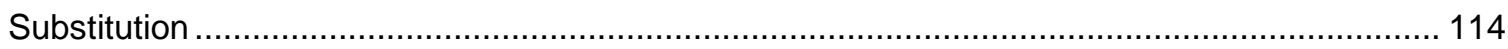

2.5.4 Kinetic Measurements with N,N-Dimethylformamide (DMF) ....................................... 115

2.5.5 Kinetic Measurements with N-Formylpyrrolidine (FPyr) ........................................ 116

2.5.6 Kinetic Measurements with Morpholine-4-carbaldehyde (FMor) .............................. 117

2.5.7 Kinetic Measurements with Piperidine-1-carbaldehyde (FPip) ................................. 118

2.5.8 Kinetic Measurements with $\mathrm{N}$-tert-Butylformamide (tBuF) ........................................ 119

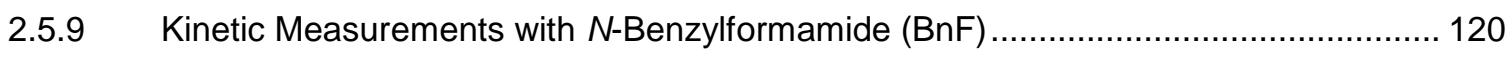

2.5.10 Kinetic Measurements with Piperazine-1,4-dicarbaldehyde (DFPiper) ........................ 121

2.5.11 Kinetic Measurements with N-Methylformamide (MF) …....................................... 122 
2.5.12 Kinetic Measurements with N,N-Di-n-butylformamide (DnBuF) .............................. 123

2.5.13 Kinetic Measurements with $N$-Benzyl- $N$-methylformamide (BnMF) ........................... 124

2.5.14 Kinetic Measurements with N,N-Dibenzylformamide (DBnF) .................................. 125

2.5.15 Kinetic Measurements with $N$-(4-Methoxyphenyl)- $N$-methylformamide ( $p M P M F) \ldots . . .126$

2.5.16 Kinetic Measurements with Azepane-1-carbaldehyde (FAz) …................................ 127

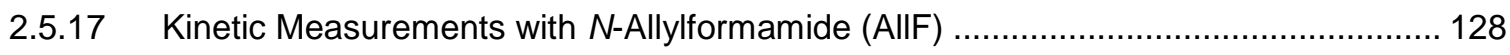

2.5.18 Kinetic Measurements with $\mathrm{N}$-Cyclohexylformamide (cHexF) ................................. 129

2.5.19 Kinetic Measurements with N-(2,2-Dimethoxyethyl)formamide (DMEF) .................... 130

2.5.20 Kinetic Measurements with N-Phenethylformamide (PEF) ................................... 131

2.5.21 Kinetic Measurements with N-Benzyl- $N$-(tert-butyl)formamide (BntBuF) .................... 132

2.5.22 Kinetic Measurements with $N$-(Naphthalen-1-ylmethyl)formamide (NaphMF) ............ 133

2.5.23 Kinetic Measurements with 3,4-Dihydroisoquinoline-2(1H)-carbaldehyde (F/soChin) 134

2.6 QuANTIFICATION OF LEWIS-BASICITY BY TITRATION OF B $\left(\mathrm{C}_{6} \mathrm{~F}_{5}\right)_{3}($ FIGURE 3) .............135

2.6.1 General Procedure for the Quantification of Lewis Basicity by Titration of $B\left(\mathrm{C}_{6} \mathrm{~F}_{5}\right)_{3}$

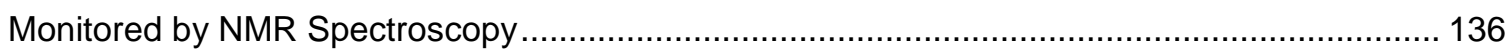

2.6.2 Titration of $\mathrm{B}\left(\mathrm{C}_{6} \mathrm{~F}_{5}\right)_{3}$ with $\mathrm{N}, \mathrm{N}$-Dimethylformamide (DMF) ...................................... 136

2.6.3 Titration of $\mathrm{B}\left(\mathrm{C}_{6} \mathrm{~F}_{5}\right)_{3}$ with Morpholine-4-carbaldehyde (FMor) ................................ 137

2.6.4 Titration of $\mathrm{B}\left(\mathrm{C}_{6} \mathrm{~F}_{5}\right)_{3}$ with Piperazine-1,4-dicarbaldehyde (FPip) .............................. 137

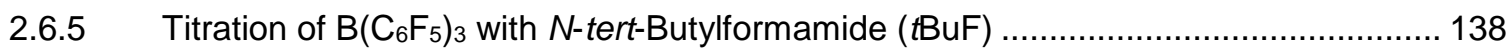

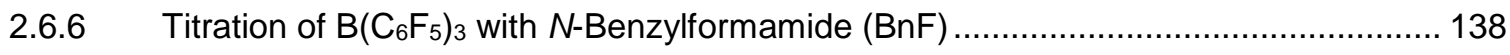

2.6.7 Titration of $\mathrm{B}\left(\mathrm{C}_{6} \mathrm{~F}_{5}\right)_{3}$ with $N, N$-Di- $n$-butylformamide (DnBuF) ................................. 139

2.6.8 Titration of $\mathrm{B}\left(\mathrm{C}_{6} \mathrm{~F}_{5}\right)_{3}$ with Piperazine-1,4-dicarbaldehyde (DFPiper) ......................... 139

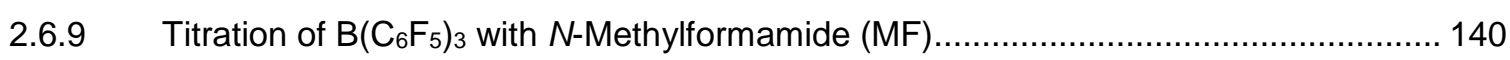

2.6.10 Titration of $\mathrm{B}\left(\mathrm{C}_{6} \mathrm{~F}_{5}\right)_{3}$ with $\mathrm{N}$-Benzyl- $\mathrm{N}$-methylformamide (BnMF) ............................ 140

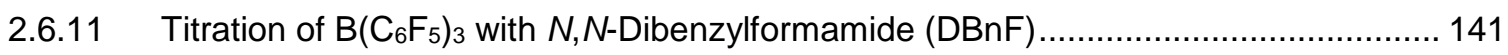

2.6.12 Titration of $\mathrm{B}\left(\mathrm{C}_{6} \mathrm{~F}_{5}\right)_{3}$ with $\mathrm{N}$-(4-Methoxyphenyl)- $\mathrm{N}$-methylformamide (pMPMF) .......... 141

2.7 LINEAR FrEe ENERGY RELATIONSHIP. ............................................................ 143

3 MULTIVARIATE LINEAR REGRESSION ANALYSIS (FIGURE 4)..........................144

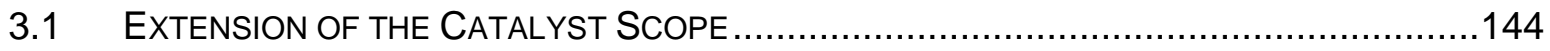

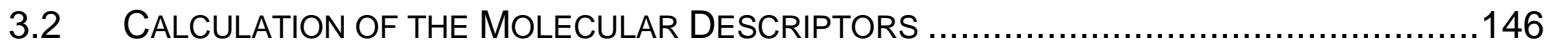

3.3 MODEL CONSTRUCTION FOR SYMMETRICAL FORMAMIDES .....................................150

3.4 AdDITION OF E-CONFIGURED FORMAMIDES TO THE MODEL …..............................153

3.5 AdDITION OF Z-CONFIGURED FORMAMIDES TO THE MODEL ....................................155 


\section{Robustness Screen and Sensitivity Assessment}

\subsection{Robustness Screen (Scheme 7 + Figure 5)}

The original truncated set of additives for the robustness screen according to Glorius were selected based on gas chromatographic retention times (Figure S1). ${ }^{[1-3]}$ Since all additives differ in their retention time, a single point batch calibration is possible using a single sample containing all additives and a suitable internal standard.

\section{Original Additives}

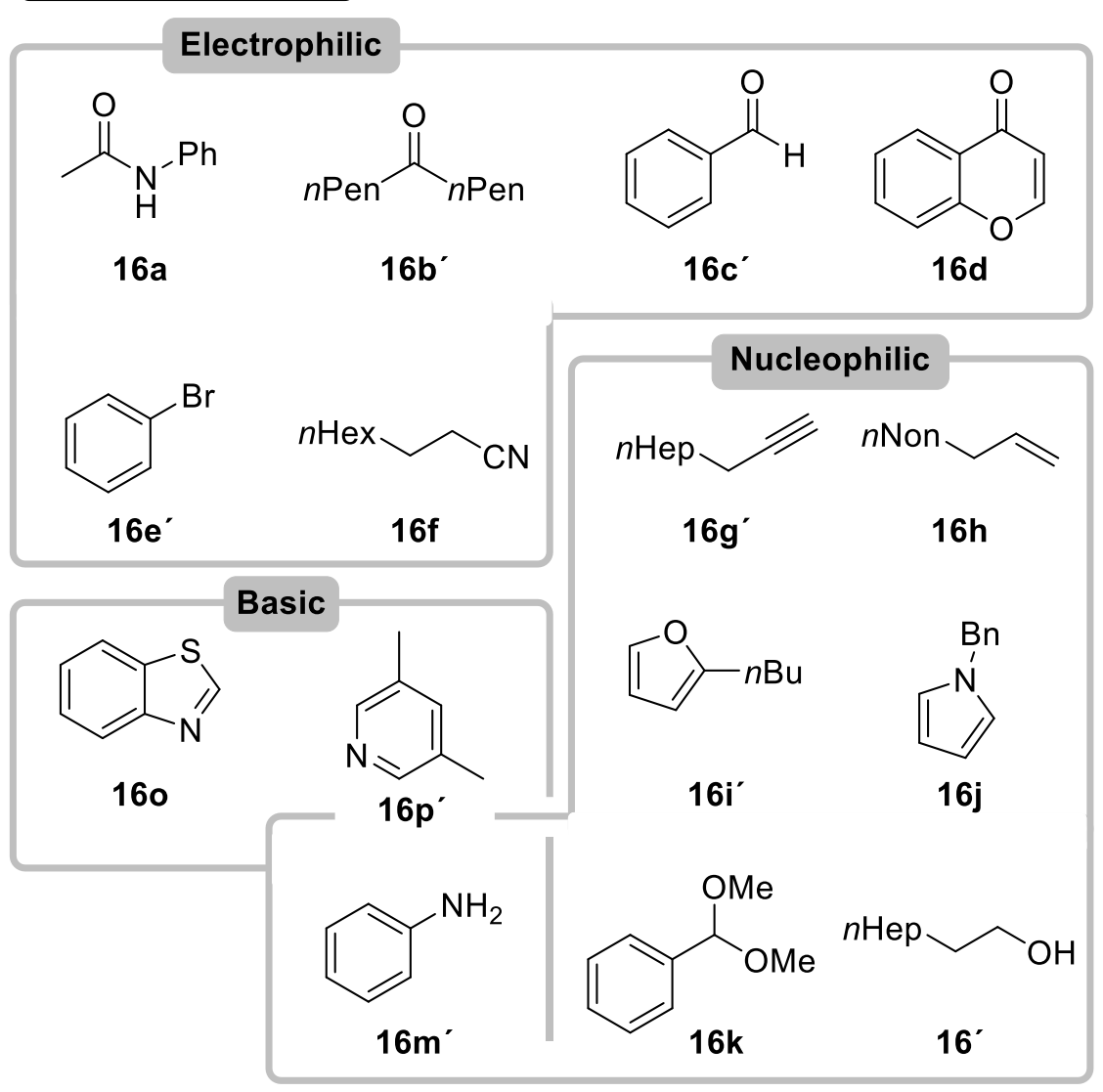

Figure S1. Original set of additives as designed by the Glorius group. [3]

In the current study, yields were determined after aqueous work up and evaporation of the solvent by ${ }^{1} \mathrm{H}$ NMR with an internal standard. Therefore, some of the additives were modified in order to accomplish higher boiling points and thus prevent a loss due to the solvent evaporation. The deviations are highlighted in Figure S2 in red.

Additives $16 \mathrm{c}, 16 \mathrm{e}$ and $16 \mathrm{~m}$ deviate from the original additives $16 \mathrm{c}^{\prime}, 16 \mathrm{e}^{\prime}$ and $16 \mathrm{~m}^{\prime}$ in a tert-butyl group in para-position. Compounds $16 \mathrm{~g}$ and $16 \mathrm{l}$ have a longer aliphatic carbon chain than $16 \mathrm{~g}^{\prime}$ and $16 \mathrm{I}^{\prime}$. Ketone $\mathbf{1 6 b}$ differs from $1 \mathbf{1 6 b}^{\prime}$ mainly through the position of the carbonyl group and is less expensive. These changes likely do not have a significant impact on the reactivity. 


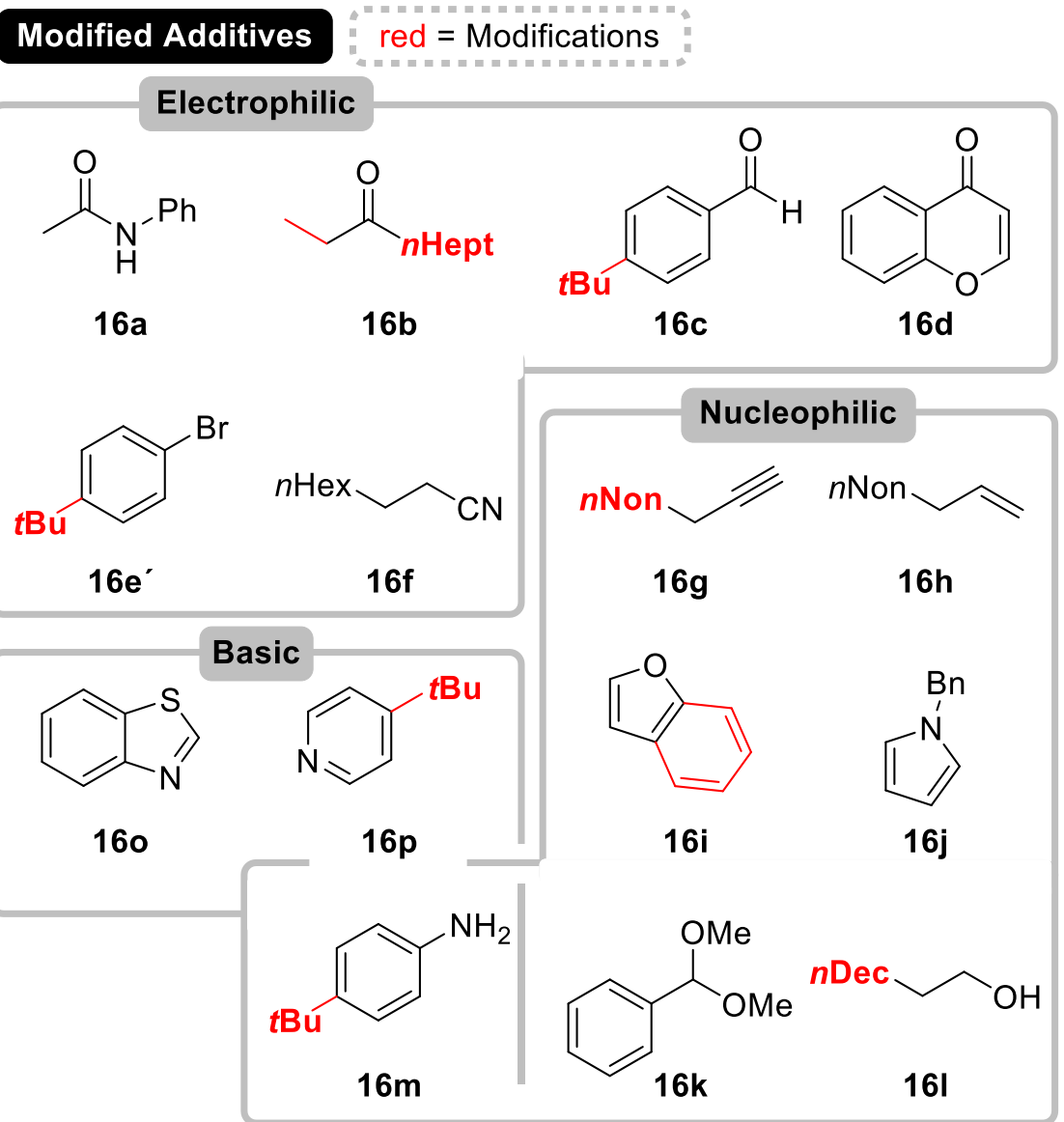

Figure S2. Altered additives with changes marked in red.

Instead of volatile 3,5-dimethylpyridine (16p'), 4-tert-butylpyridine (16p) was employed. Although 16p has no benzylic methyl groups, additive 16j possesses a reactive benzylic $\mathrm{CH}_{2}$ moiety. 2-Alkylfurane 16i' was replaced by benzofurane $16 \mathbf{i}$, since no other 2 -substituted furane with a longer carbon chain is commercially available at an acceptable price. In addition, benzofurane is more reactive towards electrophiles than $16 \mathbf{i}^{\prime}$.

In order to probe the reproducibility, the model reaction 1 I $\rightarrow$ 3l was conducted five times under identical conditions. In the experiments are listed in chronological order. The yields for 3I vary in a range of $89-93 \%$. Under consideration that the protocol comprehends an aqueous workup these experiments demonstrated reasonable levels of reproducibility. The average yield of $91 \%$ served as standard yield for the robustness screen. 
Table S1. Synthesis of 4-tert-butylbenzyl chloride $\mathbf{3 1}$.

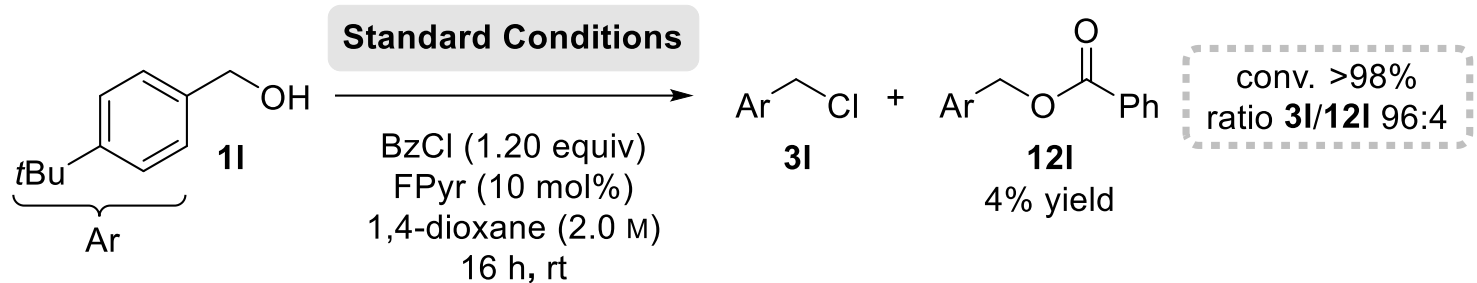

\begin{tabular}{llll}
\hline entry & NMR-standard & yield 3|[a] / \% & Journal No. \\
\hline 1 & mesitylene & 92 & $\mathrm{PH} 3976$ \\
2 & " & 91 & $\mathrm{PH} 4009$ \\
3 & “ & 89 & $\mathrm{PH} 4018$ \\
4 & “ & 89 & $\mathrm{PH} 4019$ \\
5 & " & 93 & $\mathrm{PH} 4037$ \\
\hline$\Sigma$ & & 91 &
\end{tabular}

According to general procedure 1 (chapter 2.2.1, page 14) 4-tert-butylbenzyl alcohol (0.50 mmol, 1.00 equiv) was reacted with $\mathrm{BzCl}(0.60 \mathrm{mmol}, 1.20$ equiv) in the presence of $\mathrm{FPyr}(10 \mathrm{~mol} \%)$ in 1,4-dioxane $(2.0 \mathrm{M})$ for $16 \mathrm{~h}$ at room temperature. [a] Yields were determined by means of ${ }^{1} \mathrm{H}$ NMR in $\mathrm{CDCl}_{3}$ of the crude material based on mesitylene as internal standard. Conversion and the ratio $\mathbf{3} / \mathbf{1 2}$ were calculated based on the ratio of the singlets of the $\mathrm{CH}_{2-}$ groups of the respective compounds in the ${ }^{1} \mathrm{H}$ NMR.

The results of the robustness screen are summarized in Table S2 below and in the manuscript. According to Glorius three different ranges of yield of the product $\mathbf{3} \mathbf{I}$ and the recovery yields of the additives 16 are displayed in a traffic light color code. In terms of additive 16, green is associated to yields $>67 \%$, orange to 34 to $67 \%$ and red to $<34 \%$. In the case of chloride 3 I, the ranges were adopted with respect to the mean standard yield of $91 \%$, which results in scopes of $>60 \%, 31-60 \%$ and $<31 \%$.

In addition, the additives in Table S2 are grouped into the categories electrophilic, nucleophilic and basic. Aniline 16k (entry 11) accounts to nucleophilic and basic compounds and is therefore presented in both substance classes. The average yields for each section are concluded in the manuscript in Figure 8 B. Overall, a mean yield for $3 \mathbf{3}$ of $72 \%$ was accomplished, which corresponds to $79 \%$ of the standard yield of $91 \%(=\% \circ S)$. The recovery yield is $77 \%$ over all additives.

The yield determination by means of internal NMR standard enabled the quantification of yields for benzoate 12I and of side-products like benzaldehyde (entry 11) without the necessity of calibration. The results of the robustness screen are also summarized in the main manuscript in Figure 8. 
Table S2. Robustness screen for formamide catalyzed chlorination (for additive structures see Figure S2).

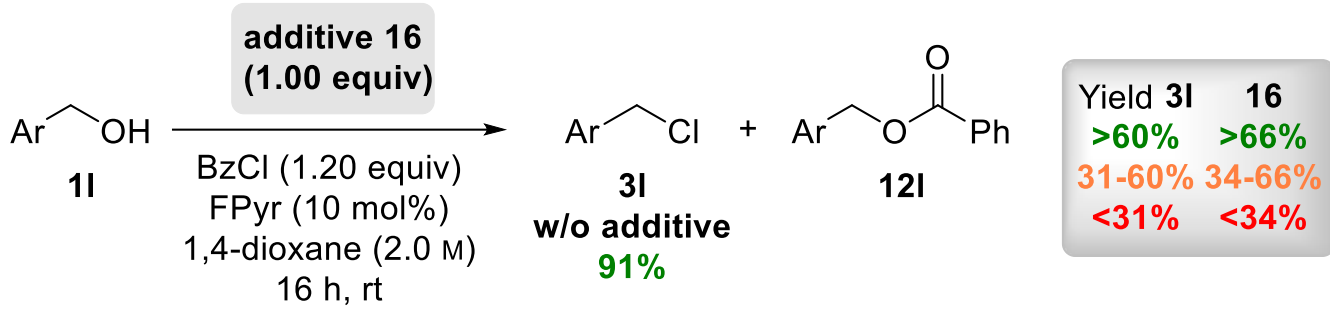

\begin{tabular}{|c|c|c|c|c|c|c|c|c|c|}
\hline \multirow{2}{*}{\multicolumn{2}{|c|}{$\begin{array}{l}\text { entry additive } \\
\text { Electrophilic }\end{array}$}} & \multicolumn{3}{|c|}{ yield of / \% } & \multicolumn{2}{|c|}{ conv. / \% ratio 3l/12I } & \multicolumn{2}{|c|}{ yield of / \% } & \multirow[t]{2}{*}{ Journal No. } \\
\hline & & & & & & & & & \\
\hline 1 & $16 a$ & 89 & 98 & 82 & $>98$ & $95: 5$ & / & 5 & PH3978 \\
\hline 2 & $16 b$ & 87 & 96 & 94 & $>98$ & $97: 3$ & / & 3 & PH3998 \\
\hline 3 & $16 c$ & 81 & 89 & 86 & $>98$ & $97: 3$ & 8 & 3 & PH3975 \\
\hline 4 & $16 d$ & 89 & 98 & 77 & $>98$ & $96: 4$ & $<1$ & 4 & PH4021 \\
\hline 5 & $16 e$ & 87 & 96 & 91 & $>98$ & $97: 3$ & $<1$ & 3 & $\mathrm{PH} 4000$ \\
\hline \multirow[t]{2}{*}{6} & $16 f$ & 90 & 99 & 100 & $>98$ & $96: 4$ & / & 4 & PH3996 \\
\hline & $\Sigma$ & 88 & 91 & 96 & & & & & \\
\hline \multicolumn{10}{|c|}{ Nucleophilic } \\
\hline 7 & $16 \mathrm{~g}$ & 81 & 89 & 87 & $>98$ & n.d. & / & n.d. & PH3997 \\
\hline 8 & $16 \mathrm{~h}$ & 87 & 96 & 84 & $>98$ & $97: 3$ & 2 & 3 & PH3974 \\
\hline 9 & $16 i$ & 85 & 93 & 76 & $>98$ & $97: 3$ & $<1$ & 3 & PH4001 \\
\hline 10 & $16 j$ & 91 & 100 & 90 & $>98$ & $96: 4$ & 1 & 3 & $\mathrm{PH} 4003$ \\
\hline $11^{\mathrm{a}}$ & $16 \mathrm{k}$ & 53 & 58 & 8 & 71 & $93: 7$ & 24 & 4 & PH3977 \\
\hline 12 & $16 I$ & 14 & 15 & 53 & 36 & $44: 56$ & 60 & 18 & PH3973 \\
\hline \multirow[t]{2}{*}{13} & $16 \mathrm{~m}$ & 43 & 47 & 32 & 50 & $92: 8$ & 47 & 4 & PH3999 \\
\hline & $\Sigma$ & 65 & 61 & 71 & & & & & \\
\hline \multicolumn{10}{|c|}{ Basic } \\
\hline 13 & $16 \mathrm{~m}$ & 43 & 47 & 32 & 50 & $92: 8$ & 47 & 4 & PH3999 \\
\hline 14 & 160 & 94 & 103 & 90 & $>98$ & $95: 5$ & / & 5 & PH4002 \\
\hline \multirow[t]{2}{*}{15} & $16 p$ & 13 & 14 & 86 & $>98$ & $15: 85$ & 2 & 78 & PH4004 \\
\hline & $\Sigma$ & 50 & 69 & 55 & & & & & \\
\hline \multicolumn{2}{|c|}{ Overall $\Sigma$} & 72 & 77 & 80 & & & & & \\
\hline
\end{tabular}

According to general procedure 1 (chapter 2.2.1, page 14) 4-tert-butylbenzyl alcohol (0.50 mmol, 1.00 equiv) was reacted with $\mathrm{BzCl}(0.60 \mathrm{mmol}, 1.20$ equiv) in the presence of $\mathrm{FPyr}(10 \mathrm{~mol} \%)$ and the respective additive 16 (1.00 equiv) in 1,4-dioxane (2.0 M) for $16 \mathrm{~h}$ at room temperature. Yields were determined by means of ${ }^{1} \mathrm{H}$ NMR of the crude material with mesitylene as internal standard in $\mathrm{CDCl}_{3}$. Conversion and the ratio $\mathbf{3} \mathbf{l} / \mathbf{1 2}$ were calculated based on the ratio of the singlets of the $\mathrm{CH}_{2}$-groups of the respective compounds in the ${ }^{1} \mathrm{H} \mathrm{NMR}$. a. benzaldehyde in 59\% yield detected. $\mathrm{Ar}=4$-tert-butylbenzyl, n.d. $=$ not determined.

The four additives $16 \mathrm{k}-\mathrm{m}$ and $16 \mathrm{p}$ were also tested under the conditions of the Appel reaction as a benchmark (Table S3 and in the manuscript). Since no aqueous workup was performed, the standard yield of $\mathbf{3} \mathbf{I}$ in the absence of additives is quantitative. 
A second robustness screen was conducted under Appel conditions using NCS and $\mathrm{PPh}_{3}$ (Table S3). As discussed in the main manuscript, the aniline $16 \mathrm{~m}$, acetale 16k and decanol $\mathbf{1 6 I}$ were not tolerated, while all other additives did not affect the yield of product $\mathbf{3} \mathbf{I}$ and were compatible with the reaction conditions.

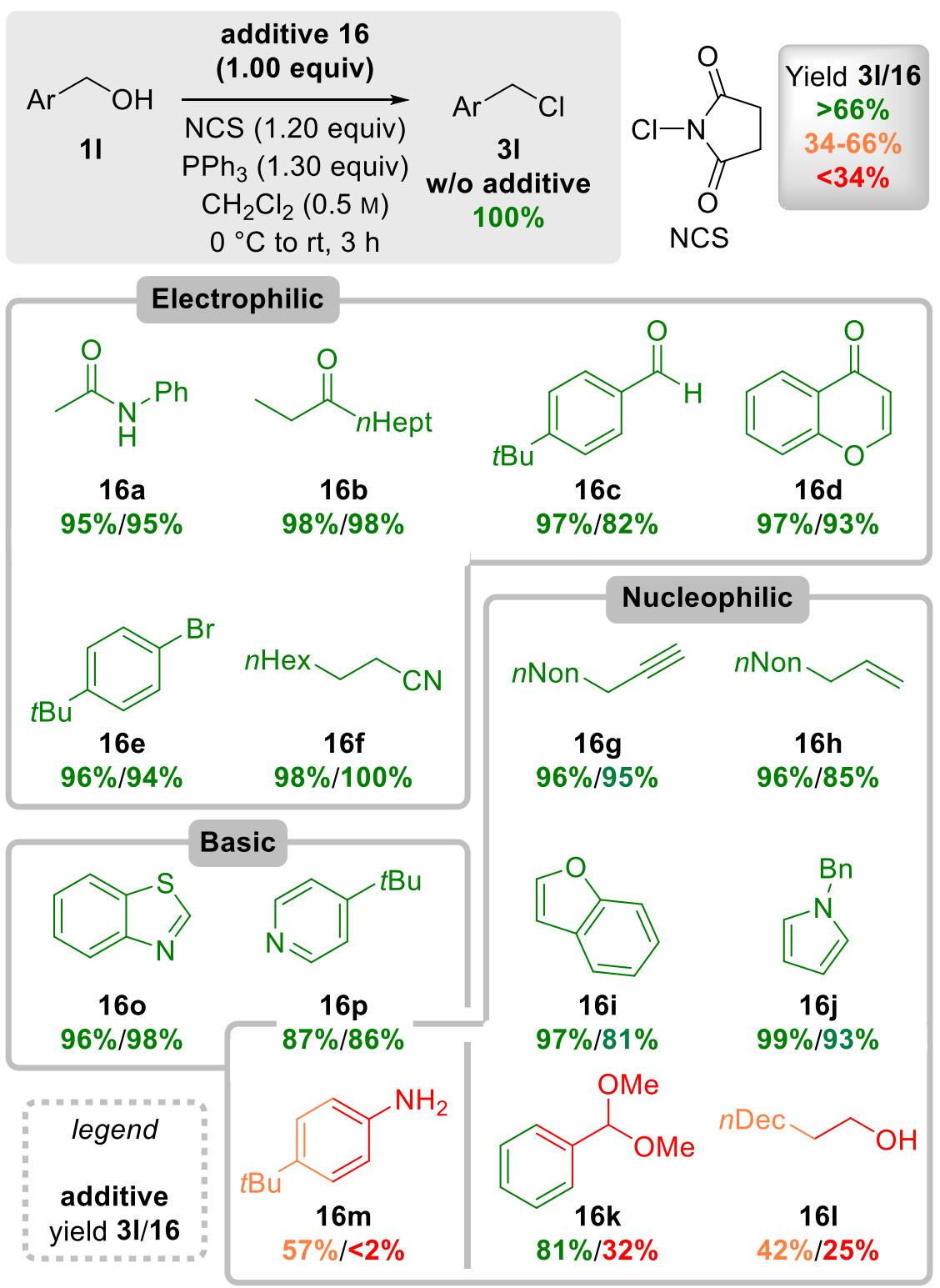

Scheme S1. Robustness screen for Appel reaction.

Since no aqueous work was performed and in most cases no side-products were formed, the standard yield of $\mathbf{3} \mathbf{I}$ in the absence of additives is quantitative. 
Table S3. Robustness screen for Appel reaction (for additive structures see Scheme S1).

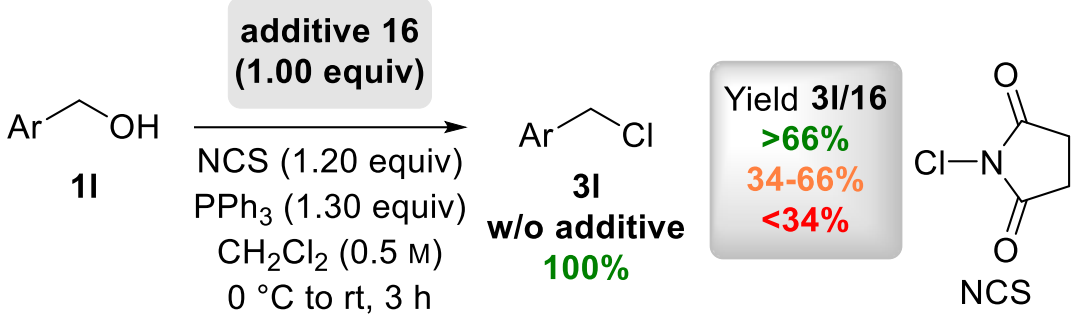

\begin{tabular}{|c|c|c|c|c|c|c|c|}
\hline \multirow{2}{*}{\multicolumn{2}{|c|}{ entry additive }} & \multicolumn{3}{|c|}{ yield of [\%] } & \multirow[b]{2}{*}{ conv. [\%] } & \multirow[b]{2}{*}{ yield of $1 \mathrm{l}$ [\%] } & \multirow[b]{2}{*}{$\begin{array}{l}\text { Journal } \\
\text { No. }\end{array}$} \\
\hline & & 31 & $\% \circ S$ & additive & & & \\
\hline \multicolumn{8}{|c|}{ Standard Yield } \\
\hline 1 & I & 100 & 100 & l & $\geq 98$ & l & PH4031 \\
\hline \multicolumn{8}{|c|}{ Electrophilic } \\
\hline 2 & $16 a$ & 95 & 95 & 95 & $\geq 98$ & / & PH4187 \\
\hline 3 & $16 b$ & 98 & 98 & 98 & $\geq 98$ & l & PH4188 \\
\hline $4^{a}$ & $16 c$ & 97 & 97 & 82 & $\geq 98$ & I & PH4189 \\
\hline 5 & $16 d$ & 97 & 97 & 93 & $\geq 98$ & / & PH4190 \\
\hline 6 & $16 e$ & 96 & 96 & 94 & $\geq 98$ & I & PH4191 \\
\hline \multirow[t]{2}{*}{7} & $16 f$ & 98 & 98 & 100 & $\geq 98$ & / & PH4192 \\
\hline & $\Sigma$ & 97 & 97 & 94 & & & \\
\hline 8 & $16 \mathrm{~g}$ & 96 & 96 & 95 & $\geq 98$ & I & $\mathrm{PH} 4193$ \\
\hline 9 & $16 h$ & 96 & 96 & 85 & $\geq 98$ & l & PH4194 \\
\hline 10 & $16 i$ & 97 & 97 & 81 & $\geq 98$ & / & PH4196 \\
\hline 11 & 16j & 99 & 99 & 93 & $\geq 98$ & / & PH4195 \\
\hline $12^{b}$ & $16 k$ & 81 & 81 & 32 & 88 & 11 & PH4030 \\
\hline $13^{c}$ & $16 I$ & 42 & 42 & 25 & 90 & 48 & PH4035 \\
\hline \multirow[t]{2}{*}{14} & $16 \mathrm{~m}$ & 57 & 57 & $\leq 2$ & 80 & 15 & PH4032 \\
\hline & $\boldsymbol{\Sigma}$ & 81 & 81 & 59 & & & \\
\hline 15 & $16 \mathrm{~m}$ & 57 & 57 & $\leq 2$ & 80 & 15 & $\mathrm{PH} 4032$ \\
\hline 15 & 160 & 96 & 96 & 98 & $\geq 98$ & / & PH4197 \\
\hline \multirow[t]{2}{*}{16} & $16 p$ & 87 & 87 & 86 & $\geq 98$ & I & PH4033 \\
\hline & $\Sigma$ & 80 & 80 & 61 & & & \\
\hline \multicolumn{2}{|c|}{ Overall $\Sigma$} & 89 & 89 & 77 & & & \\
\hline
\end{tabular}

According to general procedure 4 (chapter 2.2.4, page 16) 4-tert-butylbenzyl alcohol (0.50 mmol, 1.00 equiv) was reacted with NCS (1.20 equiv) and $\mathrm{PPh}_{3}$ (1.30 equiv) in $\mathrm{CH}_{2} \mathrm{Cl}_{2}(0.5 \mathrm{M})$ for $15 \mathrm{~min}$ at $0{ }^{\circ} \mathrm{C}$ and then for $3 \mathrm{~h}$ at room temperature. Yields were determined by means of ${ }^{1} \mathrm{H} N M R$ in $\mathrm{CDCl}_{3}$ of the crude material based on mesitylene as internal standard. a. (4-tert-butylphenyl)dichloromethane obtained in $7 \%$ yield. b. Benzaldehyde in $34 \%$ yield detected. c. 1 -Dodecyl chloride was formed in $65 \%$ yield. Ar $=4$-tert-butylbenzyl.

Moreover, a third robustness screen was performed for the thionyl chloride promoted synthesis of chloride $\mathbf{3 l}$ from alcohol $\mathbf{1 l}$ (Table S4 and Scheme S2). Thereby, $\mathrm{HCl}$ is generated as byproduct in stoichiometric quantities, which causes a highly acidic reaction medium. Nevertheless, the robustness screen showed surprisingly good results, because only three additives were not compatible. 


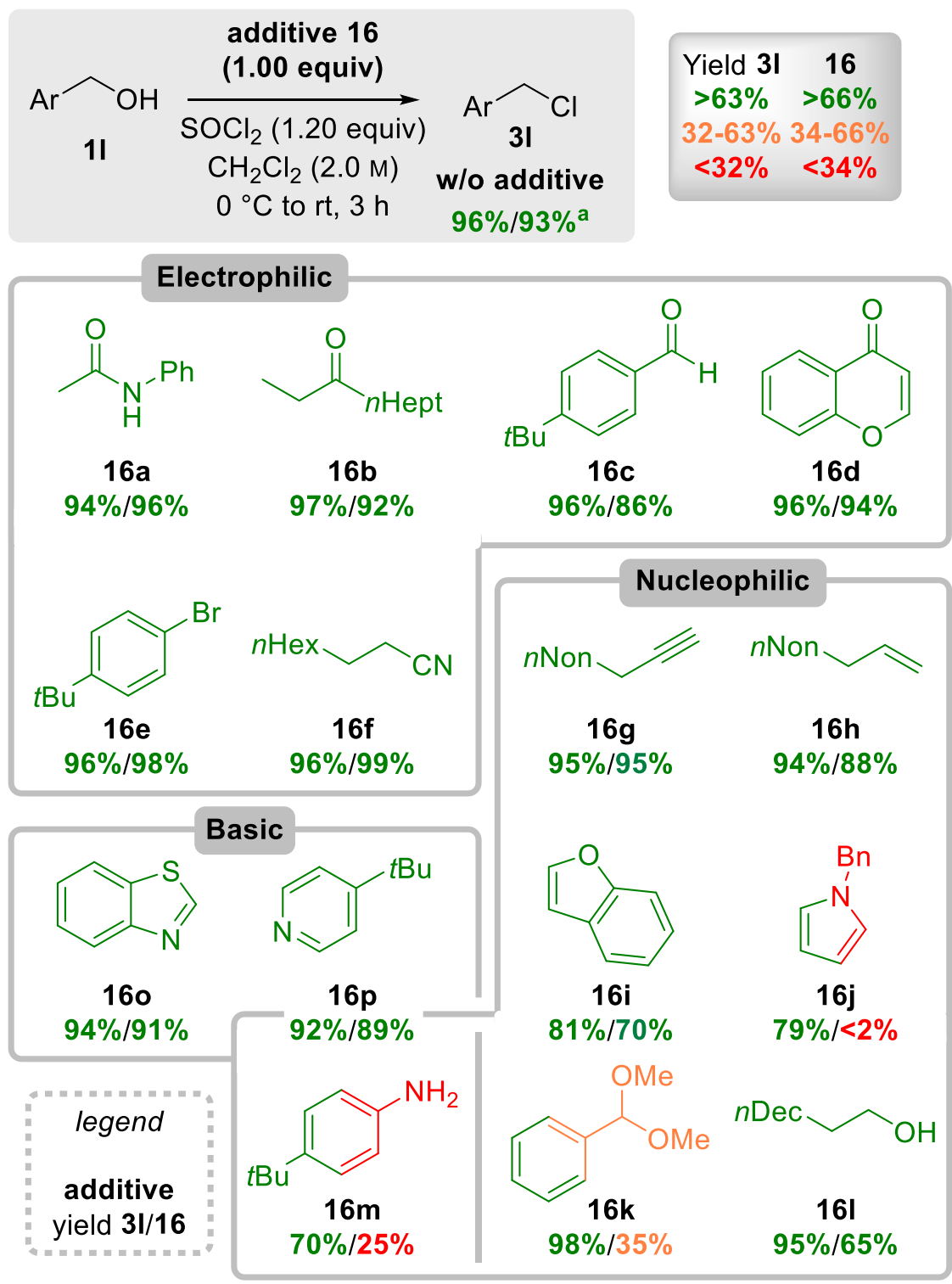

Scheme S2. Robustness Screen for chlorination using thionyl chloride.

Amongst them are aniline $\mathbf{1 6 m}$ and acetale 16k (Table S4, entries 15, 17 and 18), both of which also failed in two previous screens (see above). In addition, electron-rich pyrrole 16 $\mathbf{j}$ is incompatible (entry 14), which has been perfectly tolerate under the previously probed conditions. In fact, the reaction conditions are remarkably robust, since in all cases the yields of $\mathbf{3 l}$ are equal to or higher than $70 \%$. Surprisingly was also the chemoselective chlorination of benzylic alcohol 1I in the presence of primary aliphatic alcohol 16I (entry 15). Indeed, dodecanol $\mathbf{1 6 I}$ was not tolerated under the previously tested reaction conditions (see above).

Since $\mathrm{HCl}$ forms throughout the reactions, some of the additives were present as hydrochloride salts after solvent evaporation. In the instance of $\mathbf{1 6 0}$ the respective thiazolium salts was obtained, while $16 \mathrm{p}$ and $\mathbf{1 6 m}$ reacted to the corresponding pyridinium and anilinium hydrochlorides, respectively. The ${ }^{1} \mathrm{H}$ NMR spectra of these salts deviate from those of their neutral bases. Therefore, a basic aqueous work was carried out, to liberate the neutral Brønsted bases (entries 5, 18, 20 and 22). 
Table S4. Robustness screen for chlorination by means of thionyl chloride (for additive structures see Scheme S2).

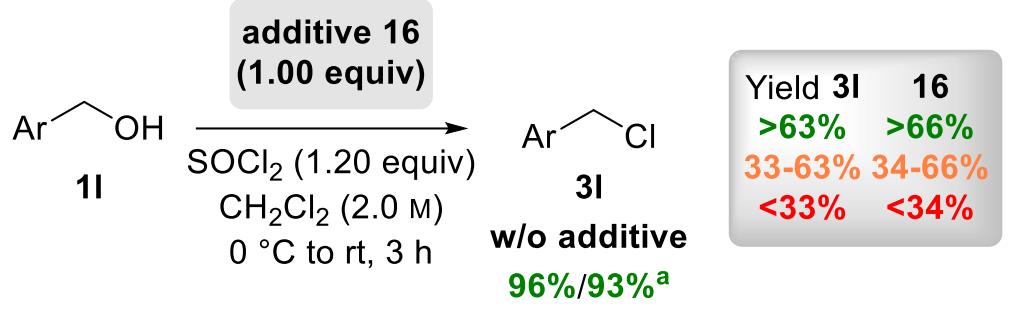

\begin{tabular}{|c|c|c|c|c|c|c|c|}
\hline \multirow{2}{*}{\multicolumn{2}{|c|}{ entry additive }} & \multicolumn{3}{|c|}{ yield of [\%] } & \multirow[b]{2}{*}{ conv. [\%] } & \multirow[b]{2}{*}{ yield of 11 [\%] } & \multirow[b]{2}{*}{ Journal No. } \\
\hline & & 31 & $\% \circ S$ & additive & & & \\
\hline \multicolumn{8}{|c|}{ Standard Yield } \\
\hline 1 & l & 95 & 99 & I & $\geq 98$ & l & PH4198 \\
\hline 2 & I & 97 & 101 & l & $\geq 98$ & l & PH4218 \\
\hline $3^{a}$ & I & 93 & 100 & l & $\geq 98$ & l & $\mathrm{PH} 4219$ \\
\hline \multicolumn{8}{|c|}{ Electrophilic } \\
\hline 4 & $16 a$ & 95 & 99 & $91^{b}$ & $\geq 98$ & l & PH4202 \\
\hline $5^{\mathrm{a}}$ & $16 a$ & 94 & 101 & 96 & $\geq 98$ & l & $\mathrm{PH} 4214$ \\
\hline 6 & $16 b$ & 97 & 101 & 92 & $\geq 98$ & l & PH4203 \\
\hline 7 & $16 c$ & 96 & 100 & 86 & $\geq 98$ & l & $\mathrm{PH} 4204$ \\
\hline 8 & $16 d$ & 96 & 100 & 94 & $\geq 98$ & l & PH4205 \\
\hline 9 & $16 e$ & 96 & 100 & 98 & $\geq 98$ & I & PH4206 \\
\hline \multirow[t]{2}{*}{10} & $16 f$ & 96 & 100 & $99^{b}$ & $\geq 98$ & l & $\mathrm{PH} 4211$ \\
\hline & $\boldsymbol{\Sigma}$ & 96 & 100 & 94 & & & \\
\hline 11 & $16 \mathrm{~g}$ & 95 & 99 & 95 & $\geq 98$ & 1 & $\mathrm{PH} 4199$ \\
\hline 12 & $16 \mathrm{~h}$ & 94 & 98 & 88 & $\geq 98$ & I & PH4200 \\
\hline 13 & $16 i$ & 81 & 84 & 70 & $\geq 98$ & I & PH4207 \\
\hline 14 & $16 j$ & 79 & 82 & $\leq 2$ & 81 & 18 & PH4201 \\
\hline $15^{c}$ & $16 \mathrm{k}$ & 98 & 102 & 35 & $\geq 98$ & I & PH4213 \\
\hline 16 & $16 I$ & 95 & 99 & $65^{b}$ & $\geq 98$ & I & PH4212 \\
\hline 17 & $16 m$ & 93 & 97 & $26^{b}$ & $\geq 98$ & I & PH4209 \\
\hline \multirow[t]{2}{*}{$18^{a}$} & $16 m$ & 70 & 75 & 25 & $\geq 98$ & I & $\mathrm{PH} 4216$ \\
\hline & $\Sigma$ & 87 & 91 & 54 & & & \\
\hline 17 & $16 \mathrm{~m}$ & 93 & 97 & $26^{b}$ & $\geq 98$ & 1 & PH4209 \\
\hline 19 & 160 & 100 & 104 & $97^{b}$ & $\geq 98$ & I & PH4210 \\
\hline $20^{a}$ & 160 & 94 & 101 & 91 & $\geq 98$ & l & $\mathrm{PH} 4217$ \\
\hline 21 & $16 p$ & 95 & 99 & $102^{b}$ & $\geq 98$ & I & PH4208 \\
\hline \multirow[t]{2}{*}{$22^{a}$} & $16 p$ & 92 & 99 & 89 & $\geq 98$ & I & PH4215 \\
\hline & $\Sigma$ & 85 & 88 & 68 & & & \\
\hline \multicolumn{2}{|c|}{$\overline{O v e r a l l} \Sigma$} & 92 & 95 & 75 & & & \\
\hline
\end{tabular}

According to general procedure 5 (chapter 2.2.5, page 17) 4-tert-butylbenzyl alcohol ( $0.50 \mathrm{mmol}, 1.00$ equiv) was reacted in the presence of the respective additive 16 (0/1.00 equiv) with $\mathrm{SOCl}_{2}\left(1.20\right.$ equiv) in $\mathrm{CH}_{2} \mathrm{Cl}_{2}(2.0 \mathrm{M})$ for $15 \mathrm{~min}$ at $0{ }^{\circ} \mathrm{C}$ and then for $3 \mathrm{~h}$ at room temperature. Yields were determined by means of ${ }^{1} \mathrm{H} \mathrm{NMR}$ in $\mathrm{CDCl}_{3}$ of the crude material based on mesitylene as internal standard. a. With aqueous work up. b. Chemical shifts changed due to $\mathrm{HCl}$ (ammonium) adduct formation. c. Benzaldehyde was observed in $40 \%$ yield. $\mathrm{Ar}=4$-tert-butylbenzyl. 
For a correct referencing, the model reaction was repeated including the same work up protocol, which furnished target compound $3 \mathbf{3}$ in 93 instead of 96\% (compare entry 3 with average of entries $1+2$ ). The results of the three robustness screens are compiled in Figure S3 as suggested in [3]. Indeed, similar levels of robustness and function group compatibility were attained with $\mathrm{SOCl}_{2}$ than in the case of the Appel reaction and our formamide catalysed alcohol dehydroxychlorination.

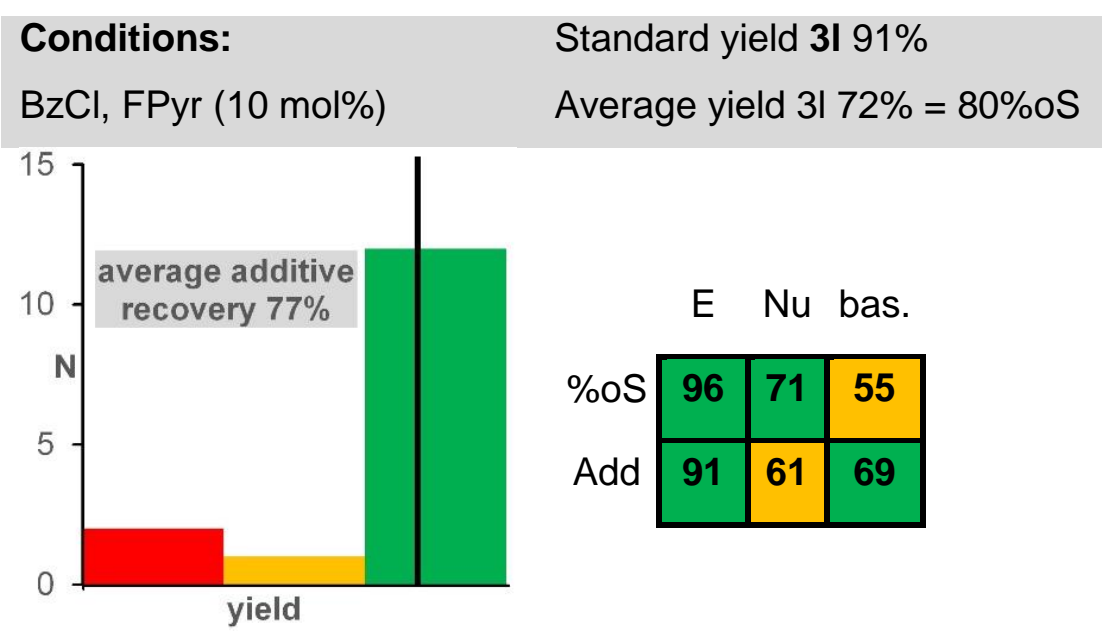

$\begin{array}{cc}\text { Yield 3I } & 16 \\ >63 \% & >66 \% \\ 33-63 \% & 34-66 \% \\ <33 \% & <34 \%\end{array}$

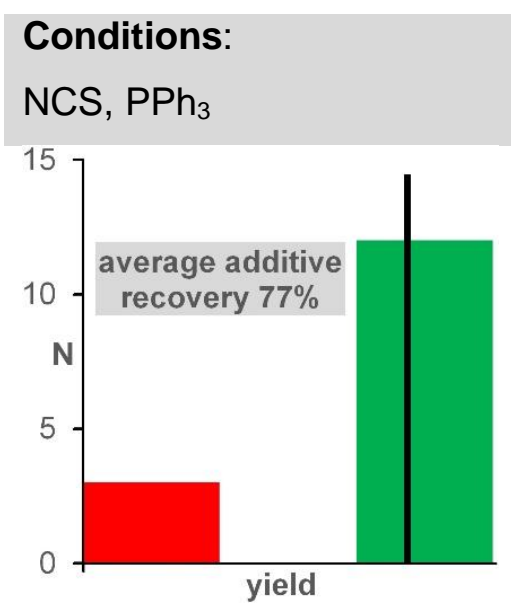

Standard yield $\mathbf{3 l}$ 100\%

Average yield $3189 \%=89 \%$ oS

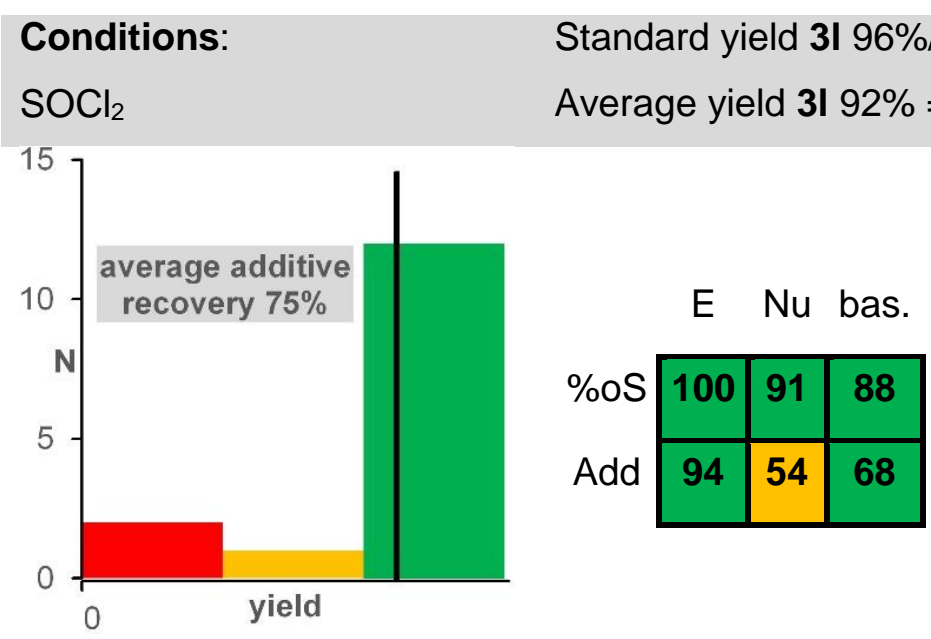

Figure S3. Summary robustness screen for the three approaches. 


\subsection{Sensitivity Assessment (Scheme 8)}

The results of the sensitivity assessment as described by Glorius ${ }^{[6]}$ are summarized in Table S5. The same conditions like in the robustness screen were chosen as zero point (see Table S1), which gave rise of benzylic chloride $3 \mathbf{I}$ in $91 \%$ average yield over 5 identical experiments (entry 1 ). In the case of high and low substrate concentrations more significant deviations from the standard conditions were selected (entries $2+3$ ), because the standard concentration of $2.0 \mathrm{M}$ is already relatively high (original difference \pm 10 vol\%). In addition, the reaction temperature $\mathbf{T}$ was varied in a range of $\pm 15^{\circ} \mathrm{C}$ instead of $\pm 10^{\circ} \mathrm{C}$ (entries $9+11$ ).

Table S5. Sensitivity assessment employing benzylic model substrate $\mathbf{1 I}$.

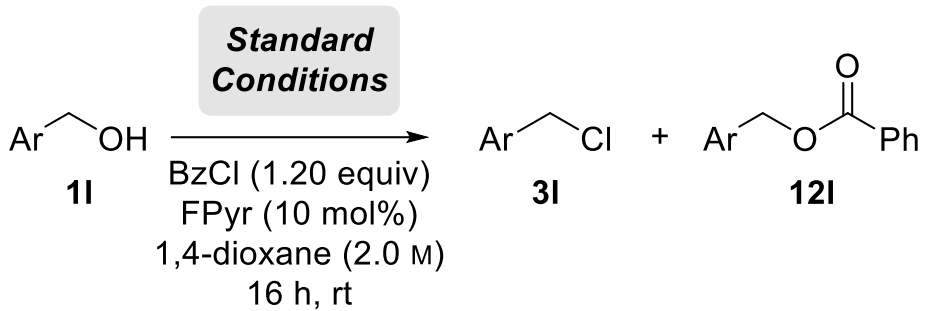

\begin{tabular}{|c|c|c|c|c|c|c|c|c|c|}
\hline entry & $\begin{array}{l}\text { deviation from } \\
\text { standard conditions }\end{array}$ & symbol & & $\begin{array}{l}\text { Id of / \% } \\
\text { deviation }\end{array}$ & $\begin{array}{l}\text { conv. } \\
/ \%\end{array}$ & $\begin{array}{l}\text { ratio } \\
31: 12 \mid\end{array}$ & 11 & 121 & $\begin{array}{l}\text { journal } \\
\text { No. }\end{array}$ \\
\hline 1 & / & / & 91 & & $>98$ & $96: 4$ & $<2$ & 4 & $\begin{array}{l}\text { see } \\
\text { Table S1 }\end{array}$ \\
\hline 2 & {$[1 \mathrm{I}]=4.0 \mathrm{M}$} & High C & 91 & \pm 0 & $>98$ & $93: 7$ & $<1$ & 7 & PH3982 \\
\hline 3 & {$[1 \mathrm{l}]=1.0 \mathrm{M}$} & Low C & 90 & -1 & $>98$ & $97: 3$ & $<1$ & 2 & PH3983 \\
\hline 4 & +1 vol\% $\mathrm{H}_{2} \mathrm{O}$ & $\mathrm{H}_{2} \mathrm{O}$ & 53 & -38 & 69 & $87: 13$ & 29 & 8 & PH4005 \\
\hline 5 & +10 vol $\% \mathrm{H}_{2} \mathrm{O}$ & I & 14 & -77 & 30 & $52: 48$ & 66 & 13 & PH3988 \\
\hline 6 & dry conditions & Low $\mathrm{O}_{2}$ & 95 & +4 & $>98$ & $96: 4$ & 1 & 4 & PH3987 \\
\hline 7 & $1 \mathrm{~atm} O 2$ & High $\mathrm{O}_{2}$ & 83 & -8 & 93 & $96: 4$ & 6 & 4 & PH4020 \\
\hline 8 & open vial & / & 44 & -47 & 55 & $90: 10$ & 42 & 5 & PH3986 \\
\hline 9 & $\mathrm{~T}=10^{\circ} \mathrm{C}$ & Low $T$ & 61 & -30 & 72 & $91: 9$ & 27 & 6 & PH4017 \\
\hline 10 & $\mathbf{T}=0^{\circ} \mathrm{C}$ & / & 13 & -78 & 27 & $60: 40$ & 70 & 9 & PH3991 \\
\hline 11 & $\mathrm{~T}=40^{\circ} \mathrm{C}$ & High T & 91 & \pm 0 & $>98$ & $97: 3$ & / & $<1$ & PH3989 \\
\hline 12 & 5 mol\% FPyr & Low Cat. & 81 & -10 & 95 & $93: 7$ & 5 & 6 & PH3984 \\
\hline 13 & $40 \mathrm{~mol} \% \mathrm{FPyr}$ & High Cat. & 95 & +4 & $>98$ & $>98: 2$ & / & 2 & PH3985 \\
\hline
\end{tabular}

According to general procedure 1 (chapter 2.2.1, page 1414) 4-tert-butylbenzyl alcohol (0.50 mmol, 1.00 equiv) was reacted with $\mathrm{BzCl}(0.60 \mathrm{mmol}, 1.20$ equiv) in the presence of $\mathrm{FPyr}(5-40 \mathrm{~mol} \%)$ in 1,4-dioxane (1.0-4.0 M) for $16 \mathrm{~h}$ at the temperature $\mathbf{T}$. Yields were determined by means of ${ }^{1} \mathrm{H} \mathrm{NMR}$ in $\mathrm{CDCl}_{3}$ of the crude material based with mesitylene internal standard. Ar = 4-tert-butylbenzyl.

The detrimental influence of water is further verified through entry 5 , in which 10 vol\% of water caused a diminished yield of $14 \%$. Water might also be the explanation, why stirring in an open vial without screw cap delivered substitution product 3 I in $44 \%$ yield (entry 8 ). That low temperatures are disadvantageous in terms of reaction velocity, is illustrated by an experiment at $0{ }^{\circ} \mathrm{C}$, which afforded $3 \mathrm{I}$ in just $13 \%$ yield (entry 10 ). These findings are illustrated clearly by 
the radar (Figure S4). A template for the radar diagram is available on the homepage of the Glorius group [6]

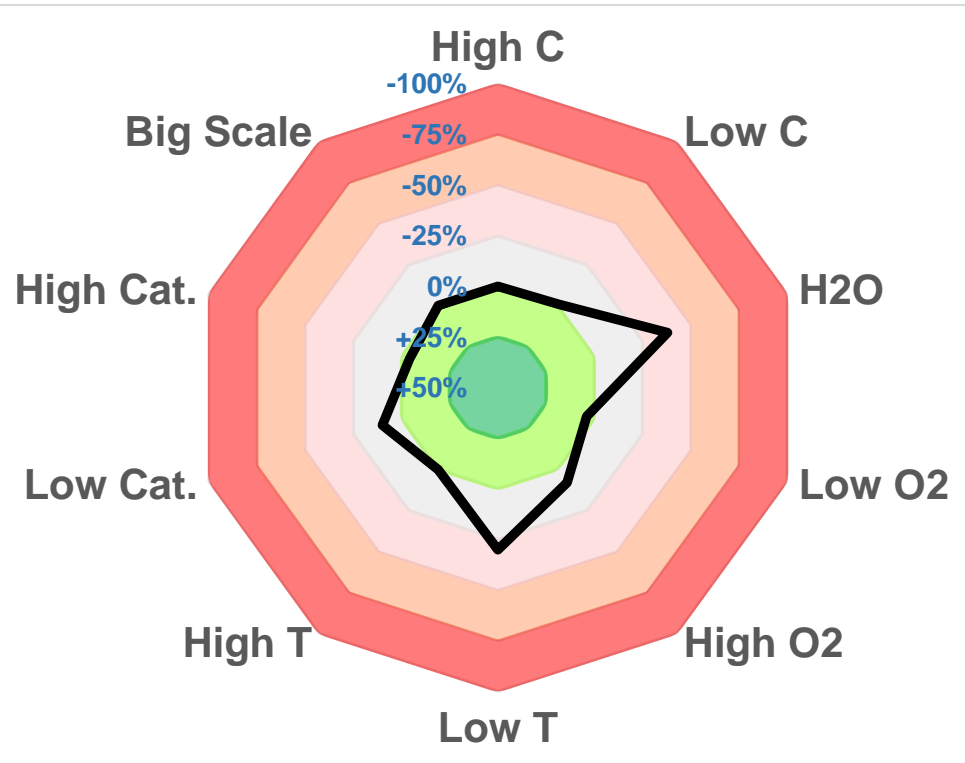

Figure S4. Radar diagram for the model transformation of benzyl alcohol $\mathbf{1 l}$ into chloride $\mathbf{3 l}$. 


\section{Experimental Part}

\subsection{General Conditions}

Unless otherwise stated all ${ }^{1} \mathrm{H},{ }^{19} \mathrm{~F}$ and ${ }^{13} \mathrm{C}$ spectra were recorded at room temperature in $\mathrm{CDCl}_{3}$ on a Bruker Avance I/ $400(400 \mathrm{MHz})$, Bruker Avance I $500(500 \mathrm{MHz})$, Bruker Fourier $300(300 \mathrm{MHz})$, Bruker Avance DRX (500 MHz) or Bruker Avance III $500(500 \mathrm{MHz})$ instruments. ${ }^{1} \mathrm{H}$ NMR spectra were measured with a delay time $\mathrm{d} 1=1.0 \mathrm{~s}$ between the scans. ${ }^{13} \mathrm{C}$ NMR spectra were recorded broadband proton decoupled. Chemical shifts $(\delta)$ are reported in parts per million (ppm) from tetramethylsilane (TMS) using the residual solvent resonance $\left({ }^{13} \mathrm{C} \mathrm{NMR}\right)$ or TMS $\left({ }^{1} \mathrm{H} \mathrm{NMR}\right)$ as the internal standard $\left(\mathrm{CDCl}_{3}: 7.26 \mathrm{ppm}\right.$ for ${ }^{1} \mathrm{H} \mathrm{NMR}, 77.0 \mathrm{ppm}$ or 77.16 ppm for ${ }^{13} \mathrm{C}$ NMR). ${ }^{[7]}$ Multiplicities are abbreviated as follows: $\mathrm{s}=$ singlet, br.s = broad singlet, $\mathrm{d}=$ doublet, $\mathrm{t}=$ triplet, $\mathrm{q}=$ quartet, sept $=$ septet, $\mathrm{m}=$ multiplet). ${ }^{11} \mathrm{~B}$ NMR spectra were recorded at room temperature in $\mathrm{CH}_{2} \mathrm{Cl}_{2}$ using a glass capillary containing $\mathrm{D}_{2} \mathrm{O}$ for locking on a Bruker Avance III 500 instrument (160 MHz), and were calibrated using an external standard $\left(\mathrm{BF}_{3} \cdot \mathrm{Et}_{2} \mathrm{O}: \delta\left({ }^{11} \mathrm{~B}\right)=0.00 \mathrm{ppm}\right)$.

IR spectra were measured at a Bruker Tensor 27 instrument or a Shimadzu IR Spirit instrument.

High resolution mass spectra were recorded either on a DFS High Resolution Magnetic Sector MS spectrometer of Thermo Fisher, on a MAT 95Q spectrometer (Cl) of Finnigan, on a Bruker SolariX 7 Tesla MALDI/ESI/APPI FTICR imaging spectrometer (ESI), or a Q-TOF Premier spectrometer of Waters (ESI).

Optical rotations were determined on a Perkin Elmer polarimeter 341 . Melting points (uncorrected) were determined on a MEL-TEMP II machine of Laboratory Devices Inc.

Analytical TLC was carried out using precoated silica gel plates (Fluka TLC plates silica gel $60 \mathrm{~F}_{254}$ on PET-foils or Merck TLC plates (Silica 60, $\mathrm{F}_{254}$ with fluorescence indicator)). TLC plates were visualized under UV irradiation $(254 \mathrm{~nm})$ or with $\mathrm{KMnO}_{4}\left(3 \mathrm{~g} \mathrm{KMnO}_{4}\right.$ and $20 \mathrm{~g}$ $\mathrm{K}_{2} \mathrm{CO}_{3}$ in $300 \mathrm{~mL}$ water) or $\mathrm{Ce}\left(\mathrm{SO}_{4}\right)_{2} / \mathrm{PMA}$ (= phosphomolybdic acid, $10 \mathrm{~g} \mathrm{Ce}\left(\mathrm{SO}_{4}\right)_{2} \cdot 4 \mathrm{H}_{2} \mathrm{O}$, $25 \mathrm{~g} \mathrm{PMA}, 80 \mathrm{~mL} 95 \% \mathrm{H}_{2} \mathrm{SO}_{4}, 920 \mathrm{~mL}$ water) stain solutions. Formamides were detected with iodine adsorbed at silica gel. Flash column chromatographic purifications were performed with technical grade silica gel M 60 from Macherey-Nagel (40-63 $\mu \mathrm{m}, 60 \mathrm{~A})$.

Gas chromatography (GC) was conducted on a GC-2010 from Shimadzu with a CPChirasil-DX CB column (length $25 \mathrm{~m}$, diameter $0.25 \mathrm{~mm}, 0.25 \mu \mathrm{m}$ layer thickness) from Agilent Technologies and a FS Supreme-5 MS column (length $25 \mathrm{~m}$, diameter $0.25 \mathrm{~mm}, 0.25 \mu \mathrm{m}$ layer thickness) from $E M R$, respectively, and nitrogen as carrier gas. Compounds were either detected by an FID (Chirasil-DX CB column) or a GCMS-QP2010 Plus mass detector from Shimadzu (Supreme $5 \mathrm{MS}$ column). The kinetic studies were performed on a Shimadzu GC-2010 Plus Series gas chromatograph with an OPTIMA-5 MS column (length: $15 \mathrm{~m}$, inner 
diameter: $0.25 \mathrm{~mm}$, film thickness: $0.25 \mu \mathrm{m}$ ) from Machery-Nagel, and with a flame ionization detector (GC parameters: injection volume $1 \mu \mathrm{L}$, injection temperature $250^{\circ} \mathrm{C}$, split ratio 1:20, start temperature $50^{\circ} \mathrm{C}$, hold time $2.0 \mathrm{~min}$, rate $20^{\circ} \mathrm{C} / \mathrm{min}$, final temperature $280^{\circ} \mathrm{C}$, hold time $1.5 \mathrm{~min}$ ). Helium was used as carrier gas. High pressure liquid chromatography (HPLC) was conducted on a D-7000 machine from Merck-Hitachi with a Chiracel OD-H column of Daicel Industries (length $250 \mathrm{~mm}$, diameter $4.6 \mathrm{~mm}$ ). Visualization was realized by a diode array UV detector (wavelength 190-300 nm).

Chemicals were purchased from Sigma-Aldrich, Acros, TCl chemicals, Carbolution and Alfa Aesar and used without further purification. Phenyl- $\lambda^{3}$-iodanediyl diacetate (PIDA) was synthesized according to a procedure of Kraszkiewicz. ${ }^{\left[{ }^{8]}\right.} \mathrm{B}\left(\mathrm{C}_{6} \mathrm{~F}_{5}\right)_{3}$ was synthesized according to a procedure of Czekelius. ${ }^{[9]}{ }^{13} \mathrm{C}$-enriched formic acid was obtained from Sigma-Aldrich (CAS [1633-56-3], isotopic purity 99 atom $\%{ }^{13} \mathrm{C}$, delivered in an ampulla), and stored under nitrogen in a Schlenk tube. Petrolether (=PE, bp. $40-60{ }^{\circ} \mathrm{C}$, technical grade) was distilled prior to usage; all other solvents were utilized without further purification. Dry solvents were purchased from Acros. Molecular sieves $(3 \AA)$ were activated heating at $200^{\circ} \mathrm{C}$ under high vacuum for at least $16 \mathrm{~h}$. THF and DMF were dried and stored over molecular sieves (3 $\AA$ ). $n$-Pentane for synthesis of $\mathrm{B}\left(\mathrm{C}_{6} \mathrm{~F}_{5}\right)_{3}$ was distilled $\left(\mathrm{CaH}_{2}\right)$ freshly under argon atmosphere. $\mathrm{CH}_{2} \mathrm{Cl}_{2}$ for NMR spectroscopic measurements was distilled $\left(\mathrm{CaH}_{2}\right)$ freshly under argon atmosphere.

Sterimol-Parameters were calculated according to the code of Verloop and Hoogenstraten. ${ }^{[10]}$

Bond lengths, bond angles and dihedral angles were determined from the energy-minimized structures with Spartan, Version 14. V1.1.0. The multivariate regression analysis was performed using JMP®, Version 13.1. SAS Institute Inc., Cary, NC, 1989-2013. xyz-Files were visualized with MERCURY 4.2.0. 


\subsection{General Procedures}

Commercial $\mathrm{N}$-formylpyrrolidine (FPyr) can be conveniently prepared from formic acid, $\mathrm{Ac}_{2} \mathrm{O}$ and pyrrolidine on a $2.00 \mathrm{~mol}$ scale using standard laboratory glassware $\leq 1 \mathrm{~L} .{ }^{[5]}$

\subsubsection{General Procedure 1: Formamide catalyzed Synthesis of 4-tert- Butylbenzyl Chloride (3I) ${ }^{[5]}$}

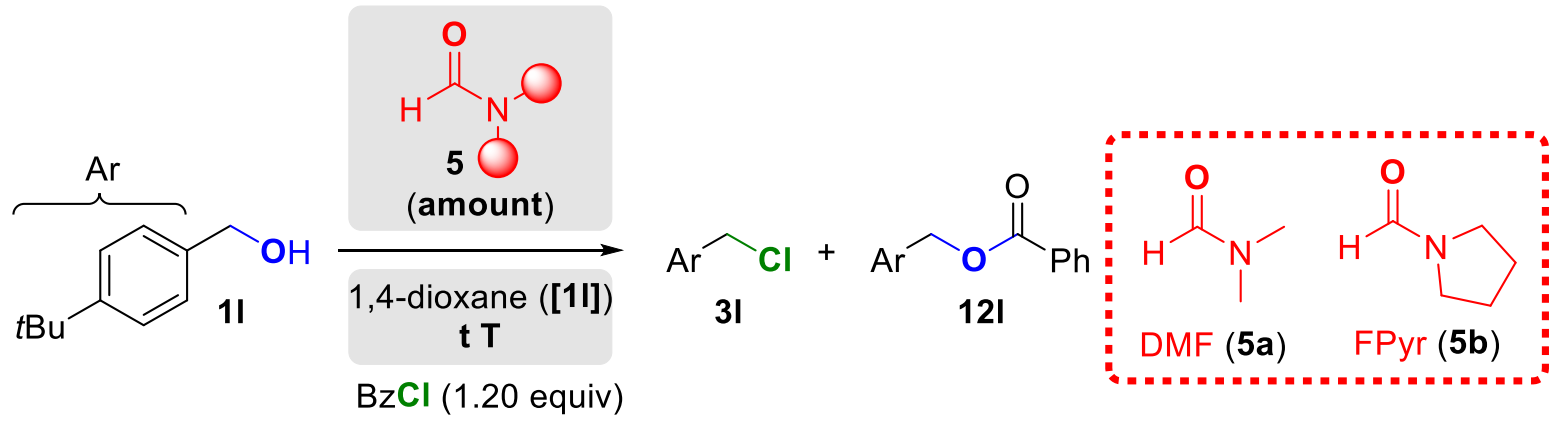

Reaction A $4 \mathrm{~mL}$ glass vial is charged with 4-tert-butylbenzyl alcohol (11, $89 \mu \mathrm{L}, 83 \mathrm{mg}$, $500 \mu \mathrm{mol}, 1.00$ equiv), formamide 5 (5-40 mol\%, 25-200 $\mu \mathrm{mol}$, mostly DMF or FPyr) and dry 1,4-dioxane (1.0-2.0 M). In the following, $\mathrm{BzCl}(70 \mu \mathrm{L}, 85 \mathrm{mg}, 600 \mu \mathrm{mol}, 1.20$ equiv) is added by means of an Eppendorf pipette at the reaction temperature $\mathbf{T}\left(<25^{\circ} \mathrm{C}\right)$ or at room temperature $\left(T \geq 25^{\circ} \mathrm{C}\right)$, the glass vial is closed with a screw cap with septum and the reaction mixture is stirred at $400 \mathrm{rpm}$ at the temperature $\mathbf{T}$ for the reaction time $\mathbf{t}$. In the case of reaction temperatures $\mathbf{T}>25^{\circ} \mathrm{C}$ an aluminum carousel is preheated to the reaction temperature. After addition of $\mathrm{BzCl}$, the reaction vial is placed into the carousel immediately.

\section{Work up}

The reaction mixture is diluted with $\mathrm{Et}_{2} \mathrm{O}(2 \mathrm{~mL})$ and taken up with a $20 \mathrm{~mL}$ syringe. $\mathrm{Next}$, the reaction vessel is rinsed with $2 \times \mathrm{Et}_{2} \mathrm{O}$ /aqueous $\mathrm{NaOH}$ solution $(1.0 \mathrm{M}, 2 \mathrm{~mL} / 2 \mathrm{~mL}$ ) and additional $\mathrm{Et}_{2} \mathrm{O}(2 \mathrm{~mL})$. The phases are separated with the aid of the $20 \mathrm{~mL}$ syringe, the organic phase is washed with brine $(1 \times 4 \mathrm{~mL})$, dried over $\mathrm{MgSO}_{4}$, filtered, concentrated under reduced pressure and the residue is dried for $5 \mathrm{~min}$ at the rotary evaporator either at $20 \mathrm{mbar}$ or at 50 mbar in the instance of volatile additives 15 in the robustness screen.

Determination of Yield

An exactly weighed amount of mesitylene $(20-40 \mathrm{mg})$ and $\mathrm{CDCl}_{3}$ (ca. $500 \mu \mathrm{L}$ ) are added to the crude material. Alternatively, the crude material is dissolved in a $0.5 \mathrm{M}$ stock solution of mesitylene (e.g., $301 \mathrm{mg}$ mesitylene in $5 \mathrm{~mL} \mathrm{CDCl}{ }_{3}$ ). Finally, a 50-100 $\mu \mathrm{l}$ aliquot of the resulting clear solution is transferred and diluted with further $\mathrm{CDCl}_{3}$ (ca. $500 \mu \mathrm{L}$ ) to an NMR tube.

Yields are determined from the ${ }^{1} \mathrm{H}$ NMR spectrum in reference to the singlets $(2 \mathrm{H})$ of benzylic alcohol $1 \mathrm{I}$ at $4.66 \mathrm{ppm}$, the chloride $\mathbf{3 l}$ at $4.57 \mathrm{ppm}$ and of benzoate $\mathbf{1 2}$ at $5.34 \mathrm{ppm}$ and the singlet $(9 \mathrm{H})$ of mesitylene at $2.27 \mathrm{ppm}$. 


\section{Robustness Screen}

(see chapter 1.1, page 1) ${ }^{[1-3]}$

The standard conditions were defined as follows: $0.2 \mathrm{M}$ FPyr in dry 1,4-dioxane (10 mol\%, $250 \mu \mathrm{L}),{ }^{(1)} 1.20$ equiv $\mathrm{BzCl}, \mathbf{t}=16 \mathrm{~h}, \mathbf{T}=$ room temperature. The NMR signals used for the determination of the recovery yield of the additives 15 are highlighted in bold in chapter 2.3.4 beginning on page 74 .

\subsubsection{General Procedure 2: Synthesis of Alkoxyiminium Chlorides (II) ${ }^{[5]}$}

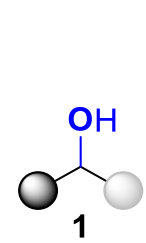

(aliphatic)

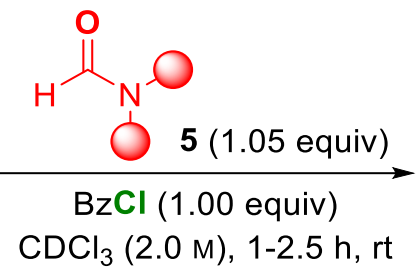

A $4 \mathrm{~mL}$ glass vial with a stirring bar is loaded with alcohol 1 (1.00 mmol, 1.00 equiv), a $2.0 \mathrm{M}$ stock solution of formamide 5 in $\mathrm{CDCl}_{3}(525 \mu \mathrm{L}, 1.05 \mathrm{mmol}, 1.05$ equiv, ca. $2.0 \mathrm{M}$ with respect to 1). ${ }^{(2)} \mathrm{Next}, \mathrm{BzCl}(117 \mu \mathrm{L}, 142 \mathrm{mg}, 1.00 \mathrm{mmol}, 1.05$ equiv) is added at room temperature under stirring $(400 \mathrm{rpm})$ and the resulting solution was stirred for the indicated time period (1-2.5 h). Then, a small aliquot of the reaction solution $(50 \mu \mathrm{L})$ was withdrawn using an Eppendorf pipette, diluted with further $\mathrm{CDCl}_{3}(500 \mu \mathrm{L})$, and immediately characterized by NMR. Both, longer and shorter reaction times resulted in lower conversions into II. After full characterization by means of NMR, another ${ }^{1} \mathrm{H} N M R$ is recorded to check the remaining amount of II, whereby typically only little decomposition ( $\leq 10 \%$ of II) was observed.

\subsubsection{General Procedure 3: Synthesis of Alkoxyiminium Hexafluorophosphates}

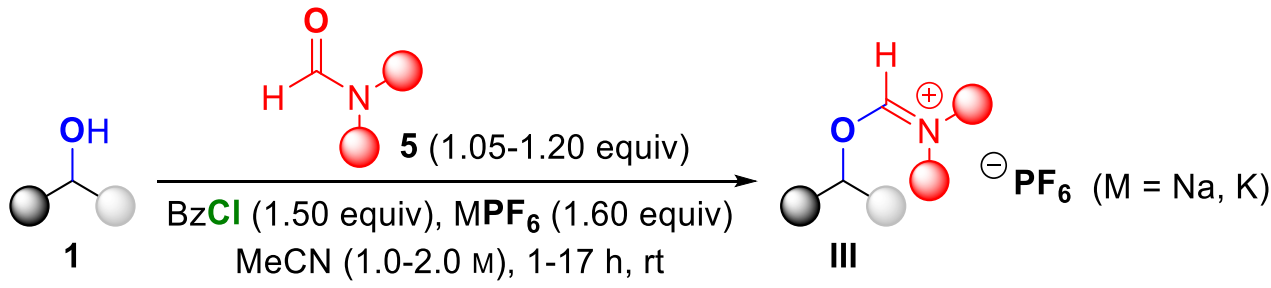

A $4 \mathrm{~mL}$ glass vial with a stirring bar was charged with either $\mathrm{NaPF}_{6}$ or $\mathrm{KPF}_{6}$ (134 mg or $147 \mathrm{mg}$, $0.80 \mathrm{mmol}, 1.60$ equiv), ${ }^{(3)}$ the alcohol 1 (500 $\mu \mathrm{mol}, 1.00$ equiv), the formamide 5 (525-

(1) Prepared from FPyr (39.3 $\mu \mathrm{L}, 40.8 \mathrm{mg}, 400 \mu \mathrm{mol})$ in $2 \mathrm{~mL}$ dioxane (dry).

(2) While FPyr, DMF and $\mathrm{N}$-methylformamide allowed for the preparation of iminium phosphates III, dimethylacetamide was less appropriate with conversions to type III species around $10 \%$.

(3) Sodium and potassium hexafluorophosphate allow for higher conversions to III than the respective tetrafluoroborate salts. In general, the respective sodium salt was found to be superior in the instance of reactive benzylic and allylic alcohols. Both, the use of $\mathrm{AgPF}_{6}$ and $\mathrm{NaBPh}_{4}$ resulted in lower amounts of III. 
$600 \mu \mathrm{mol}, 1.05-1.20$ equiv) and dry $\operatorname{MeCN}(250 / 500 \mu \mathrm{L}, 2.0 / 1.0 \mathrm{M}$, aliphatic/benzylic and allylic substrates 1). ${ }^{(4)} \mathrm{Next}, \mathrm{BzCl}$ ( $88 \mu \mathrm{L}, 107 \mathrm{mg}, 0.75 \mathrm{mmol}, 1.50$ equiv) is added at room temperature under stirring (600 rpm). After $1-17 \mathrm{~h}^{(5)}$ a $100 \mu \mathrm{L}$ aliquot is withdrawn by the means of an Eppendorf pipette ${ }^{(6)}$ from the reaction suspension, transferred to a $10 \mathrm{~mL}$ pear shaped flask and dried for $5 \mathrm{~min}$ at $20 \mathrm{mbar}$ at the rotary evaporator. The residue is taken up with $\mathrm{CHCl}_{3}(1 \mathrm{~mL})$, concentrated and dried for $5 \mathrm{~min}$ at $20 \mathrm{mbar}$ at the rotary evaporator to remove residual MeCN. As an alternative in the case of aliphatic starting materials $\mathbf{1}$, the residue can be dried in high vacuum for $30 \mathrm{~min}$. Eventually, the remaining solid is suspended in $\mathrm{CDCl}_{3}(500 \mu \mathrm{L})$ and the resulting suspension is filtered through a disposable glass pipette with a plug of wool and a ca. $5 \mathrm{~mm}$ layer of $\mathrm{MgSO}_{4}$ directly into an NMR-tube. After full characterization by means of NMR, another ${ }^{1} \mathrm{H}$ NMR is recorded to check the remaining amount of III, whereby typically only little decomposition ( $\leq 5 \%$ of III) was observed.

\subsubsection{General Procedure 4: Appel type Synthesis of 4-tert-Butylbenzyl Chloride ${ }^{[5]}$}
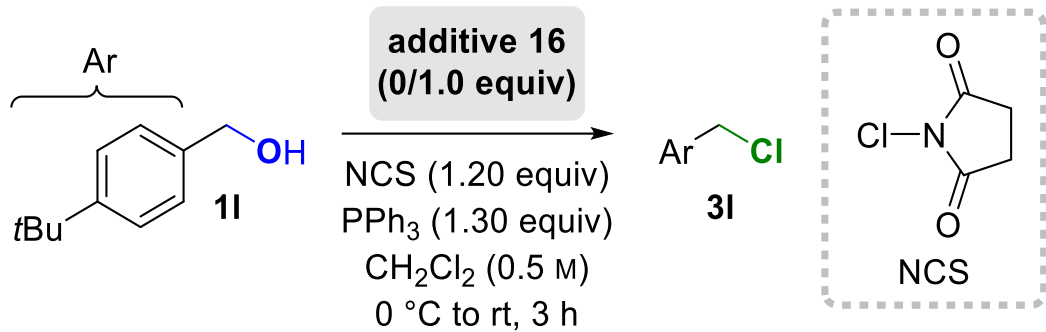

A $4 \mathrm{~mL}$ glass vial with a stir bar is charged with $\mathrm{PPh}_{3}(170 \mathrm{mg}, 0.65 \mathrm{mmol}, 1.30$ equiv), the respective additive $16(0-500 \mu \mathrm{mol}, 0$ or 1.00 equiv), the starting alcohol 11 (89 $\mu \mathrm{L}, 83 \mathrm{mg}$, $500 \mu \mathrm{mol}, 1.00$ equiv) and dry $\mathrm{CH}_{2} \mathrm{Cl}_{2}(1.0 \mathrm{~mL}, 0.5 \mathrm{M})$. The resulting solution is cooled in an ice bath and NCS ( $80 \mathrm{mg}, 600 \mu \mathrm{mol}, 1.30$ equiv) is added in one portion under stirring (600 rpm). After $15 \mathrm{~min}$ of stirring the cooling bath is removed and the reaction mixture is allowed to stir for $3 \mathrm{~h}$ at ambient temperature.

Next, the reaction solution is transferred to a $10 \mathrm{~mL}$ flask with $\mathrm{CH}_{2} \mathrm{Cl}_{2}(2 \mathrm{~mL})$, the reaction vial is rinsed with $\mathrm{CH}_{2} \mathrm{Cl}_{2}(2 \times 2 \mathrm{~mL})$, the collected $\mathrm{CH}_{2} \mathrm{Cl}_{2}$ phases are concentrated and dried at the rotary evaporator for $5 \mathrm{~min}$ at $50 \mathrm{mbar}$. The yield is determined with mesitylene as

(4) The preparation of salts III is also possible in acetone, albeit conversions to III are lower presumably due to a higher amount of water in the solvent. $\mathrm{CDCl}_{3}$ is less feasible, likely because of low solubility of $\mathrm{NaPF}_{6}$ and $\mathrm{KPF}_{6}$.

(5) In the case of activated benzylic and allylic alcohols short reaction durations around $1 \mathrm{~h}$ enable the highest conversions to iminium salts III. With less reactive aliphatic alcohols longer reaction times $\geq 3 \mathrm{~h}$ allow for the best results.

(6) A part of the plastic tip for the Eppendorf pipette was cut off to increase the opening diameter. This avoids cloaking of the tip through the solid precipitate of the reaction mixture $(\mathrm{NaCl} / \mathrm{KCl})$. 
internal NMR standard as delineated in general procedure 1 (chapter 2.2.1 on page 14). After addition of mesitylene and $\mathrm{CDCl}_{3}$ to the crude product, the mixture is carefully heated with a heat gun to obtain a clear solution.

\subsubsection{General Procedure 5: Synthesis of 4-tert-Butylbenzyl Chloride with} Thionylchloride

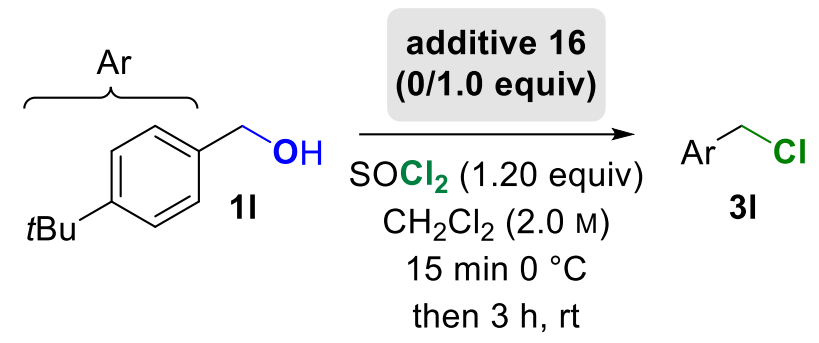

A $4 \mathrm{~mL}$ glass vial is charged with the additive (0/1.0 equiv), 4-tert-butylbenzylalcohol (89 $\mu \mathrm{L}$, $83 \mathrm{mg}, 500 \mu \mathrm{mol}, 1.00$ equiv), dry $\mathrm{CH}_{2} \mathrm{Cl}_{2}(250 \mu \mathrm{L}, 2.0 \mathrm{M})$ and is cooled in an ice bath. Next, thionylchloride ( $44 \mu \mathrm{L}, 600 \mu \mathrm{mol}, 1.20$ equiv) is added dropwise under stirring ( $400 \mathrm{rpm}$ ) with the aid of $50 \mu \mathrm{L}$ Hamilton syringe, whereby a gas evolution can typically be observed. The reaction vessel is closed with a screw cap with septum and stirred for 15 min at $0{ }^{\circ} \mathrm{C}$ and after removal of the ice bath for $3 \mathrm{~h}$ at room temperature.

Non-aqueous work up: Next, the reaction vial is opened carefully, whereat a gas evolution occurs, the reaction mixture is transferred to a $10 \mathrm{~mL}$ flask with $\mathrm{CH}_{2} \mathrm{Cl}_{2}(2 \mathrm{~mL})$, the reaction vial is rinsed with $\mathrm{CH}_{2} \mathrm{Cl}_{2}(2 \times 2 \mathrm{~mL})$, the collected $\mathrm{CH}_{2} \mathrm{Cl}_{2}$ phases are concentrated and dried at the rotary evaporator for $5 \mathrm{~min}$ at $50 \mathrm{mbar}$. The yield is determined with mesitylene as internal NMR standard as delineated in general procedure 1 (chapter 2.2.1 on page 14).

Aqueous work up: This work up procedure was applied in the case of basic additives such as 4-tert-butylpyridine (16p), benzothiazol (16o), and 4-tert-butylaniline (16m). With $\mathrm{SOCl}_{2}$ one equivalent of $\mathrm{HCl}$ is formed, which protonates these bases. The ${ }^{1} \mathrm{H} N M R$ spectra of the respective hydrochloride salts differ from their neutral forms.

The reaction vial is opened carefully, whereat a gas evolution occurs, $\mathrm{CH}_{2} \mathrm{Cl}_{2}(2 \mathrm{~mL})$ and $2 \mathrm{~N} \mathrm{NaOH}$ solution in water is added $(1 \mathrm{~mL})$ and the mixture is taken up with a $20 \mathrm{~mL}$ syringe. The reaction vial is rinsed with additional $\mathrm{CH}_{2} \mathrm{Cl}_{2} / 2 \mathrm{~N} \mathrm{NaOH}$ solution $(1 \times 2 \mathrm{~mL} / 1 \mathrm{~mL})$, the phases are separate with the aid of the syringe, the organic phase is dried over $\mathrm{MgSO}_{4}$, concentrated and dried at the rotary evaporator for $5 \mathrm{~min}$ at $50 \mathrm{mbar}$. The yields are determined as described above. 


\subsubsection{General Procedure 6: Synthesis of Formamides 5 via PIDA-catalyzed} amidation

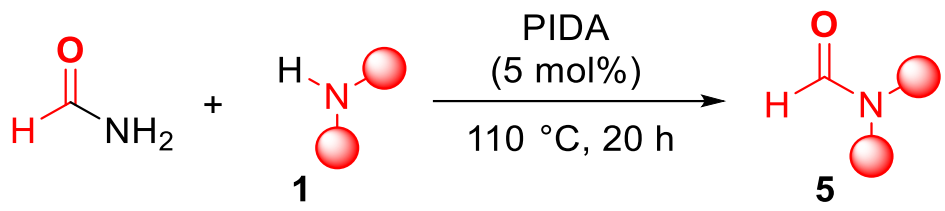

1.0 equiv $\quad 1.0$ equiv

Reaction According to a procedure of Singh, ${ }^{[11]}$ a Schlenk flask is charged with amine (1.00 equiv) and formamide (1.00 equiv) under argon atmosphere. Then, PIDA ( $5 \mathrm{~mol} \%$ ) is added and the reaction mixture is stirred at $110^{\circ} \mathrm{C}$ for $20 \mathrm{~h}$.

\section{Work up}

After cooling to room temperature, water is added carefully. The phases are separated, and the aqueous layer is extracted with EtOAc (15-20 times). Then, the combined organic layers are dried $\left(\mathrm{MgSO}_{4}\right)$, filtered, and concentrated under reduced pressure.

Chromatographic Purification

Eventually, the crude product is purified by filtering through a small plug of silica gel. 


\subsection{Experimental Procedures and Analytical Data}

\subsubsection{Synthesis of Alkoxyiminium Chlorides II (Scheme 3 A)}

The synthesis and analytical data of the following compounds have been included in our previous publication, ${ }^{[5]}$ of which the supporting information are available free of charge at https://onlinelibrary.wiley.com/doi/full/10.1002/anie.201604921:

$N$-((2-Ethylhexyloxy)methylidene) pyrrolidinium chloride (Ila)

$N$-((2-Ethylhexyloxy)methylidene) dimethyliminium chloride (IIb)

$N$-((2-Ethylhexyloxy)methylidene) methyliminium chloride (Ilc)

$\mathrm{N}$-((1-Octyloxy)methylidene) dimethyliminium chloride (IId)

rac- $N$-((4-Phenylbut-2-yloxy)methylidene) dimethyliminium chloride (IIg)

$N-(($ Cyclohexyloxy)methylidene) dimethyliminium chloride (IIh)

In this reference the protocols for the transformation of lle (86\% conversion) into 1-chlorododecane (3e) und dodecyl formate (13e) are given, too.

\subsubsection{Synthesis of $\boldsymbol{N}$-((1-Dodecyloxy)methylidene) dimethyliminium chloride (lle)}

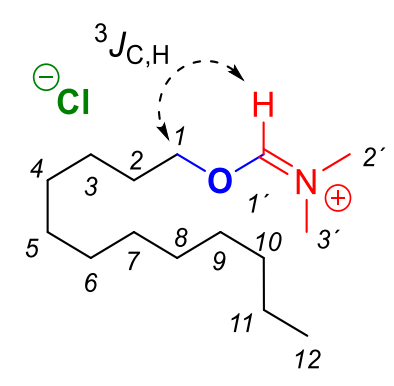

Ile

PH4284: According to general procedure 2 (chapter 2.2.2, page 15) the title compound was prepared form 1-dodecanol $(225 \mu \mathrm{L}$, $188 \mathrm{mg}, 1.00 \mathrm{mmol}, 1.00$ equiv), a $2.0 \mathrm{M}$ solution of DMF in $\mathrm{CDCl}_{3}$ (525 $\mu \mathrm{L}, 1.05 \mathrm{mmol}, 1.05$ equiv) and $\mathrm{BzCl}$ (1.00 equiv). A sample withdrawn after $1 \mathrm{~h}$ showed a 72:7:2:12:7 mixture of lle with respect to dodecyl benzoate, dodecyl formate, 1-dodecanol and 1-dodecyl chloride according to ${ }^{1} \mathrm{H}$ NMR.

$\mathbf{M}\left(\mathrm{C}_{15} \mathrm{H}_{32} \mathrm{CINO}\right)=277.88 \mathrm{~g} / \mathrm{mol} ;{ }^{1} \mathrm{H}$ NMR $\left(500 \mathrm{MHz}, \mathrm{CDCl}_{3}\right) \delta[\mathrm{ppm}]=10.36\left(\mathrm{~s}, 1 \mathrm{H}, 1^{\prime}-\mathrm{H}\right), 4.88$ $\left(\mathrm{t},{ }^{3} J_{1,2}=6.7 \mathrm{~Hz}, 2 \mathrm{H}, 1-\mathrm{H}\right), 3.62\left(\mathrm{~s}, 3 \mathrm{H}, 2^{\prime}-\mathrm{H}\right), 3.22\left(\mathrm{~s}, 3 \mathrm{H}, 3^{\prime}-\mathrm{H}\right), 1.88-1.83(\mathrm{~m}, 2 \mathrm{H}, 2-\mathrm{H}), 1.40-$ $1.35(\mathrm{~m}, 2 \mathrm{H}, 3-\mathrm{H}), 1.31-1.23(\mathrm{~m}, 16 \mathrm{H}, 4-\mathrm{H}$ to $11-\mathrm{H}), 0.89-0.87(\mathrm{~m}, 3 \mathrm{H}, 12-\mathrm{H}) ;{ }^{13} \mathrm{C}$ NMR $\left(125 \mathrm{MHz}, \mathrm{CDCl}_{3}\right) \delta[\mathrm{ppm}]=168.93\left(\mathrm{C}-1^{\prime}\right), 79.47$ (C-1), 41.55 (C-2'), 35.99 (C-3'), 31.78 (C-10 or C-11), 29.48, 29.38, 29.27, 29.21, 29.16 (C-4 to C-9), 28.96 (C-2), 25.04 (C-3), 22.56 (C-10 or C-11), 14.00 (C-12). 


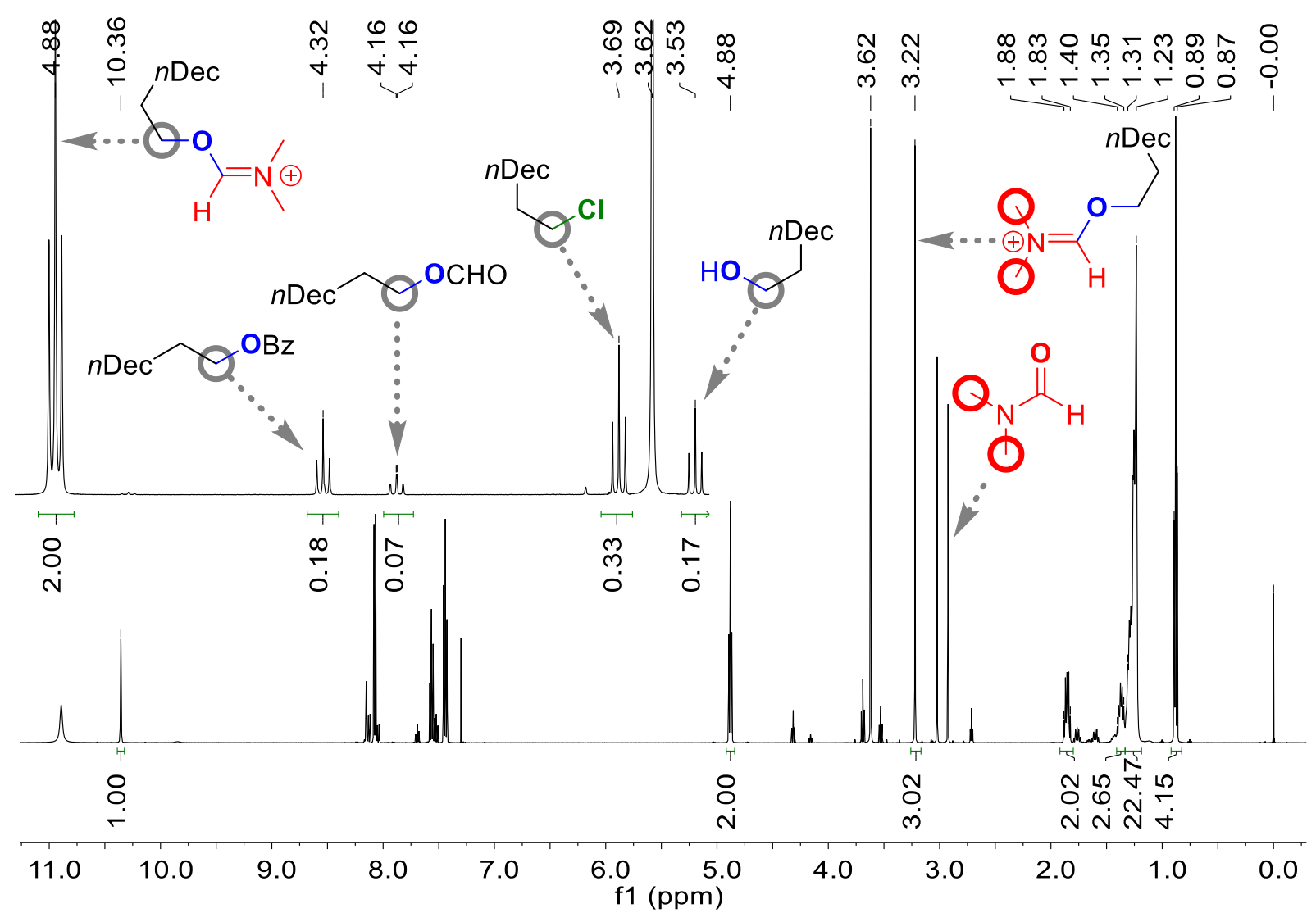

${ }^{1} \mathrm{H}$ NMR spectrum of $\mathrm{N}$-((1-dodecyloxy)methylidene) dimethyliminium chloride $(400 \mathrm{MHz}$, $\mathrm{CDCl}_{3}$ ).

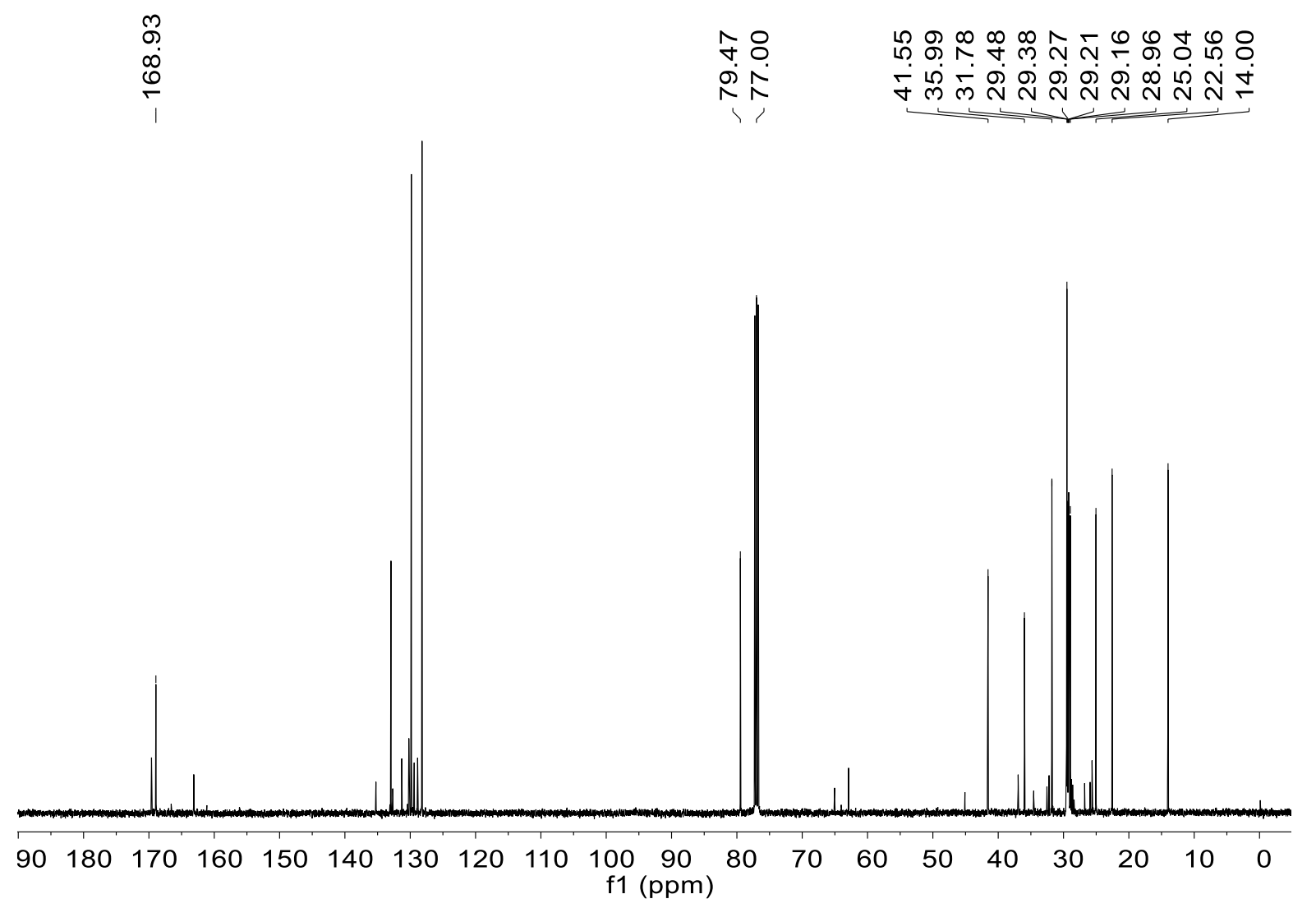

${ }^{13} \mathrm{C}$ NMR spectrum of $\mathrm{N}-((1$-dodecyloxy)methylidene) dimethyliminium chloride $(100 \mathrm{MHz}$, $\mathrm{CDCl}_{3}$ ). 


\subsubsection{Synthesis of rac $N$-((2-Octyloxy)methylidene) dimethyliminium chloride (IIf)}

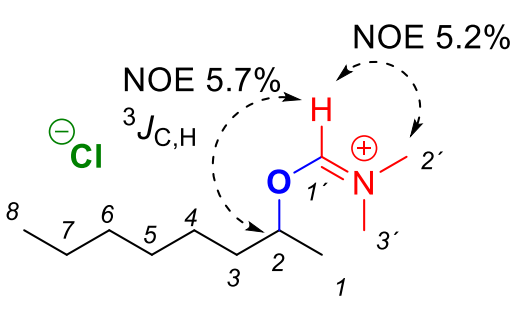

IIf

PH1123: According to general procedure 2 (chapter 2.2.2, page 15) racemic 2-octanol (166 $\mu \mathrm{L}, 134 \mathrm{mg}, 1.00 \mathrm{mmol}$, 1.00 equiv) was combined with a $2.0 \mathrm{M}$ solution of DMF in $\mathrm{CDCl}_{3}(525 \mu \mathrm{L}, 1.05 \mathrm{mmol}, 1.05$ equiv), which had been prepared from $155 \mu \mathrm{L} / 146 \mathrm{mg} \mathrm{DMF}$ and $1 \mathrm{~mL}$ of $\mathrm{CDCl}_{3}$, and $\mathrm{BzCl}$ (117 $\mu \mathrm{L}, 142 \mathrm{mg}, 1.00 \mathrm{mmol}, 1.05$ equiv). After $2.5 \mathrm{~h}$ of stirring, ${ }^{1} \mathrm{H}$ NMR showed a composition of Ilf and 2-octanol in a ratio of 83:17.

$\mathbf{M}\left(\mathrm{C}_{11} \mathrm{H}_{24} \mathrm{CINO}\right)=221.77 \mathrm{~g} / \mathrm{mol} ;{ }^{1} \mathbf{H}$ NMR $\left(400 \mathrm{MHz}, \mathrm{CDCl}_{3}\right) \delta[\mathrm{ppm}]=10.64\left(\mathrm{~s}, 1 \mathrm{H}, 1^{\prime}-\mathrm{H}\right)$, 5.41-5.33 (m, 1H, 2-H), $3.64\left(\mathrm{~s}, 3 \mathrm{H}, 2^{\prime}-\mathrm{H}\right), 3.21\left(\mathrm{~s}, 3 \mathrm{H}, 3^{\prime}-\mathrm{H}\right), 1.88-1.79\left(\mathrm{~m}, 1 \mathrm{H}, 3-\mathrm{H}_{\mathrm{a}}\right), 1.76-$ $1.67\left(\mathrm{~m}, 1 \mathrm{H}, 3-\mathrm{H}_{\mathrm{b}}\right), 1.54\left(\mathrm{~d},{ }^{3} \mathrm{~J}_{1,2}=6.3 \mathrm{~Hz}, 3 \mathrm{H}, 1-\mathrm{H}\right), 1.38-1.23(\mathrm{~m}, 8 \mathrm{H}, 4-\mathrm{H}$ to $7-\mathrm{H}) 0.87-0.83$ $(\mathrm{m}, 3 \mathrm{H}, 8-\mathrm{H}) ;^{\prime 13} \mathrm{C}$ NMR (100 MHz, $\left.\mathrm{CDCl}_{3}\right) \delta[\mathrm{ppm}]=168.51\left(\mathrm{C}-1^{\prime}\right), 89.21$ (C-2), 41.57 (C-2'), 36.06 (C-3'), 35.93 (C-3), 31.43 (C-6 or C-7), 28.86 (C-5), 24.68 (C-4), 22.39 (C-6 or C-7), $20.70(\mathrm{C}-1), 13.90(\mathrm{C}-8)$.

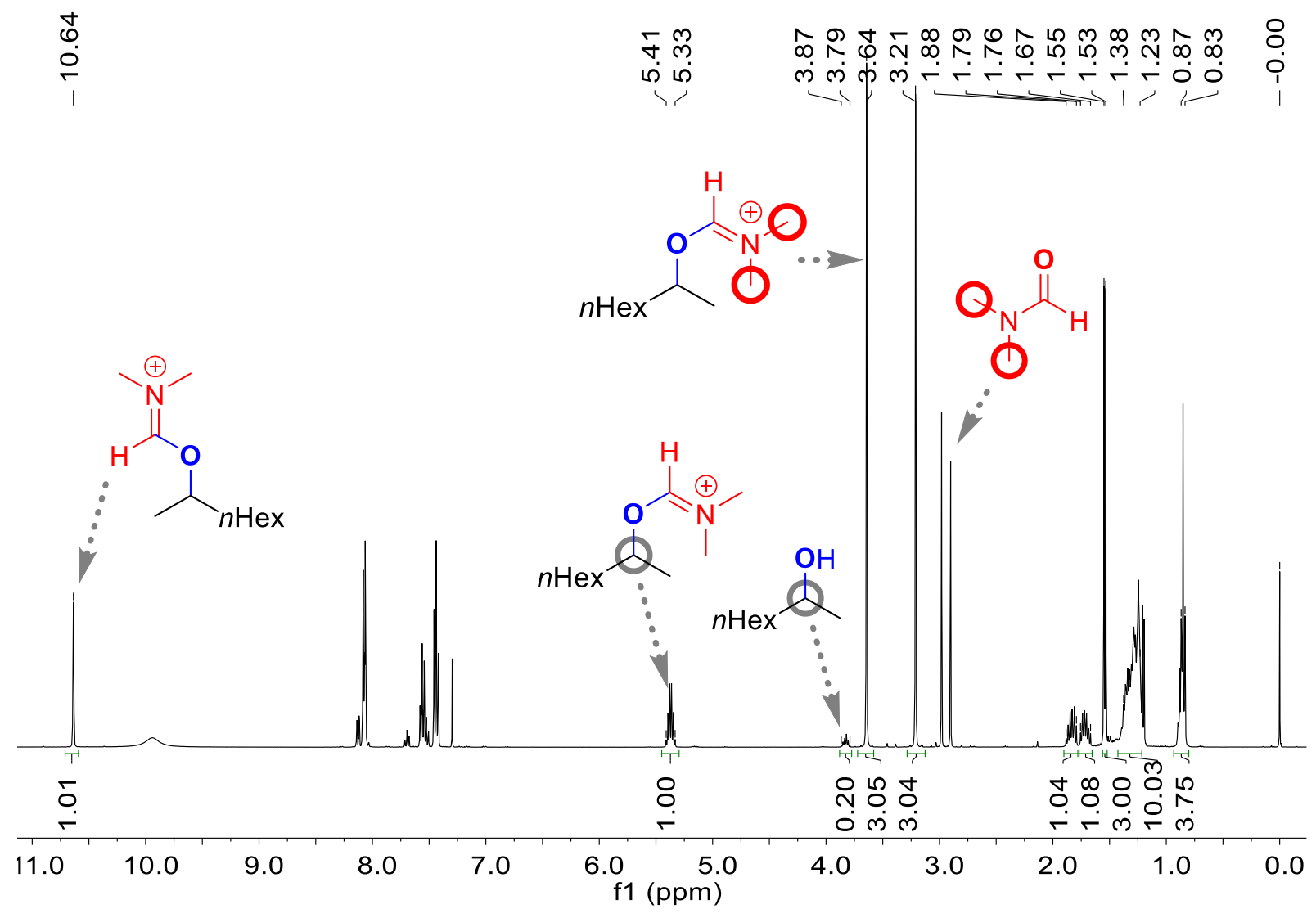

${ }^{1} \mathrm{H}$ NMR spectrum of rac $\mathrm{N}$-((2-octyloxy)methylidene) dimethyliminium chloride $(400 \mathrm{MHz}$, $\left.\mathrm{CDCl}_{3}\right)$. 


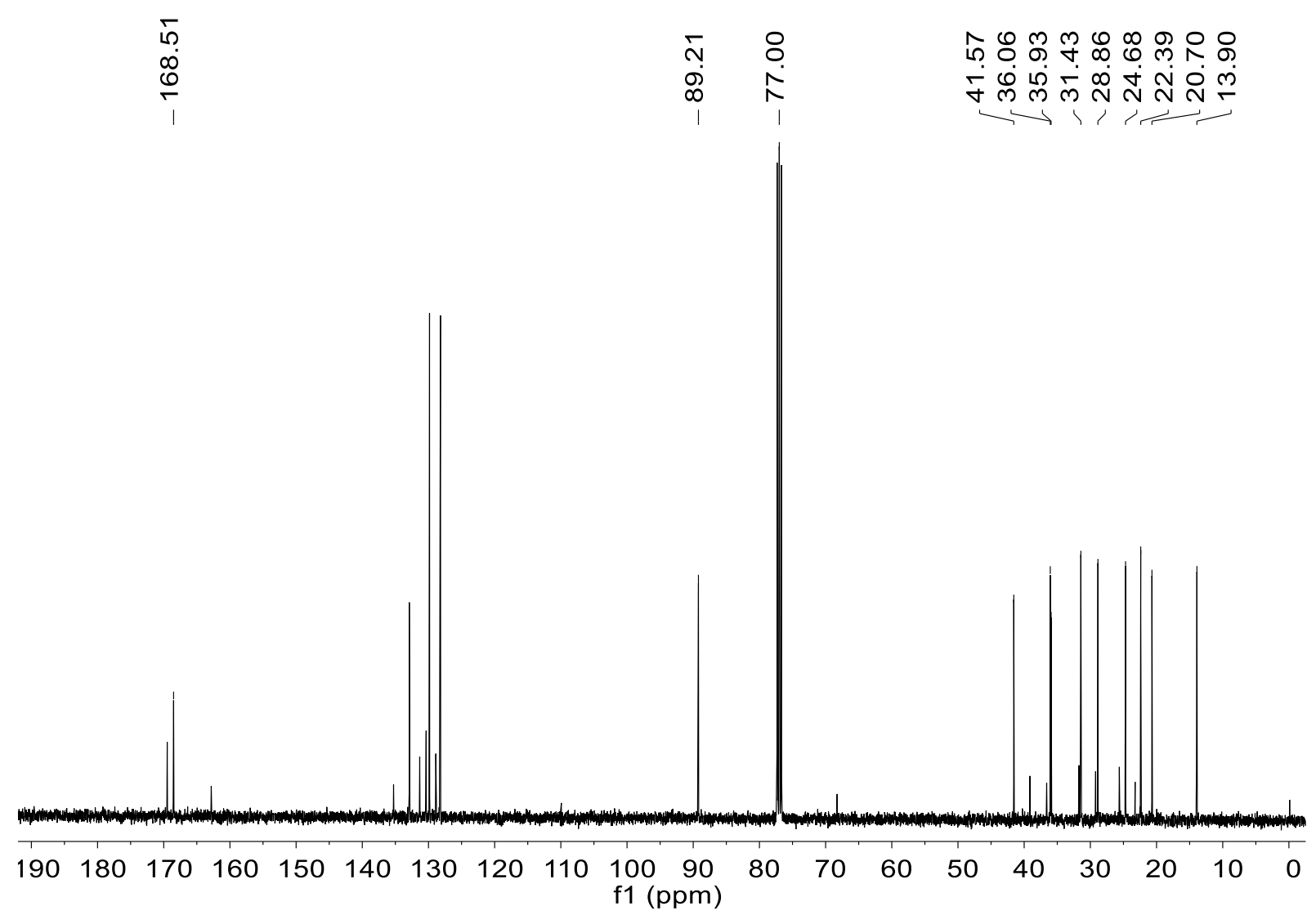

${ }^{13} \mathrm{C}$ NMR spectrum of rac $\mathrm{N}$-((2-octyloxy)methylidene) dimethyliminium chloride $(100 \mathrm{MHz}$, $\left.\mathrm{CDCl}_{3}\right)$.

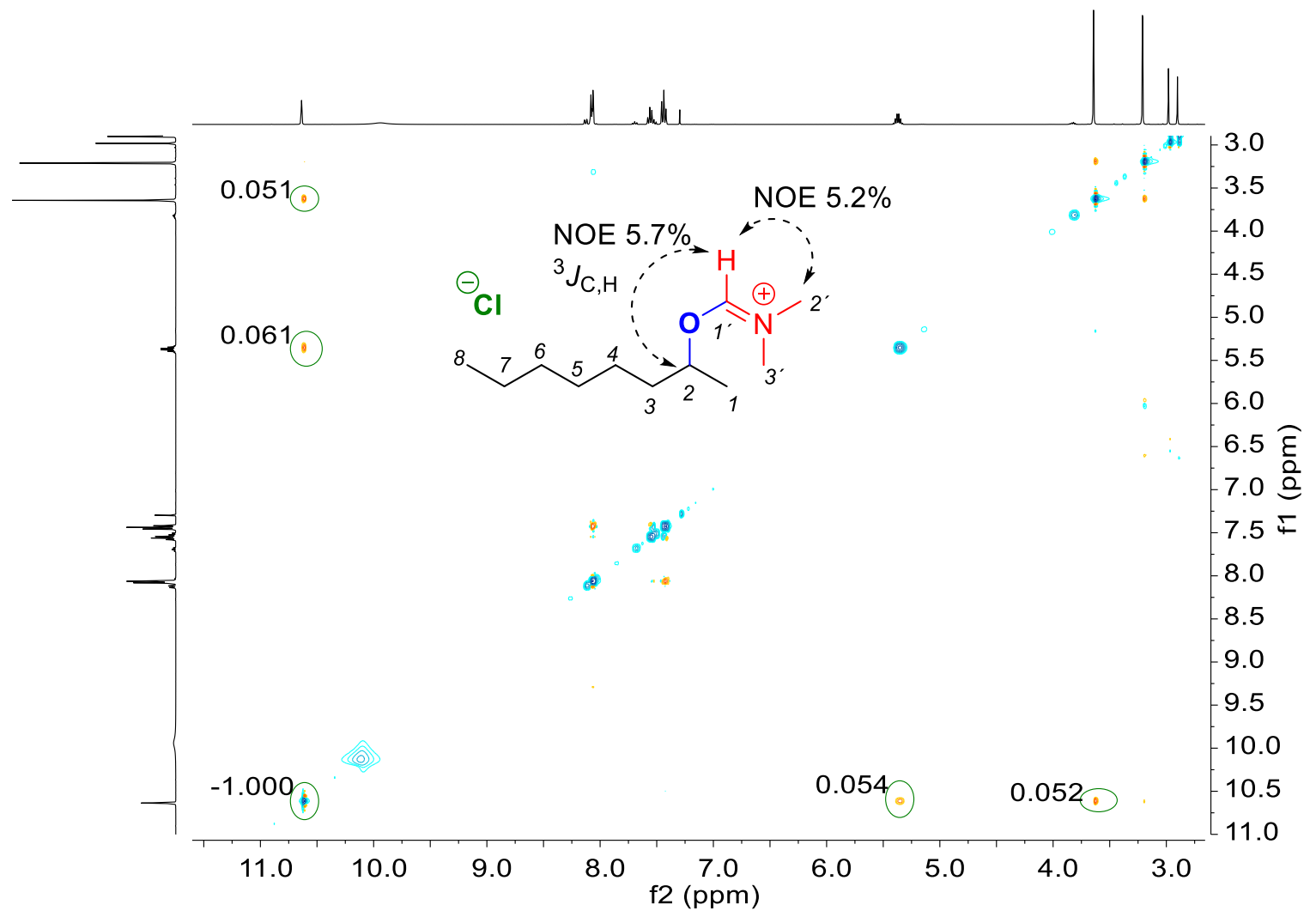

NOESY spectrum of rac $\mathrm{N}$-((2-octyloxy)methylidene) dimethyliminium chloride $(400 \mathrm{MHz}$, $\left.\mathrm{CDCl}_{3}\right)$. 


\subsubsection{Synthesis of Alkoxyiminium Hexafluorophosphates III (Scheme 3 B)}

Analytical data for 1-dodecyl chloride, benzoate, and formate, 4-tert-butylbenzyl chloride, benzoate, and formate, E-2-octenyl-1-chloride, and 2-chloro-4-phenyl butane, and FPyr are included in our previous publication ${ }^{[5]}$ in the supporting information. The following additional analytical data can be found in:

1-dodecanol in chapter 2.3.6.2 (page 91)

4-tert-butylbenzyl alcohol in chapter 2.3.6.4 (page 100)

E-2-octen-1-ol, 4-phenyl-2-butanol and DMF in chapter 2.3.2.8 (page 46)

\subsubsection{Synthesis of $N$-((1-Dodecyloxy)methylidene) pyrrolidinium hexafluoro-} phosphate (IIIi)

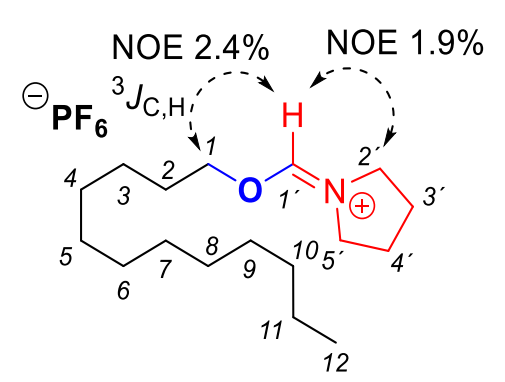

IIIi

PH1954: According to general procedure 3 (chapter 2.2.3, page 15), $\mathrm{KPF}_{6}$ (1.60 equiv), 1-dodecanol (113 $\mu \mathrm{L}, 94 \mathrm{mg}$, $500 \mu \mathrm{mol}, 1.00$ equiv), FPyr $(59 \mu \mathrm{L}, 61 \mathrm{mg}, 0.60 \mathrm{mmol}$, 1.20 equiv), $\mathrm{MeCN}(2.0 \mathrm{M})$ and $\mathrm{BzCl}$ (1.50 equiv) were combined. After $5.5 \mathrm{~h}$ of stirring, ${ }^{1} \mathrm{H}$ NMR verified full conversion to iminium species Illi.

M $\left(\mathrm{C}_{17} \mathrm{H}_{34} \mathrm{~F}_{6} \mathrm{NOP}\right)=413.43 \mathrm{~g} / \mathrm{mol} ;{ }^{1} \mathrm{H}$ NMR $\left(400 \mathrm{MHz}, \mathrm{CDCl}_{3}\right)$ $\delta[\mathrm{ppm}]=8.55\left(\mathrm{~s}, 1 \mathrm{H}, 1^{\prime}-\mathrm{H}\right), 4.64\left(\mathrm{t},{ }^{3} \mathrm{~J}_{1,2}=7.2 \mathrm{~Hz}, 2 \mathrm{H}, 1-\mathrm{H}\right), 4.00-3.97\left(\mathrm{~m}, 2 \mathrm{H}, 2^{\prime}-\mathrm{H}\right), 3.68-3.65$ $\left(\mathrm{m}, 2 \mathrm{H}, 5^{\prime}-\mathrm{H}\right), 2.13-2.05\left(\mathrm{~m}, 4 \mathrm{H}, 3^{\prime}-\mathrm{H}, 4^{\prime}-\mathrm{H}\right), 1.86-1.79\left(\mathrm{~m} \mathrm{2H}, 2^{\prime}-\mathrm{H}\right), 1.38-1.25(\mathrm{~m}, 18 \mathrm{H}, 3-\mathrm{H}$ to $11-\mathrm{H}), 0.90-0.86(\mathrm{~m}, 3 \mathrm{H}, 12-\mathrm{H}) ;{ }^{19} \mathrm{~F}$ NMR $\left(376 \mathrm{MHz}, \mathrm{CDCl}_{3} \delta[\mathrm{ppm}]=-72.62\left(\mathrm{~d},{ }^{1} \mathrm{~J}_{\mathrm{F}, \mathrm{P}}=\right.\right.$ $714 \mathrm{~Hz}$ ); ${ }^{31} \mathbf{P}$ NMR $\left(162 \mathrm{MHz}, \mathrm{CDCl}_{3} \delta[\mathrm{ppm}]=-144.36\right.$ (sept, ${ }^{1} \mathrm{~J}_{\mathrm{P}, \mathrm{F}}=713 \mathrm{~Hz}$ ); ${ }^{13} \mathbf{C} \mathbf{~ N M R}$ $\left(100 \mathrm{MHz}, \mathrm{CDCl}_{3}\right) \delta[\mathrm{ppm}]=164.51\left(\mathrm{C}-1^{\prime}\right), 79.65$ (C-1), 51.16 (C-2'), 47.85 (C-5'), 31.85 (C-10 or C-11), 29.57, 29.56, 29.48, 29.35, 29.29 (C-4 to C-9), 28.99 (C-2), 25.06 (C-3), 24.37 (C-3'), 23.84 (C-4'), 22.63 (C-10 or C-11), 14.05 (C-12').

As apparent from the ${ }^{1} \mathrm{H}$ NMR spectrum of IIli below, 1-dodecyl chloride (3i) and 1-dodecanol (1i) show no distinct multiplets. However, a ratio of the t at $4.64 \mathrm{ppm}(1-\mathrm{H}$ of $\mathrm{Illi})$ and the multiplet at 0.90-0.86 ppm (12-H of all products and substrate 1i) of 2.01:3.00 verifies that both compounds are basically not present. As consequence, the conversion of $\mathbf{1 i}$ to Illi is $\geq 98 \%$. 


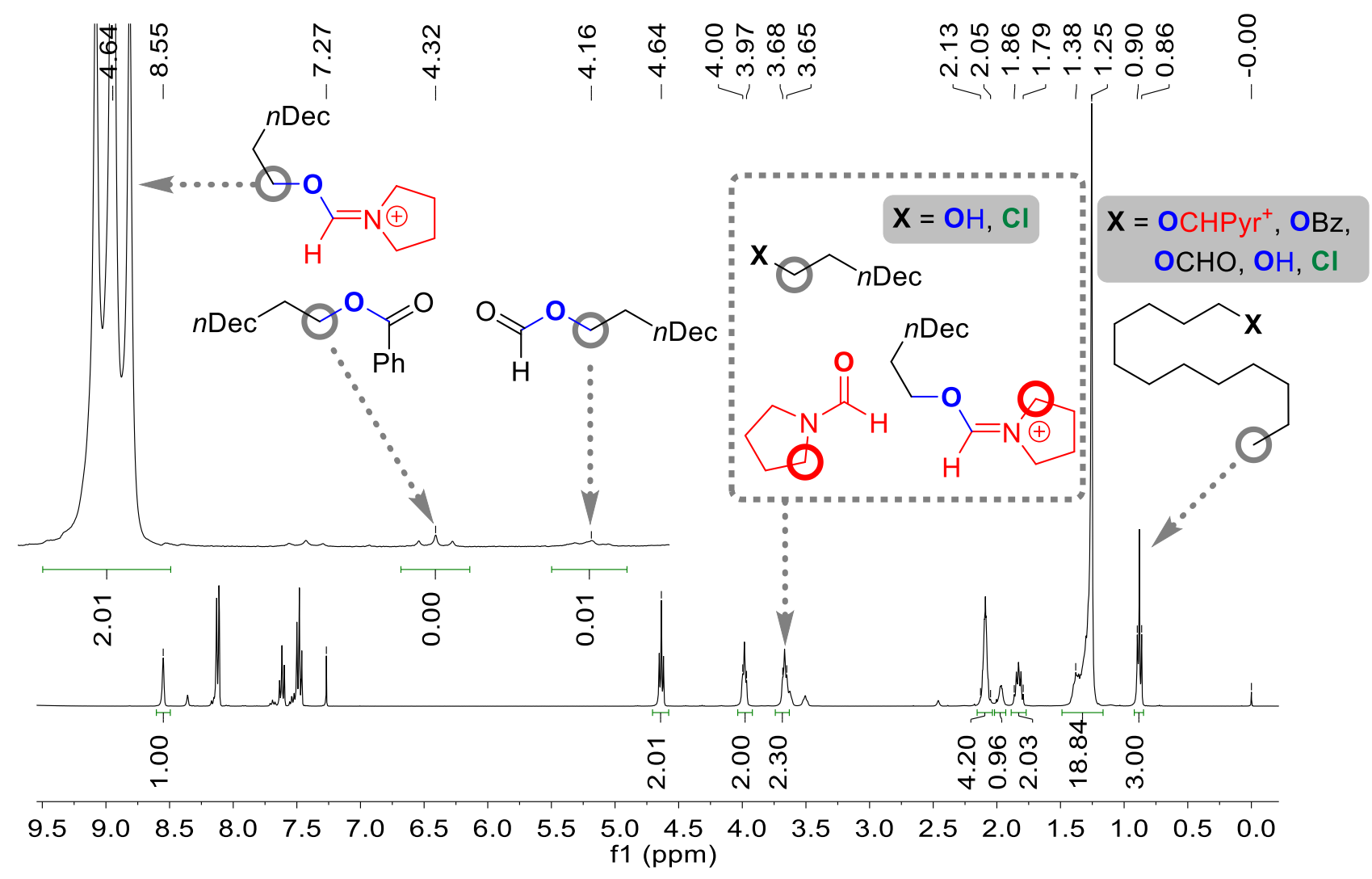

${ }^{1} \mathrm{H}$ NMR spectrum of $\mathrm{N}$-((1-dodecyloxy)methylidene) pyrrolidinium hexafluorophosphate (400 MHz, $\left.\mathrm{CDCl}_{3}\right)$.

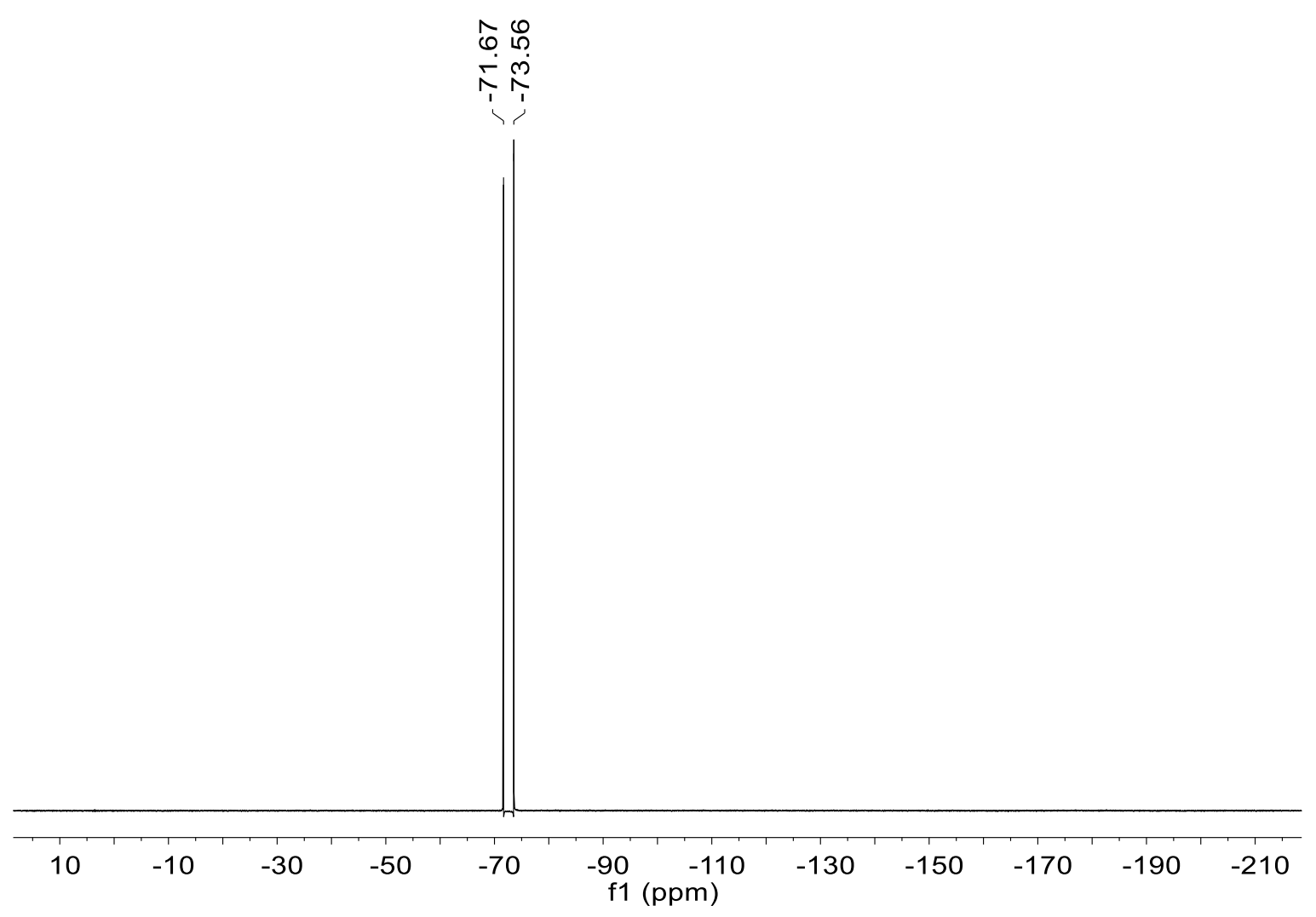

${ }^{19} \mathrm{~F}$ NMR spectrum of $\mathrm{N}$-((1-dodecyloxy)methylidene) pyrrolidinium hexafluorophosphate (376 $\left.\mathrm{MHz}, \mathrm{CDCl}_{3}\right)$. 


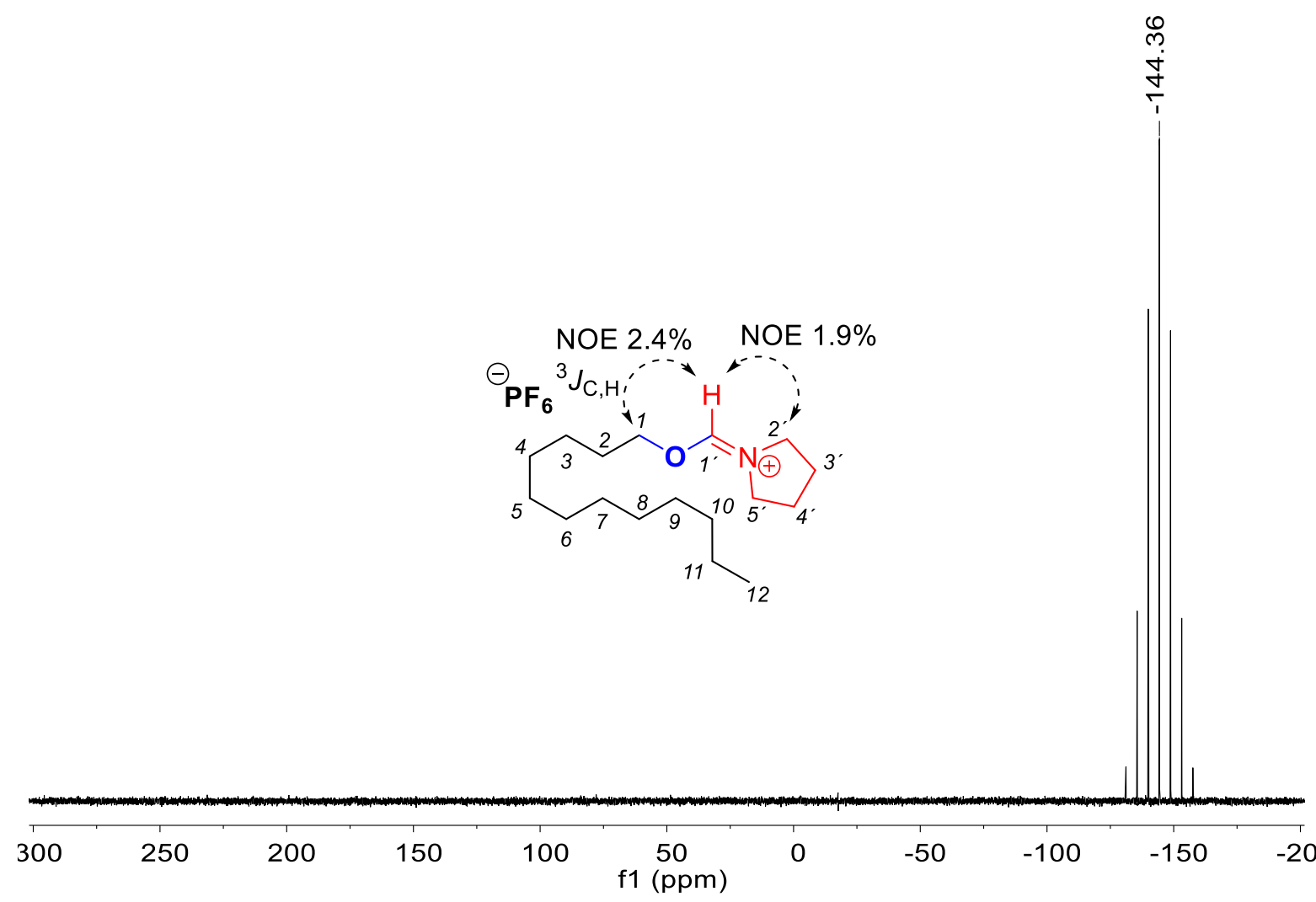

${ }^{31} \mathrm{P}$ NMR spectrum of $\mathrm{N}$-((1-dodecyloxy)methylidene) pyrrolidinium hexafluorophosphate (162 $\left.\mathrm{MHz}, \mathrm{CDCl}_{3}\right)$.

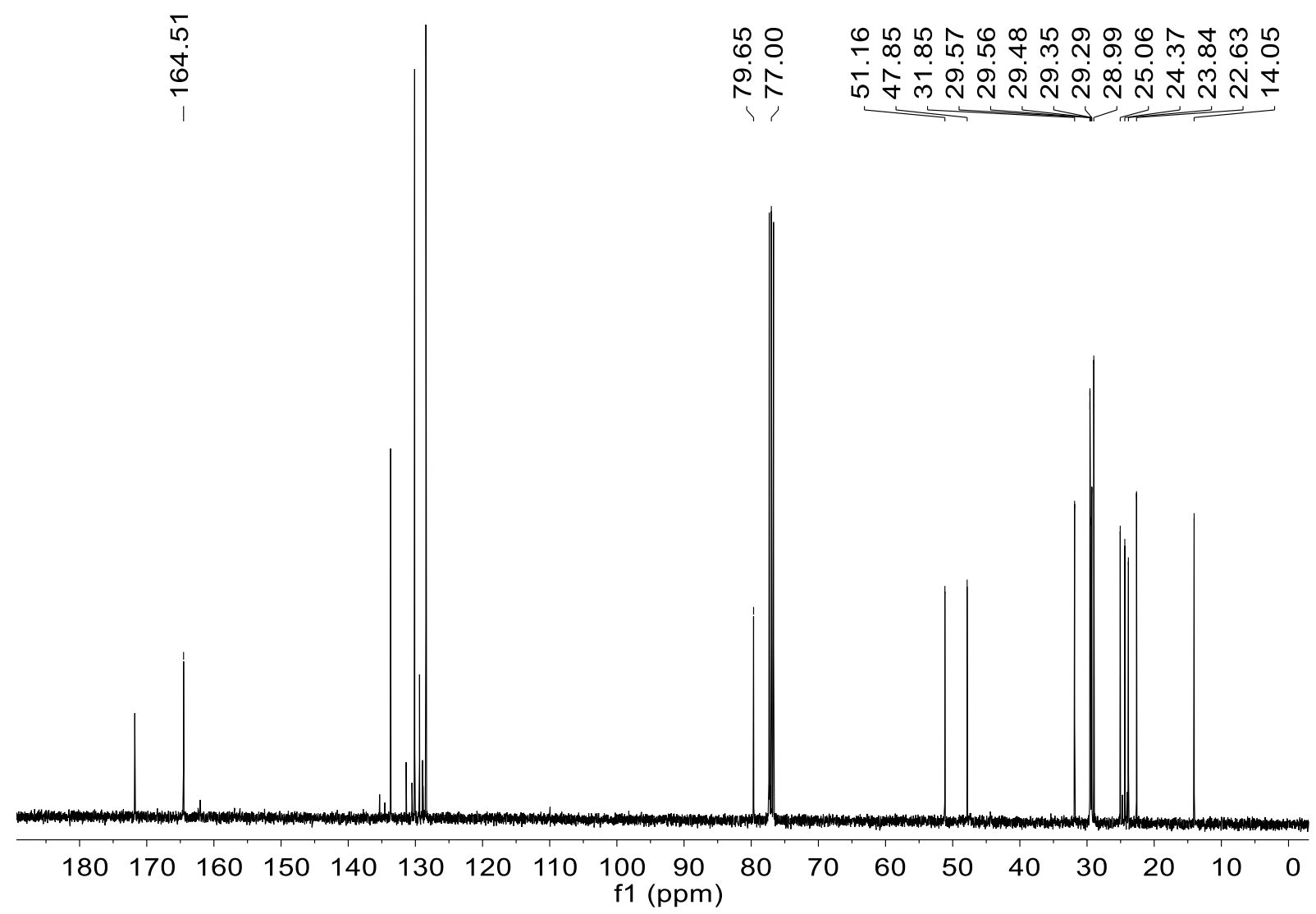

${ }^{13} \mathrm{C}$ NMR spectrum of $\mathrm{N}$-((1-dodecyloxy)methylidene) pyrrolidinium hexafluorophosphate $\left(100 \mathrm{MHz}, \mathrm{CDCl}_{3}\right)$. 


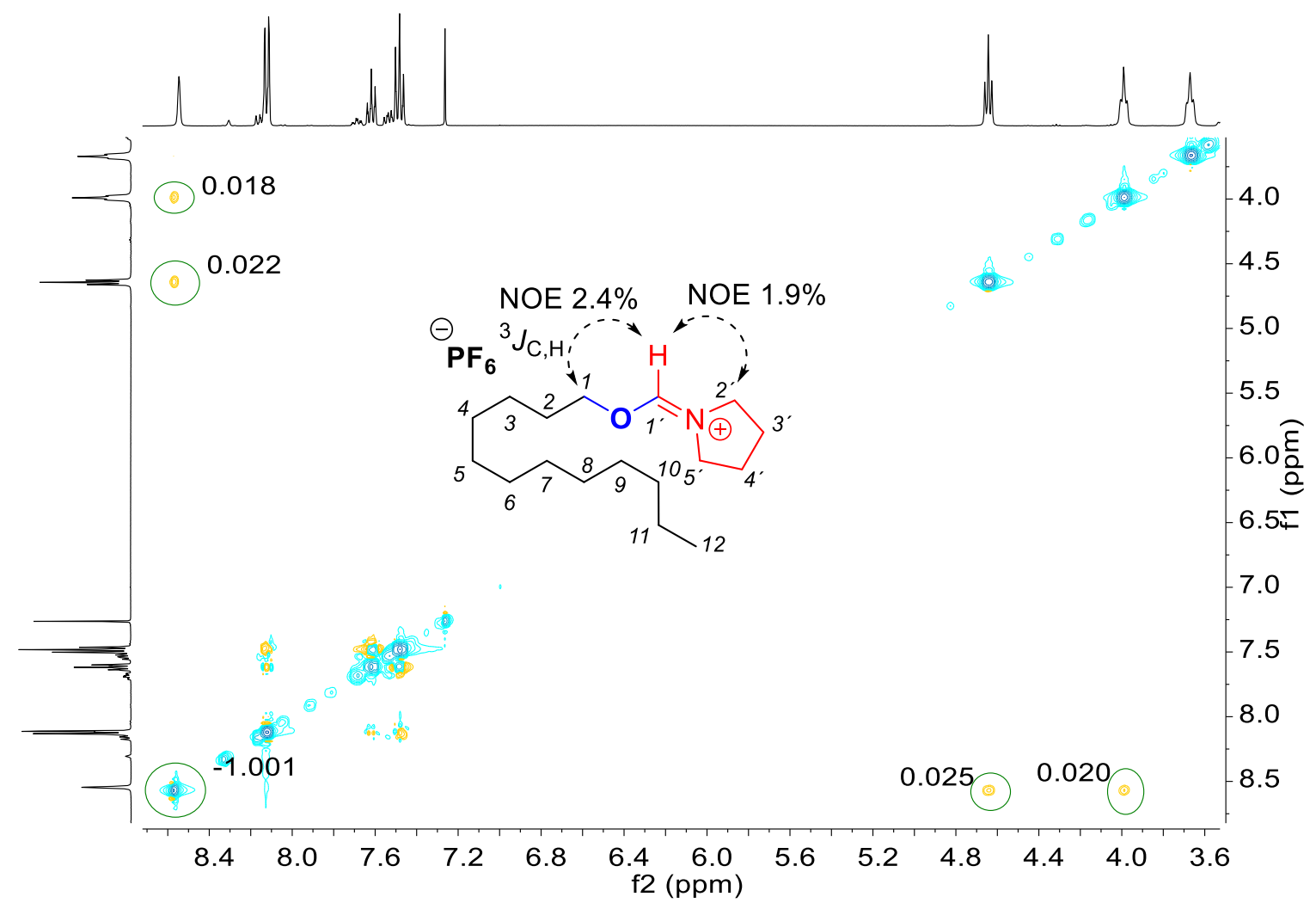

NOESY spectrum of $\mathrm{N}$-((1-dodecyloxy)methylidene) pyrrolidinium hexafluorophosphate (400 MHz, $\left.\mathrm{CDCl}_{3}\right)$.

\subsubsection{Synthesis of $\mathrm{N}$-((1-Dodecyloxy)methylidene) dimethyliminium hexafluoro- phosphate (III)}

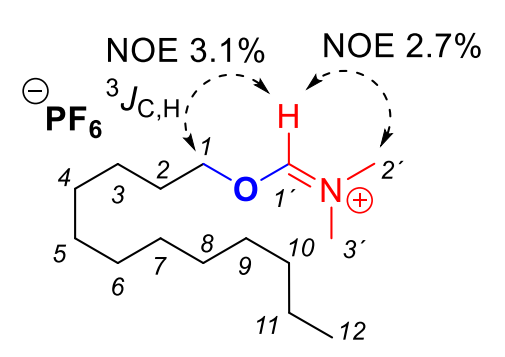

IIIj

PH1953: According to general procedure 3 (chapter 2.2.3, page 15), 1-dodecanol ( $113 \mu \mathrm{L}, 94 \mathrm{mg}, 500 \mu \mathrm{mol}, 1.00$ equiv) was allowed to react with DMF $(46 \mu \mathrm{L}, 44 \mathrm{mg}, 0.60 \mathrm{mmol}$, 1.20 equiv), $\mathrm{BzCl}$ ( 1.50 equiv) and $\mathrm{KPF}_{6}$ (1.60 equiv) in $\mathrm{MeCN}$ (2.0 M) for $5.5 \mathrm{~h} .{ }^{1} \mathrm{H}$ NMR attested a conversion of dodecanol to title compound of $\geq 98 \%$.

M $\left(\mathrm{C}_{15} \mathrm{H}_{32} \mathrm{~F}_{6} \mathrm{NOP}\right)=387.39 \mathrm{~g} / \mathrm{mol} ;{ }^{1} \mathrm{H}$ NMR $\left(400 \mathrm{MHz}, \mathrm{CDCl}_{3}\right)$ $\delta[\mathrm{ppm}]=8.42\left(\mathrm{~s}, 1 \mathrm{H}, 1^{\prime}-\mathrm{H}\right), 4.66\left(\mathrm{t},{ }^{3} J_{1,2}=6.8 \mathrm{~Hz}, 2 \mathrm{H}, 1-\mathrm{H}\right), 3.42\left(\mathrm{~s}, 3 \mathrm{H}, 2^{\prime}-\mathrm{H}\right), 3.22(\mathrm{~s}, 3 \mathrm{H}$, $\left.3^{\prime}-\mathrm{H}\right), 1.87-1.80(\mathrm{~m}, 2 \mathrm{H}, 2-\mathrm{H}), 1.40-1.25(\mathrm{~m}, 18 \mathrm{H}, 3-\mathrm{H}$ to $11-\mathrm{H}), 0.90-0.86(\mathrm{~m}, 3 \mathrm{H}, 12-\mathrm{H})$; ${ }^{19} \mathrm{~F} \mathrm{NMR}\left(376 \mathrm{MHz}, \mathrm{CDCl}_{3} \delta[\mathrm{ppm}]=-72.49\left(\mathrm{~d},{ }^{1} \mathrm{~J}_{\mathrm{F}, \mathrm{P}}=712 \mathrm{~Hz}\right) ;{ }^{31} \mathbf{P}\right.$ NMR $\left(162 \mathrm{MHz}, \mathrm{CDCl}_{3} \delta\right.$ [ppm] = -144.4 (sept, $\left.{ }^{1} \mathrm{JP}_{\mathrm{P}, \mathrm{F}} 713 \mathrm{~Hz}\right) ;{ }^{13} \mathrm{C}$ NMR $\left(100 \mathrm{MHz}, \mathrm{CDCl}_{3}\right) \delta[\mathrm{ppm}]=164.51\left(\mathrm{C}-1^{\prime}\right), 79.65$ (C-1), 51.16 (C-2'), 47.85 (C-3'), 31.85 (C-10 or C-11), 29.57, 29.56, 29.48, 29.35, 29.29 (C-4 to C-9), 28.99 (C-2), 25.06 (C-3), 22.63 (C-10 or C-11), 14.05 (C-12). 


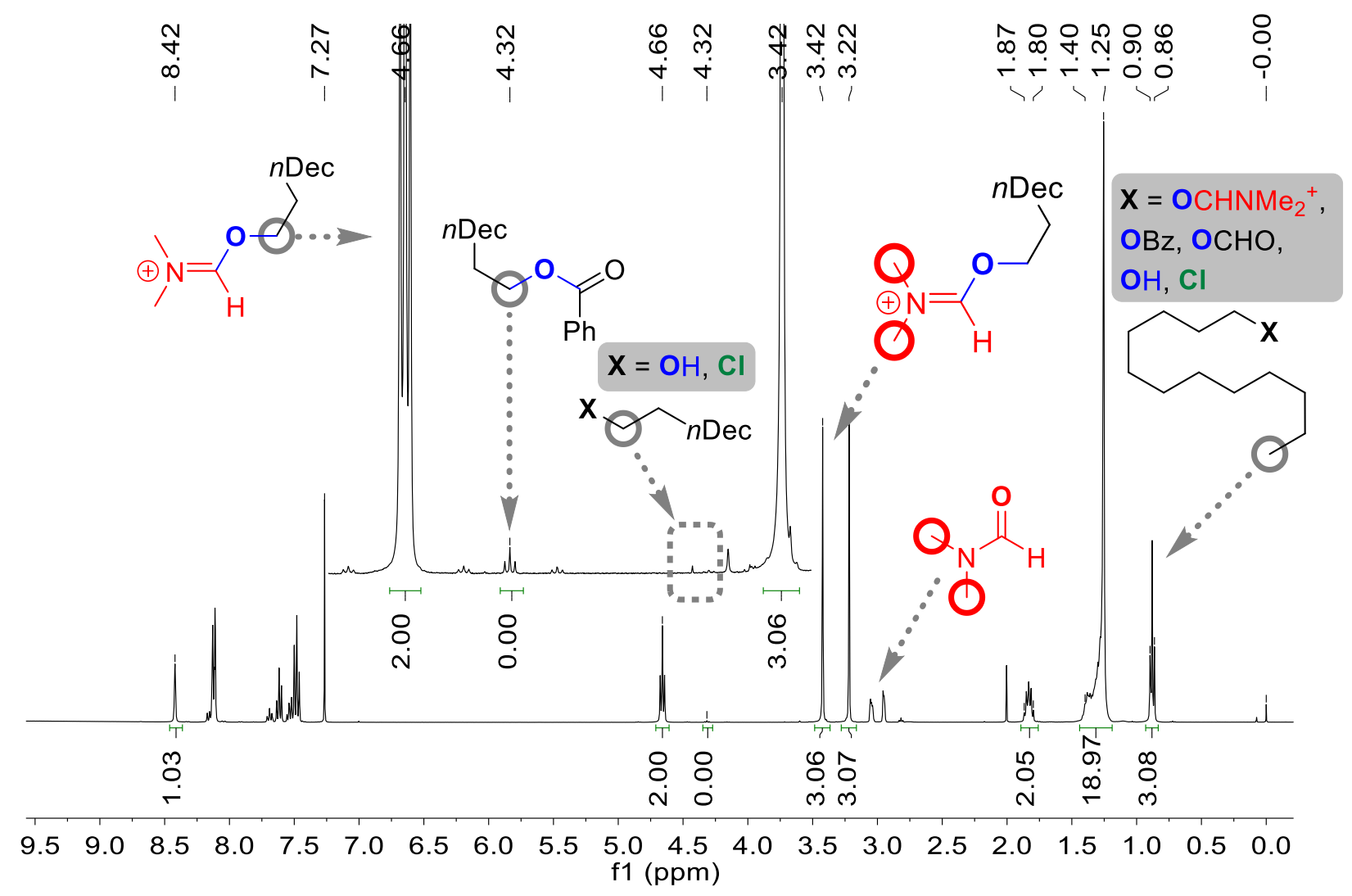

${ }^{1} \mathrm{H}$ NMR spectrum of $\mathrm{N}-((1$-dodecyloxy)methylidene) dimethyliminium hexafluorophosphate (400 MHz, $\mathrm{CDCl}_{3}$ ).

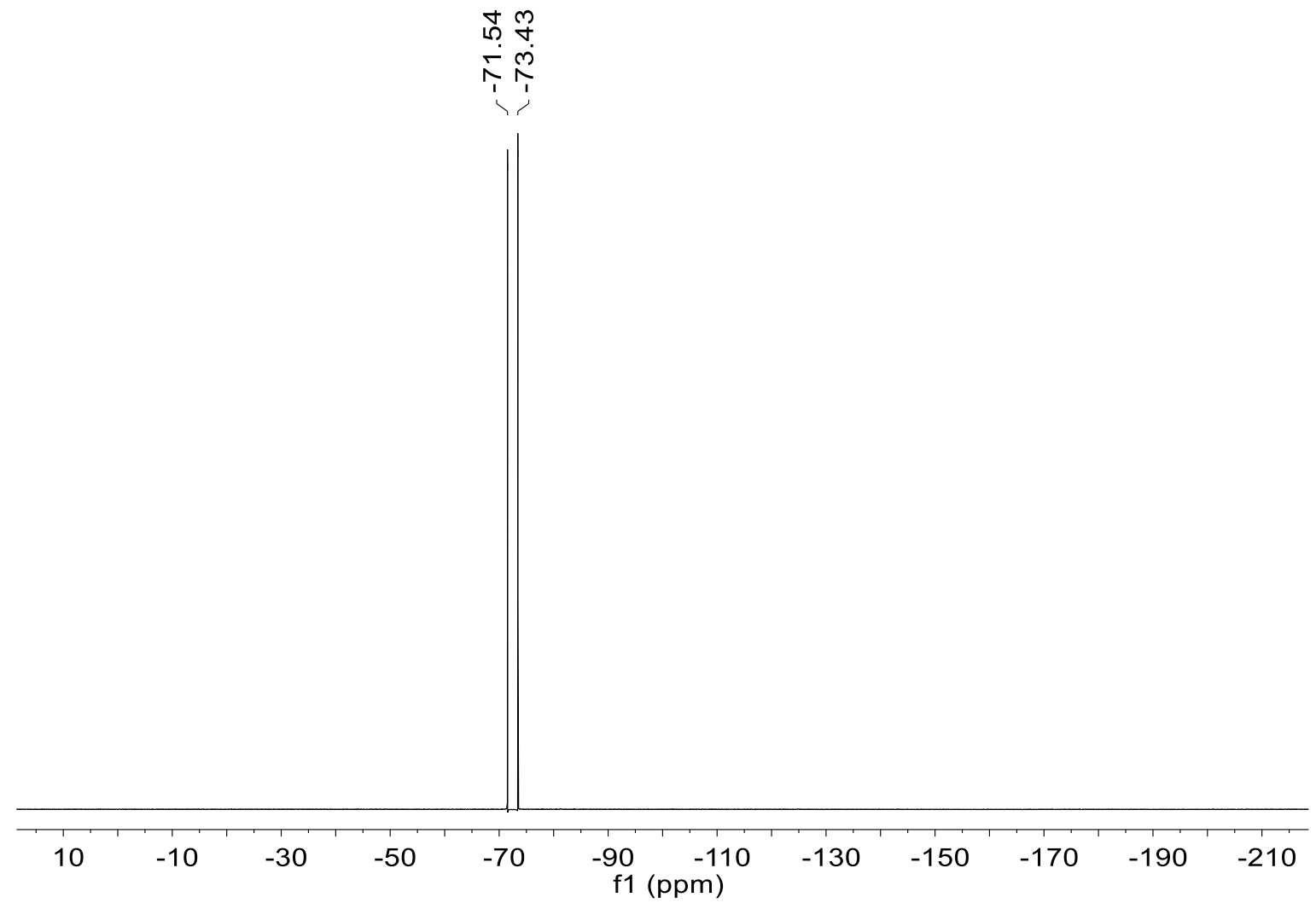

${ }^{19} \mathrm{~F}$ NMR spectrum of $\mathrm{N}$-((1-dodecyloxy)methylidene) dimethyliminium hexafluorophosphate (376 $\left.\mathrm{MHz}, \mathrm{CDCl}_{3}\right)$. 


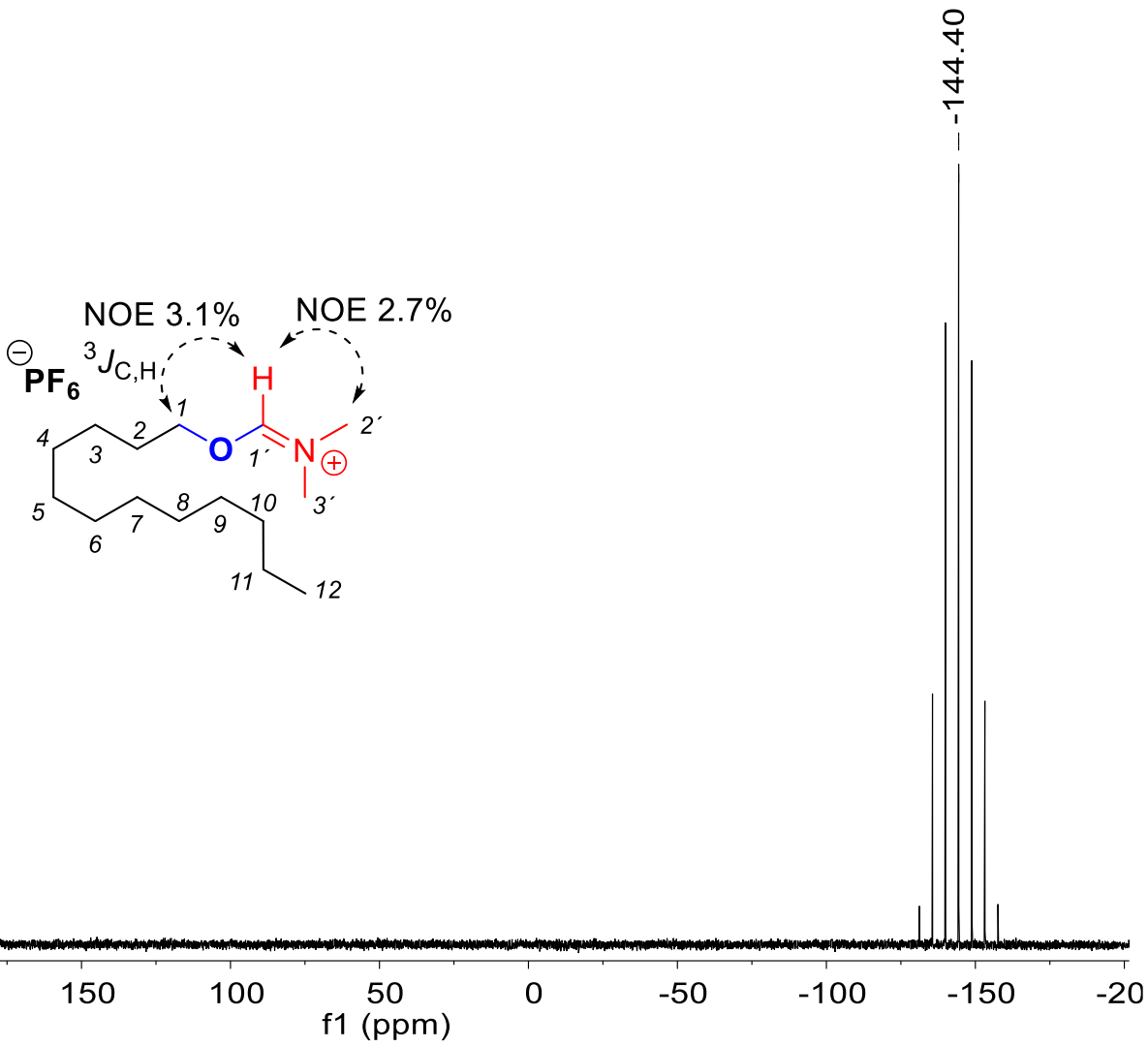

${ }^{31} \mathrm{P}$ NMR spectrum of $\mathrm{N}-((1$-dodecyloxy)methylidene) dimethyliminium hexafluorophosphate (162 $\left.\mathrm{MHz}, \mathrm{CDCl}_{3}\right)$.

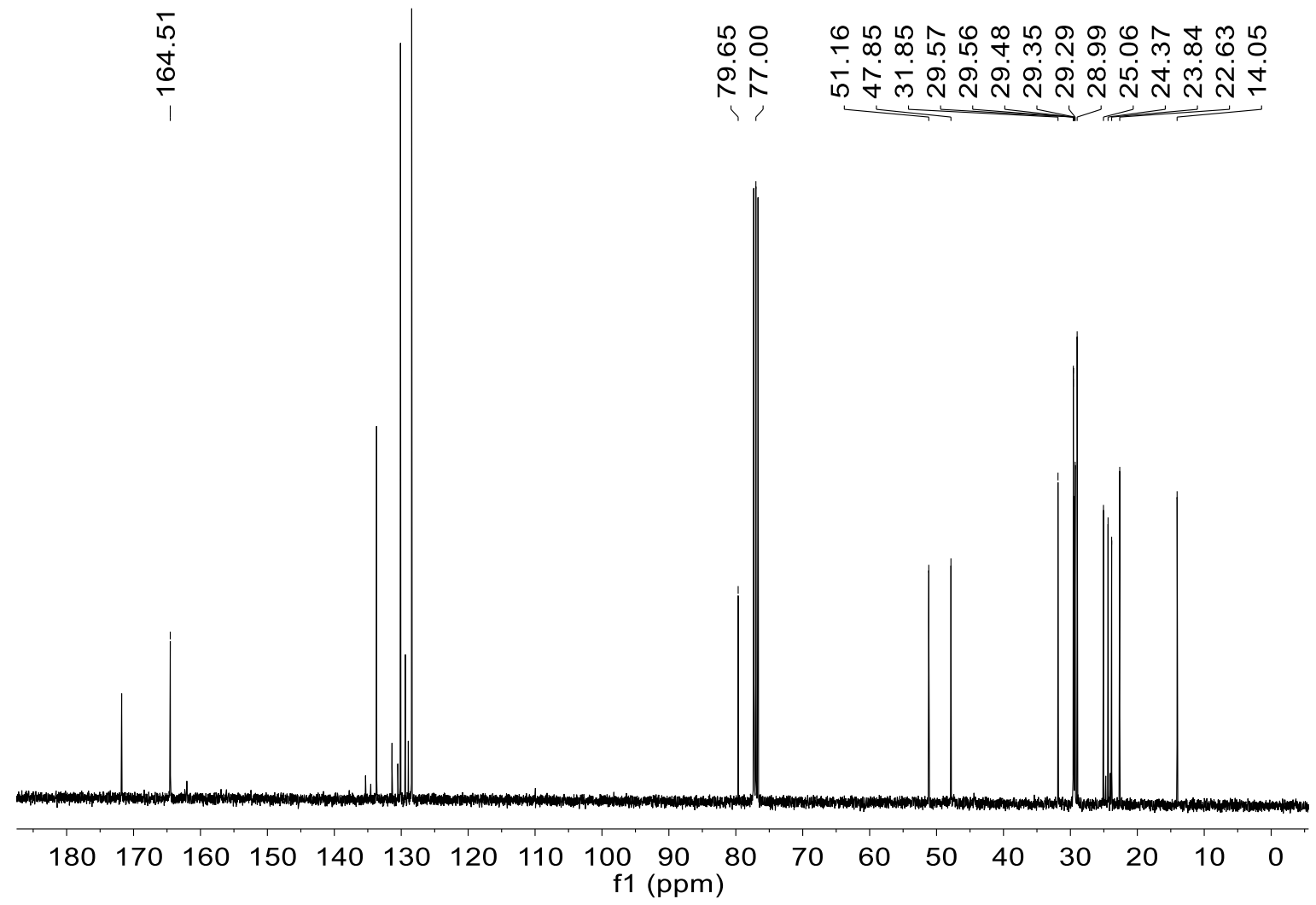

${ }^{13} \mathrm{C}$ NMR spectrum of $\mathrm{N}-((1$-dodecyloxy)methylidene) dimethyliminium hexafluorophosphate (100 $\mathrm{MHz}, \mathrm{CDCl}_{3}$ ). 


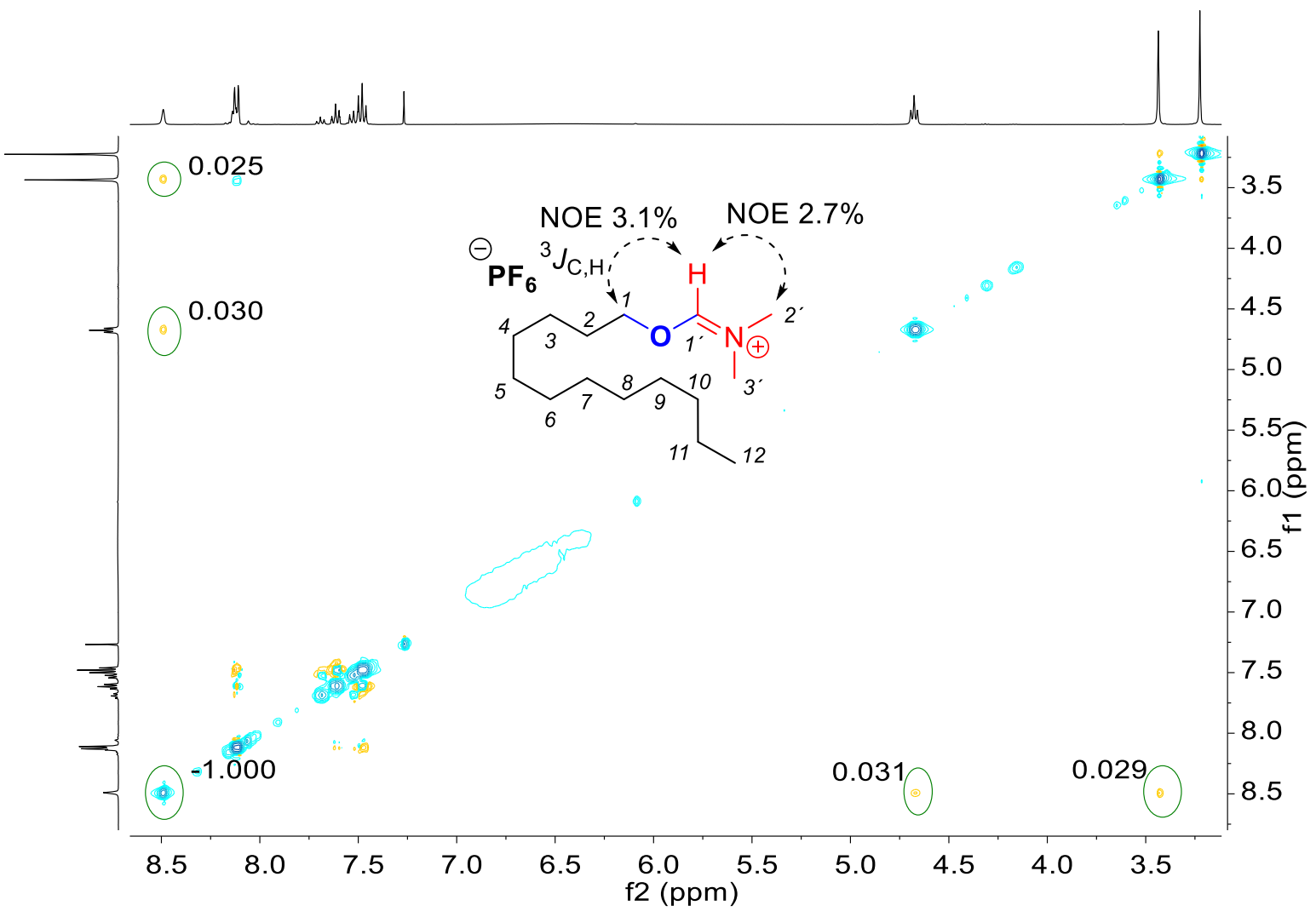

NOESY spectrum of $\mathrm{N}$-((1-dodecyloxy)methylidene) dimethyliminium hexafluorophosphate $\left(400 \mathrm{MHz}, \mathrm{CDCl}_{3}\right)$.

\subsubsection{Synthesis of $N$-((1-Dodecyloxy)methylidene) methyliminium hexafluoro- phosphate and Difluorophosphate (IIIk/IVk)}

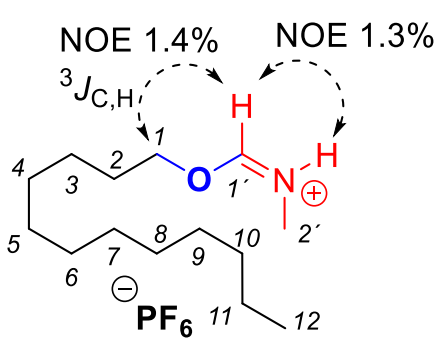

IIIk

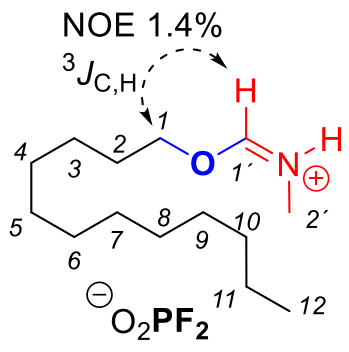

IVk
In contrast to the respective chloride salt IIc, ${ }^{[5]}$ the phosphate salt IIIk showed a double set of signals in the ${ }^{1} \mathrm{H},{ }^{19} \mathrm{~F},{ }^{31} \mathrm{P}$ and ${ }^{13} \mathrm{C}$ NMR spectrum when $\mathrm{NaPF}_{6}$ was used in the synthesis. In particular, the appearance of a triplet at $-18.83 \mathrm{ppm}$ and the strong difference in terms of chemical shift between this triplet and the septet at $-144.33 \mathrm{ppm}(\Delta \delta=125.5 \mathrm{ppm})$ in the ${ }^{13} \mathrm{P} \mathrm{NMR}$ spectrum suggest a difluorophosphate salt as minor component. Indeed, the ${ }^{19} \mathrm{~F}$ and ${ }^{31} \mathrm{P}$ NMR-data compares well to literature values of $\mathrm{NMe}_{4}{ }^{+}-\mathrm{O}_{2} \mathrm{PF}_{2}$ in DMSO- $\mathrm{d}_{6}{ }^{[5]}$

The strong impact of the counterion in the ${ }^{1} \mathrm{H}$ and ${ }^{13} \mathrm{C}$ NMR might be reasoned by chelation via the formyl $\mathrm{H}$ - and the $\mathrm{NH}$ proton, which results in a very close proximity. With $\mathbf{K P F}_{6} \mathbf{I V k}$ was formed in minor traces, while Illk was observed as main product. The hydrolysis of hexafluorophosphate to $\mathrm{F}_{2} \mathrm{PO}_{2}{ }^{-}$could be rationalized by the poorer solubility of $\mathrm{NaF}$ compared to $\mathrm{KF}$ in $\mathrm{MeCN}$. The relative 
Z-configuration of the main component was verified by a clear NOE between the $\mathrm{NH}$ and formyl proton and is identical with that of iminium chloride Ilc.

With $\mathrm{NaPF}_{6}$ (PH1952): Following general protocol 3 (chapter 2.2.3, page 15) the title compound was synthesized from 1-dodecanol (113 $\mu \mathrm{L}, 94 \mathrm{mg}, 500 \mu \mathrm{L}, 1.00$ equiv), $\mathrm{N}$-methylformamide ( $35 \mu \mathrm{L}, 36 \mathrm{mg}, 0.60 \mathrm{mmol}, 1.20$ equiv), $\mathrm{BzCl}$ ( 1.50 equiv) and $\mathrm{NaPF}_{6}$ (1.60 equiv) in MeCN (2.0 M). After stirring for $5.5 \mathrm{~h}{ }^{1} \mathrm{H}$ NMR displayed iminium phosphate IIIk in $\geq 98 \%$ conversion and a IVk/IIlk ratio of $21: 79$.

With $\mathrm{KPF}_{6}\left(\mathrm{PH}_{1903):}\right.$ In accordance with general protocol 3 (chapter 2.2.3, page 15) the hexafluorophosphate IIIk was synthesized from 1-dodecanol (500 $\mu \mathrm{mol}, 1.00$ equiv), $N$-methylformamide (1.20 equiv), $\mathrm{BzCl}$ (1.50 equiv) and $\mathrm{KPF}_{6}$ (1.60 equiv) in $\mathrm{MeCN}(2.0 \mathrm{M})$. After $10 \mathrm{~h}{ }^{1} \mathrm{H}$ NMR of a reaction sample indicated $\geq 98 \%$ conversion and a ratio IIIk/IVk of $\geq 98: 2$. The selectivity IIIk/IIIn was reproduced in two additional experiments.

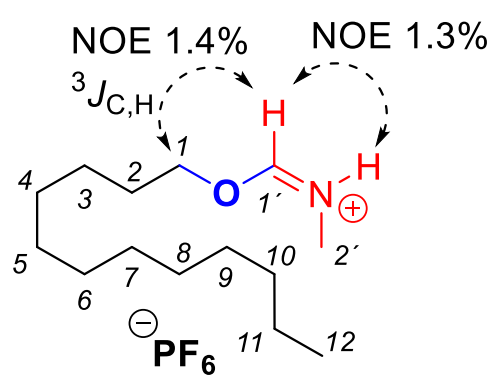

$N$-((1-Dodecyloxy)methylidene)

methyliminium hexafluoro-phosphate (IIIk)

M $\left(\mathrm{C}_{14} \mathrm{H}_{30} \mathrm{~F}_{6} \mathrm{NOP}\right)=373.36 \mathrm{~g} / \mathrm{mol} ;{ }^{1} \mathrm{H}$ NMR $\left(400 \mathrm{MHz}, \mathrm{CDCl}_{3}\right)$ $\delta[\mathrm{ppm}]=9.82(\mathrm{br} . \mathrm{s}, 1 \mathrm{H}, \mathrm{NH}), 8.38\left(\mathrm{~s}, 1 \mathrm{H}, 1^{\prime}-\mathrm{H}\right), 4.67\left(\mathrm{t},{ }^{3} J_{1,2}=\right.$ 6.7 Hz, 2H, 1-H, Illk/lVk= 79:21), $3.13\left(\mathrm{~d},{ }^{3} \mathrm{~J}_{2}, \mathrm{NH}=5.2 \mathrm{~Hz}, 1 \mathrm{H}\right.$, $2^{\prime}-\mathrm{H}$, IIIk/lVk = 77:23), 1.87-1.75 (m, 2H, 2-H), 1.40-1.26 (m, $18 \mathrm{H}, 3-\mathrm{H}$ to $11-\mathrm{H}), 0.90-0.86(\mathrm{~m}, 3 \mathrm{H}, 12-\mathrm{H}) ;{ }^{19} \mathrm{~F} \mathrm{NMR}\left(376 \mathrm{MHz}, \mathrm{CDCl}_{3} \delta[\mathrm{ppm}]=-71.92(\mathrm{~d}\right.$, $\left.{ }^{1} J_{F, P}=711 \mathrm{~Hz}\right) ;{ }^{31} \mathbf{P}$ NMR $\left(162 \mathrm{MHz}, \mathrm{CDCl}_{3} \delta[\mathrm{ppm}]=-144.33\left(\mathrm{sept},{ }^{1} \mathrm{~J}_{\mathrm{P}, \mathrm{F}}=712 \mathrm{~Hz}\right) ;{ }^{13} \mathrm{C}\right.$ NMR $\left(100 \mathrm{MHz}, \mathrm{CDCl}_{3}\right) \delta[\mathrm{ppm}]=168.14\left(\mathrm{C}-1^{\prime}\right), 80.03(\mathrm{C}-1), 31.88$ (C-10 or C-11), 29.58, 29.49, 29.35, 29.31, 29.05, 29.00 (C-4 to C-9), 28.70 (C-2'), 25.00 (C-3), 22.65 (C-10 or C-11), 14.07 (C-12).

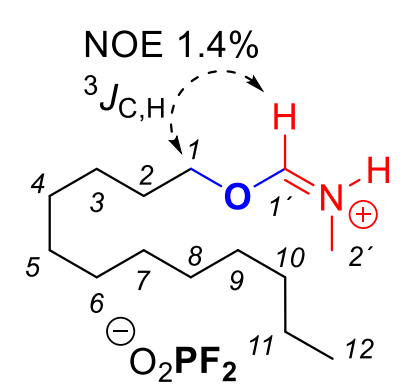

$N$-((1-Dodecyloxy)methylidene)

methyliminium difluorophosphate (IVk)

Only distinct ${ }^{13} \mathrm{C}$ NMR signals are listed.

$\mathbf{M}\left(\mathrm{C}_{14} \mathrm{H}_{30} \mathrm{~F}_{6} \mathrm{NOP}\right)=373.36 \mathrm{~g} / \mathrm{mol} ;{ }^{1} \mathrm{H}$ NMR $\left(400 \mathrm{MHz}, \mathrm{CDCl}_{3}\right) \delta[\mathrm{ppm}]$ $=9.50$ (br.s, $1 \mathrm{H}, \mathrm{NH}), 8.21\left(\mathrm{~s}, 1 \mathrm{H}, 1^{\prime}-\mathrm{H}\right), 4.53\left(\mathrm{t},{ }^{3} J_{1,2}=6.7 \mathrm{~Hz}, 2 \mathrm{H}\right.$, $1-\mathrm{H}, \mathbf{I V k} / \mathbf{I I I} \mathbf{k}=21: 79), 3.29\left(\mathrm{~d},{ }^{3} \mathrm{~J}^{\prime}, \mathrm{NH}=5.1 \mathrm{~Hz}, 1 \mathrm{H}, 2^{\prime}-\mathrm{H}, \mathbf{I V k} / \mathbf{I I I} \mathbf{k}=\right.$ 23:77), $1.87-1.75(\mathrm{~m}, 2 \mathrm{H}, 2-\mathrm{H}), 1.40-1.26(\mathrm{~m}, 18 \mathrm{H}, 3-\mathrm{H}$ to $11-\mathrm{H}), 0.90-0.86(\mathrm{~m}, 3 \mathrm{H}, 12-\mathrm{H}) ;{ }^{19} \mathbf{F}$ NMR $\left(376 \mathrm{MHz}, \mathrm{CDCl}_{3} \delta[\mathrm{ppm}]=--83.36\left(\mathrm{~d},{ }^{1} \mathrm{~J}_{\mathrm{F}, \mathrm{P}}=970 \mathrm{~Hz}\right) ;{ }^{31} \mathbf{P} \mathbf{N M R}\left(162 \mathrm{MHz}, \mathrm{CDCl}_{3} \delta[\mathrm{ppm}]\right.\right.$ $=-18.83\left(\mathrm{t},{ }^{1} \mathrm{~J}_{\mathrm{P}, \mathrm{F}}=970 \mathrm{~Hz}\right) ;{ }^{13} \mathrm{C}$ NMR $\left(100 \mathrm{MHz}, \mathrm{CDCl}_{3}\right) \delta[\mathrm{ppm}]=168.45\left(\mathrm{C}-1^{\prime}\right), 78.36(\mathrm{C}-1)$, $32.70\left(\mathrm{C}-2^{\prime}\right), 24.93(\mathrm{C}-3)$. 


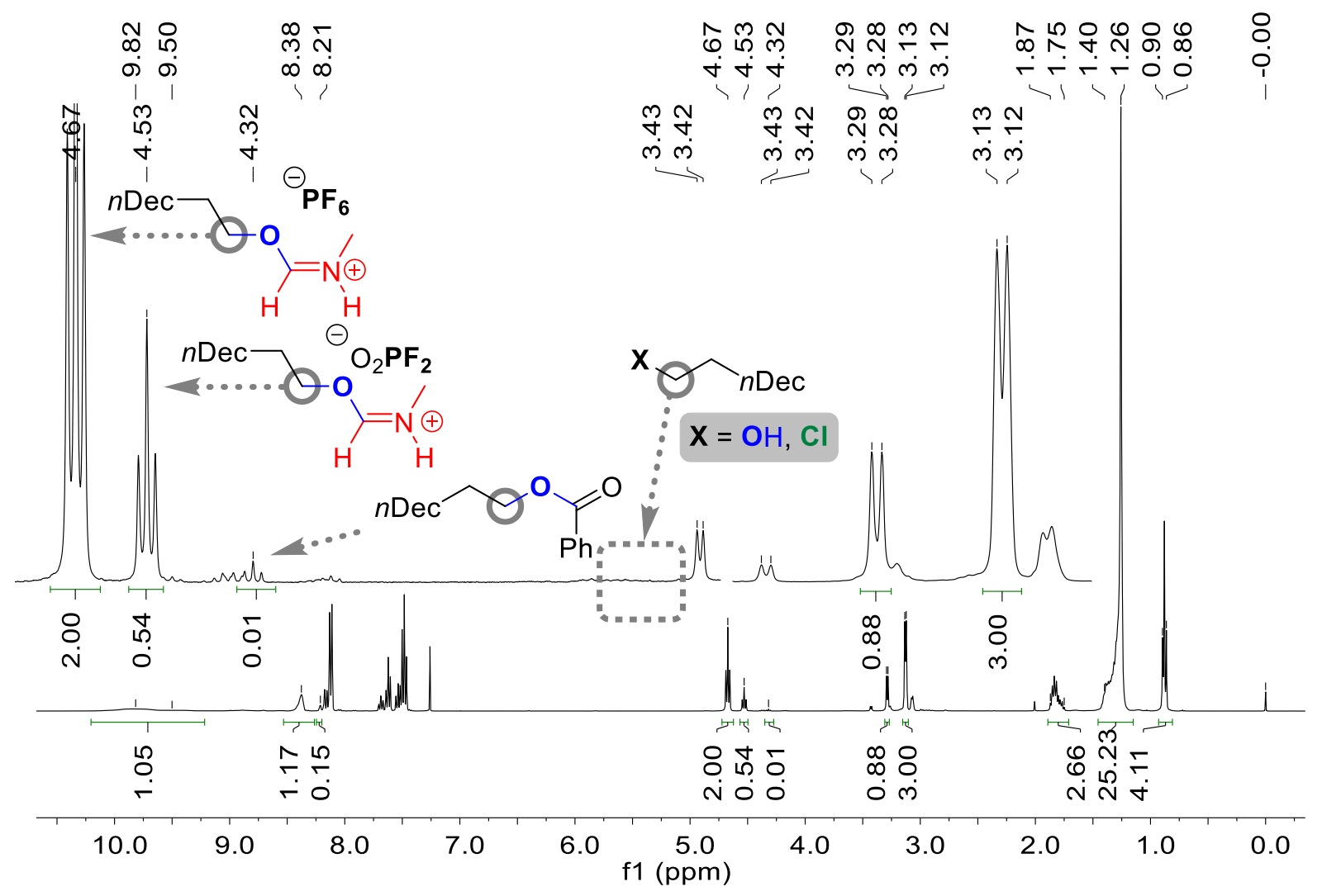

${ }^{1} \mathrm{H}$ NMR spectrum of $\mathrm{N}$-((1-dodecyloxy)methylidene) methyliminium hexafluorophosphate and difluorophosphate prepared with $\mathrm{NaPF}_{6}\left(400 \mathrm{MHz}, \mathrm{CDCl}_{3}\right)$.

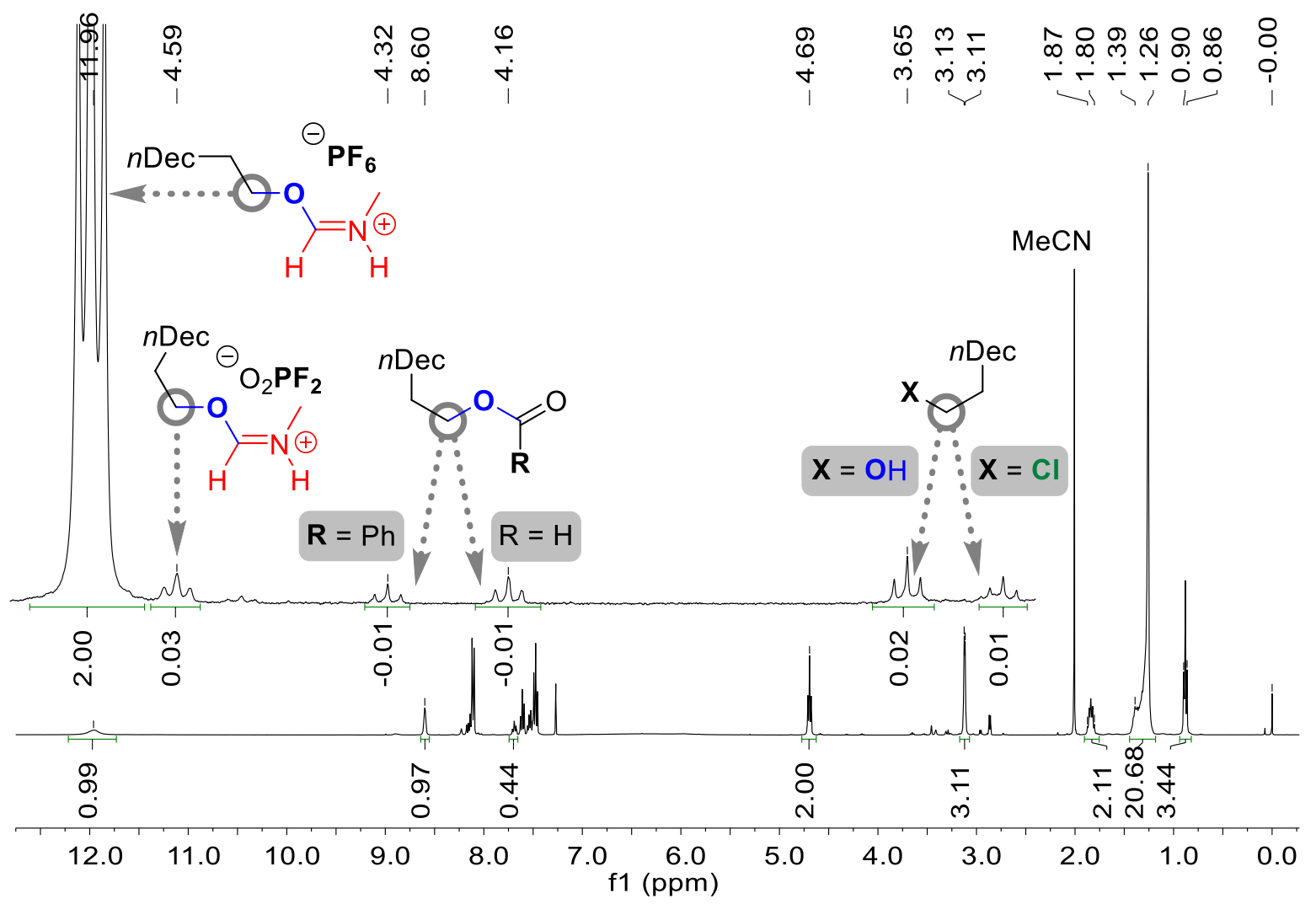

${ }^{1} \mathrm{H}$ NMR spectrum of $\mathrm{N}$-((1-dodecyloxy)methylidene) methyliminium hexafluorophosphate prepared with $\mathrm{KPF}_{6}\left(400 \mathrm{MHz}, \mathrm{CDCl}_{3}\right)$. 


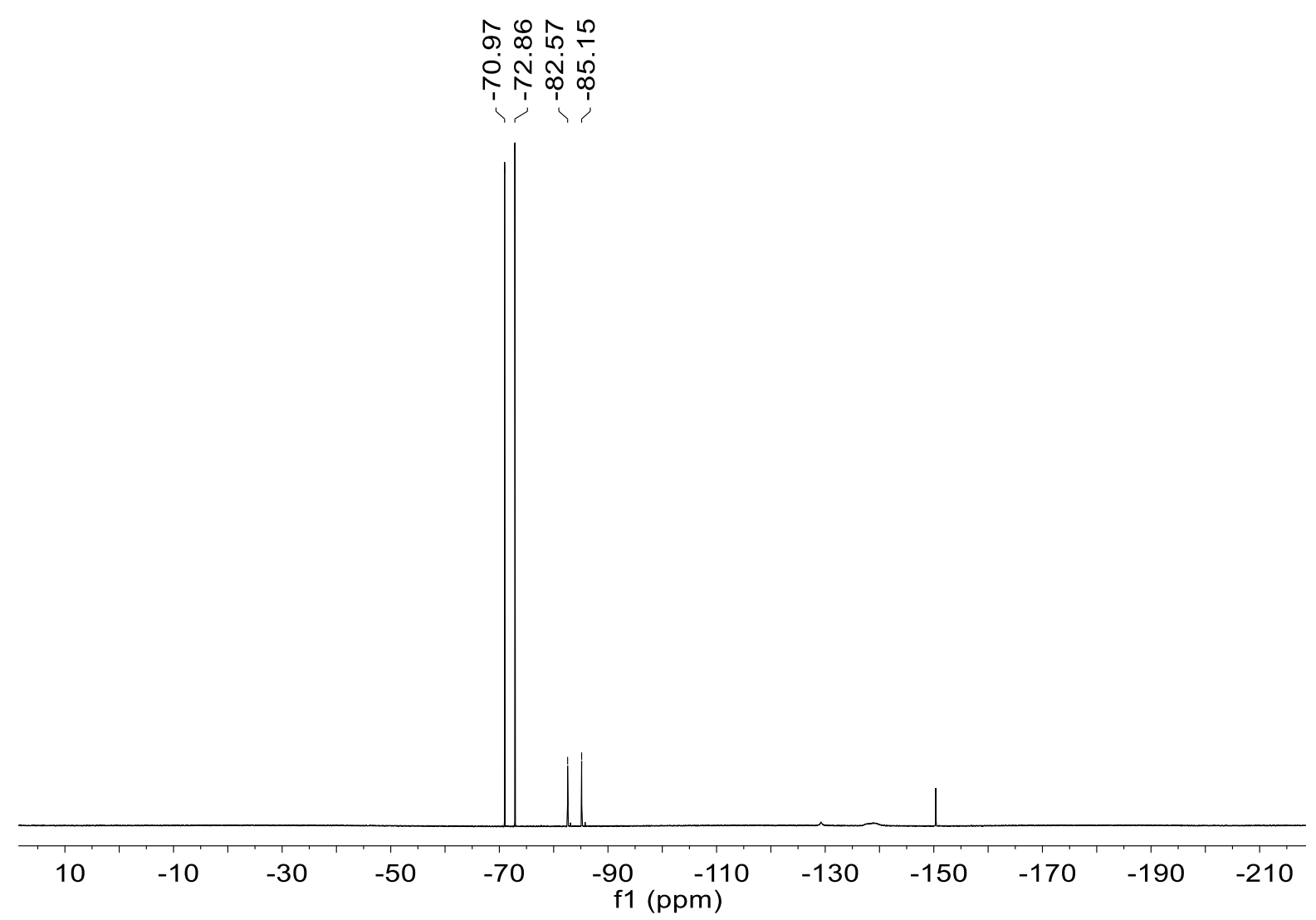

${ }^{19} \mathrm{~F}$ NMR spectrum of $\mathrm{N}$-((1-dodecyloxy)methylidene) methyliminium hexafluorophosphate and difluorophosphate prepared with $\mathrm{NaPF}_{6}\left(376 \mathrm{MHz}, \mathrm{CDCl}_{3}\right)$.
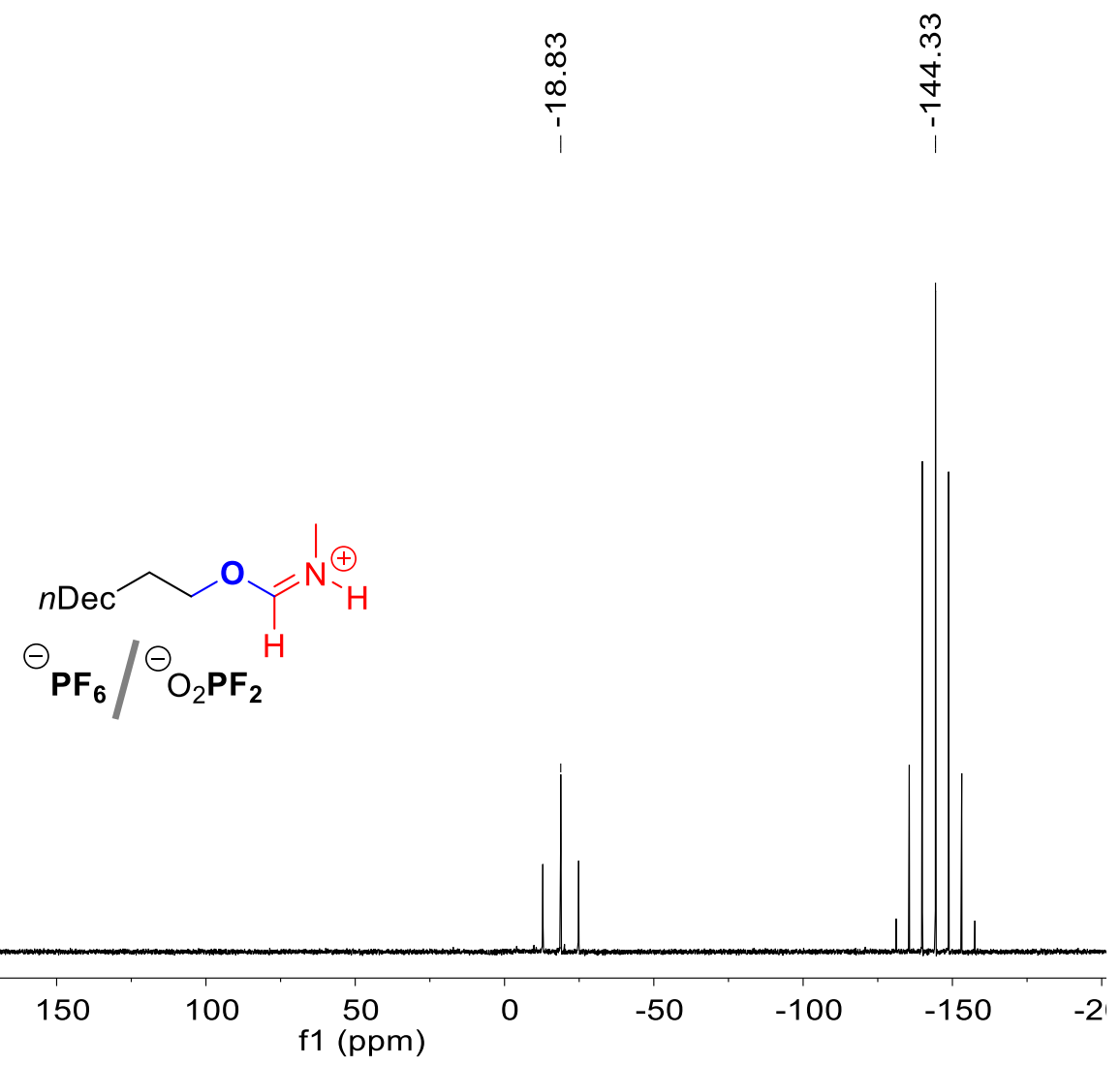

$\frac{m}{\mathfrak{j}}$

${ }^{31} \mathrm{P}$ NMR spectrum of $\mathrm{N}$-((1-dodecyloxy)methylidene) methyliminium hexafluorophosphate and difluorophosphate prepared with $\mathrm{NaPF}_{6}\left(162 \mathrm{MHz}, \mathrm{CDCl}_{3}\right)$. 

$\stackrel{4}{4}$

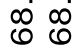
mo
i̊n

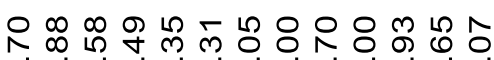
$\stackrel{\infty}{\sim}$
ஸึ่

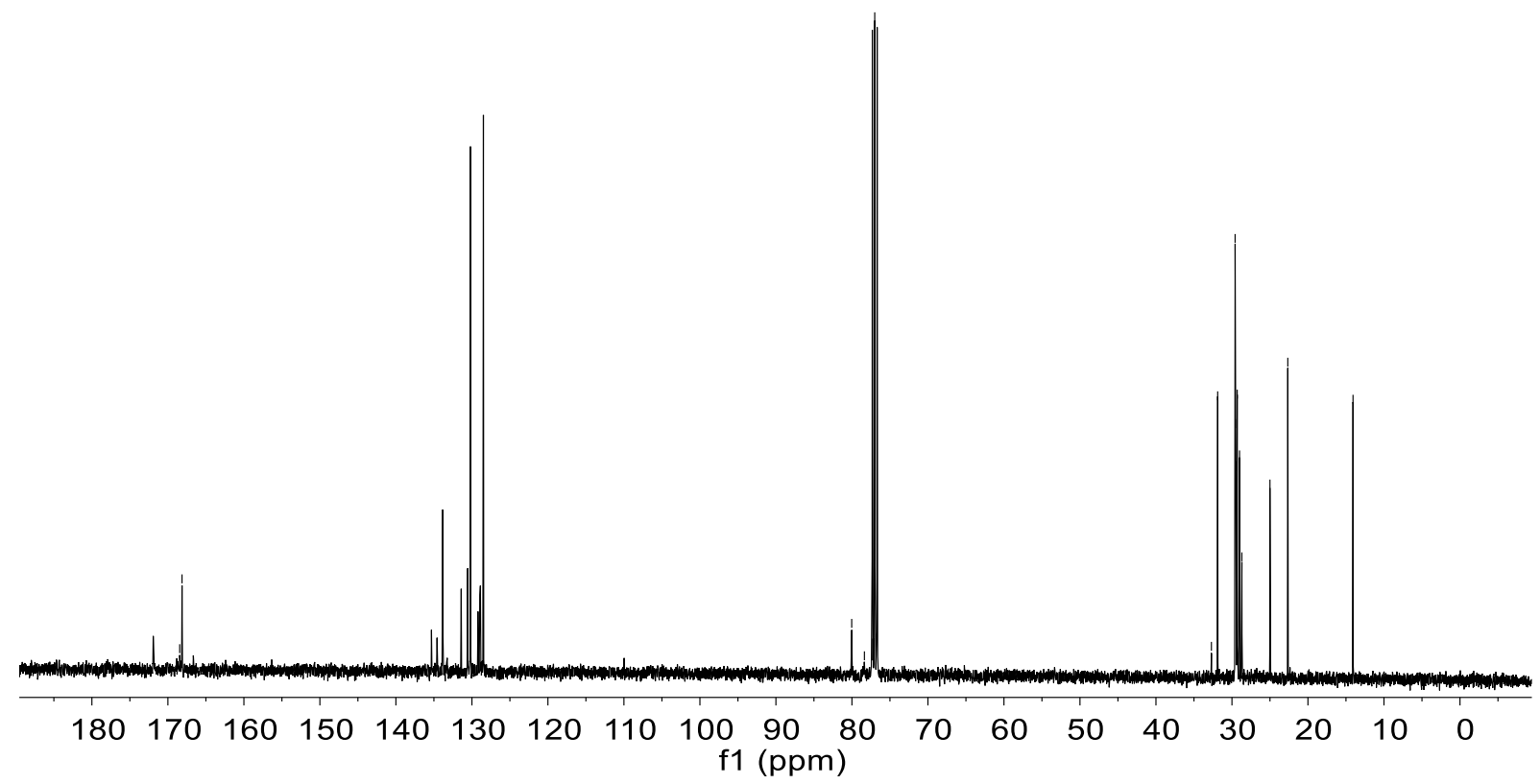

${ }^{13} \mathrm{C}$ NMR spectrum of $\mathrm{N}$-((1-dodecyloxy)methylidene) pyrrolidinium hexafluorophosphate and difluorophosphate prepared with $\mathrm{NaPF}_{6}\left(100 \mathrm{MHz}, \mathrm{CDCl}_{3}\right)$.

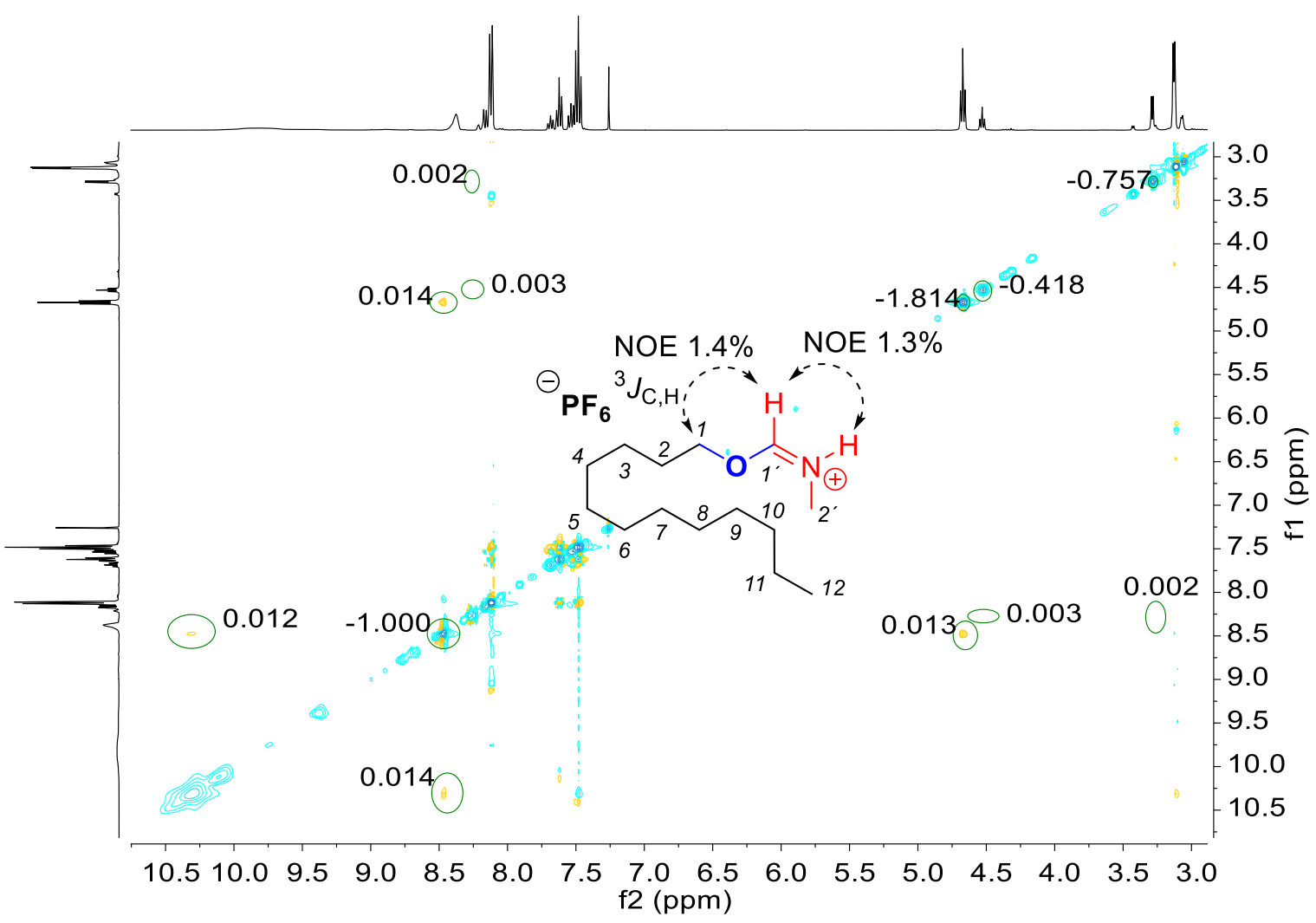

NOESY spectrum of $\mathrm{N}-((1$-dodecyloxy)methylidene) pyrrolidinium hexafluorophosphate and difluorophosphate prepared with $\mathrm{NaPF}_{6}\left(400 \mathrm{MHz}, \mathrm{CDCl}_{3}\right)$. 


\subsubsection{Synthesis of $\mathrm{N}$-((4-tert-Butylbenzyloxy)methylidene) dimethyliminium hexa-} fluorophosphate (IIII)

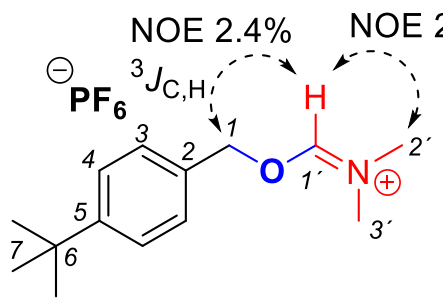

IIII

PH1963: According to general procedure 3 (chapter 2.2.3, page 15), 4-tert-butylbenzyl alcohol $(89 \mu \mathrm{L}, 83 \mathrm{mg}$, $500 \mu \mathrm{mol}, 1.00$ equiv) was reacted with DMF $(46 \mu \mathrm{L}$, $44 \mathrm{mg}, 0.60 \mathrm{mmol}, 1.20$ equiv), $\mathrm{BzCl}$ (1.50 equiv) and $\mathrm{NaPF}_{6}$ (1.60 equiv) in dry MeCN $(1.0 \mathrm{M}) .{ }^{(7)}$ After $1 \mathrm{~h}$, ${ }^{1} \mathrm{H}$ NMR attested full conversion of the starting material to

iminium salt IIII.

$\mathbf{M}\left(\mathrm{C}_{14} \mathrm{H}_{22} \mathrm{~F}_{6} \mathrm{NOP}\right)=365.300 \mathrm{~g} / \mathrm{mol} ;{ }^{1} \mathbf{H}$ NMR $\left(400 \mathrm{MHz}, \mathrm{CDCl}_{3}\right) \delta[\mathrm{ppm}]=8.51\left(\mathrm{~s}, 1 \mathrm{H} 1^{\prime}-\mathrm{H}\right)$, 7.44-7.39 (m, 4H, 3-H, 4-H), $5.60(\mathrm{~s}, 2 \mathrm{H}, 1-\mathrm{H}), 3.43\left(\mathrm{~s}, 3 \mathrm{H}, 2^{\prime}-\mathrm{H}\right), 3.19\left(\mathrm{~s}, 3 \mathrm{H}, 3^{\prime}-\mathrm{H}\right), 1.30$ (s, $9 \mathrm{H}, 7-\mathrm{H}) ;{ }^{19} \mathrm{~F} \mathrm{NMR}\left(376 \mathrm{MHz}, \mathrm{CDCl}_{3} \delta[\mathrm{ppm}]=-72.09\left(\mathrm{~d},{ }^{1} \mathrm{~J}_{\mathrm{F}, \mathrm{P}}=710 \mathrm{~Hz}\right) ;{ }^{31} \mathbf{P}\right.$ NMR $(162 \mathrm{MHz}$, $\mathrm{CDCl}_{3} \delta[\mathrm{ppm}]=-144.24\left(\mathrm{~d},{ }^{1} \mathrm{~J}_{\mathrm{F}, \mathrm{P}^{\prime}}=715 \mathrm{~Hz}\right) ;{ }^{13} \mathrm{C} \mathrm{NMR}\left(100 \mathrm{MHz}, \mathrm{CDCl}_{3}\right) \delta[\mathrm{ppm}]=166.68$ (C-5), 153.62 (C-5), 131.39 (C-2), 130.16 (C-3), 128.47 (C-4), 80.82 (C-1), 41.74 (C-2'), 36.38 (C-3'), 34.75 (C-6), $31.12(\mathrm{C}-7)$.

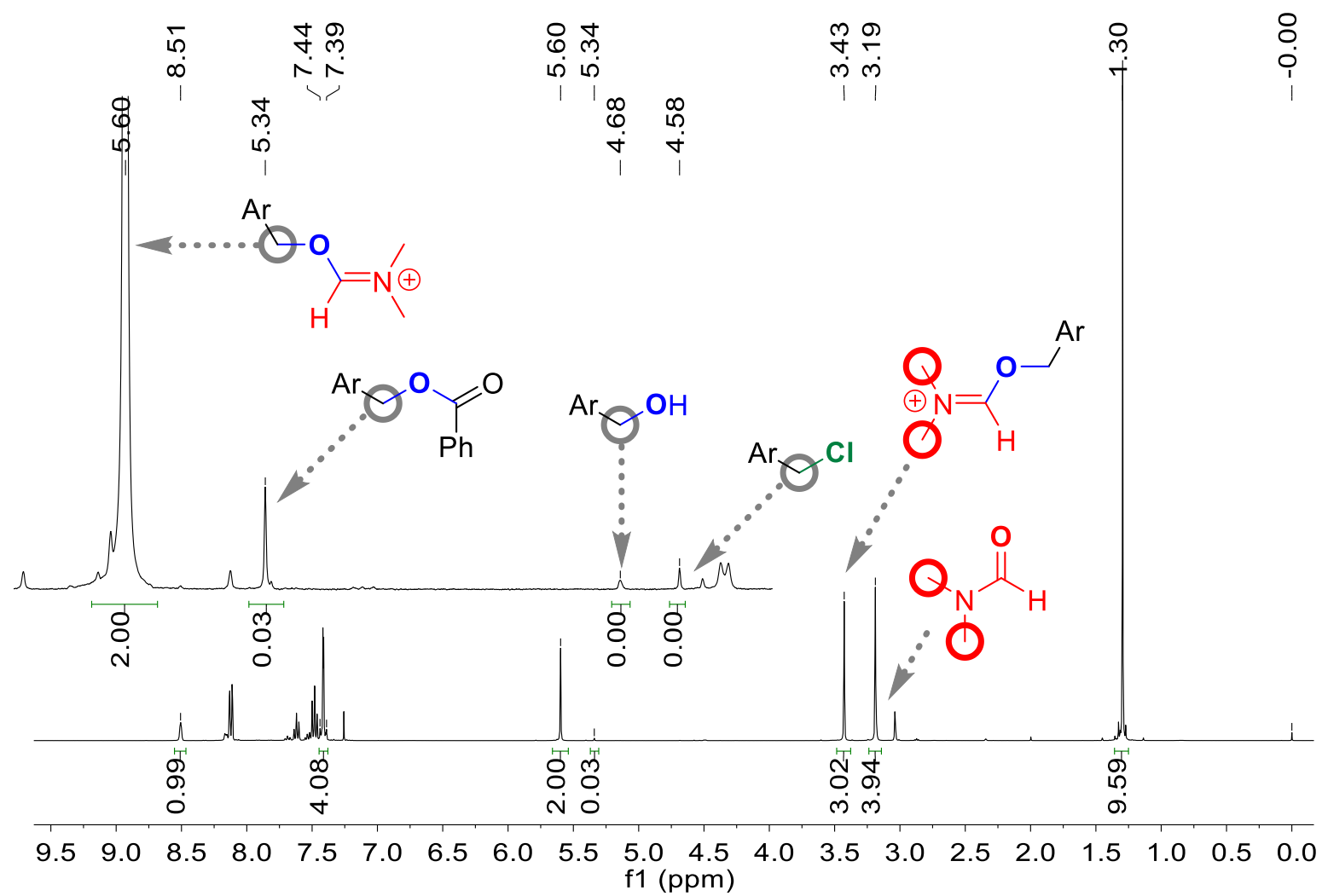

${ }^{1} \mathrm{H}$ NMR spectrum of $\mathrm{N}$-((4-tert-butylbenzyloxy)methylidene) dimethyliminium hexafluorophosphate $\left(400 \mathrm{MHz}, \mathrm{CDCl}_{3}\right) . \mathrm{Ar}=4$-tert-butylphenyl.

(7) Utilization of $\mathrm{KPF}_{6}, \mathrm{NaBF}_{4}$ and $\mathbf{A g B F}_{4}$ instead of $\mathrm{NaPF}_{6}$ resulted in lower conversions of the current substrate to iminium phosphate IIII of $\leq 89 \%, \leq 68 \%$ and $\leq 49 \%$, respectively. Addition of a $\mathrm{BzCl}$ solution by means of a syringe pump over 30 min did not improve the conversions significantly. Usage of acetone affected lower conversions. 


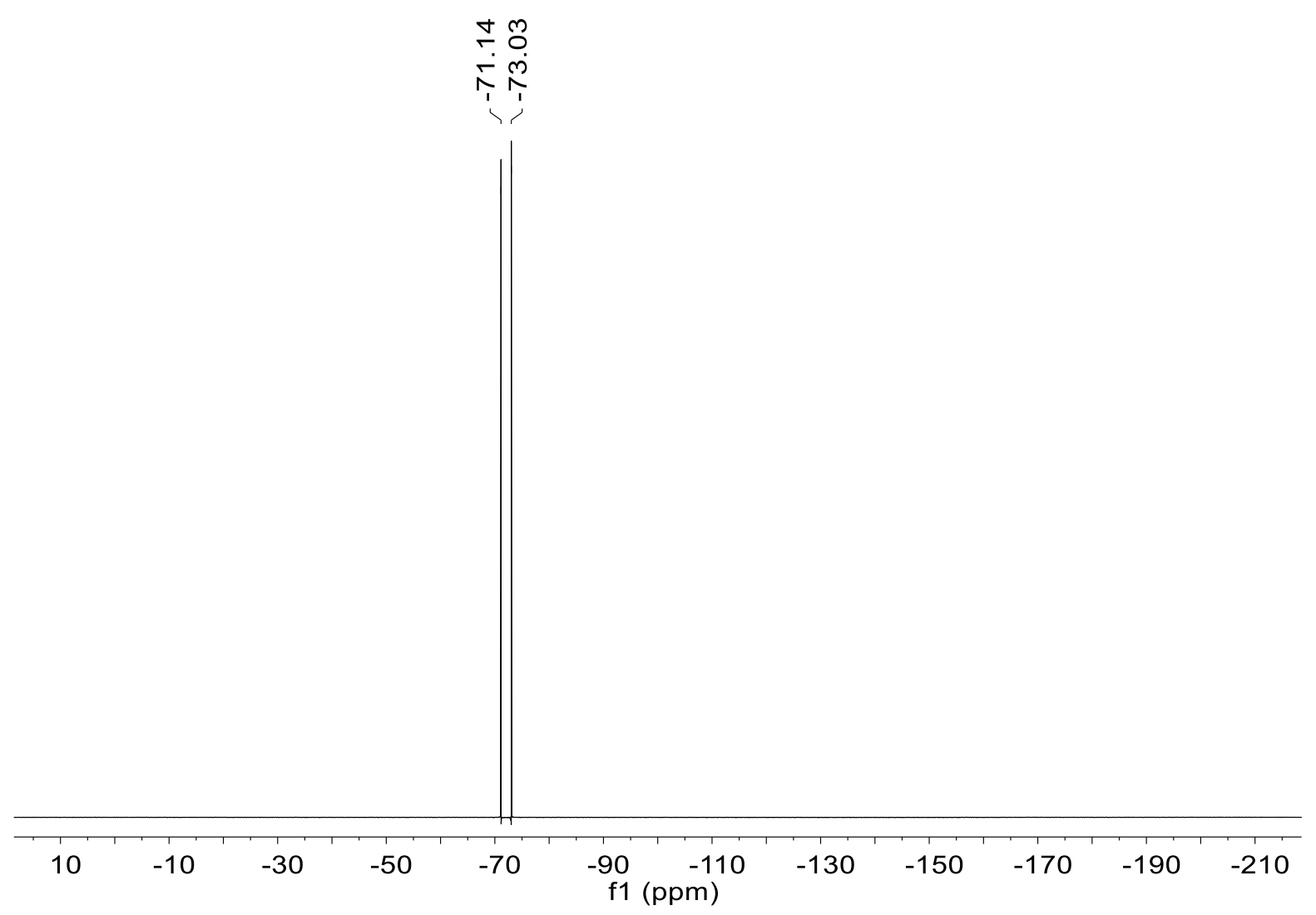

${ }^{19} \mathrm{~F}$ NMR spectrum of $\mathrm{N}$-((4-tert-butylbenzyloxy)methylidene) dimethyliminium hexafluorophosphate $\left(376 \mathrm{MHz}, \mathrm{CDCl}_{3}\right)$.

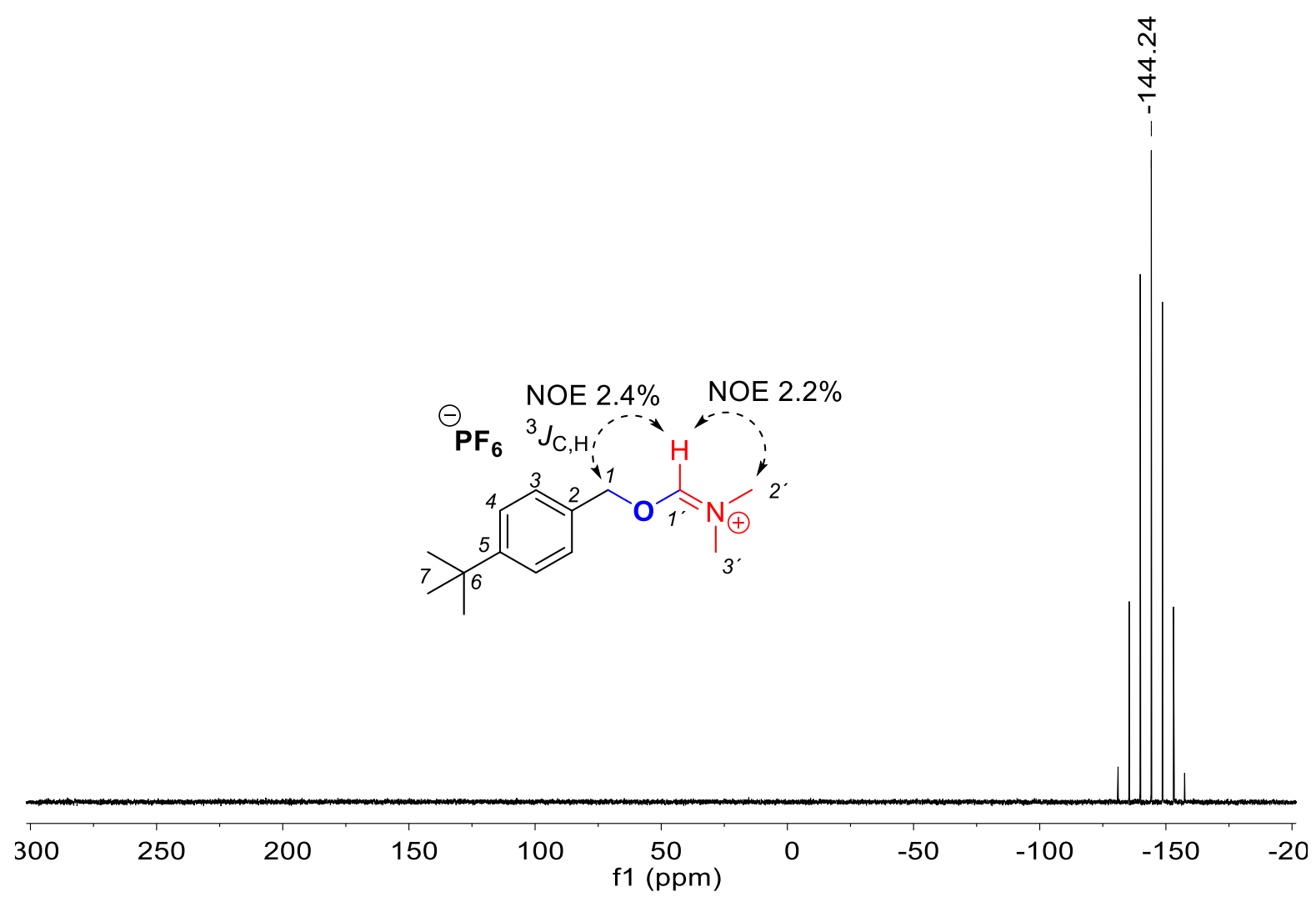

${ }^{31} \mathrm{P}$ NMR spectrum of $\mathrm{N}$-((4-tert-butylbenzyloxy)methylidene) dimethyliminium hexafluorophosphate (162 MHz, $\mathrm{CDCl}_{3}$ ). 


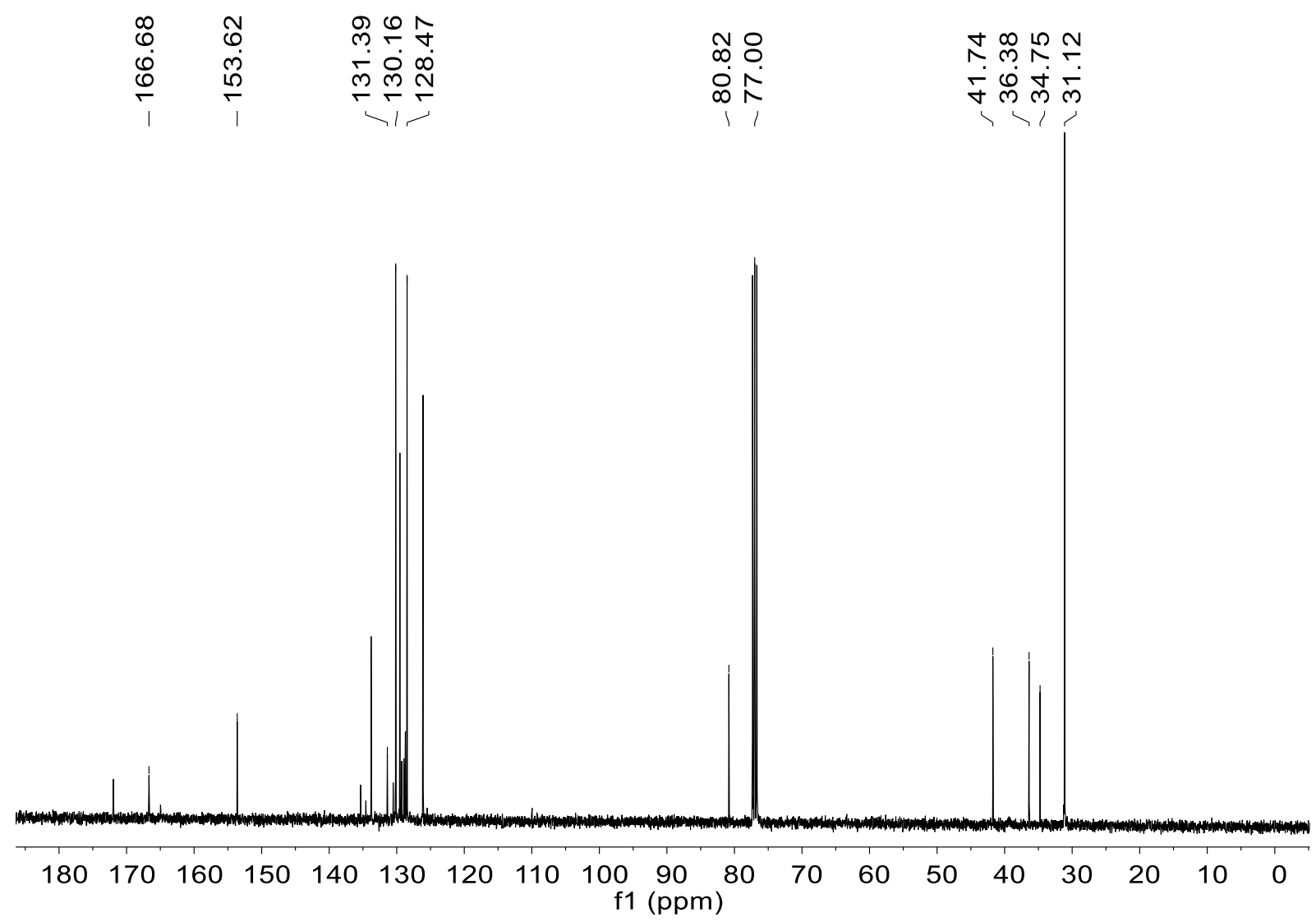

${ }^{13} \mathrm{C}$ NMR spectrum of $\mathrm{N}$-((4-tert-butylbenzyloxy)methylidene) dimethyliminium hexafluorophosphate $\left(100 \mathrm{MHz}, \mathrm{CDCl}_{3}\right)$.

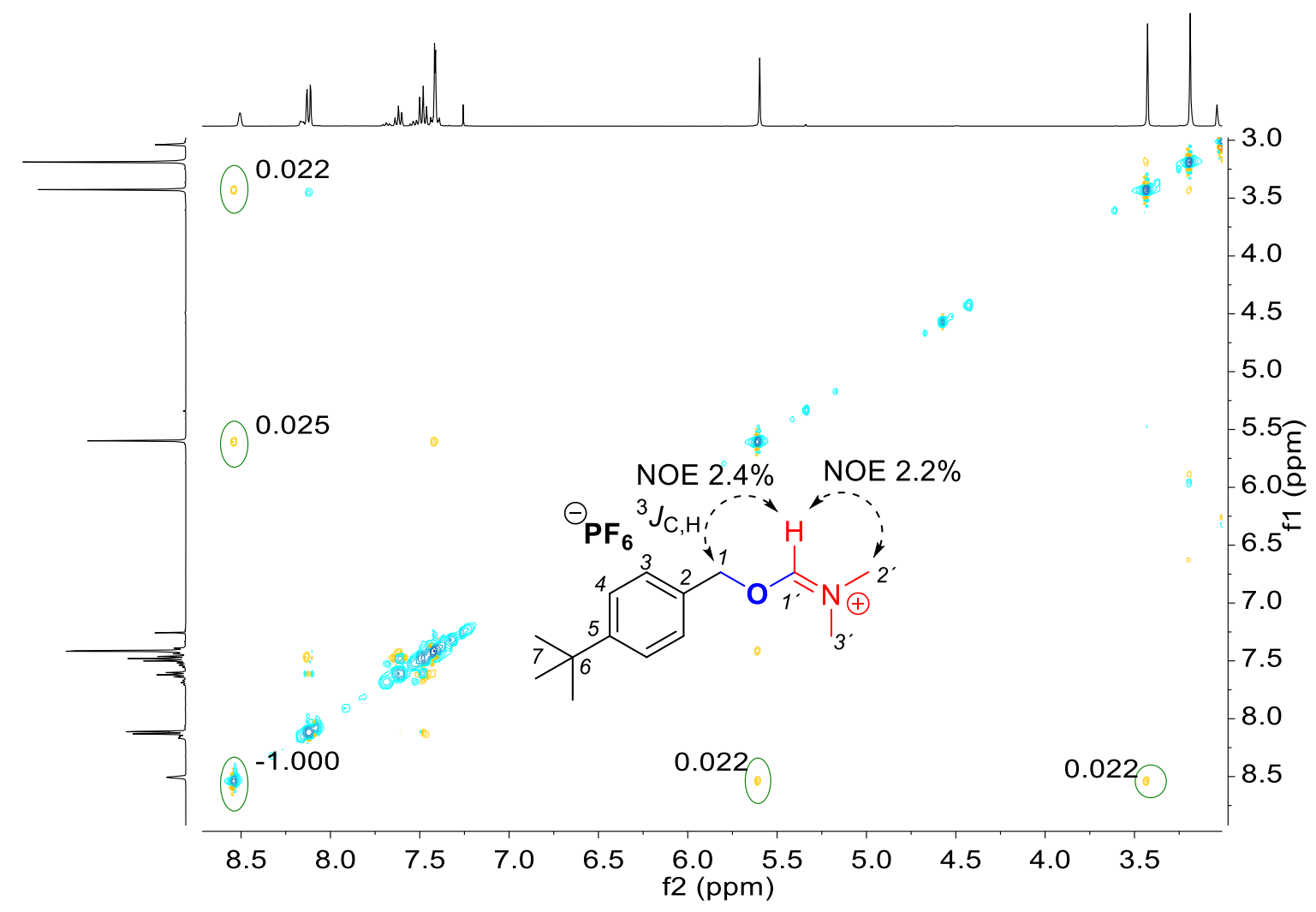

NOESY spectrum of $\mathrm{N}$-((4-tert-butylbenzyloxy)methylidene) dimethyliminium hexafluorophosphate $\left(400 \mathrm{MHz}, \mathrm{CDCl}_{3}\right)$. 


\subsubsection{Synthesis of $N$-((E-2-octen-1-yloxy)methylidene) dimethyliminium hexafluoro-} phosphate and difluorophosphate (IIIm/IVm)

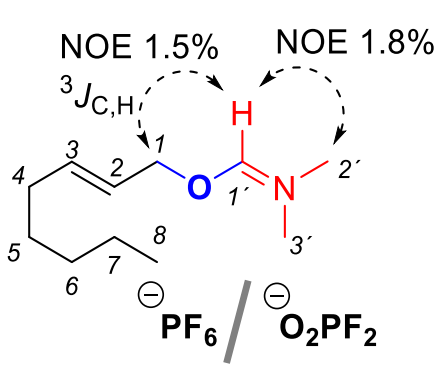

IIIm/IVm

In contrast to alkoxyiminium species IIIk, $\mathrm{PF}_{6}^{-}$and $\mathrm{O}_{2} \mathrm{PF}_{2}^{-}$salts do not show two distinct set of signals in ${ }^{1} \mathrm{H}$ and ${ }^{13} \mathrm{C}$ NMR spectrum. The double set of multiplets in the instance of Illk (chapter 2.3.2.3 on page 29) is likely explained by chelation of these counterions and thus a very close proximity of the phosphate anion to the iminium cation. Whereas usage of $\mathrm{NaPF}_{6}$ mainly causes formation of difluorophosphate IVm, application of $\mathrm{KPF}_{6}$ results in hexafluorophosphate IIIm as main compound.

With $\mathrm{NaPF}_{6}$ (PH1915): As described in general procedure 3 (chapter 2.2.3, page 15), NaPF 6 (134 mg, 0.80 mmol, 1.60 equiv), ${ }^{(8)} E$-2-octen-1-ol (77 $\mu \mathrm{L}, 65 \mathrm{mg}, 500 \mu \mathrm{mol}, 1.00$ equiv), DMF (41 $\mu \mathrm{L}, 38 \mathrm{mg}, 525 \mu \mathrm{mol}, 1.05$ equiv), $\mathrm{MeCN}(1.0 \mathrm{M})$ and $\mathrm{BzCl}$ (1.50 equiv) were combined and stirred at ambient temperature. After $1.5 \mathrm{~h}$ of stirring, ${ }^{1} \mathrm{H}$ NMR of a reaction sample verified $\geq 98 \%$ consumption of the starting material. ${ }^{19} \mathrm{~F}$ and ${ }^{13} \mathrm{P}$ NMR unraveled difluorophosphate IVm as main product.

With $\mathrm{KPF}_{6}\left(\mathrm{PH}_{1915)::}\right.$ According to general procedure 3 (chapter 2.2.3, page 15), the title compound was produced from $E$-2-octen-1-ol (500 $\mu$ mol, 1.00 equiv), DMF (1.05 equiv), $\mathrm{BzCl}$ (1.50 equiv) and $\mathrm{KPF}_{6}$ (1.60 equiv) in $\mathrm{MeCN}(1.0 \mathrm{M}) .{ }^{1} \mathrm{H}$ NMR of a sample taken after $30 \mathrm{~min}$ showed a mixture of IIIm and E-2-ocent-1-yl chloride in a ratio of 86:14. ${ }^{19} \mathrm{~F}$ and ${ }^{13} \mathrm{P}$ NMR indicated both, hexa- and difluorophosphate IIIm and IVm, whilst the first one was formed as main component in a ratio of $90: 10$ according to ${ }^{19} \mathrm{~F}$ NMR.

\section{N-((E-2-octen-1-yloxy)methylidene) dimethyliminium difluorophosphate (IVm)}

M $\left(\mathrm{C}_{11} \mathrm{H}_{22} \mathrm{~F}_{6} \mathrm{NOP}\right)=329.27 \mathrm{~g} / \mathrm{mol} ;{ }^{1} \mathrm{H}$ NMR $\left(400 \mathrm{MHz}, \mathrm{CDCl}_{3}\right) \delta[\mathrm{ppm}]=8.40\left(\mathrm{~s}, 1 \mathrm{H}, 1^{\prime}-\mathrm{H}\right)$, $6.10\left(\mathrm{dt},{ }^{3} J_{3,2}=15.3 \mathrm{~Hz},{ }^{3} J_{3,4}=6.7 \mathrm{~Hz}, 1 \mathrm{H}, 3-\mathrm{H}\right), 5.71-5.64(\mathrm{~m}, 1 \mathrm{H}, 2-\mathrm{H}), 5.07\left(\mathrm{~d},{ }^{3} J_{1,2}=7.4 \mathrm{~Hz}\right.$, $2 \mathrm{H}, 1-\mathrm{H}$ ), 3.44 (s, 3H, 2'-H), $3.23\left(\mathrm{~s}, 3 \mathrm{H}, 3^{\prime}-\mathrm{H}\right), 2.11$ (dt, $\left.{ }^{3} J_{3,2}={ }^{3} J_{3,4}=7.2 \mathrm{~Hz}, 2 \mathrm{H}, 4-\mathrm{H}\right), 1.44-$ $1.37(\mathrm{~m}, 2 \mathrm{H}, 5-\mathrm{H}), 1.34-1.25(\mathrm{~m}, 4 \mathrm{H}, 6-\mathrm{H}, 7-\mathrm{H}), 0.90-0.87(\mathrm{~m}, 3 \mathrm{H}, 8-\mathrm{H}) ;{ }^{19} \mathrm{~F}$ NMR $(376 \mathrm{MHz}$, $\mathrm{CDCl}_{3} \delta[\mathrm{ppm}]=83.98\left(\mathrm{~d},{ }^{1} \mathrm{~J}_{\mathrm{F} . \mathrm{P}}=971 \mathrm{~Hz}\right) ;{ }^{31} \mathrm{P} \mathbf{N M R}\left(162 \mathrm{MHz}, \mathrm{CDCl}_{3} \delta[\mathrm{ppm}]=-18.63\left(\mathrm{t},{ }^{1} \mathrm{JP}_{\mathrm{F}}\right.\right.$ $=973 \mathrm{~Hz}) ;{ }^{\prime 3} \mathrm{C}$ NMR $\left(100 \mathrm{MHz}, \mathrm{CDCl}_{3}\right) \delta[\mathrm{ppm}]=167.29$ (C-1', extracted from HMBC NMR), 144.03 (C-3), 120.37 (C-2), 80.26 (C-1), 41.74 (C-2'), 36.37 (C-3'), 32.23 (C-4), 31.29 (C-6), 27.97 (C-5), 22.38 (C-7), 13.94 (C-8).

(8) Use of $\mathrm{KPF}_{6}$ instead of $\mathrm{NaPF}_{6}$ furnished iminium species $\leq 91 \%$ conversion. 


\section{$\mathrm{N}$-((E-2-octen-1-yloxy)methylidene) dimethyliminium hexafluorophosphate (IIIm)}

Only separate signals are compiled.

$\mathbf{M}\left(\mathrm{C}_{11} \mathrm{H}_{22} \mathrm{~F}_{6} \mathrm{NOP}\right)=329.27 \mathrm{~g} / \mathrm{mol} ;{ }^{19} \mathrm{~F} \mathrm{NMR}\left(376 \mathrm{MHz}, \mathrm{CDCl}_{3} \delta[\mathrm{ppm}]-72.97\left(\mathrm{~d},{ }^{1} \mathrm{~J}_{\mathrm{F}, \mathrm{P}}=711 \mathrm{~Hz}\right)\right.$; ${ }^{31} \mathbf{P}$ NMR $\left(162 \mathrm{MHz}, \mathrm{CDCl}_{3} \delta[\mathrm{ppm}]=-144.40\left(\psi-\mathrm{t},{ }^{1} J_{\mathrm{P}, \mathrm{F}}=715 \mathrm{~Hz}, \boldsymbol{P F}_{6}{ }^{-}\right)\right.$.

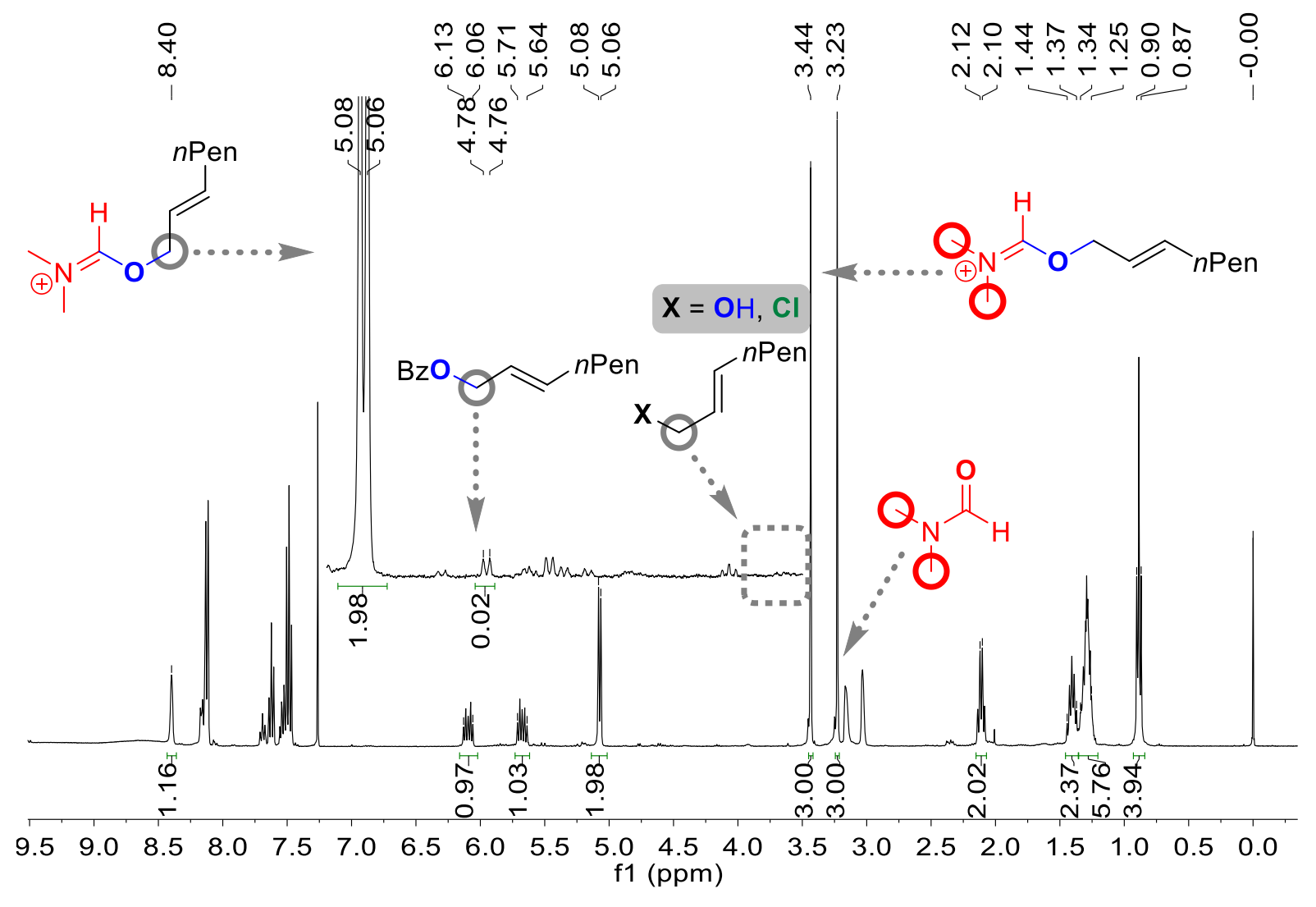

${ }^{1} \mathrm{H}$ NMR spectrum of $\mathrm{N}$-((E-2-octen-1-yloxy)methylidene) dimethyliminium hexafluorophosphate and difluorophosphate, synthesis with $\mathrm{NaPF}_{6}\left(400 \mathrm{MHz}, \mathrm{CDCl}_{3}\right)$. 


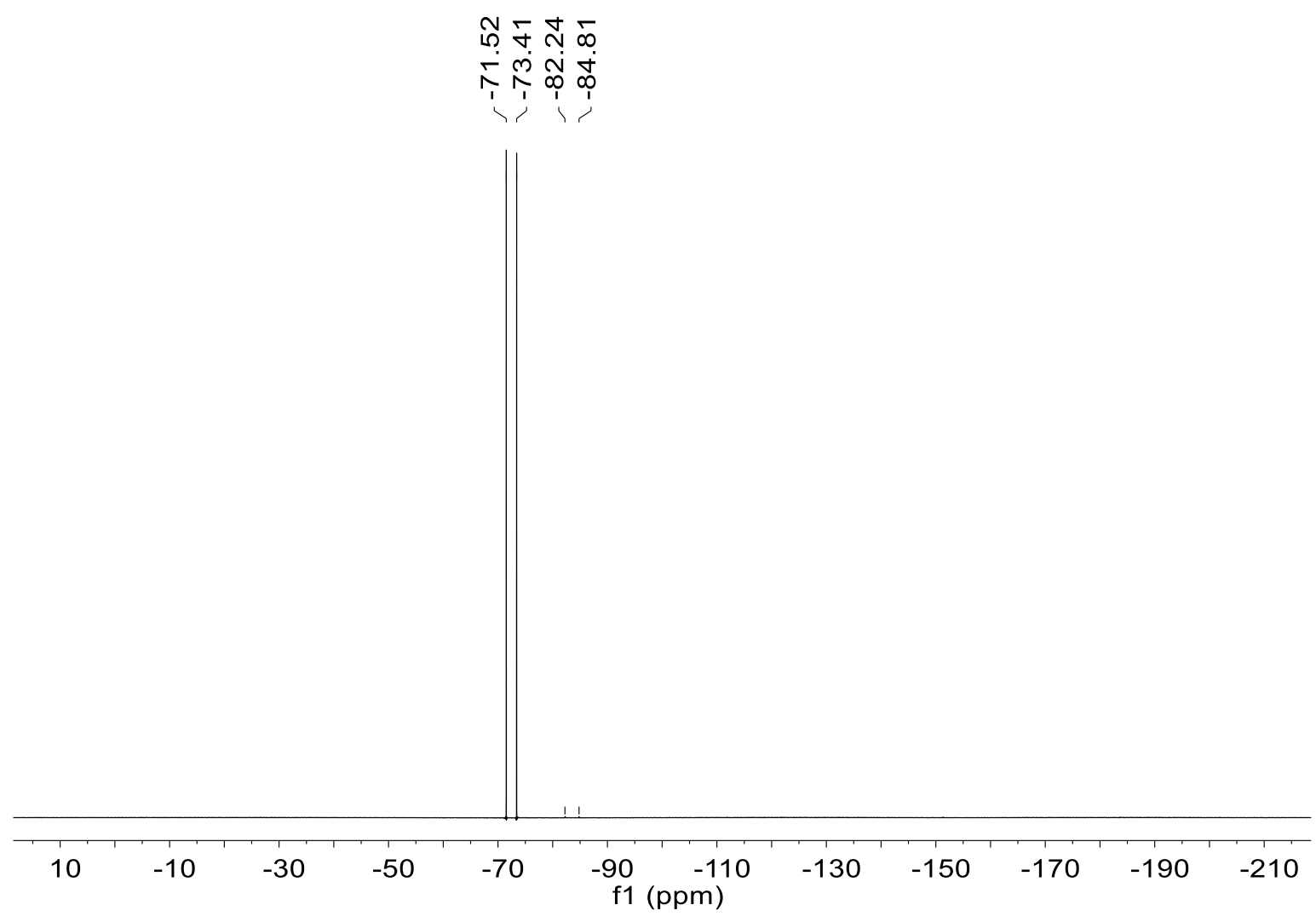

${ }^{19} \mathrm{~F}$ NMR spectrum of $\mathrm{N}$-((E-2-octen-1-yloxy)methylidene) dimethyliminium hexafluorophosphate and difluorophosphate, synthesis with $\mathrm{KPF}_{6}\left(376 \mathrm{MHz}, \mathrm{CDCl}_{3}\right.$ ).

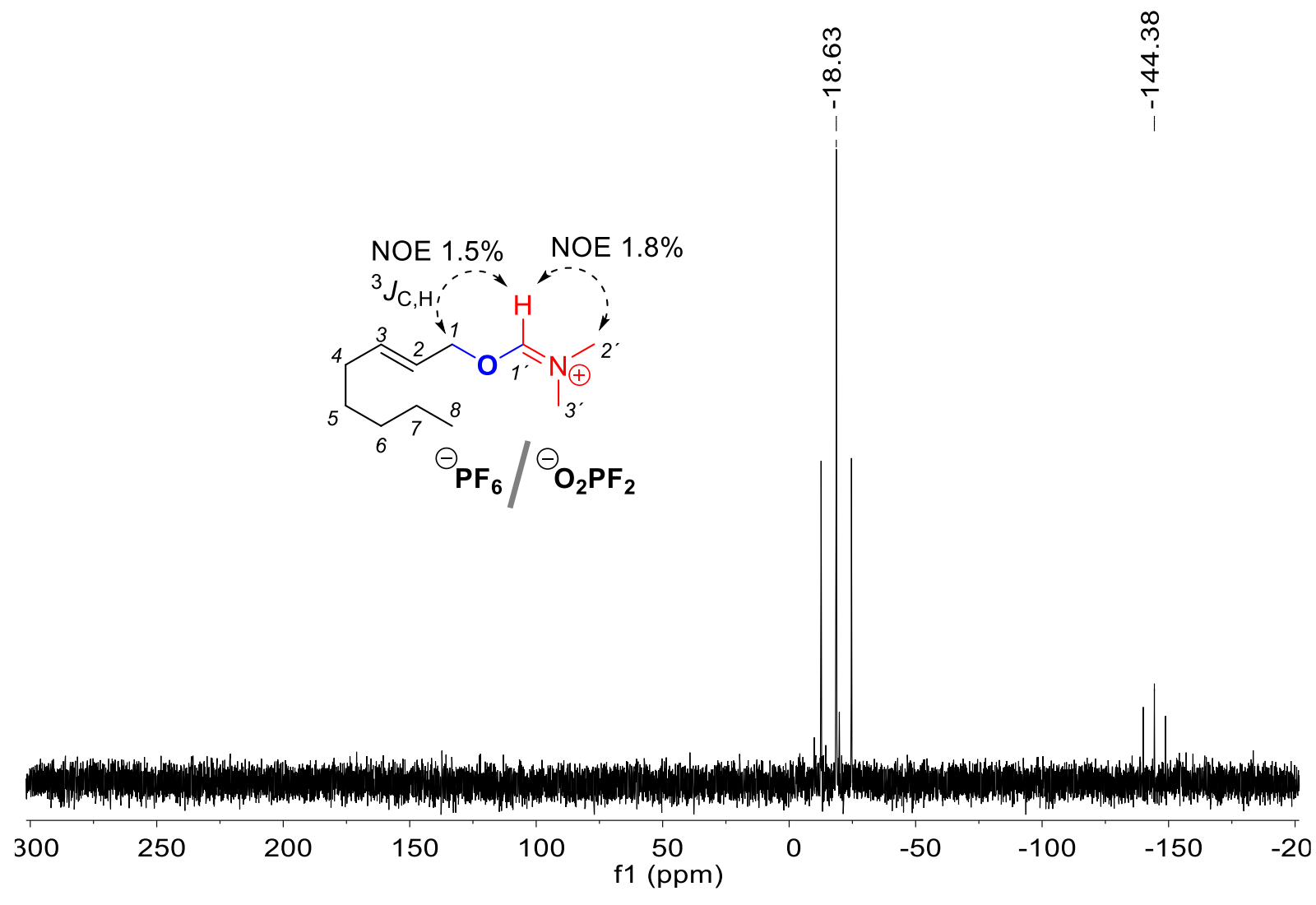

${ }^{31} \mathrm{P}$ NMR spectrum of $\mathrm{N}$-((E-2-octen-1-yloxy)methylidene) dimethyliminium hexafluorophosphate and difluorophosphate, synthesis with $\mathrm{NaPF}_{6}\left(162 \mathrm{MHz}, \mathrm{CDCl}_{3}\right.$ ). 


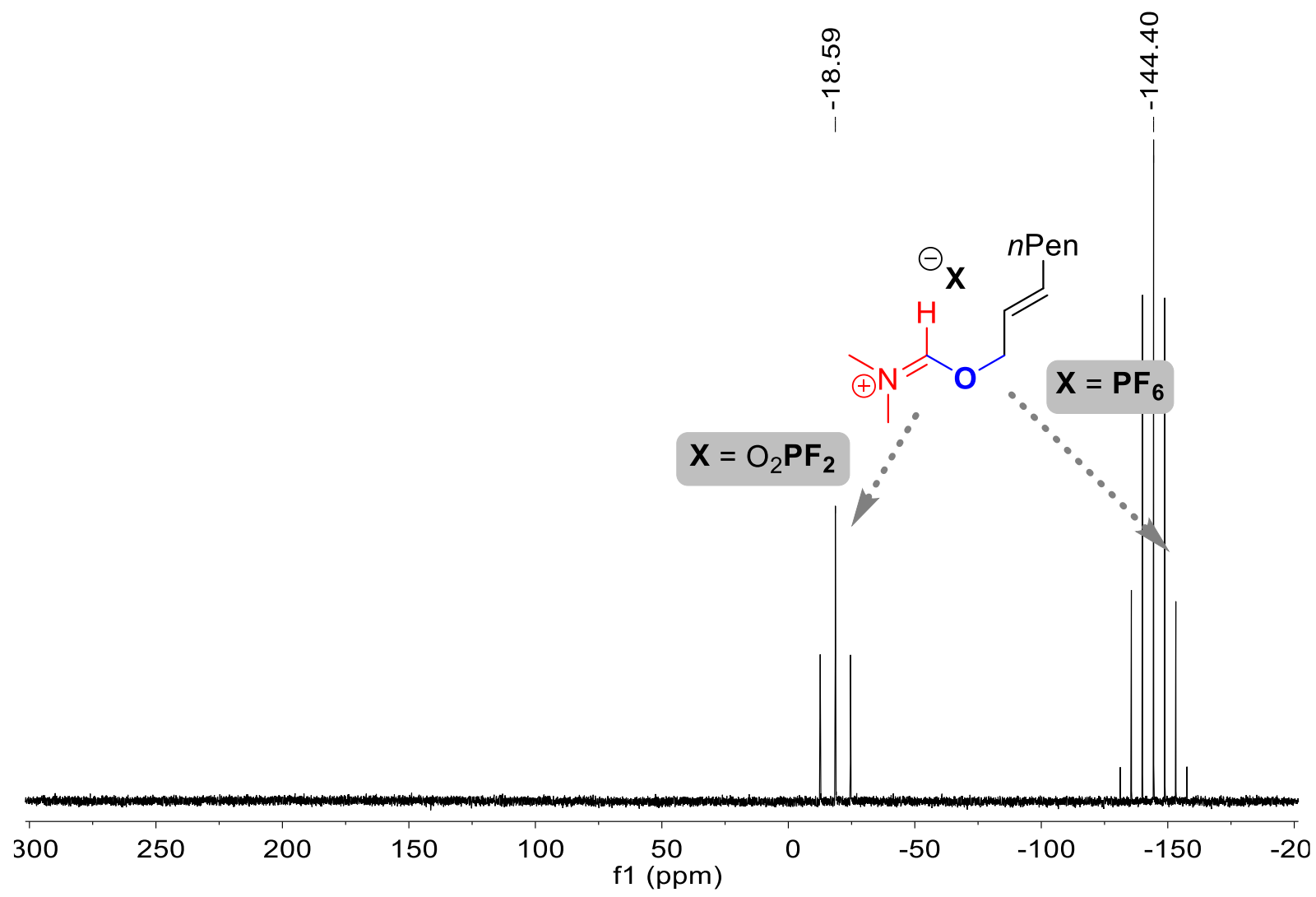

${ }^{31} \mathrm{P}$ NMR spectrum of $N$-((E-2-octen-1-yloxy)methylidene) dimethyliminium hexafluorophosphate and difluorophosphate, synthesis with $\mathrm{KPF}_{6}\left(162 \mathrm{MHz}, \mathrm{CDCl}_{3}\right)$.

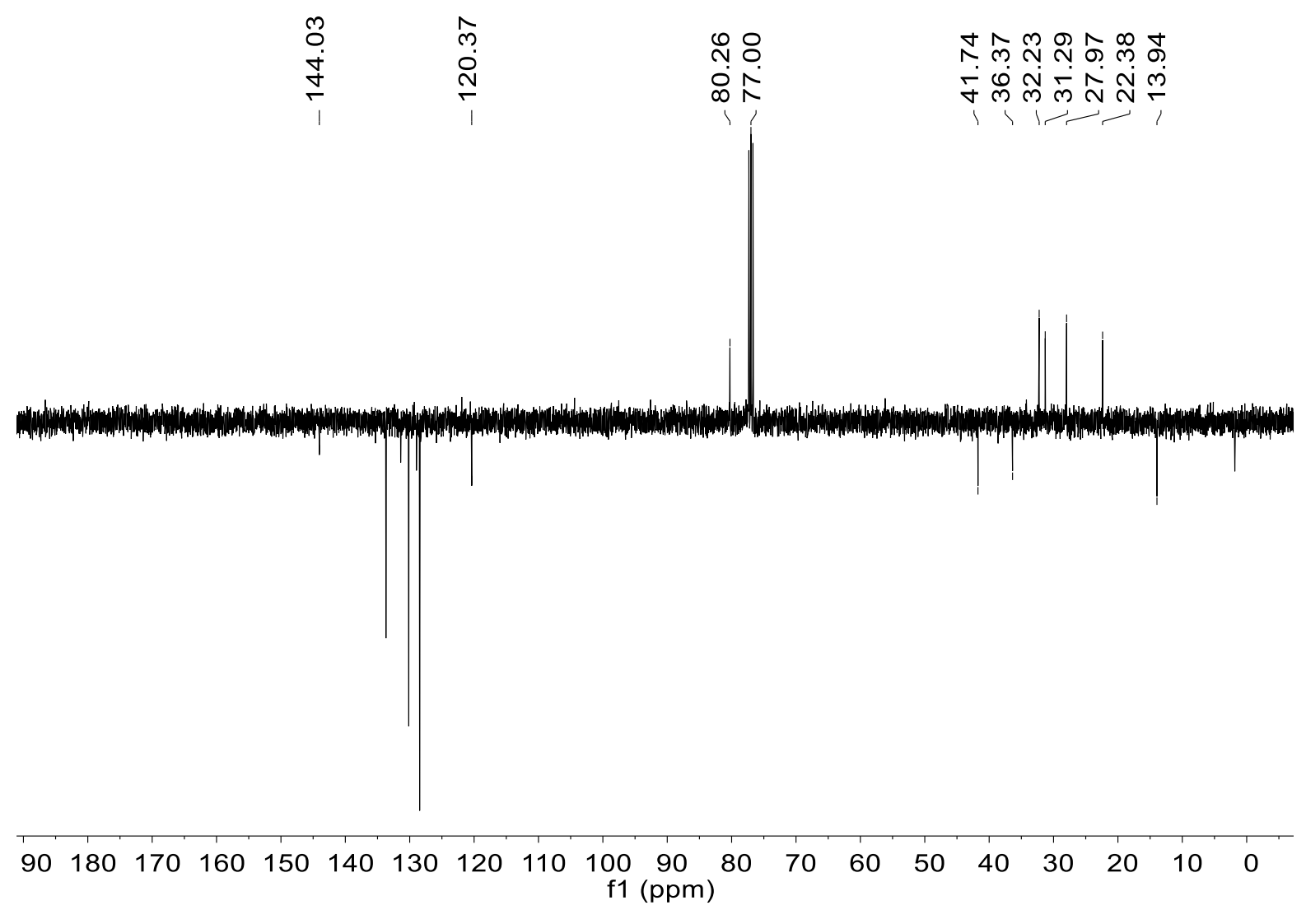

${ }^{13} \mathrm{C}$ NMR spectrum of $\mathrm{N}$-((E-2-octen-1-yloxy)methylidene) dimethyliminium hexafluorophosphate and difluorophosphate, synthesis with $\mathrm{NaPF}_{6}\left(100 \mathrm{MHz}, \mathrm{CDCl}_{3}\right)$. 


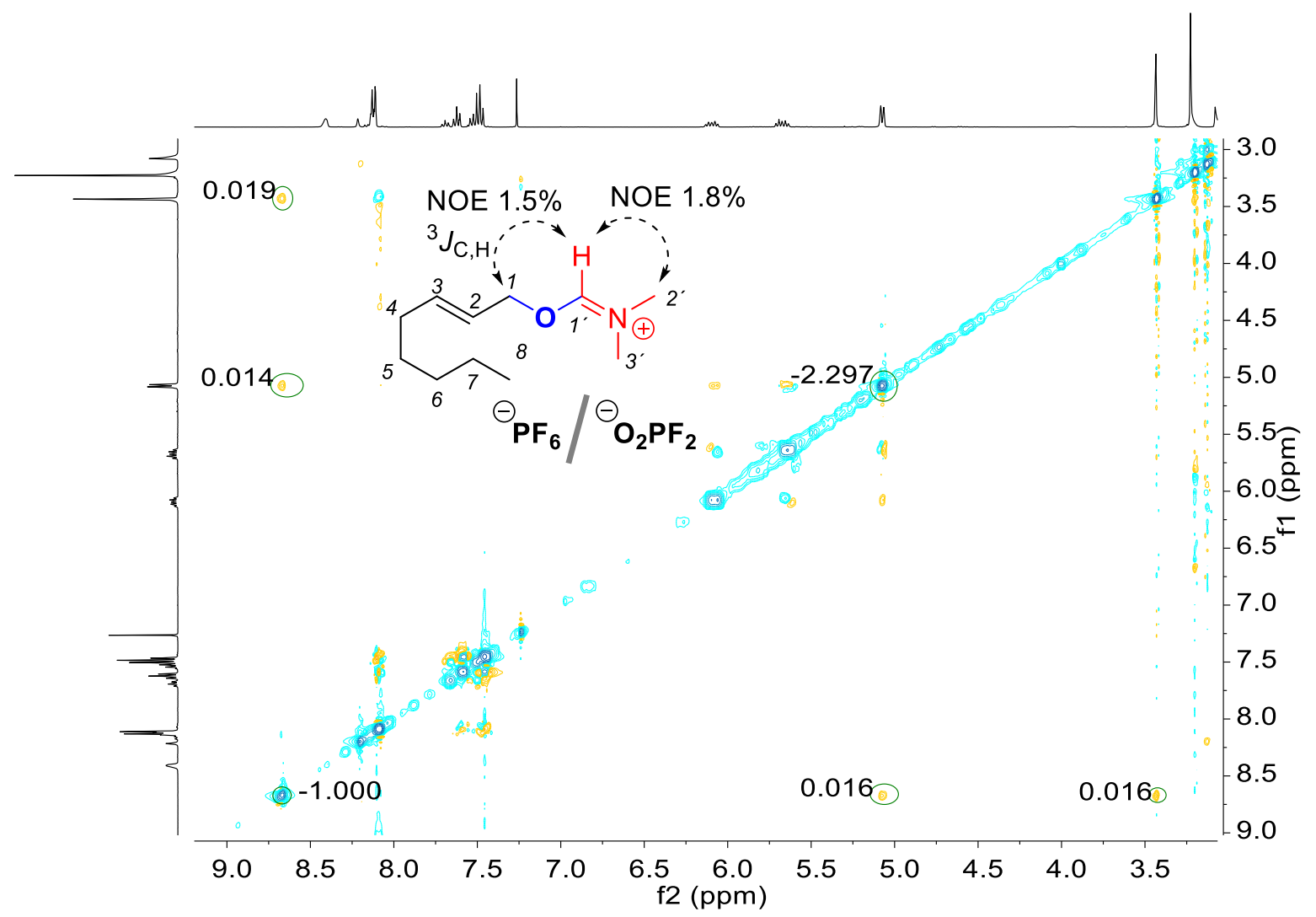

NOESY spectrum of $N$-((E-2-octen-1-yloxy)methylidene) dimethyliminium hexafluorophosphate and difluorophosphate, synthesis with $\mathrm{NaPF}_{6}\left(400 \mathrm{MHz}, \mathrm{CDCl}_{3}\right)$.

\subsubsection{Synthesis of rac- $N$-((4-Phenylbut-2-yloxy)methylidene) dimethyliminium hexa-} fluorophosphate (IIIg)

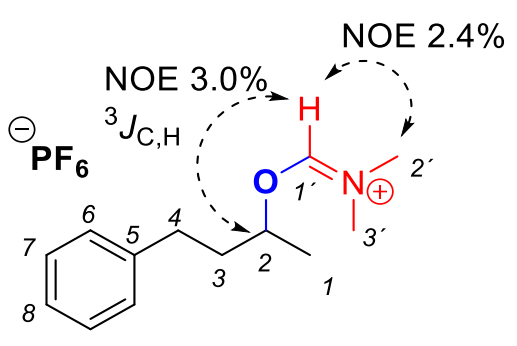

IIIg

PH1955: As given in general procedure 3 (chapter 2.2.3, page 15), racemic 4-phenyl-2-butanol (78 $\mu \mathrm{L}, 76 \mathrm{mg}$, $500 \mu \mathrm{L}, 1.00$ equiv) was allowed to react with $\mathrm{BzCl}$ (1.50 equiv), DMF (46 $\mu \mathrm{L}, 44 \mathrm{mg}, 0.60 \mathrm{mmol}, 1.20$ equiv) and $\mathrm{KPF}_{6}$ (1.60 equiv) in MeCN (1.0 M) for $5.5 \mathrm{~h} .{ }^{1} \mathrm{H}$ NMR confirmed a conversion $\geq 98 \%$ to the title compound.

$\mathbf{M}\left(\mathrm{C}_{13} \mathrm{H}_{20} \mathrm{~F}_{6} \mathrm{NOP}\right)=351.27 \mathrm{~g} / \mathrm{mol} ;{ }^{1} \mathrm{H}$ NMR $\left(400 \mathrm{MHz}, \mathrm{CDCl}_{3}\right) \delta[\mathrm{ppm}]=8.31\left(\mathrm{~s}, 1 \mathrm{H}, 1^{\prime}-\mathrm{H}\right)$, 7.29-7.25 (m, 2H, 7-H), 7.20-7.16 (m, 3H, 6-H, 8-H), 5.02-4.94 (m, 1H, 2-H)), 3.31 (s, 3H, 2'- $\mathrm{H})$, $3.02\left(\mathrm{~s}, 3 \mathrm{H}, 3^{\prime}-\mathrm{H}\right), 2.72\left(\psi-\mathrm{t},{ }^{3} \mathrm{~J}_{4,3}=7.7 \mathrm{~Hz}, 2 \mathrm{H}, 4-\mathrm{H}\right) 2.21-2.11\left(\mathrm{~m}, 1 \mathrm{H}, 3-\mathrm{H}_{\mathrm{a}}\right), 2.08-1.99(\mathrm{~m}, 1 \mathrm{H}$, $\left.3-\mathrm{H}_{\mathrm{b}}\right), 1.50\left(\mathrm{~d},{ }^{3} \mathrm{~J}_{1,2}=6.3 \mathrm{~Hz}, 3 \mathrm{H}, 1-\mathrm{H}\right) ;{ }^{19} \mathrm{~F} \mathbf{N M R}\left(376 \mathrm{MHz}, \mathrm{CDCl}_{3} \delta[\mathrm{ppm}]=-72.08\left(\mathrm{~d},{ }^{1} \mathrm{~J}_{\mathrm{F}, \mathrm{P}}=\right.\right.$ $712 \mathrm{~Hz}) ;{ }^{31} \mathbf{P}$ NMR $\left(162 \mathrm{MHz}, \mathrm{CDCl}_{3} \delta[\mathrm{ppm}]=-144.4\right.$ (sept, ${ }^{1} \mathrm{~J}_{\mathrm{P}, \mathrm{F}}=712 \mathrm{~Hz}$ ); ${ }^{13} \mathrm{C} \mathbf{~ N M R}$ $\left(100 \mathrm{MHz}, \mathrm{CDCl}_{3}\right) \delta[\mathrm{ppm}]=165.62\left(\mathrm{C}-1^{\prime}\right), 140.39(\mathrm{C}-5), 128.58(\mathrm{C}-7), 128.35(\mathrm{C}-6), 126.18$ (C-8), 89.78 (C-2), 41.51 (C-2'), 37.15 (C-3), 36.03 (C-3'), 31.14 (C-4), 20.37 (C-1). 


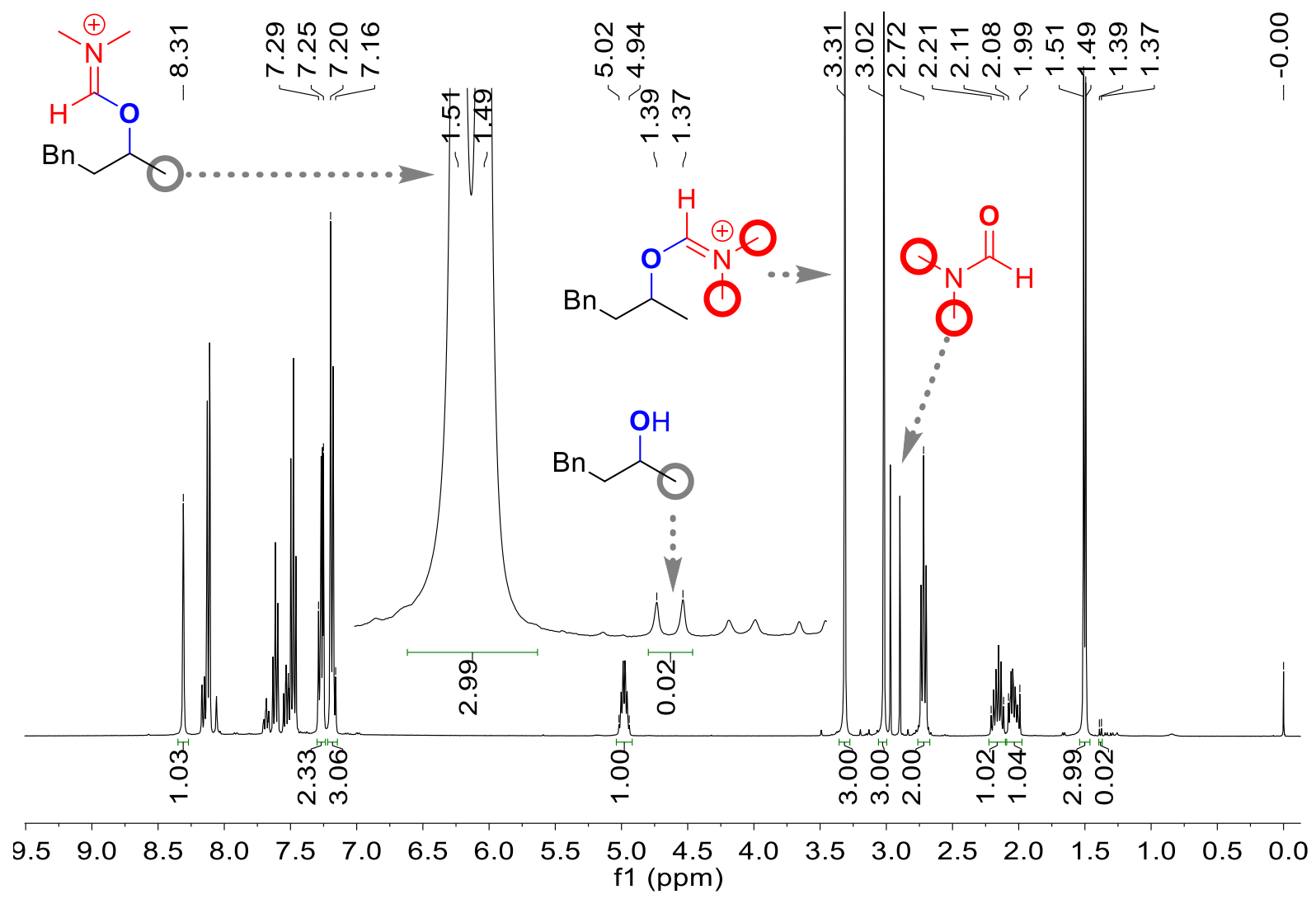

${ }^{1} \mathrm{H}$ NMR spectrum of rac- $\mathrm{N}-((4-$ phenylbut-2-yloxy)methylidene) dimethyliminium hexafluorophosphate $\left(400 \mathrm{MHz}, \mathrm{CDCl}_{3}\right)$.

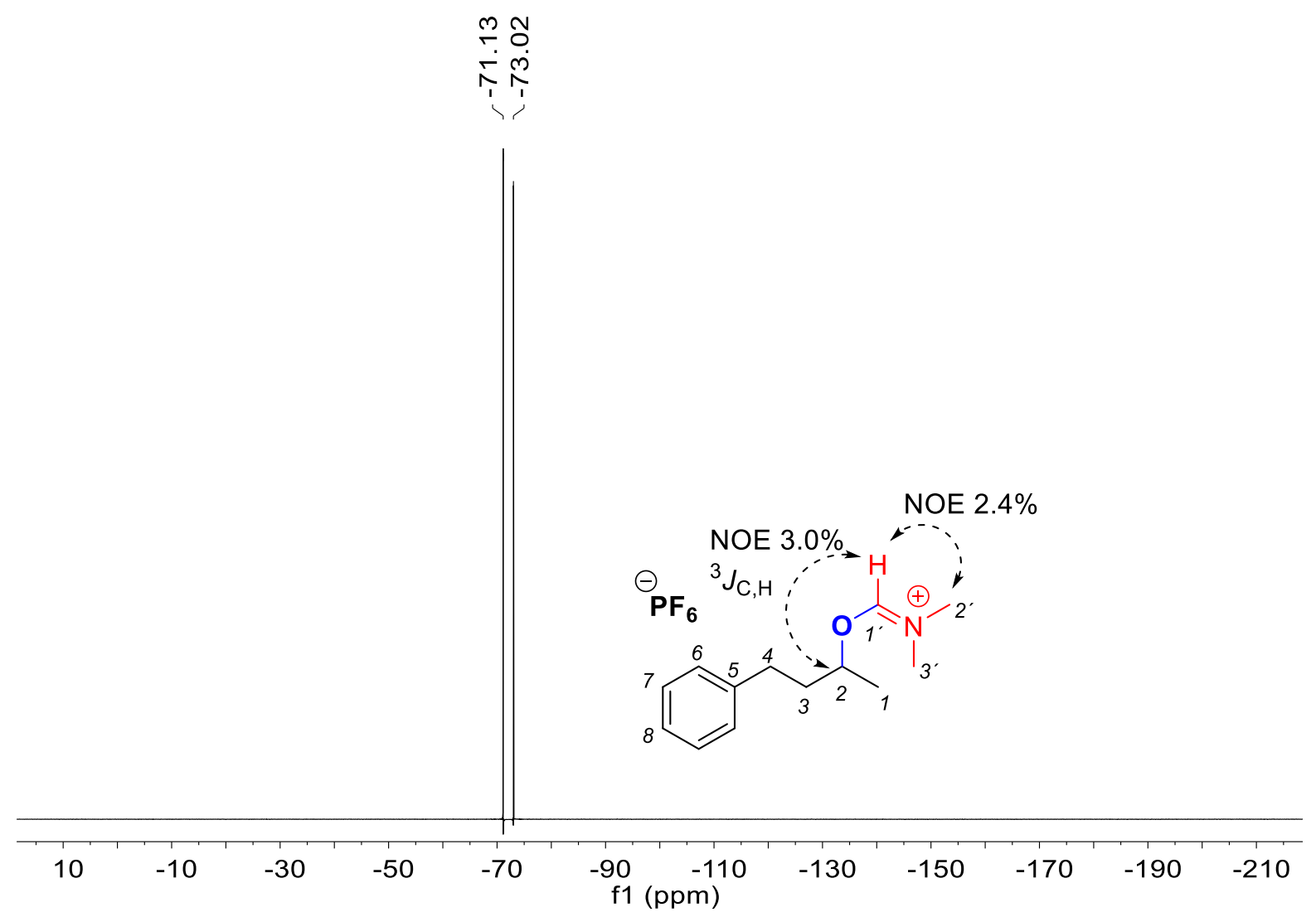

${ }^{19} \mathrm{~F}$ NMR spectrum of rac- $N$-((4-phenylbut-2-yloxy)methylidene) dimethyliminium hexafluorophosphate $\left(376 \mathrm{MHz}, \mathrm{CDCl}_{3}\right)$. 


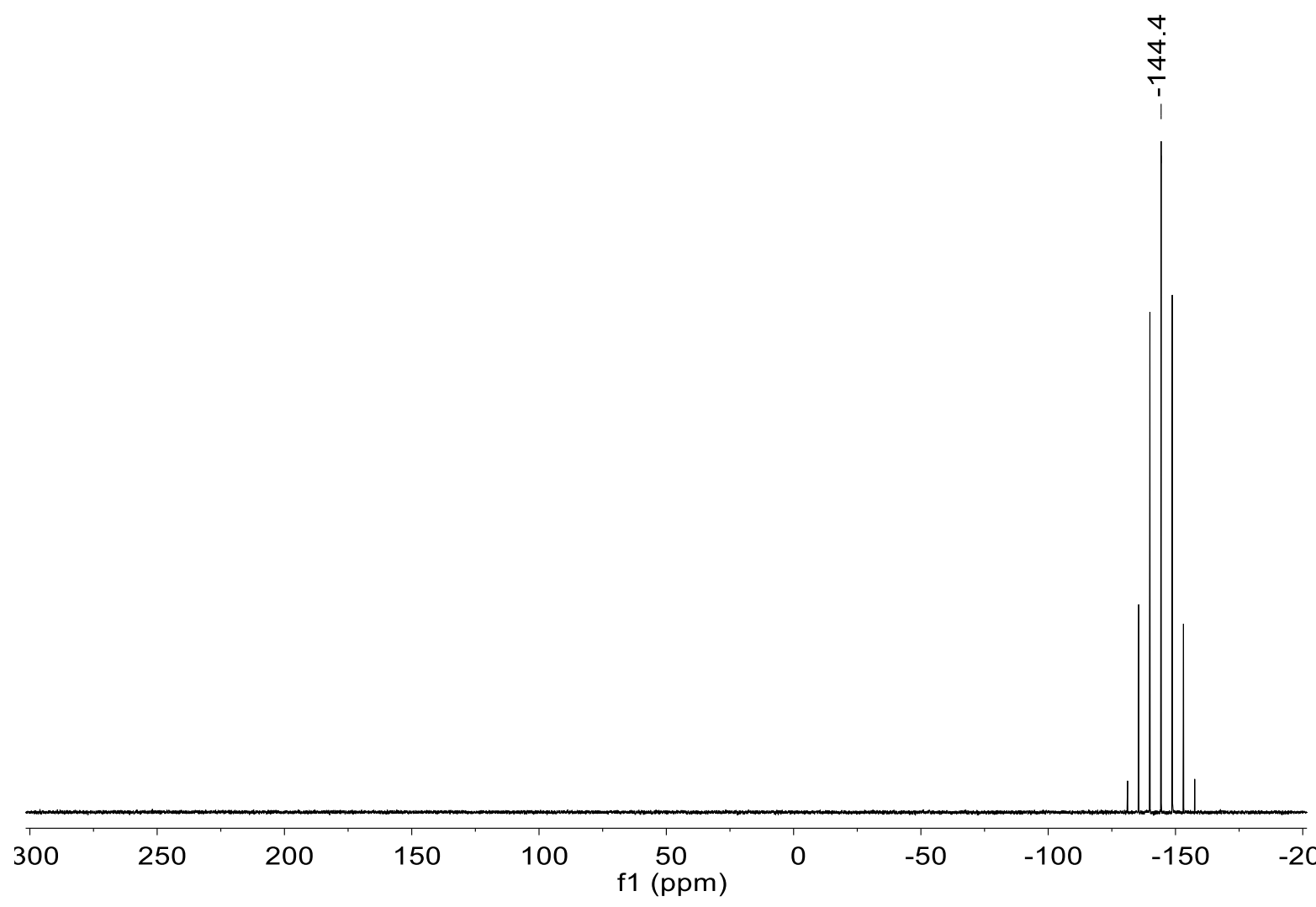

${ }^{31} \mathrm{P}$ NMR spectrum of rac- $N-((4-p h e n y l b u t-2-y l o x y)$ methylidene) dimethyliminium hexafluorophosphate $\left(162 \mathrm{MHz}, \mathrm{CDCl}_{3}\right)$.
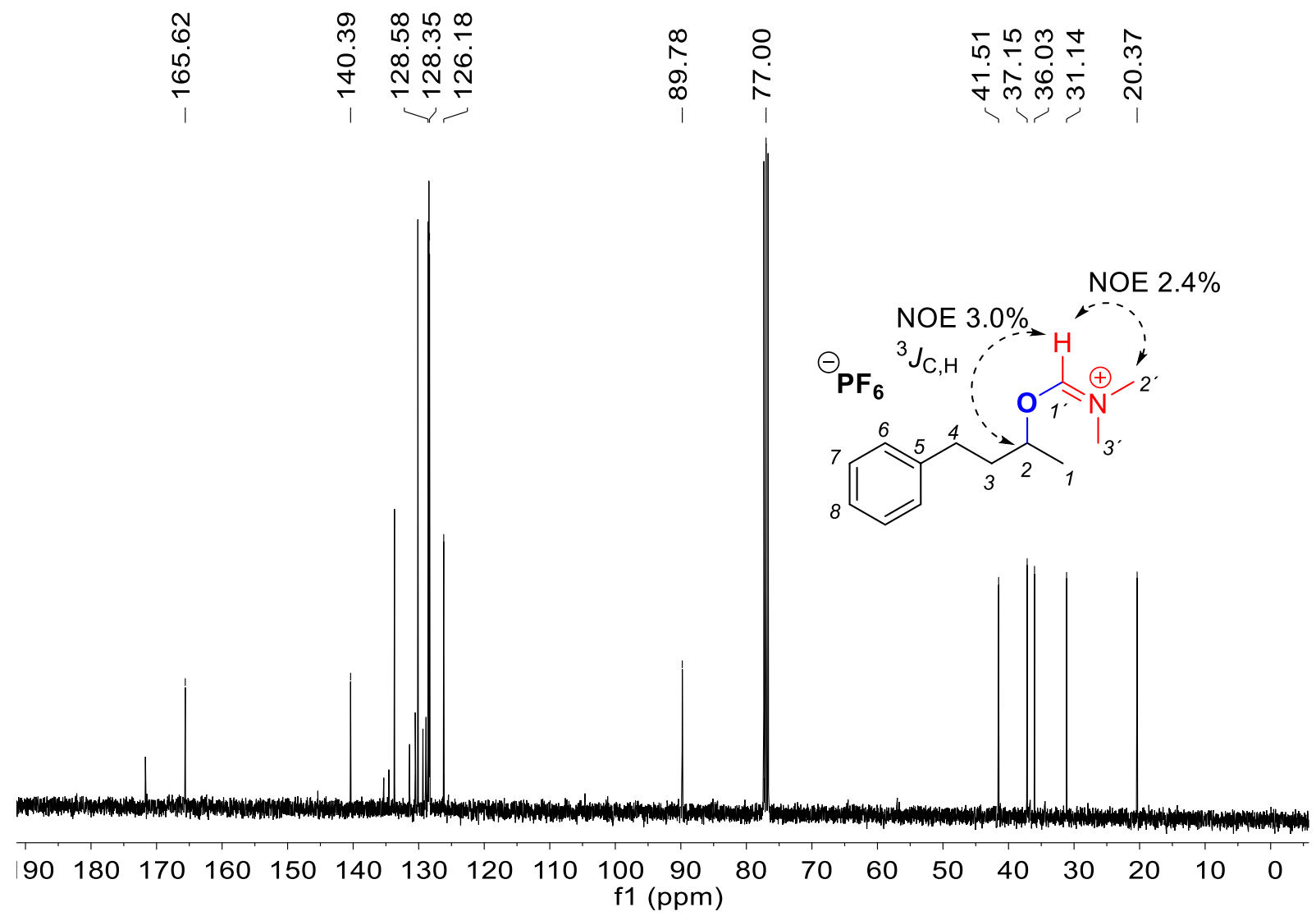

${ }^{13} \mathrm{C}$ NMR spectrum of rac-N-((4-phenylbut-2-yloxy)methylidene) dimethyliminium hexafluorophosphate $\left(100 \mathrm{MHz}, \mathrm{CDCl}_{3}\right)$. 


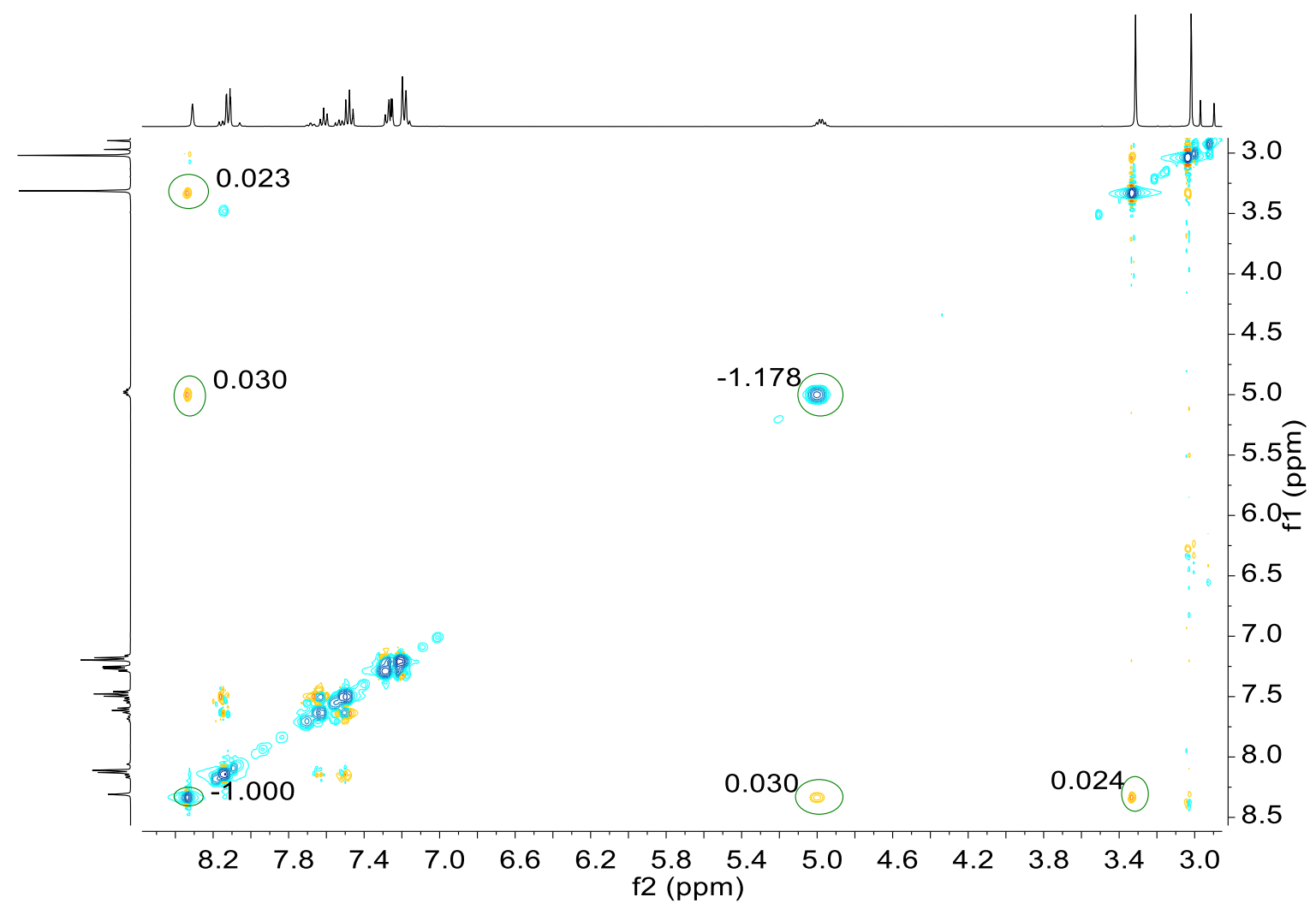

NOESY spectrum of $\mathrm{N}$-((1-dodecyloxy)methylidene) pyrrolidinium hexafluorophosphate (400 MHz, $\left.\mathrm{CDCl}_{3}\right)$.

\subsubsection{Transformation of Iminium Hexafluorophosphate IIII into 4-tert-Butylbenzyl} Chloride (3I) and formate 13I

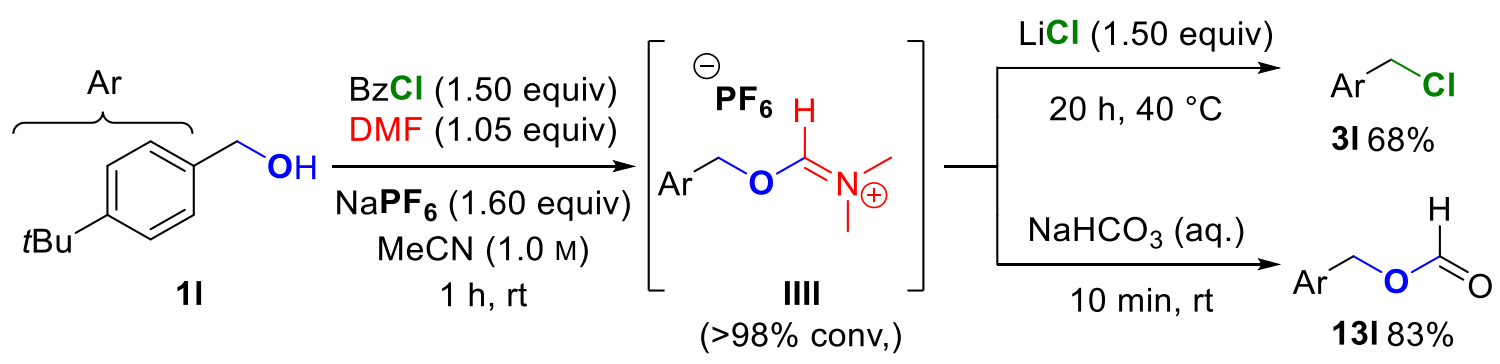

Iminium hexafluorophosphate IIII was prepared from 4-tert-butylbenzyl alcohol ( $89 \mu \mathrm{L}, 83 \mathrm{mg}$, $500 \mu \mathrm{L}, 1.00$ equiv), $\mathrm{NaPF}_{6}$ (1.60 equiv), DMF (41 mg, $39 \mu \mathrm{L}, 525 \mu \mathrm{mol}, 1.05$ equiv) and $\mathrm{BzCl}$ (1.50 equiv) in MeCN (500 $\mu \mathrm{L}, 1.0 \mathrm{M})$ under stirring for $1 \mathrm{~h}$ as stated in general procedure 3 (chapter 2.2.3, page 15).

Conversion to Chloride 3I (PH1925): Next, dry LiCl (32 mg, $0.75 \mathrm{mmol}, 1.50$ equiv) was added to the reaction suspension and the resulting mixture was heated to $40{ }^{\circ} \mathrm{C}$ for $20 \mathrm{~h}$. After cooling down to ambient temperature, the reaction mixture was taken up with $\mathrm{Et}_{2} \mathrm{O}(6 \mathrm{~mL})$ and $\mathrm{NaOH}(1.0 \mathrm{M})$ solution in water $(3 \mathrm{~mL})$, the phases were separated with the aid of a $20 \mathrm{~mL}$ syringe, the organic phase was dried over $\mathrm{MgSO}_{4}$, filtered, concentrated, and dried at 20 mbar 
for $5 \mathrm{~min}$ at the rotary evaporator. ${ }^{1} \mathrm{H}$ NMR with naphthalene as internal standard as described in general procedure 1 (chapter 2.2.1, page 14) showed chloride $3 \mathbf{l}$ in a yield of $68 \%$ alongside with 4-tert-butylbenzyl benzoate in $3 \%$. In addition, a second side-product was observed in $22 \%$ yield, which is most likely 4-tert-butylbenzyl acetamide. This amide could be formed through Ritter reaction of IIII and the solvent MeCN. Reaction with $\mathrm{LiCl}$ for $20 \mathrm{~h}$ at room temperature afforded chloride $\mathbf{3 l}$ in only $57 \%$ yield.

Conversion to Formate 13I (PH1940): To the reaction suspension was added saturated $\mathrm{NaHCO}_{3}$-solution (aq., $3 \mathrm{~mL}$ ) and the mixture was allowed to stir for $10 \mathrm{~min}$ at room temperature. Next, the mixture was diluted with $\mathrm{Et}_{2} \mathrm{O}(6 \mathrm{~mL})$ and water $(1 \mathrm{~mL})$, the organic phase was dried over $\mathrm{MgSO}_{4}$, filtered, concentrated under reduced pressure, and dried for $5 \mathrm{~min}$ at $20 \mathrm{mbar}$ at the rotary evaporator. ${ }^{1} \mathrm{H}$ NMR with naphthalene as internal standard showed formate $13 \mathrm{I}$ in $83 \%$ yield alongside with traces of starting material $1 \mathrm{ll}(8 \%)$ and 4-tertbutylbenzyl benzoate (2\%). 


\subsubsection{Analytical data of Miscellaneous Compounds}

Analytical data was obtained from commercial samples.

E-2-Octen-1-ol (1m)<smiles>CCCCCCCCC</smiles>

IIIg

$\mathbf{M}\left(\mathrm{C}_{8} \mathrm{H}_{16} \mathrm{O}\right)=128.22 \mathrm{~g} / \mathrm{mol}^{\prime}{ }^{1} \mathrm{H}$ NMR $\left(400 \mathrm{MHz}, \mathrm{CDCl}_{3}\right) \delta[\mathrm{ppm}]=5.74-5.59$ (m, 2H, 2-H, 3-H), 4.10-4.08 (m, 2H, 1-H), 2.07-2.01 (m, 2H, 4-H), 1.42-1.26 (m, 7H, 5- $\mathrm{H}, 6-\mathrm{H}, 7-\mathrm{H}, \mathrm{OH}), 0.91-0.87(\mathrm{~m}, 3 \mathrm{H}, 8-\mathrm{H})$.

\section{4-Phenyl-2-butanol (1g)}

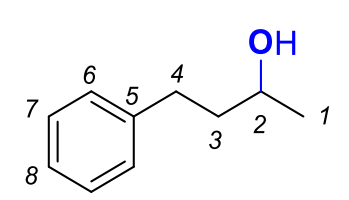

$\lg$

$\mathbf{M}\left(\mathrm{C}_{10} \mathrm{H}_{14} \mathrm{O}\right)=150.22 \mathrm{~g} / \mathrm{mol} ;{ }^{1} \mathbf{H}$ NMR $\left(400 \mathrm{MHz}, \mathrm{CDCl}_{3}\right) \delta[\mathrm{ppm}]=7.31$ $7.27(\mathrm{~m}, 2 \mathrm{H}, 7-\mathrm{H}), 7.21-7.16(\mathrm{~m}, 3 \mathrm{H}, 6-\mathrm{H}, 8-\mathrm{H}), 3.87-3.79(\mathrm{~m}, 1 \mathrm{H}, 2-\mathrm{H})$, 2.80-2.72 $\left(\mathrm{m}, 1 \mathrm{H}, 3-\mathrm{H}_{\mathrm{a}}\right), 2.71-2.63\left(\mathrm{~m}, 1 \mathrm{H}, 3-\mathrm{H}_{\mathrm{b}}\right), 1.84-1.72(\mathrm{~m}, 2 \mathrm{H}$, 4-H), 1.43 (br.s, $1 \mathrm{H}, \mathrm{OH}), 1.23,\left(\mathrm{~d},{ }^{3} J_{1,2}=6.2 \mathrm{~Hz}, 3 \mathrm{H}, 1-\mathrm{H}\right)$.

\section{$\mathrm{N}, \mathrm{N}$-Dimethylformamide (DMF)}<smiles>CN(C)C=O</smiles>

$\mathbf{M}\left(\mathrm{C}_{3} \mathrm{H}_{7} \mathrm{O}\right)=73.10 \mathrm{~g} / \mathrm{mol} ;{ }^{1} \mathrm{H}$ NMR $\left(400 \mathrm{MHz}, \mathrm{CDCl}_{3}\right) \delta[\mathrm{ppm}]=8.02(\mathrm{~s}, 1 \mathrm{H}$, $1-\mathrm{H}), 2.97(\mathrm{~s}, 3 \mathrm{H}, 2-\mathrm{H}), 2.88(\mathrm{~s}, 3 \mathrm{H}, 3-\mathrm{H}) ;{ }^{13} \mathrm{C} \mathrm{NMR}\left(100 \mathrm{MHz}, \mathrm{CDCl}_{3}\right) \delta[\mathrm{ppm}]=$ 162.33 (C-1), 36.31 (C-2), 31.23 (C-3). 


\subsubsection{Synthesis of Formamides}

The following formamides were obtained from commercial sources:

$N, N$-Dimethylformamide, [68-12-2]

Di-iso-propylformamide [2700-30-3]

$N$-Formylpyrrolidine ([3760-54-1]) was produced as described in our previous publication ${ }^{[5]}$ and is also commercially available.

\subsubsection{Synthesis of Morpholine-4-carbaldehyde (FMor)}<smiles>O=CN1CCOCC1</smiles>
Morpholine (1.31 mL, $15.0 \mathrm{mmol}, 1.00$ equiv), formamide $(0.60 \mathrm{~mL}, 15.0 \mathrm{mmol}$, 1.00 equiv) and PIDA (242 mg, $0.75 \mathrm{mmol}, 5 \mathrm{~mol} \%$ ) were reacted according to general procedure 6 (chapter 2.2.6, page 18). Purification by a small plug of silica gel ( $n$-pentane:EtOAc $=5: 1 \rightarrow n$-pentane:EtOAc $=1: 1$ ) furnished the title formamide as pale yellow oil (621 mg, $5.40 \mathrm{mmol}, 36 \%$ ).

$\mathbf{M}\left(\mathrm{C}_{5} \mathrm{H}_{9} \mathrm{NO}_{2}\right)=115.13 \mathrm{~g} / \mathrm{mol} ;{ }^{1} \mathrm{H}$ NMR $\left(300 \mathrm{MHz}, \mathrm{CDCl}_{3}\right) \delta[\mathrm{ppm}]=8.06(\mathrm{~s}, 1 \mathrm{H}), 3.83-3.62(\mathrm{~m}$, $4 \mathrm{H}), 3.61-3.52(\mathrm{~m}, 2 \mathrm{H}), 3.44-3.34(\mathrm{~m}, 2 \mathrm{H}) ;{ }^{13} \mathrm{C} \mathrm{NMR}\left(75 \mathrm{MHz}, \mathrm{CDCl}_{3}\right) \delta[\mathrm{ppm}]=160.95$, $67.27,66.47,45.86,40.66$.

The NMR data are in agreement with reported data. ${ }^{[11]}$
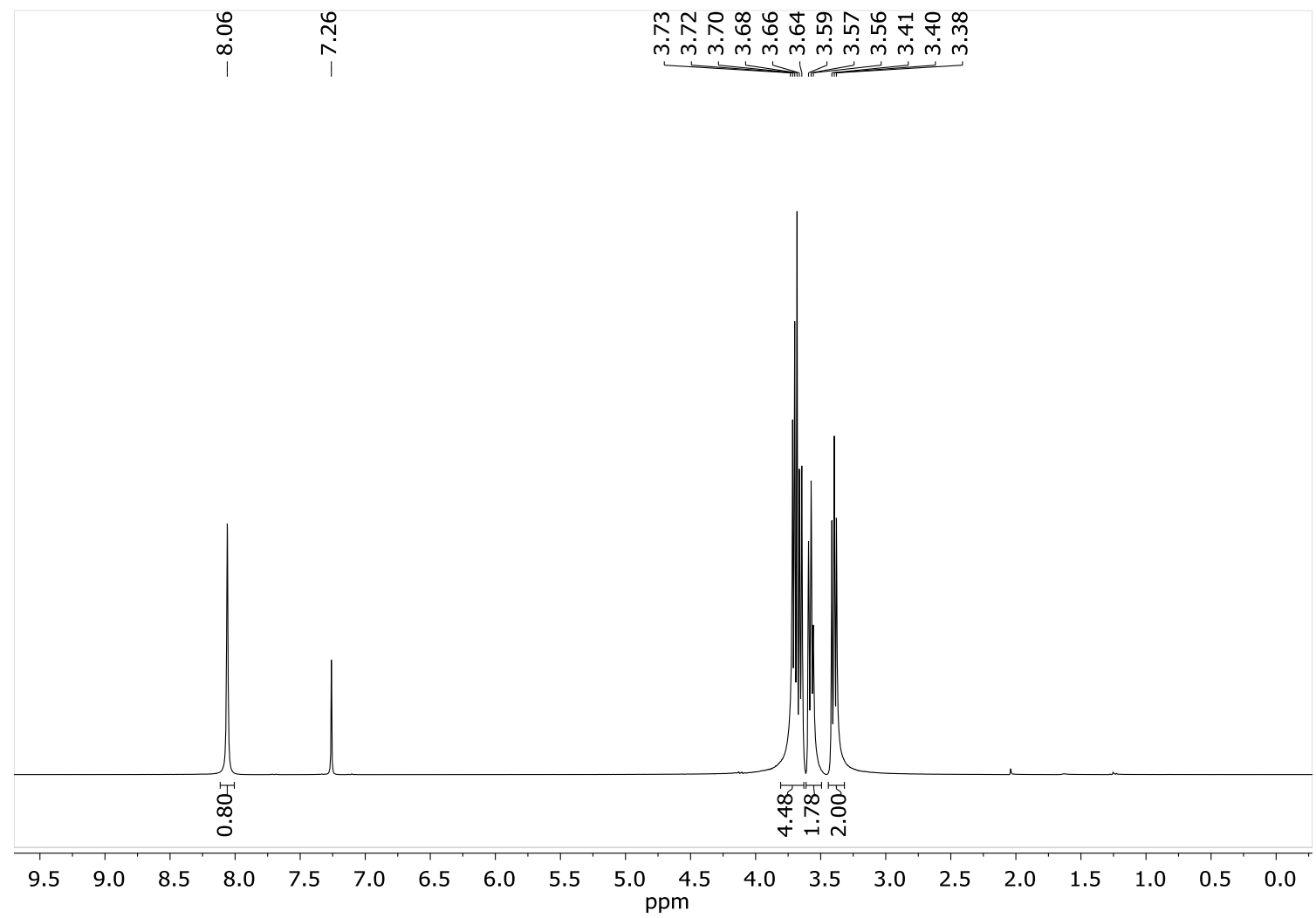

${ }^{1} \mathrm{H}$ NMR spectrum of morpholine-4-carbaldehyde (300 MHz, $\left.\mathrm{CDCl}_{3}\right)$. 


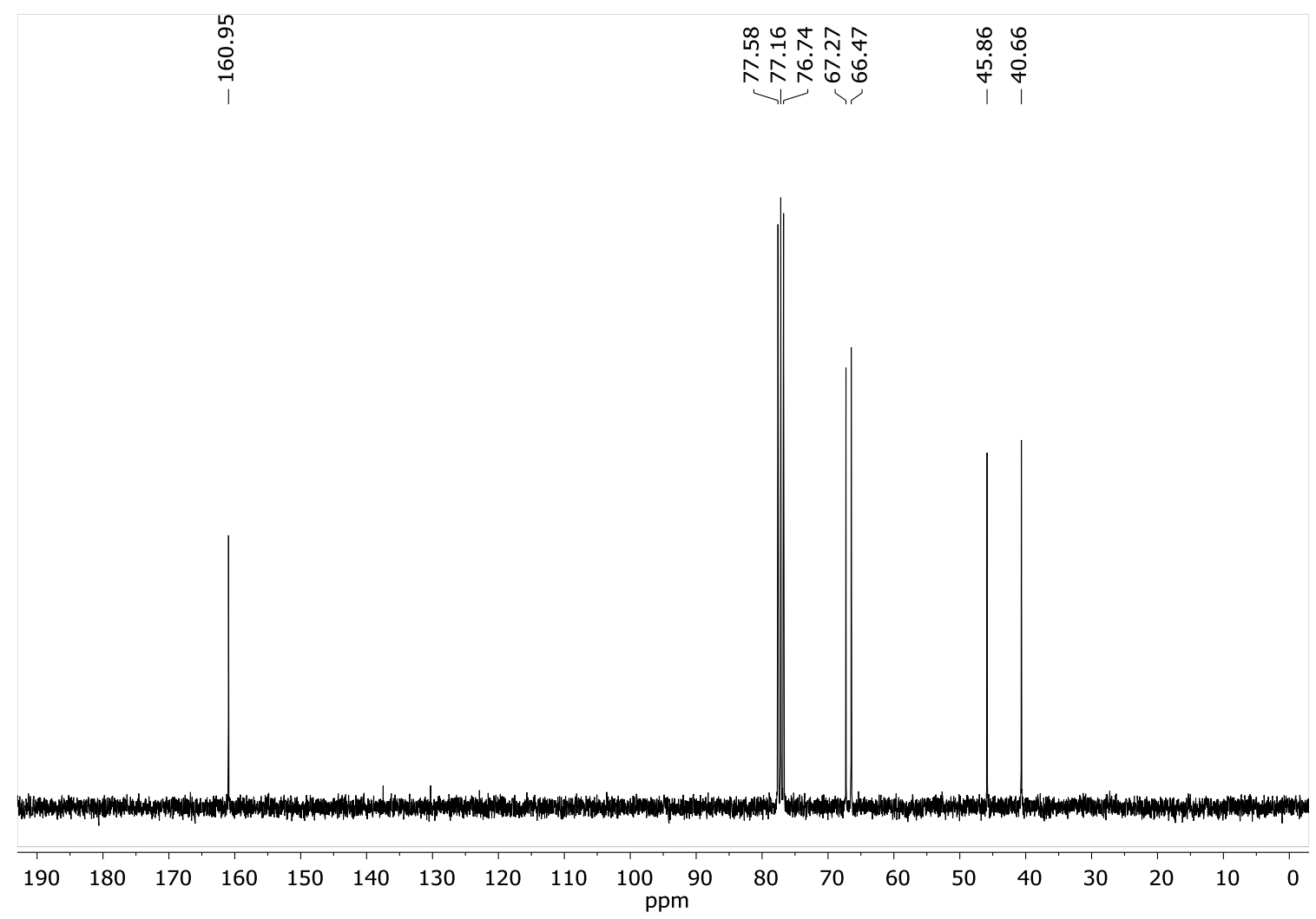

${ }^{13} \mathrm{C}$ NMR spectrum of morpholine-4-carbaldehyde $\left(75 \mathrm{MHz}, \mathrm{CDCl}_{3}\right)$.

\subsubsection{Synthesis of Piperidine-1-carbaldehyde (FPip)}

o Piperidine (1.49 mL, $15.0 \mathrm{mmol}, 1.00$ equiv), formamide $(0.60 \mathrm{~mL}, 15.0 \mathrm{mmol}$, 1.00 equiv) and PIDA (242 mg, $0.75 \mathrm{mmol}, 5 \mathrm{~mol} \%$ ) were reacted according to general procedure 6 (chapter 2.2.6, page 18). Purification by a small plug of silica gel ( $n$-pentane:EtOAc $=5: 1 \rightarrow n$-pentane:EtOAc $=2: 1$ ) furnished the title formamide as pale yellow oil ( $810 \mathrm{mg}, 7.20 \mathrm{mmol}, 48 \%)$.

$\mathbf{M}\left(\mathrm{C}_{6} \mathrm{H}_{11} \mathrm{NO}\right)=113.16 \mathrm{~g} / \mathrm{mol} ;{ }^{1} \mathrm{H}$ NMR $\left(300 \mathrm{MHz}, \mathrm{CDCl}_{3}\right) \delta[\mathrm{ppm}]=7.93(\mathrm{~s}, 1 \mathrm{H}), 3.44-3.38(\mathrm{~m}$, $2 \mathrm{H}), \quad 3.27-3.21(\mathrm{~m}, 2 \mathrm{H}),{ }_{1.66-1.58}(\mathrm{~m}, 2 \mathrm{H}), 1.55-1.43(\mathrm{~m}, 4 \mathrm{H}),{ }^{13} \mathrm{C}$ NMR $(75 \mathrm{MHz}$, $\left.\mathrm{CDCl}_{3}\right) \delta[\mathrm{ppm}]=160.75,46.79,40.60,26.60,25.09,24.73$.

The NMR data are in agreement with reported data. ${ }^{[11]}$ 


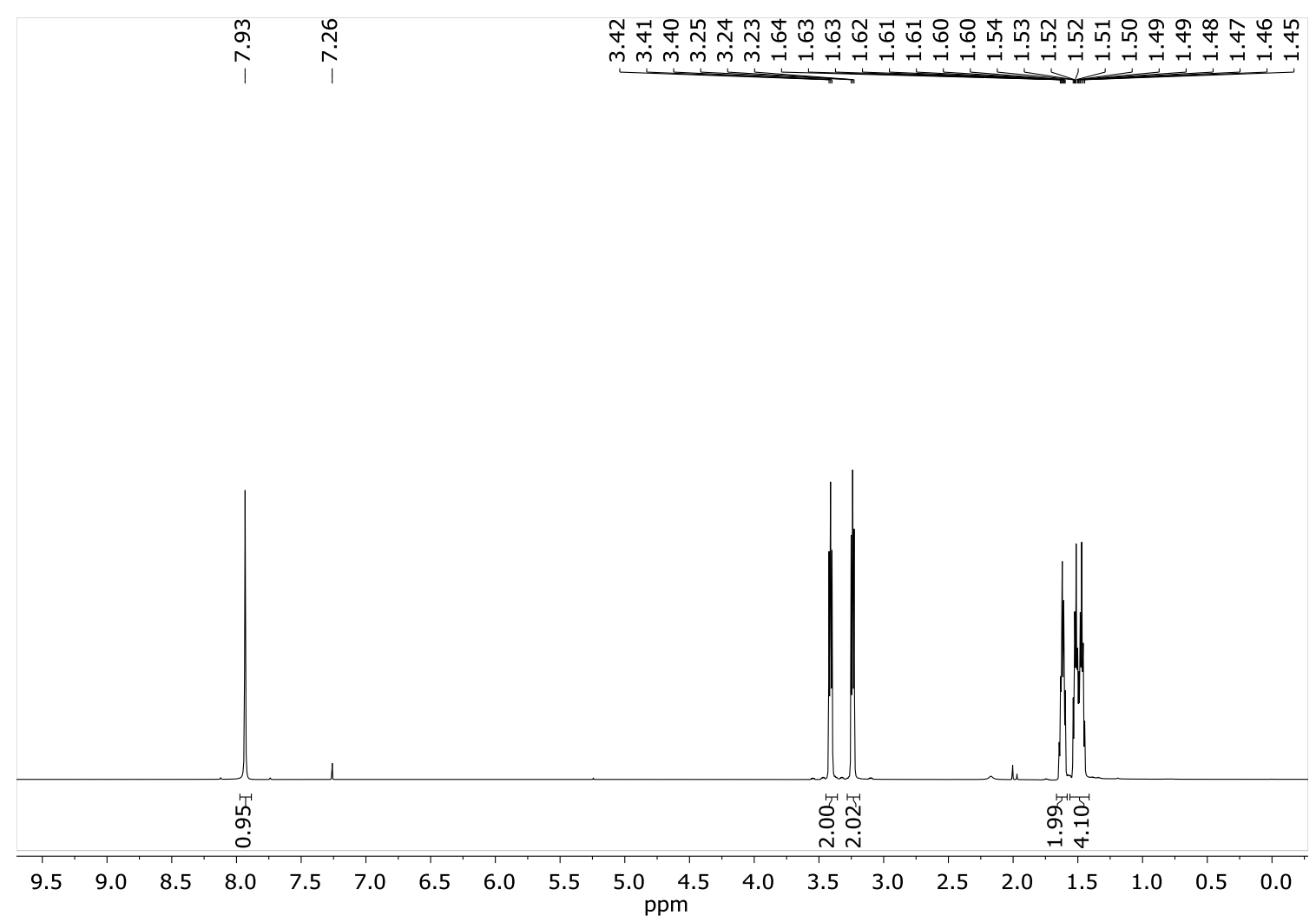

${ }^{1} \mathrm{H}$ NMR spectrum of piperidine-1-carbaldehyde $\left(300 \mathrm{MHz}, \mathrm{CDCl}_{3}\right)$.

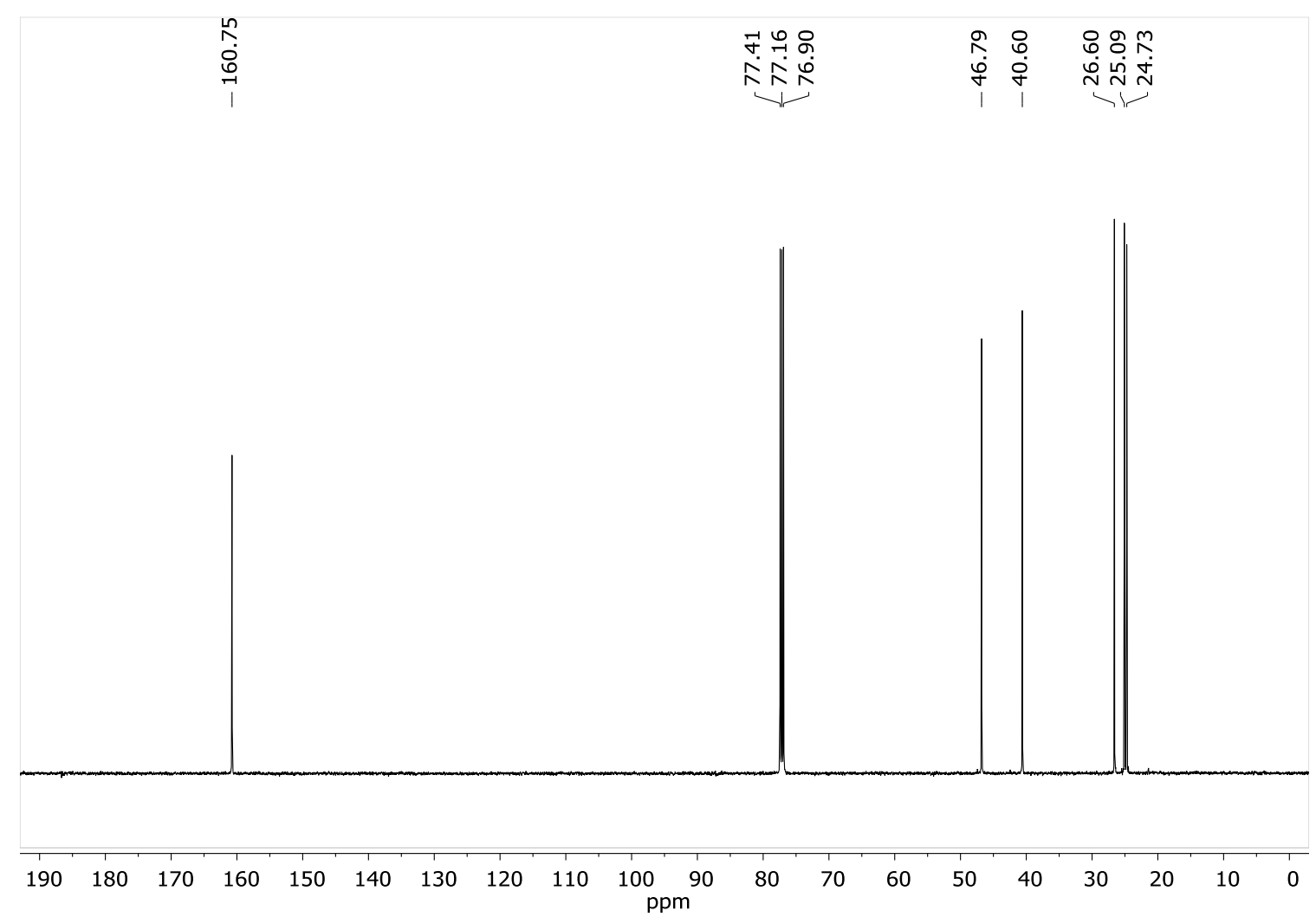

${ }^{13} \mathrm{C}$ NMR spectrum of piperidine-1-carbaldehyde (75 $\left.\mathrm{MHz}, \mathrm{CDCl}_{3}\right)$. 


\subsubsection{Synthesis of $\mathbf{N}$-tert-Butylformamide (tBuF)}

o tert-Butylamine $(21.0 \mathrm{~mL}, 200 \mathrm{mmol}, 1.00$ equiv), formamide $(7.97 \mathrm{~mL}$, $\mathrm{H}_{\mathrm{H}}{ }^{-t \mathrm{Bu}} 200 \mathrm{mmol}, 1.00$ equiv) and PIDA (3.22 g, $10.0 \mathrm{mmol}, 5 \mathrm{~mol} \%$ ) were reacted according to general procedure 6 (chapter 2.2.6, page 18). Purification by a small plug of silica gel ( $n$-pentane:EtOAc $=5: 1 \rightarrow n$-pentane:EtOAc $=2: 1$ ) furnished the title formamide as pale yellow oil (12.4 g, $123 \mathrm{mmol}, 62 \%)$.

$\mathbf{M}\left(\mathrm{C}_{5} \mathrm{H}_{11} \mathrm{NO}\right)=101.15 \mathrm{~g} / \mathrm{mol}$; the formamide $t \mathrm{BuF}$ was obtained as mixture of rotamers $52: 48$; resolved signals of the major rotamer: ${ }^{1} \mathbf{H} \mathbf{~ N M R}\left(300 \mathrm{MHz}, \mathrm{CDCl}_{3}\right) \delta[\mathrm{ppm}]=8.25$ (d, $J=12.4 \mathrm{~Hz}, \quad 1 \mathrm{H}), \quad 5.39 \quad$ (br.s, $\quad 1 \mathrm{H}, \quad \mathrm{NH}), \quad 1.32 \quad(\mathrm{~s}, \quad 9 \mathrm{H}) ; \quad{ }^{13} \mathrm{C} \mathbf{N M R} \quad(75 \mathrm{MHz}$, $\left.\mathrm{CDCl}_{3}\right) \delta[\mathrm{ppm}]=163.09,51.47,31.01$; resolved signals of the minor rotamer: ${ }^{1} \mathbf{H} \mathbf{N M R}$ $\left(300 \mathrm{MHz}, \mathrm{CDCl}_{3}\right) \delta[\mathrm{ppm}]=8.02(\mathrm{~d}, J=1.20 \mathrm{~Hz}, 1 \mathrm{H}), 6.16$ (br.s, $\left.1 \mathrm{H}, \mathrm{NH}\right), 1.36(\mathrm{~s}, 9 \mathrm{H})$; ${ }^{13} \mathrm{C} \mathrm{NMR}\left(75 \mathrm{MHz}, \mathrm{CDCl}_{3}\right) \delta[\mathrm{ppm}]=160.65,50.45,29.03$.

The NMR data are in agreement with reported data. ${ }^{[12]}$

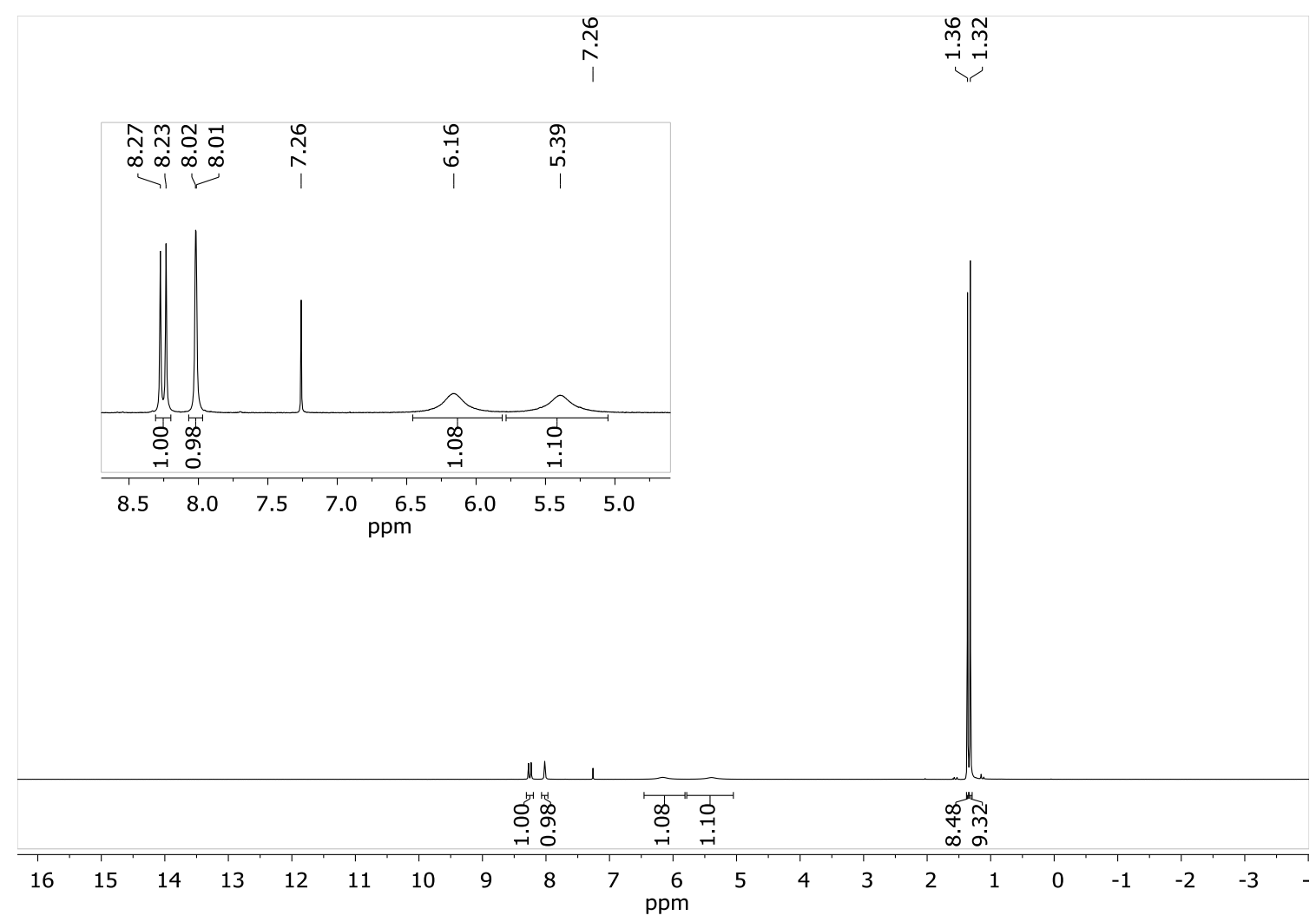

${ }^{1} \mathrm{H}$ NMR spectrum of $\mathrm{N}$-tert-butylformamide $\left(300 \mathrm{MHz}, \mathrm{CDCl}_{3}\right)$. 


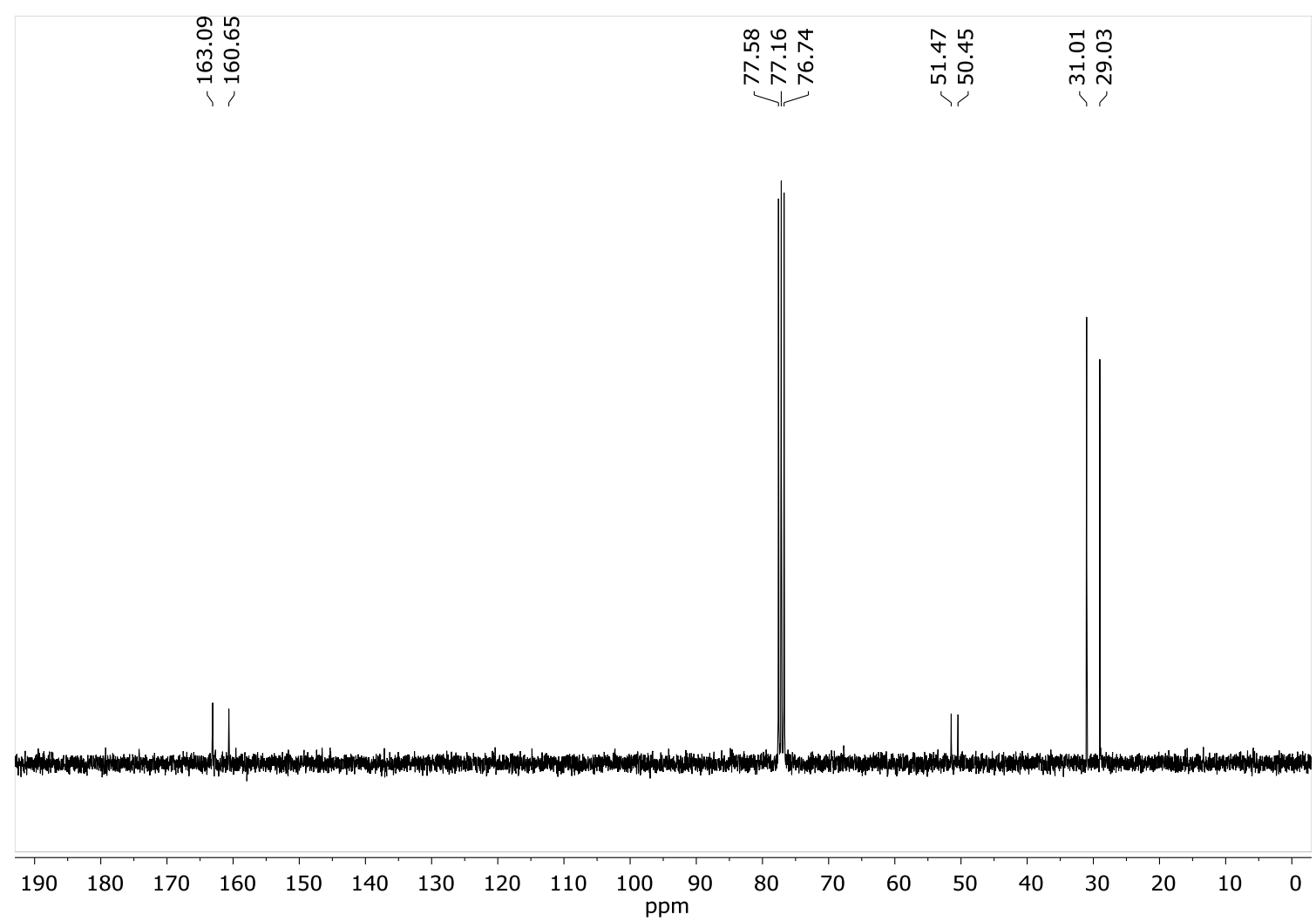

${ }^{13} \mathrm{C}$ NMR spectrum of $\mathrm{N}$-tert-butylformamide $\left(75 \mathrm{MHz}, \mathrm{CDCl}_{3}\right)$.

\subsubsection{Synthesis of $N$-Benzylformamide (BnF)}<smiles>O=CNCc1ccccc1</smiles>

Benzylamine $(0.33 \mathrm{~mL}, 3.00 \mathrm{mmol}, 1.00$ equiv), formamide $(0.12 \mathrm{~mL}$, $3.00 \mathrm{mmol}, 1.00$ equiv) and PIDA (48.3 mg, $0.15 \mathrm{mmol}, 5 \mathrm{~mol} \%)$ were reacted according to general procedure 6 (chapter 2.2.6, page 18). Purification by a small plug of silica gel ( $n$-pentane:EtOAc $=5: 1 \rightarrow n$-pentane:EtOAc $=1: 1$ ) furnished the title formamide as yellow solid (297 mg, $2.20 \mathrm{mmol}, 73 \%$ ).

$\mathbf{M}\left(\mathrm{C}_{8} \mathrm{H}_{9} \mathrm{NO}\right)=135.17 \mathrm{~g} / \mathrm{mol}$; the formamide $\mathrm{BnF}$ was obtained as mixture of rotamers $84: 16$; resolved signals of the major rotamer: ${ }^{1} \mathbf{H}$ NMR $\left(300 \mathrm{MHz}, \mathrm{CDCl}_{3}\right) \delta[\mathrm{ppm}]=8.28(\mathrm{~s}, 1 \mathrm{H}), 7.42-$ $4.22(\mathrm{~m}, 5 \mathrm{H}), 5.83$ (br.s, $1 \mathrm{H}, \mathrm{NH}), 4.50$ (d, J=5.9 Hz, 2H); ${ }^{13} \mathrm{C} \mathrm{NMR}(75 \mathrm{MHz}$, $\left.\mathrm{CDCl}_{3}\right) \delta[\mathrm{ppm}]=161.10,137.67,128.92,127.94,127.84,42.31$; resolved signals of the minor rotamer: ${ }^{1} \mathrm{H}$ NMR $\left(300 \mathrm{MHz}, \mathrm{CDCl}_{3}\right) \delta[\mathrm{ppm}]=8.20(\mathrm{~d}, J=12.0 \mathrm{~Hz}, J=2.3 \mathrm{~Hz}, 1 \mathrm{H}), 4.34(\mathrm{~d}$, $J=6.5 \mathrm{~Hz}, 2 \mathrm{H}) ;{ }^{13} \mathrm{C}$ NMR $\left(75 \mathrm{MHz}, \mathrm{CDCl}_{3}\right) \delta[\mathrm{ppm}]=164.74,129.06,128.11,127.08$.

The NMR data are in agreement with reported data. ${ }^{[11]}$ 


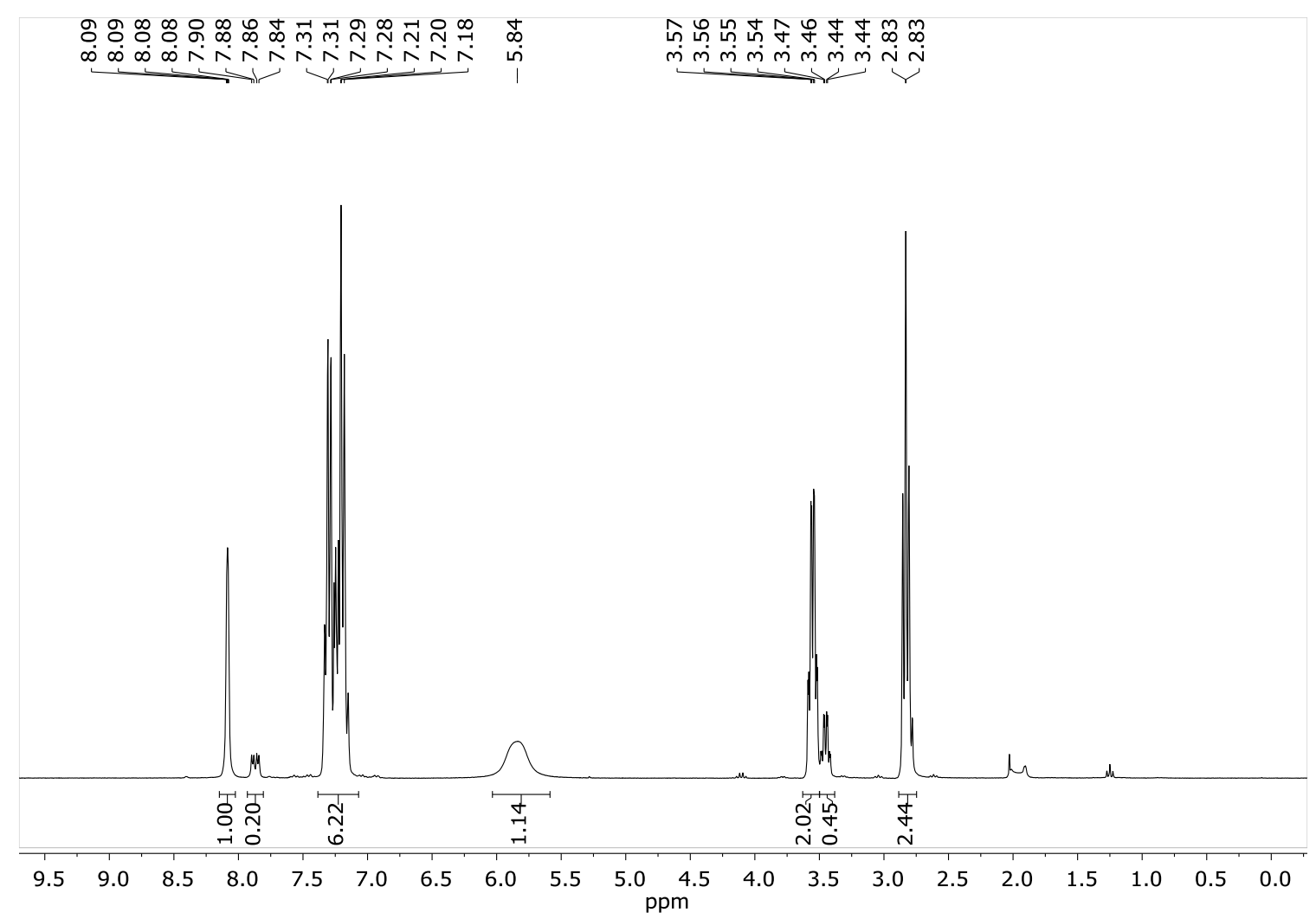

${ }^{1} \mathrm{H}$ NMR spectrum of $\mathrm{N}$-benzylformamide $\left(300 \mathrm{MHz}, \mathrm{CDCl}_{3}\right)$.

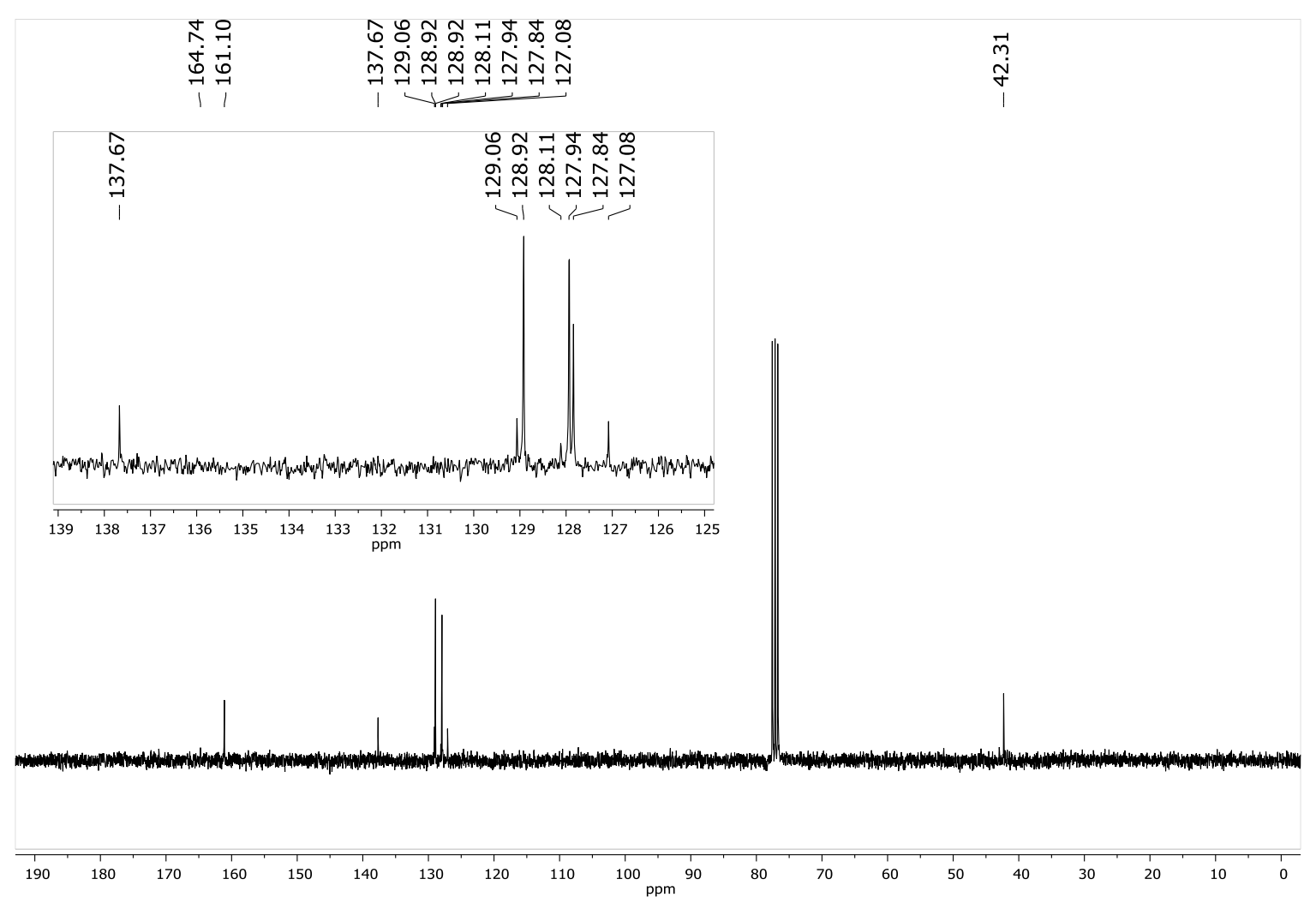

${ }^{13} \mathrm{C}$ NMR spectrum of $\mathrm{N}$-benzylformamide $\left(75 \mathrm{MHz}, \mathrm{CDCl}_{3}\right)$. 


\subsubsection{Synthesis of $N, N$-Di- $n$-butylformamide (DnBuF)}

o Di- $n$-butylamine ( $3.37 \mathrm{~mL}, 20.0 \mathrm{mmol}, 1.00$ equiv), formamide $(0.80 \mathrm{~mL}$, $20.0 \mathrm{mmol}, 1.00$ equiv) and PIDA (322 mg, $1.00 \mathrm{mmol}, 5 \mathrm{~mol} \%$ ) were reacted according to general procedure 6 (chapter 2.2.6, page 18). Purification by a small plug of silica gel ( $n$-pentane:EtOAc $=5: 1 \rightarrow n$-pentane:EtOAc $=1: 1$ ) furnished the title formamide as pale yellow oil (1.60 g, $10.2 \mathrm{mmol}, 51 \%$ ).

M $\left(\mathrm{C}_{9} \mathrm{H}_{19} \mathrm{NO}\right)=157.26 \mathrm{~g} / \mathrm{mol} ;{ }^{1} \mathrm{H}$ NMR $\left(300 \mathrm{MHz}, \mathrm{CDCl}_{3}\right) \delta[\mathrm{ppm}]=8.04(\mathrm{~s}, 1 \mathrm{H}), 3.28$ (t, $J=7.6 \mathrm{~Hz}, 2 \mathrm{H}), 3.19(\mathrm{t}, J=7.1 \mathrm{~Hz}, 2 \mathrm{H}), 1.58-1.43(\mathrm{~m}, 4 \mathrm{H}), 1.40-1.21(\mathrm{~m}, 4 \mathrm{H}), 0.93$ (td, $J=7.2 \mathrm{~Hz}, 1.9 \mathrm{~Hz}, 6 \mathrm{H}),{ }^{13} \mathrm{C}$ NMR $\left(75 \mathrm{MHz}, \mathrm{CDCl}_{3}\right) \delta[\mathrm{ppm}]=162.77,47.26,41.95,30.84$, 29.50, 20.27, 19.75, 13.92, 13.76 .

The NMR data are in agreement with reported data. ${ }^{[13]}$

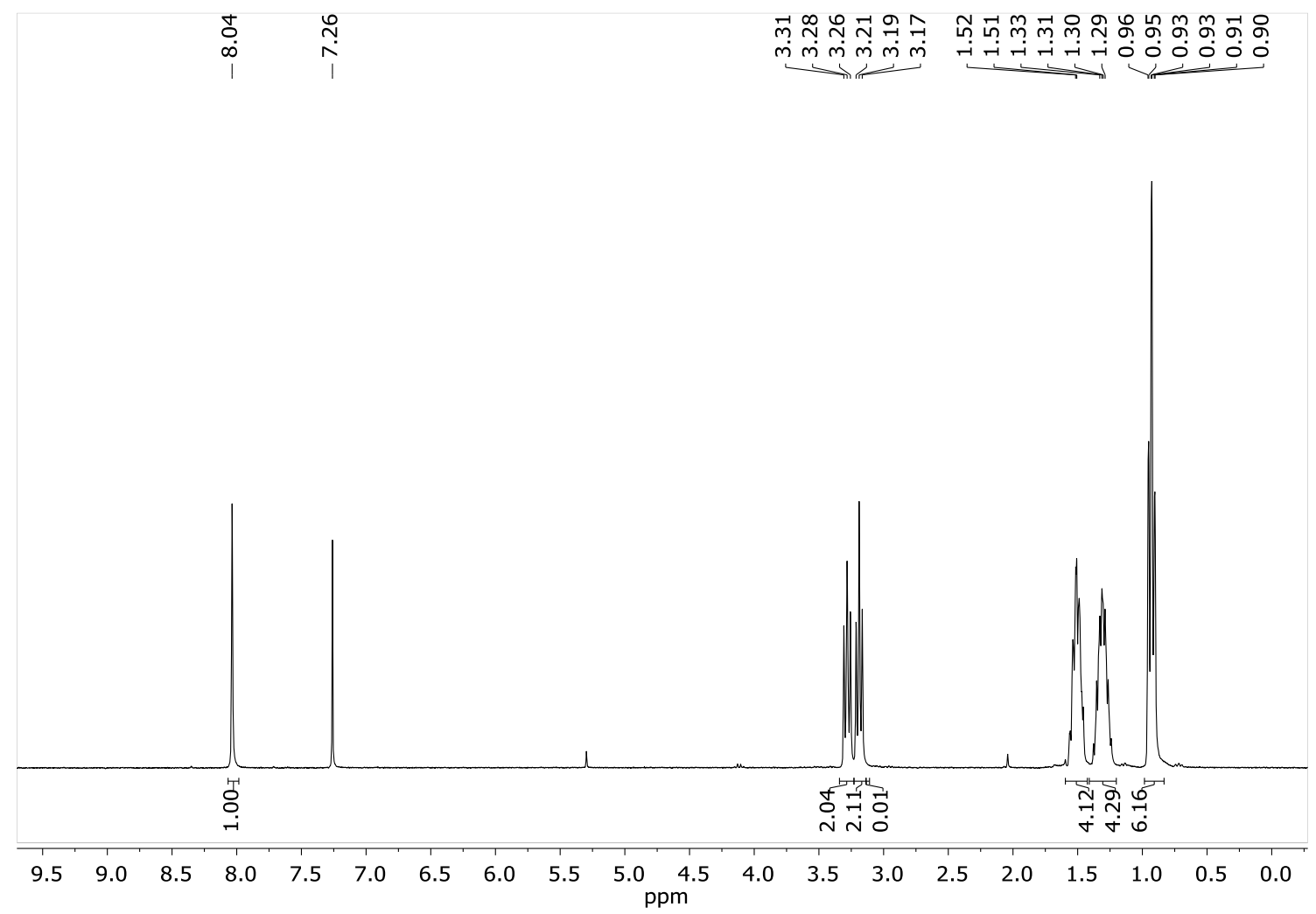

${ }^{1} \mathrm{H}$ NMR spectrum of $N, N$-Di- $n$-butylformamide $\left(300 \mathrm{MHz}, \mathrm{CDCl}_{3}\right)$. 


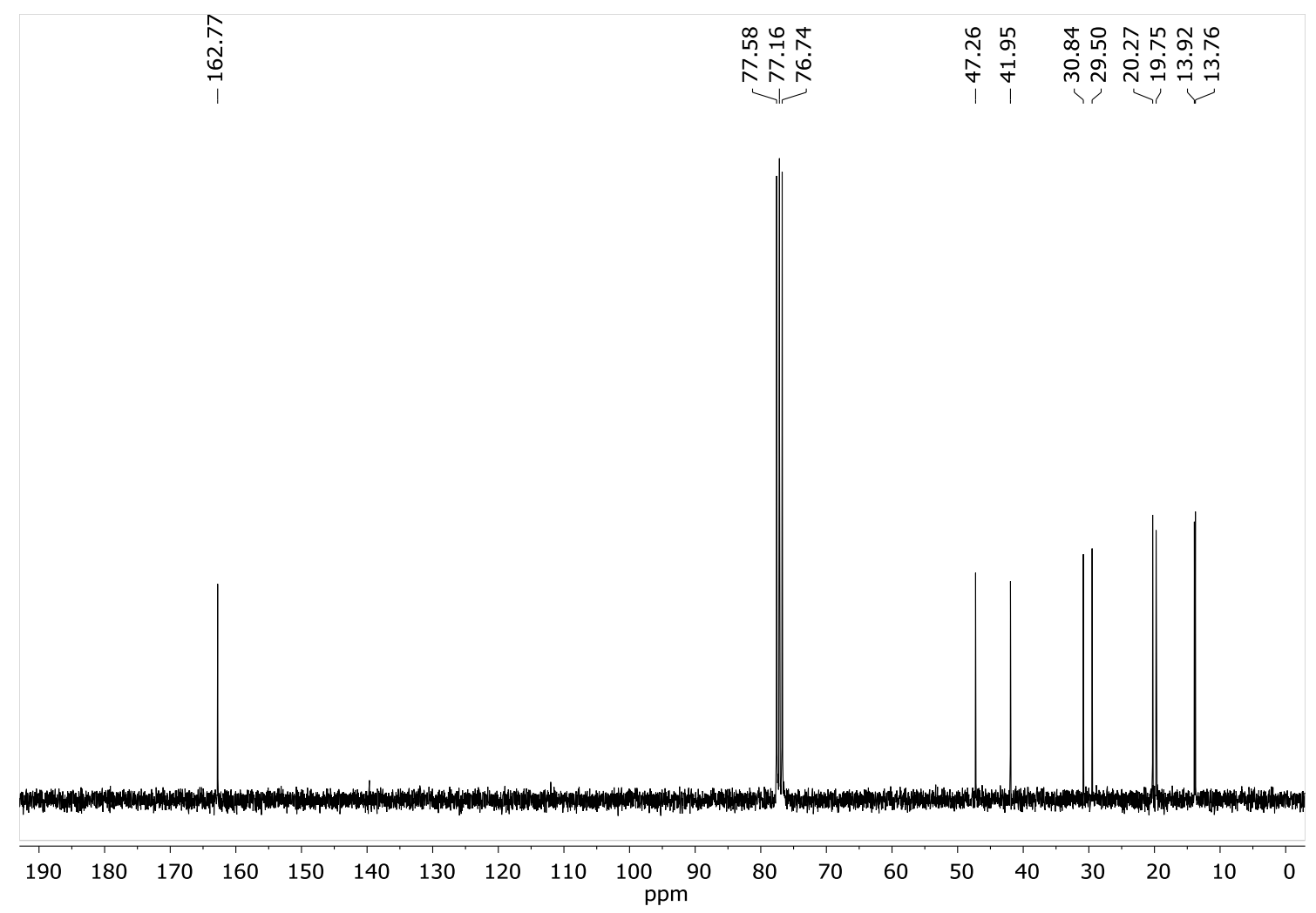

${ }^{13} \mathrm{C}$ NMR spectrum of $N, N$-Di-n-butylformamide $\left(75 \mathrm{MHz}, \mathrm{CDCl}_{3}\right)$.

\subsubsection{Synthesis of Piperazine-1,4-dicarbaldehyde (DFPiper)}<smiles>O=CN1CCN(C=O)CC1</smiles>

Piperazine (1.38 mL, $17.6 \mathrm{mmol}, 1.00$ equiv), formamide $(1.40 \mathrm{~mL}$, $35.2 \mathrm{mmol}, 2.00$ equiv) and PIDA (567 mg, $1.76 \mathrm{mmol}, 10 \mathrm{~mol} \%$ ) were reacted according to general procedure 6 (chapter 2.2.6, page 18). For crystallization, the crude product was dissolved in $\mathrm{CH}_{2} \mathrm{Cl}_{2}$ and overlapped with $n$-pentane. The title formamide was obtained as colorless solid (1.07 g, $7.53 \mathrm{mmol}, 43 \%)$.

M $\left(\mathrm{C}_{6} \mathrm{H}_{10} \mathrm{~N}_{2} \mathrm{O}_{2}\right)=142.16 \mathrm{~g} / \mathrm{mol} ;{ }^{1} \mathbf{H}$ NMR $\left(300 \mathrm{MHz}, \mathrm{CDCl}_{3}\right) \delta[\mathrm{ppm}]=8.12-8.03(\mathrm{~m}, 2 \mathrm{H})$, 3.65-3.48 (m, 4H), 3.46-3.29 (m, 4H); ${ }^{13} \mathrm{C}$ NMR $\left(75 \mathrm{MHz}, \mathrm{CDCl}_{3}\right) \delta[\mathrm{ppm}]=161.03,160.88$, 46.15, 45.03, 40.58, 39.54.

The NMR data are in agreement with reported data. ${ }^{[14]}$ 


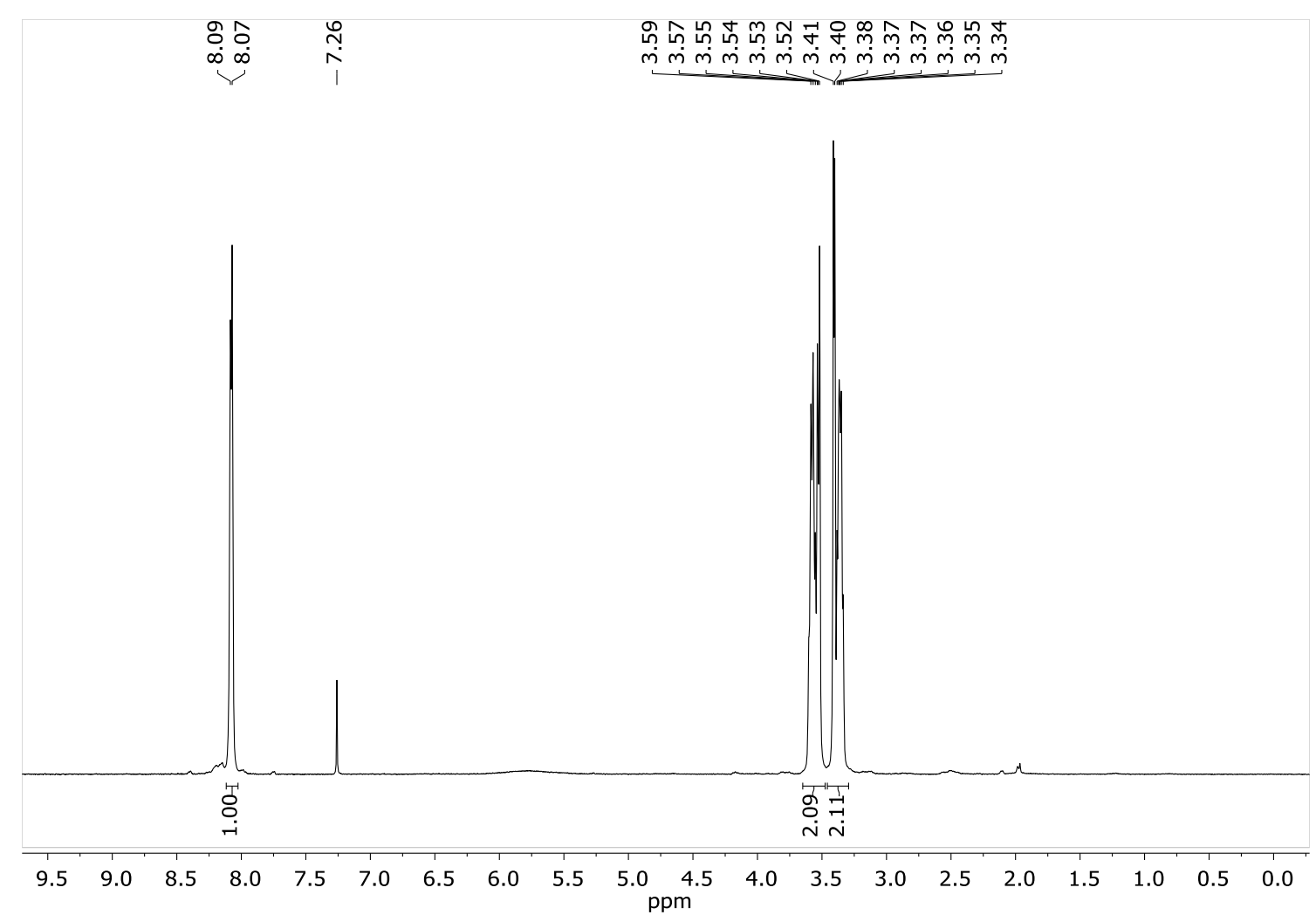

${ }^{1} \mathrm{H}$ NMR spectrum of piperazine-1,4-dicarbaldehyde (300 MHz, $\left.\mathrm{CDCl}_{3}\right)$.

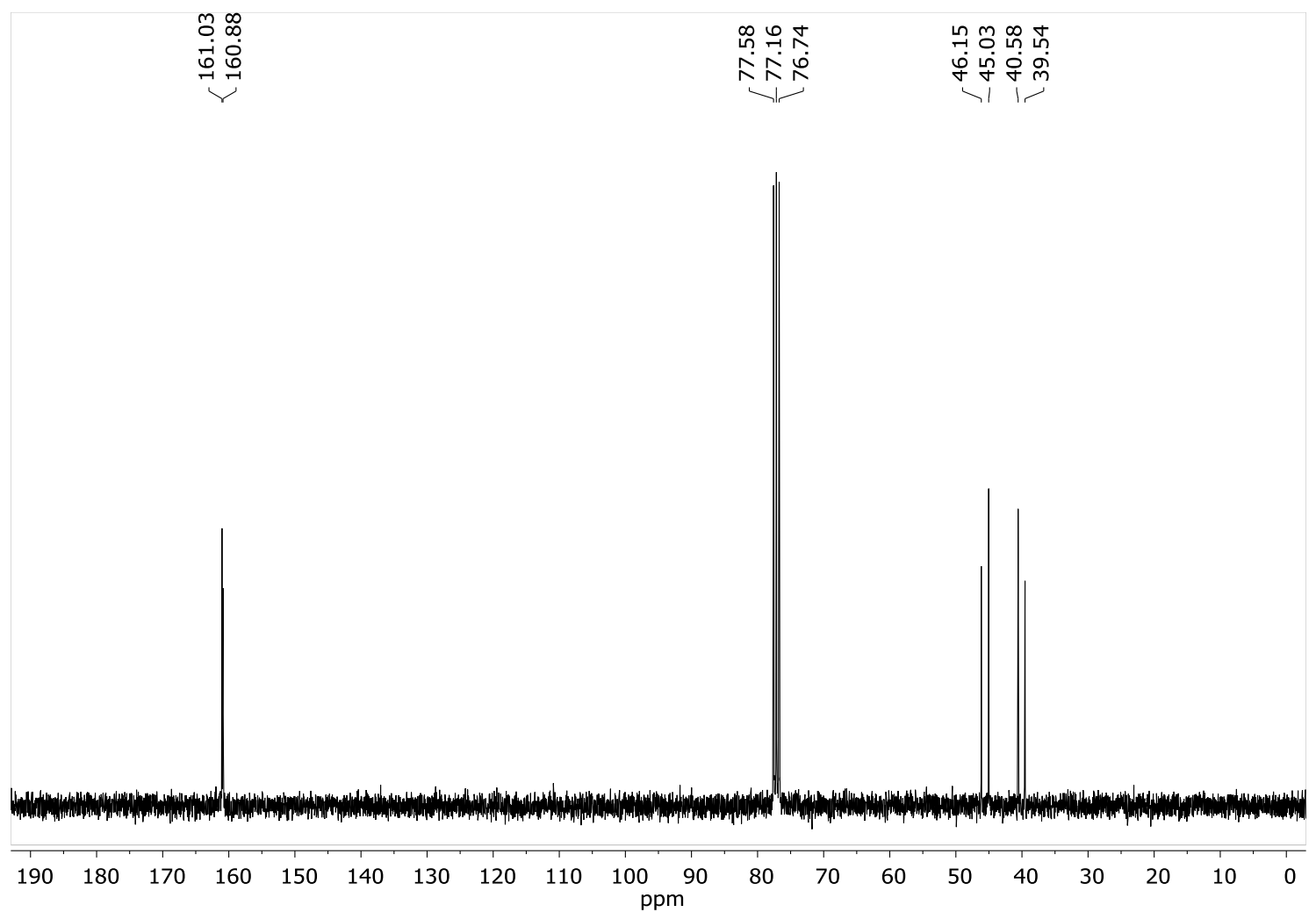

${ }^{13} \mathrm{C}$ NMR spectrum of piperazine-1,4-dicarbaldehyde (75 MHz, $\mathrm{CDCl}_{3}$ ). 


\subsubsection{Synthesis of $N$-Benzyl- $N$-methylformamide (BnMF)}<smiles>CN(C=O)Cc1ccccc1</smiles>

$N$-Benzylmethylamine $(1.90 \mathrm{~mL}, 15.0 \mathrm{mmol}, 1.00$ equiv $)$, formamide ( $0.60 \mathrm{~mL}, 15.0 \mathrm{mmol}, 1.00$ equiv) and PIDA (242 mg, $0.75 \mathrm{mmol}, 5 \mathrm{~mol} \%$ ) were reacted according to general procedure 6 (chapter 2.2.6, page 18). Purification by a small plug of silica gel ( $n$-pentane:EtOAc $=5: 1$ $\rightarrow n$-pentane:EtOAc = 1:1) furnished the title formamide as yellow tough oil $(2.08 \mathrm{~g}$, $13.9 \mathrm{mmol}, 93 \%)$.

M $\left(\mathrm{C}_{9} \mathrm{H}_{11} \mathrm{NO}\right)=149.19 \mathrm{~g} / \mathrm{mol}$; the formamide $\mathrm{BnMF}$ was obtained as mixture of rotamers 57:43; resolved signals of the major rotamer: ${ }^{1} \mathbf{H}$ NMR $\left(300 \mathrm{MHz}, \mathrm{CDCl}_{3}\right) \delta[\mathrm{ppm}]=8.26(\mathrm{~s}$, $1 \mathrm{H}), 7.41-7.14(\mathrm{~m}, 5 \mathrm{H}), 4.37(\mathrm{~s}, 2 \mathrm{H}), 2.76(\mathrm{~s}, 3 \mathrm{H}) ;{ }^{13} \mathrm{C} \mathrm{NMR}\left(75 \mathrm{MHz}, \mathrm{CDCl}_{3}\right) \delta[\mathrm{ppm}]=162.70$, $135.77,128.86,128.20,127.37,53.40,34.01$; resolved signals of the minor rotamer: ${ }^{1} \mathbf{H}$ NMR $\left(300 \mathrm{MHz}, \mathrm{CDCl}_{3}\right) \delta[\mathrm{ppm}]=8.14(\mathrm{~s}, 1 \mathrm{H}), 7.41-7.14(\mathrm{~m}, 5 \mathrm{H}), 4.50(\mathrm{~s}, 2 \mathrm{H}), 2.82(\mathrm{~s}, 3 \mathrm{H})$; ${ }^{13} \mathrm{C}$ NMR $\left(75 \mathrm{MHz}, \mathrm{CDCl}_{3}\right) \delta[\mathrm{ppm}]=162.53,136.02,128.65,128.05,127.59,47.68,29.38$.

The NMR data are in agreement with reported data. ${ }^{[15]}$

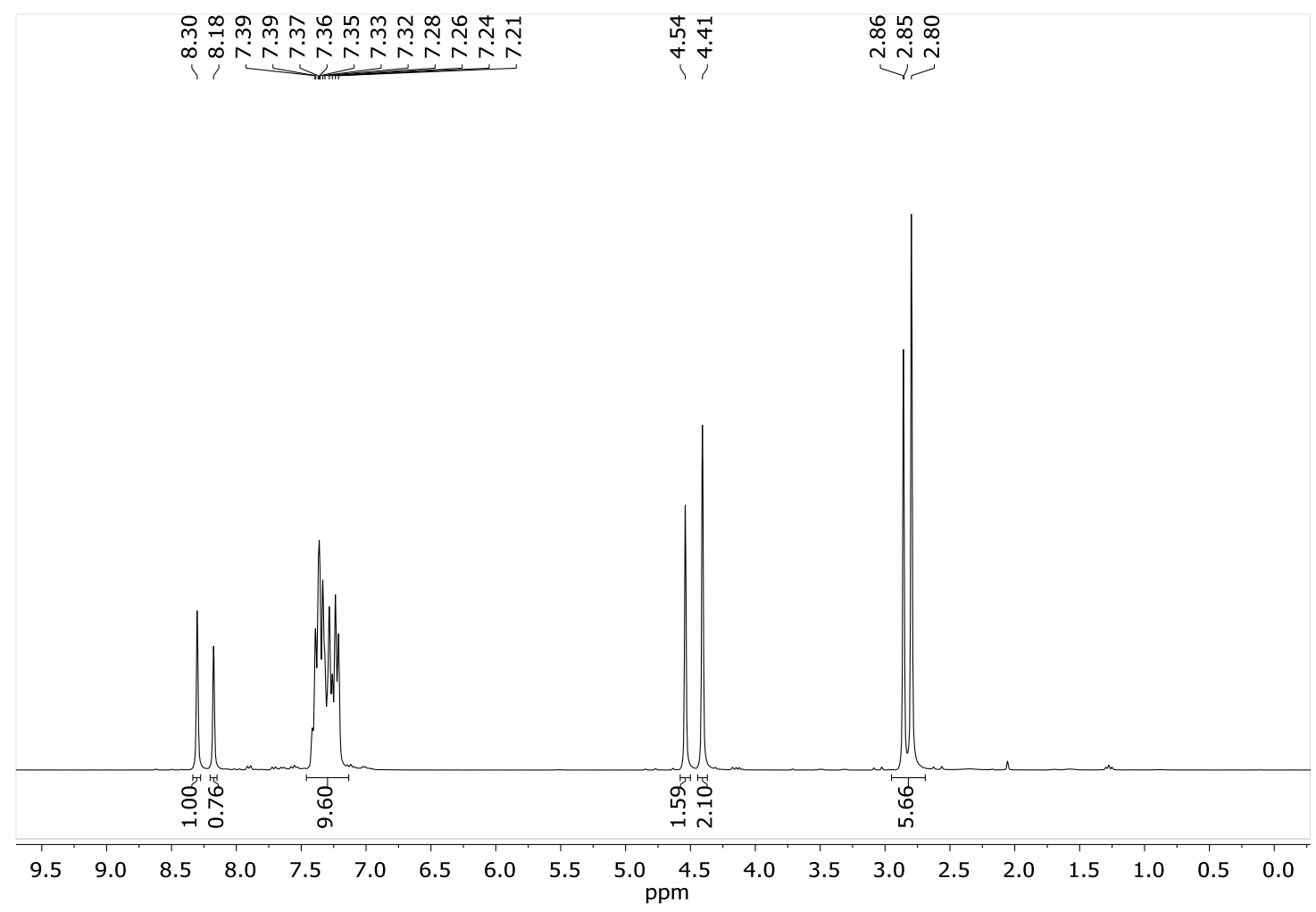

${ }^{1} \mathrm{H}$ NMR spectrum of $\mathrm{N}$-benzyl- $\mathrm{N}$-methylformamide $\left(300 \mathrm{MHz}, \mathrm{CDCl}_{3}\right)$. 


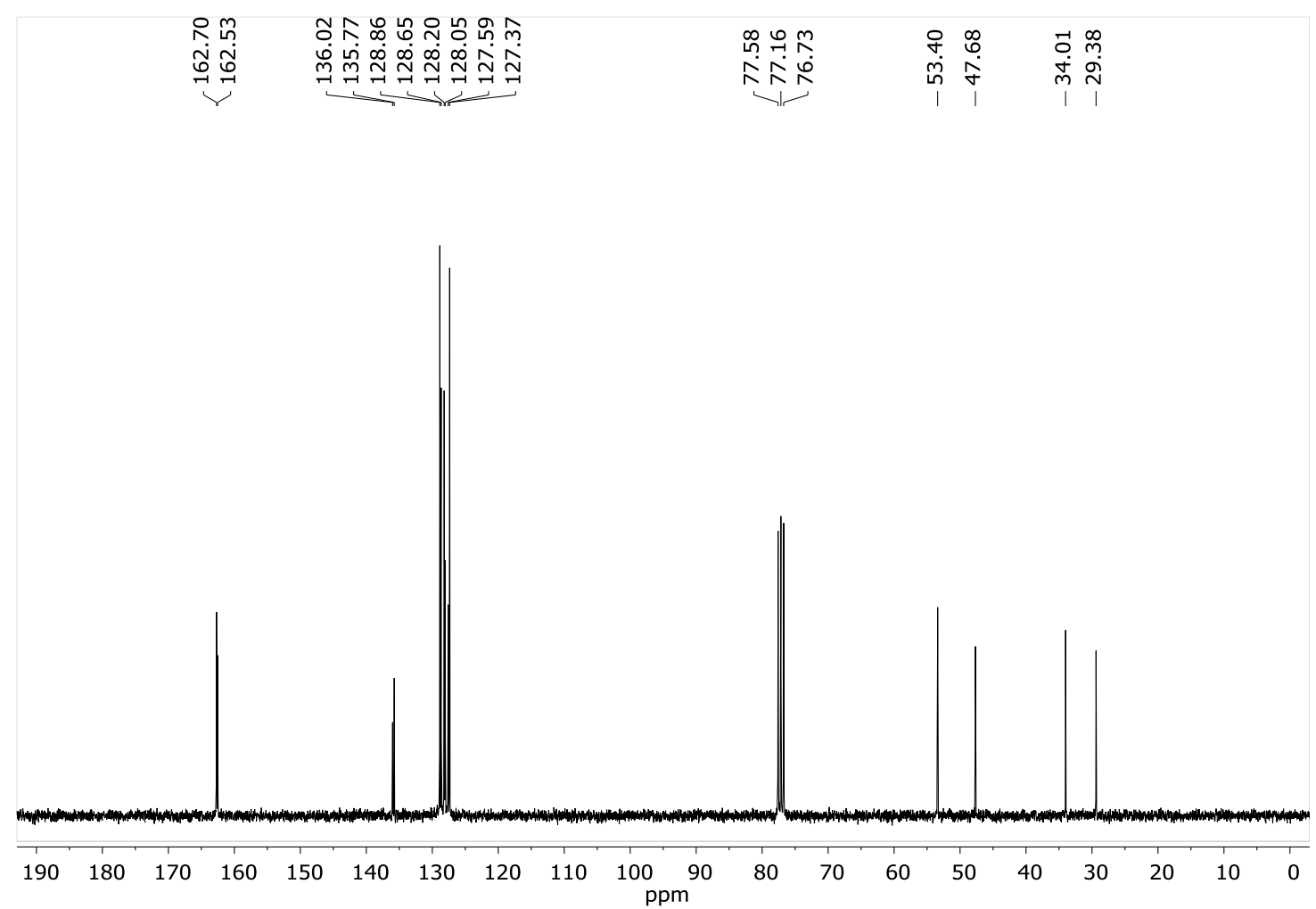

${ }^{13} \mathrm{C}$ NMR spectrum of $\mathrm{N}$-benzyl- $\mathrm{N}$-methylformamide (75 $\mathrm{MHz}, \mathrm{CDCl}_{3}$ ).

\subsubsection{Synthesis of $N, N$-Dibenzylformamide (DBnF)}<smiles></smiles>

Dibenzylamine (3.83 mL, $20.0 \mathrm{mmol}, 1.00$ equiv), formamide $(0.80 \mathrm{~mL}$, $20.0 \mathrm{mmol}, 1.00$ equiv) and PIDA (322 $\mathrm{mg}, 1.00 \mathrm{mmol}, 5 \mathrm{~mol} \%$ ) were reacted according to general procedure 6 (chapter 2.2.6, page 18). Purification by a small plug of silica gel ( $n$-pentane:EtOAc $=5: 1 \rightarrow n$-pentane:EtOAc $=1: 1$ ) furnished the title formamide as yellow solid (3.52 g, $13.9 \mathrm{mmol}, 78 \%$ ).

$\mathbf{M}\left(\mathrm{C}_{15} \mathrm{H}_{15} \mathrm{NO}\right)=225.29 \mathrm{~g} / \mathrm{mol} ;{ }^{1} \mathbf{H}$ NMR $\left(300 \mathrm{MHz}, \mathrm{CDCl}_{3}\right) \delta[\mathrm{ppm}]=8.45(\mathrm{~s}, 1 \mathrm{H}), 7.45-7.26$ $(\mathrm{m}, 6 \mathrm{H}), 7.26-7.15(\mathrm{~m}, 4 \mathrm{H}), 4.44(\mathrm{~s}, 2 \mathrm{H}), 4.29(\mathrm{~s}, 2 \mathrm{H}) ;{ }^{13} \mathrm{C} \mathbf{N M R}\left(75 \mathrm{MHz}, \mathrm{CDCl}_{3}\right)$ $\delta[\mathrm{ppm}]=162.92,136.12,135.74,129.03,128.80,128.63,128.25,127.81,127.77,50.33$, 44.72.

The NMR data are in agreement with reported data. ${ }^{[13]}$ 


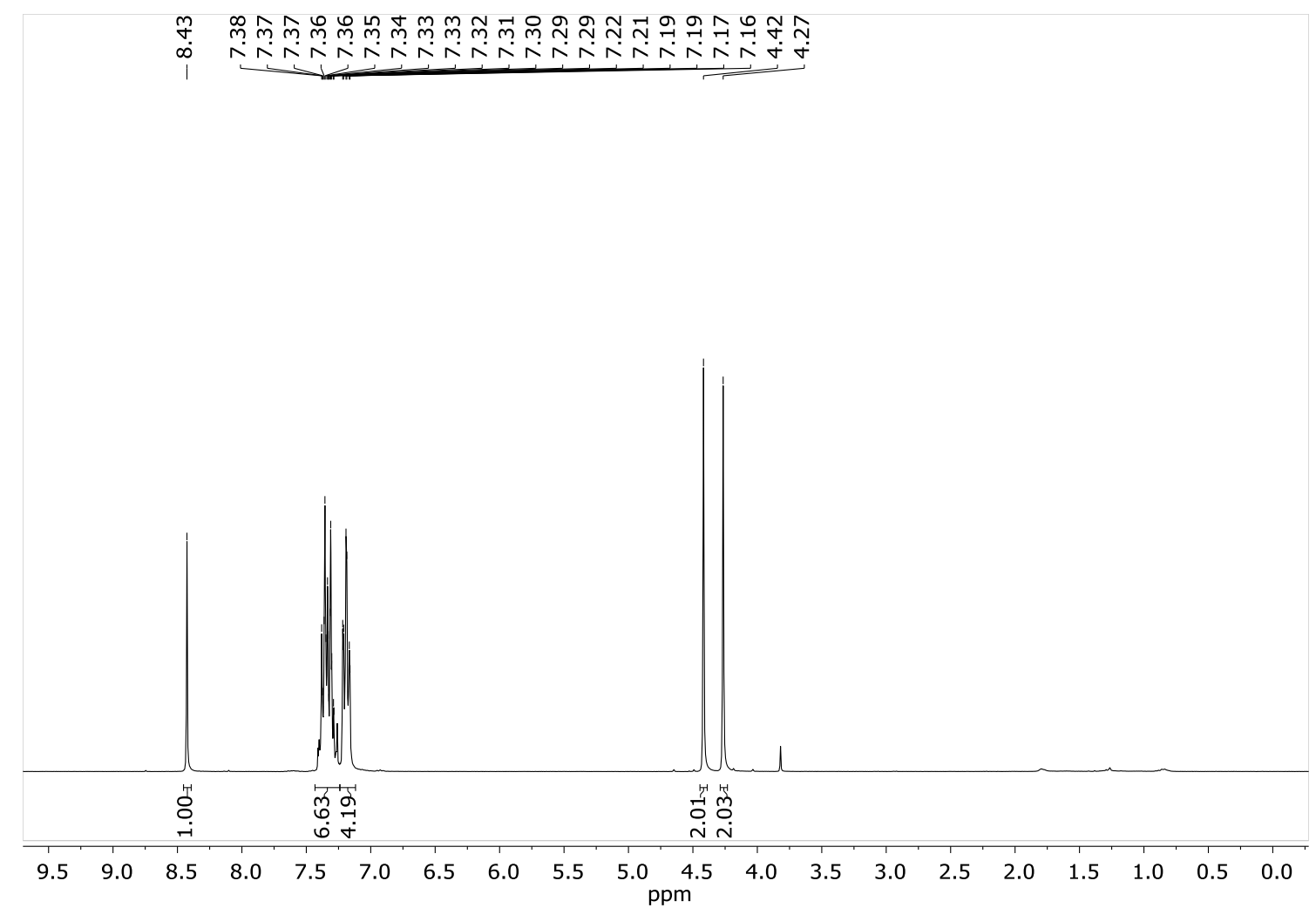

${ }^{1} \mathrm{H}$ NMR spectrum of $\mathrm{N}, \mathrm{N}$-dibenzylformamide $\left(300 \mathrm{MHz}, \mathrm{CDCl}_{3}\right)$.

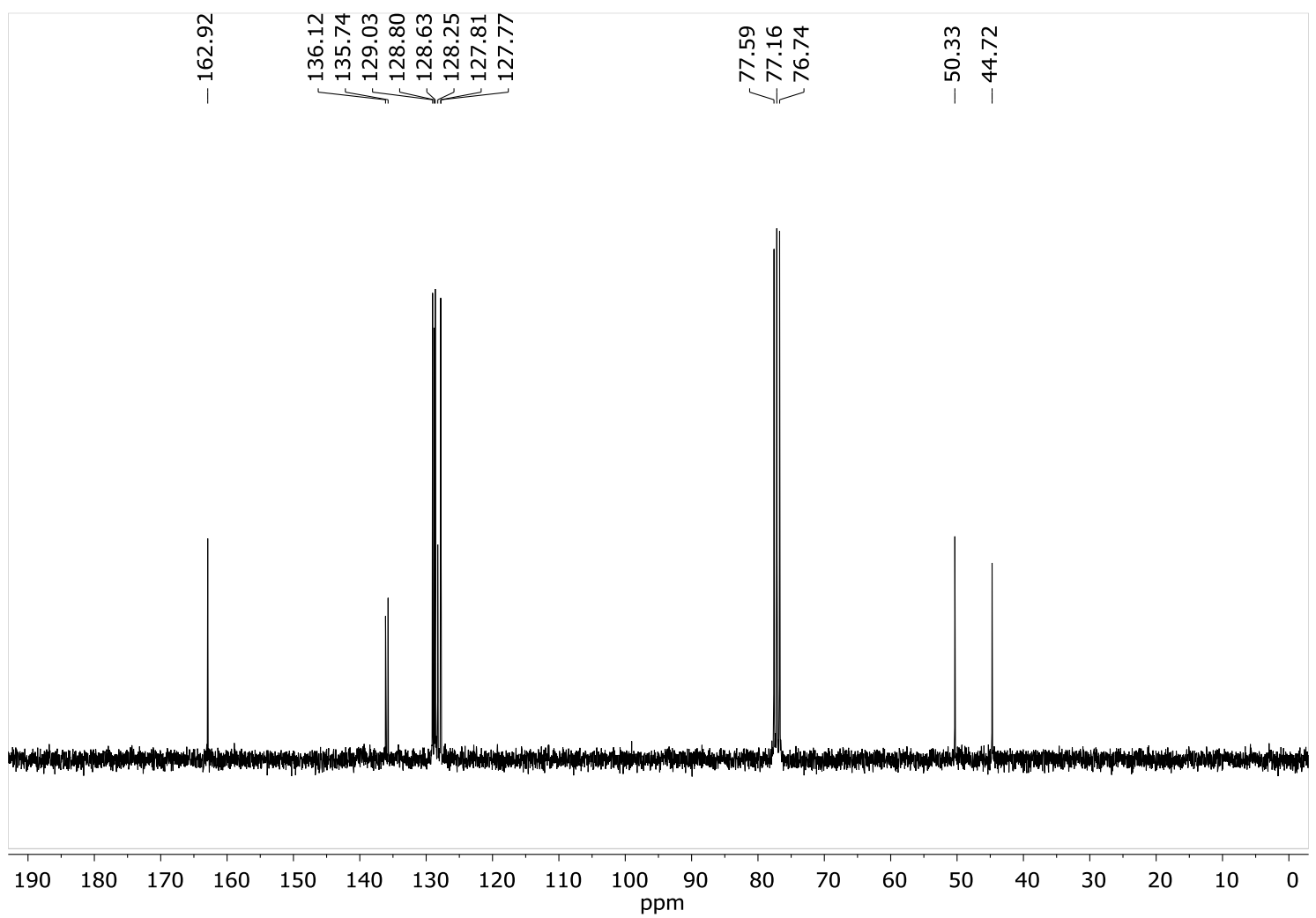

${ }^{13} \mathrm{C}$ NMR spectrum of $\mathrm{N}, \mathrm{N}$-dibenzylformamide $\left(75 \mathrm{MHz}, \mathrm{CDCl}_{3}\right)$. 


\subsubsection{Synthesis of $N$-(4-Methoxyphenyl)- $N$-methylformamide (pMPMF)}<smiles>COc1ccc(N(C)C=O)cc1</smiles>

According to a procedure of Buchwald, ${ }^{[16]}$ under argon atmosphere, a Schlenk flask was charged with Cul (11.4 mg, $60.0 \mu \mathrm{mol}, 1.2 \mathrm{~mol} \%)$ and $\mathrm{K}_{3} \mathrm{PO}_{4}(2.15 \mathrm{~g}, 10.1 \mathrm{mmol}, 2.03$ equiv). Then, the flask was evacuated three times, backfilled with argon and abs. 1,4-dioxane $(9.60 \mathrm{~mL})$ was added. 4-lodoanisole $(1.17 \mathrm{~g}, 5.00 \mathrm{mmol}, 1.00$ equiv), $N$-methylformamide $(0.35 \mathrm{~mL}, \quad 6.00 \mathrm{mmol}, 1.20$ equiv) und cyclohexane-1,2-diamine $(376 \mu \mathrm{L}, 3.13 \mathrm{mmol}$, $0.63 \mathrm{~mol} \%$ ) were added, and the reaction mixture was stirred $23 \mathrm{~h}$ at $110^{\circ} \mathrm{C}$. Once again, $\mathrm{Cul}$ (11.4 mg, $60.0 \mu \mathrm{mol}, 1.2 \mathrm{~mol} \%)$ and cyclohexane-1,2-diamine $(376 \mu \mathrm{L}, \quad 3.13 \mathrm{mmol}$, $0.63 \mathrm{~mol} \%$ ) were added and the mixture was stirred $3 \mathrm{~h}$ at $110^{\circ} \mathrm{C}$. After complete conversion of the aryl iodide (monitored by GC-MS), the mixture was filtered through a small plug of silica gel (EtOAc) and the solvent was removed at reduced pressure. Purification by column chromatography on silica gel ( $n$-pentane:EtOAc $=3: 1$ ) furnished the title formamide as yellow oil (747 mg, $4.50 \mathrm{mmol}, 90 \%)$.

M $\left(\mathrm{C}_{9} \mathrm{H}_{11} \mathrm{NO}_{2}\right)=165.19 \mathrm{~g} / \mathrm{mol}$; the formamide pMPMF was obtained as mixture of rotamers 98:2; resolved signals of the major rotamer: ${ }^{1} \mathbf{H}$ NMR $\left(300 \mathrm{MHz}, \mathrm{CDCl}_{3}\right) \delta[\mathrm{ppm}]=8.34(\mathrm{~s}, 1 \mathrm{H})$, 7.14-7.05 (m, 2H), 6.97-9.86 (m, 2H), $3.82(\mathrm{~s}, 3 \mathrm{H}), 3.27(\mathrm{~s}, 3 \mathrm{H}) ;{ }^{13} \mathrm{C} \mathrm{NMR}(75 \mathrm{MHz}$, $\left.\mathrm{CDCl}_{3}\right) \delta[\mathrm{ppm}]=162.57,158.40,135.38,124.78,114.87,55.68,32.82$; resolved signals of the minor rotamer: ${ }^{1} \mathbf{H}$ NMR $\left(300 \mathrm{MHz}, \mathrm{CDCl}_{3}\right) \delta[\mathrm{ppm}]=8.31(\mathrm{~s}, 1 \mathrm{H}), 7.14-7.05(\mathrm{~m}, 2 \mathrm{H}), 3.80$ (s, 3H), $3.31(\mathrm{~s}, 3 \mathrm{H})$; IR (ATR) $\tilde{v}\left[\mathrm{~cm}^{-1}\right]=2871(\mathrm{w}), 1669(\mathrm{~s}), 1582(\mathrm{w}), 1510(\mathrm{~s}), 1463(\mathrm{~m})$, $1445(m), 1401(w), 1344(m), 1283(m), 1243(s), 1175(m), 1112(m), 1025(m), 979(m)$, $836(s), 780(w), 724(w), 636(w), 613(m) ; M S(E I) ~ m / z ~[u](\%)=165\left(\left[\mathrm{M}^{+}\right], 100\right), 136(20)$, 124 (50), 122 (65), 108 (15), 94 (20), 77 (5), 65 (10), 52 (5); HRMS (El) calculated for $\mathrm{C}_{9} \mathrm{H}_{11} \mathrm{NO}_{2}{ }^{+}: \mathrm{m} / \mathrm{z}[\mathrm{u}]=165.0784$; found: $\mathrm{m} / \mathrm{z}[\mathrm{u}]=165.0782$. 


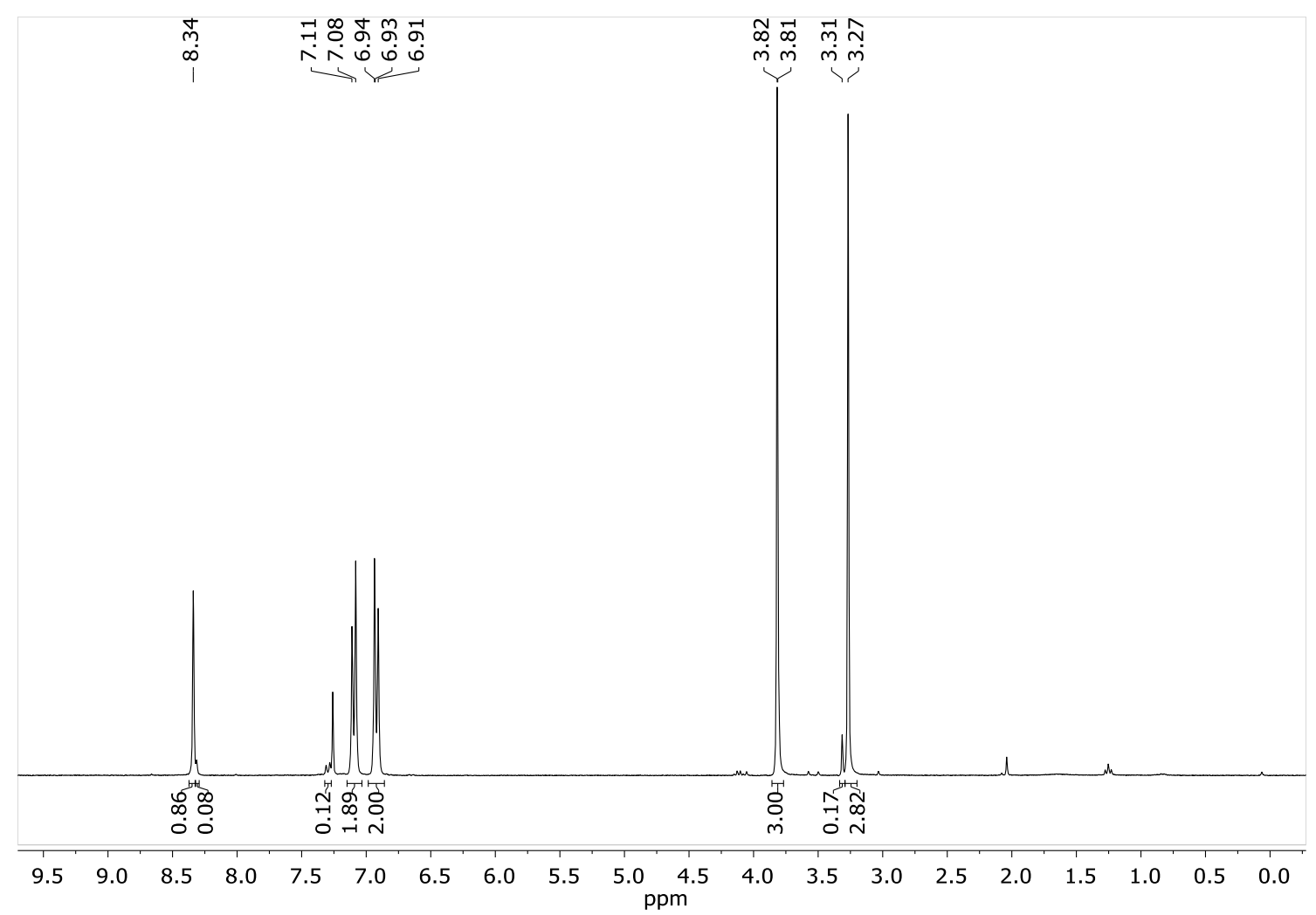

${ }^{1} \mathrm{H}$ NMR spectrum of $\mathrm{N}$-(4-methoxyphenyl)- $\mathrm{N}$-methylformamide (300 $\left.\mathrm{MHz}, \mathrm{CDCl}_{3}\right)$.

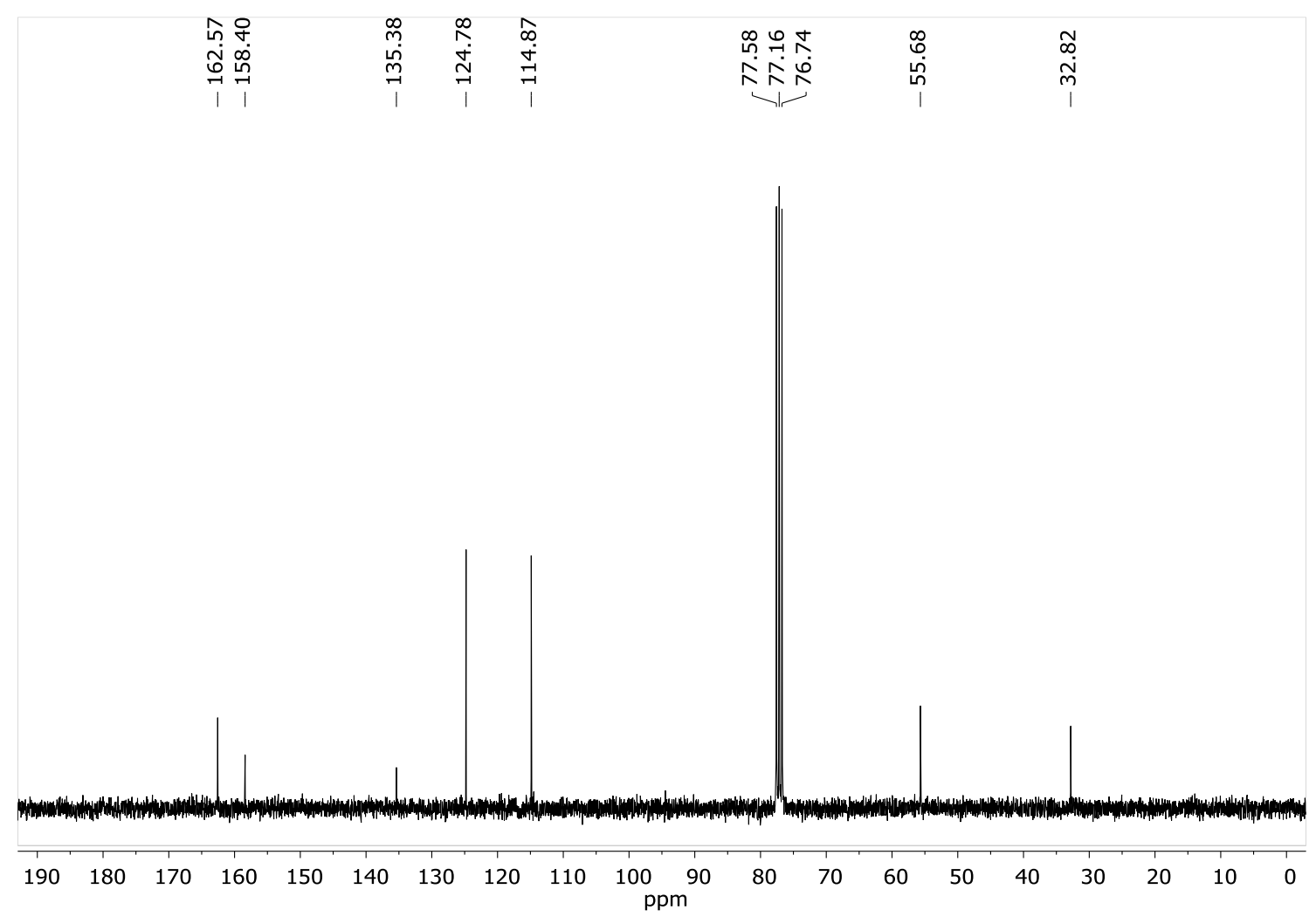

${ }^{13} \mathrm{C}$ NMR spectrum of $\mathrm{N}$-(4-methoxyphenyl)- $\mathrm{N}$-methylformamide $\left(75 \mathrm{MHz}, \mathrm{CDCl}_{3}\right)$. 


\subsubsection{Synthesis of Azepane-1-carbaldehyde (FAz)}

o Azepane (1.69 mL, $15.0 \mathrm{mmol}, 1.00$ equiv), formamide $(0.60 \mathrm{~mL}, 15.0 \mathrm{mmol}$, 1.00 equiv) and PIDA (242 mg, $0.75 \mathrm{mmol}, 5 \mathrm{~mol} \%$ ) were reacted according to general procedure 6 (chapter 2.2.6, page 18). Purification by a small plug of silica gel ( $n$-pentane:EtOAc $=5: 1 \rightarrow n$-pentane:EtOAc $=1: 1$ ) furnished the title formamide as pale yellow oil $(1.61 \mathrm{~g}, 12.7 \mathrm{mmol})$.

$\mathbf{M}\left(\mathrm{C}_{7} \mathrm{H}_{13} \mathrm{NO}\right)=127.19 \mathrm{~g} / \mathrm{mol} ;{ }^{1} \mathrm{H}$ NMR $\left(300 \mathrm{MHz}, \mathrm{CDCl}_{3}\right) \delta[\mathrm{ppm}]=7.89(\mathrm{~s}, 1 \mathrm{H}), 3.44-3.18(\mathrm{~m}$, $4 \mathrm{H}), 1.76-1.37(\mathrm{~m}, 8 \mathrm{H}) ;{ }^{13} \mathrm{C}$ NMR $\left(75 \mathrm{MHz}, \mathrm{CDCl}_{3}\right) \delta[\mathrm{ppm}]=162.76,47.59,43.29,30.17$, 27.87, 26.85, 26.77.

The NMR data are in agreement with reported data. ${ }^{[15]}$

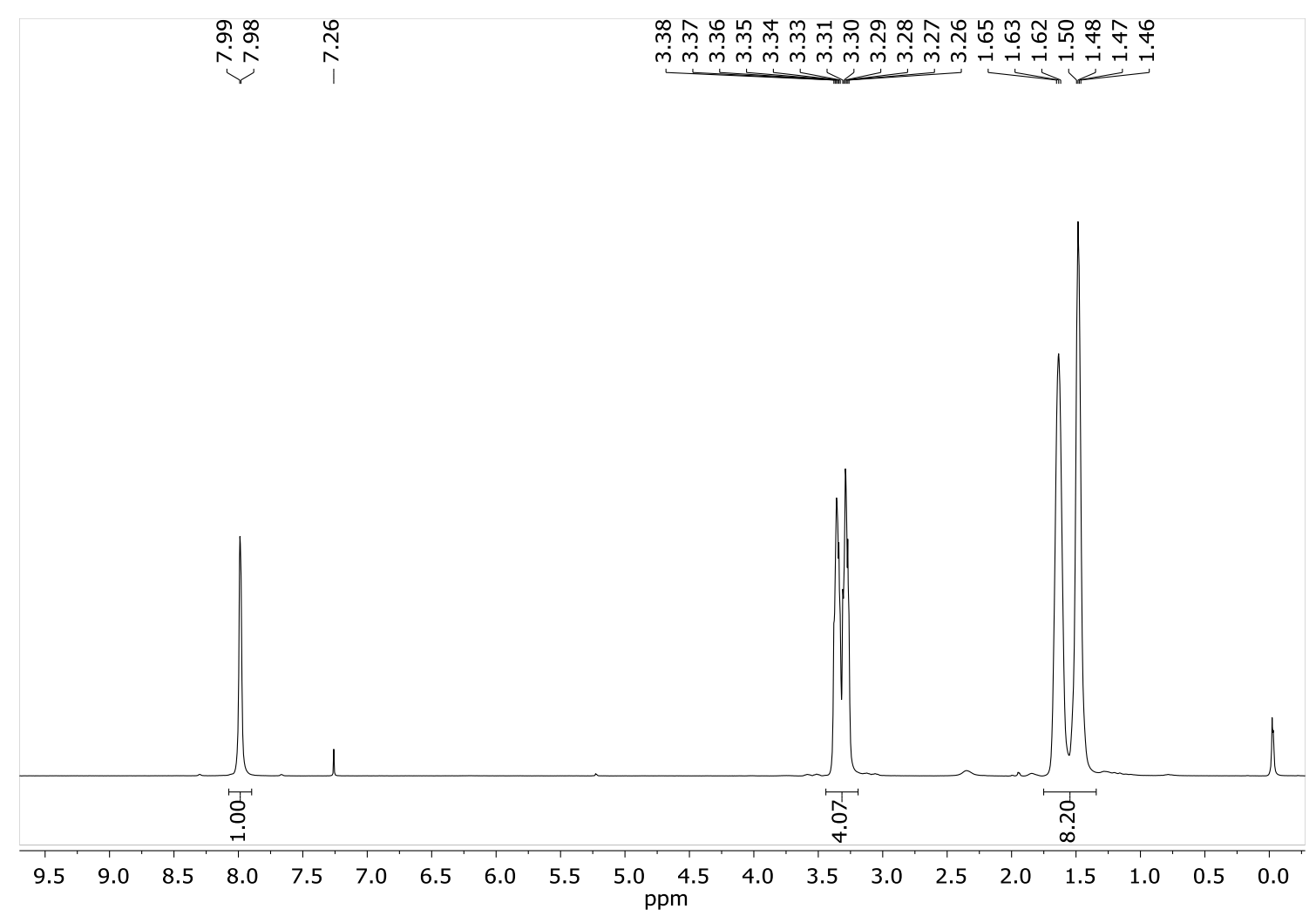

${ }^{1} \mathrm{H}$ NMR spectrum of azepane-1-carbaldehyde $\left(75 \mathrm{MHz}, \mathrm{CDCl}_{3}\right)$. 


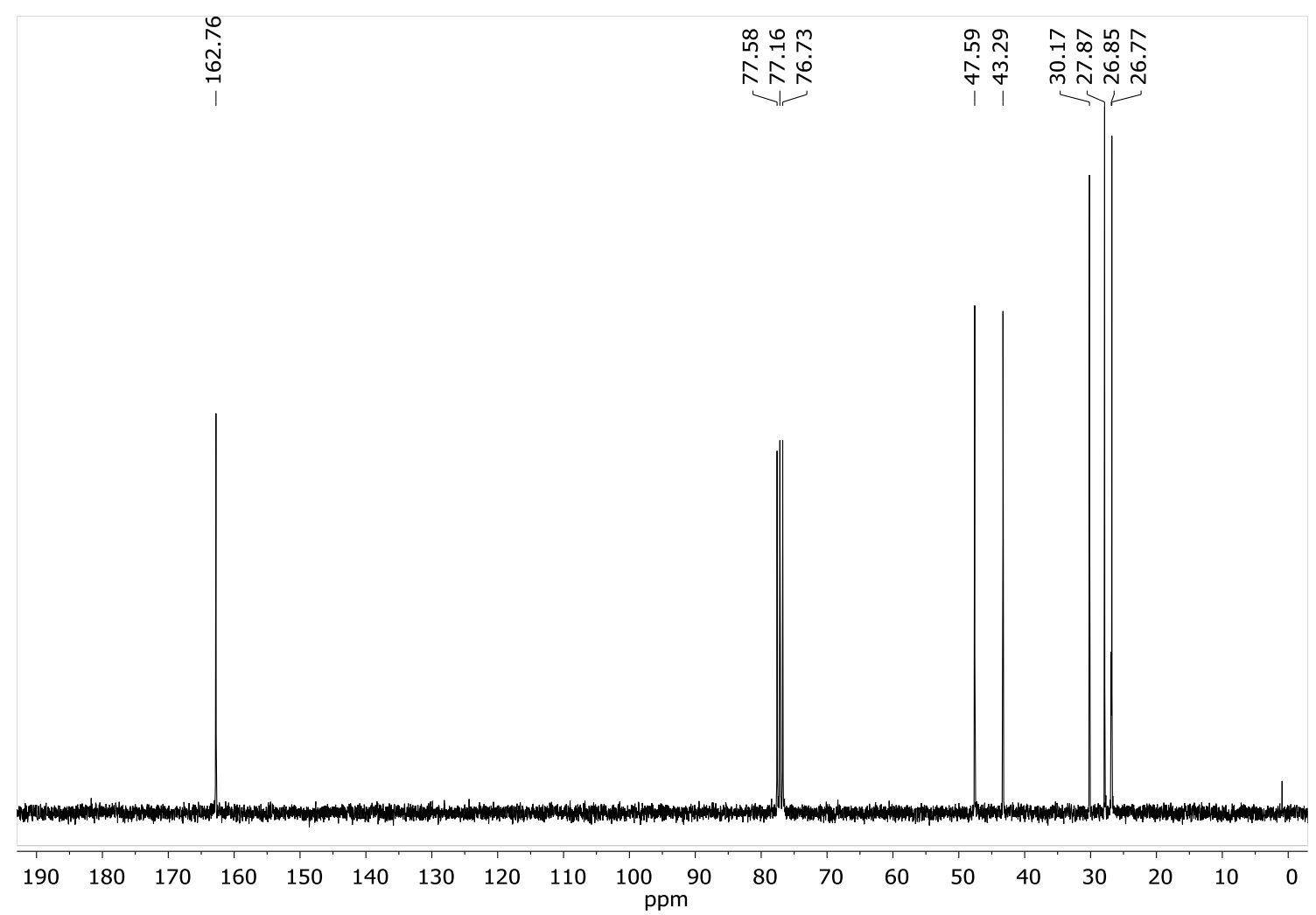

${ }^{13} \mathrm{C}$ NMR spectrum of azepane-1-carbaldehyde $\left(75 \mathrm{MHz}, \mathrm{CDCl}_{3}\right)$.

\subsubsection{Synthesis of $N$-Allylformamide (AllF)}<smiles>C=CCNC(=O)CC</smiles>

Allylamine $\quad(2.25 \mathrm{~mL}, \quad 30.0 \mathrm{mmol}, \quad 1.00$ equiv), formamide $\quad(1.20 \mathrm{~mL}$, $30.0 \mathrm{mmol}, 1.00$ equiv) and PIDA (480 mg, $1.50 \mathrm{mmol}, 5 \mathrm{~mol} \%$ ) were reacted according to general procedure 6 (chapter 2.2.6, page 18). Purification by a small plug of silica gel ( $n$-pentane:EtOAc $=5: 1 \rightarrow n$-pentane:EtOAc $=1: 1$ ) furnished the title formamide as pale yellow oil (1.58 g, $18.6 \mathrm{mmol}, 62 \%)$.

$\mathbf{M}\left(\mathrm{C}_{4} \mathrm{H}_{7} \mathrm{NO}\right)=85.11 \mathrm{~g} / \mathrm{mol}$; the formamide AllF was obtained as mixture of rotamers $83: 17$; resolved signals of the major rotamer: ${ }^{1} \mathrm{H}$ NMR $\left(300 \mathrm{MHz}, \mathrm{CDCl}_{3}\right) \delta[\mathrm{ppm}]=8.20(\mathrm{~s}, 1 \mathrm{H}), 5.93$ (br.s, $1 \mathrm{H}, 1 \mathrm{NH}), 5.92-5.71(\mathrm{~m}, 1 \mathrm{H}), 5.30-5.05(\mathrm{~m}, 2 \mathrm{H}), 3.99-3.86(\mathrm{~m}, 2 \mathrm{H}) ;{ }^{13} \mathrm{C}$ NMR $(75 \mathrm{MHz}$, $\left.\mathrm{CDCl}_{3}\right): \delta=161.28,133.61,116.68,40.51$; resolved signals of the major rotamer: ${ }^{1} \mathbf{H}$ NMR $\left(300 \mathrm{MHz}, \mathrm{CDCl}_{3}\right) \delta[\mathrm{ppm}]=8.03(\mathrm{~d}, J=11.9 \mathrm{~Hz}, 1 \mathrm{H}), 5.92-5.71(\mathrm{~m}, 1 \mathrm{H}), 5.30-5.05(\mathrm{~m}, 2 \mathrm{H})$, 3.86-3.79 (m, 2H); ${ }^{13} \mathrm{C}$ NMR $\left(75 \mathrm{MHz}, \mathrm{CDCl}_{3}\right) \delta[\mathrm{ppm}]=164.97,134.44,116.93,44.02$. The NMR data are in agreement with reported data. ${ }^{[17]}$ 


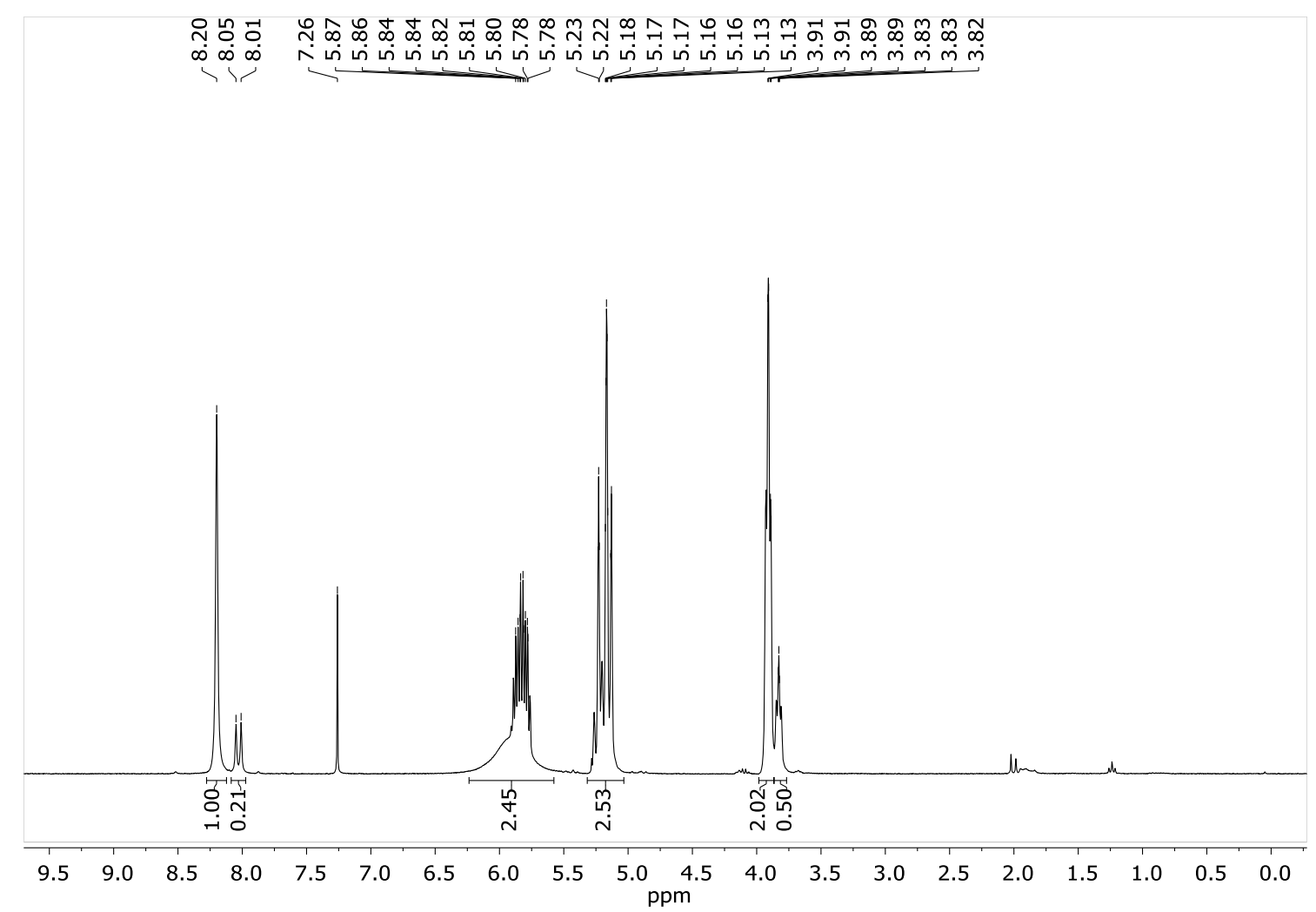

${ }^{1} \mathrm{H}$ NMR spectrum of $\mathrm{N}$-allylformamide $\left(300 \mathrm{MHz}, \mathrm{CDCl}_{3}\right)$.

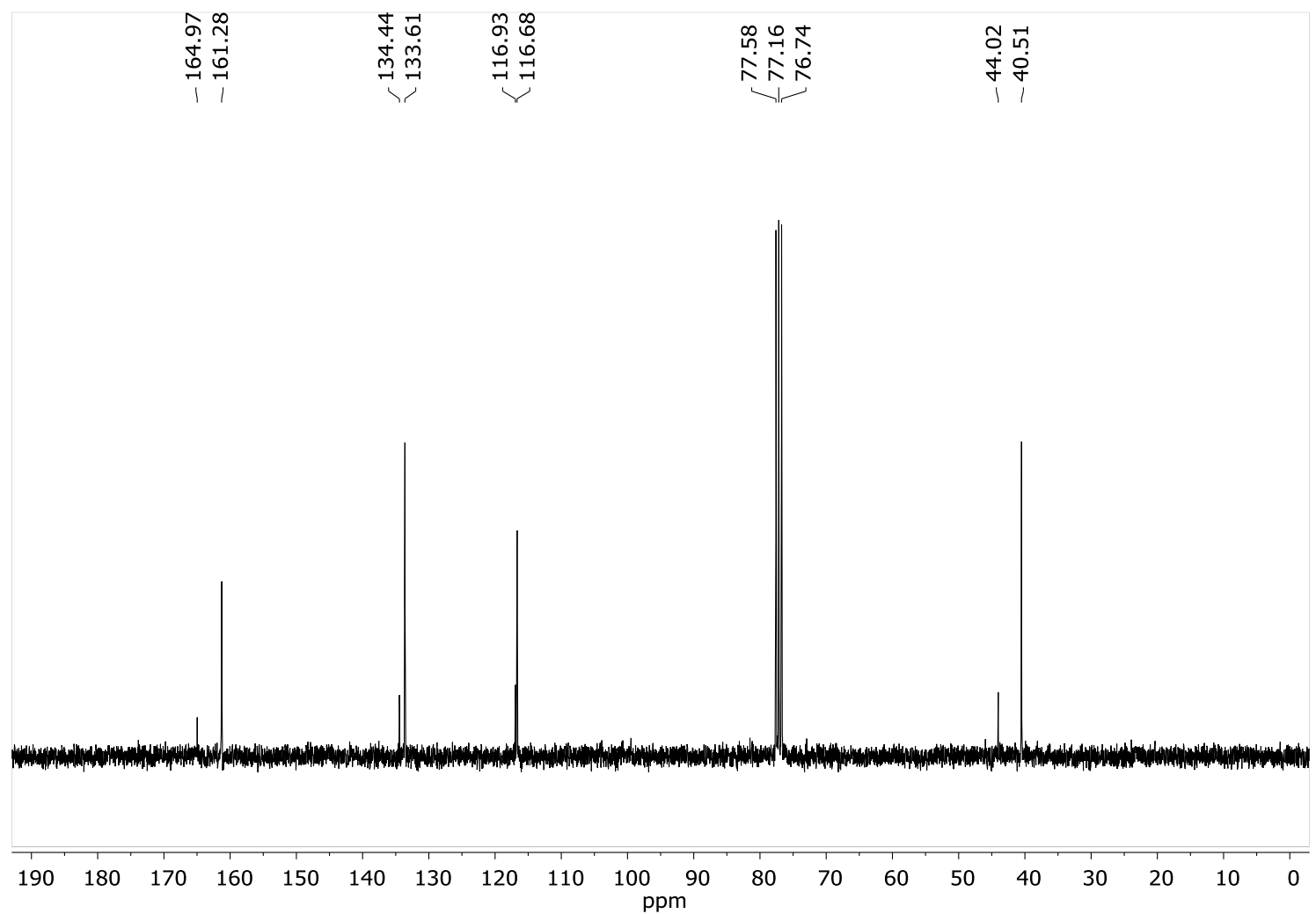

${ }^{13} \mathrm{C}$ NMR spectrum of $\mathrm{N}$-allylformamide $\left(75 \mathrm{MHz}, \mathrm{CDCl}_{3}\right)$. 


\subsubsection{Synthesis of $\mathrm{N}$-Cyclohexylformamide (cHexF)}<smiles>O=CNC1CC2CCCCC21</smiles>

Cyclohexylamine ( $3.43 \mathrm{~mL}, 30.0 \mathrm{mmol}, 1.00$ equiv), formamide $(1.20 \mathrm{~mL}$, $30.0 \mathrm{mmol}, 1.00$ equiv) and PIDA (480 mg, $1.50 \mathrm{mmol}, 5 \mathrm{~mol} \%$ ) were reacted according to general procedure 6 (chapter 2.2.6, page 18). Purification by a small plug of silica gel ( $n$-pentane:EtOAc $=5: 1 \rightarrow n$-pentane:EtOAc $=1: 1$ ) furnished the title formamide as yellow oil (3.45 g, $27.1 \mathrm{mmol}, 91 \%)$.

M $\left(\mathrm{C}_{7} \mathrm{H}_{13} \mathrm{NO}\right)=127.19 \mathrm{~g} / \mathrm{mol}$; the formamide $\mathrm{cHexF}$ was obtained as mixture of rotamers 83:17; resolved signals of the major rotamer: ${ }^{1} \mathrm{H}$ NMR $\left(300 \mathrm{MHz}, \mathrm{CDCl}_{3}\right) \delta[\mathrm{ppm}]=8.07$ (s, $1 \mathrm{H}), 5.72$ (br.s, $1 \mathrm{H}, 1 \mathrm{NH}), 3.97-3.69(\mathrm{~m}, 1 \mathrm{H}), 1.99-0.99(\mathrm{~m}, 10 \mathrm{H}) ;{ }^{13} \mathrm{C} \mathrm{NMR}(75 \mathrm{MHz}$, $\left.\mathrm{CDCl}_{3}\right) \delta[\mathrm{ppm}]=160.44,47.18,33.12,25.52,24.84$; resolved signals of the minor rotamer: ${ }^{1} \mathrm{H}$ NMR $\left(300 \mathrm{MHz}, \mathrm{CDCl}_{3}\right) \delta[\mathrm{ppm}]=8.11(\mathrm{~s}, 1 \mathrm{H}), 5.95$ (br.s, $\left.1 \mathrm{H}, \mathrm{NH}\right), 3.38-3.17(\mathrm{~m}, 1 \mathrm{H})$, $1.99-0.99(\mathrm{~m}, 10 \mathrm{H}) ;{ }^{13} \mathrm{C}$ NMR $\left(75 \mathrm{MHz}, \mathrm{CDCl}_{3}\right) \delta[\mathrm{ppm}]=51.10,34.78,25.12,24.81$.

The NMR data are in agreement with reported data. ${ }^{[18]}$

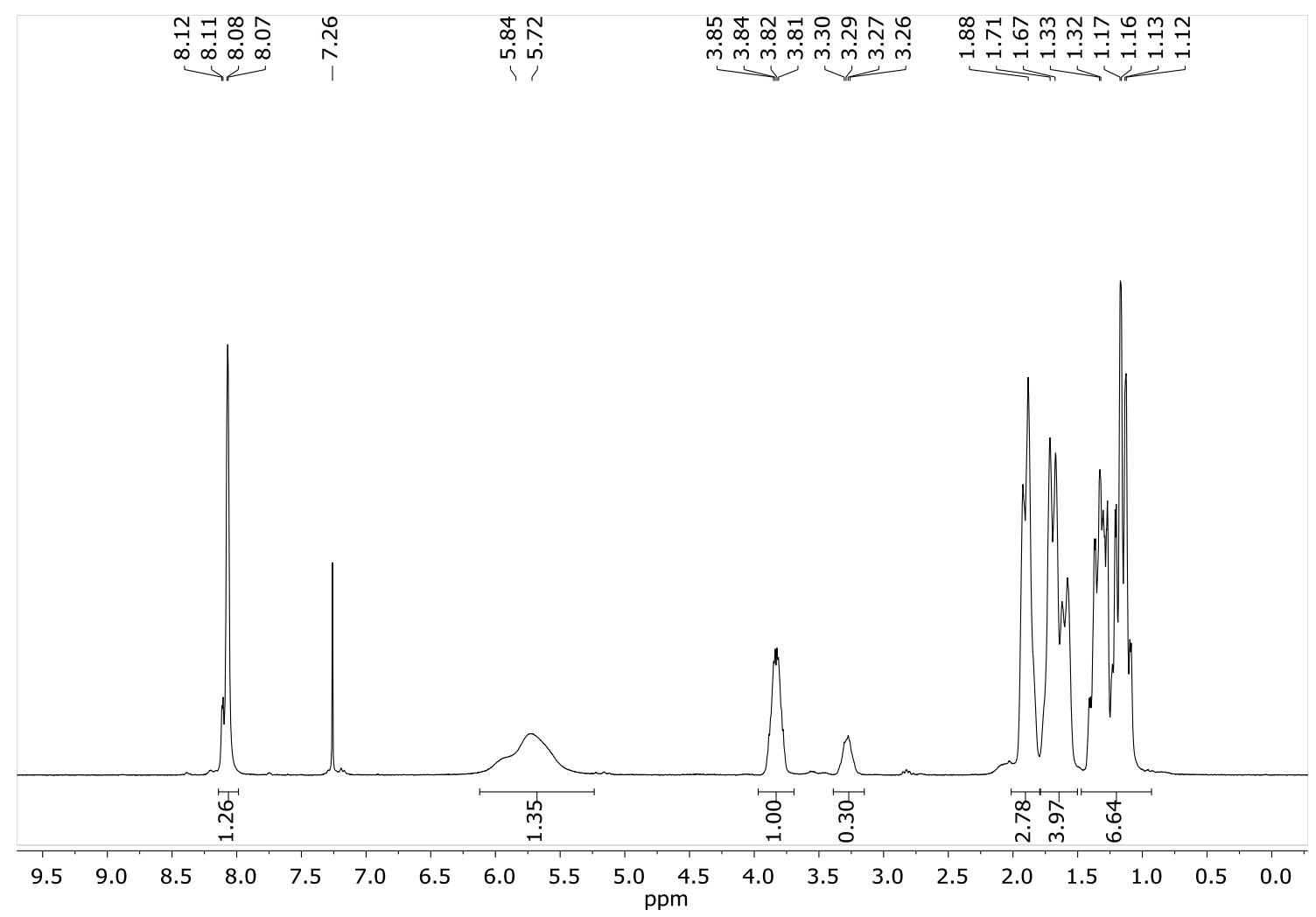

${ }^{1} \mathrm{H}$ NMR spectrum of $\mathrm{N}$-cyclohexylformamide (300 $\left.\mathrm{MHz}, \mathrm{CDCl}_{3}\right)$. 


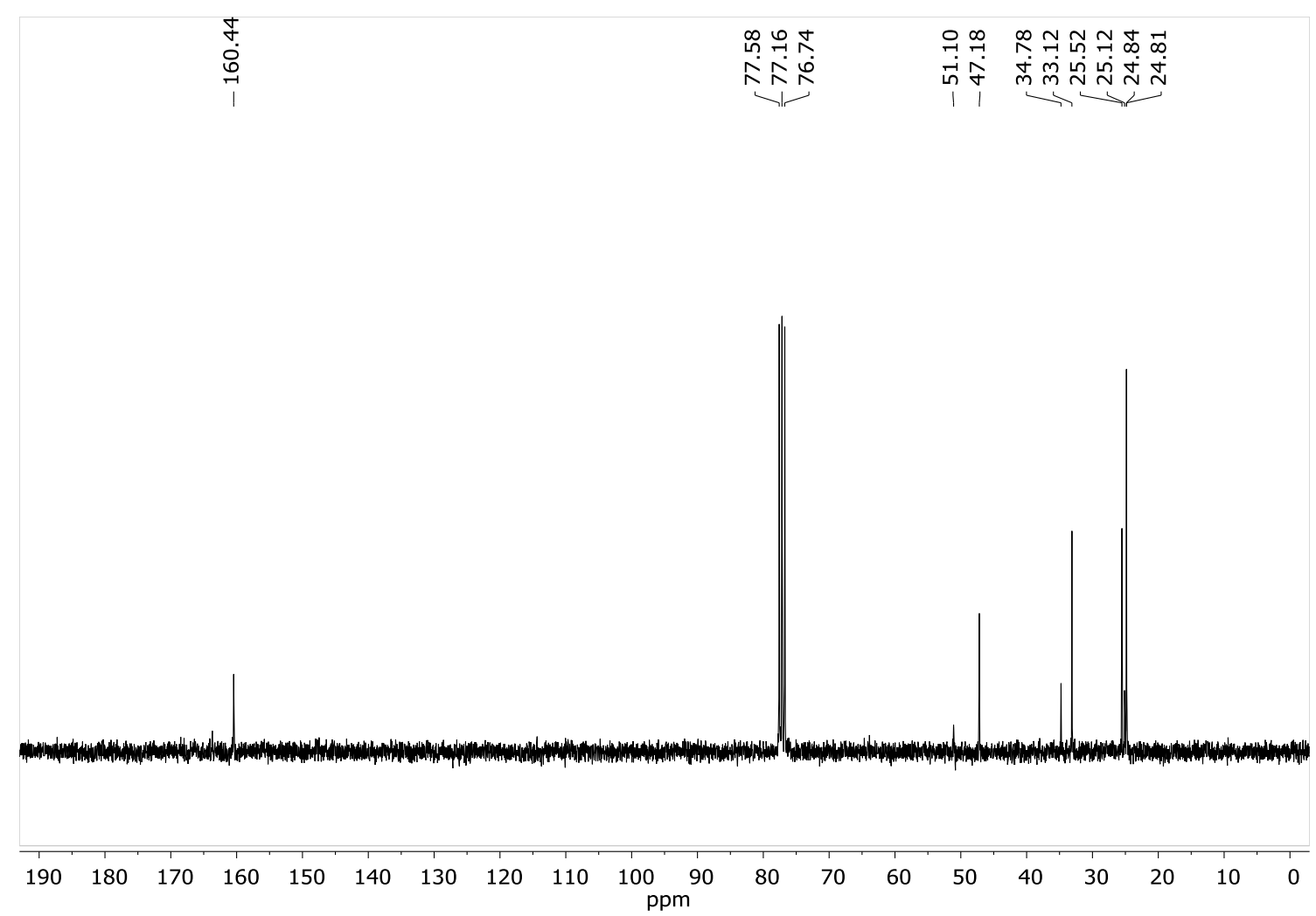

${ }^{13} \mathrm{C}$ NMR spectrum of $\mathrm{N}$-cyclohexylformamide $\left(75 \mathrm{MHz}, \mathrm{CDCl}_{3}\right)$.

\subsubsection{Synthesis of $\mathbf{N}$-(2,2-Dimethoxyethyl)formamide (DMEF)}<smiles>COC(CNC=O)OC</smiles>

(3.27 mL, $\quad 30.0 \mathrm{mmol}, \quad 1.00$ equiv), formamide $(1.20 \mathrm{~mL}, \quad 30.0 \mathrm{mmol}, 1.00$ equiv) and PIDA (480 mg, $1.50 \mathrm{mmol}, 5 \mathrm{~mol} \%)$ were reacted according to general procedure 6 (chapter 2.2.6, page 18). Purification by a small plug of silica gel ( $n$-pentane:EtOAc $=5: 1 \rightarrow$ $n$-pentane:EtOAc = 2:1) furnished the title formamide as yellow oil (3.33 g, $25.0 \mathrm{mmol}, 83 \%)$.

M $\left(\mathrm{C}_{5} \mathrm{H}_{11} \mathrm{NO}_{3}\right)=133.15 \mathrm{~g} / \mathrm{mol}$; the formamide DMEF was obtained as mixture of rotamers 86:14; resolved signals of the major rotamer: ${ }^{1} \mathbf{H}$ NMR $\left(300 \mathrm{MHz}, \mathrm{CDCl}_{3}\right) \delta[\mathrm{ppm}]=8.18(\mathrm{~s}$, $1 \mathrm{H}), 5.90$ (br.s, $1 \mathrm{H}, \mathrm{NH}), 4.38$ (td, $J=5.2 \mathrm{~Hz}, J=2.1 \mathrm{HZ}, 1 \mathrm{H}), 3.42-3.34(\mathrm{~m}, 8 \mathrm{H}) ;{ }^{13} \mathrm{C}$ NMR $\left(75 \mathrm{MHz}, \mathrm{CDCl}_{3}\right) \delta[\mathrm{ppm}]=161.46,102.43,54.42,39.49$; resolved signals of the minor rotamer: ${ }^{1} \mathrm{H}$ NMR $\left(300 \mathrm{MHz}, \mathrm{CDCl}_{3}\right) \delta[\mathrm{ppm}]=8.02(\mathrm{dd}, J=12.1 \mathrm{~Hz}, J=3.6 \mathrm{~Hz}, 1 \mathrm{H}), 4.31$ (td, $J=5.2 \mathrm{~Hz}, J=2.5 \mathrm{HZ}, 1 \mathrm{H}), 3.84-3.42(\mathrm{~m}, 6 \mathrm{H}), 3.32-3.25(\mathrm{~m}, 2 \mathrm{H}) ;{ }^{13} \mathrm{C}$ NMR $(75 \mathrm{MHz}$, $\left.\mathrm{CDCl}_{3}\right) \delta[\mathrm{ppm}]=165.10,103.54,54.81,43.75$.

The NMR data are in agreement with reported data. ${ }^{[12]}$ 


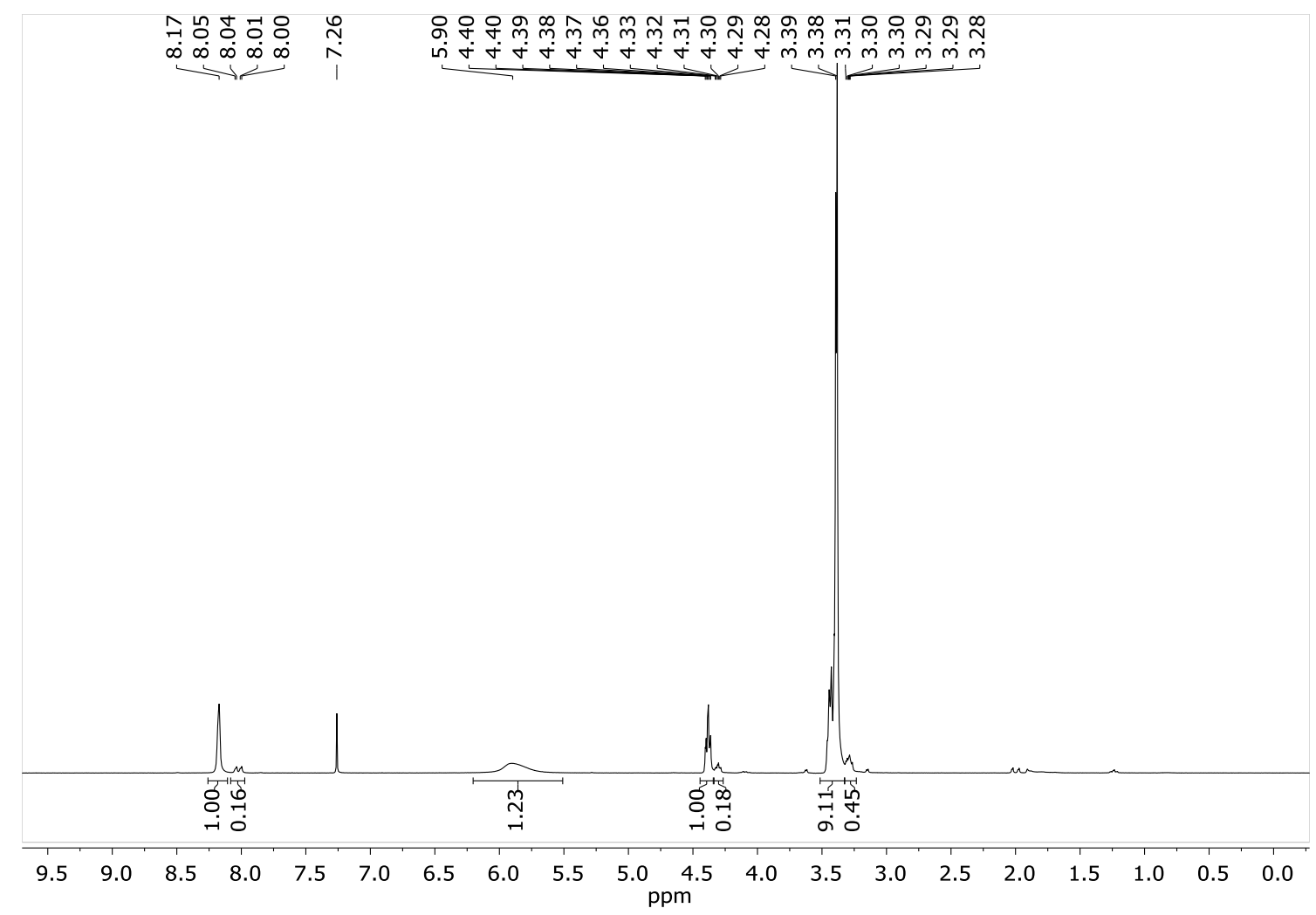

${ }^{1} \mathrm{H}$ NMR spectrum of $\mathrm{N}$-(2,2-dimethoxyethyl)formamide (300 MHz, $\mathrm{CDCl}_{3}$ ).

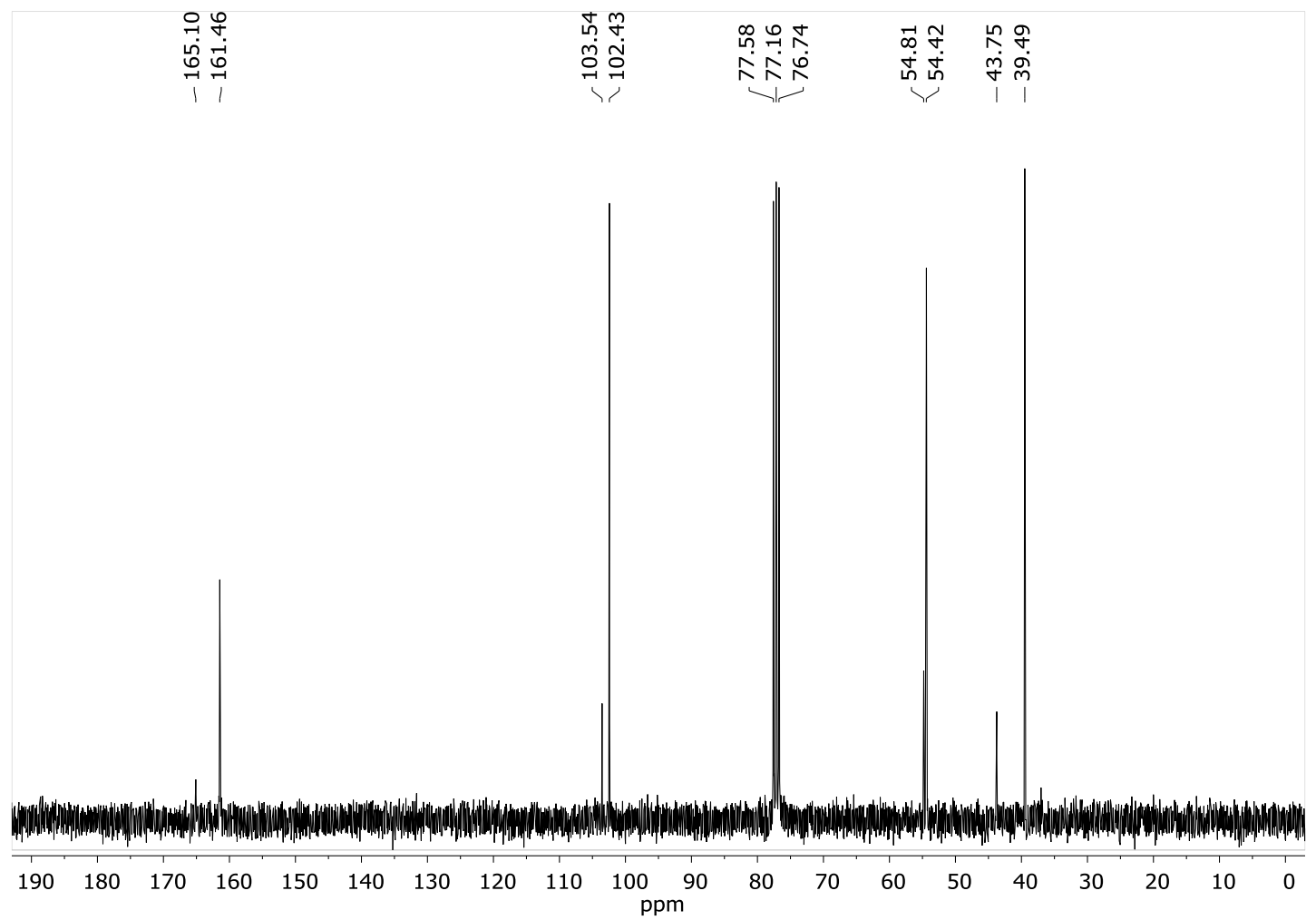

${ }^{13} \mathrm{C}$ NMR spectrum of $\mathrm{N}$-(2,2-dimethoxyethyl)formamide (75 $\mathrm{MHz}, \mathrm{CDCl}_{3}$ ). 


\subsubsection{Synthesis of $\mathbf{N}$-Phenethylformamide (PEF)}

2-Phenylethan-1-amine $(3.78 \mathrm{~mL}, \quad 30.0 \mathrm{mmol}, 1.00$ equiv), formamide
$(1.20 \mathrm{~mL}, 30.0 \mathrm{mmol}, 1.00$ equiv) were reacted according to general procedure
6 (chapter 2.2.6, page 18). Purification by a small plug of silica gel ( $n$-pentane:EtOAc $=5: 1 \rightarrow n$-pentane:EtOAc $=2: 1$ ) furnished the title formamide as yellow oil (3.18 g, $21.3 \mathrm{mmol}, 71 \%)$.

$\mathbf{M}\left(\mathrm{C}_{9} \mathrm{H}_{11} \mathrm{NO}\right)=149.19 \mathrm{~g} / \mathrm{mol}$; the formamide PEF was obtained as mixture of rotamers 83:17; resolved signals of the major rotamer: ${ }^{1} \mathbf{H} \mathbf{N M R}\left(300 \mathrm{MHz}, \mathrm{CDCl}_{3}\right) \delta[\mathrm{ppm}]=8.10$ (d, $J=1.4 \mathrm{~Hz}, 1 \mathrm{H}$ ), 7.40-7.12 (m, 5H), 5.86 (br.s, $1 \mathrm{H}, \mathrm{NH}$ ), 3.57 (qd, $J=6.8 \mathrm{~Hz}, J=2.3 \mathrm{~Hz}, 2 \mathrm{H}$ ), $2.85(\mathrm{t}, J=7.4 \mathrm{~Hz}, 2 \mathrm{H}) ;{ }^{13} \mathrm{C}$ NMR $\left(75 \mathrm{MHz}, \mathrm{CDCl}_{3}\right) \delta[\mathrm{ppm}]=161.30,138.59,128.81,128.75$, 126.70, 39.27, 35.57; resolved signals of the minor rotamer: ${ }^{1} \mathbf{H} \mathbf{N M R}(300 \mathrm{MHz}$, $\left.\mathrm{CDCl}_{3}\right) \delta[\mathrm{ppm}]=7.89(\mathrm{dd}, J=12.0 \mathrm{~Hz}, J=5.1 \mathrm{~Hz}, 1 \mathrm{H}), 7.40-7.12(\mathrm{~m}, 5 \mathrm{H}), 3.47$ (qd, $J=6.8 \mathrm{~Hz}, J=2.3 \mathrm{~Hz}, 2 \mathrm{H}) ;{ }^{13} \mathrm{C}$ NMR $\left(75 \mathrm{MHz}, \mathrm{CDCl}_{3}\right) \delta[\mathrm{ppm}]=164.54,137.69,128.91$, 126.97, 43.21, 37.80 .

The NMR data are in agreement with reported data. ${ }^{[19]}$

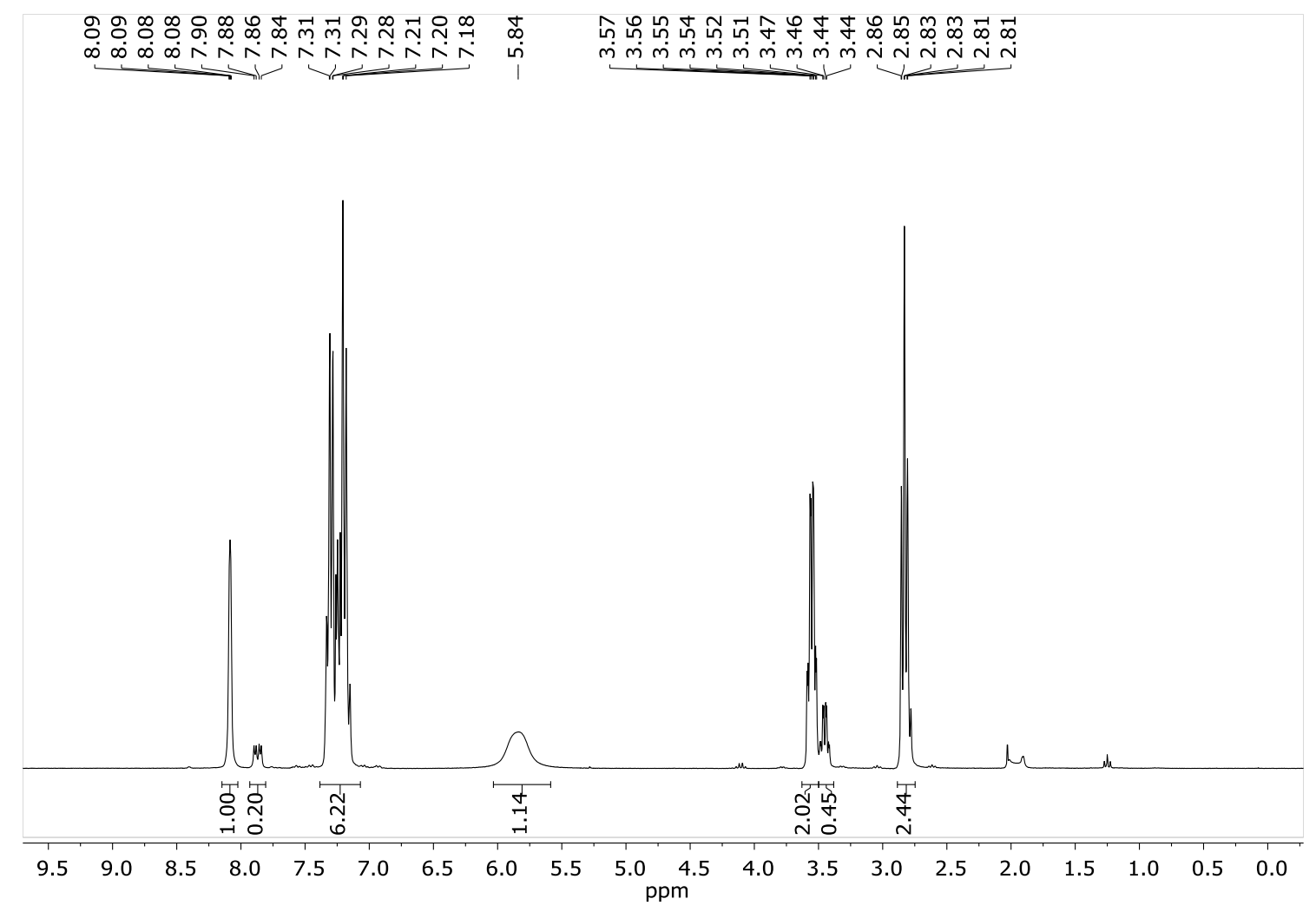

${ }^{1} \mathrm{H}$ NMR spectrum of $\mathrm{N}$-phenethylformamide $\left(300 \mathrm{MHz}, \mathrm{CDCl}_{3}\right)$. 


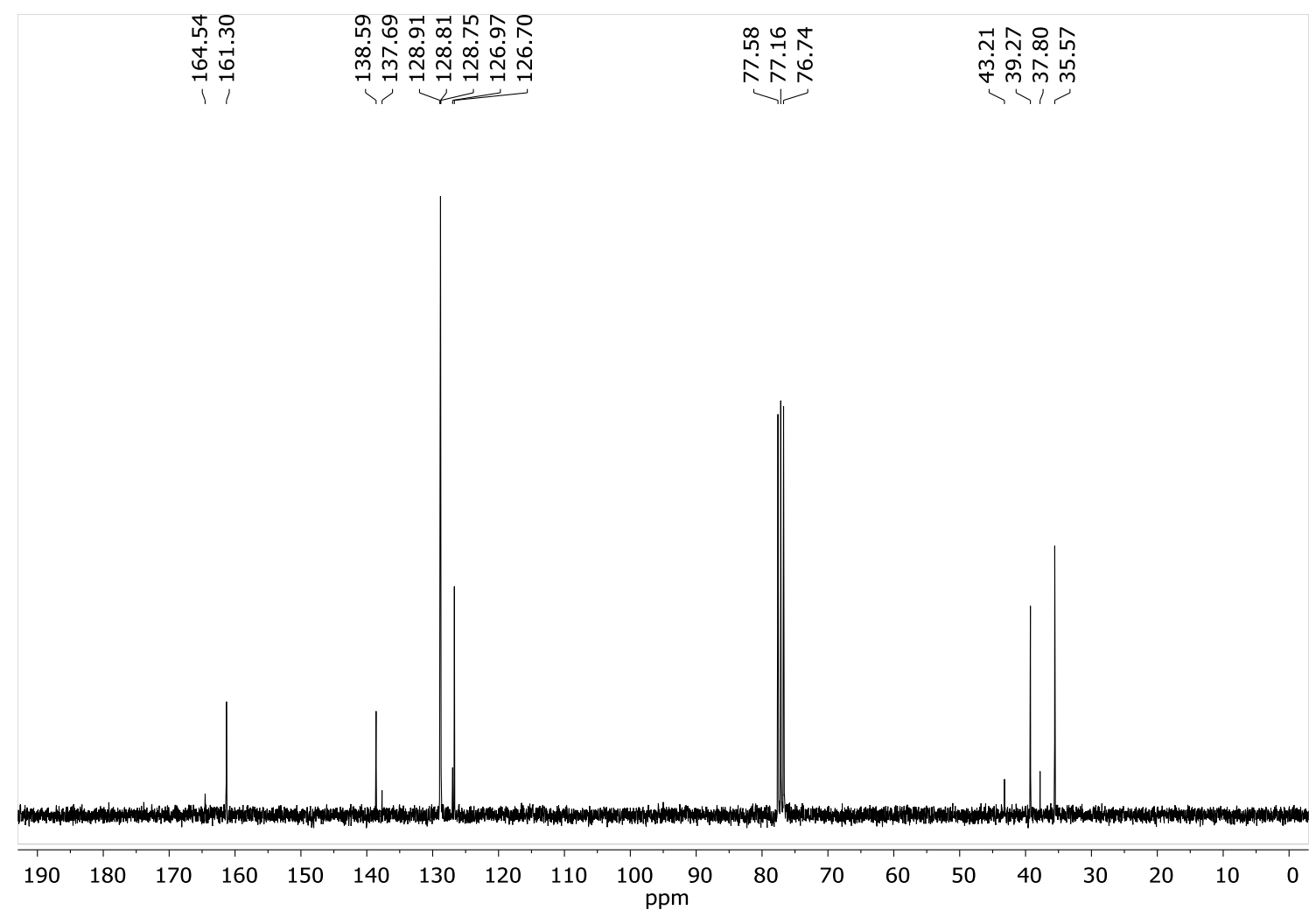

${ }^{13} \mathrm{C}$ NMR spectrum of $\mathrm{N}$-phenethylformamide $\left(75 \mathrm{MHz}, \mathrm{CDCl}_{3}\right)$.

\subsubsection{Synthesis of $N$-Benzyl- $N$-(tert-butyl)formamide (BntBuF)}<smiles>O=C(Br)c1ccccc1CN1CCCCC1</smiles>

According to a procedure of Huy, ${ }^{[20]} \mathrm{Ac}_{2} \mathrm{O}$ (2.40 mL, $25.0 \mathrm{mmol}, 2.50$ equiv) was cooled to $0^{\circ} \mathrm{C}$. Formic acid $(1.50 \mathrm{~mL}, 40.0 \mathrm{mmol}, 4.00$ equiv) was added dropwise and the reaction mixture was stirred at $55^{\circ} \mathrm{C}$ for $3 \mathrm{~h}$. Then, the mixture was cooled to $0{ }^{\circ} \mathrm{C}$ and added dropwise to a solution of $\mathrm{N}$-benzyl-2-methylpropan2-amine (1.81 mL, $10.0 \mathrm{mmol}, 1.00$ equiv) in THF (20.0 mL). After stirring at $0{ }^{\circ} \mathrm{C}$ for $5 \mathrm{~min}$ the reaction mixture was allowed to reach room temperature, and was stirred at this temperature for $5 \mathrm{~d}$. The mixture was diluted with $\mathrm{CH}_{2} \mathrm{Cl}_{2}$ and a saturated aqueous $\mathrm{NaHCO}_{3}$ solution was added to adjust a $\mathrm{pH}=9$. The phases were separated, and the organic phase was extracted with $\mathrm{CH}_{2} \mathrm{Cl}_{2}$. The combined organic phase was dried $\left(\mathrm{MgSO}_{4}\right)$, filtered and the solvent was removed under reduced pressure. Purification by column chromatography on silica gel ( $n$-pentane:EtOAc = 7:1) furnished the title formamide as colorless oil $(1.42 \mathrm{~g}, 7.42 \mathrm{mmol}$, $75 \%)$.

M $\left(\mathrm{C}_{12} \mathrm{H}_{17} \mathrm{NO}\right)=191.13 \mathrm{~g} / \mathrm{mol}$; the formamide BntBuF was obtained as mixture of rotamers 94:6; resolved signals of the major rotamer: ${ }^{1} \mathbf{H}$ NMR $\left(300 \mathrm{MHz}, \mathrm{CDCl}_{3}\right) \delta[\mathrm{ppm}]=8.69(\mathrm{~s}, 1 \mathrm{H})$, 7.39-7.12 (m, 5H), $4.64(\mathrm{~s}, 2 \mathrm{H}), 1.35(\mathrm{~s}, 9 \mathrm{H}) ;{ }^{13} \mathrm{C} \mathrm{NMR}\left(75 \mathrm{MHz}, \mathrm{CDCl}_{3}\right) \delta[\mathrm{ppm}]=162.53$, $138.98,128.48,126.88,126.79,55.98,44.09,30.14$; resolved signals of the minor rotamer: ${ }^{1} \mathrm{H}$ NMR $\left(300 \mathrm{MHz}, \mathrm{CDCl}_{3}\right) \delta[\mathrm{ppm}]=8.31(\mathrm{~s}, 1 \mathrm{H}), 7.39-7.12(\mathrm{~m}, 5 \mathrm{H}), 4.47(\mathrm{~s}, 2 \mathrm{H}), 1.39(\mathrm{~s}$, 
9H); ${ }^{13} \mathrm{C} \mathrm{NMR}\left(75 \mathrm{MHz}, \mathrm{CDCl}_{3}\right) \delta[\mathrm{ppm}]=164.81,139.53,128.81,127.55,126.40,56.75$, 50.24, 28.25; IR (ATR) $\tilde{v}\left[\mathrm{~cm}^{-1}\right]=3030(w), 2977(m), 2930(w), 1651(s), 1470(m), 1454(m)$, $1429(w), 1399(w), 1380(s), 1353(s), 1287(w), 1267(w), 1202(m), 1193(m), 1097(w)$, $1030(m), 961(\mathrm{~m}), 724(\mathrm{~s}), 696(\mathrm{~s}), 620(\mathrm{~m}), 584(\mathrm{~m}), 503(\mathrm{~m})$; MS (El) m/z [u] $(\%)=165\left(\left[\mathrm{M}^{+}\right]\right.$, 19), 176 (3), 146 (2), 136 (4), 135 (31), 134 (73), 107 (6), 106 (36), 104 (4), 92 (9), 91 (100), 86 (5), 84 (9), 79 (15), 77 (10), 70 (2), 65 (17), 63 (3), 57 (15), 51 (8), 49 (15), 42 (7); HRMS (EI) calculated for $\mathrm{C}_{12} \mathrm{H}_{17} \mathrm{NO}^{+}: \mathrm{m} / \mathrm{z}[\mathrm{u}]=191.1305$; found: $\mathrm{m} / \mathrm{z}[\mathrm{u}]=191.1301$.

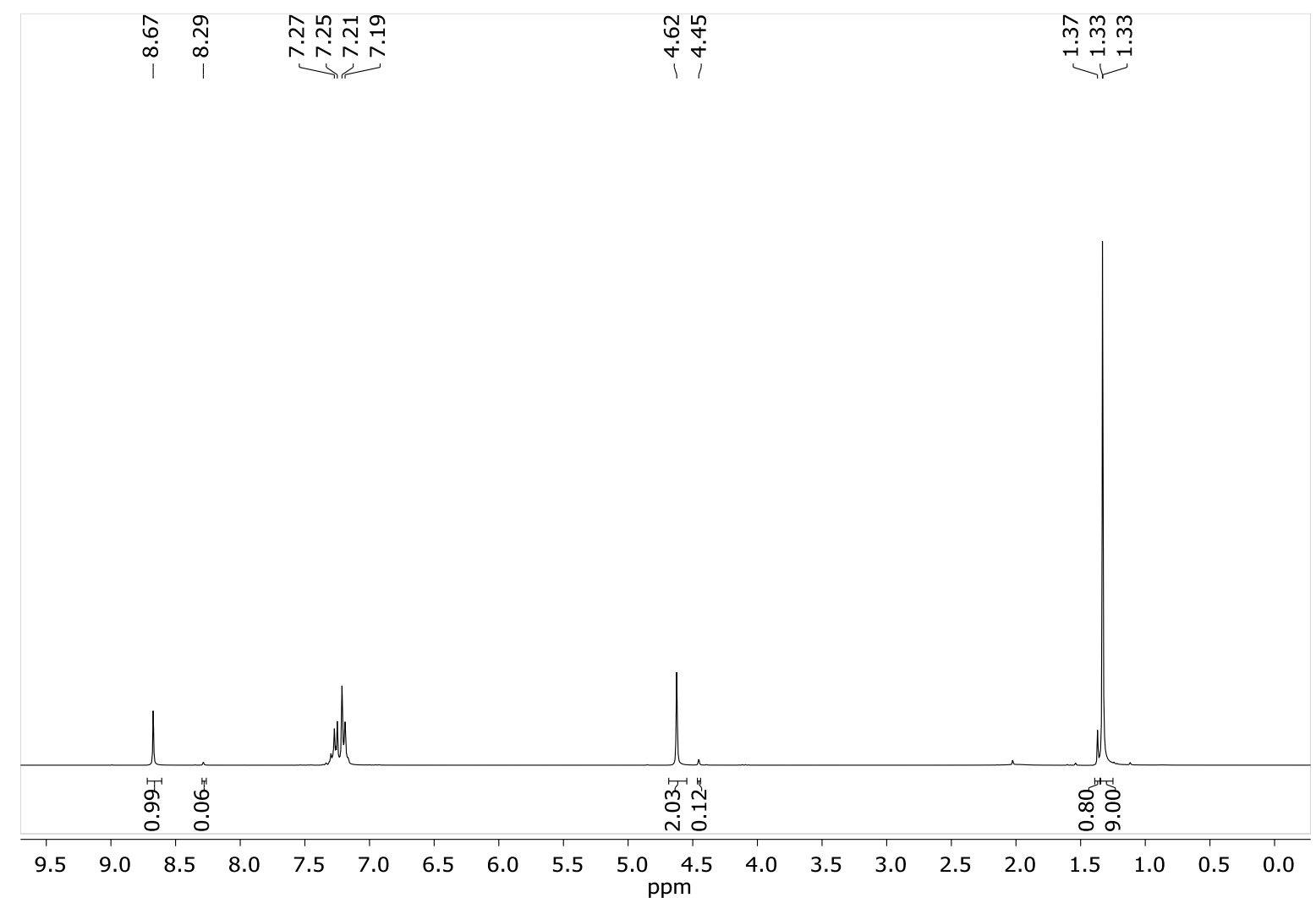

${ }^{1} \mathrm{H}$ NMR spectrum of $\mathrm{N}$-benzyl- $\mathrm{N}$-(tert-butyl)formamide $\left(300 \mathrm{MHz}, \mathrm{CDCl}_{3}\right)$. 


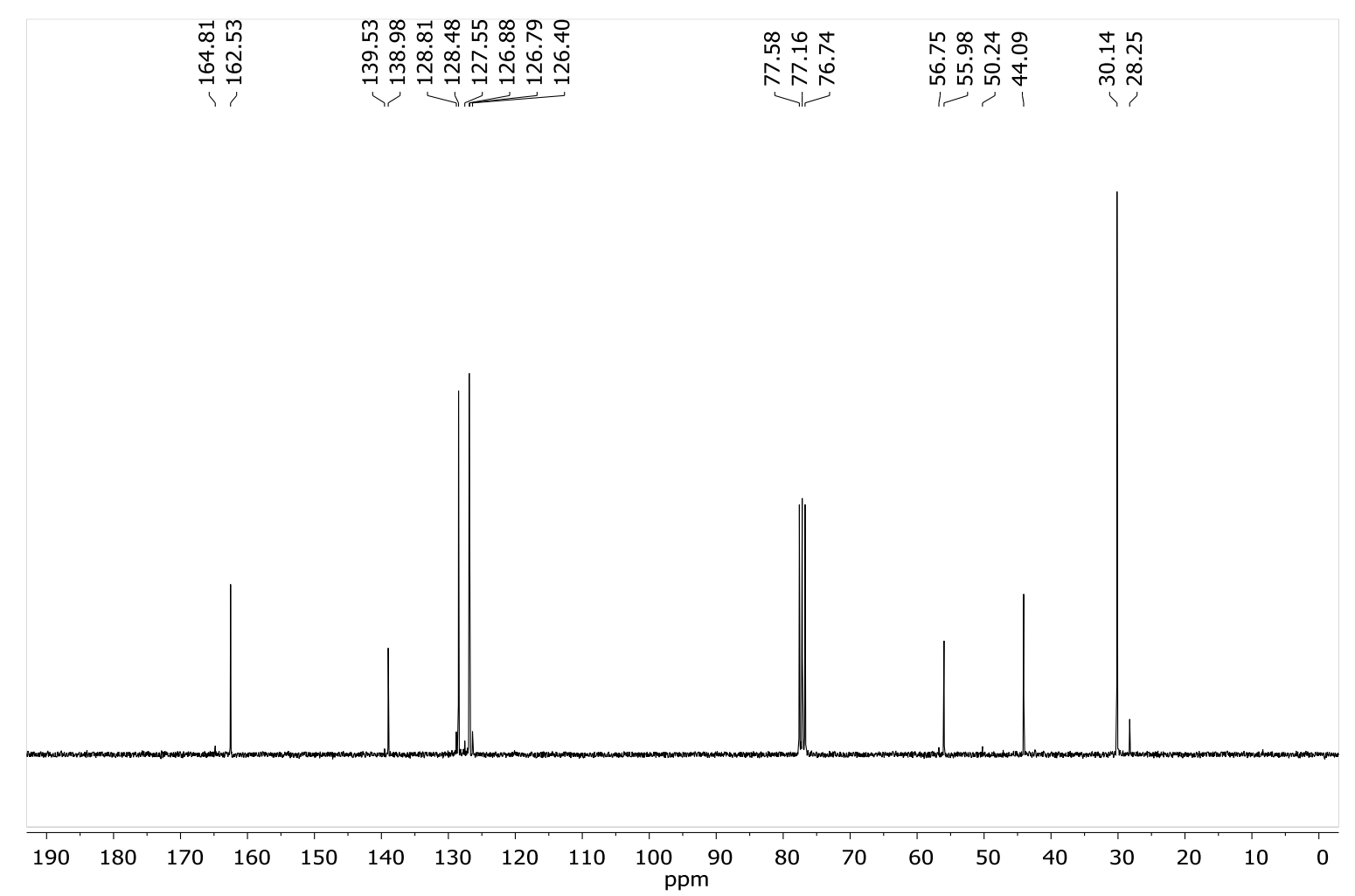

${ }^{13} \mathrm{C}$ NMR spectrum of $\mathrm{N}$-benzyl- $\mathrm{N}$-(tert-butyl)formamide $\left(75 \mathrm{MHz}, \mathrm{CDCl}_{3}\right.$ ).

\subsubsection{Synthesis of $\boldsymbol{N}$-(Naphthalen-1-ylmethyl)formamide (NaphMF)}<smiles>O=CNCc1cccc2ccccc12</smiles>

Naphthalen-1-ylmethanamine $\quad(0.72 \mathrm{~mL}, \quad 5.00 \mathrm{mmol}, \quad 1.00$ equiv $)$, formamide $(0.20 \mathrm{~mL}, 5.00 \mathrm{mmol}, 1.00$ equiv) and PIDA $(80.0 \mathrm{mg}$, $1.00 \mathrm{mmol}, 5 \mathrm{~mol} \%)$ were reacted according to general procedure 6 (chapter 2.2.6, page 18). For crystallization, the crude product was dissolved in $\mathrm{CH}_{2} \mathrm{Cl}_{2}$ and overlapped with $n$-pentane. The title formamide was obtained as yellow solid (723 mg, $3.91 \mathrm{mmol}, 78 \%$ ).

$\mathbf{M}\left(\mathrm{C}_{12} \mathrm{H}_{11} \mathrm{NO}\right)=185.23 \mathrm{~g} / \mathrm{mol}$; the formamide NaphMF was obtained as mixture of rotamers 90:10; resolved signals of the major rotamer: ${ }^{1} \mathbf{H}$ NMR $\left(300 \mathrm{MHz}, \mathrm{CDCl}_{3}\right) \delta[\mathrm{ppm}]=8.22(\mathrm{~s}$, $1 \mathrm{H}), 8.00(\mathrm{~d}, J=8.2 \mathrm{~Hz}, 1 \mathrm{H}), 7.91-7.86(\mathrm{~m}, 1 \mathrm{H}), 7.85-7.80(\mathrm{~m}, 1 \mathrm{H}), 7.59-4.49(\mathrm{~m}, 2 \mathrm{H}), 7.47-$ $7.39(\mathrm{~m}, 2 \mathrm{H}), 5.84$ (br.s, $1 \mathrm{H}, \mathrm{NH}), 4.91(\mathrm{~d}, J=5.6 \mathrm{~Hz}, 2 \mathrm{H}) ;{ }^{13} \mathrm{C} \mathbf{N M R} \quad(75 \mathrm{MHz}$, $\left.\mathrm{CDCl}_{3}\right) \delta[\mathrm{ppm}]=160.88,133.94,132.92,131.32,128.91,128.88,126.87,126.80,126.17$, 125.46, 123.43, 40.33; resolved signals of the minor rotamer: ${ }^{1} \mathrm{H}$ NMR $(300 \mathrm{MHz}$, $\left.\mathrm{CDCl}_{3}\right) \delta[\mathrm{ppm}]=8.04(\mathrm{~d}, J=8.4 \mathrm{~Hz}, 2 \mathrm{H}), 7.93-7.90(\mathrm{~m}, 1 \mathrm{H}), 4.85(\mathrm{~d}, J=5.6 \mathrm{~Hz}, 2 \mathrm{H})$; ${ }^{13} \mathrm{C} \mathrm{NMR}\left(75 \mathrm{MHz}, \mathrm{CDCl}_{3}\right) \delta[\mathrm{ppm}]=164.76,125.46,43.38$.

The NMR data are in agreement with reported data. ${ }^{[1]}$ 


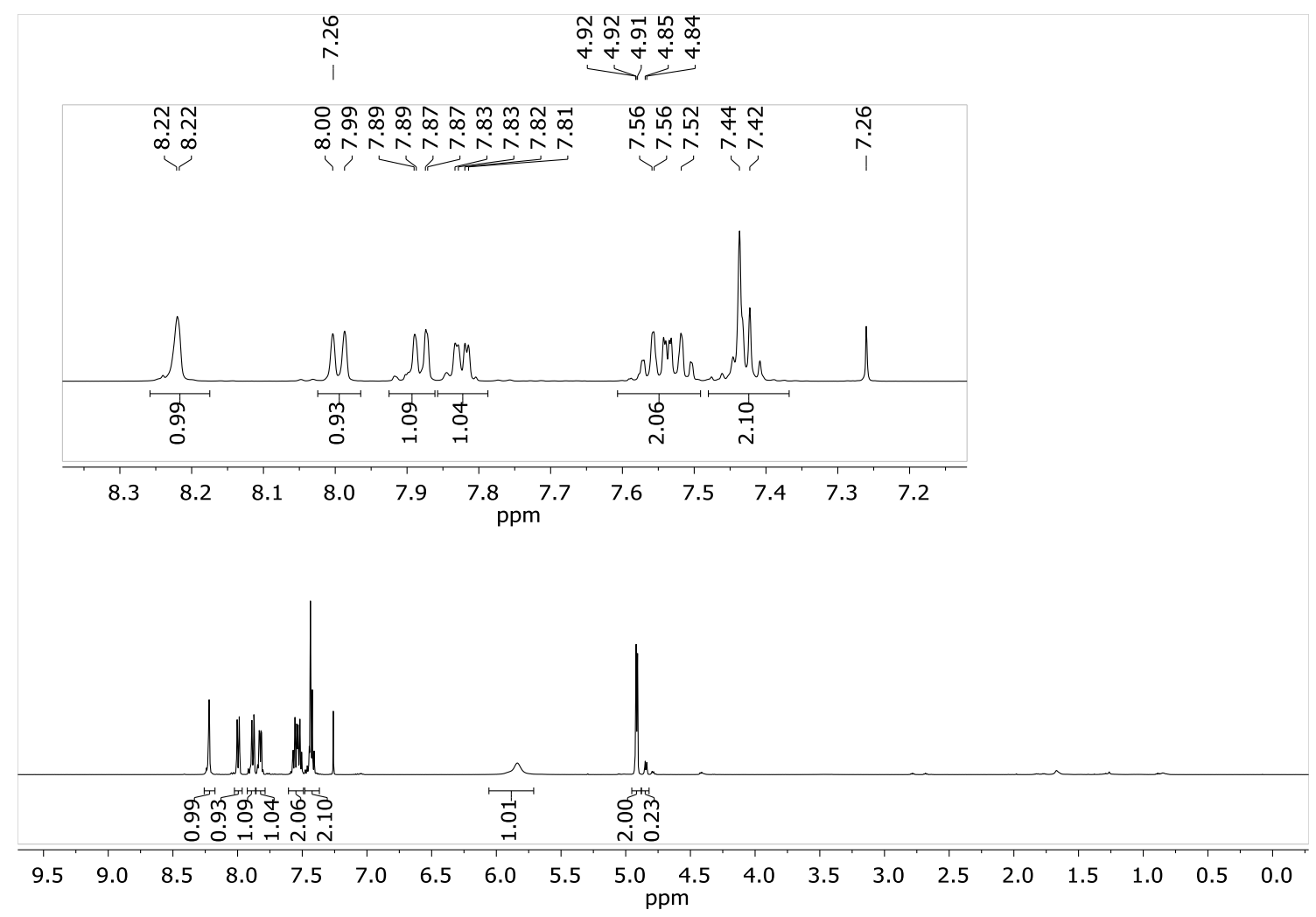

${ }^{1} \mathrm{H}$ NMR spectrum of $\mathrm{N}$-(naphthalen-1-ylmethyl)formamide (300 $\mathrm{MHz}, \mathrm{CDCl}_{3}$ ).

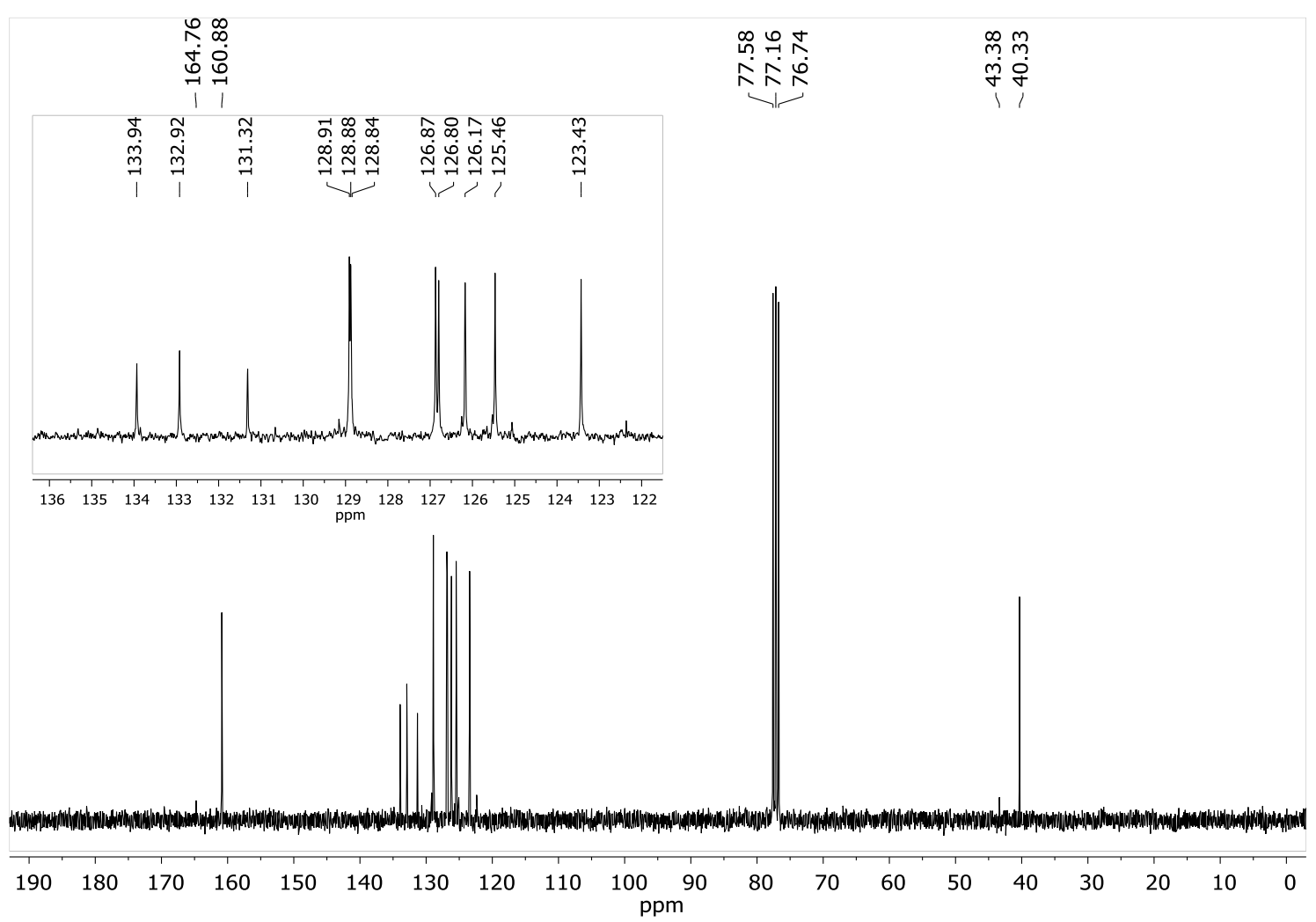

${ }^{13} \mathrm{C}$ NMR spectrum of $\mathrm{N}$-(naphthalen-1-ylmethyl)formamide $\left(75 \mathrm{MHz}, \mathrm{CDCl}_{3}\right)$. 


\subsubsection{Synthesis of 3,4-Dihydroisoquinoline-2(1 H)-carbaldehyde (F/soChin)}

o 1,2,3,4-Tetrahydroisoquinoline (2.54 mL, $20.0 \mathrm{mmol}, \quad 1.00$ equiv),<smiles>C=CN1CCc2ccccc2C1</smiles>
formamide $(0.80 \mathrm{~mL}, 20.0 \mathrm{mmol}, 1.00$ equiv) and PIDA (322 mg, $1.00 \mathrm{mmol}, 5 \mathrm{~mol} \%)$ were reacted according to general procedure 6 (chapter 2.2.6, page 18). Purification by a small plug of silica gel ( $n$-pentane:EtOAc $=5: 1 \rightarrow$ $n$-pentane:EtOAc = 1:1) furnished the title formamide as orange oil $(3.26 \mathrm{~g}, 20.0 \mathrm{mmol}$, quantitative).

M $\left(\mathrm{C}_{10} \mathrm{H}_{11} \mathrm{NO}\right)=161.20 \mathrm{~g} / \mathrm{mol}$; the formamide F/soChin was obtained as mixture of rotamers 62:38; resolved signals of the major rotamer: ${ }^{1} \mathbf{H}$ NMR $\left(300 \mathrm{MHz}, \mathrm{CDCl}_{3}\right) \delta[\mathrm{ppm}]=8.19(\mathrm{~s}$, $1 \mathrm{H}), 7.24-7.04(\mathrm{~m}, 4 \mathrm{H}), 4.68(\mathrm{~s}, 2 \mathrm{H}), 3.64(\mathrm{t}, J=5.9 \mathrm{~Hz}, 2 \mathrm{H}), 2.96-2.81(\mathrm{~m}, 2 \mathrm{H}) ;{ }^{13} \mathrm{C}$ NMR $\left(75 \mathrm{MHz}, \mathrm{CDCl}_{3}\right) \delta[\mathrm{ppm}]=161.71,133.63,131.87,128.99,126.81,126.71,43.30,42.39$, 29.83; resolved signals of the minor rotamer: ${ }^{1} \mathbf{H}$ NMR $\left(300 \mathrm{MHz}, \mathrm{CDCl}_{3}\right) \delta[\mathrm{ppm}]=8.25$ (s, $1 \mathrm{H}), 7.24-7.04(\mathrm{~m}, 4 \mathrm{H}), 4.53(\mathrm{~s}, 2 \mathrm{H}), 3.78(\mathrm{t}, J=6.2 \mathrm{~Hz}, 2 \mathrm{H}), 2.96-2.81(\mathrm{~m}, 2 \mathrm{H}) ;{ }^{13} \mathrm{C}$ NMR $\left(75 \mathrm{MHz}, \mathrm{CDCl}_{3}\right) \delta[\mathrm{ppm}]=161.21,134.53,132.33,129.27,127.17,126.57,125.96,47.39$, 38.07, 28.02.

The NMR data are in agreement with reported data. ${ }^{[22]}$

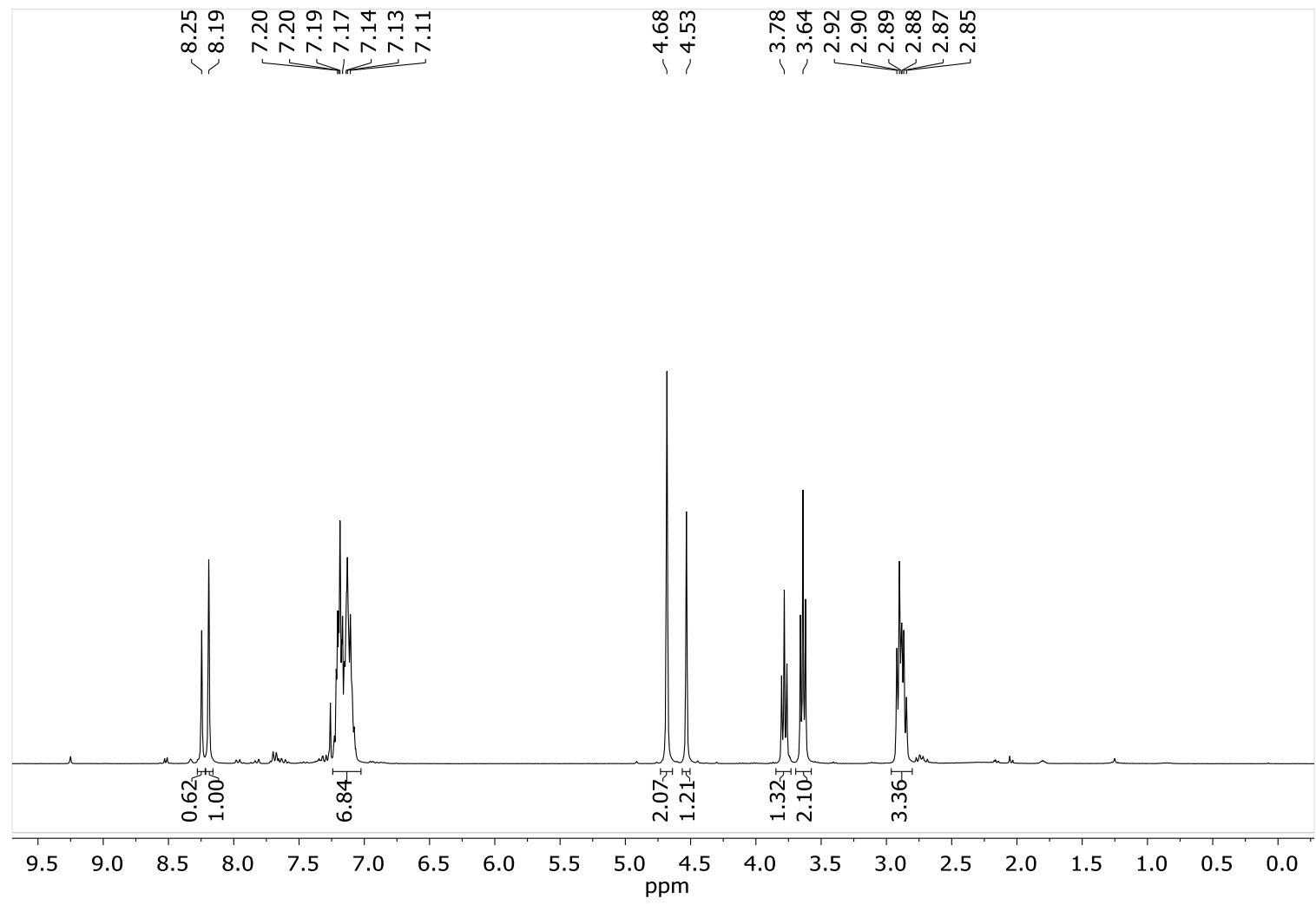

${ }^{1} \mathrm{H}$ NMR spectrum of 3,4-dihydroisoquinoline-2(1H)-carbaldehyde $\left(300 \mathrm{MHz}, \mathrm{CDCl}_{3}\right)$. 


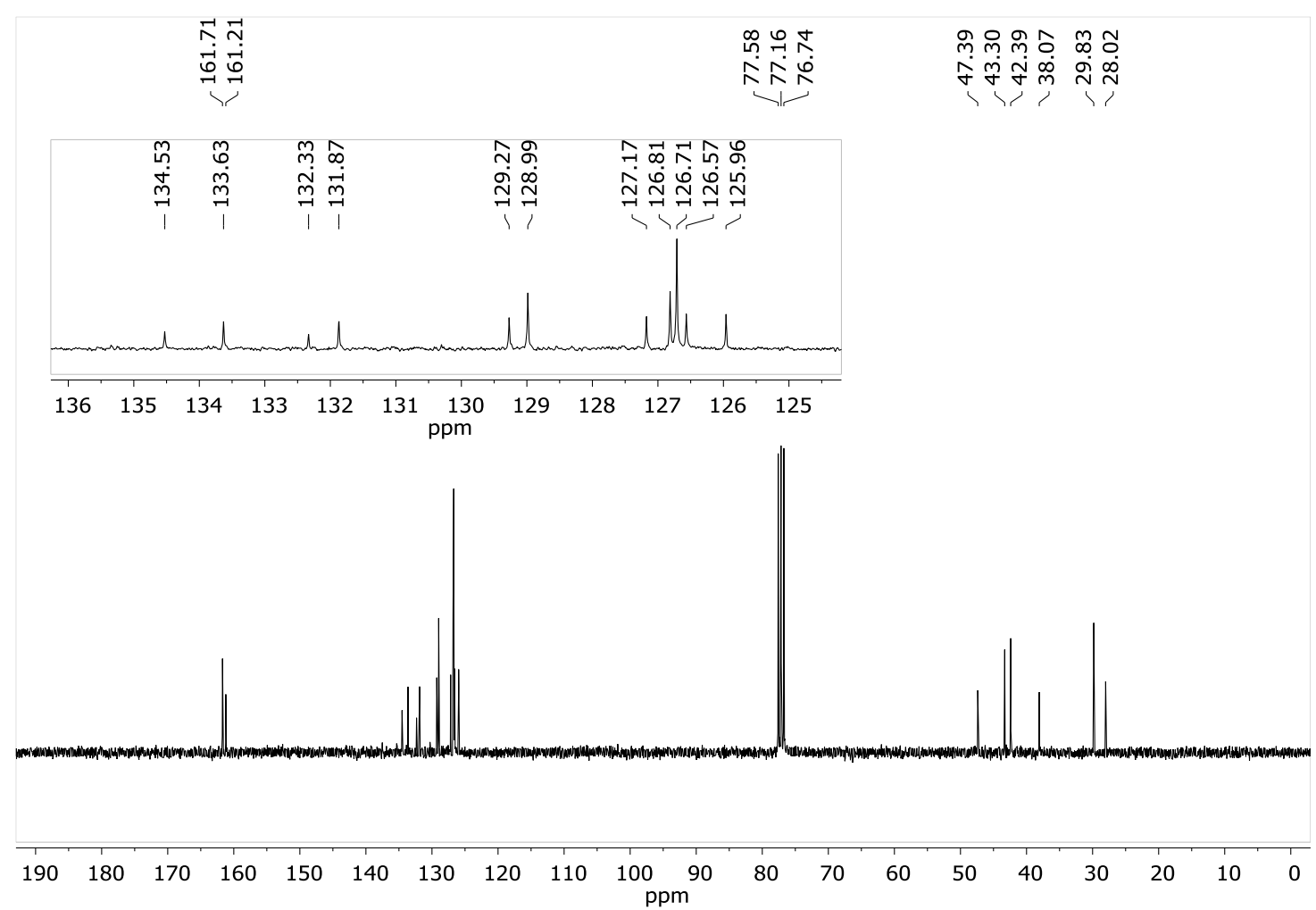

${ }^{13} \mathrm{C}$ NMR spectrum of 3,4-dihydroisoquinoline-2(1H)-carbaldehyde $\left(75 \mathrm{MHz}, \mathrm{CDCl}_{3}\right)$. 


\subsubsection{Synthesis of Chloro Alklanes}

Alkyl chlorides were prepared using the respective alcohol (300 $\mu \mathrm{mol}, 1.0$ equiv) instead of 4-tert-butylbenzyl alcohol (1) with $\mathbf{B z C l}$ in the presence of FPyr according to general procedure I and II in our previous manuscript ${ }^{[5]}$ (2) using NCS and $\mathbf{P P h}$ as described in general protocol 4 (chapter 2.2.4 on page 16) and (3) with $\mathrm{SOCl}_{2}$ as given in general procedure 5 (section 2.2.5, page 17). For work up the reaction mixture was either the non-aqueous or the aqueous protocol of general procedure 5 were applied.

\subsubsection{Synthesis of 1-(tert-Butoxycarbonyl)-3-(chloromethyl)indole (30)}

The procedure for the synthesis of the starting material 1-(tert-butoxycarbonyl)-3(hydroxylmethyl)indole (10) is given in chapter 2.3.5.1 (page 79).

With BzCl and FPyr (PH4223): The title compound was synthesized from 1-(tertbutoxycarbonyl)-3-(hydroxylmethyl)indole (10, $74.2 \mathrm{mg}, 300 \mu \mathrm{mol}, 1.0$ equiv) with $\mathrm{BzCl}$ $(42 \mu \mathrm{L}, 51 \mathrm{mg})$ in the presence of FPyr $(3.0 \mu \mathrm{L}, 3.1 \mathrm{mg}, 10 \mathrm{~mol} \%)$ in dry dioxane $(300 \mu \mathrm{L}$, $1.0 \mathrm{M})$ under stirring for $17 \mathrm{~h}$ at room temperature. ${ }^{1} \mathrm{H}$ NMR of the crude material with mesitylene as internal standard after non-aqueous work up unravelled chloride 30 in $87 \%$ yield and complete consumption of the starting materials.

With $\mathrm{SOCl}_{2}$ (PH4222): Substrate 10 (300 $\mu \mathrm{mol}, 1.00$ equiv) was allowed to react with thionyl chloride (27 $\mu \mathrm{L}, 360 \mu \mathrm{mol}, 1.20$ equiv) in dry $\mathrm{CH}_{2} \mathrm{Cl}_{2}(300 \mu \mathrm{L}, 1 \mathrm{M})$ for $15 \mathrm{~min}$ at $0{ }^{\circ} \mathrm{C}$ and $17 \mathrm{~h}$ at room temperature. ${ }^{1} \mathrm{H}$ NMR of the crude product past non-aqueous work up indicated the title chloride in $13 \%$ yield, while no starting material remained.

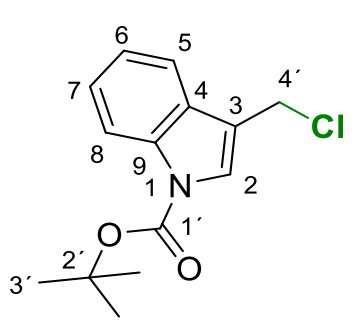

With $\mathrm{BzCl}$ and FPyr (PH1187), isolated yield: According to general procedure II in our previous publication [5]. Benzoyl chloride $(216 \mu \mathrm{L}$, $261 \mathrm{mg}, 1.84 \mathrm{mmol}, 1.20$ equiv) was added at $0{ }^{\circ} \mathrm{C}$ to a solution of 1 (tert-butoxycarbonyl)-3-(hydroxymethyl)indole (10, $373 \mathrm{mg}$, $1.51 \mathrm{mmol}, 1.00$ equiv), FPyr $(15.0 \mu \mathrm{L}, 15.6 \mathrm{mg}, 0.150 \mathrm{mmol}$, $10 \mathrm{~mol} \%$ ) in dioxane ( $1 \mathrm{~mL}, 2.0 \mathrm{M})$. After $15 \mathrm{~min}$ of stirring, the cooling bath was removed and the reaction solution was allowed to stir for $24 \mathrm{~h}$ at room temperature. ${ }^{1} \mathrm{H}$ NMR of the crude material (447 mg, brownish solid) showed $\geq 98 \%$. Next the, crude product was dissolved in a minimum amount of toluene $(0.5 \mathrm{~mL})$ at $40^{\circ} \mathrm{C}$ in a water bath. ${ }^{(9)}$ This solution was applied to column chromatography on silica gel (mass crude material/SiO $\mathrm{S}_{2} 1: 9$ )

\footnotetext{
${ }^{9}$ The crude material is virtually insoluble in the eluent. Adsorption on silica gel or isolute prior to chromatography results in diminished yields and low purities.
} 
with $\mathrm{Et}_{2} \mathrm{O} / n$ Pen 10:90. After concentration with chloroform $(2 \times 2 \mathrm{~mL})$ and drying for $20 \mathrm{~min}$ at $20 \mathrm{mbar}$ the chloride 30 was isolated as colourless solid in $81 \%$ yield (326 mg, $1.23 \mathrm{mmol}$ ). M $\left(\mathrm{C}_{14} \mathrm{H}_{16} \mathrm{CINO}_{2}\right)=265.735 \mathrm{~g} / \mathrm{mol} ; \mathbf{m p} .84-86^{\circ} \mathrm{C} ; \mathbf{r}_{\mathbf{f}}\left(\mathrm{SiO}_{2}, \mathrm{Et}_{2} \mathrm{O} / n\right.$ Pen $\left.10: 90\right)=0.55+0.00$ (additional weak spots at 0.36+0.26+0.17+0.11); ${ }^{1} \mathrm{H} \mathrm{NMR}\left(400 \mathrm{MHz}, \mathrm{CDCl}_{3}\right) \delta[\mathrm{ppm}]=8.15$ (d, $J=8.0 \mathrm{~Hz}, 1 \mathrm{H}, 8-\mathrm{H}), 7.68-7.65(\mathrm{~m}, 2 \mathrm{H}, 2-\mathrm{H}, 5-\mathrm{H}), 7.38-7.34(\mathrm{~m}, 1 \mathrm{H}, 7-\mathrm{H}), 7.32-7.27(\mathrm{~m}, 1 \mathrm{H}$, $6-\mathrm{H}), 4.78\left(\mathrm{~d}, J=0.8 \mathrm{~Hz}, 4^{\prime}-\mathrm{H}\right), 1.67\left(\mathrm{~s}, 9 \mathrm{H}, 3^{\prime}-\mathrm{H}\right) ;{ }^{13} \mathrm{C}$ NMR $\left(100 \mathrm{MHz}, \mathrm{CDCl}_{3}\right) \delta[\mathrm{ppm}]=149.39$ (C-1'), 135.71 (C-9), 128.68 (C-3 or C-4), 124.94 (C-2), 122.88 (C-6), 119.21 (C-5), 117.06 (C-3 or C-4), 115.41 (C-8), 84.05 (C-2'), 37.75 (C-4'), 28.14 (C-3'); GC-MS (El, 70 eV) m/z [u] $(\%)=267\left(1,\left[\mathrm{C}_{14} \mathrm{H}_{16} \mathrm{Cl}^{37} \mathrm{NO}_{2}\right]^{+}\right), 265\left(3,\left[\mathrm{C}_{14} \mathrm{H}_{16} \mathrm{Cl}^{35} \mathrm{NO}_{2}\right]^{+}\right), 211\left(3, \quad\left[\mathrm{C}_{14} \mathrm{H}_{16} \mathrm{Cl}^{37} \mathrm{NO}_{2^{-}}\right.\right.$ $\left.\left.\mathrm{CH}_{2}=\mathrm{C}\left(\mathrm{CH}_{3}\right)_{2}\right]^{+}\right), 209\left(9,\left[\mathrm{C}_{14} \mathrm{H}_{16} \mathrm{Cl}^{35} \mathrm{NO}_{2}-\mathrm{CH}_{2}=\mathrm{C}\left(\mathrm{CH}_{3}\right)_{2}\right]^{+}\right), 192\left(3,\left[\mathrm{C}_{14} \mathrm{H}_{16} \mathrm{Cl}^{35} \mathrm{NO}_{2}-\mathrm{OtBu}\right]^{+}\right), 174$ $\left.\left(15,\left[\mathrm{M}-\mathrm{Cl}-\mathrm{CH}_{2}=\mathrm{C}\left(\mathrm{CH}_{3}\right)_{2}\right]^{+}\right), 130\left(70 \text {, [indole } \mathrm{CH}_{2}\right]^{+}\right), 102$ (15), 57 (100, [tBu] $\left.]^{+}\right)$; HR-MS (Cl, $\left[\mathrm{C}_{14} \mathrm{H}_{16} \mathrm{Cl}^{35} \mathrm{NO}_{2}\right]^{+}$) calc. 265.0870 u found $265.0836 \mathrm{u}$.

The ${ }^{1} \mathrm{H}$ NMR data was in agreement with literature data. ${ }^{[23]}$

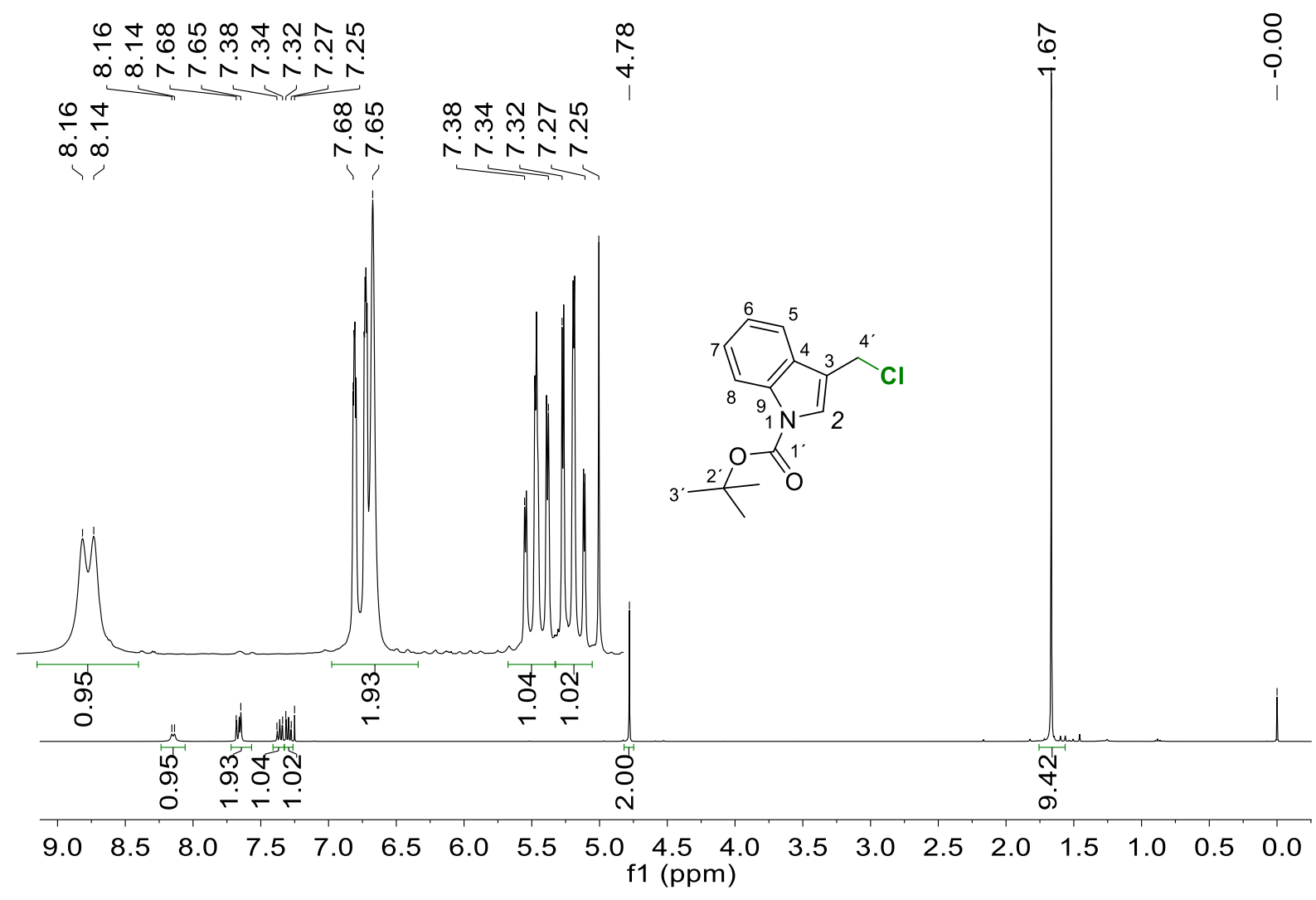

${ }^{1} \mathrm{H}$ NMR spectrum of 1-(tert-butoxycarbonyl)-3-(chloromethyl)indole (3a) $\left(400 \mathrm{MHz}, \mathrm{CDCl}_{3}\right.$ ). 


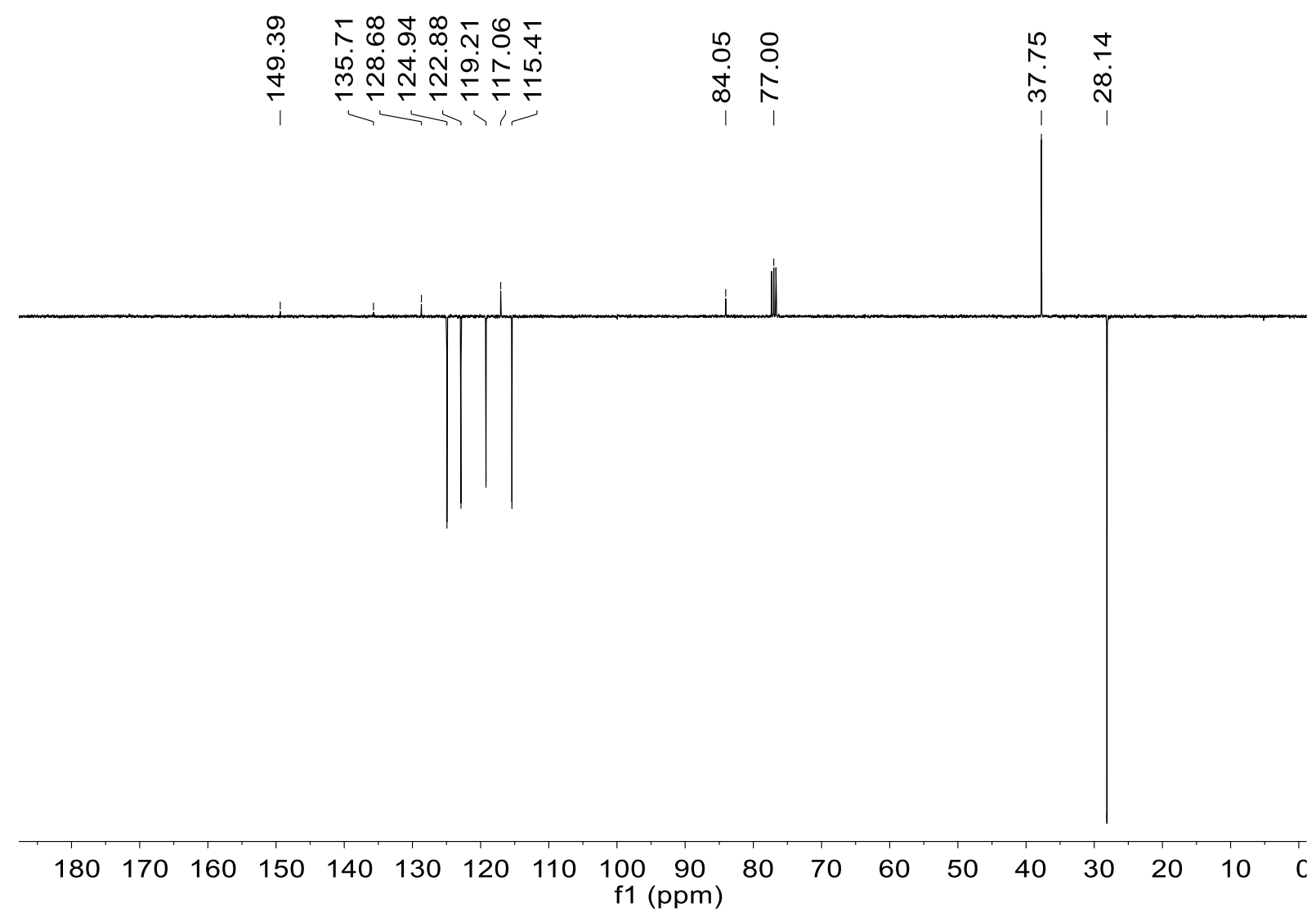

APT-NMR spectrum of 1-(tert-butoxycarbonyl)-3-(chloromethyl)indole (3o, $100 \mathrm{MHz}, \mathrm{CDCl}_{3}$ ).

Peak\#:1 R.Time:16.389(Scan\#:4318

MassPeaks:197

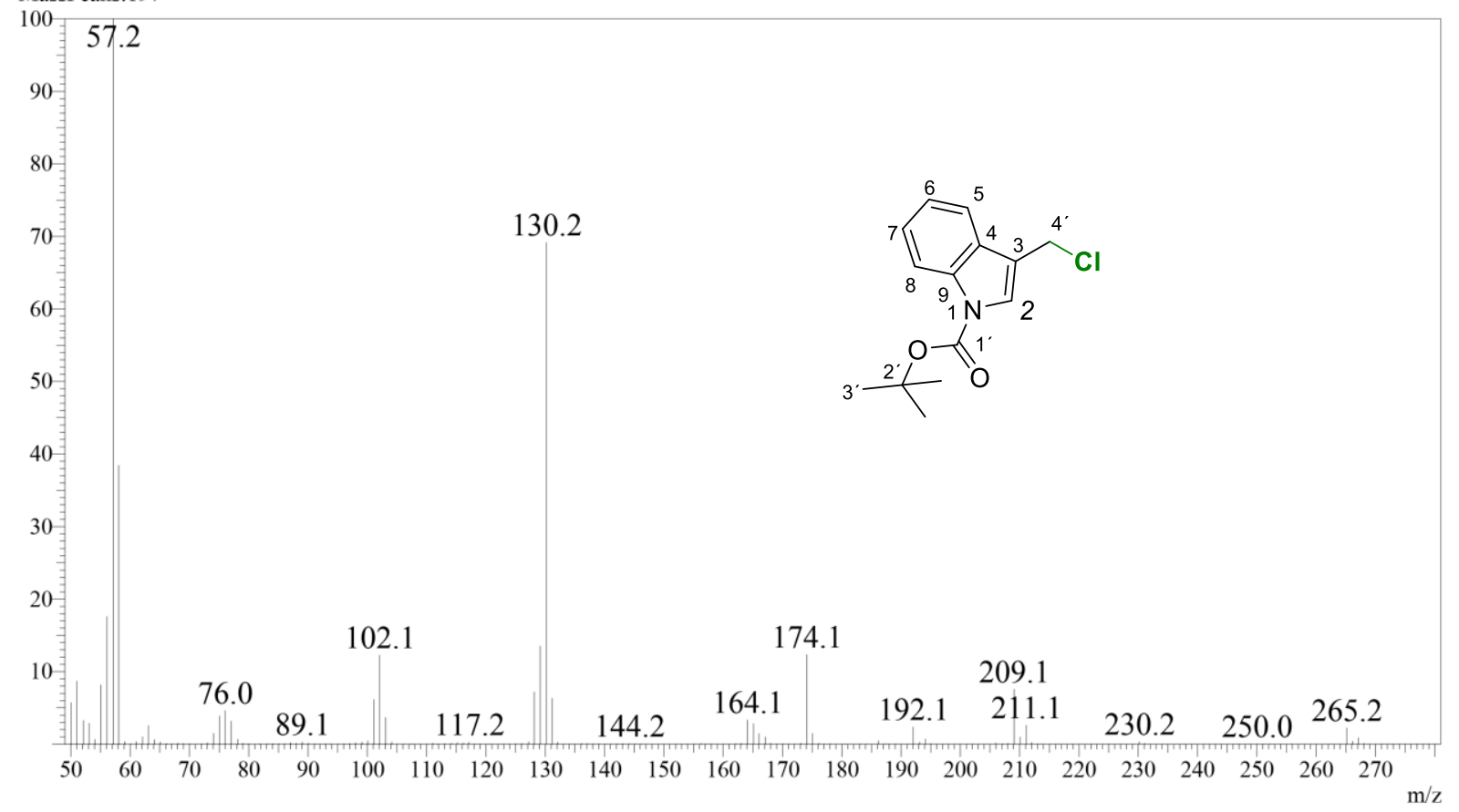

Mass spectrum of 1-(tert-butoxycarbonyl)-3-(chloromethyl)indole (3o, El, $70 \mathrm{eV}$ ). 


\subsubsection{Synthesis of tert-Butyl 2-(3-(chloromethyl)phenyl)acetate (3p)}

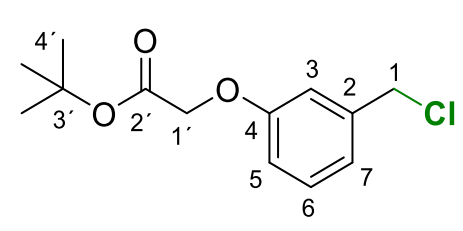

tert-Butyl 2-(3-(hydroxymethyl)phenyl)acetate (1p) was prepared as detailed in reference. ${ }^{[5]}$ Analytical data of and the procedure for the production of the title ester in $86 \%$ isolated yield are also located in reference. ${ }^{[5]}$

With $\mathrm{BzCl}$ and FPyr (PH4237): The title compound was accessed from alcohol (1p, $74.2 \mathrm{mg}$, $300 \mu \mathrm{mol}, 1.00$ equiv) using $\mathrm{BzCl}(42 \mu \mathrm{L}, 51 \mathrm{mg})$ and FPyr (3.0 $\mu \mathrm{L}, 3.1 \mathrm{mg}, 10 \mathrm{~mol} \%)$ in dry dioxane $(300 \mu \mathrm{L}, 1.0 \mathrm{M})$ under stirring for $19 \mathrm{~h}$ at room temperature. ${ }^{1} \mathrm{H}$ NMR of the crude product with mesitylene as internal standard after aqueous work up showed chloride $3 p$ in $94 \%$ yield. No residual starting material could be detected.

With $\mathrm{SOCl}_{2}$ (PH4236): Substrate $1 \mathrm{p}$ (300 $\mu \mathrm{mol}, 1.00$ equiv) was reacted with thionyl chloride (27 $\mu \mathrm{L}, 360 \mu \mathrm{mol}, 1.20$ equiv) in dry $\mathrm{CH}_{2} \mathrm{Cl}_{2}(300 \mu \mathrm{L}, 1.0 \mathrm{M})$ for $15 \mathrm{~min}$ at $0{ }^{\circ} \mathrm{C}$ and $19 \mathrm{~h}$ at ambient temperature. After aqueous work up, ${ }^{1} \mathrm{H}$ NMR of the crude product verified the title chloro alkane in $12 \%$ yield ( $\geq 98 \%$ conversion).

\subsubsection{Synthesis of Chloro-2,3:5,6-Di-O-iso-propylidene-D-mannofuranoside (3q)}

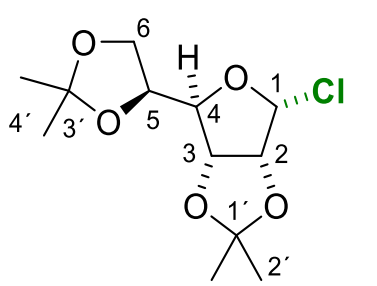

Analytical data of and the experimental procedure for the synthesis of the title acetale in $89 \%$ isolated yield with 40 mol\% TCT are given in reference. ${ }^{[4]}$

With $\mathrm{BzCl}$ and $\mathrm{FPyr}$ at $40^{\circ} \mathbf{C}$ (PH4235): 2,3:5,6-Di-O-isopropylidene- $D$-mannofuranoside (1q, $78.1 \mathrm{mg}, 300 \mu \mathrm{mol}, 1.00$ equiv) was combined with FPyr (5.9 $\mu \mathrm{L}, 6.1 \mathrm{mg}, 60 \mu \mathrm{mol}, 20 \mathrm{~mol} \%)$, dry dioxane $(300 \mu \mathrm{L}, 1.0 \mathrm{M})$ and $\mathrm{BzCl}(42 \mu \mathrm{L}, 51 \mathrm{mg}$, $360 \mu \mathrm{mol}, 1.20$ equiv) and stirred for $20 \mathrm{~h}$ at $40^{\circ} \mathrm{C}$. Next, aqueous work up and ${ }^{1} \mathrm{H}$ NMR with mesitylene as internal standard showed the title compound in $67 \%$ yield beside residual starting material in $4 \%$ yield (95\% conversion).

With $\mathrm{BzCl}$ and FPyr at room temperature (PH4233): The current experiment was carried out as described above with the difference that the reaction mixture was stirred at room temperature for $20 \mathrm{~h}$. Eventually, ${ }^{1} \mathrm{H}$ NMR indicated the chloro ether $3 \mathrm{q}$ in $49 \%$ yield alongside with $4 \%$ remaining substrate 1 q (93\% conversion).

With $\mathrm{SOCl}_{2}$ (PH4232): A suspension of hemiacetale 1q $\left(300 \mu \mathrm{mol}, 1.00\right.$ equiv) in $\mathrm{CH}_{2} \mathrm{Cl}_{2}$ $(300 \mu \mathrm{L}, 1 \mathrm{M})$ was treated with thionyl chloride $\left(27 \mu \mathrm{L}, 360 \mu \mathrm{L}, 1.20\right.$ equiv) at $0{ }^{\circ} \mathrm{C}$ and subsequently stirred for $15 \mathrm{~min}$ at $0^{\circ} \mathrm{C}$ and for $20 \mathrm{~h}$ at room temperature. In the following, aqueous work up and ${ }^{1} \mathrm{H}$ NMR with mesitylene as internal standard showed the title compound in ca. $25 \%$ and as mixture of stereoisomers. Due to signal overlap the ratio of the isomers could not be determined. 


\subsubsection{Synthesis of 3-(tert-Butyldimethylsilyloxy)benzyl chloride (3r)}

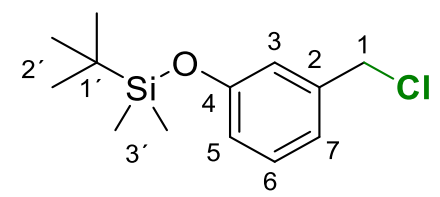

3-(tert-Butyldimethylsilyloxy)phenyl methanol (1r) was synthesized as previously described. ${ }^{[5]}$ The experimental procedure for the preparation of the title compound in $84 \%$ isolated yield and analytical data is compiled in reference,,$^{[5]}$ too.

With $\mathrm{BzCl}$ and FPyr (PH4225): The alcohol 1r (71.5 mg, $300 \mu \mathrm{mol}, 1.00$ equiv) was allowed to react with $\mathrm{BzCl}(42 \mu \mathrm{L}, 51 \mathrm{mg}, 360 \mu \mathrm{mol}, 1.20$ equiv) in the presence of FPyr $(3.0 \mu \mathrm{L}$, $3.1 \mathrm{mg}, 30 \mu \mathrm{mol}, 10 \mathrm{~mol} \%)$ in dioxane $(300 \mu \mathrm{mol}, 1.0 \mathrm{M})$ for $17 \mathrm{~h}$ at room temperature. Subsequently, non-aqueous work up and ${ }^{1} \mathrm{H}$ NMR harnessing mesitylene as internal standard uncovered the title benzylic chloride $3 r$ in $84 \%$ yield together with starting material $\mathbf{~} r$ in $5 \%$ yield, which corresponds to a conversion of $95 \%$.

With $\mathrm{SOCl}_{2}$ (PH4224): Reaction of the starting material 1r (300 $\mu \mathrm{mol}, 1.00$ equiv) with thionyl chloride (27 $\mu \mathrm{mol}, 360 \mu \mathrm{mol}, 1.20$ equiv) for $15 \mathrm{~min}$ at $0{ }^{\circ} \mathrm{C}$ and for $17 \mathrm{~h}$ at room temperature afforded the title compound in $84 \%$ after non-aqueous work up as determined by ${ }^{1} \mathrm{H}$ NMR with mesitylene as internal standard.

\subsubsection{Synthesis of Geranyl chloride (3n)}

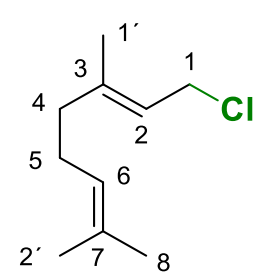

Analytical data and experimental procedures for the preparation of the title compound with $\mathrm{BzCl}$ and $\mathrm{SOCl}_{2}$ have been reported in reference. ${ }^{[5]}$

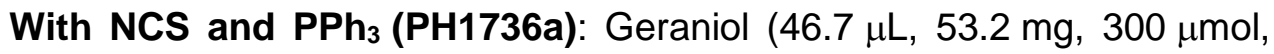
1.00 equiv) was combined with $\mathrm{PPh}_{3}(104 \mathrm{mg}, 390 \mu \mathrm{mol}, 1.30$ equiv), $\mathrm{CH}_{2} \mathrm{Cl}_{2}(1.0 \mathrm{~mL}, 0.3 \mathrm{M})$ and NCS (50 mg, $360 \mu \mathrm{mol}, 1.20$ equiv) and stirred for $15 \mathrm{~min}$ at $0{ }^{\circ} \mathrm{C}$ and for $28 \mathrm{~h}$ at room temperature. After non-aqueous work up, ${ }^{1} \mathrm{H}$ NMR with dodecane as internal standard verified geranyl chloride in $93 \%$ yield and a regioselectivity linear/branched of $\geq 98: 2$. Since no starting material was detected, $\geq 98 \%$ conversion were accomplished. 


\subsubsection{Synthesis of Alcohols}

\subsubsection{Synthesis of 1-(tert-Butoxycarbonyl)-3-(chloromethyl)indole (10)}

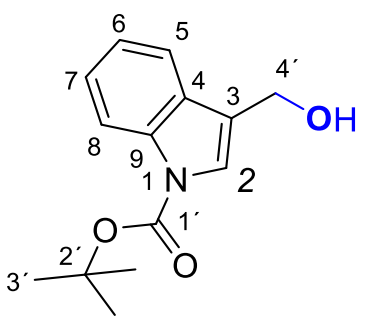

PH1164: 3-Formylindole ( $1.452 \mathrm{~g}, 10.0 \mathrm{mmol}, 1.00$ equiv) was suspended in $\mathrm{CH}_{2} \mathrm{Cl}_{2}(10 \mathrm{~mL}, 1.0 \mathrm{M})$, cooled to $0^{\circ} \mathrm{C}$ and $\mathrm{Boc}_{2} \mathrm{O}$ (3.0 mL, $13.0 \mathrm{mmol}, 1.30$ equiv) and DMAP (122 mg, $1.00 \mathrm{mmol}$, 10 mol\%) were added subsequently. After DMAP addition a strong $\mathrm{CO}_{2}$-evolution was observed and a clear solution formed. The reaction solution was allowed to stir for $30 \mathrm{~min}$ at $0{ }^{\circ} \mathrm{C}$ and for $30 \mathrm{~min}$ at ambient temperature, whereupon TLC control indicated full consumption of 3-formylindole.

Next, the reaction mixture was diluted with $\mathrm{MeOH}\left(10 \mathrm{~mL}, \mathrm{MeOH} / \mathrm{CH}_{2} \mathrm{Cl}_{2} 1: 1,0.5 \mathrm{M}\right)$, cooled in an ice bath and $\mathrm{NaBH}_{4}$ (502 mg, $13.0 \mathrm{mmol}, 1.30$ equiv) was added portion wise within $10 \mathrm{~min}$. After $15 \mathrm{~min}$ of stirring the ice bath was removed and after $2 \mathrm{~h}$ of stirring at room temperature the reaction suspension was diluted with $\mathrm{Et}_{2} \mathrm{O}(10 \mathrm{~mL})$ and $1 \mathrm{~N} \mathrm{HCl}$ solution in water $\left(20 \mathrm{~mL}\right.$ ) was added carefully at $0{ }^{\circ} \mathrm{C}$. Subsequently, the aqueous phase was extracted with further $\mathrm{Et}_{2} \mathrm{O}(2 \times 10 \mathrm{~mL})$, the combined organic phases were washed with brine $(2 \mathrm{x}$ $10 \mathrm{~mL}$ ), dried over $\mathrm{MgSO}_{4}$ and concentrated under reduced pressure. In the following the crude alcohol 10 (3.14 g) was dissolved in $\mathrm{Et}_{2} \mathrm{O} / \mathrm{PE}$ 65:35 (ca. $2 \mathrm{~mL}$ ) and loaded onto a silica gel column (mass crude material/SiO ${ }_{2}$ 1:10). Elution with $\mathrm{Et}_{2} \mathrm{O} / \mathrm{PE}$ 65:35 and drying in high vacuum afforded the indole derivative 10 as a colourless, viscous oil in $83 \%$ yield $(2.055 \mathrm{~g}$, $8.31 \mathrm{mmol})$.

$\mathbf{M}\left(\mathrm{C}_{14} \mathrm{H}_{17} \mathrm{NO}_{3}\right)=247.290 \mathrm{~g} / \mathrm{mol} ; \mathbf{r}_{\mathbf{f}}\left(\mathrm{SiO}_{2}, \mathrm{Et}_{2} \mathrm{O} / \mathrm{PE} 65: 35\right)=0.38 ;{ }^{1} \mathbf{H} \mathbf{N M R}\left(400 \mathrm{MHz}, \mathrm{CDCl}_{3}\right.$, $\mathrm{PH} 11642) \delta[\mathrm{ppm}]=8.14$ (br.s, $1 \mathrm{H}), 7.65(\mathrm{~d}, J=7.8 \mathrm{~Hz}, 1 \mathrm{H}), 7.58(\mathrm{~s}, 1 \mathrm{H}), 7.36-7.32(\mathrm{~m}, 1 \mathrm{H})$, 7.28-7.24 (m, 1H), $4.84\left(\mathrm{~s}, 2 \mathrm{H}, 4^{\prime}-\mathrm{H}\right) 1.67$ (s, 9H, 3'-H), 1.58 (br.s, $\left.1 \mathrm{H}, \mathrm{OH}\right) ;{ }^{13} \mathrm{C}$ NMR $\left(100 \mathrm{MHz}, \mathrm{CDCl}_{3}, \mathrm{PH} 11642\right) \delta[\mathrm{ppm}]=149.66\left(\mathrm{C}-1^{\prime}\right), 135.75(\mathrm{C}-9), 129.14\left(\mathrm{C}_{\mathrm{q}}\right), 124.64(\mathrm{CH})$, $123.70(\mathrm{CH}), 122.68(\mathrm{CH}), 120.41\left(\mathrm{C}_{\mathrm{q}}\right), 119.26(\mathrm{CH}), 115.29(\mathrm{CH}), 83.73\left(\mathrm{C}^{\prime} 4^{\prime}\right), 57.14\left(\mathrm{C}-4^{\prime}\right)$, 28.15 (C-3'); GC-MS (El, 70 eV) m/z [u] (\%) = 247 (3, [M] $\left.{ }^{+}\right), 191$ (15, [M-butene] $\left.]^{+}\right), 174$ (4, [M$\left.\left.\left.\mathrm{OtBu}]^{+}\right), 147\left(6,{\mathrm{M}-\mathrm{CO}_{2}-\text { butene] }}^{+}\right), 130\left(21, \text { indoleCH}_{2}\right]^{+}\right), 118(10 \text {, [indoleH }]^{+}\right), 102$ (10), 57 $\left(100,[t \mathrm{Bu}]^{+}\right), 56\left(60,[\text { butene }]^{+}\right)$.

The ${ }^{1} \mathrm{H}$ NMR data was in agreement with literature data. ${ }^{[23]}$ 


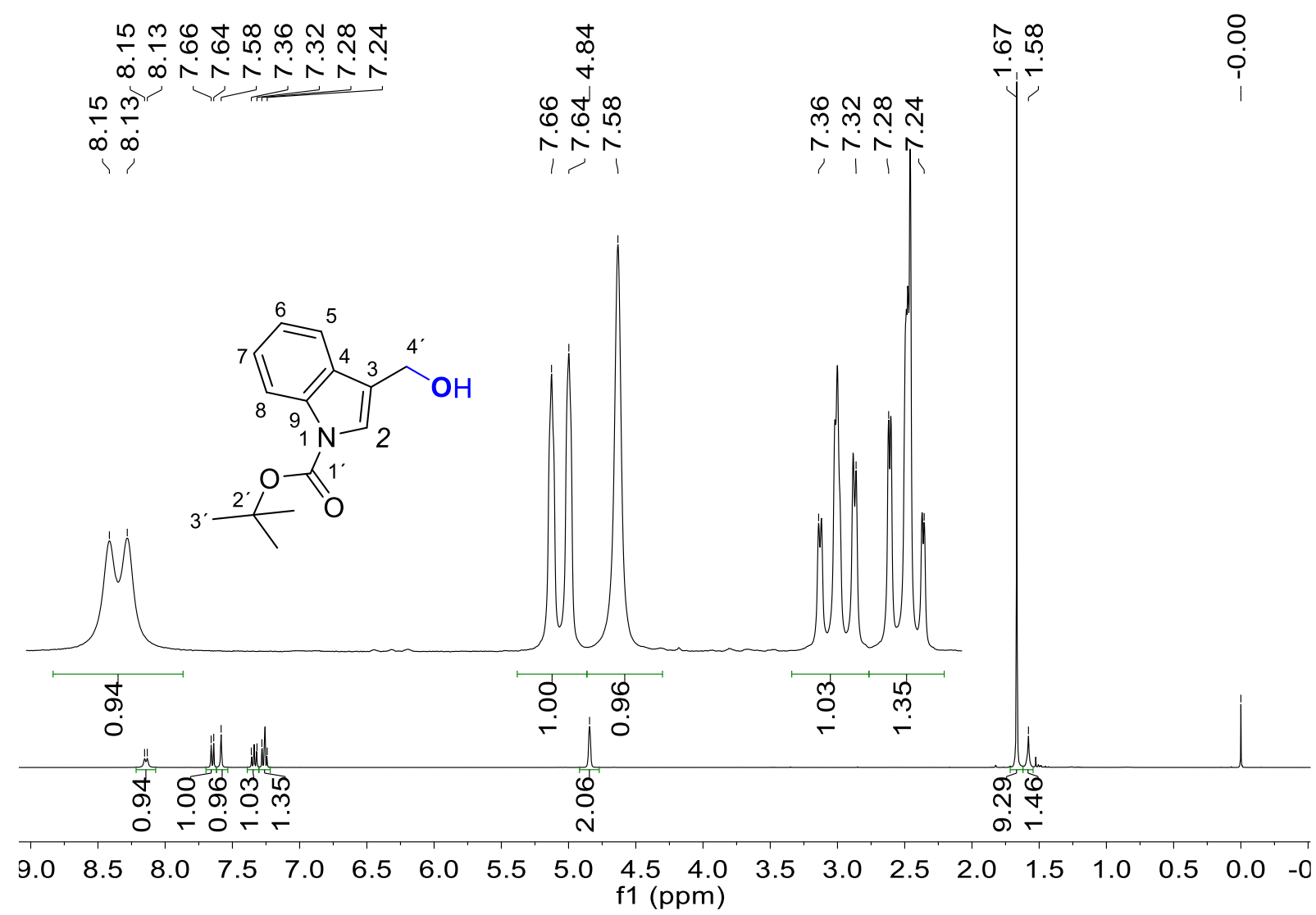

${ }^{1} \mathrm{H}$ NMR spectrum of 1-(tert-Butoxycarbonyl)-3-(chloromethyl)indole $\left(400 \mathrm{MHz}, \mathrm{CDCl}_{3}\right)$.

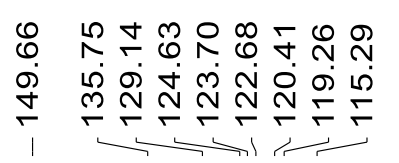

$\begin{array}{ll}m & 8 \\ & 0 \\ 0 & \hat{1}\end{array}$

$\frac{5}{5}$

$\stackrel{\infty}{\stackrel{\infty}{N}}$

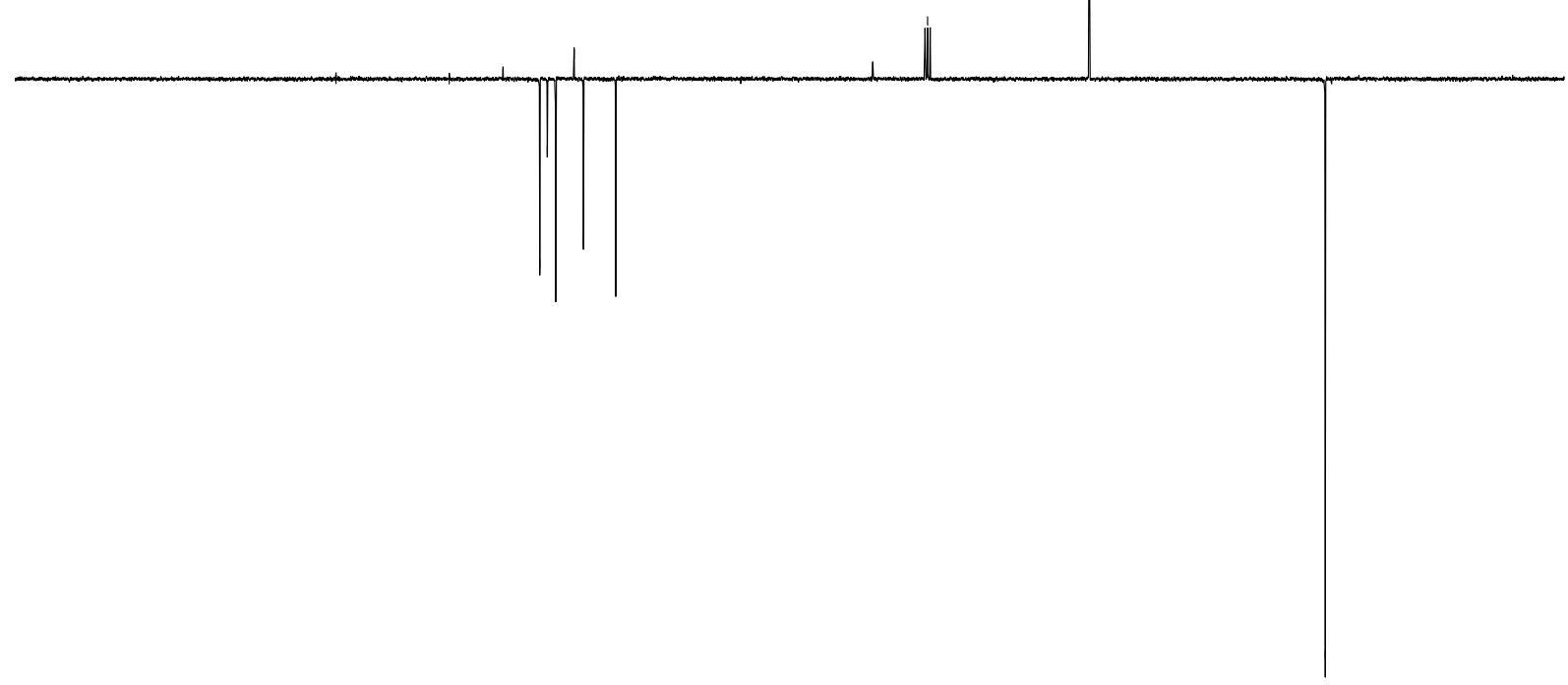

$\begin{array}{lllllllllllllllllll}180 & 170 & 160 & 150 & 140 & 130 & 120 & 110 & 100 & 90 & 80 & 70 & 60 & 50 & 40 & 30 & 20 & 10 & \mathrm{C}\end{array}$ f1 (ppm)

${ }^{13} \mathrm{C}$ NMR spectrum of 1-(tert-Butoxycarbonyl)-3-(chloromethyl)indole $\left(100 \mathrm{MHz}, \mathrm{CDCl}_{3}\right)$. 


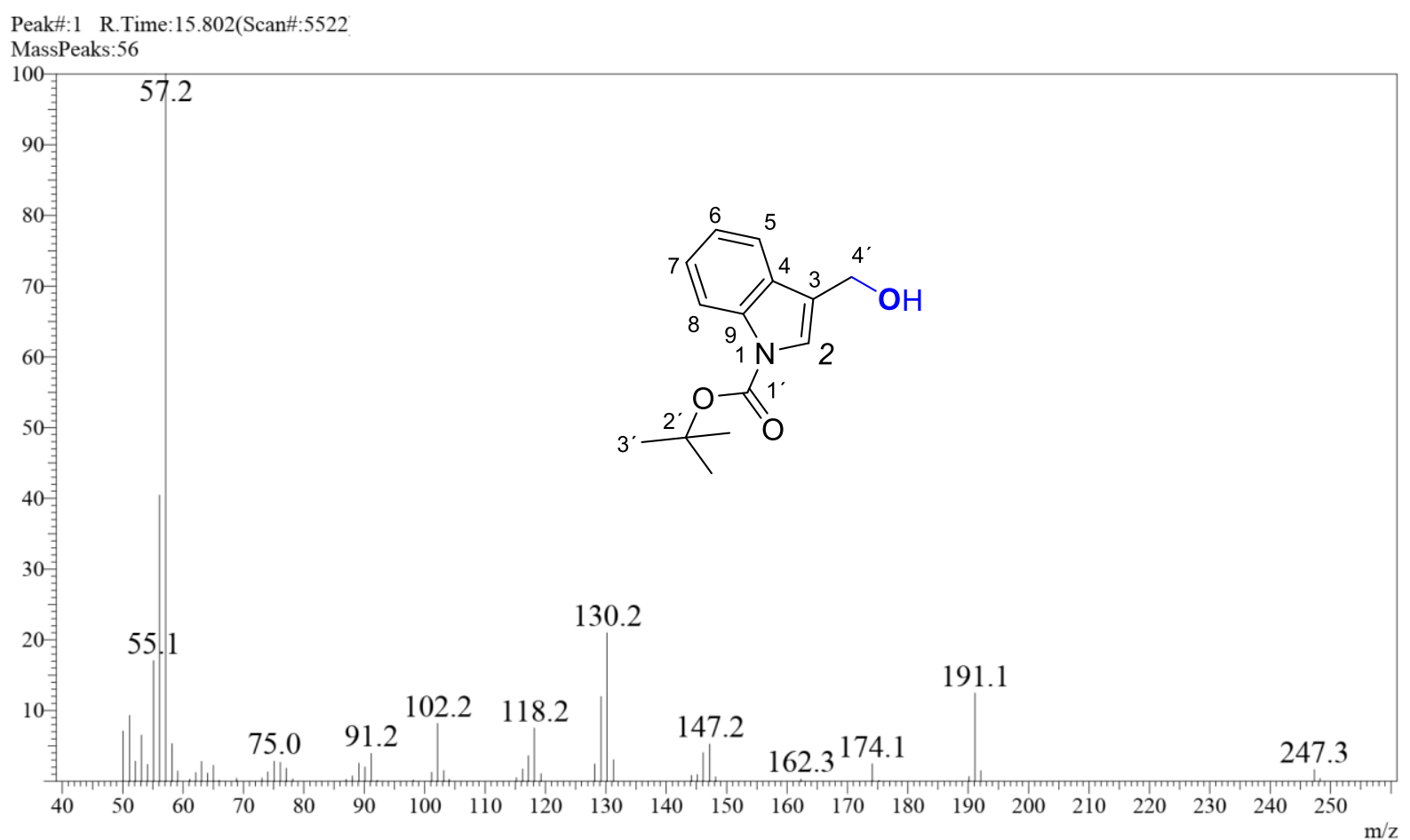

Mass spectrum of spectrum of 1-(tert-butoxycarbonyl)-3-(chloromethyl)indole (EI, $70 \mathrm{eV})$. 


\subsubsection{Analytical Data of the Additives (16) and Internal Standard for the Robustness Screen}

All additives are commercially available and analytical data were obtained from commercial samples. Molecular weights, densities, boiling points and CAS numbers are compiled in Figure S5. In the following, the signals used to determine the recovery yield are highlighted in bold and red. ${ }^{1} \mathrm{H}$ NMR spectra for all additives are available on the homepage of Peter Huy under https://www.peterhuylab.de/publications/.

Modified Additives red $=$ Modifications

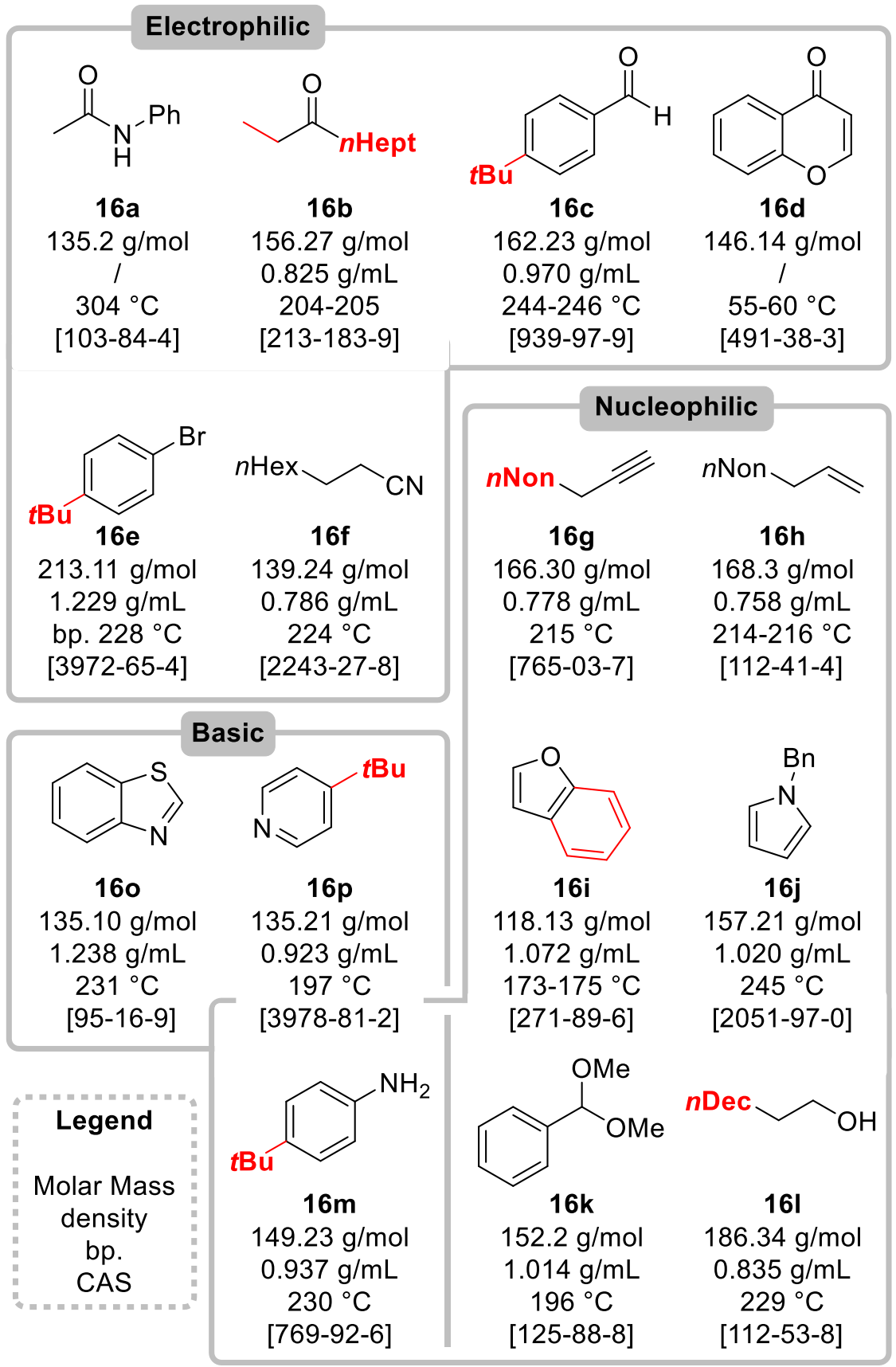

Figure S5. Overview over additives. Differences from original additives are illustrated in red. Data obtained from "https://www.sigmaaldrich.com/". 
Analytical data for 4-tert-butylbenzyl chloride $\mathbf{3 l}$ and benzoate $\mathbf{1 2}$ and 1-dodecyl chloride are available in reference. ${ }^{[5]}$

\subsubsection{Electrophilic Additives (16)}

\section{Acetanilide, $\mathrm{N}$-Phenylethanoic amide (16a)}<smiles>CC(=O)Nc1ccc(F)cc1</smiles>

$16 a$

$\mathbf{M}\left(\mathrm{C}_{8} \mathrm{H}_{9} \mathrm{NO}\right)=135.17 \mathrm{~g} / \mathrm{mol} ;{ }^{1} \mathbf{H}$ NMR $\left(400 \mathrm{MHz}, \mathrm{CDCl}_{3}\right) \delta[\mathrm{ppm}]=7.78$ (br.s, $1 \mathrm{H}, \mathrm{NH}), 7.50\left(\mathrm{~d},{ }^{3} \mathrm{~J}=8.0 \mathrm{~Hz}, 2 \mathrm{H}, 2^{\prime}-\mathrm{H}\right.$, or $\left.3^{\prime}-\mathrm{H}\right), 7.31-7.27(\mathrm{~m}, 2 \mathrm{H}$, $2^{\prime}-\mathrm{H}$, or $\left.3^{\prime}-\mathrm{H}\right), 7.09\left(\psi-\mathrm{t},{ }^{3} \mathrm{~J}=7.4 \mathrm{~Hz}, 1 \mathrm{H}, 4^{\prime}-\mathrm{H}\right), 2.15$ (s, 3H, 2-H).

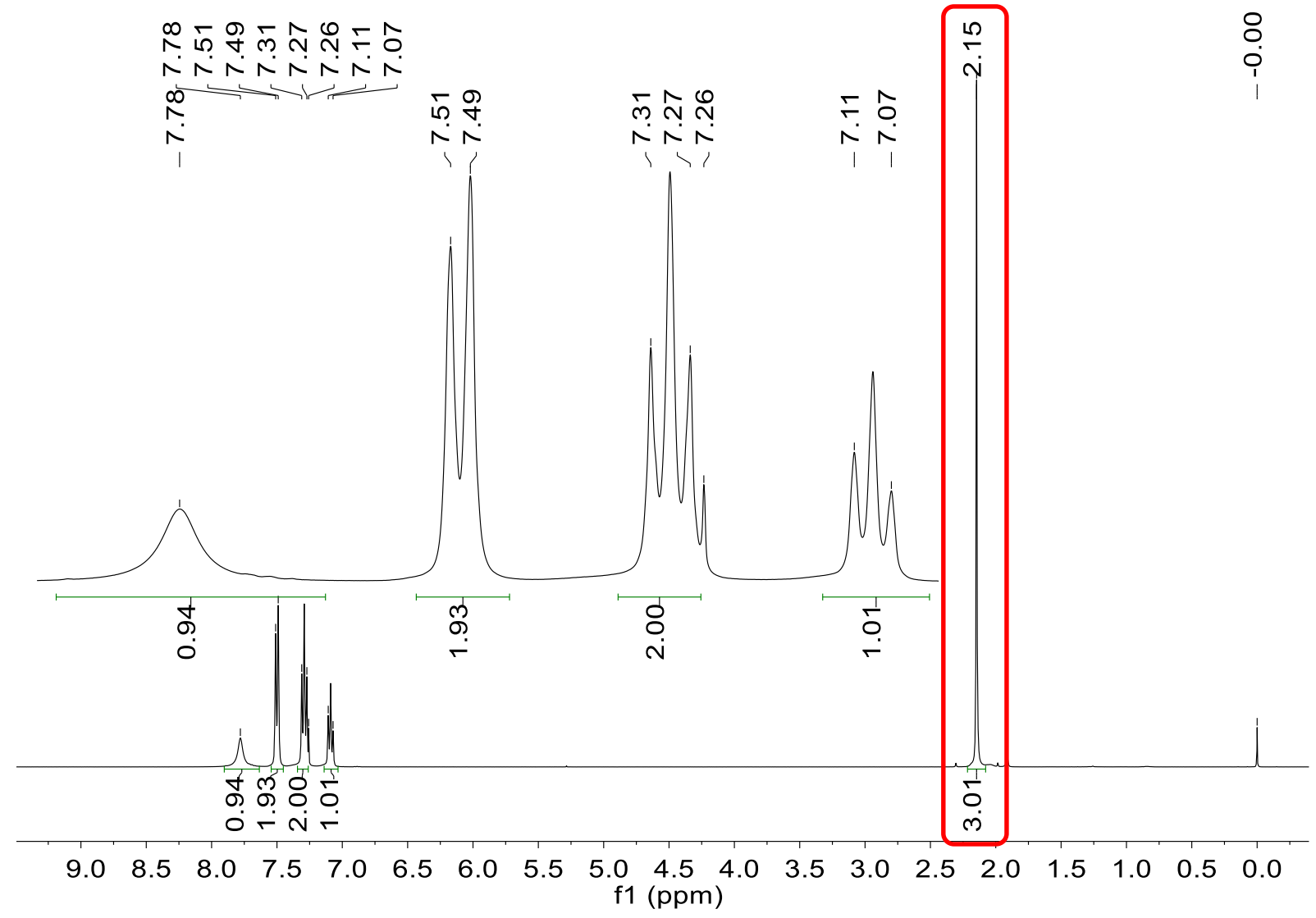

${ }^{1} \mathrm{H}$ NMR spectrum of acetanilide, multiplet used for yield determination highlighted by a red box $\left(400 \mathrm{MHz}, \mathrm{CDCl}_{3}\right)$. 


\section{3-Decanone (16b)}

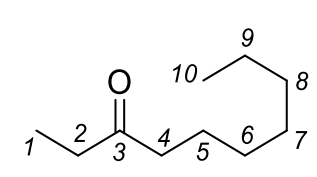

$\mathbf{M}\left(\mathrm{C}_{10} \mathrm{H}_{20} \mathrm{O}\right)=156.27 \mathrm{~g} / \mathrm{mol} ;{ }^{1} \mathbf{H}$ NMR $\left(400 \mathrm{MHz}, \mathrm{CDCl}_{3}\right) \delta[\mathrm{ppm}]=\mathbf{2 . 4 4 -}$ $2.37(\mathrm{~m}, 4 \mathrm{H}, 2-\mathrm{H}, 4-\mathrm{H}), 1.60-1.53(\mathrm{~m}, 2 \mathrm{H}, 5-\mathrm{H}), 1.33-1.27(\mathrm{~m}, 8 \mathrm{H}, 6-\mathrm{H}$, $7-\mathrm{H}, 8-\mathrm{H}, 9-\mathrm{H}), 1.05\left(\mathrm{t},{ }^{3} J_{1,2}=7.4 \mathrm{~Hz}, 3 \mathrm{H}, 1-\mathrm{H}\right), 0.89-0.86(\mathrm{~m}, 3 \mathrm{H}, 10-\mathrm{H})$. $16 b$

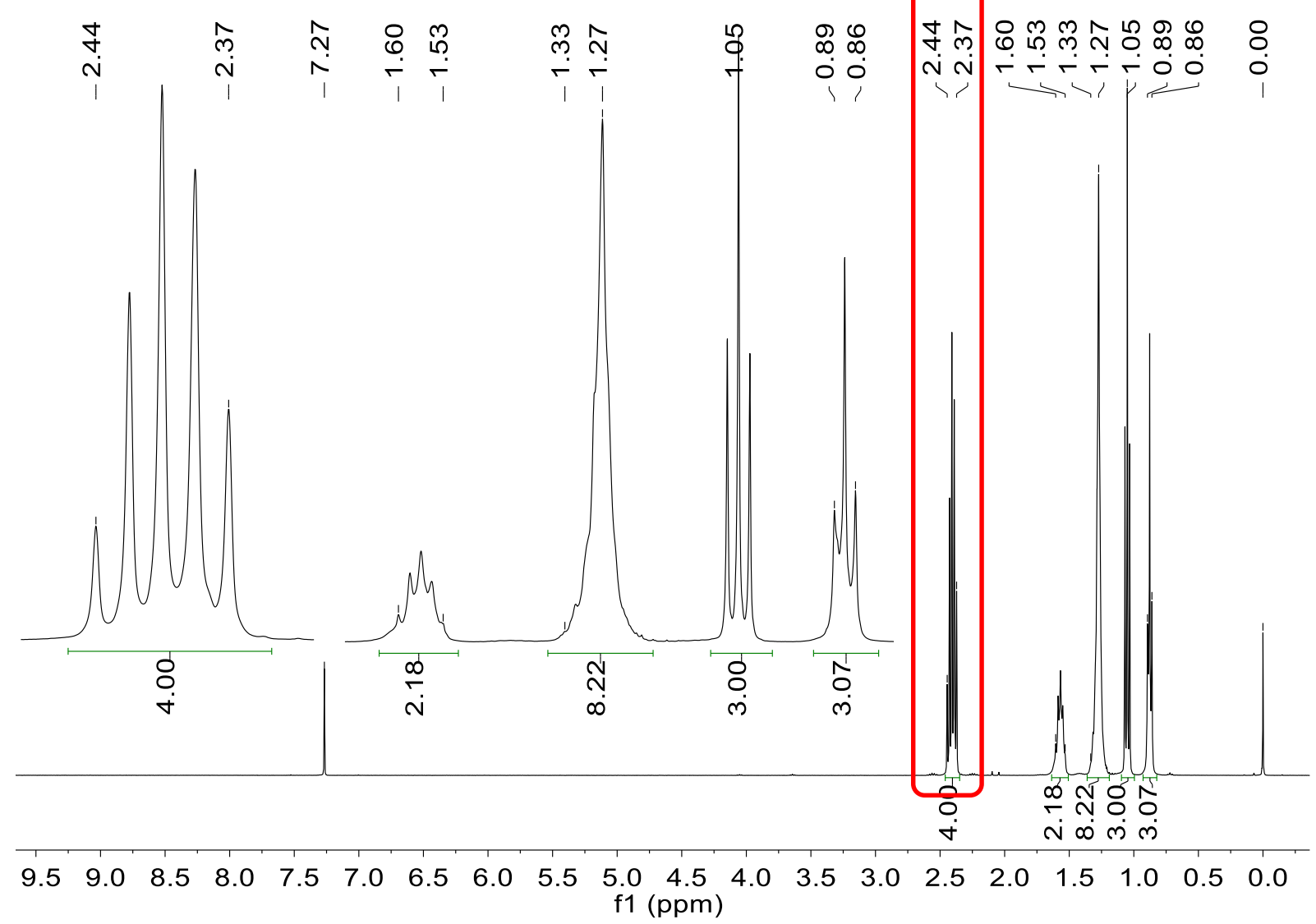

${ }^{1} \mathrm{H}$ NMR spectrum of 3-decanone, multiplet used for yield determination highlighted by a red box $\left(400 \mathrm{MHz}, \mathrm{CDCl}_{3}\right)$. 


\section{4-tert-butylbenzalydehyde (16c)}<smiles>CC(C)(C)c1ccc(C=O)cc1</smiles>

$16 c$

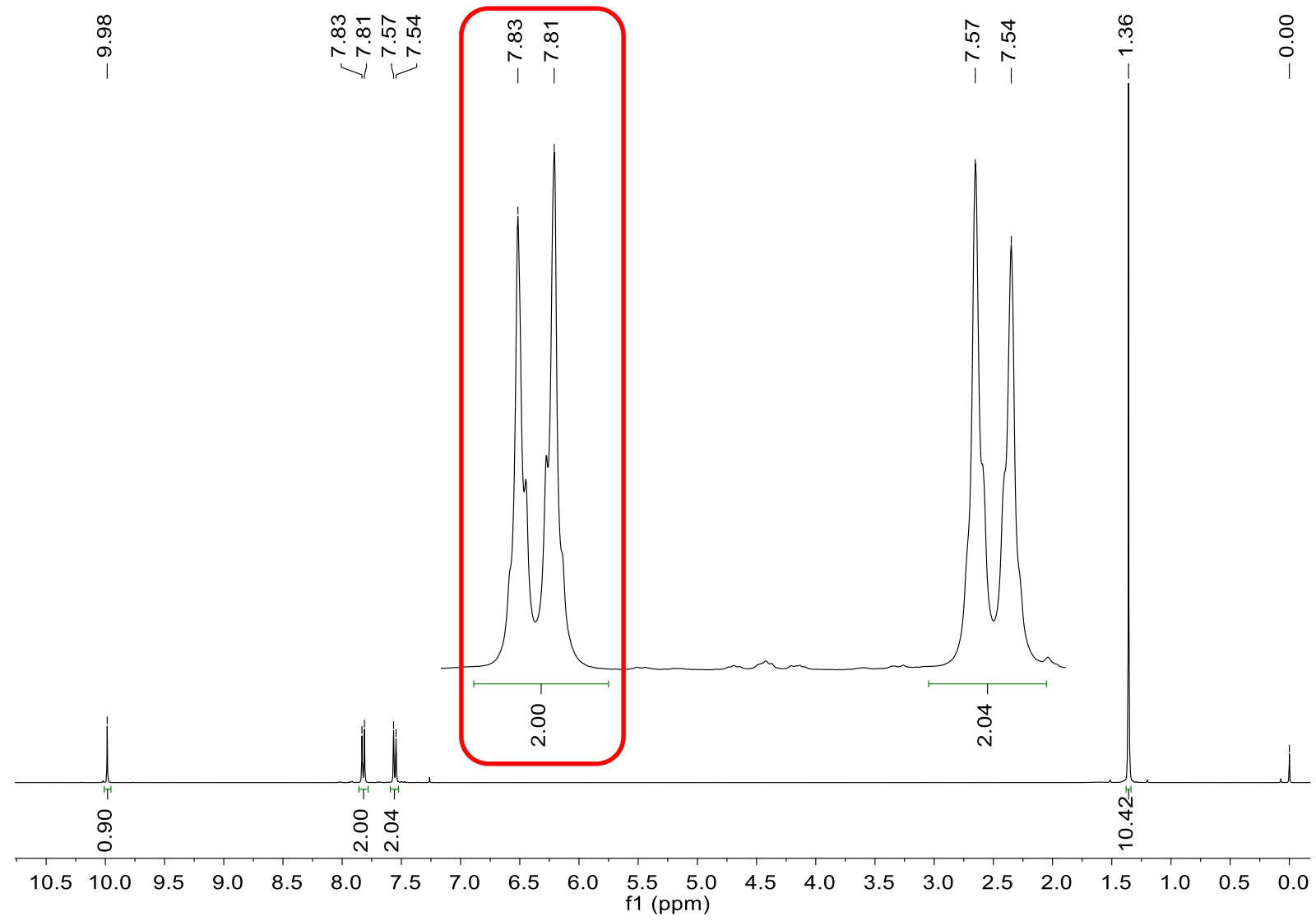

${ }^{1} \mathrm{H}$ NMR spectrum of 4-tert-butylbenzaldehyde, multiplet used for yield determination highlighted by a red box $\left(400 \mathrm{MHz}, \mathrm{CDCl}_{3}\right)$.

$\mathbf{M}\left(\mathrm{C}_{11} \mathrm{H}_{14} \mathrm{O}\right)=162.10 \mathrm{~g} / \mathrm{mol} ;{ }^{1} \mathbf{H}$ NMR $\left(400 \mathrm{MHz}, \mathrm{CDCl}_{3}\right) \delta[\mathrm{ppm}]=9.98$ (s, $1 \mathrm{H}, 1-\mathrm{H}), 7.83-7.81(\mathrm{~m}, 2 \mathrm{H}, 3-\mathrm{H}$ or $4-\mathrm{H}), 7.57-7.54(\mathrm{~m}, 2 \mathrm{H}, 3-\mathrm{H}$ or 4-H), 1.36 (s, 9H, 7-H). 


\section{Chromone (16d)}

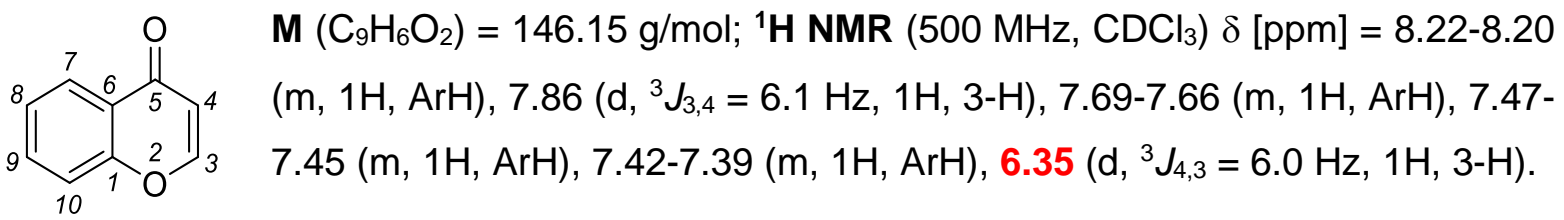

\section{6d}

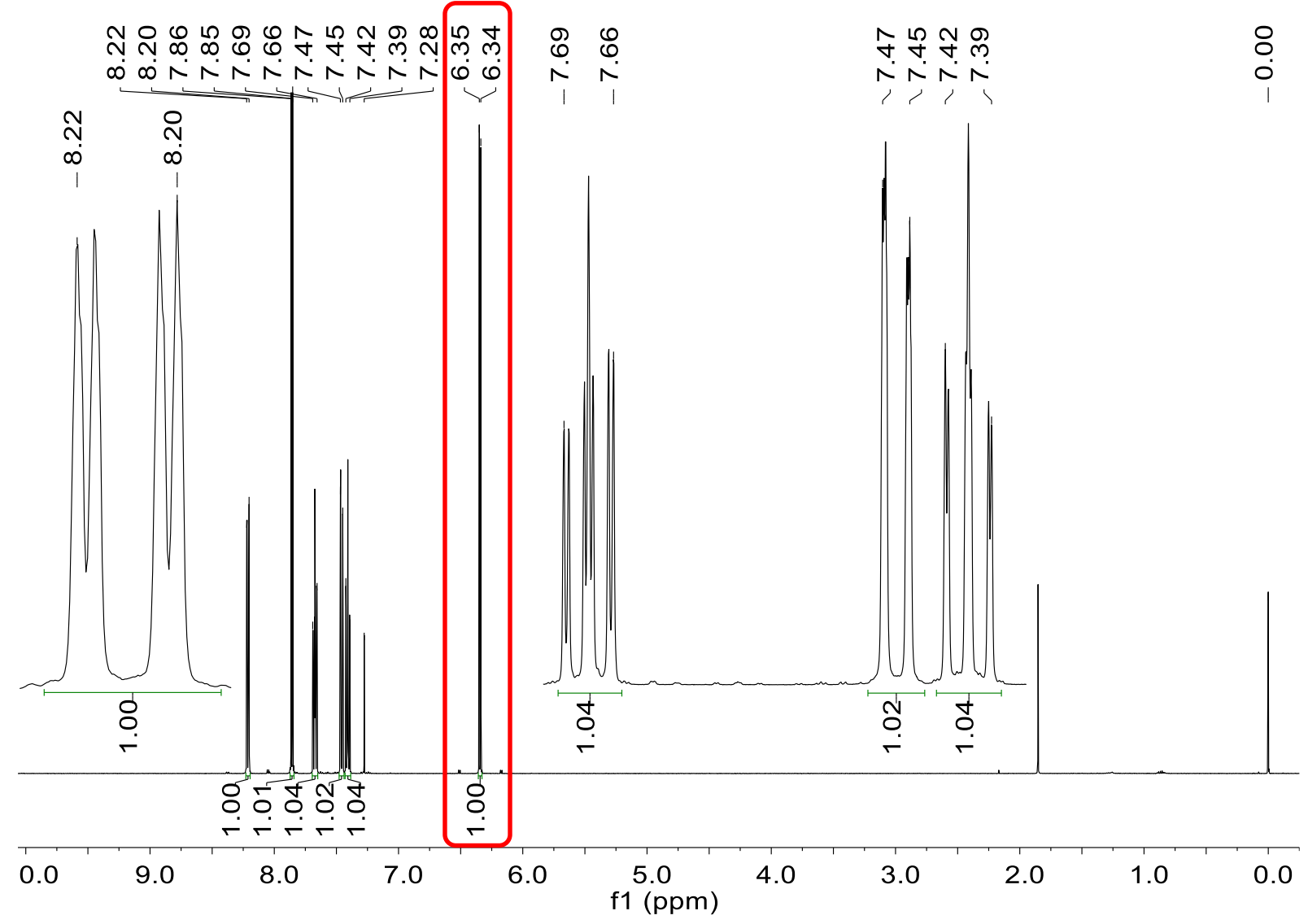

${ }^{1} \mathrm{H}$ NMR spectrum of Chromone, multiplet used for yield determination highlighted by a red box $\left(500 \mathrm{MHz}, \mathrm{CDCl}_{3}\right)$. 


\section{1-Bromo-4-tert-butylbenzene (16e)}<smiles>CC(C)(C)c1ccc(Br)cc1</smiles>

$\mathbf{M}\left(\mathrm{C}_{10} \mathrm{H}_{13} \mathrm{Br}\right)=213.12 \mathrm{~g} / \mathrm{mol} ;{ }^{1} \mathbf{H}$ NMR $\left(400 \mathrm{MHz}, \mathrm{CDCl}_{3}\right) \delta[\mathrm{ppm}]=7.42-$ 7.39 (m 2H, ArH), 7.27-7.23 (m, 2H, ArH), 1.30 (s, 9H, 6-H).

$16 e$

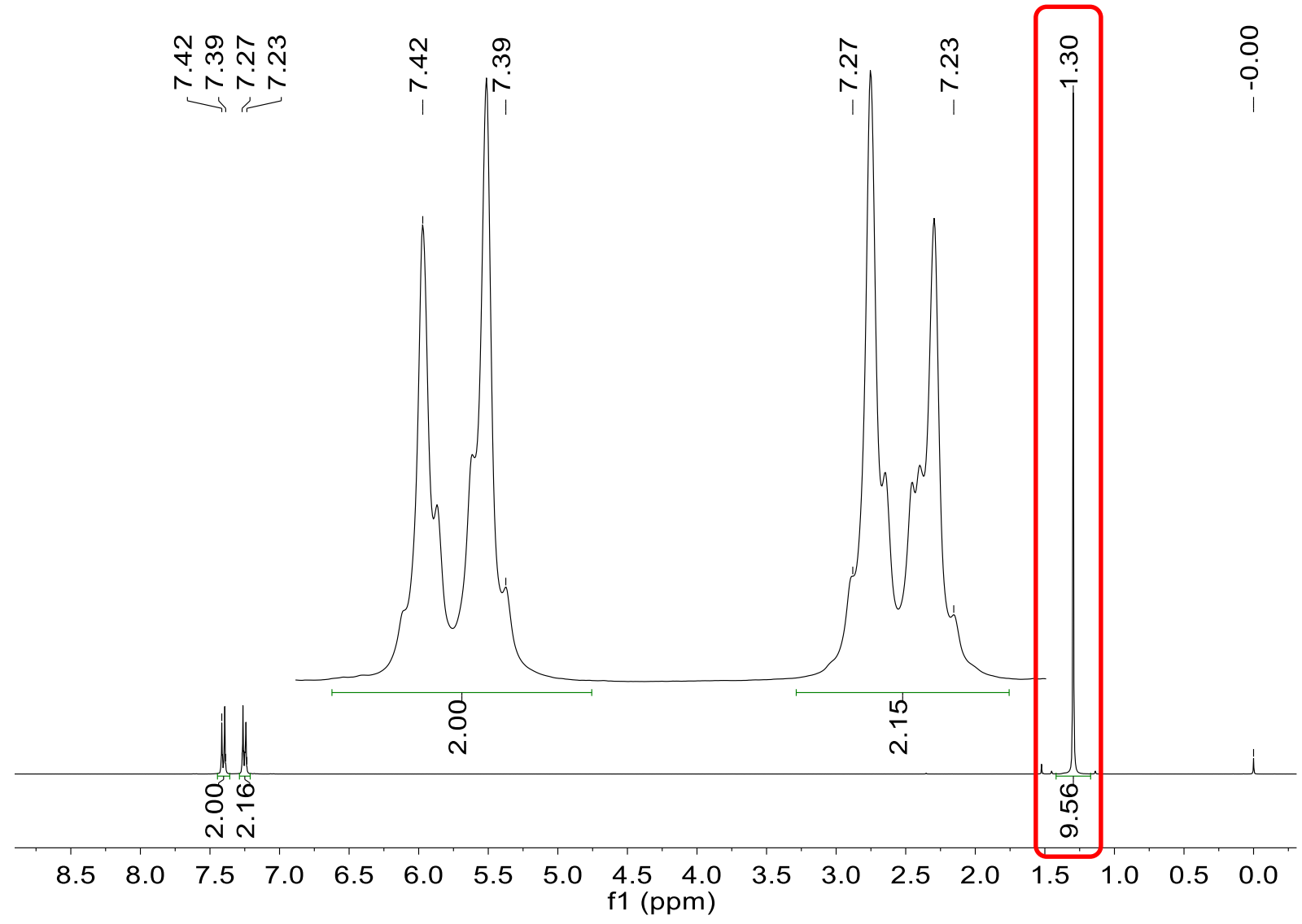

${ }^{1} \mathrm{H}$ NMR spectrum of 1-bromo-4-tert-butylbenzene, multiplet used for yield determination highlighted by a red box $\left(400 \mathrm{MHz}, \mathrm{CDCl}_{3}\right)$.

Albeit the singlets of the tert-butyl groups of 1-bromo-4-tert-butylbenzene and 4-tertbutylbenzyl chloride are close to each other, the separation is reasonable (see below, right). The singlets can be assigned by integration of the area with the singlets of the $\mathrm{CH}_{2}$ groups for 4-tert-butylbenzyl chloride and benzoate (left). 


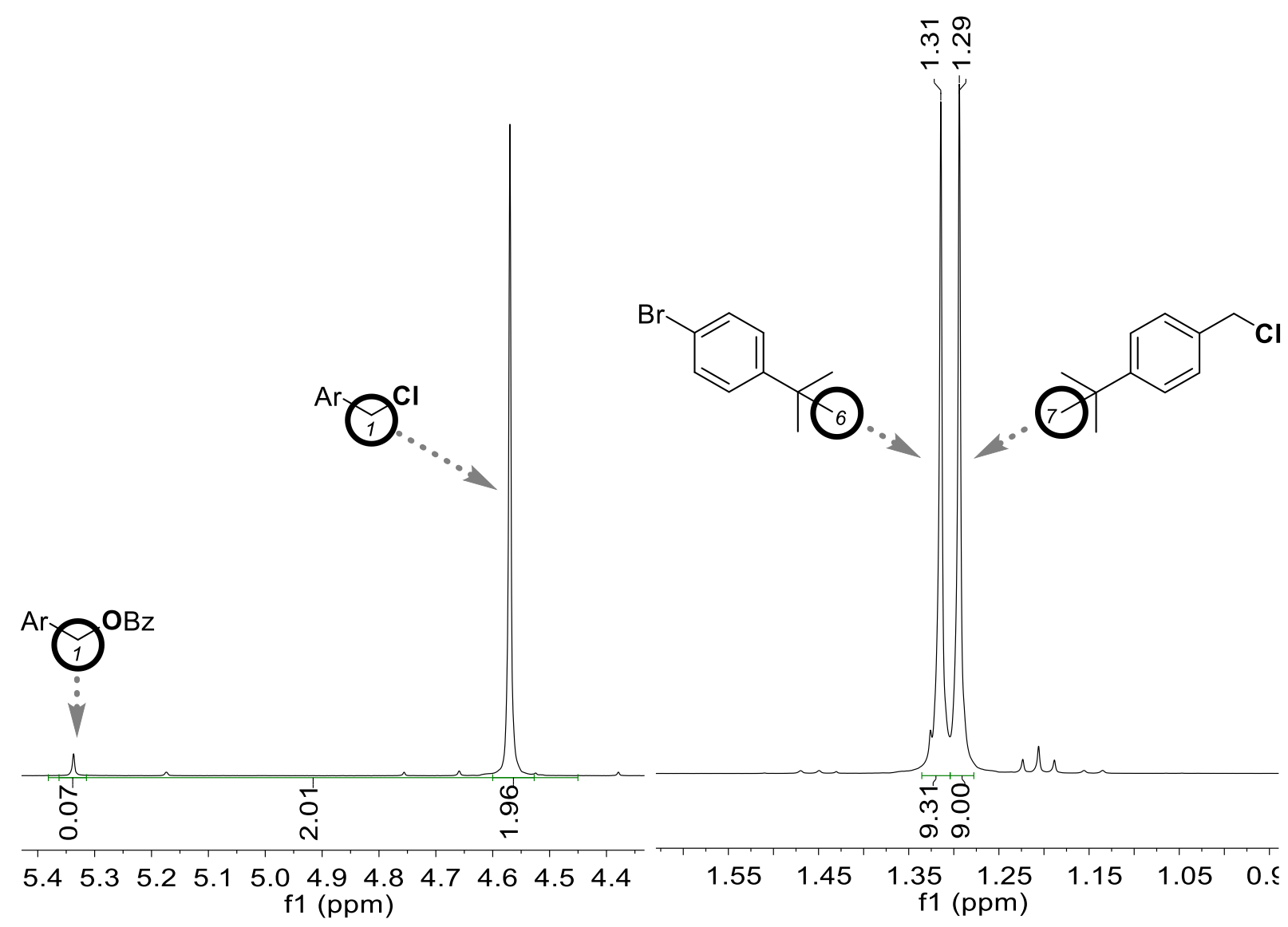

Two extracts of the ${ }^{1} \mathrm{H}$ NMR spectrum of the crude reaction product of the model reaction in the presence of 1-bromo-4-tert-butylbenzene (400 $\left.\mathrm{MHz}, \mathrm{CDCl}_{3}\right)$. 


\section{Nonanenitrile (16f)}

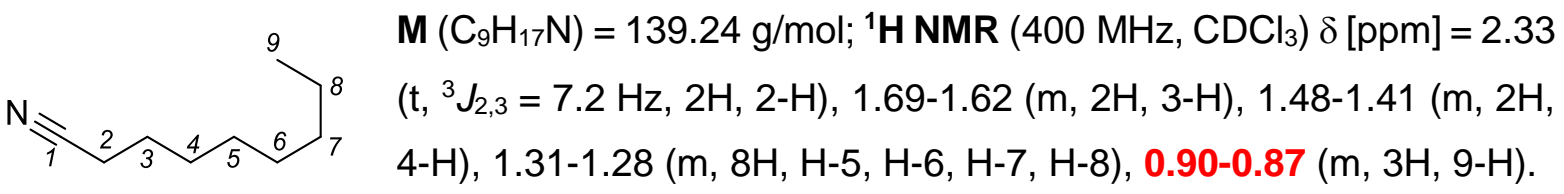

$16 f$

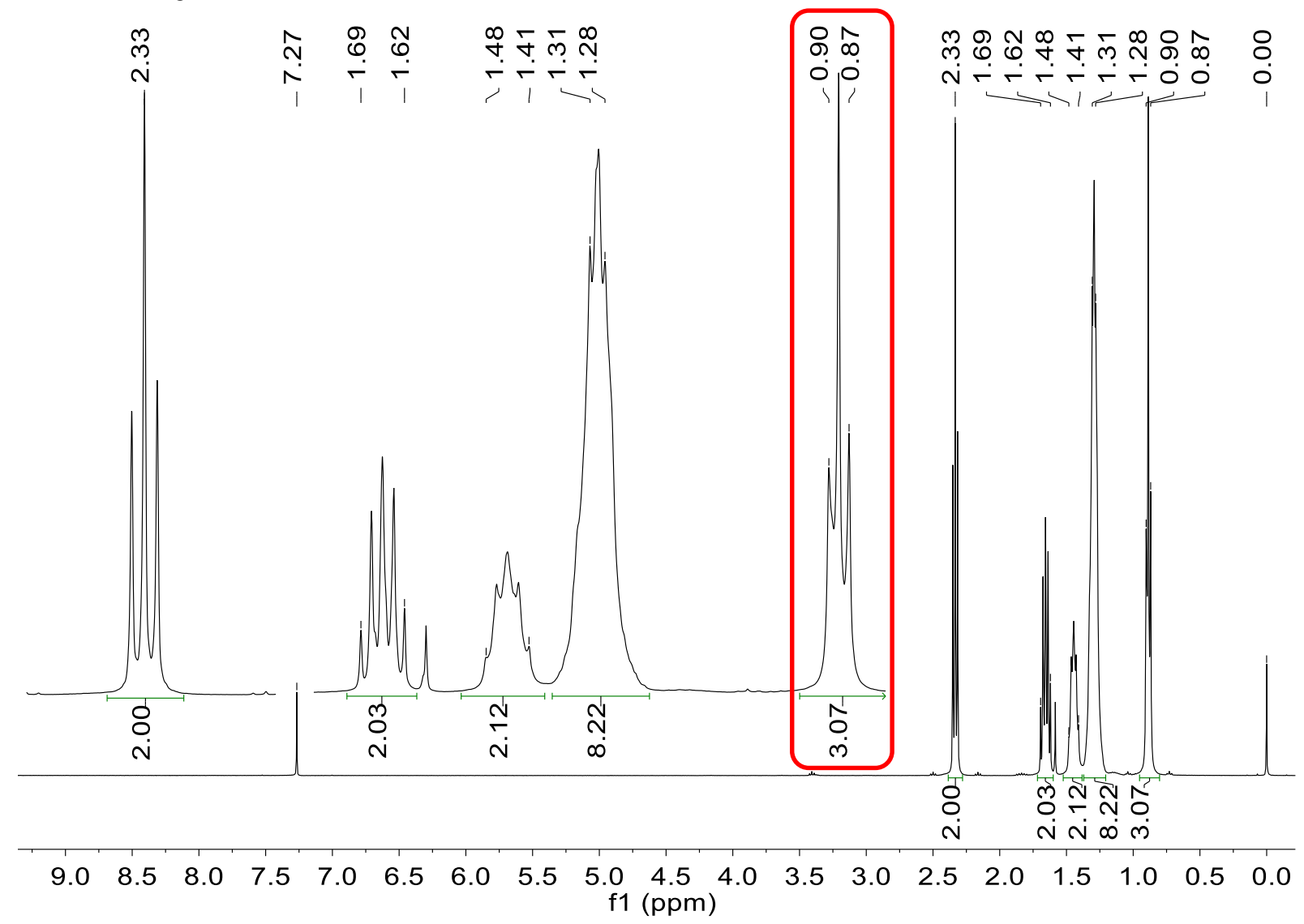

${ }^{1} \mathrm{H}$ NMR spectrum of nonanenitrile, multiplet used for yield determination highlighted by a red box $\left(400 \mathrm{MHz}, \mathrm{CDCl}_{3}\right)$.

As only example of the current robustness screen, in the crude ${ }^{1} \mathrm{H}$ NMR of the model reaction $11 \rightarrow 3$ I with nonanenitrile as additive the essential signal of the internal standard mesitylene overlapped with one of the signals of the additive as shown below (Table S2, page 4, entry 6). Nevertheless, the yield was calculated referring to the multiplet at $0.90-0.87$ for the methyl group of nonanenitril. The area of the singlet of mesitylene at $2.27 \mathrm{ppm}$ a (mesitylene) can be calculated as follows:

$$
\begin{gathered}
a(\text { mesitylene })=a(\text { m at } 2.34-2.32)-a(\text { m at } 0.90-0.87) \frac{3}{2} \\
=6.76-3.00 \frac{3}{2}=4.76
\end{gathered}
$$


For the other components the integral of the mesitylene singlet can be calculated accordingly. As an alternative, the reaction can be performed with a different NMR standard. The NMR standard may have an influence on the calculated yield, because of different longitudinal relaxation times $T_{1}$. Therefore, the model reaction without an additive has to be repeated with the new NMR standard in order to create a new reference yield for the product.

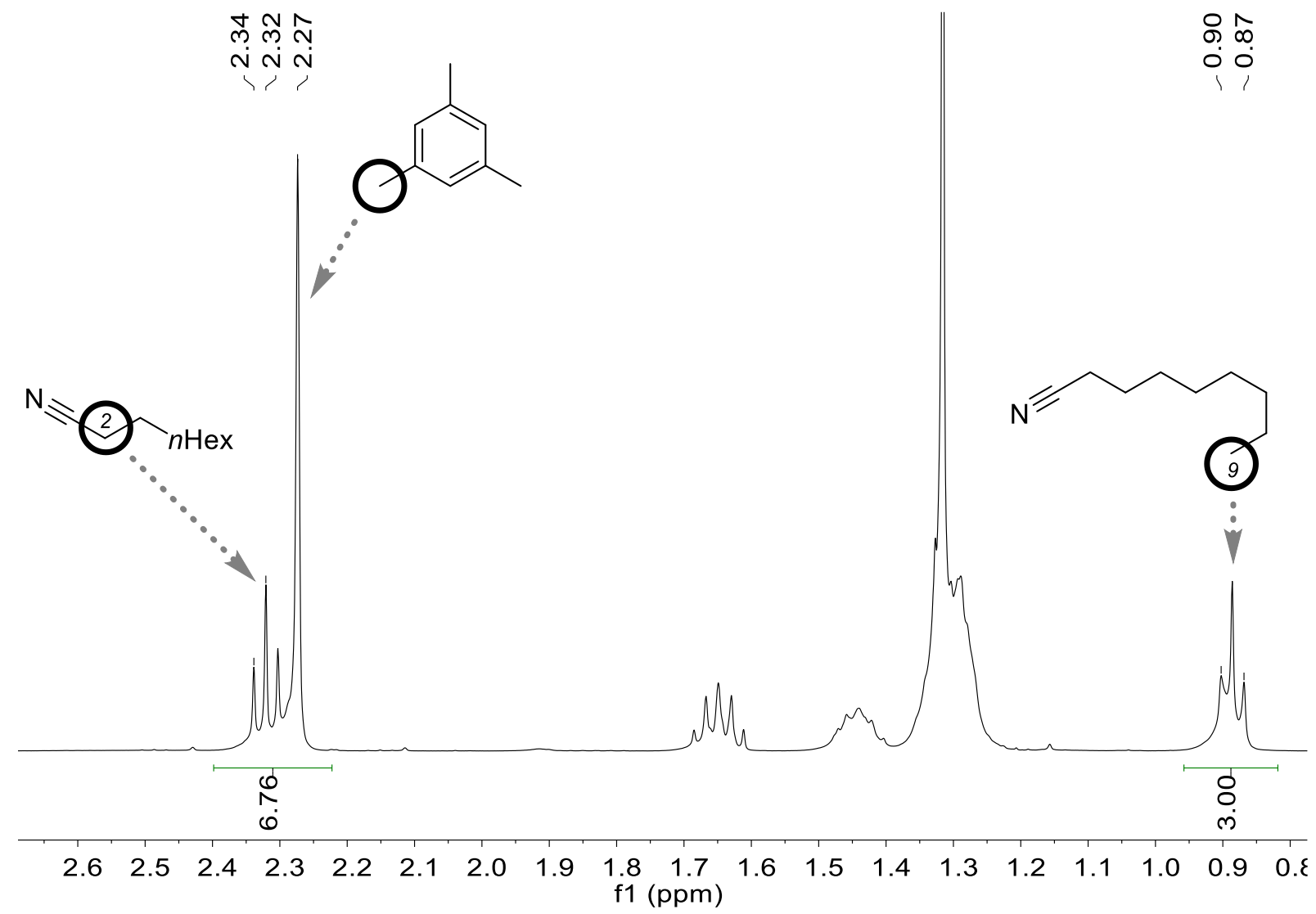

Extract of the ${ }^{1} \mathrm{H}$ NMR spectrum of the crude reaction product of the model reaction in the presence of nonanenitrile (experiment $\mathrm{PH} 3996,400 \mathrm{MHz}, \mathrm{CDCl}_{3}$ ). 


\subsubsection{Nucleophilic Additives (16)}

\section{1-Dodecyne (15g)}

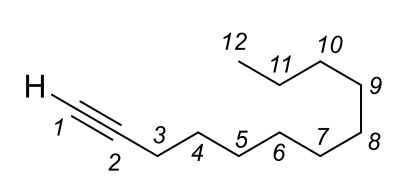

$\mathbf{M}\left(\mathrm{C}_{12} \mathrm{H}_{22}\right)=166.31 \mathrm{~g} / \mathrm{mol} ;{ }^{1} \mathbf{H}$ NMR $\left(400 \mathrm{MHz}, \mathrm{CDCl}_{3}\right) \delta[\mathrm{ppm}]=$ $2.18\left(\mathrm{td},{ }^{3} J_{3,1}=7.1 \mathrm{~Hz},{ }^{4} J_{3,1}=2.6 \mathrm{~Hz}, 2 \mathrm{H}, 3-\mathrm{H}\right), 1.93\left(\mathrm{t},{ }^{4} J_{1,3}=2.7 \mathrm{~Hz}\right.$, $1 \mathrm{H}, 1-\mathrm{H}), 1.56-1.49(\mathrm{~m}, 2 \mathrm{H}, 4-\mathrm{H}), 1.41-1.37(\mathrm{~m}, 2 \mathrm{H}, 5-\mathrm{H}), 1.32-1.27$

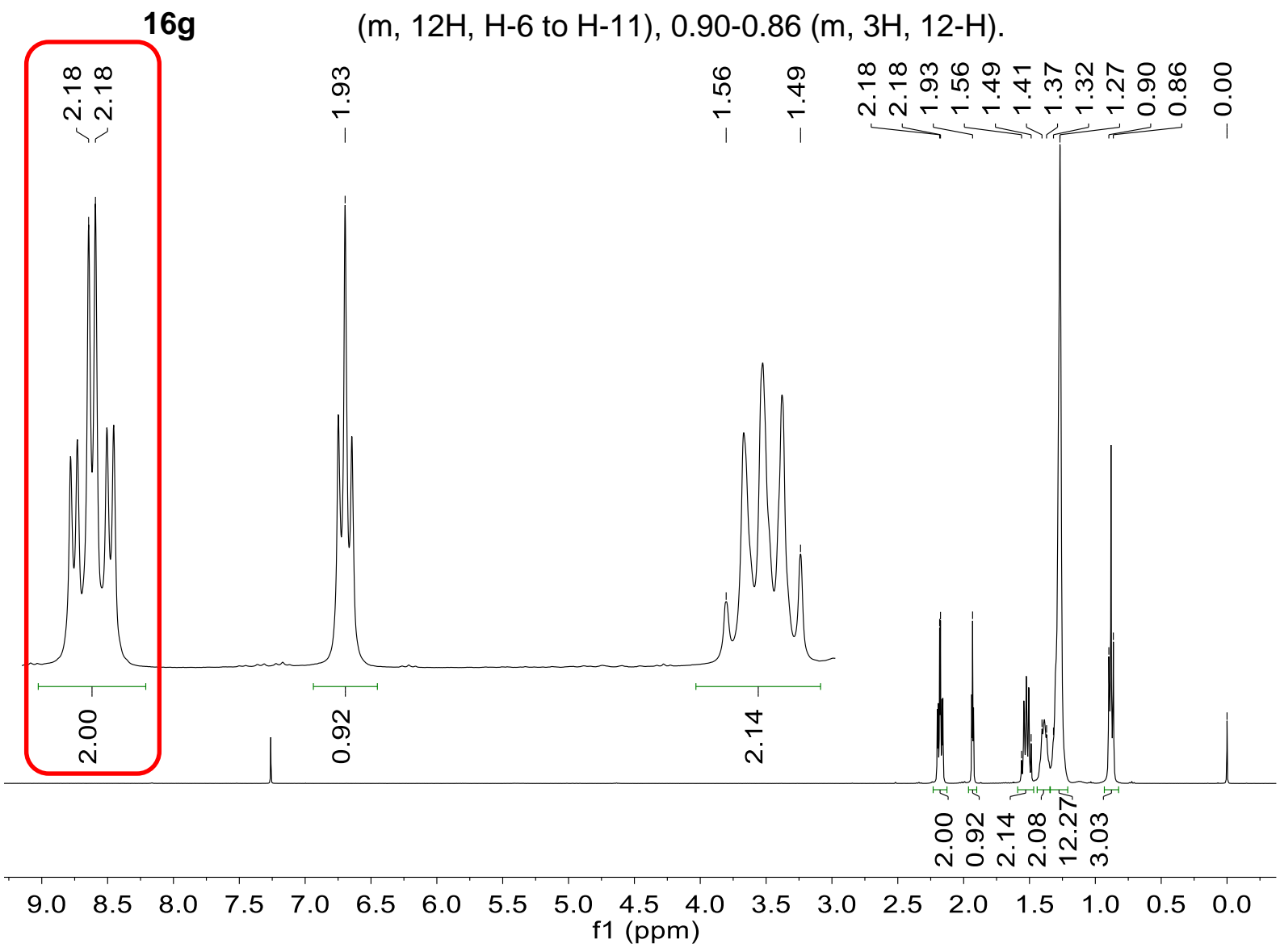

${ }^{1} \mathrm{H}$ NMR spectrum of 1-dodecyne, multiplet used for yield determination highlighted by a red box $\left(400 \mathrm{MHz}, \mathrm{CDCl}_{3}\right)$. 


\section{1-Dodecene (16h)}

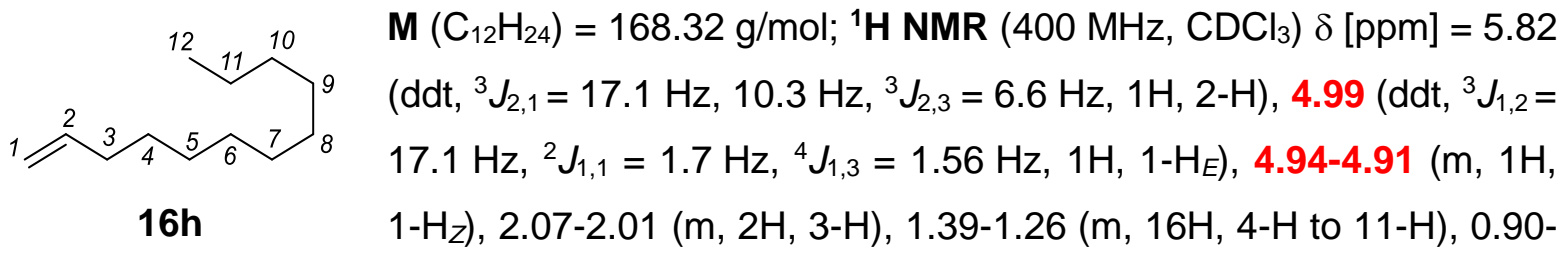
$0.86(\mathrm{~m}, 3 \mathrm{H}, 12-\mathrm{H})$.

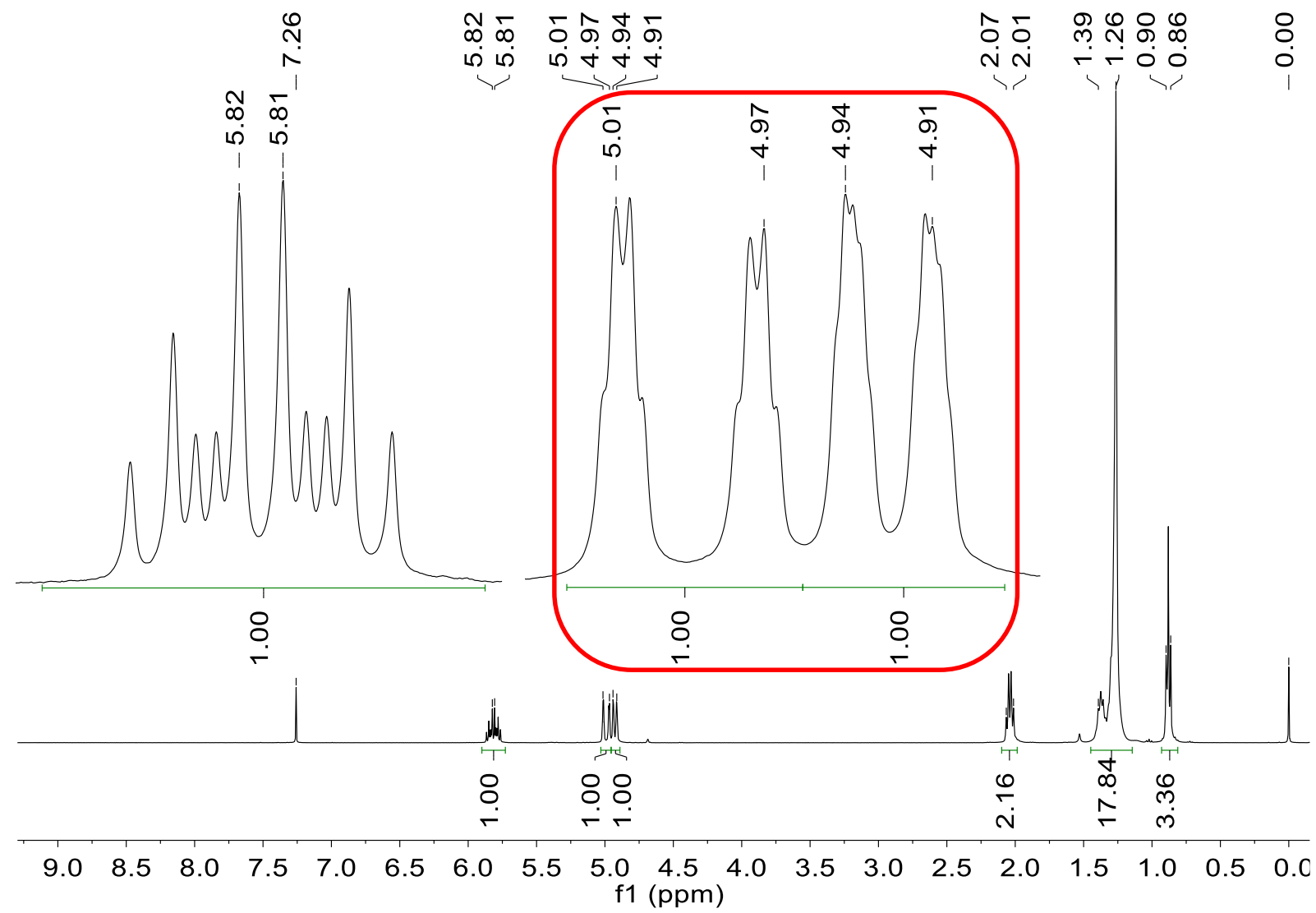

${ }^{1} \mathrm{H}$ NMR spectrum of 1 -dodecene, multiplet used for yield determination highlighted by a red box $\left(400 \mathrm{MHz}, \mathrm{CDCl}_{3}\right)$. 


\section{2,3-Benzofurane (16i)}

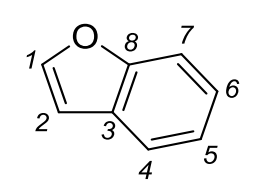

$16 \mathbf{i}$

$\mathbf{M}\left(\mathrm{C}_{8} \mathrm{H}_{6} \mathrm{O}\right)=118.14 \mathrm{~g} / \mathrm{mol} ;{ }^{1} \mathbf{H}$ NMR $\left(400 \mathrm{MHz}, \mathrm{CDCl}_{3}\right) \delta[\mathrm{ppm}]=7.61-7.59$

$(\mathrm{m}, 2 \mathrm{H}), 7.51\left(\mathrm{~d},{ }^{3} J_{1,2}=8.2 \mathrm{~Hz}, 1 \mathrm{H}, 1-\mathrm{H}\right), 7.31-7.27(\mathrm{~m}, 1 \mathrm{H}), 7.25-7.21(\mathrm{~m}$, $1 \mathrm{H}), 6.76$ (br. $\psi-\mathrm{s}, 1 \mathrm{H}, 2-\mathrm{H})$.

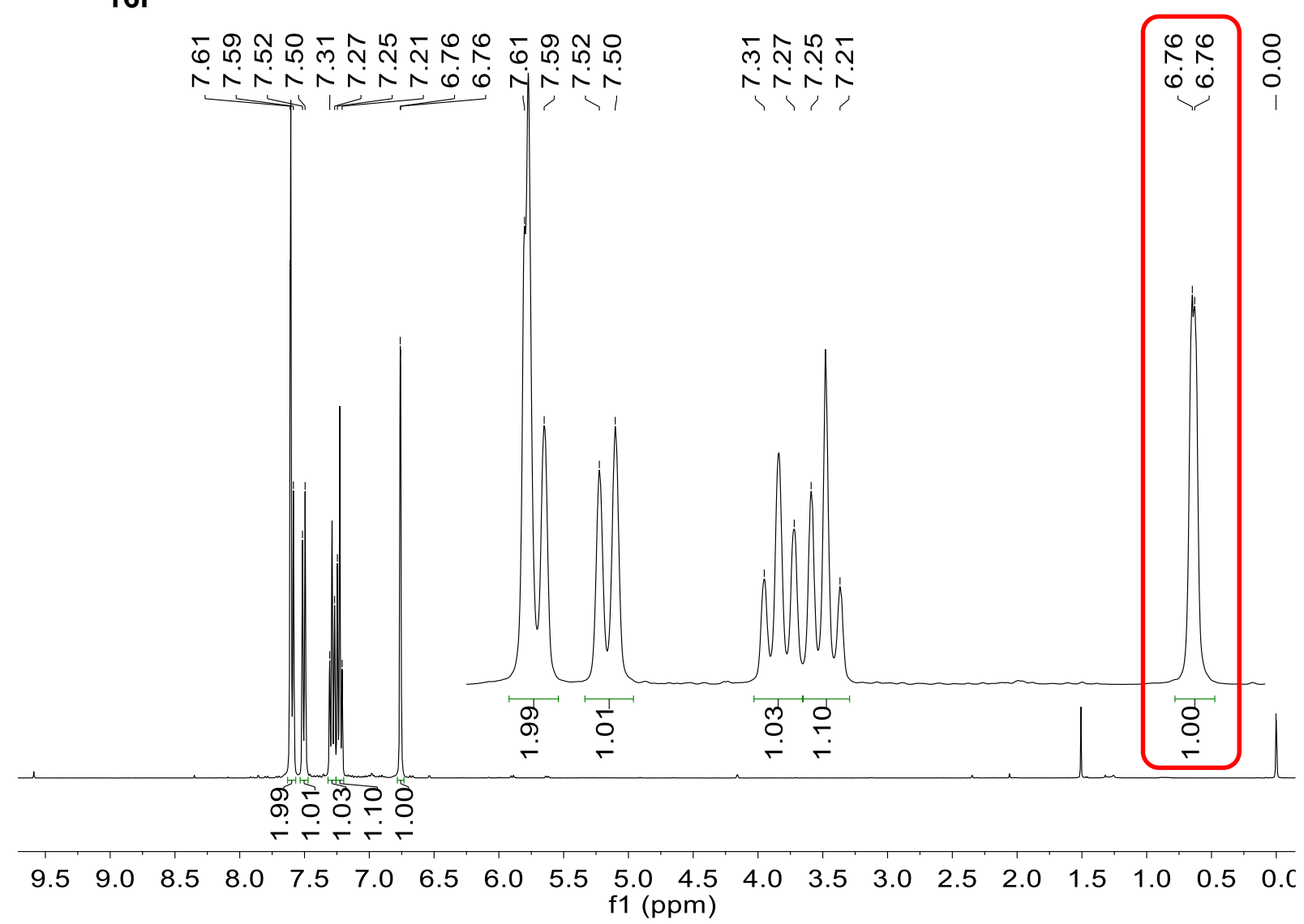

${ }^{1} \mathrm{H}$ NMR spectrum of 2,3-benzofurane, multiplet used for yield determination highlighted by a red box $\left(400 \mathrm{MHz}, \mathrm{CDCl}_{3}\right)$. 


\section{N-Benzylpyrrol (16j)}

$\mathbf{M}\left(\mathrm{C}_{11} \mathrm{H}_{11} \mathrm{~N}\right)=157.22 \mathrm{~g} / \mathrm{mol} ;{ }^{1} \mathbf{H}$ NMR $\left(400 \mathrm{MHz}, \mathrm{CDCl}_{3}\right) \delta[\mathrm{ppm}]=7.33-7.24$ $\left(\mathrm{m}, 3 \mathrm{H}, 4^{\prime}-\mathrm{H}, 5^{\prime}-\mathrm{H}\right), 7.10\left(\mathrm{~d},{ }^{3} \mathrm{~J}_{3^{\prime}, 4^{\prime}}=7.1 \mathrm{~Hz}, 2 \mathrm{H}, 3^{\prime}-\mathrm{H}\right), 6.69-6.68(\mathrm{~m}, 2 \mathrm{H}$, $2-\mathrm{H}), 6.19-6.18(\mathrm{~m}, 2 \mathrm{H}, 3-\mathrm{H}), 5.06\left(\mathrm{~s}, 2 \mathrm{H}, 1^{\prime}-\mathrm{H}\right)$.

16j

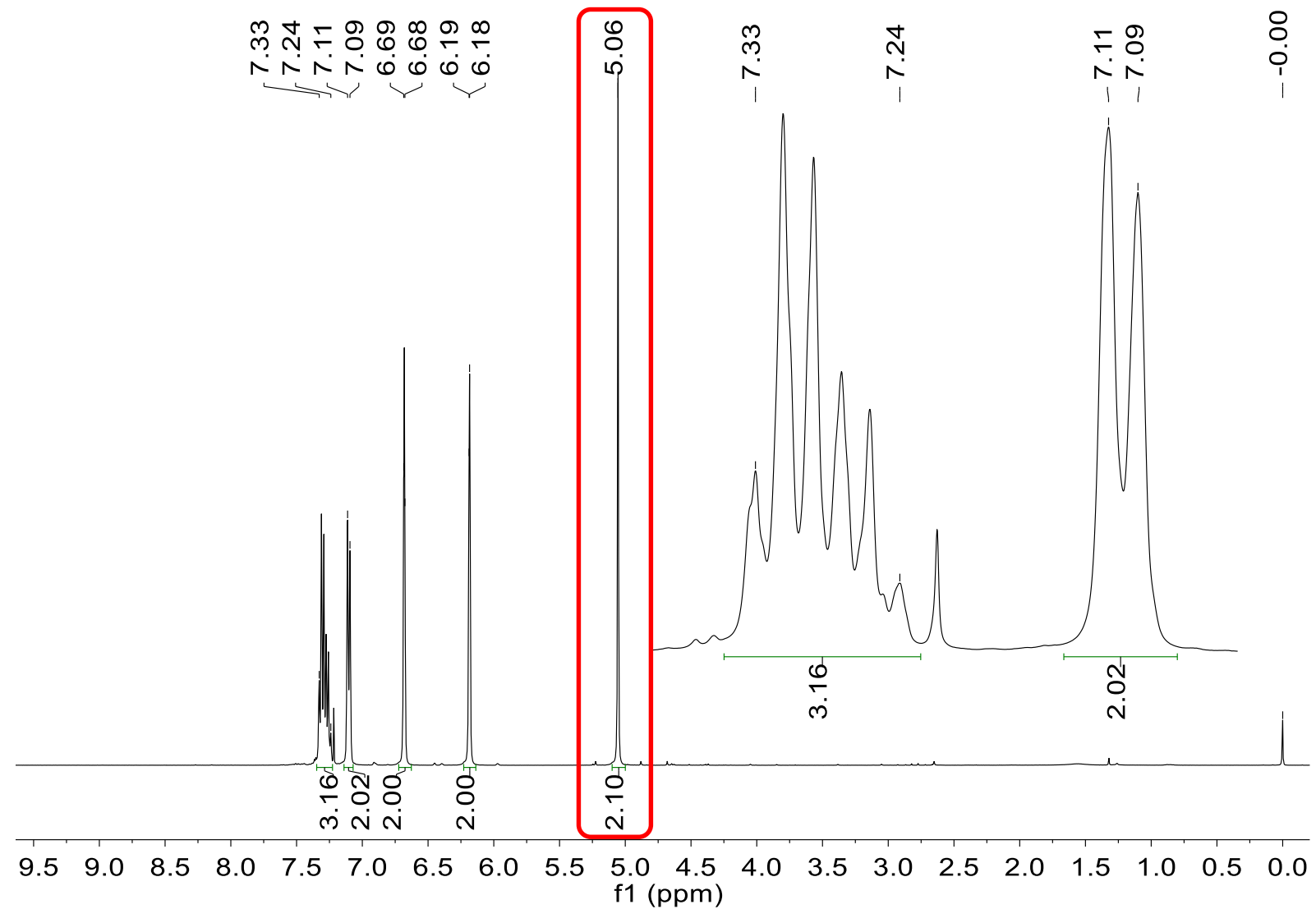

${ }^{1} \mathrm{H}$ NMR spectrum of $\mathrm{N}$-benzylpyrrol, multiplet used for yield determination highlighted by a red box $\left(400 \mathrm{MHz}, \mathrm{CDCl}_{3}\right)$. 


\section{Benzaldehyde dimethylacetale (16k)}<smiles>COC(OC)c1ccccc1</smiles>

16j

The current sample contained traces of benzaldehyde.

$\mathbf{M}\left(\mathrm{C}_{9} \mathrm{H}_{12} \mathrm{O}_{2}\right)=152.19 \mathrm{~g} / \mathrm{mol} ;{ }^{1} \mathbf{H}$ NMR $\left(400 \mathrm{MHz}, \mathrm{CDCl}_{3}\right) \delta[\mathrm{ppm}]=7.46-7.44$ (m, 2H, 4-H), 7.39-7.30 (m, 3H, 3-H, 5-H), $5.39(\mathrm{~s}, 1 \mathrm{H}, 1-\mathrm{H}), 3.33(\mathrm{~s}, 6 \mathrm{H}$, $\left.1^{\prime}-\mathrm{H}\right)$.

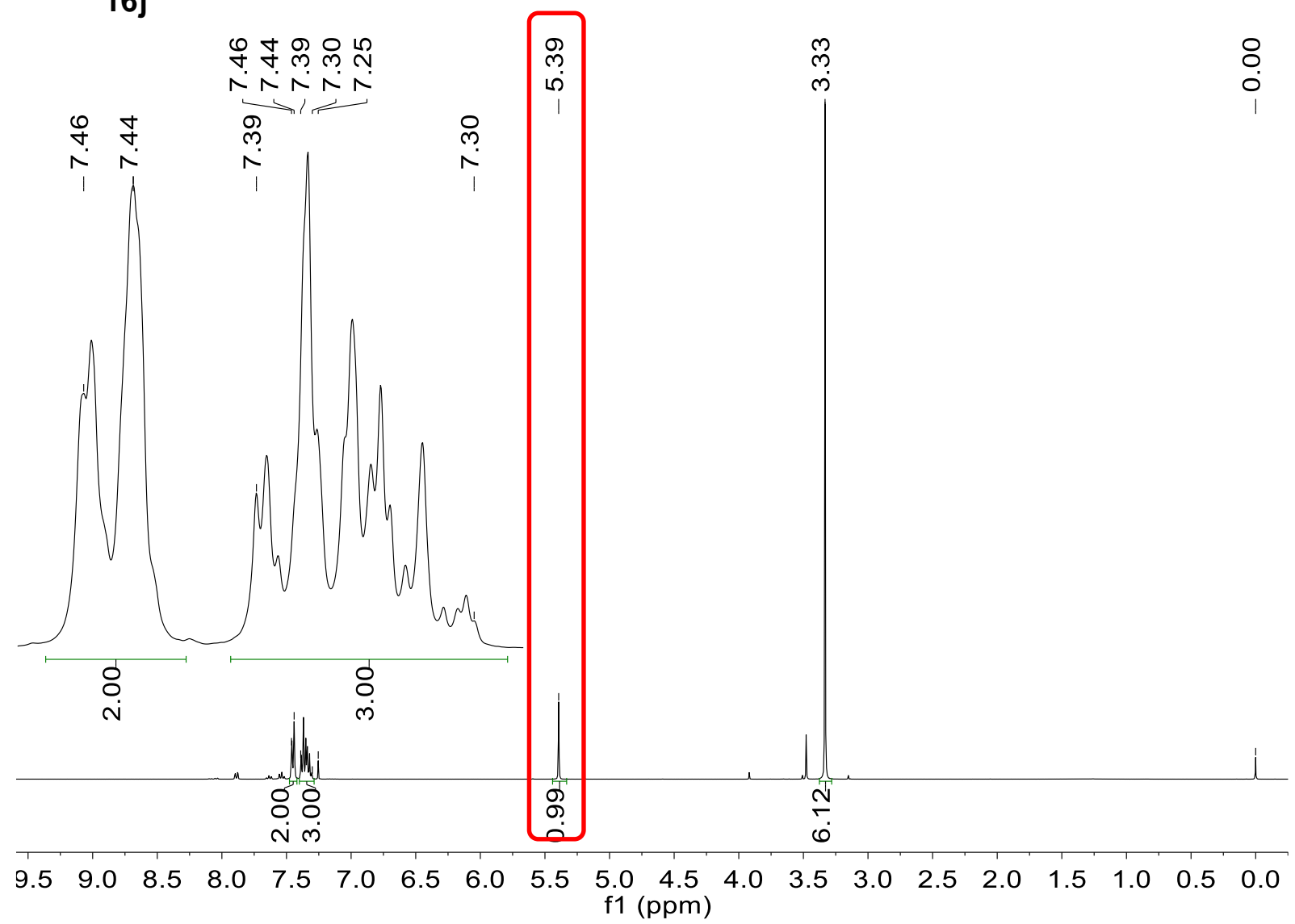

${ }^{1} \mathrm{H}$ NMR spectrum of benzaldehyde dimethylacetale, multiplet used for yield determination highlighted by a red box $\left(400 \mathrm{MHz}, \mathrm{CDCl}_{3}\right)$.

\section{Benzaldehyde}

NMR data obtained from a commercial sample.

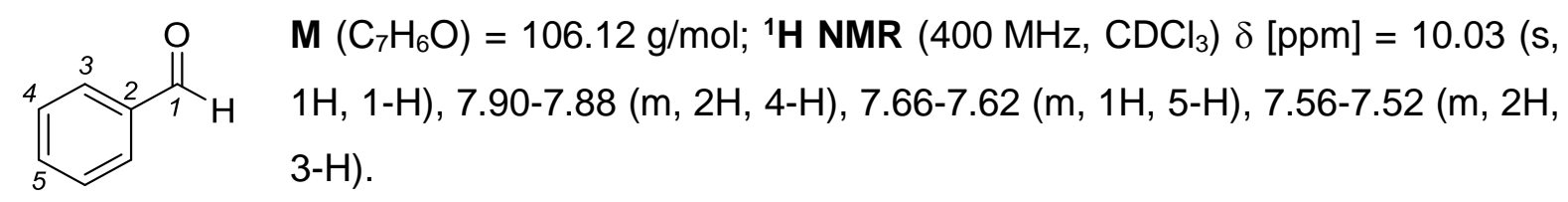




\section{1-Dodecanol (16l)}

$$
\begin{aligned}
& { }^{12}{ }^{11}{ }^{10} \quad \mathbf{M}\left(\mathrm{C}_{12} \mathrm{H}_{26} \mathrm{O}\right)=186.34 \mathrm{~g} / \mathrm{mol} ;{ }^{1} \mathbf{H} \mathbf{N M R}\left(400 \mathrm{MHz}, \mathrm{CDCl}_{3}\right) \delta[\mathrm{ppm}] \\
& \mathrm{HO} \underbrace{2}_{4} \underbrace{3}_{6}=3.66-3.61(\mathrm{~m}, 2 \mathrm{H}, 1-\mathrm{H}), 1.60-1.53(\mathrm{~m}, 2 \mathrm{H}, 2-\mathrm{H}), 1.36-1.26(\mathrm{~m} \text {, }
\end{aligned}
$$
16I

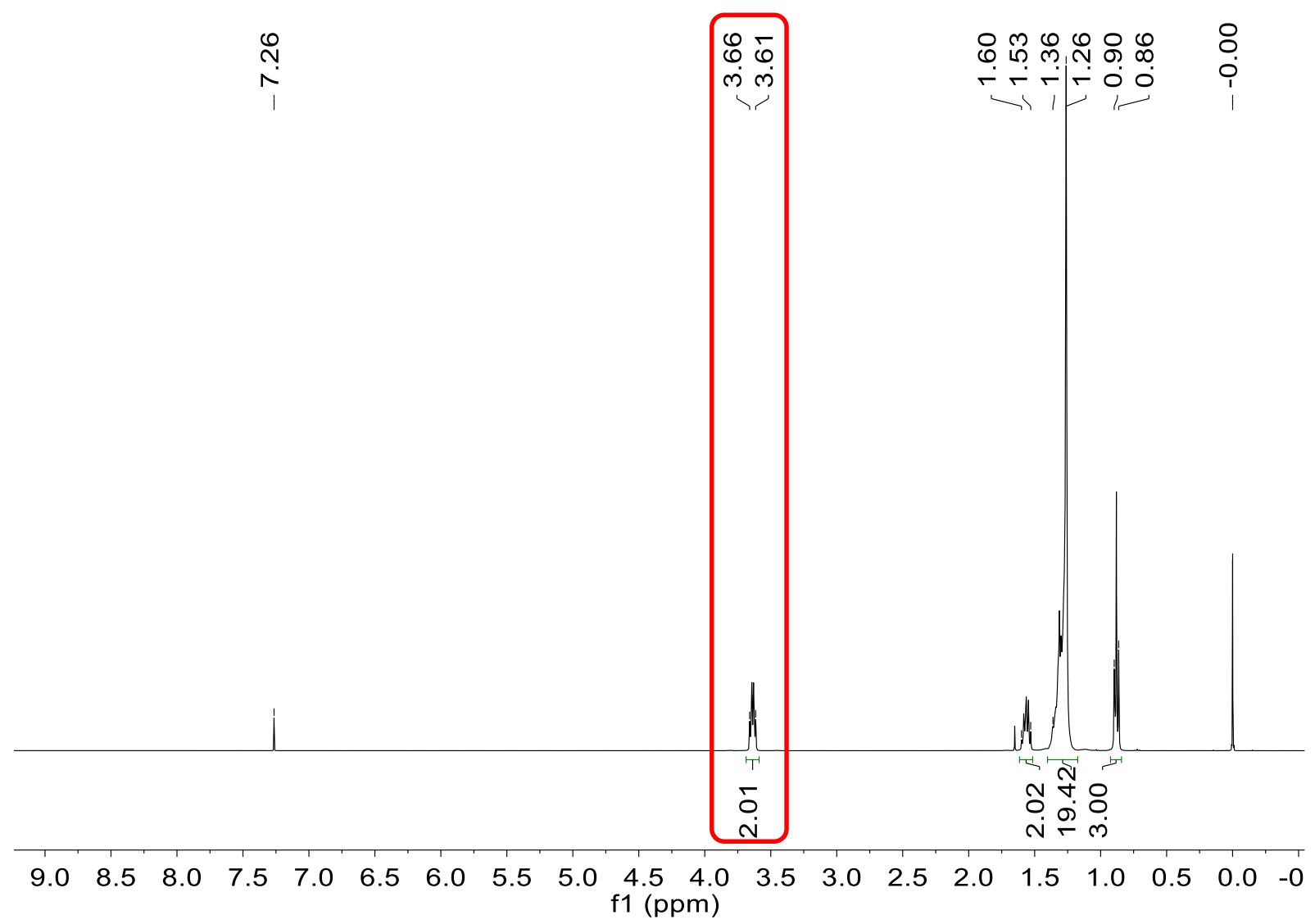

${ }^{1} \mathrm{H}$ NMR spectrum of 1-dodecanol, multiplet used for yield determination highlighted by a red box $\left(400 \mathrm{MHz}, \mathrm{CDCl}_{3}\right)$. 


\section{4-tert-Butylaniline (16m)}<smiles>CC(C)(C)c1ccc(N)cc1</smiles>

$\mathbf{M}\left(\mathrm{C}_{10} \mathrm{H}_{15} \mathrm{~N}\right)=149.24 \mathrm{~g} / \mathrm{mol} ;{ }^{1} \mathbf{H}$ NMR $\left(400 \mathrm{MHz}, \mathrm{CDCl}_{3}\right) \delta[\mathrm{ppm}]=7.20-$ 7.16 (m, 2H, ArH), 6.66-6.62 (m, 2H, ArH), 3.53 (br.s, 2H, NH $\mathrm{N}_{2}$ ), 1.27 (s, $9 \mathrm{H}, 6-\mathrm{H})$.

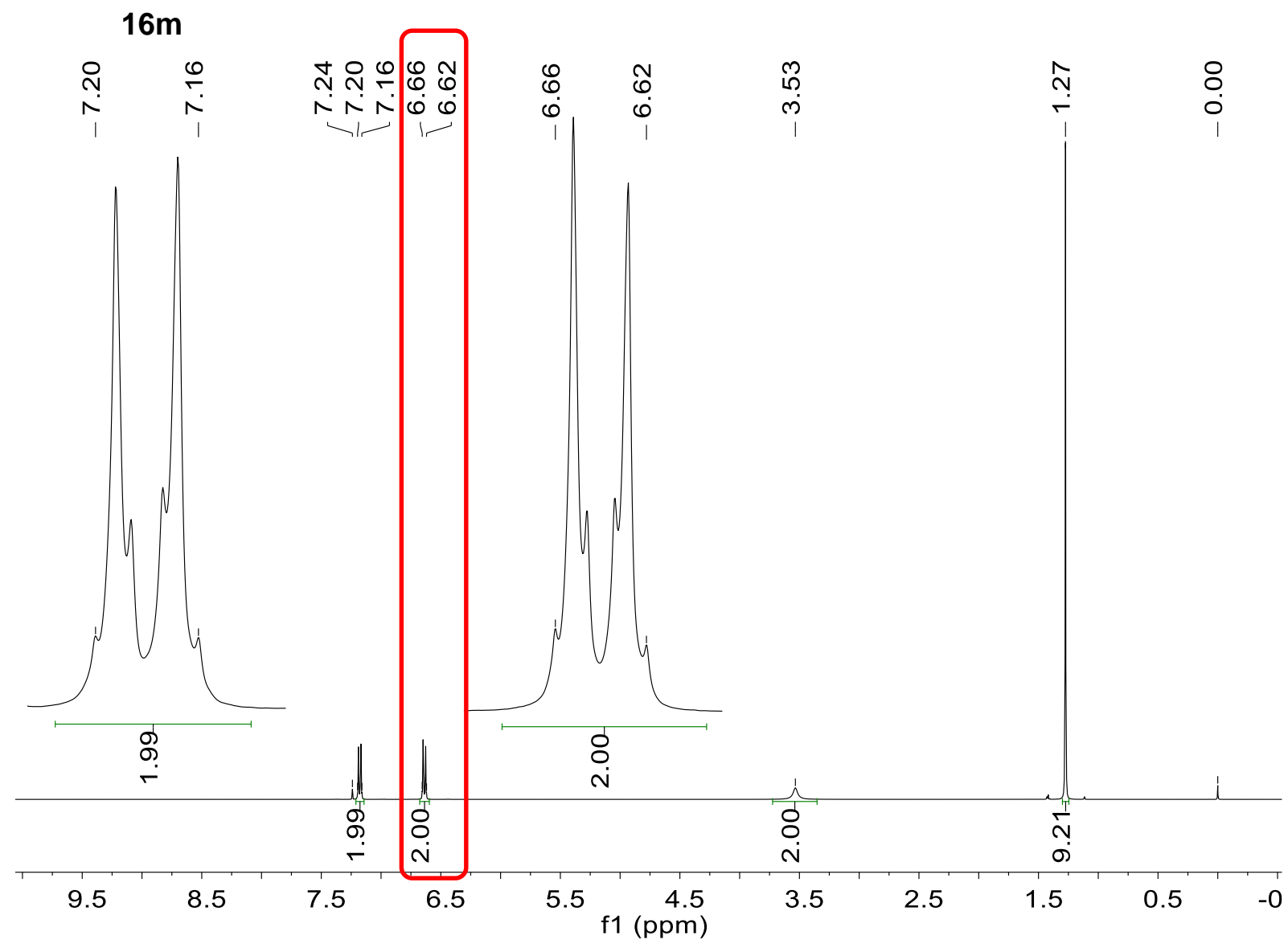

${ }^{1} \mathrm{H}$ NMR spectrum of 4-tert-butylaniline, multiplet used for yield determination highlighted by a red box $\left(400 \mathrm{MHz}, \mathrm{CDCl}_{3}\right)$. 


\subsubsection{Basic Additives (16)}

\section{Benzothiazol (160)}<smiles>c1ccc2scnc2c1</smiles>

$\mathbf{M}\left(\mathrm{C}_{7} \mathrm{H}_{5} \mathrm{NS}\right)=135.18 \mathrm{~g} / \mathrm{mol} ;{ }^{1} \mathbf{H}$ NMR $\left(400 \mathrm{MHz}, \mathrm{CDCl}_{3}\right) \delta[\mathrm{ppm}]=8.99(\mathrm{~s}$, $1 \mathrm{H}, 2-\mathrm{H}), 8.15\left(\mathrm{~d},{ }^{3} \mathrm{~J}=8.3 \mathrm{~Hz}, 1 \mathrm{H}\right), 7.96\left(\mathrm{~d},{ }^{3} \mathrm{~J}=8.0 \mathrm{~Hz}, 1 \mathrm{H}\right), 7.54-7.50(\mathrm{~m}$, $1 \mathrm{H}), 7.46-7.42(\mathrm{~m}, 1 \mathrm{H})$.

160

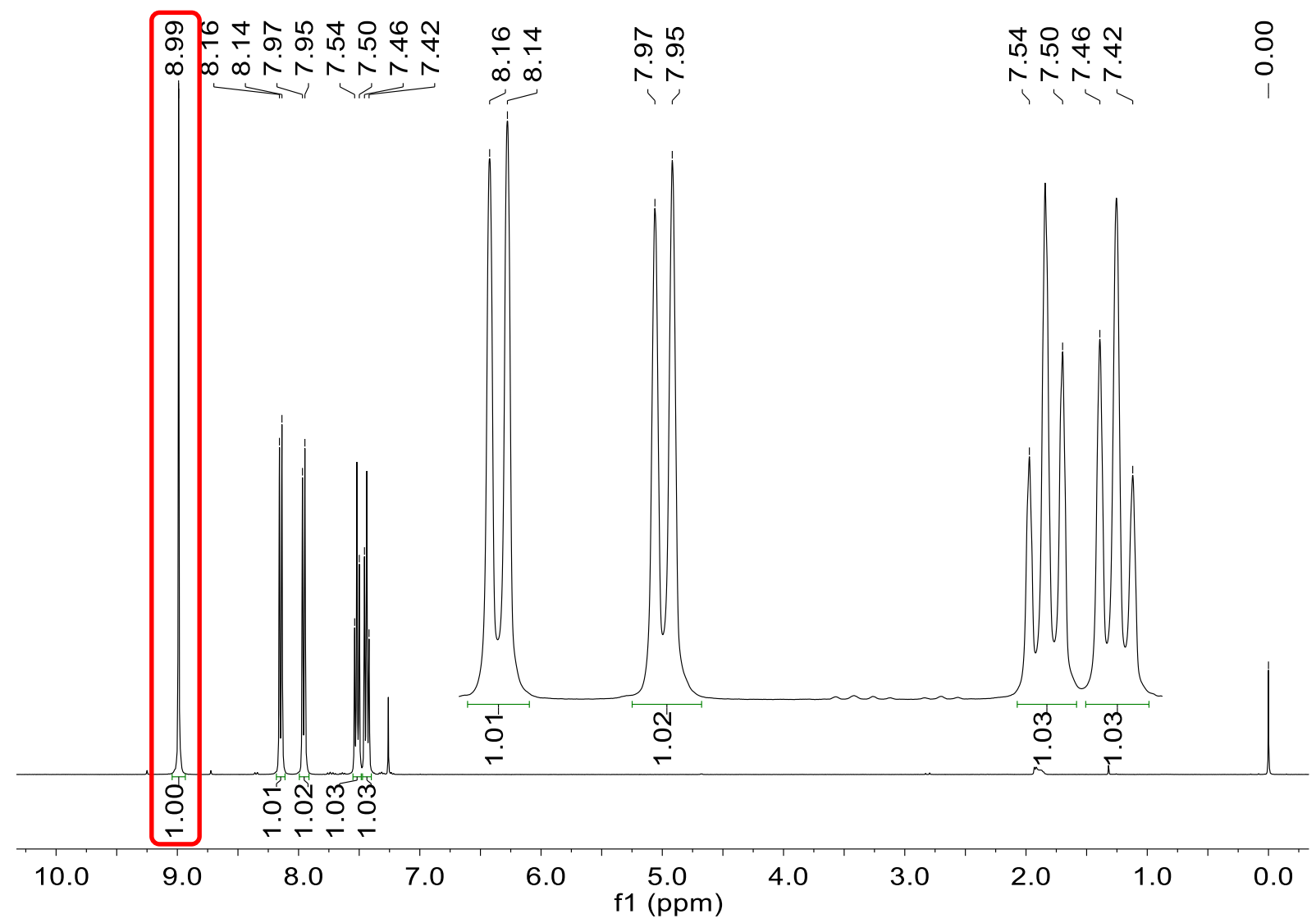

${ }^{1} \mathrm{H}$ NMR spectrum of benzothiazol, multiplet used for yield determination highlighted by a red box $\left(400 \mathrm{MHz}, \mathrm{CDCl}_{3}\right)$. 


\section{4-tert-Butylpyridine (16p)}
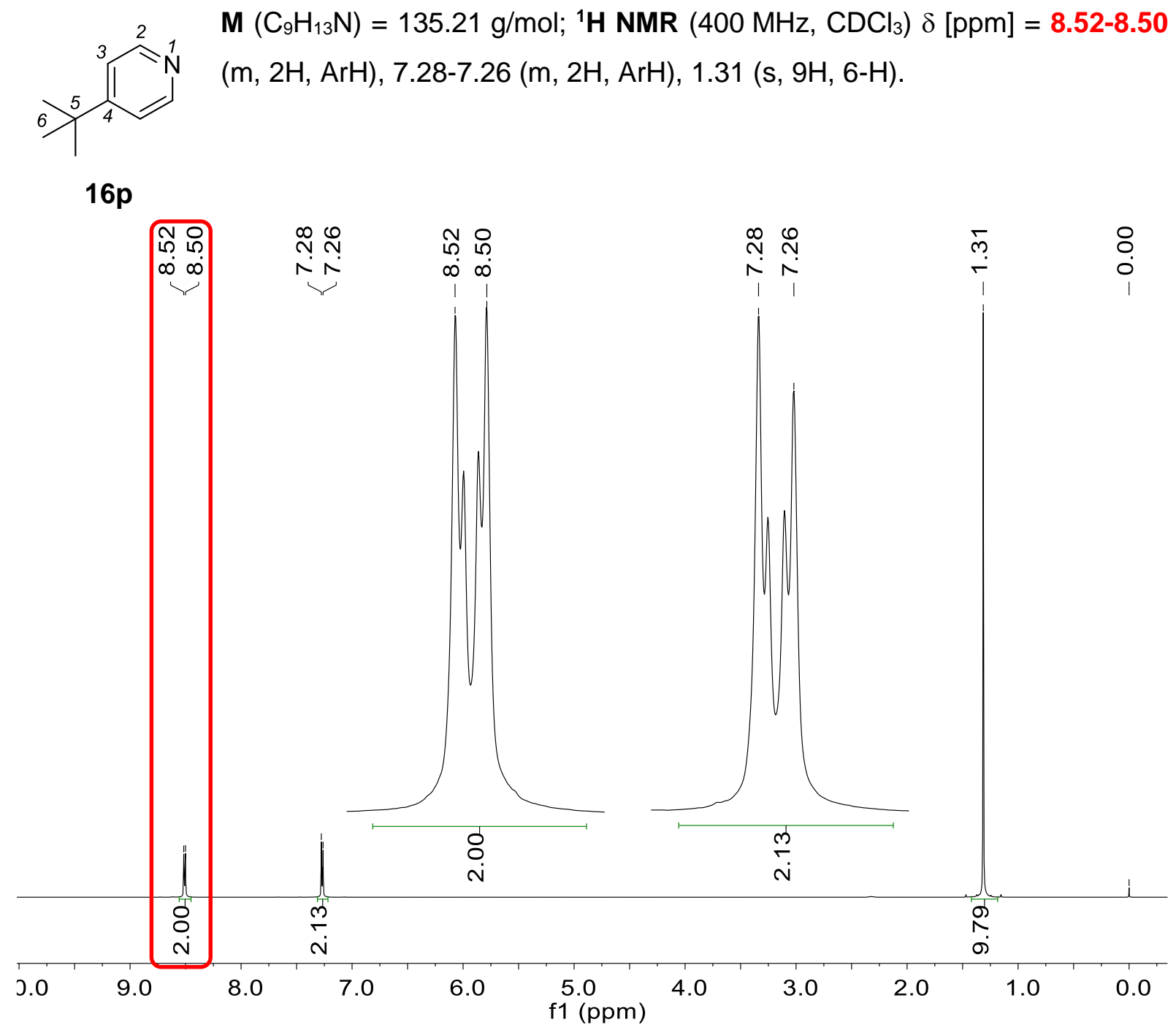

${ }^{1} \mathrm{H}$ NMR spectrum of 4-tert-butylpyridine, multiplet used for yield determination highlighted by a red box $\left(400 \mathrm{MHz}, \mathrm{CDCl}_{3}\right)$. 


\subsubsection{Internal Standard and Starting Material}

\section{1,3,5-Mesitylene}

$\mathbf{M}\left(\mathrm{C}_{9} \mathrm{H}_{12}\right)=120.20 \mathrm{~g} / \mathrm{mol} ;{ }^{1} \mathbf{H}$ NMR $\left(400 \mathrm{MHz}, \mathrm{CDCl}_{3}\right) \delta[\mathrm{ppm}]=6.80(\mathrm{~s}, 3 \mathrm{H}$, $2-\mathrm{H}), 2.28$ (s, 9H, $\left.1^{\prime}-\mathrm{H}\right)$.

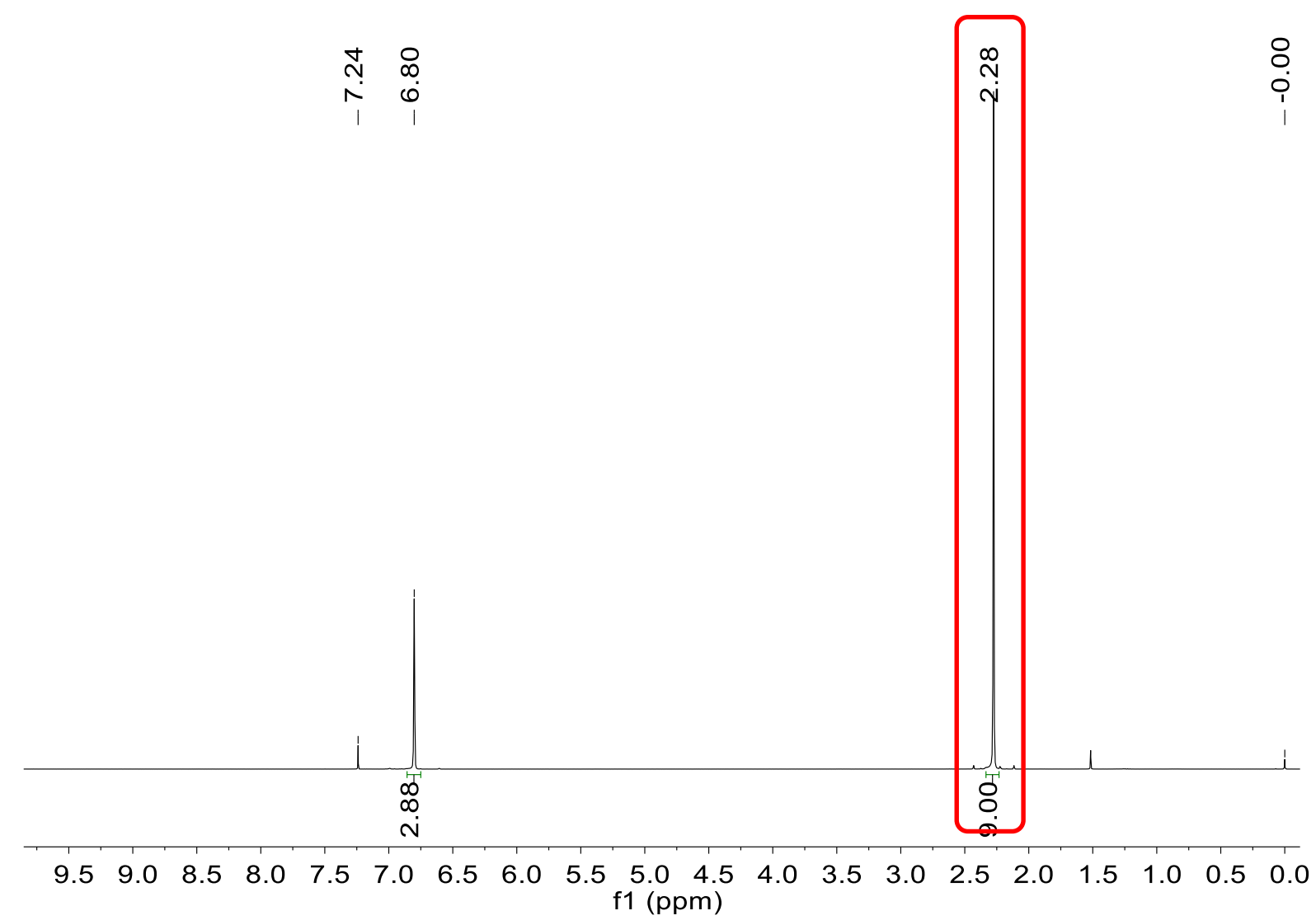

${ }^{1} \mathrm{H}$ NMR spectrum of mesitylene, multiplet used for yield determination highlighted by a red box $\left(400 \mathrm{MHz}, \mathrm{CDCl}_{3}\right)$.

Due to a higher longitudinal relaxation delay $T_{1}$ the integral of the singlet of the aromatic protons at $6.80 \mathrm{ppm}$ is smaller than 3.0. Therefore, yields were calculated in reference to the singlet of the methyl $\mathrm{H}$-atoms at $2.28 \mathrm{ppm}$. 


\section{4-tert-Butylbenzyl alcohol (1I)}<smiles>CC(C)(C)c1ccc(CO)cc1</smiles>

11

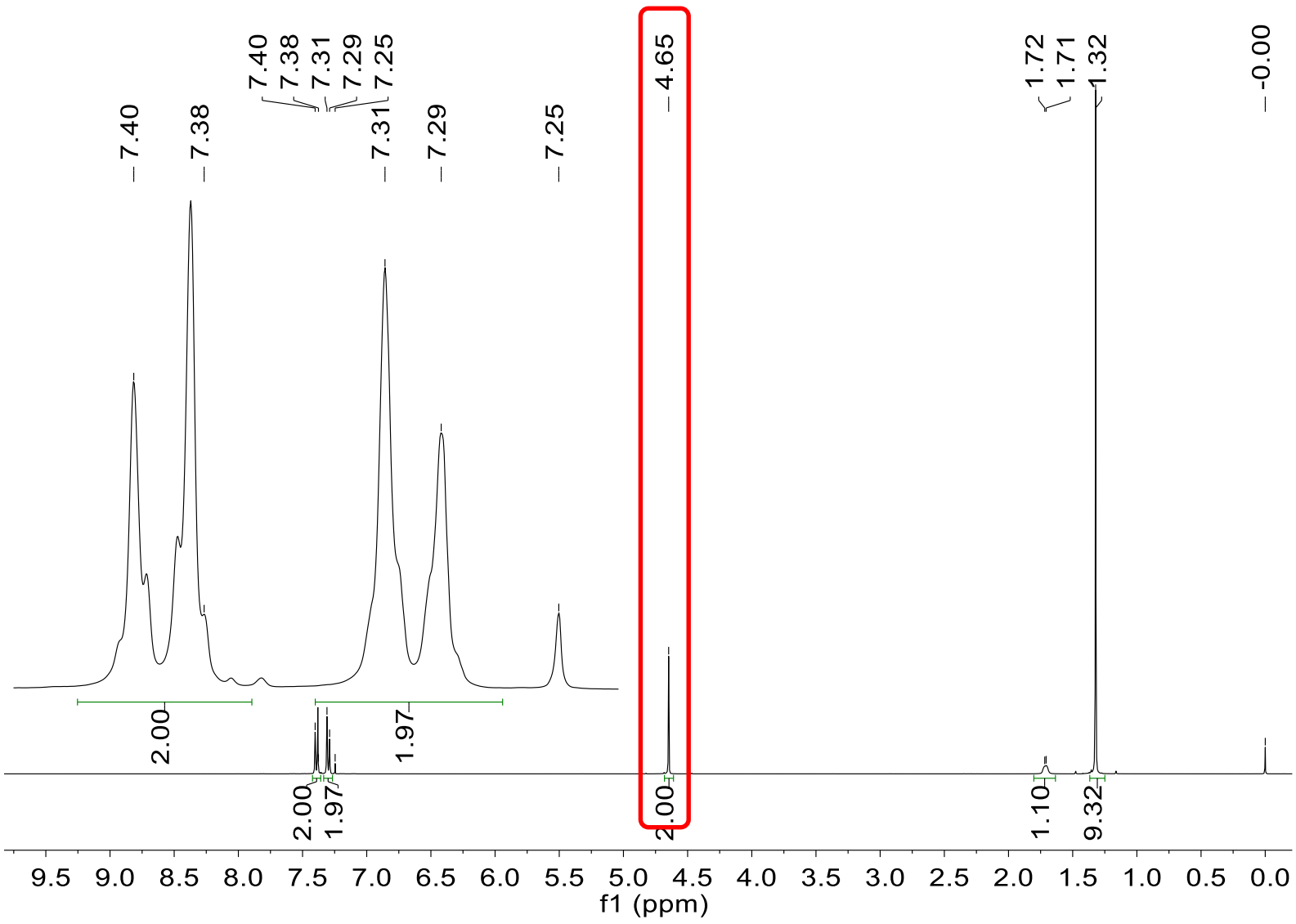

$\mathbf{M}\left(\mathrm{C}_{11} \mathrm{H}_{16} \mathrm{O}\right)=164.25 \mathrm{~g} / \mathrm{mol} ;{ }^{1} \mathbf{H}$ NMR $\left(400 \mathrm{MHz}, \mathrm{CDCl}_{3}\right) \delta[\mathrm{ppm}]=7.40-$ $7.38(\mathrm{~m}, 2 \mathrm{H}, \mathrm{ArH}), 7.31-7.29(\mathrm{~m}, 2 \mathrm{H}, \mathrm{ArH}), 4.65$ (s, 2H, 1-H), 1.72-1.71 $(\mathrm{m}, 1 \mathrm{H}, \mathrm{OH}), 1.32(\mathrm{~s}, 9 \mathrm{H}, 7-\mathrm{H})$.

${ }^{1} \mathrm{H}$ NMR spectrum of 4-tert-butylbenzyl alcohol, multiplet used for yield determination highlighted by a red box $\left(400 \mathrm{MHz}, \mathrm{CDCl}_{3}\right)$.

\subsubsection{Sensitivity Assessment ${ }^{[6]}$}

The standard conditions, which serve as zero point, are the same as in the robustness screen. In the following, deviations from the general procedure 1 (chapter 2.2.1, page 14) are listed in reference to the entries in Table S5 on page 10:

Entry 2, Difference [1I] = $4.0 \mathrm{M}$ : For this experiment a $0.4 \mathrm{M}$ stock solution of FPyr in dioxane (10 mol\%, $125 \mu \mathrm{mol})$ was used.

Entry 3, Deviation [11] = $1.0 \mathrm{M}$ : Herein, a $0.1 \mathrm{M}$ solution of FPyr in dioxane was utilized (10 mol\%, $500 \mu \mathrm{L}$ ).

Entry 4, Deviation +1 vol\% $\mathrm{H}_{2} \mathrm{O}$ : Water $(2.5 \mu \mathrm{L}, 2.5 \mathrm{mg}, 1$ vol\%) was added to the reaction solution before $\mathrm{BzCl}$. 
Entry 5, Difference $+\mathbf{1 0}$ vol\% $\mathbf{H}_{\mathbf{2}} \mathrm{O}$ : Water $(25 \mu \mathrm{L}, 25 \mathrm{mg}, 1$ vol\%) was added to the reaction solution prior to $\mathrm{BzCl}$.

Entry 6, Deviation dry conditions: The reaction was performed in a $10 \mathrm{~mL}$ Schlenk-tube, which had been heated in an oven to $80^{\circ} \mathrm{C}$ overnight. After introduction of the alcohol, the reaction apparatus was purged for several minutes with nitrogen, closed with a septum and all other reaction components were added through the septum. The reaction solution was stirred under $1 \mathrm{~atm}$ of $\mathrm{N}_{2}$.

Entry 7, Difference $1 \mathrm{~atm} \mathrm{O}_{2}$ : The reaction was conducted in a $10 \mathrm{~mL}$ Schlenk-Tube. After addition of all reaction components, the reaction apparatus was purged with oxygen with the aid of a balloon, closed with a well-greased glass stopper, which was subsequently fixed with a metal clamp and the reaction solution was stirred under a positive oxygen pressure for $16 \mathrm{~h}$. Entry 8, Deviation open vial: This experiment was conducted in a $4 \mathrm{~mL}$ glass, which was not closed with a screw cap.

Entry 9, Difference $\mathbf{T}=\mathbf{1 0}^{\circ} \mathbf{C}$ : The reaction was performed in a $10 \mathrm{~mL}$ Schlenk-tube, which was stirred in a cooling bath with an immersion cooler at a temperature of $10^{\circ} \mathrm{C}$. To avoid condensation of water, the reaction tube was sealed with a greased glass stopper and the glass tap was closed.

Entry 10, Deviation $\mathbf{T}=10^{\circ} \mathbf{C}$ : The current experiment was conducted as given in entry 9 at $0{ }^{\circ} \mathrm{C}$.

Entry 12, 5 mol\% FPyr: Herein, a $0.1 \mathrm{M}$ solution of FPyr in dioxane was used $(5 \mathrm{~mol} \%$, $250 \mu \mathrm{L}$.

Entry 13, 10 mol\% FPyr: The current experiment was conducted with FPyr (19.7 $\mu \mathrm{L}, 20.4$ mg, $100 \mu \mathrm{mol}, 20 \mathrm{~mol} \%)$ and dioxane $(250 \mu \mathrm{mol}, 2.0 \mathrm{M})$.

For more details on the sensitivity assessment see chapter 1.2 (page 10). 


\subsection{Mechanistic Studies}

\subsubsection{Determination of Reaction Order by Variable Time Normalization Analysis (VTNA)}

In order to investigate the reaction order of each reaction component, we employed variable time normalization analysis (VTNA), ${ }^{[24]}$ because this method permits to ignore the induction period analyzing the entire course of the reaction. Therefore, the concentration data of the induction was discarded. Only data after the maximum of induction period were collected and used to generate the normalized concentration profiles. All measurements were conducted using DMF as catalyst. Overall, three experiments with varying start concentration of the explored reaction component were performed. Afterwards, the concentration profiles were constructed applying the following equation:

$$
\mathrm{f}\left(t_{\mathrm{n}}\right)=\int_{0}^{t_{\mathrm{n}}}[\mathrm{A}]^{\alpha} \mathrm{dt} \approx \sum_{\mathrm{i}=0}^{\mathrm{n}}\left(\frac{[\mathrm{A}]_{\mathrm{i}}+[\mathrm{A}]_{\mathrm{i}-1}}{2}\right)^{\alpha}\left(t_{\mathrm{i}}-t_{\mathrm{i}-1}\right) .
$$

[A] is the concentration of the explored reaction component in which the reaction order is determined and $\alpha$ is the normalization factor. Subsequently, $\alpha$ was varied until the concentration profiles overlay, so that $\alpha$ corresponds with the reaction order of $A$.

\section{Reaction Order of 4-(tert-Butyl)phenyl)methanol (1I)}

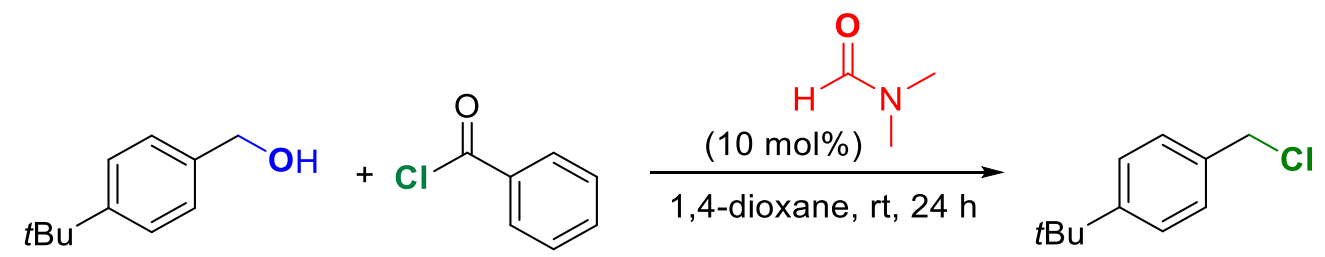

$1.6 \mathrm{M}-2.4 \mathrm{M}, 1 \mathrm{I}$

$2.4 \mathrm{M}, 9 \mathrm{~d}$

3I

(4-(tert-Butyl)phenyl)methanol $(1.6 \mathrm{M}, 131.1 \mathrm{mg}, 0.80 \mathrm{mmol}, 0.80$ equiv; $2.0 \mathrm{M}, 164 \mathrm{mg}$, $1.00 \mathrm{mmol}, 1.00$ equiv or $2.4 \mathrm{M}, 197.0 \mathrm{mg}, 1.20 \mathrm{mmol}, 1.20$ equiv), mesitylene (139 $\mu \mathrm{L}$, $1.00 \mathrm{mmol}, 1.00$ equiv) as internal standard and DMF (1.0 M in 1,4-dioxane, $100 \mu \mathrm{mol}$, $10 \mathrm{~mol} \%$ ) were dissolved in 1,4-dioxane (2.0 M). To ensure the accuracy of the catalyst loading the formamide was added as stock solution (1.0 $\mathrm{M}$ in 1,4-dioxane) by using an Eppendorf pipette. Lastly, $\mathrm{BzCl}(139 \mu \mathrm{L}, 1.20 \mathrm{mmol}, 1.00$ equiv) was added to the reaction mixture. The course of the reaction was monitored by GC analysis. The first 60 min every 5 min an aliquot of the reaction mixture was withdrawn, filtered through a small amount of basic aluminum oxide in a disposable Pasteur pipette (MTBE), and the prepared sample was measured by GC 
analysis. Then, after $70 \mathrm{~min}, 80 \mathrm{~min}, 90 \mathrm{~min}, 105 \mathrm{~min}, 120 \mathrm{~min}, 3 \mathrm{~h}, 4 \mathrm{~h}, 6 \mathrm{~h}, 8 \mathrm{~h}, 10 \mathrm{~h}$ and after $24 \mathrm{~h}$ an aliquot of the reaction mixture was withdrawn and prepared for $\mathrm{GC}$ analysis.

In the following, selective reaction profiles with different $\alpha$ are shown.
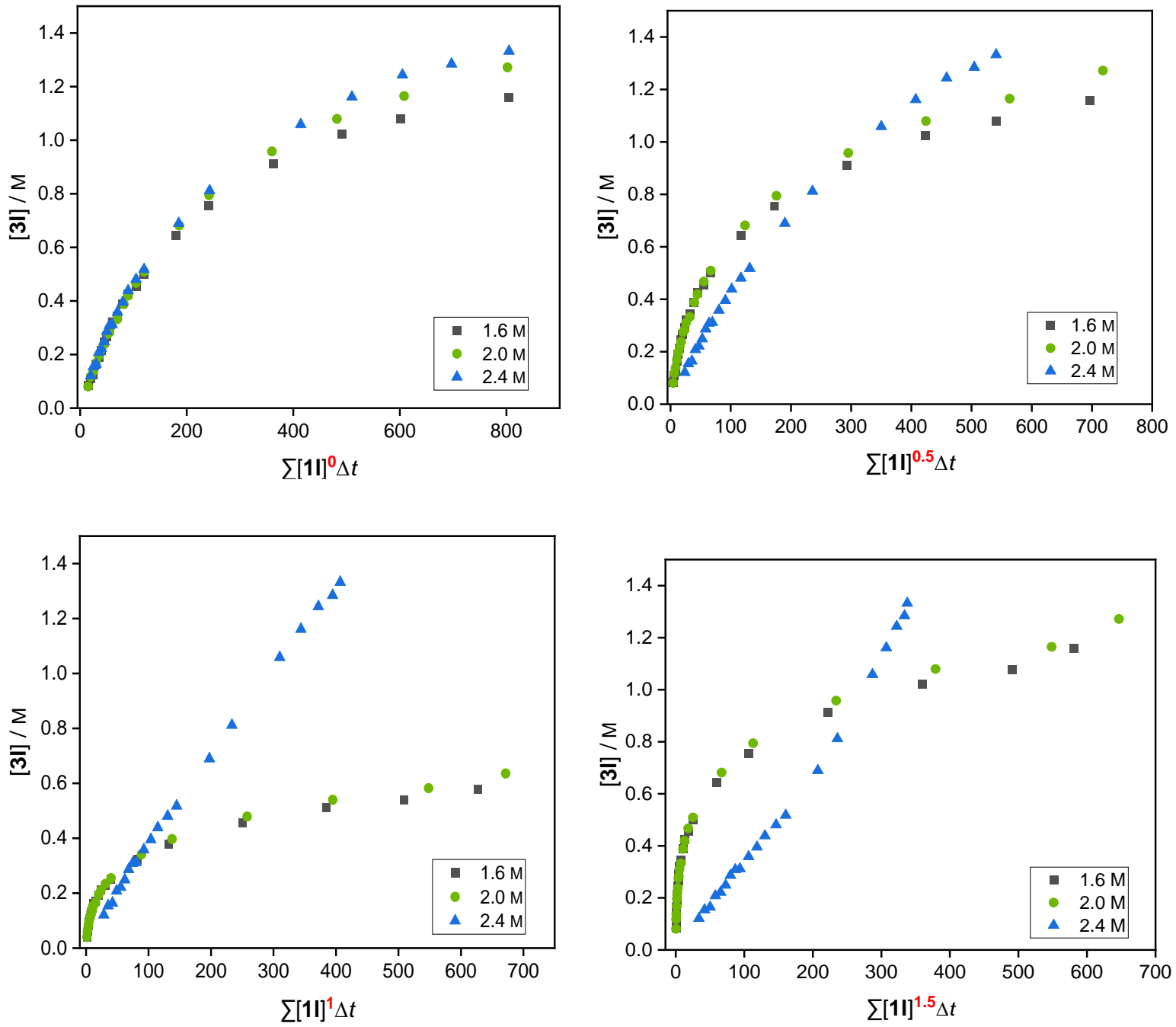

Figure S6. VTNA to determine reaction order of 4-(tert-butyl)phenyl)methanol (11).

Despite deviation of the reaction profile at higher conversion, a zero-order dependence on (4-(tert-butyl)phenyl)methanol (1I) was observed, because the best overlay could be achieved with $\alpha=0$. The deviation can be explained due to formation of stochiometric amounts of benzoic acid (2a), which could coordinate the formamide and therefore inactivate the catalyst toward an off cycle-equilibrium. 


\section{Reaction Order of DMF}

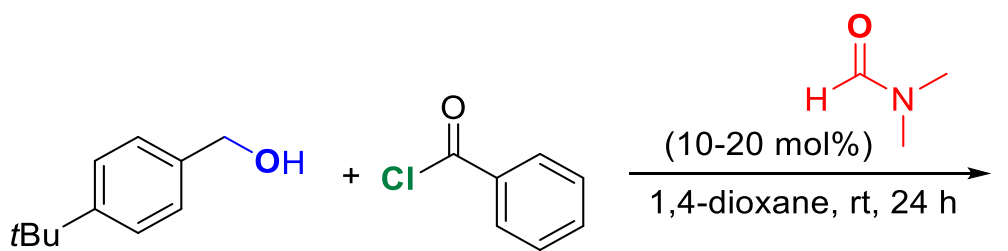

$2.0 \mathrm{M}, 1 \mathrm{I}$<smiles>CC(C)(C)c1ccc(CCl)cc1</smiles>

3!

(4-(tert-Butyl)phenyl)methanol (2.0 M, $164 \mathrm{mg}, 1.00 \mathrm{mmol}, 1.00$ equiv), mesitylene (139 $\mu \mathrm{L}$, $1.00 \mathrm{mmol}, 1.00$ equiv) as internal standard and DMF $(1.0 \mathrm{M}$ in 1,4-dioxane, $100 \mu \mathrm{mol}$, $10 \mathrm{~mol} \%$; $150 \mu \mathrm{mol}, 15 \mathrm{~mol} \%$ or $200 \mu \mathrm{mol}, 20 \mathrm{~mol} \%$ ) were dissolved in 1,4-dioxane (2.0 M). To ensure the accuracy of the catalyst loading the formamide was added as stock solution (1.0 M in 1,4-dioxane) by using an Eppendorf pipette. Lastly, BzCl was (9d, $139 \mu \mathrm{L}, 1.20 \mathrm{mmol}$, 1.00 equiv) was added to the reaction mixture. The course of the reaction was monitored by GC analysis. The first $60 \mathrm{~min}$ every $5 \mathrm{~min}$ an aliquot of the reaction mixture was withdrawn, filtered through a small amount of basic aluminum oxide in a disposable Pasteur pipette (MTBE), and the prepared sample was measured by GC analysis. Then, after $70 \mathrm{~min}, 80 \mathrm{~min}$, $90 \mathrm{~min}, 105 \mathrm{~min}, 120 \mathrm{~min}, 3 \mathrm{~h}, 4 \mathrm{~h}, 6 \mathrm{~h}, 8 \mathrm{~h}, 10 \mathrm{~h}, 13 \mathrm{~h}$ and after $24 \mathrm{~h}$ an aliquot of the reaction mixture was withdrawn and prepared for GC analysis. 
In the following, selective reaction profile with different $\alpha$ are shown.
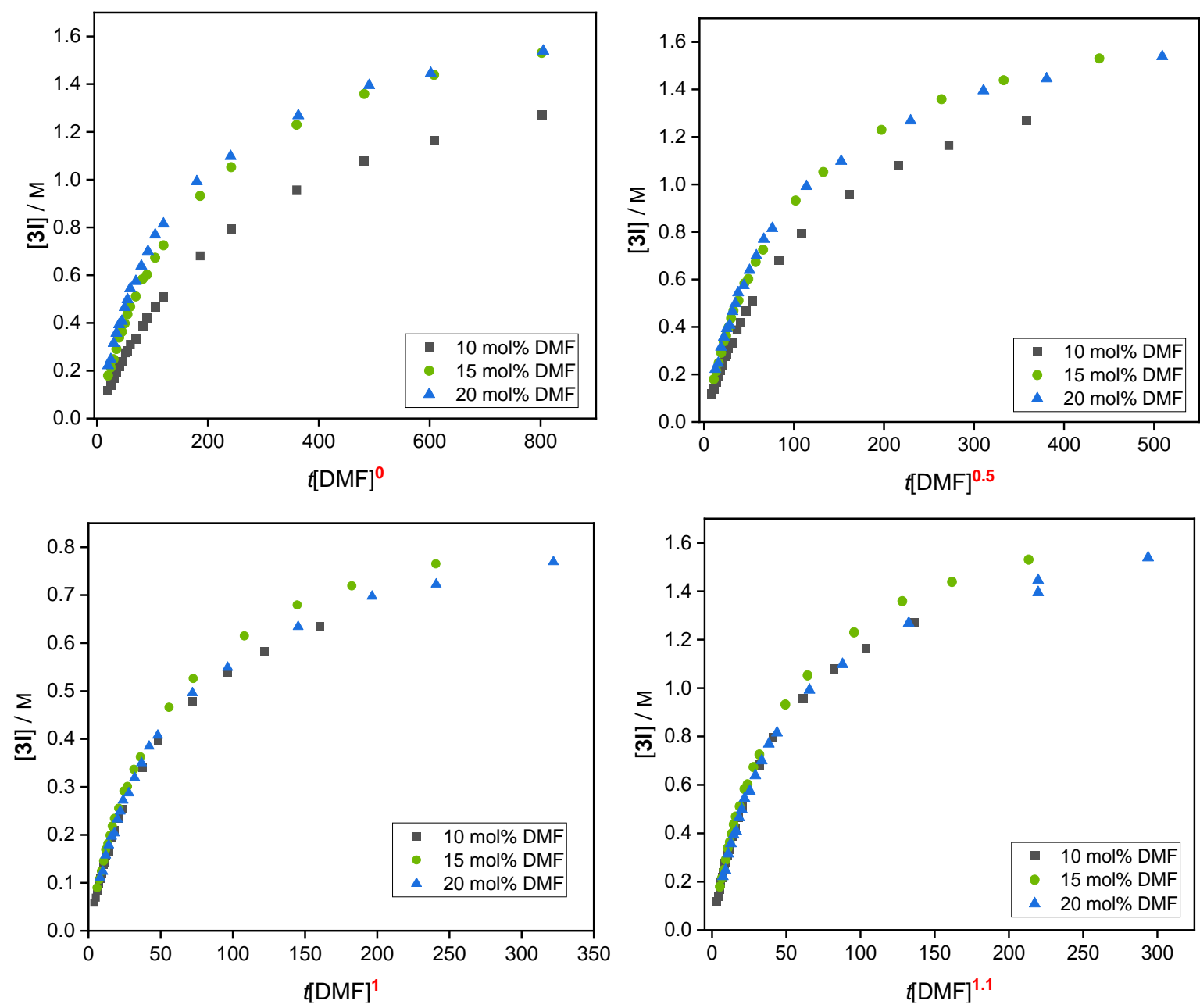

Figure S7. VTNA to determine reaction order of DMF.

Best overlay of the concentration profiles was achieved with a normalization factor $\alpha=1.1$. Based on this, for DMF a reaction order of 1.1 was obtained. The deviation of the profiles at higher conversation could be attributed to an off-cycle equilibrium between the formamide and benzoic acid $(\mathbf{2 a})$. 


\subsubsection{Determination of Reaction Order of $\mathrm{BzCl}$ by an Initial Rate Dependence}

In order to obtain the reaction order of $\mathrm{BzCl}$, an initial rate dependence was applied, because $\mathrm{BzCl}$ is not detectable by $\mathrm{GC}$ analysis, and therefore its concentration cannot be determined during the course of reaction. As show in Figure S8, the induction period of formamide catalyzed nucleophilic substitution could be supressed by performing the reaction at elevated temperature $\left(40^{\circ} \mathrm{C}\right)$.

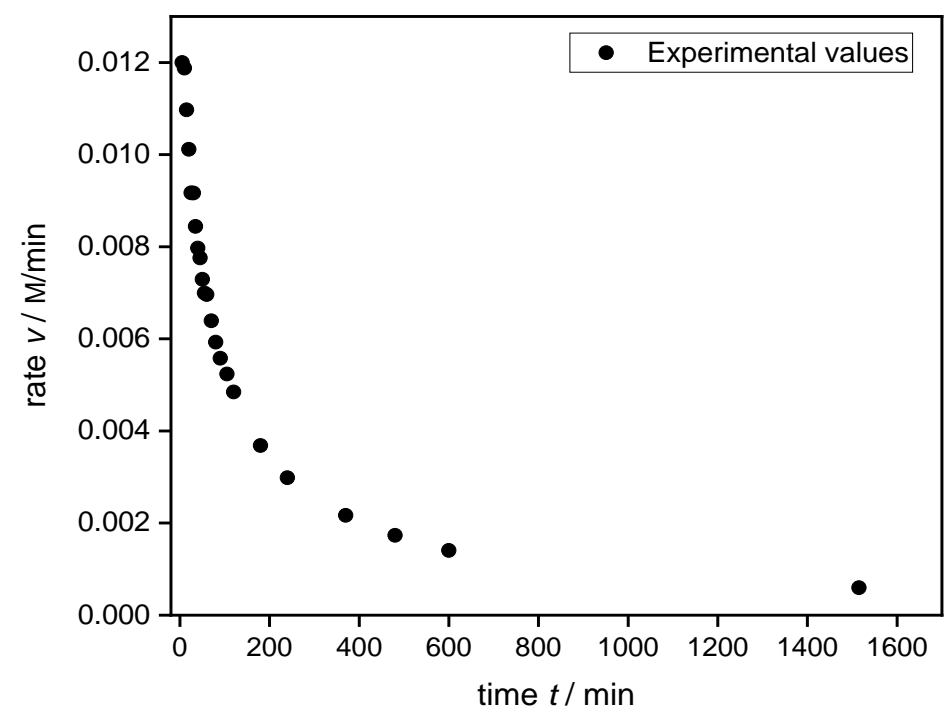

Figure S8. Plot of rate $v$ against time $t$ for formamide catalyzed nucleophilic substitution at $40{ }^{\circ} \mathrm{C}$ reaction temperature.

Overall, five experiments with different start concentration of $\mathrm{BzCl}$, ranging from $2.0 \mathrm{M}$ to $2.8 \mathrm{M}$, were performed. The measurements were conducted at $40^{\circ} \mathrm{C}$ reaction temperature.

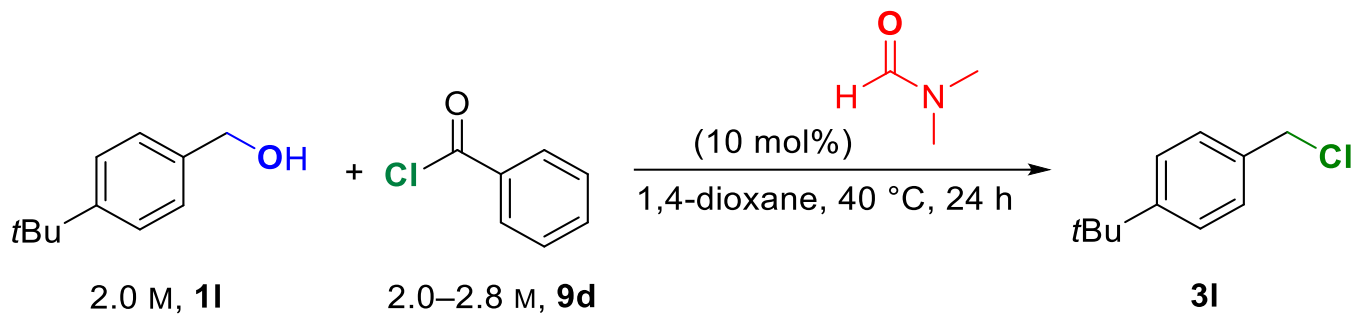

(4-(tert-Butyl)phenyl)methanol (2.0 M, $164 \mathrm{mg}, 1.00 \mathrm{mmol}, 1.00$ equiv), mesitylene (139 $\mu \mathrm{L}$, $1.00 \mathrm{mmol}, 1.00$ equiv) as internal standard and $N, N$-dimethylformamide (1.0 $\mathrm{M}$ in 1,4-dioxane, $100 \mu \mathrm{mol}, 10 \mathrm{~mol} \%$; $150 \mu \mathrm{mol}, 15 \mathrm{~mol} \%$ or $200 \mu \mathrm{mol}, 20 \mathrm{~mol} \%$ ) were dissolved in 1,4-dioxane $(2.0 \mathrm{M})$. To ensure the accuracy of the catalyst loading the formamide was added as stock solution (1.0 M in 1,4-dioxane) by using an Eppendorf pipette. After heating at $40{ }^{\circ} \mathrm{C}, \mathrm{BzCl}$ $(116 \mu \mathrm{L}, 1.00 \mathrm{mmol} ; 128 \mu \mathrm{L}, 1.10 \mathrm{mmol} ; 139 \mu \mathrm{L}, 1.20 \mathrm{mmol} ; 151 \mu \mathrm{L}, 1.30 \mathrm{mmol} ; 163 \mu \mathrm{L}$, $1.40 \mathrm{mmol}$ ) was added to the reaction mixture. The course of the reaction was monitored by $\mathrm{GC}$ analysis. The first $60 \mathrm{~min}$ every $5 \mathrm{~min}$ an aliquot of the reaction mixture was withdrawn, 
filtered through a small amount of basic aluminum oxide in a disposable Pasteur pipette (MTBE), and the prepared sample was measured by GC analysis. Then, after $70 \mathrm{~min}, 80 \mathrm{~min}$, $90 \mathrm{~min}, 105 \mathrm{~min}, 120 \mathrm{~min}, 3 \mathrm{~h}, 4 \mathrm{~h}, 6 \mathrm{~h}, 8 \mathrm{~h}, 10 \mathrm{~h}, 13 \mathrm{~h}$ and after $24 \mathrm{~h}$ an aliquot of the reaction mixture was withdrawn and prepared for GC analysis.

Table S6. Initial rate dependence to determine the reaction order of $\mathrm{BzCl}$.

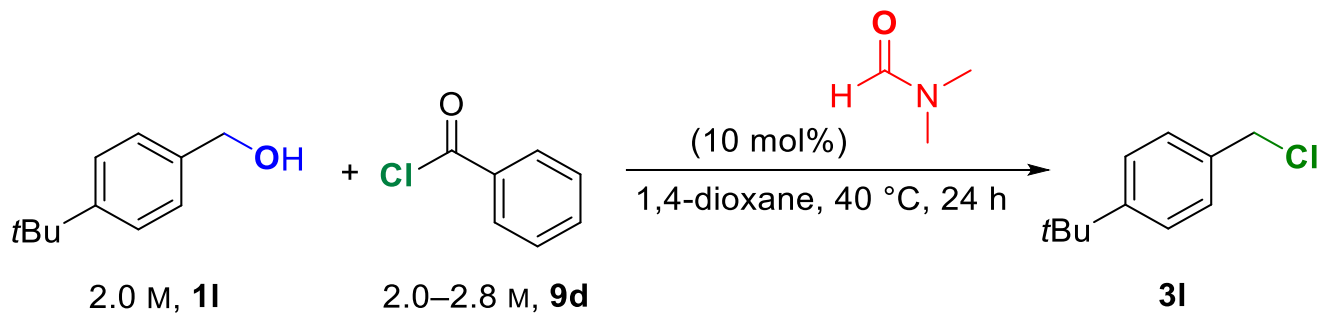

\begin{tabular}{llllll}
\hline entry & $\mathbf{B z C l}$ & {$[\mathrm{BzCl}] / \mathbf{M}$} & $\mathbf{I g}([\mathrm{BzCl}]$ & $\boldsymbol{v}_{\mathbf{0}} / \mathbf{1 0}^{-\mathbf{5}} \mathbf{M} \cdot \mathbf{s}^{-\mathbf{1}}$ & $\mathbf{I g}\left(\boldsymbol{v}_{\mathbf{0}}\right)$ \\
\hline $\mathbf{1}$ & 1.00 equiv & 2.00 & 0.3010 & 7.6939 & -4.1139 \\
$\mathbf{2}$ & 1.10 equiv & 2.20 & 0.3424 & 8.4749 & -4.0719 \\
$\mathbf{3}$ & 1.20 equiv & 2.40 & 0.3802 & 8.8411 & -4.0535 \\
$\mathbf{4}$ & 1.30 equiv & 2.60 & 0.4150 & 9.4319 & -4.0254 \\
$\mathbf{5}$ & 1.40 equiv & 2.80 & 0.4472 & 10.6197 & -3.9739
\end{tabular}

Plotting the logarithm of initial rate $v_{0}$ versus logarithm of concentration of $\mathrm{BzCl}$ revealed a reaction order of 0.9 (Figure S9).

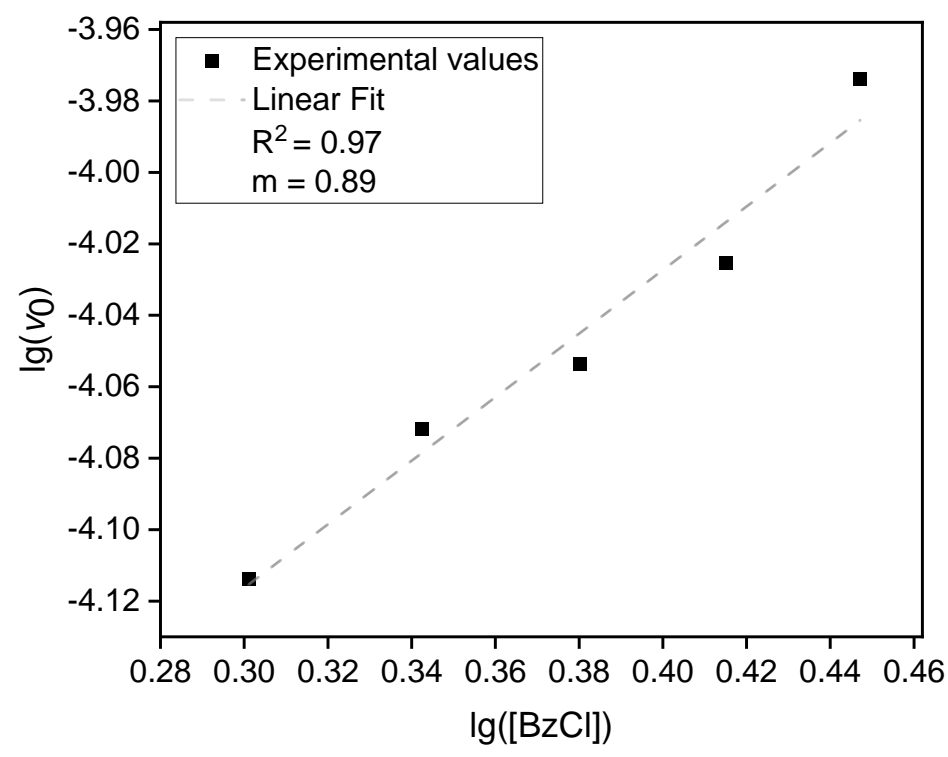

Figure S9. Initial rate dependence. 


\subsubsection{Effect on Reaction Rate Adding Different Additives}

\section{Chloride lons as Additive}

(4-(tert-Butyl)phenyl)methanol (164 mg, $1.00 \mathrm{mmol}, 1.00$ equiv), mesitylene (139 $\mu \mathrm{L}$, $1.00 \mathrm{mmol}, 1.00$ equiv) as internal standard and $N, N$-dimethylformamide (1.0 M in 1,4-dioxane, $100 \mu \mathrm{mol}, 10 \mathrm{~mol} \%$ ) were dissolved in 1,4-dioxane $(2.0 \mathrm{M})$. To ensure the accuracy of the catalyst loading the formamide was added as stock solution (1.0 M in 1,4-dioxane) by using an Eppendorf pipette. Lastly, $\mathrm{LiCl}\left(42.1 \mathrm{mg}, 1.00 \mathrm{mmol}, 1.00\right.$ equiv) or $\mathrm{Bu}_{4} \mathrm{NCl}$ (278 mg, $1.00 \mathrm{mmol}, 1.00$ equiv) and $\mathrm{BzCl}(139 \mu \mathrm{L}, 1.20 \mathrm{mmol}, 1.00$ equiv) were added to the reaction mixture. The course of the reaction was monitored by GC analysis. The first 60 min every 5 min an aliquot of the reaction mixture was withdrawn, filtered through a small amount of basic aluminum oxide in a disposable Pasteur pipette (MTBE), and the prepared sample was measured by GC analysis. Then, after $70 \mathrm{~min}, 80 \mathrm{~min}, 90 \mathrm{~min}, 105 \mathrm{~min}, 120 \mathrm{~min}, 3 \mathrm{~h}, 4 \mathrm{~h}, 6 \mathrm{~h}$, $8 \mathrm{~h}, 10 \mathrm{~h}, 13 \mathrm{~h}$ and after $24 \mathrm{~h}$ an aliquot of the reaction mixture was withdrawn and prepared for GC analysis.

Figure S10. Experiment with additional chloride ions.

\begin{tabular}{llll} 
entry & chloride ion & $\boldsymbol{k}_{\text {start }} / \mathbf{1 0}^{-\mathbf{3}} \mathbf{~ m i n}^{-\mathbf{1}}$ & $\boldsymbol{k}_{\text {total }} / \mathbf{1 0}^{\mathbf{- 3}} \mathbf{~ m i n}^{\mathbf{- 1}}$ \\
\hline $\mathbf{1}$ & - & 3.77 & 3.02 \\
$\mathbf{2}$ & 1.00 equiv $\mathrm{LiCl}$ & 4.22 & 2.68 \\
$\mathbf{3}$ & 1.00 equiv $\mathrm{Bu}{ }_{4} \mathrm{NCl}$ & 4.42 & 2.49
\end{tabular}

\section{Benzoic Acid (2a) as Additive}

(4-(tert-Butyl)phenyl)methanol (164 mg, $1.00 \mathrm{mmol}, 1.00$ equiv), mesitylene (139 $\mu \mathrm{L}$, $1.00 \mathrm{mmol}, 1.00$ equiv) as internal standard and $N, N$-dimethylformamide ( $1.0 \mathrm{M}$ in 1,4-dioxane, $100 \mu \mathrm{mol}, 10 \mathrm{~mol} \%$ ) were dissolved in 1,4-dioxane $(2.0 \mathrm{M})$. To ensure the accuracy of the catalyst loading the formamide was added as stock solution (1.0 M in 1,4-dioxane) by using an Eppendorf pipette. Lastly, benzoic acid (1.00 equiv, 2.00 equiv, 3.00 equiv or 5.00 equiv) and $\mathrm{BzCl}(39 \mu \mathrm{L}, 1.20 \mathrm{mmol}, 1.00$ equiv) were added to the reaction mixture. The course of the reaction was monitored by GC analysis. The first $60 \mathrm{~min}$ every $5 \mathrm{~min}$ an aliquot of the reaction mixture was withdrawn, filtered through a small amount of basic aluminum oxide in a disposable Pasteur pipette (MTBE), and the prepared sample was measured by GC analysis. Then, after $70 \mathrm{~min}, 80 \mathrm{~min}, 90 \mathrm{~min}, 105 \mathrm{~min}, 120 \mathrm{~min}, 3 \mathrm{~h}, 4 \mathrm{~h}, 6 \mathrm{~h}, 8 \mathrm{~h}, 10 \mathrm{~h}, 13 \mathrm{~h}$ and after $24 \mathrm{~h}$ an aliquot of the reaction mixture was withdrawn and prepared for $\mathrm{GC}$ analysis. 
Table S7. Experiment with additional different equivalent benzoic acid (2a).

\begin{tabular}{llll}
\hline entry & benzoic acid (2a) & $\boldsymbol{k}_{\text {start }} / \mathbf{1 0}^{-\mathbf{3}} \mathbf{~ m i n}^{\mathbf{1}}$ & $\boldsymbol{k}_{\text {total }} / \mathbf{1 0}^{\mathbf{3}} \mathbf{~ m i n}^{\mathbf{- 1}}$ \\
\hline 1 & - & 3.77 & 3.02 \\
2 & 1.00 equiv & 3.08 & 2.84 \\
3 & 2.00 equiv & 2.37 & 2.09 \\
4 & 3.00 equiv & 2.42 & 2.29 \\
5 & 5.00 equiv & 1.90 & 1.92 \\
\hline
\end{tabular}

\section{2,6-Di-tert-butylpyridine (14) as Additive}

(4-(tert-Butyl)phenyl)methanol (164 mg, 1.00 mmol, 1.00 equiv), mesitylene (139 $\mu \mathrm{L}$, $1.00 \mathrm{mmol}, 1.00$ equiv) as internal standard and $N, N$-dimethylformamide (1.0 $\mathrm{M}$ in 1,4-dioxane, $100 \mu \mathrm{mol}, 10 \mathrm{~mol} \%)$ were dissolved in 1,4-dioxane (2.0 M). To ensure the accuracy of the catalyst loading the formamide was added as stock solution (1.0 $\mathrm{M}$ in 1,4-dioxane) by using an Eppendorf pipette. Lastly, 2,6-di-tert-butylpyridine (225 $\mu \mathrm{L}, 1.00 \mathrm{mmol}, 1.00$ equiv) and $\mathrm{BzCl}$ (139 $\mu \mathrm{L}, 1.20 \mathrm{mmol}, 1.00$ equiv) were added to the reaction mixture. The course of the reaction was monitored by $\mathrm{GC}$ analysis. The first $60 \mathrm{~min}$ every $5 \mathrm{~min}$ an aliquot of the reaction mixture was withdrawn, filtered through a small amount of basic aluminum oxide in a disposable Pasteur pipette (MTBE), and the prepared sample was measured by GC analysis. Then, after $70 \mathrm{~min}, 80 \mathrm{~min}, 90 \mathrm{~min}, 105 \mathrm{~min}, 120 \mathrm{~min}, 3 \mathrm{~h}, 4 \mathrm{~h}, 6 \mathrm{~h}, 8 \mathrm{~h}, 10 \mathrm{~h}, 13 \mathrm{~h}$ and after $24 \mathrm{~h}$ an aliquot of the reaction mixture was withdrawn and prepared for GC analysis.

Table S8. Experiment with additional different equivalent 2,6-di-tert-butylpyridine (14).

\begin{tabular}{llll} 
entry & $\mathbf{1 4}$ & $\boldsymbol{k}_{\text {start }} / \mathbf{1 0}^{-\mathbf{3}} \mathbf{~ m i n}^{-\mathbf{1}}$ & $\boldsymbol{k}_{\text {total }} / \mathbf{1 0}^{-\mathbf{3}} \mathbf{~ m i n}^{-\mathbf{1}}$ \\
\hline 1 & - & 3.77 & 3.02 \\
2 & 1.00 equiv & 4.14 & 2.92
\end{tabular}




\subsection{Kinetic Analysis of Formamide Catalyzed Nucleophilic Substitution Monitored by GC}

\subsubsection{Synthesis of 4-(tert-Butyl)benzaldehyde}<smiles>CC(C)(C)c1ccc(C=O)cc1</smiles>

According to a procedure of Yang, ${ }^{[25]}$ under argon atmosphere 1-bromo-4tert-butylbenzene ( $3.41 \mathrm{~mL}, 20.0 \mathrm{mmol}, 1.00$ equiv) was dissolved in abs. THF (100 mL). After cooling to $-78^{\circ} \mathrm{C}, n$-BuLi (2.5 M in $n$-hexane, $9.28 \mathrm{~mL}$, $23.2 \mathrm{mmol}, 1.16$ equiv) was added dropwise and the reaction mixture was stirred at this temperature for $1 \mathrm{~h}$. Abs. DMF (4.02 mL, $52.0 \mathrm{mmol}, 2.60$ equiv) was added and the mixture was stirred for another $1 \mathrm{~h}$. Then, the mixture was allowed to reach room temperature and stirred overnight. A saturated aqueous solution of $\mathrm{NH}_{4} \mathrm{Cl}$ was added and the phases were separated. The aqueous phase was extracted with $\mathrm{CH}_{2} \mathrm{Cl}_{2}$. The combined organic phases were dried $\left(\mathrm{MgSO}_{4}\right)$, filtered and the solvent was removed under reduced pressure. Purification of the crude product by column chromatography on silica gel ( $n$-pentane: $\left.\mathrm{Et}_{2} \mathrm{O}=10: 1\right)$ furnished the title formamide as yellow oil $(3.45 \mathrm{~g}, 27.1 \mathrm{mmol}, 91 \%$ ).

M $\left(\mathrm{C}_{11} \mathrm{H}_{14} \mathrm{O}\right)=162.23 \mathrm{~g} / \mathrm{mol} ;{ }^{1} \mathbf{H}$ NMR $\left(300 \mathrm{MHz}, \mathrm{CDCl}_{3}\right) \delta[\mathrm{ppm}]=9.98(\mathrm{~s}, 1 \mathrm{H}), 7.82(\mathrm{~d}$, $J=8.4 \mathrm{~Hz}, \quad 2 \mathrm{H}), \quad 7.55 \quad(\mathrm{~d}, \quad J=8.4 \mathrm{~Hz}, \quad 2 \mathrm{H}), \quad 1.35 \quad(\mathrm{~s}, \quad 9 \mathrm{H}) ; \quad{ }^{13} \mathrm{C} \mathrm{NMR} \quad(75 \mathrm{MHz}$, $\left.\mathrm{CDCl}_{3}\right) \delta[\mathrm{ppm}]=192.20,158.57,134.17,129.82,126.11,35.47,31.18$.

The NMR data is in agreement with reported data. ${ }^{26]}$

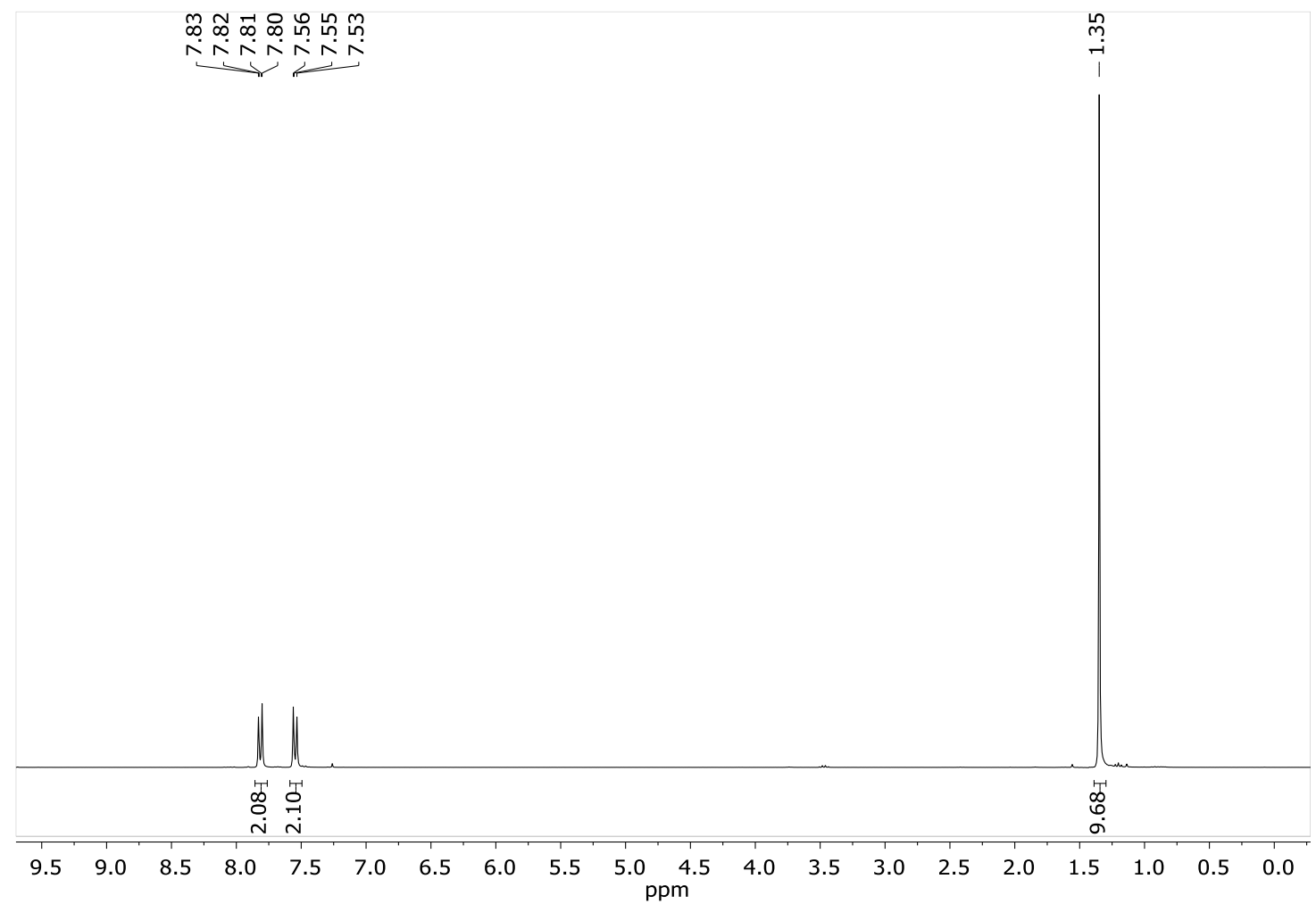

${ }^{1} \mathrm{H}$ NMR spectrum of 4-(tert-butyl)benzaldehyde (300 $\left.\mathrm{MHz}, \mathrm{CDCl}_{3}\right)$. 


\begin{tabular}{|c|c|c|c|c|}
\hline 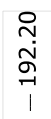 & 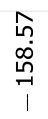 & 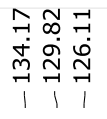 & 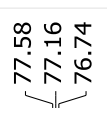 & 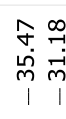 \\
\hline
\end{tabular}

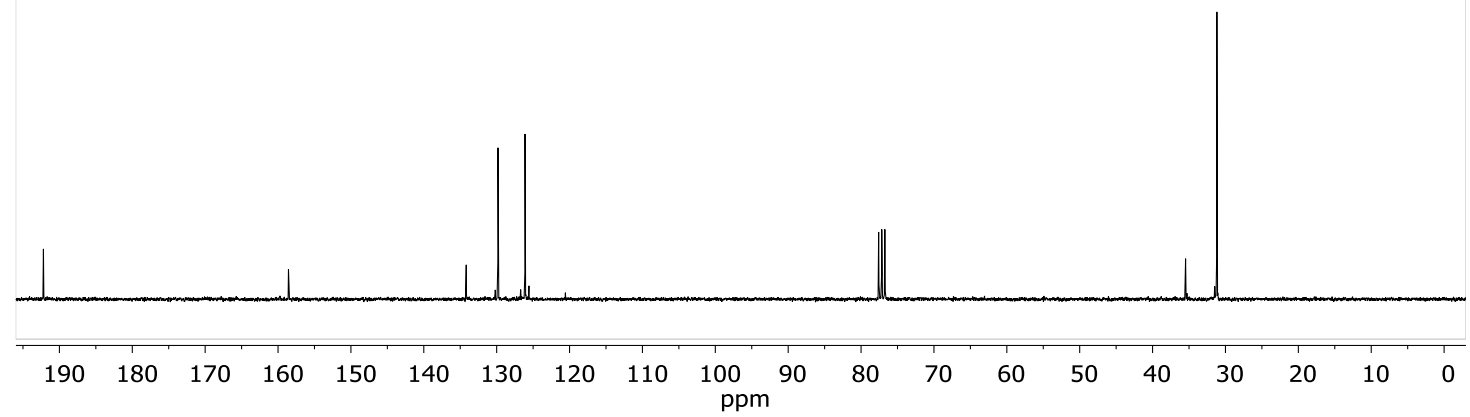

${ }^{13} \mathrm{C}$ NMR spectrum of 4 -(tert-butyl)benzaldehyde $\left(75 \mathrm{MHz}, \mathrm{CDCl}_{3}\right)$.

\subsubsection{Synthesis of 4-(tert-Butyl)phenyl)methanol (1I)}<smiles>CC(C)(C)c1ccc(CO)cc1</smiles>

According to a procedure of Weaver, ${ }^{[27]}$ under argon atmosphere 4-(tertbutyl)benzaldehyde ( $2.83 \mathrm{~g}, 17.4 \mathrm{mmol}, 1.00$ equiv) was dissolved in abs. EtOH $(35.0 \mathrm{~mL})$ and cooled to $0{ }^{\circ} \mathrm{C}$. $\mathrm{NaBH}_{4}(987 \mathrm{mg}, 26.1 \mathrm{mmol}$, 1.50 equiv) was added in one portion and the reaction mixture was stirred at room temperature for $45 \mathrm{~min}$. An aqueous solution of $\mathrm{NaOH}(2.0 \mathrm{M})$ was added, the phases were separated, and the aqueous phase was extracted with $\mathrm{CH}_{2} \mathrm{Cl}_{2}$. Then, the combined organic phases were washed with a saturated aqueous solution of $\mathrm{NaHCO}_{3}$, dried $\left(\mathrm{MgSO}_{4}\right)$ and filtered. After removal of the solvent the crude product was used without further purification. The title product was obtained as pale-yellow oil (2.30 g, $14.0 \mathrm{mmol}, 93 \%)$.

M $\left(\mathrm{C}_{11} \mathrm{H}_{16} \mathrm{O}\right)=164.25 \mathrm{~g} / \mathrm{mol} ;{ }^{1} \mathbf{H}$ NMR $\left(300 \mathrm{MHz}, \mathrm{CDCl}_{3}\right) \delta[\mathrm{ppm}]=7.41(\mathrm{~d}, J=8.4 \mathrm{~Hz}, 2 \mathrm{H})$, $7.31(\mathrm{~d}, J=8.4 \mathrm{~Hz}, 2 \mathrm{H}), 4.66(\mathrm{~s}, 2 \mathrm{H}), 1.81(\mathrm{~s}, 1 \mathrm{H}), 1.33(\mathrm{~s}, 9 \mathrm{H},) ;{ }^{13} \mathrm{C} \mathrm{NMR}(75 \mathrm{MHz}$, $\left.\mathrm{CDCl}_{3}\right) \delta[\mathrm{ppm}]=150.83,138.05,127.03,125.62,65.28,34.67,31.47$.

The NMR data are in agreement with reported data. ${ }^{[28]}$ 


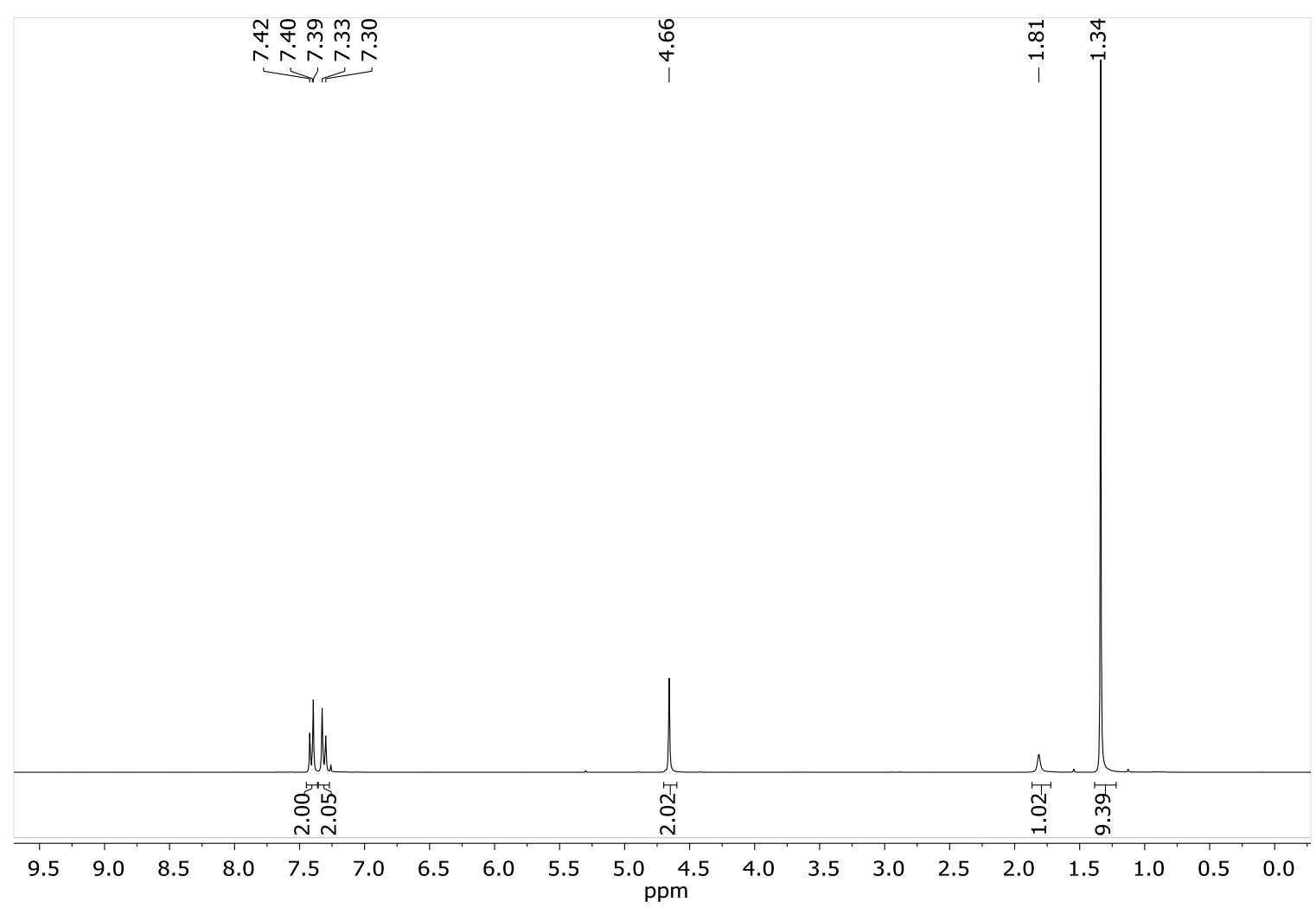

${ }^{1} \mathrm{H}$ NMR spectrum of 4-(tert-butyl)phenyl)methanol (300 MHz, $\left.\mathrm{CDCl}_{3}\right)$.

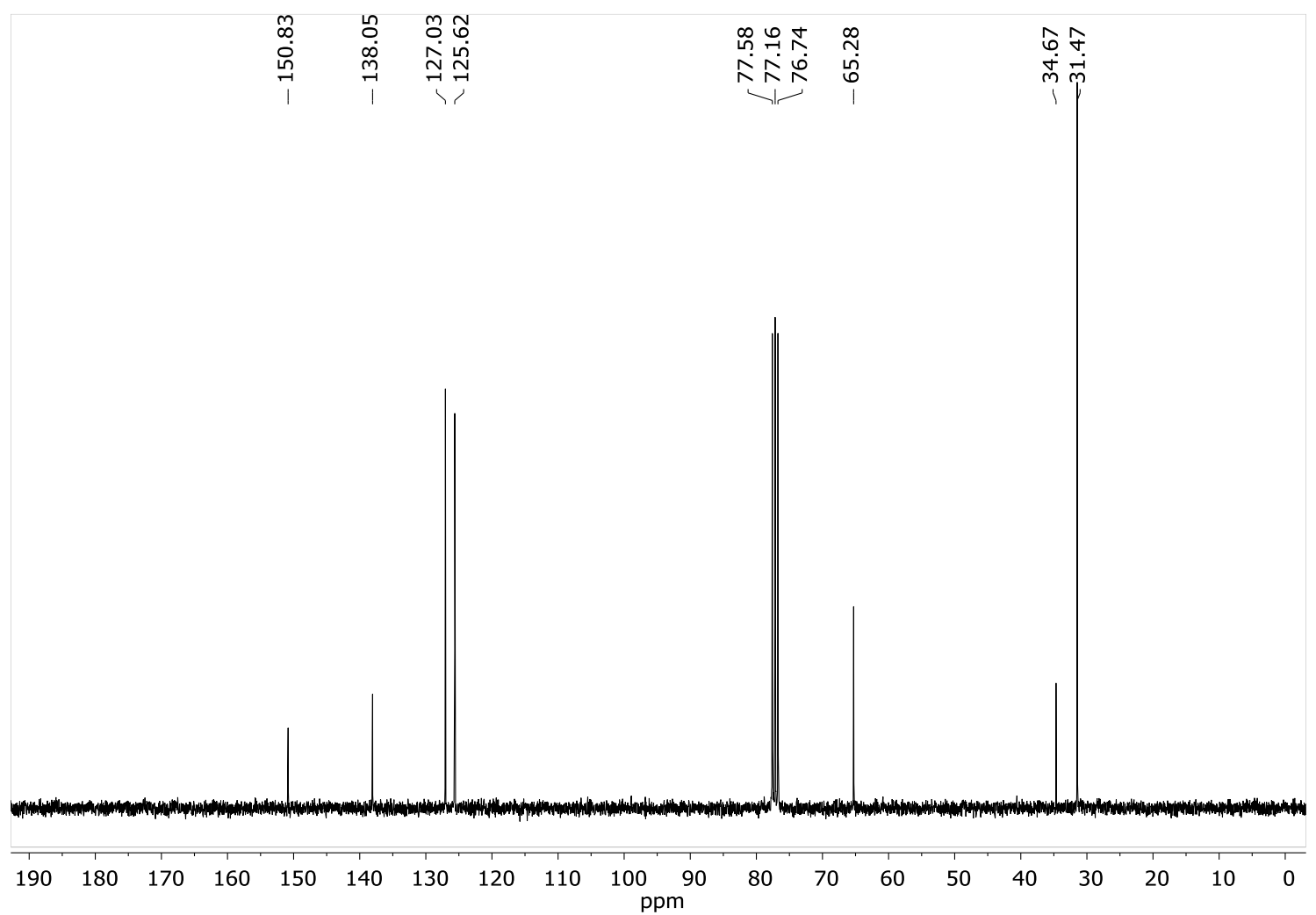

${ }^{13} \mathrm{C}$ NMR spectrum of 4-(tert-butyl)phenyl)methanol (75 MHz, $\mathrm{CDCl}_{3}$ ). 


\subsubsection{General Procedure for the Kinetic Analysis of Formamide Catalyzed}

\section{Nucleophilic Substitution}<smiles>CC(C)(C)c1ccc(CO)cc1</smiles>

(4-(tert-Butyl)phenyl)methanol $(1.00 \mathrm{mmol}$ or $0.50 \mathrm{mmol}$, 1.00 equiv), mesitylene $(1.00 \mathrm{mmol}$ or $0.50 \mathrm{mmol}, 1.00$ equiv) as internal standard and the corresponding formamide catalyst $(100 \mu \mathrm{mol}$ or $50.0 \mu \mathrm{mol}, 10 \mathrm{~mol} \%)$ are dissolved in 1,4-dioxane $(2.0 \mathrm{M})$. To ensure the accuracy of the catalyst loading, liquid formamides are added as stock solution $(1.0 \mathrm{M}$ in 1,4-dioxane) by using an Eppendorf pipette and solid formamides are weighted as solid. Finally, $\mathrm{BzCl}(0.20 \mathrm{mmol}$ or $0.60 \mathrm{mmol}, 1.00$ equiv) are added by means of an Eppendorf pipette. The course of the reaction is monitored by GC analysis. During the first $60 \mathrm{~min}$, an aliquot of the reaction mixture is withdrawn every $5 \mathrm{~min}$ and filtered through a small amount of basic aluminum oxide in a disposable Pasteur pipette (MTBE). The prepared probe is measured by GC analysis. Then, after $70 \mathrm{~min}, 80 \mathrm{~min}, 90 \mathrm{~min}, 105 \mathrm{~min}, 120 \mathrm{~min}, 3 \mathrm{~h}, 4 \mathrm{~h}, 6 \mathrm{~h}$, $8 \mathrm{~h}, 10 \mathrm{~h}$, and after $24 \mathrm{~h}$ an aliquot of the reaction is withdrawn and prepared for GC analysis. 


\subsubsection{Kinetic Measurements with $\mathbf{N}, \mathbf{N}$-Dimethylformamide (DMF)}

o (4-(tert-Butyl)phenyl)methanol (164 mg, $1.00 \mathrm{mmol}, 1.00$ equiv), $N, N$-dimethyl$\mathrm{H}_{\mathrm{N}}$ - formamide $(1.0 \mathrm{M}$ in 1,4-dioxane, $100 \mu \mathrm{L}, 0.10 \mathrm{mmol}, 10 \mathrm{~mol} \%)$, mesitylene (138 $\mu \mathrm{L}, 1.00 \mathrm{mmol}, 1.00$ equiv) and $\mathrm{BzCl}(139 \mu \mathrm{L}, 1.20 \mathrm{mmol}, 1.20$ equiv) in 1,4-dioxane $(0.50 \mathrm{~mL})$ were reacted according to general procedure 7 (chapter 2.5.3, page 114).

Table S9. Determination of $k_{\text {start }}$ and $k_{\text {total. }}$.

\begin{tabular}{llll} 
Measurement & $\boldsymbol{K}_{\text {start }} / \mathbf{1 0}^{\mathbf{- 3}} \mathbf{~ m i n}^{-\mathbf{1}}$ & $\boldsymbol{k}_{\text {total }} / \mathbf{1 0}^{\mathbf{- 3}} \mathbf{~ m i n}^{\mathbf{- 1}}$ & Induction Period / $\mathbf{~ i n ~}$ \\
\hline 1 & 4.69 & 4.25 & 20 \\
2 & 4.63 & 4.38 & 20 \\
$\varnothing$ & 4.66 & 4.32 & -
\end{tabular}
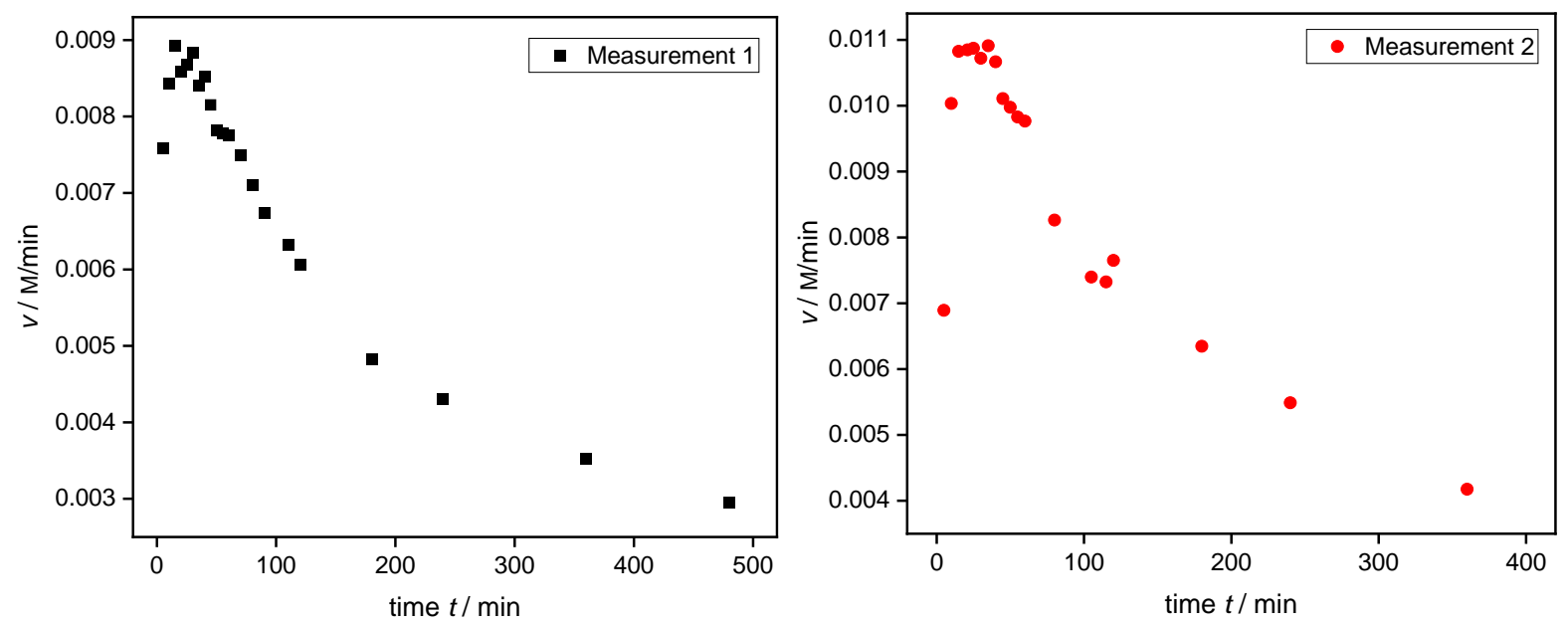

Figure S11. Reaction profiles plotting reaction rate $v$ versus time $t$.
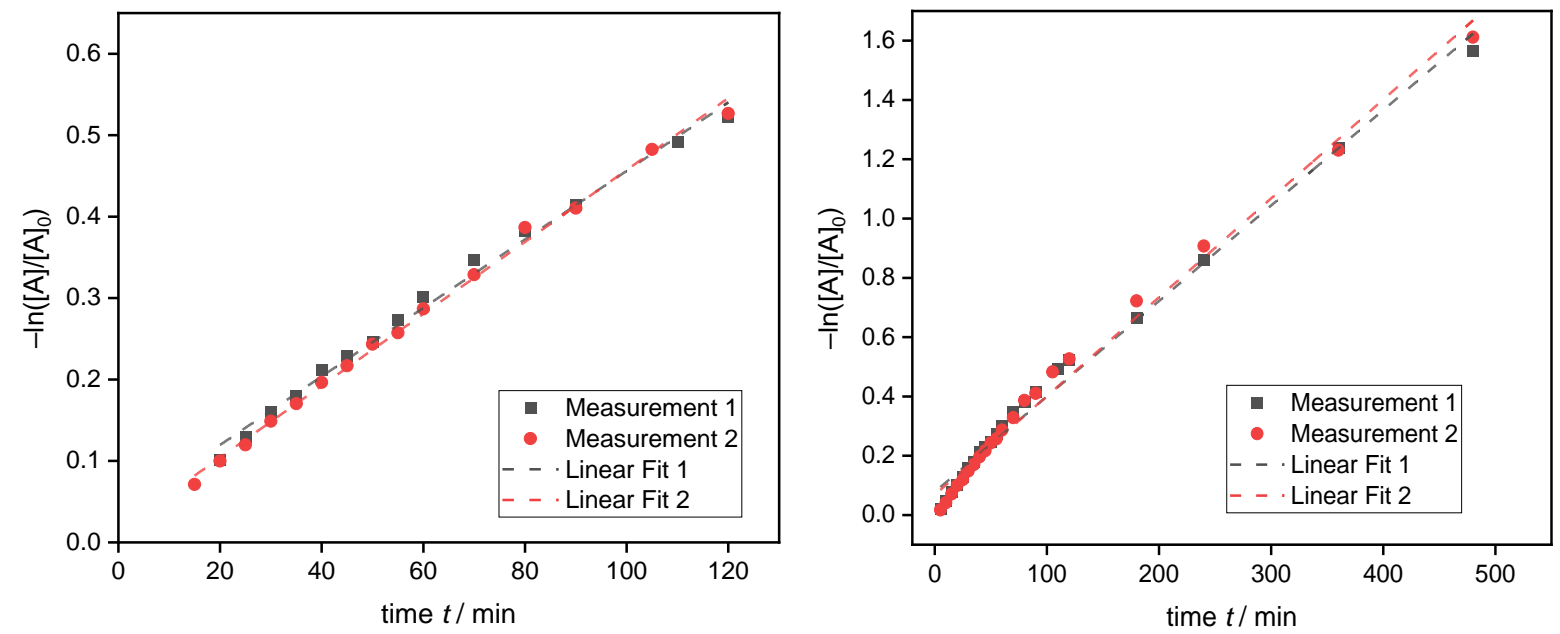

Figure S12. Logarithmic plots to determine $k_{\text {start }}\left(\right.$ left) and $k_{\text {total }}$ (right). 


\subsubsection{Kinetic Measurements with N-Formylpyrrolidine (FPyr)}<smiles>O=C1CC2CCC1C2</smiles>
(4-(tert-Butyl)phenyl)methanol

(164 mg,

$1.00 \mathrm{mmol}$, 1.00 equiv), $N$-formylpyrrolidine (1.0 $\mathrm{M}$ in 1,4-dioxane, $100 \mu \mathrm{L}, 0.10 \mathrm{mmol}, 10 \mathrm{~mol} \%)$, mesitylene $(138 \mu \mathrm{L}, 1.00 \mathrm{mmol}, 1.00$ equiv) and $\mathrm{BzCl}(139 \mu \mathrm{L}, 1.20 \mathrm{mmol}$, 1.20 equiv) in 1,4-dioxane $(0.50 \mathrm{~mL})$ were reacted according to general procedure 7 (chapter 2.5.3, page 114).

Table S10. Determination of $k_{\text {start }}$ and $k_{\text {total }}$.

\begin{tabular}{llll} 
Measurement & $\boldsymbol{k}_{\text {start }} / \mathbf{1 0}^{\mathbf{- 3}} \mathbf{~ m i n}^{\mathbf{- 1}}$ & $\boldsymbol{k}_{\text {total }} / \mathbf{1 0}^{\mathbf{- 3}} \mathbf{~ m i n}^{\mathbf{- 1}}$ & Induction Period $/ \mathbf{~} \mathbf{~ i n}$ \\
\hline 1 & 5.83 & 5.06 & 15 \\
2 & 6.06 & 4.78 & 25 \\
$\varnothing$ & 5.95 & 4.92 & -
\end{tabular}
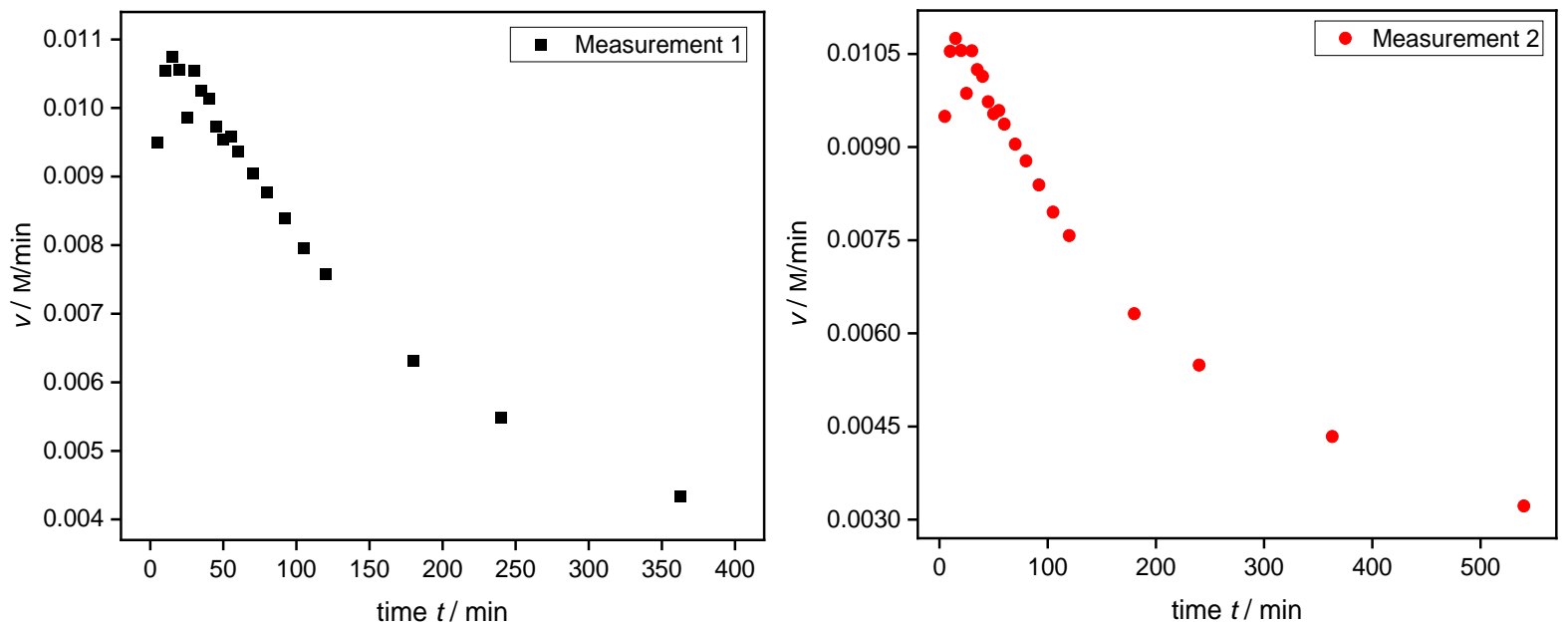

Figure S13. Reaction profiles plotting reaction rate $v$ versus time $t$.
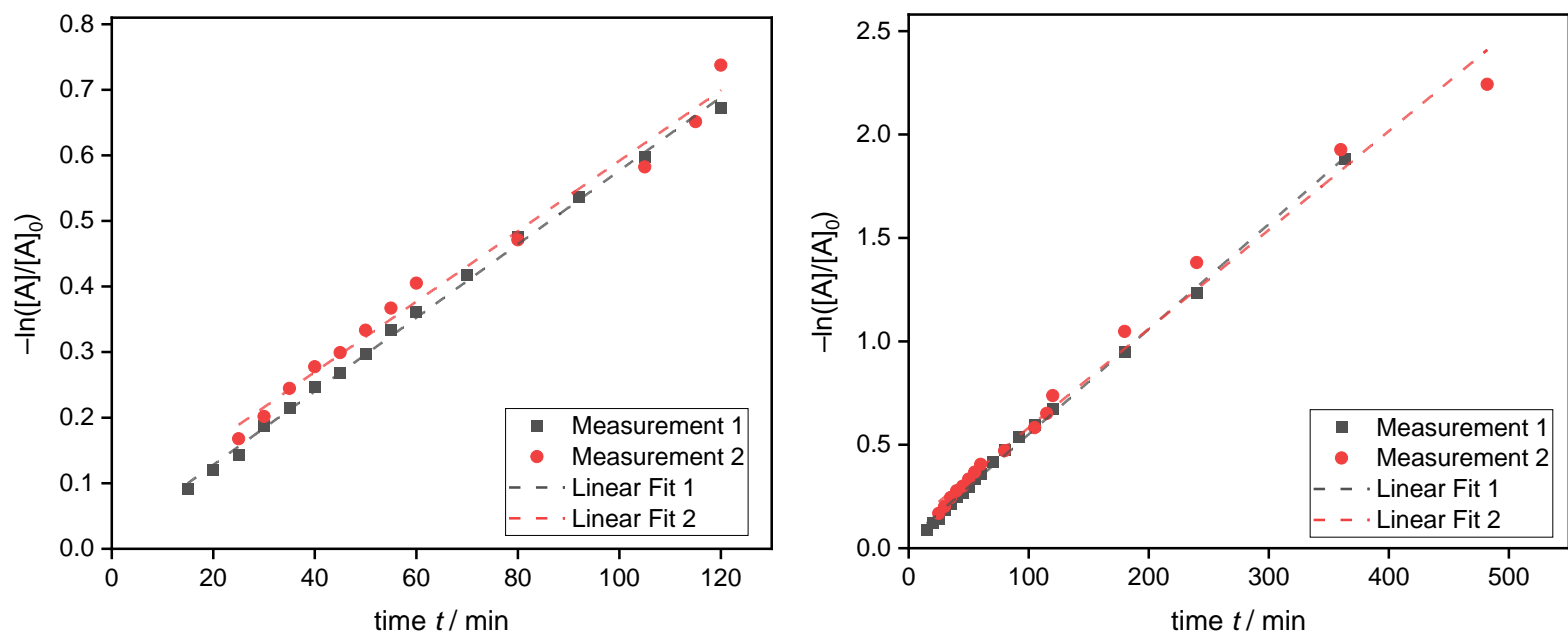

Figure S14. Logarithmic plots to determine $k_{\text {start }}$ (left) and $k_{\text {total }}$ (right). 


\subsubsection{Kinetic Measurements with Morpholine-4-carbaldehyde (FMor)}<smiles>O=C1CC2CCCCOC1C2</smiles>

(4-(tert-Butyl)phenyl)methanol (164 mg, $1.00 \mathrm{mmol}, 1.00$ equiv), morpholine-4carbaldehyde (1.0 M in 1,4-dioxane, $100 \mu \mathrm{L}, 0.10 \mathrm{mmol}, 10 \mathrm{~mol} \%$ ), mesitylene (138 $\mu \mathrm{L}, 1.00 \mathrm{mmol}, 1.00$ equiv) and $\mathrm{BzCl}(139 \mu \mathrm{L}, 1.20 \mathrm{mmol}, 1.20$ equiv) in 1,4-dioxane $(0.50 \mathrm{~mL})$ were reacted according to general procedure 7 (chapter 2.5.3, page 114).

Table S11. Determination of $k_{\text {start }}$ and $k_{\text {total }}$.

\begin{tabular}{llll} 
Measurement & $\boldsymbol{k}_{\text {start }} / \mathbf{1 0}^{\mathbf{- 3}} \mathbf{~ m i n}^{-\mathbf{1}}$ & $\boldsymbol{k}_{\text {total }} / \mathbf{1 0}^{\mathbf{- 3}} \mathbf{~ m i n}^{-\mathbf{1}}$ & Induction Period / $\mathbf{~ i n ~}$ \\
\hline 1 & 2.71 & 2.23 & 35 \\
2 & 2.36 & 2.16 & 45 \\
$\varnothing$ & 2.54 & 2.20 & -
\end{tabular}
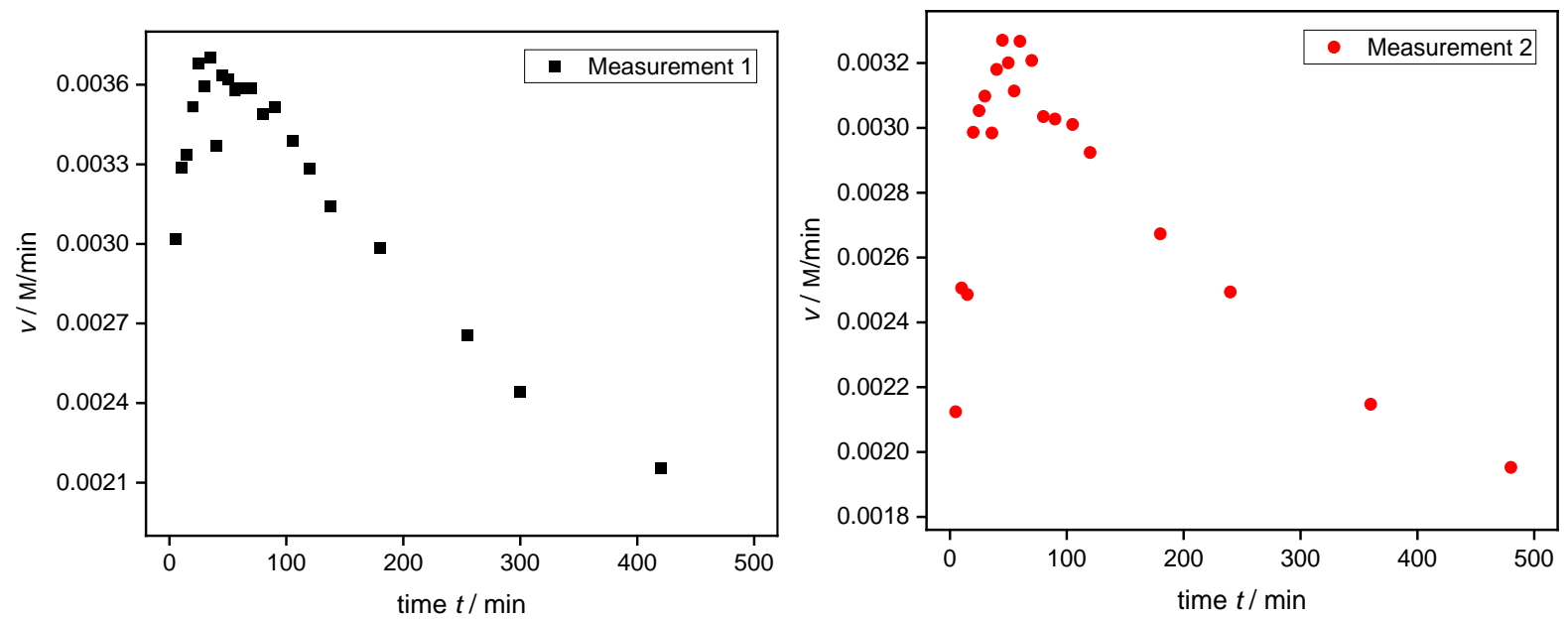

Figure S15. Reaction profiles plotting reaction rate $v$ versus time $t$.
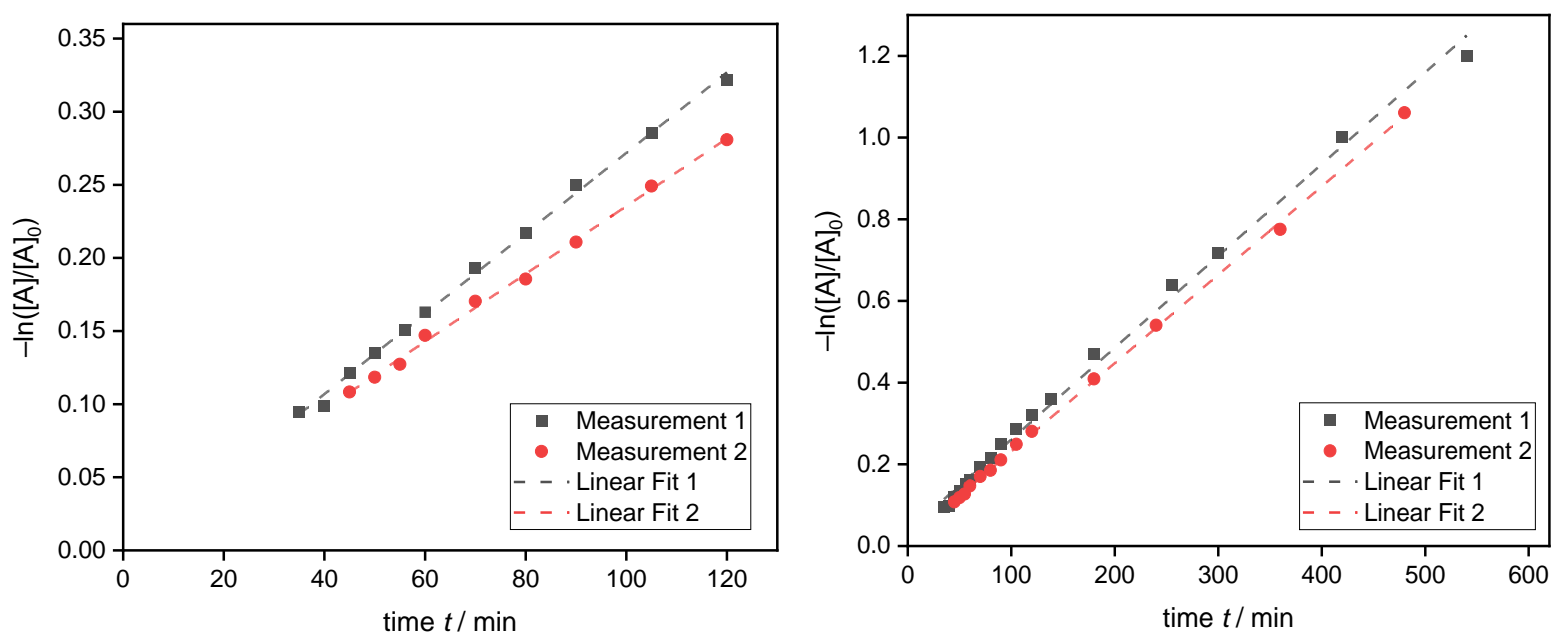

Figure S16. Logarithmic plots to determine $k_{\text {start }}$ (left) and $k_{\text {total }}$ (right). 


\subsubsection{Kinetic Measurements with Piperidine-1-carbaldehyde (FPip)}<smiles>O=C1CC2CCCC1C2</smiles>

(4-(tert-Butyl)phenyl)methanol (164 mg, $1.00 \mathrm{mmol}, 1.00$ equiv), piperidine-1carbaldehyde (1.0 M in 1,4-dioxane, $100 \mu \mathrm{L}, 0.10 \mathrm{mmol}, 10 \mathrm{~mol} \%)$, mesitylene (138 $\mu \mathrm{L}, 1.00 \mathrm{mmol}, 1.00$ equiv) and $\mathrm{BzCl}(139 \mu \mathrm{L}, 1.20 \mathrm{mmol}, 1.20$ equiv) in 1,4-dioxane $(0.50 \mathrm{~mL})$ were reacted according to general procedure 7 (chapter

\subsection{3, page 114)}

Table S12. Determination of $k_{\text {start }}$ and $k_{\text {total. }}$.

\begin{tabular}{llll} 
Measurement & $\boldsymbol{k}_{\text {start }} / \mathbf{1 0}^{\mathbf{- 3}} \mathbf{~} \mathbf{n}^{\mathbf{- 1}}$ & $\boldsymbol{k}_{\text {total }} / \mathbf{1 0}^{-\mathbf{3}} \mathbf{~ m i n}^{\mathbf{- 1}}$ & Induction Period / $\mathbf{~ i n ~}$ \\
\hline 1 & 5.99 & 3.70 & 20 \\
2 & 5.16 & 3.49 & 20 \\
$\varnothing$ & 5.58 & 3.60 & -
\end{tabular}
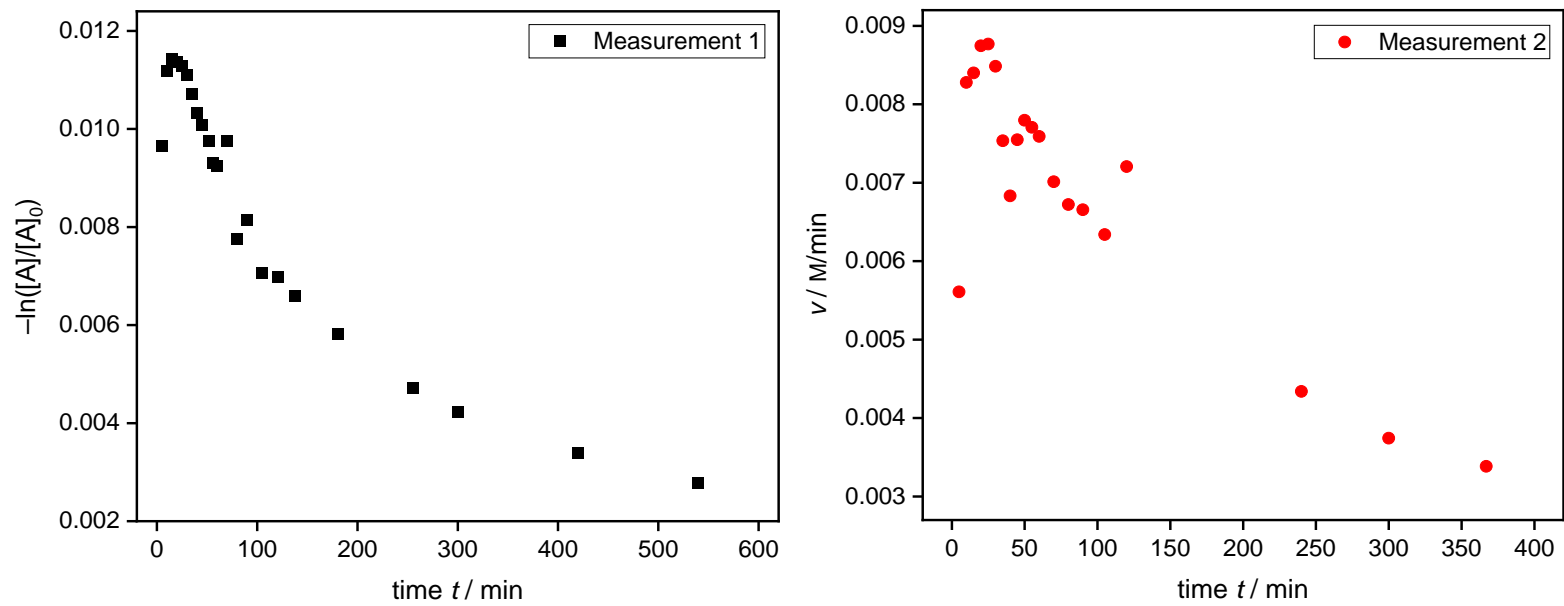

Figure S17. Reaction profiles plotting reaction rate $v$ versus time $t$.
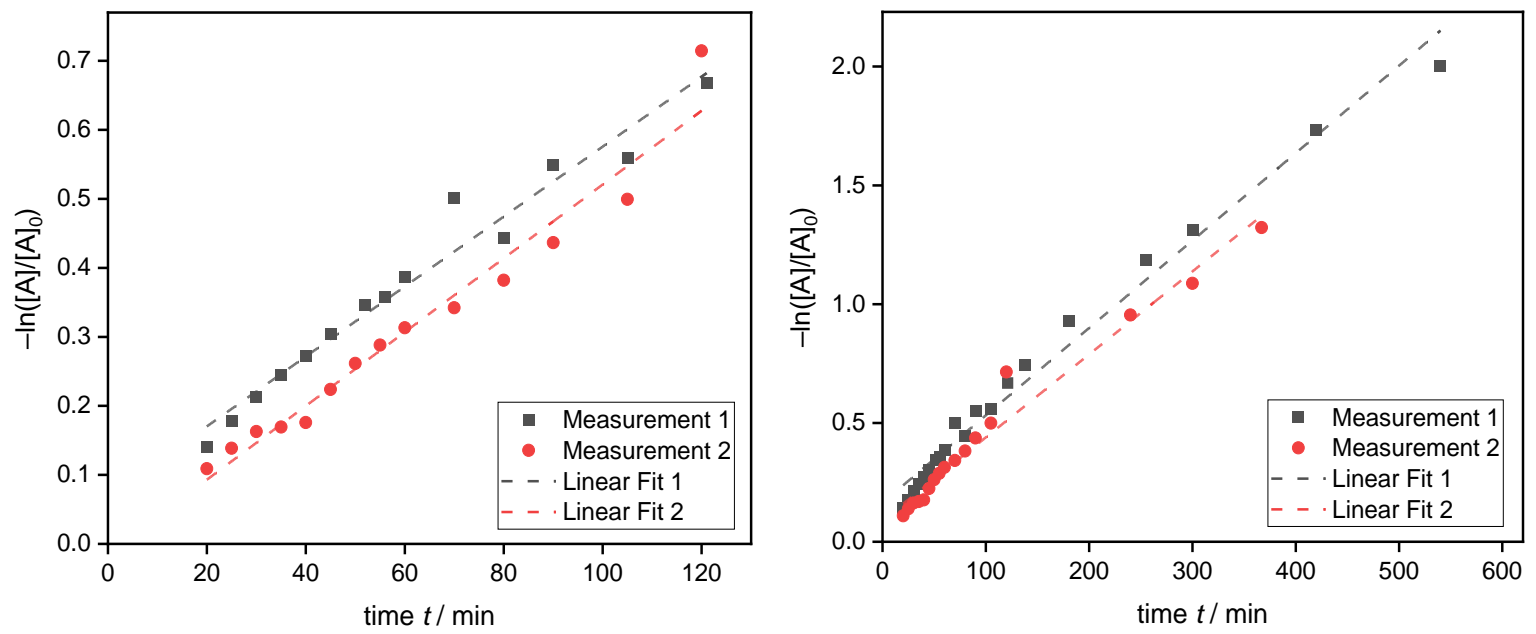

Figure S18. Logarithmic plots to determine $k_{\text {start }}\left(\right.$ left) and $k_{\text {total }}$ (right). 


\subsubsection{Kinetic Measurements with $N$-tert-Butylformamide (tBuF)}

o (4-(tert-Butyl)phenyl)methanol (164 mg, $1.00 \mathrm{mmol}, 1.00$ equiv), $\quad \mathrm{N}$-tert$\mathrm{H}_{\mathrm{H}}{ }^{-t \mathrm{Bu}}$ butylformamide $(1.0 \mathrm{M}$ in 1,4-dioxane, $100 \mu \mathrm{L}, 0.10 \mathrm{mmol}, 10 \mathrm{~mol} \%)$, mesitylene 1,4-dioxane $(0.50 \mathrm{~mL})$ were reacted according to general procedure 7 (chapter 2.5.3, page 114).

Table S13. Determination of $k_{\text {start }}$ and $k_{\text {total. }}$

\begin{tabular}{llll} 
Measurement & $\boldsymbol{k}_{\text {start }} / \mathbf{1 0}^{\mathbf{- 3}} \mathbf{~ m i n}^{\mathbf{- 1}}$ & $\boldsymbol{k}_{\text {total }} / \mathbf{1 0}^{-\mathbf{3}} \mathbf{~ m i n}^{-\mathbf{1}}$ & Induction Period $/ \mathbf{~} \mathbf{m i n}$ \\
\hline 1 & 4.83 & 4.74 & 20 \\
2 & 4.50 & 3.76 & 20 \\
$\varnothing$ & 4.66 & 5.25 & -
\end{tabular}
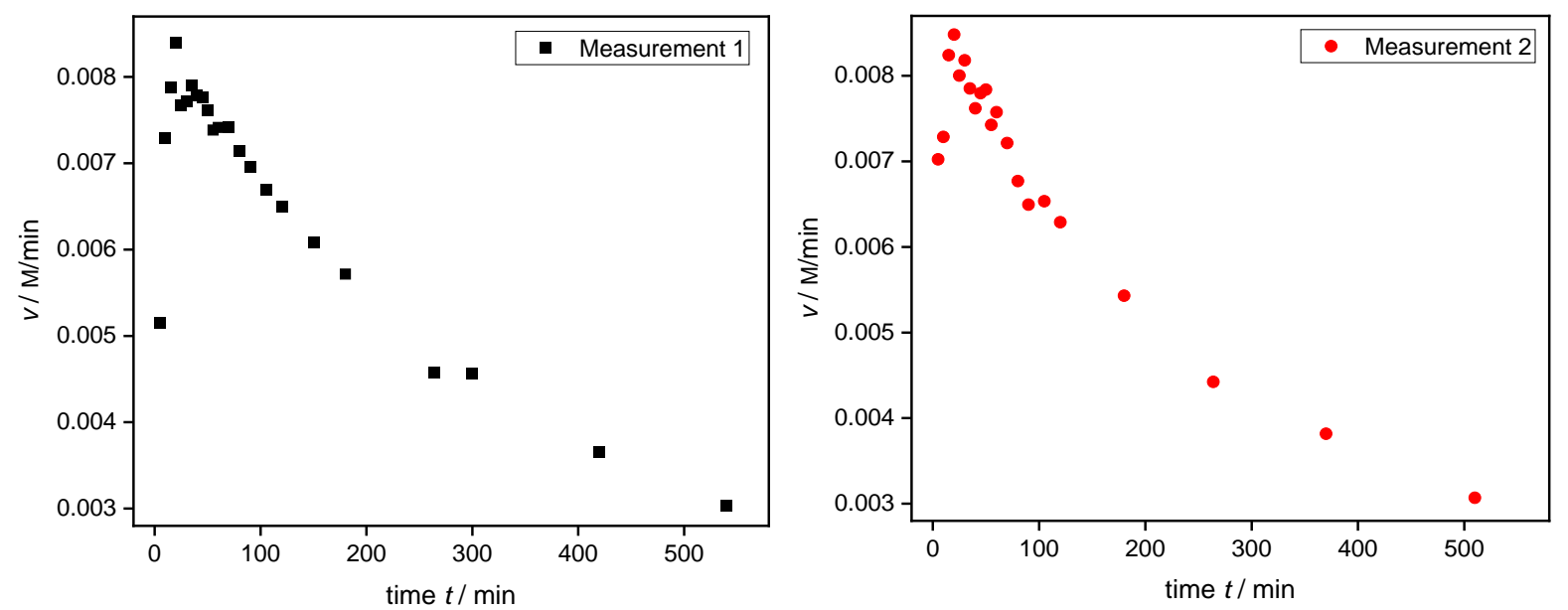

Figure S19. Reaction profiles plotting reaction rate $v$ versus time $t$.
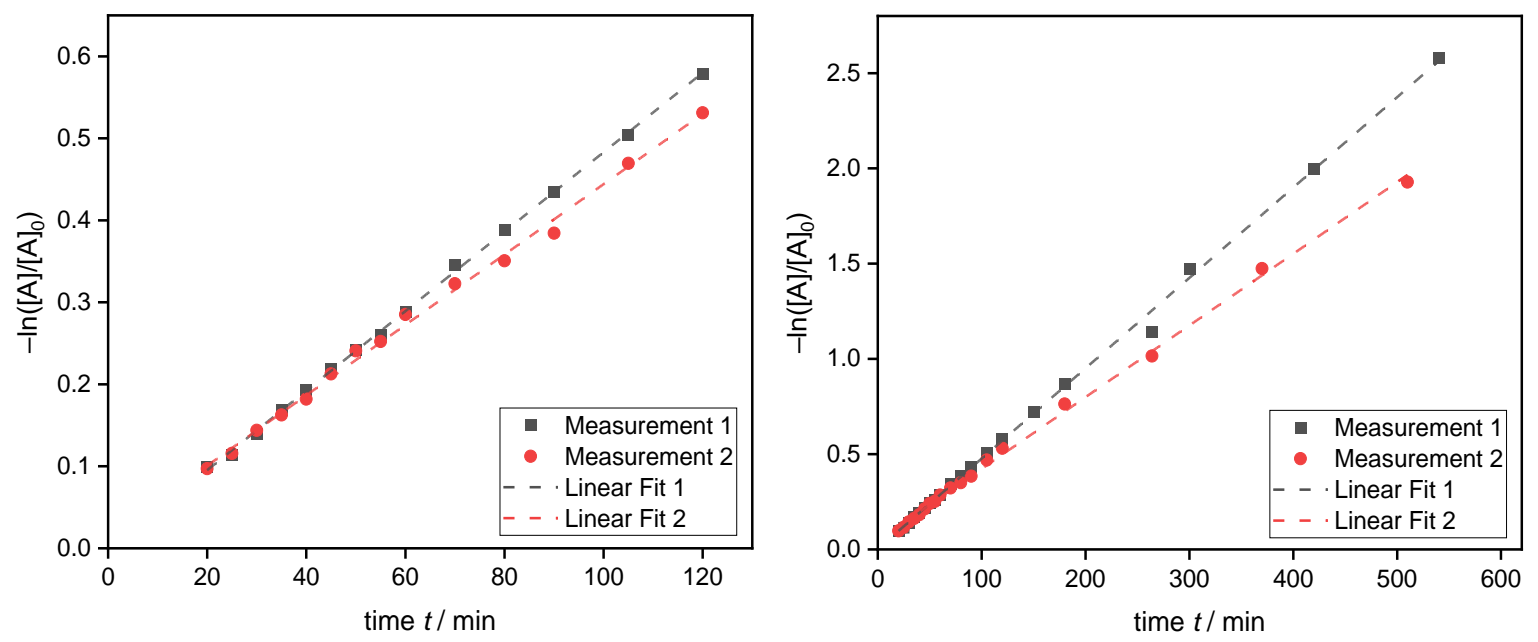

Figure S20. Logarithmic plots to determine $k_{\text {start }}$ (left) and $k_{\text {total }}$ (right). 


\subsubsection{Kinetic Measurements with $\mathrm{N}$-Benzylformamide (BnF)}<smiles>O=CNCc1ccccc1</smiles>

(4-(tert-Butyl)phenyl)methanol (164 mg, $1.00 \mathrm{mmol}, \quad 1.00$ equiv), $\mathrm{N}$-benzylformamide (1.0 M in 1,4-dioxane, $100 \mu \mathrm{L}, 0.10 \mathrm{mmol}, 10 \mathrm{~mol} \%$ ), mesitylene (138 $\mu \mathrm{L}, 1.00 \mathrm{mmol}, 1.00$ equiv) and $\mathrm{BzCl}(139 \mu \mathrm{L}, 1.20 \mathrm{mmol}$, 1.20 equiv) in 1,4-dioxane $(0.50 \mathrm{~mL})$ were reacted according the general procedure 7 (chapter 2.5.3, page 114).

Table S14. Determination of $k_{\text {start }}$ and $k_{\text {total }}$.

\begin{tabular}{llll} 
Measurement & $\boldsymbol{k}_{\text {start }} / \mathbf{1 0}^{\mathbf{- 3}} \mathbf{~} \mathbf{i n}^{\mathbf{- 1}}$ & $\boldsymbol{k}_{\text {total }} / \mathbf{1 0}^{\mathbf{- 3}} \mathbf{~} \mathbf{n i n}^{\mathbf{- 1}}$ & Induction Period / $\mathbf{~ i n ~}$ \\
\hline 1 & 3.63 & 3.42 & 45 \\
2 & 3.81 & 3.00 & 40 \\
$\varnothing$ & 3.72 & 3.21 & -
\end{tabular}
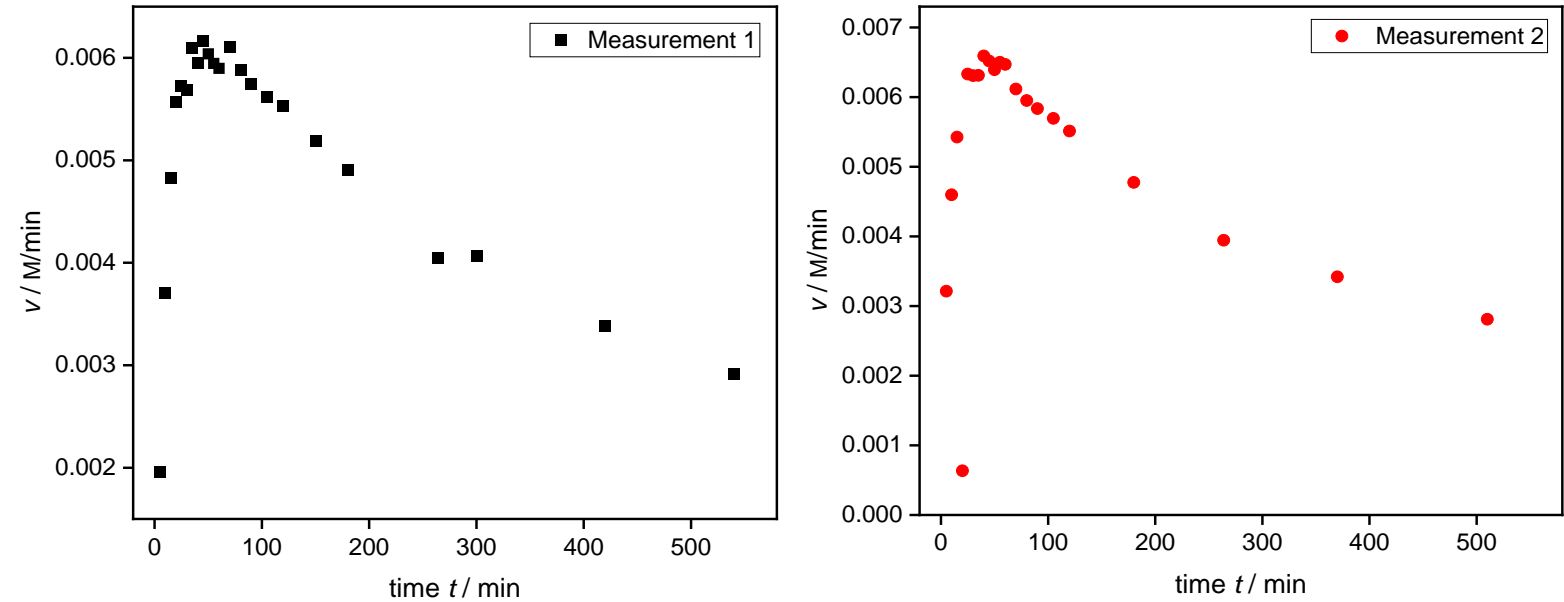

Figure S21. Reaction profiles plotting reaction rate $v$ versus time $t$.
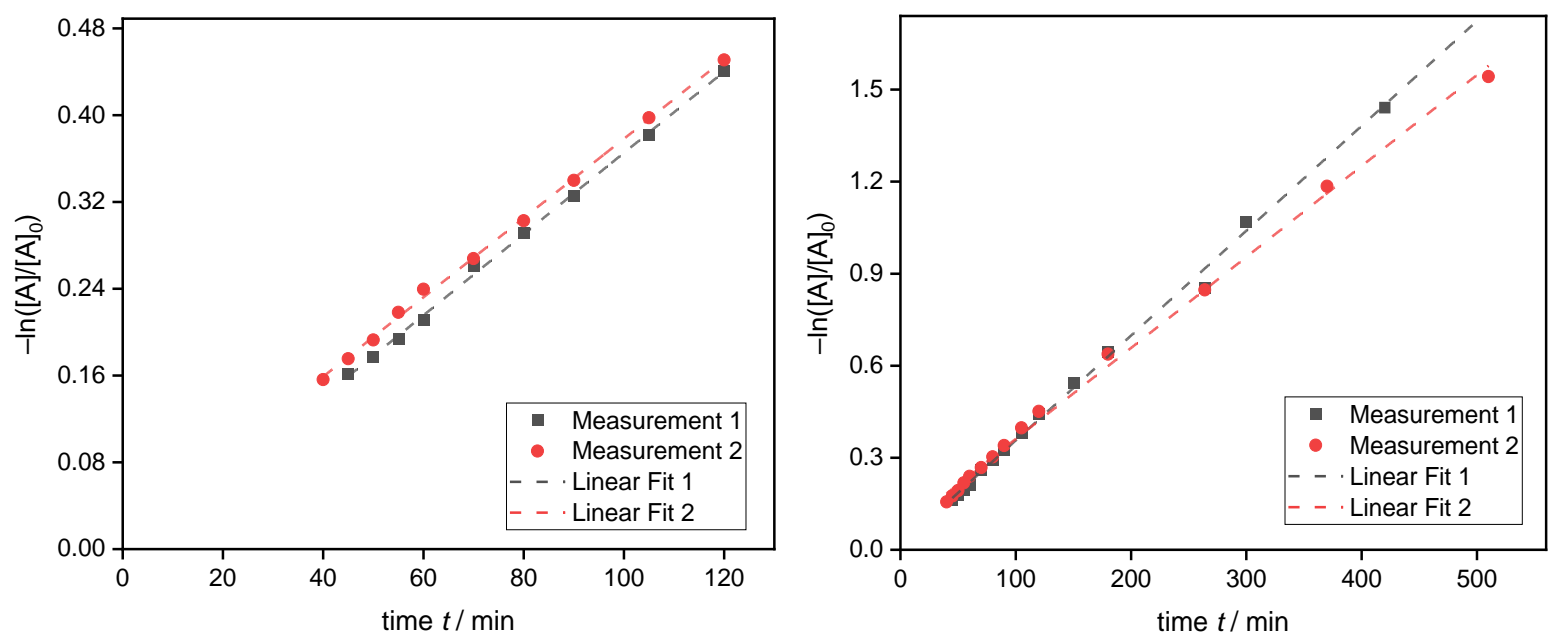

Figure S22. Logarithmic plots to determine $k_{\text {start }}$ (left) and $k_{\text {total }}$ (right). 


\subsubsection{Kinetic Measurements with Piperazine-1,4-dicarbaldehyde (DFPiper)}

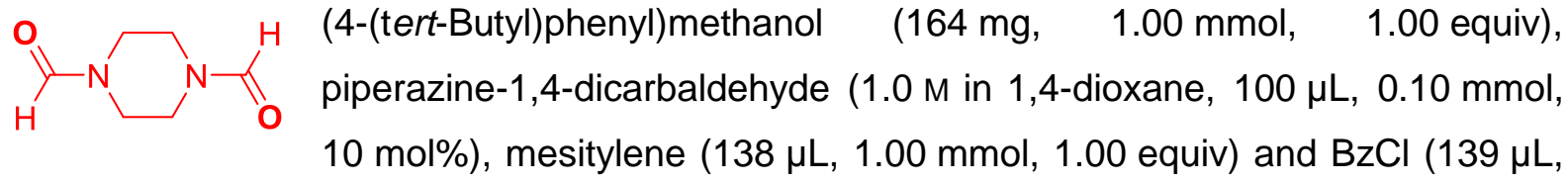
$1.20 \mathrm{mmol}, 1.20$ equiv) in 1,4-dioxane $(0.50 \mathrm{~mL})$ were reacted according to general procedure 7 (chapter 2.5.3, page 114).

Table S15. Determination of $k_{\text {start }}$ and $k_{\text {total }}$.

\begin{tabular}{llll} 
Measurement & $\boldsymbol{k}_{\text {start }} / \mathbf{1 0}^{\mathbf{- 3}} \mathbf{~ m i n}^{-\mathbf{1}}$ & $\boldsymbol{k}_{\text {total }} / \mathbf{1 0}^{\mathbf{- 3}} \mathbf{~ m i n}^{-\mathbf{1}}$ & Induction Period / $\mathbf{~ i n ~}$ \\
\hline 1 & 2.24 & 2.38 & 60 \\
2 & 2.80 & 2.93 & 50 \\
$\varnothing$ & 2.52 & 2.66 & -
\end{tabular}
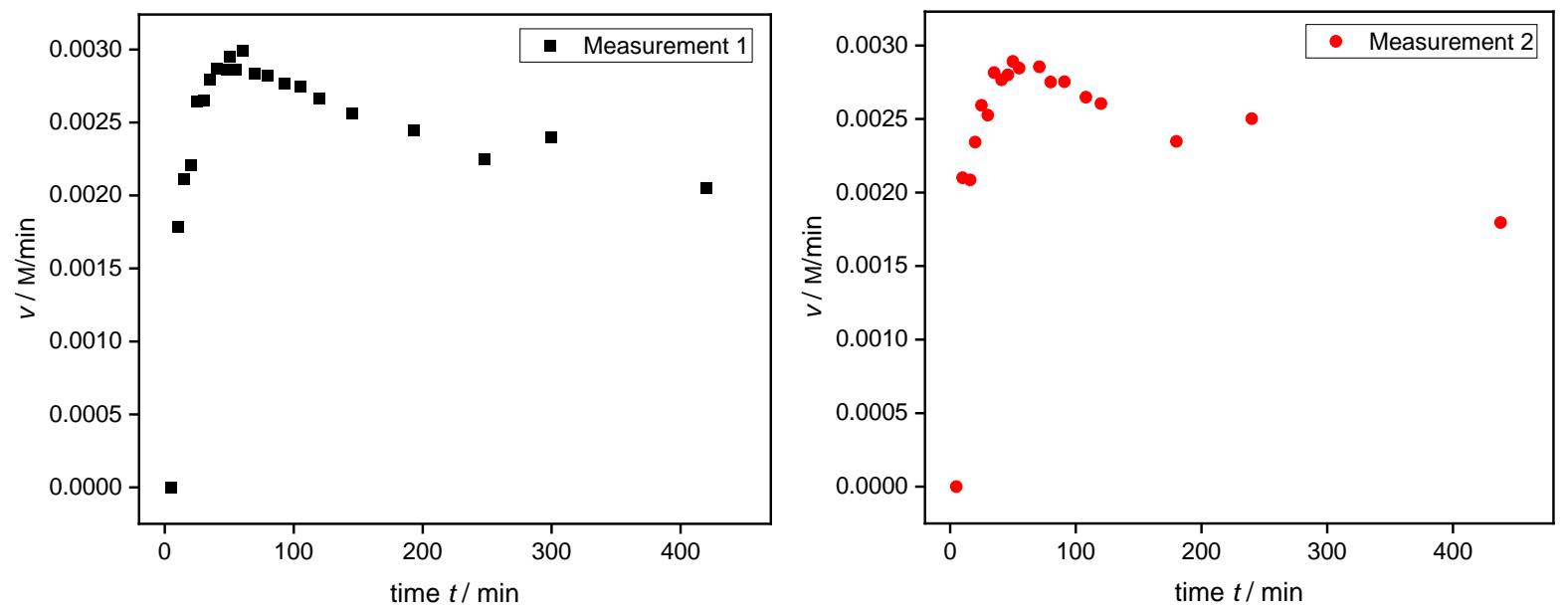

Figure $\mathbf{S}$ 23. Reaction profiles plotting reaction rate $v$ versus time $t$.
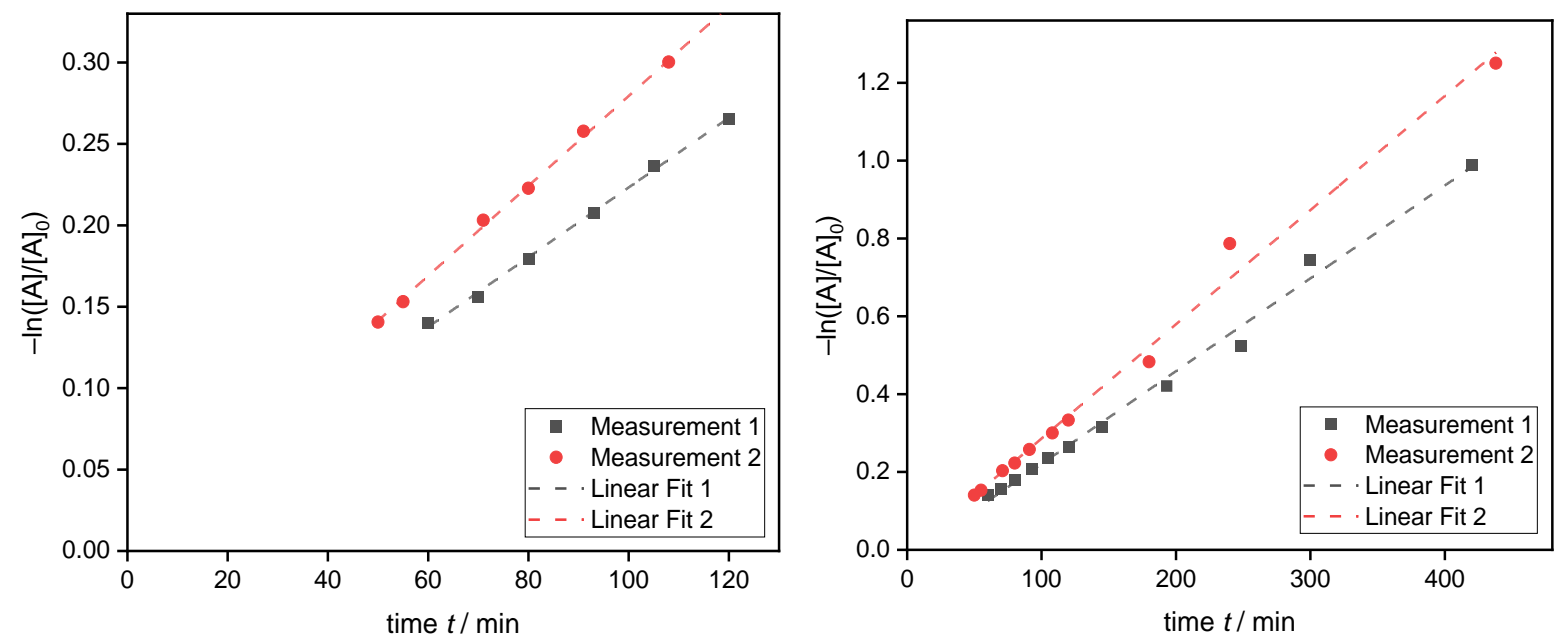

Figure S 24. Logarithmic plots to determine $k_{\text {start }}$ (left) and $k_{\text {total }}$ (right). 


\subsubsection{Kinetic Measurements with $\mathrm{N}$-Methylformamide (MF)}

$\mathrm{H}_{\mathrm{H}^{-}}^{\mathrm{O}}$

(4-(tert-Butyl)phenyl)methanol

(164 mg,

$1.00 \mathrm{mmol}$,

1.00 equiv),

$\mathrm{N}$-Methylformamide $(1.0 \mathrm{M}$ in 1,4-dioxane, $100 \mu \mathrm{L}, 0.10 \mathrm{mmol}, 10 \mathrm{~mol} \%)$, mesitylene (138 $\mu \mathrm{L}, 1.00 \mathrm{mmol}, 1.00$ equiv) and $\mathrm{BzCl}(139 \mu \mathrm{L}, 1.20 \mathrm{mmol}$, 1.20 equiv) in 1,4-dioxane $(0.50 \mathrm{~mL})$ were reacted according to general procedure 7 (chapter 2.5.3, page 114).

Table S16. Determination of $k_{\text {start }}$ and $k_{\text {total }}$.

\begin{tabular}{llll} 
Measurement & $\boldsymbol{k}_{\text {start }} / \mathbf{1 0}^{\mathbf{- 3}} \mathbf{~ m i n}^{\mathbf{- 1}}$ & $\boldsymbol{k}_{\text {total }} / \mathbf{1 0}^{\mathbf{- 3}} \mathbf{~ m i n}^{-\mathbf{1}}$ & Induction Period / $\mathbf{~ i n ~}$ \\
\hline 1 & 6.48 & 8.87 & 80 \\
2 & 6.93 & 7.66 & 90 \\
$\varnothing$ & 6.71 & 8.27 & -
\end{tabular}
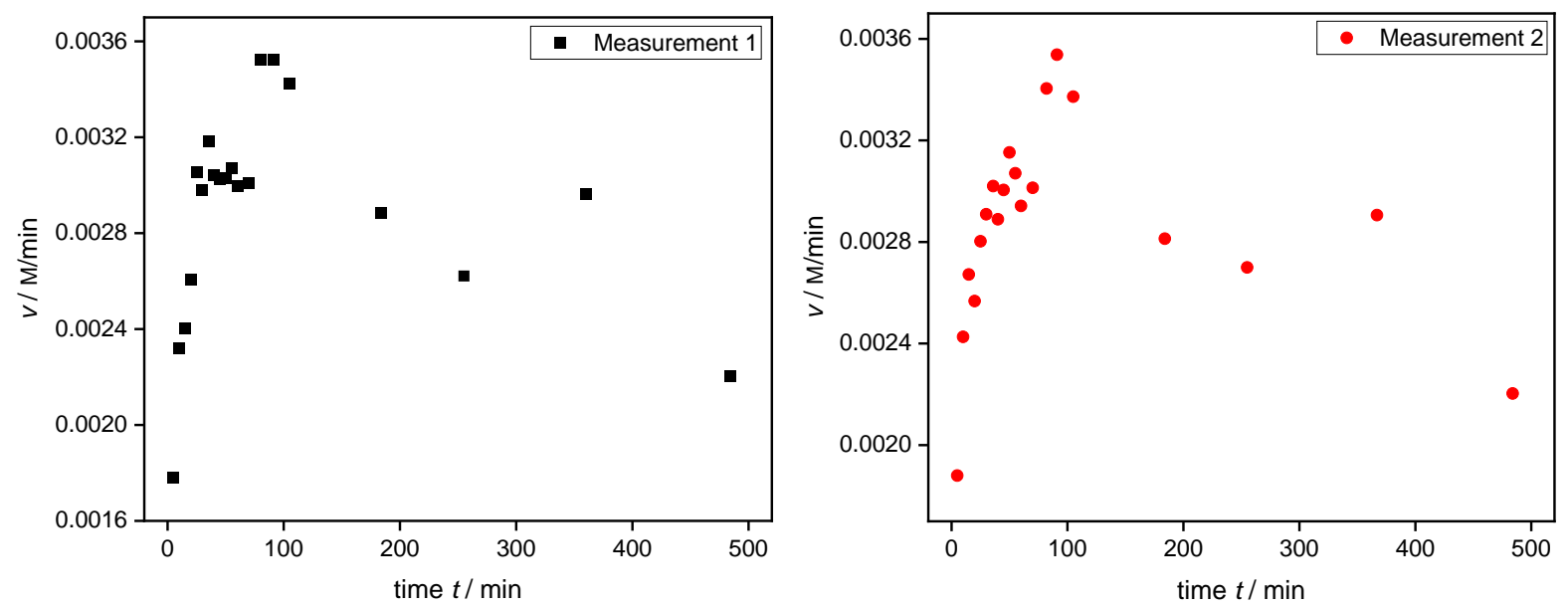

Figure S25. Reaction profiles plotting reaction rate $v$ versus time $t$.
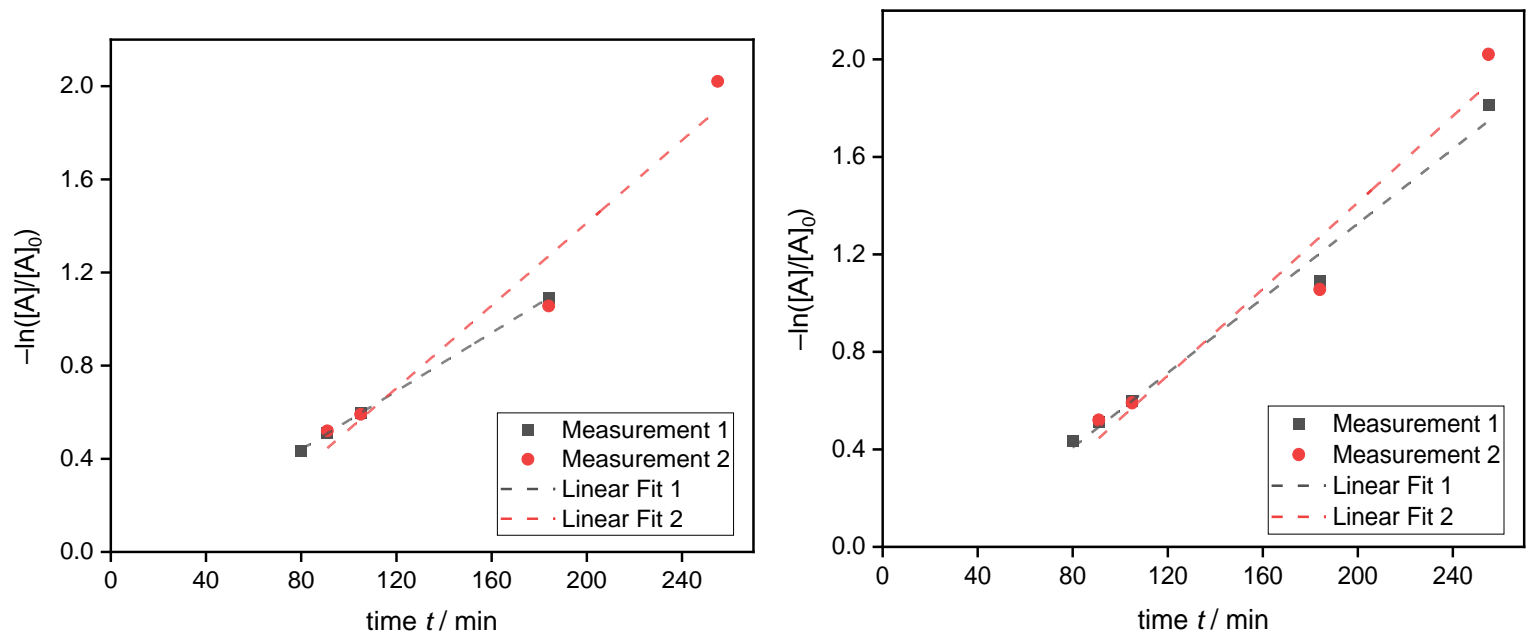

Figure S26. Logarithmic plots to determine $k_{\text {start }}$ (left) and $k_{\text {total }}$ (right). 


\subsubsection{Kinetic Measurements with $N, N$-Di- $n$-butylformamide (DnBuF)}<smiles>CCCCN(C=O)CCCC</smiles>
(4-(tert-Butyl)phenyl)methanol (164 mg, $1.00 \mathrm{mmol}, 1.00$ equiv), $\mathrm{N}, \mathrm{N}$-di$n$-butylformamide (1.0 M in 1,4-dioxane, $100 \mu \mathrm{L}, 0.10 \mathrm{mmol}, 10 \mathrm{~mol} \%$ ), mesitylene $(138 \mu \mathrm{L}, 1.00 \mathrm{mmol}, 1.00$ equiv $)$ and $\mathrm{BzCl}(139 \mu \mathrm{L}$, $1.20 \mathrm{mmol}, 1.20$ equiv) in 1,4-dioxane $(0.50 \mathrm{~mL})$ were reacted according to general procedure 7 (chapter 2.5.3, page 114).

Table S17. Determination of $k_{\text {start }}$ and $k_{\text {total }}$.

\begin{tabular}{llll} 
Measurement & $\boldsymbol{k}_{\text {start }} / \mathbf{1 0}^{\mathbf{- 3}} \mathbf{~ m i n}^{-\mathbf{1}}$ & $\boldsymbol{k}_{\text {total }} / \mathbf{1 0}^{\mathbf{- 3}} \mathbf{~ m i n}^{-\mathbf{1}}$ & Induction Period / $\mathbf{~ i n ~}$ \\
\hline 1 & 2.88 & 2.12 & 15 \\
2 & 3.18 & 2.29 & 40 \\
$\varnothing$ & 3.03 & 2.21 & -
\end{tabular}
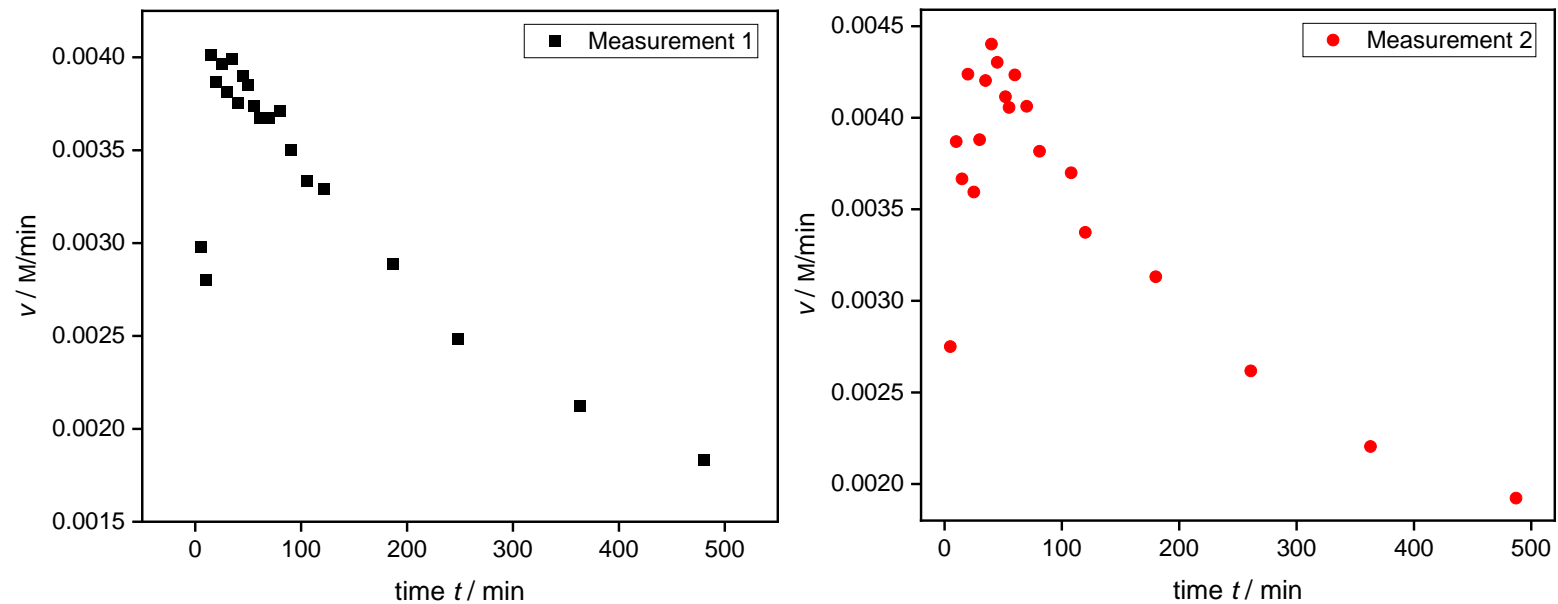

Figure S27. Reaction profiles plotting reaction rate $v$ versus time $t$.
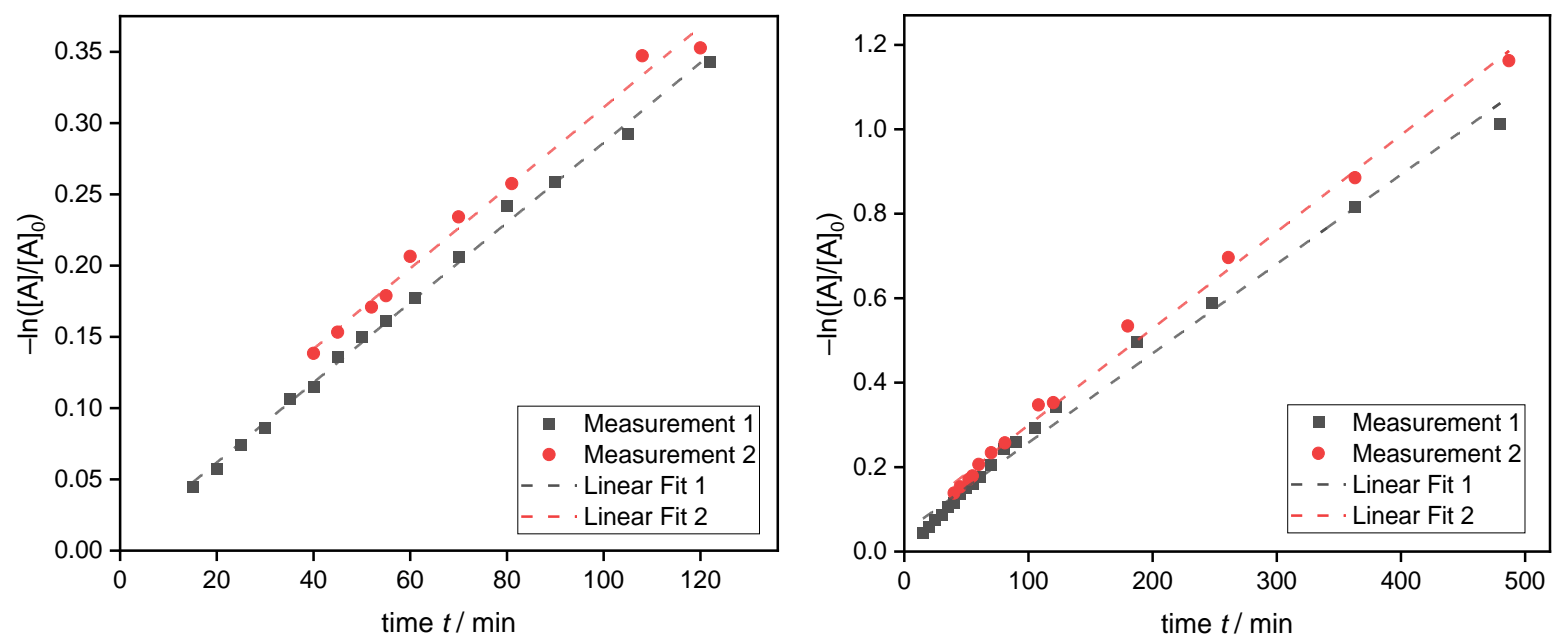

Figure S28. Logarithmic plots to determine $k_{\text {start }}$ (left) and $k_{\text {total }}$ (right). 


\subsubsection{Kinetic Measurements with $\mathrm{N}$-Benzyl- $\mathrm{N}$-methylformamide (BnMF)}<smiles>CN(C=O)Cc1ccccc1</smiles>

(4-(tert-Butyl)phenyl)methanol (164 mg, $1.00 \mathrm{mmol}, 1.00$ equiv), $\mathrm{N}$-benzyl$\mathrm{N}$-methylformamide (1.0 M in 1,4-dioxane, $100 \mu \mathrm{L}, 0.10 \mathrm{mmol}, 10 \mathrm{~mol} \%$ ), mesitylene (138 $\mu \mathrm{L}, 1.00 \mathrm{mmol}, 1.00$ equiv) and $\mathrm{BzCl}(139 \mu \mathrm{L}, 1.20 \mathrm{mmol}$, 1.20 equiv) in 1,4 -dioxane $(0.50 \mathrm{~mL})$ were reacted according to general procedure 7 (chapter 2.5.3, page 114).

Table S18. Determination of $k_{\text {start }}$ and $k_{\text {total }}$.

\begin{tabular}{|c|c|c|c|}
\hline Measurement & $k_{\text {start }} / 10^{-3} \mathrm{~min}^{-1}$ & $k_{\text {total }} / 10^{-3} \min ^{-1}$ & Induction Period / min \\
\hline 1 & 2.72 & 2.44 & 20 \\
\hline 2 & 2.53 & 2.30 & 15 \\
\hline$\varnothing$ & 2.62 & 2.37 & - \\
\hline
\end{tabular}
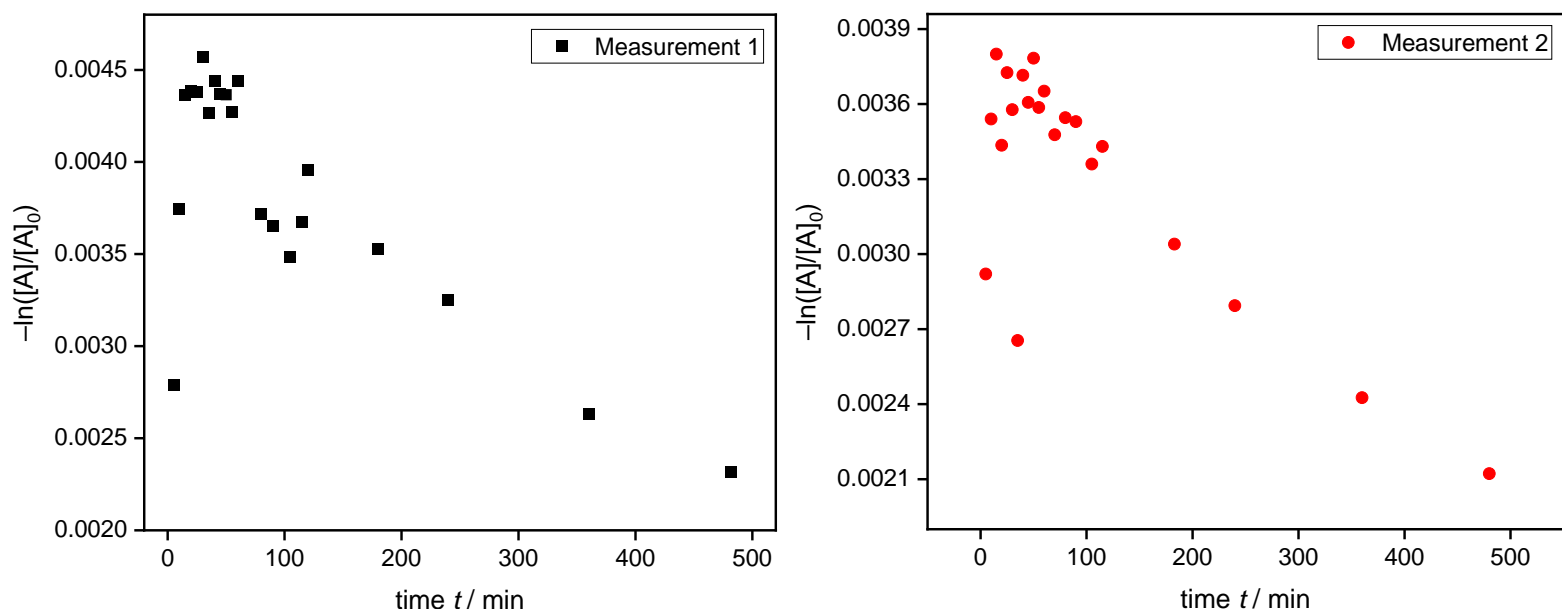

Figure S29. Reaction profiles plotting reaction rate $v$ versus time $t$.
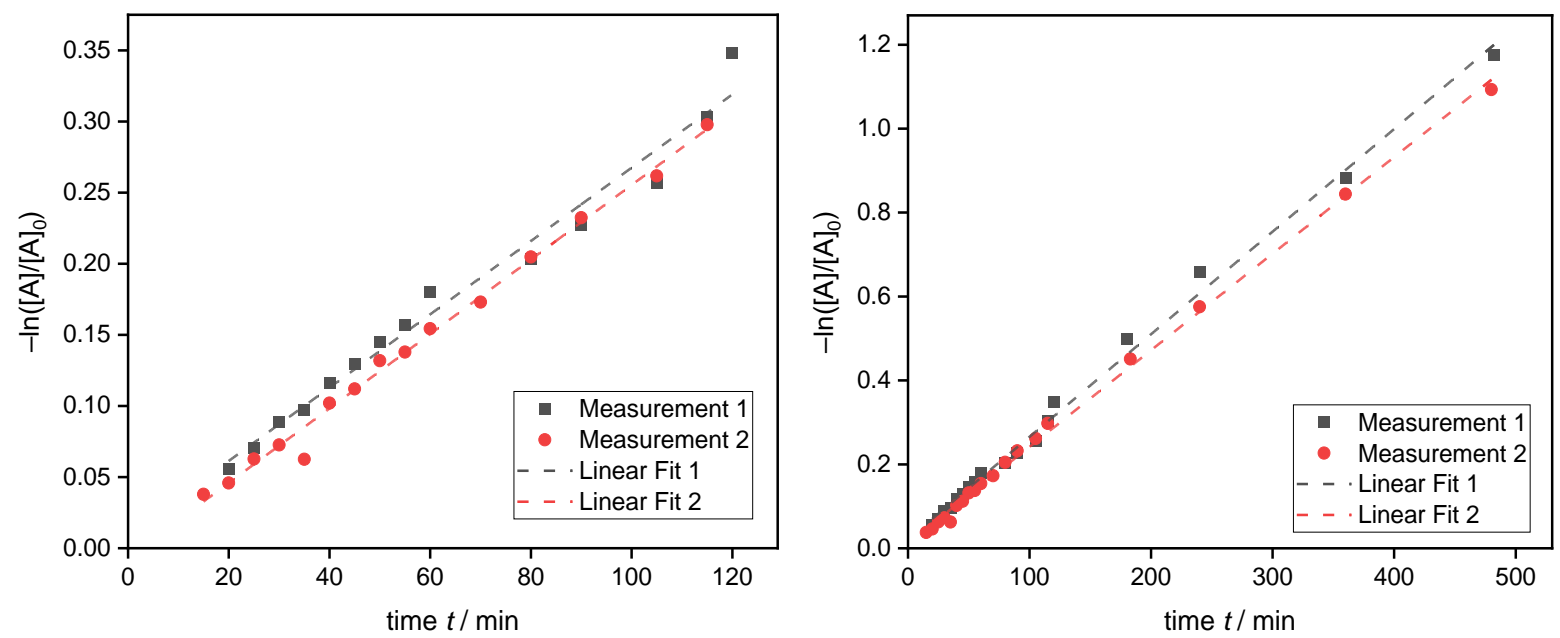

Figure S30. Logarithmic plots to determine $k_{\text {start }}$ (left) and $k_{\text {total }}$ (right). 


\subsubsection{Kinetic Measurements with $N, N$-Dibenzylformamide (DBnF)}<smiles>O=C(Cc1ccccc1)NCc1ccccc1</smiles>

(4-(tert-Butyl)phenyl)methanol (164 mg, $1.00 \mathrm{mmol}, 1.00$ equiv), $N, N$-dibenzylformamide (1.0 $\mathrm{M}$ in 1,4-dioxane, $100 \mu \mathrm{L}, 0.10 \mathrm{mmol}, 10 \mathrm{~mol} \%$ ), mesitylene (138 $\mu \mathrm{L}, 1.00 \mathrm{mmol}, 1.00$ equiv) and $\mathrm{BzCl}(139 \mu \mathrm{L}, 1.20 \mathrm{mmol}, 1.20$ equiv) in 1,4-dioxane $(0.50 \mathrm{~mL})$ were reacted according to general procedure 7 (chapter 2.5.3, page 114).

Table S19. Determination of $k_{\text {start }}$ and $k_{\text {total. }}$

\begin{tabular}{llll} 
Measurement & $\boldsymbol{k}_{\text {start }} / \mathbf{1 0}^{\mathbf{- 3}} \mathbf{~ m i n}^{\mathbf{- 1}}$ & $\boldsymbol{k}_{\text {total }} / \mathbf{1 0}^{-\mathbf{3}} \mathbf{~ m i n}^{\mathbf{- 1}}$ & Induction Period / $\mathbf{~ i n ~}$ \\
\hline 1 & 1.67 & 1.58 & 60 \\
2 & 1.97 & 2.02 & 25 \\
$\varnothing$ & 1.82 & 1.80 & - \\
\hline
\end{tabular}
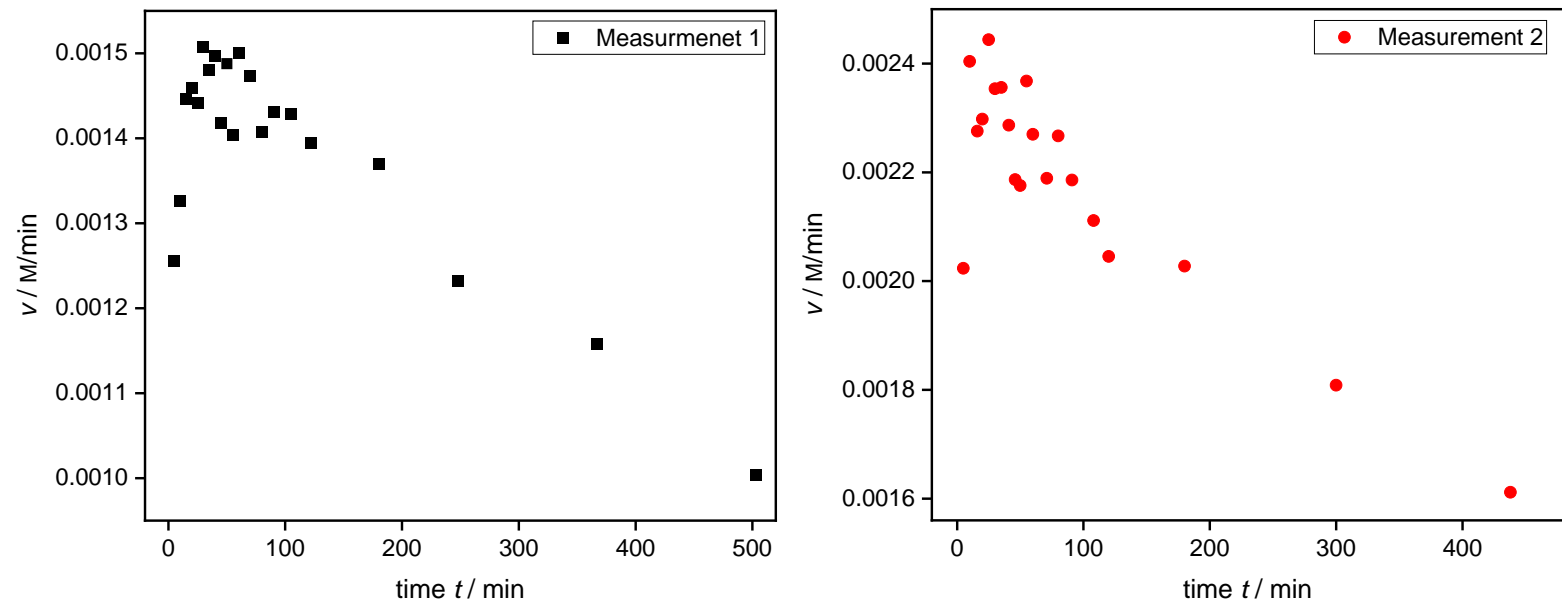

Figure S31. Reaction profiles plotting reaction rate $v$ versus time $t$.
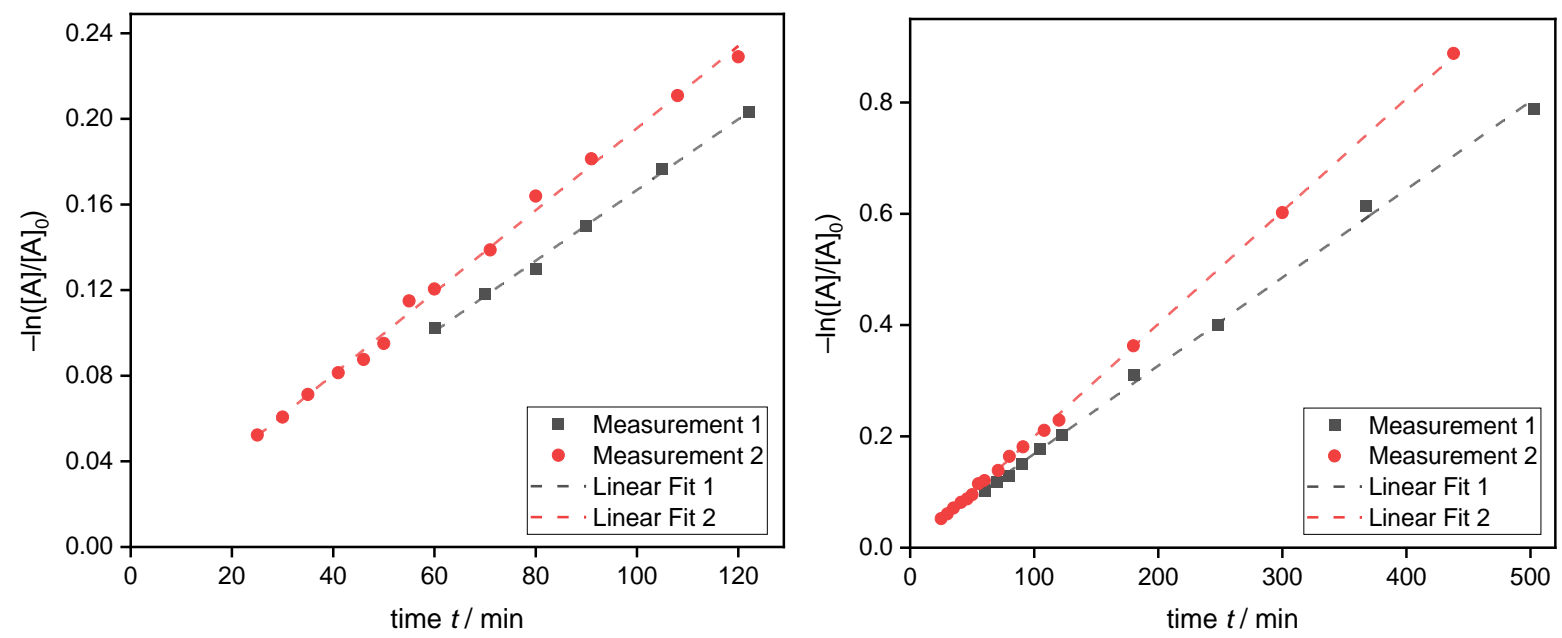

Figure S32. Logarithmic plots to determine $k_{\text {start }}$ (left) and $k_{\text {total }}$ (right). 


\subsubsection{Kinetic Measurements with $N$-(4-Methoxyphenyl)- $N$-methylformamide (pMPMF)}<smiles>COc1ccc(N(C)C=O)cc1</smiles>

(4-(tert-Butyl)phenyl)methanol (164 mg, $1.00 \mathrm{mmol}, 1.00$ equiv), $\mathrm{N}$-(4-methoxyphenyl)- $\mathrm{N}$-methylformamide $\quad(1.0 \mathrm{M}$ in 1,4 -dioxane, $100 \mu \mathrm{L}, \quad 0.10 \mathrm{mmol}, 10 \mathrm{~mol} \%)$, mesitylene $(138 \mu \mathrm{L}, 1.00 \mathrm{mmol}$, 1.00 equiv) and $\mathrm{BzCl}(139 \mu \mathrm{L}, 1.20 \mathrm{mmol}, 1.20$ equiv) in 1,4-dioxane $(0.50 \mathrm{~mL})$ were reacted according to general procedure 7 (chapter 2.5.3, page 114).

Table S20. Determination of $k_{\text {start }}$ and $k_{\text {total. }}$

\begin{tabular}{llll} 
Measurement & $\boldsymbol{k}_{\text {start }} / \mathbf{1 0}^{-\mathbf{3}} \mathbf{~ m i n}^{-\mathbf{1}}$ & $\boldsymbol{k}_{\text {total }} / \mathbf{1 0}^{-\mathbf{3}} \mathbf{~ m i n}^{-\mathbf{1}}$ & Induction Period $/ \mathbf{~} \mathbf{m i n}$ \\
\hline 1 & 1.20 & 1.38 & 105 \\
2 & 1.05 & 1.33 & 60 \\
$\varnothing$ & 1.13 & 1.36 & -
\end{tabular}
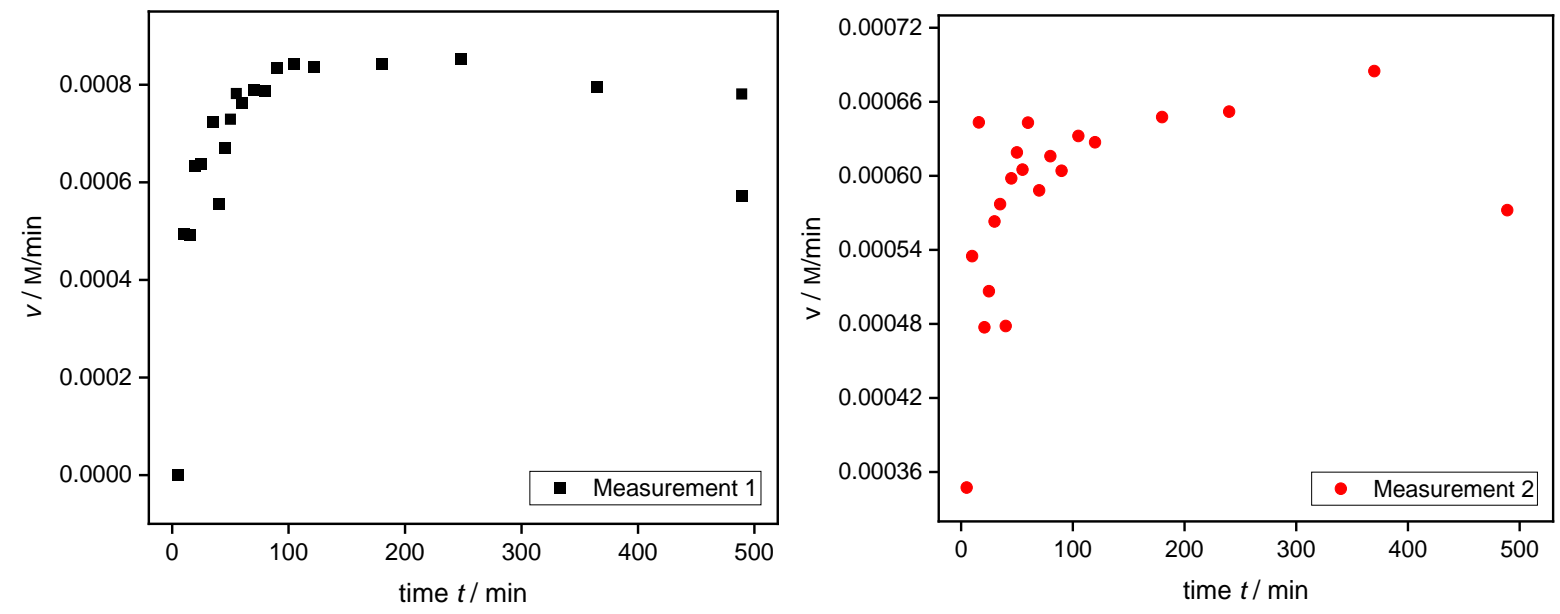

Figure S33. Reaction profiles plotting reaction rate $v$ versus time $t$.
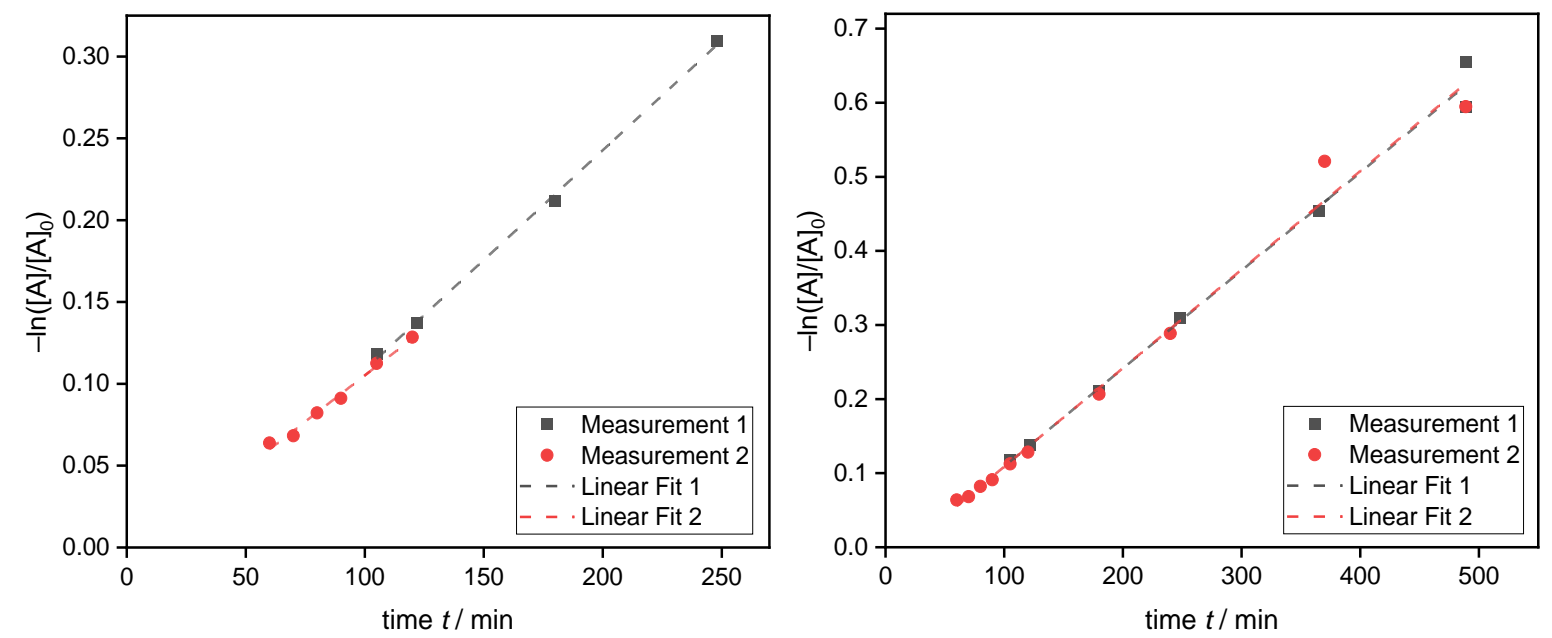

Figure S34. Logarithmic plots to determine $k_{\text {start }}\left(\right.$ left) and $k_{\text {total }}$ (right). 


\subsubsection{Kinetic Measurements with Azepane-1-carbaldehyde (FAz)}<smiles>O=CN1CCCCCC1</smiles>

(4-(tert-Butyl)phenyl)methanol (164 mg, $1.00 \mathrm{mmol}, 1.00$ equiv), azepane-1carbaldehyde (1.0 M in 1,4-dioxane, $100 \mu \mathrm{L}, 0.10 \mathrm{mmol}, 10 \mathrm{~mol} \%$ ), mesitylene (138 $\mu \mathrm{L}, 1.00 \mathrm{mmol}, 1.00$ equiv) and $\mathrm{BzCl}$ (139 $\mu \mathrm{L}, 1.20 \mathrm{mmol}, 1.20$ equiv) in 1,4-dioxane $(0.50 \mathrm{~mL})$ were reacted according to general procedure 7 (chapter 2.5.3, page 114).

Table S21. Determination of $k_{\text {start }}$ and $k_{\text {total }}$.

\begin{tabular}{|llll} 
Measurement & $\boldsymbol{K}_{\text {start }} / \mathbf{1 0}^{\mathbf{- 3}} \mathbf{~ m i n}^{\mathbf{- 1}}$ & $\boldsymbol{K}_{\text {total }} / \mathbf{1 0}^{-\mathbf{3}} \mathbf{~ m i n}^{\mathbf{- 1}}$ & Induction Period / $\mathbf{~ i n ~}$ \\
\hline 1 & 4.23 & 2.54 & 30 \\
\hline 2 & 4.17 & 2.47 & 10 \\
$\varnothing$ & 4.20 & 2.51 & - \\
\hline
\end{tabular}
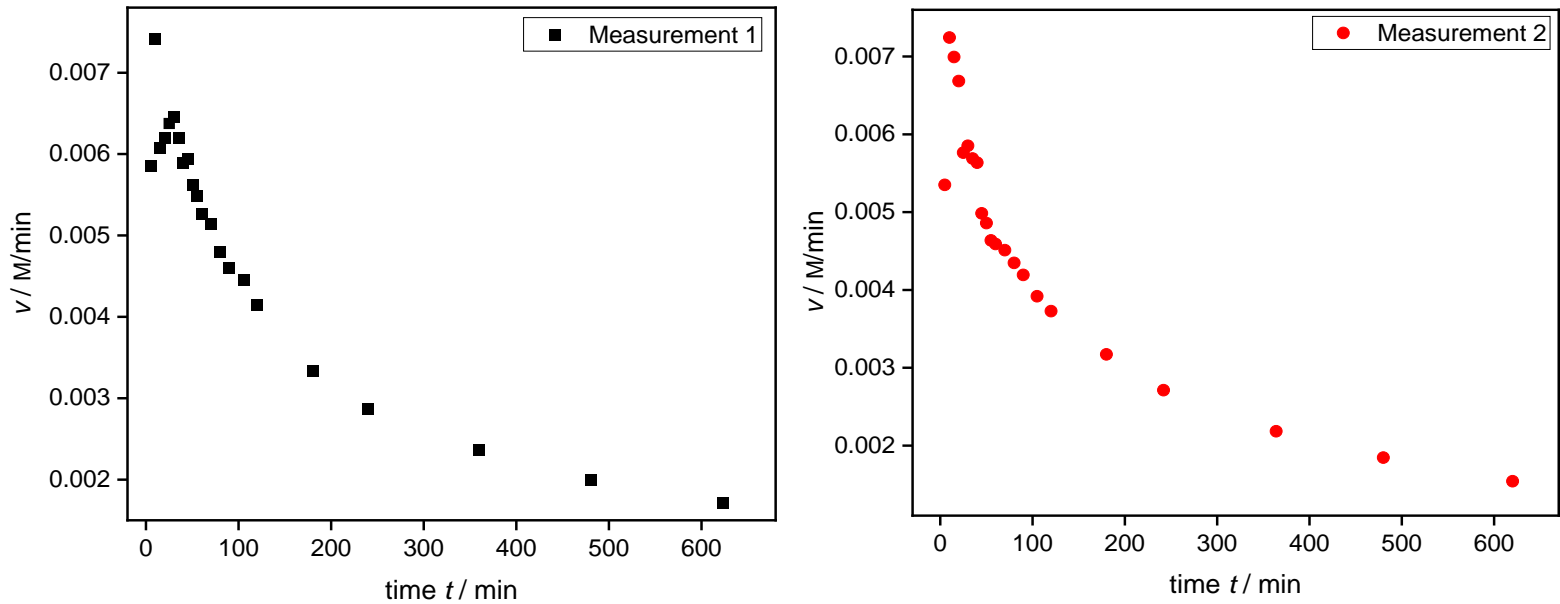

Figure S35. Reaction profiles plotting reaction rate $v$ versus time $t$.
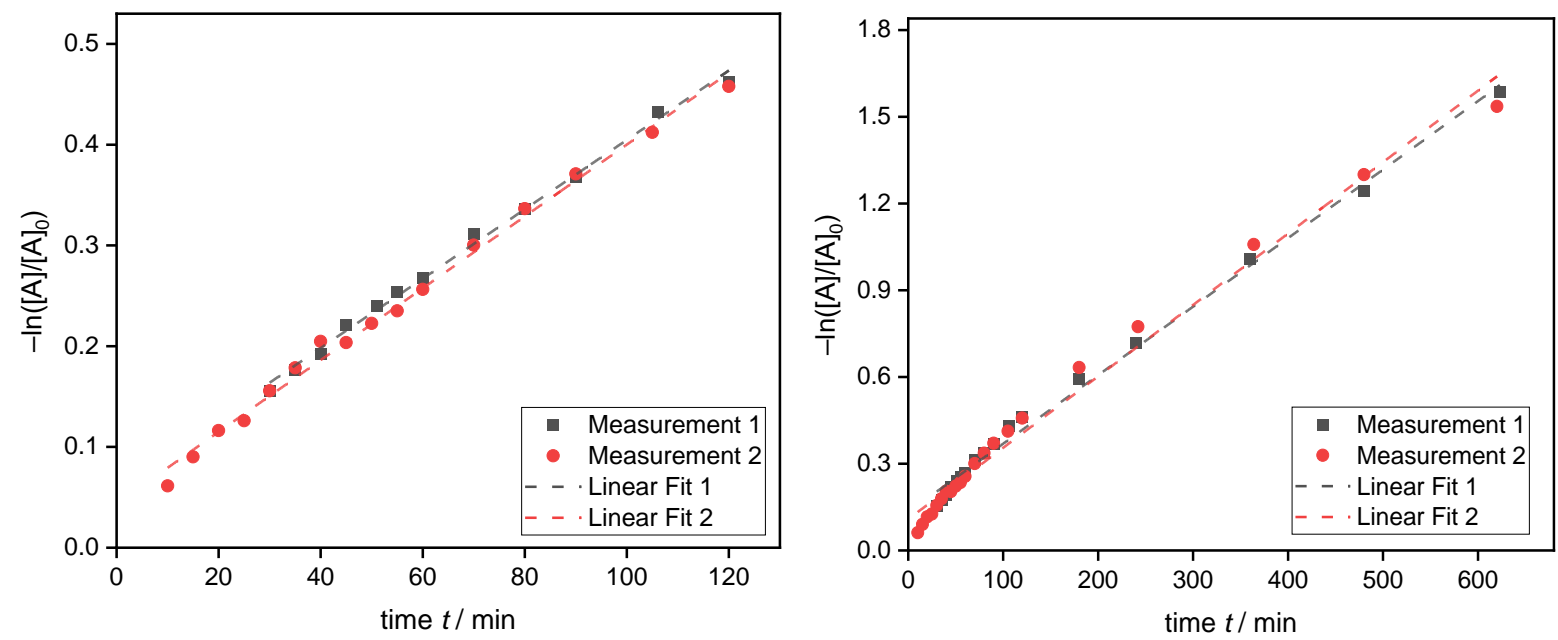

Figure S36. Logarithmic plots to determine $k_{\text {start }}$ (left) and $k_{\text {total }}$ (right). 


\subsubsection{Kinetic Measurements with $\mathrm{N}$-Allylformamide (AIIF)}

$\stackrel{\mathrm{H}}{\mathrm{O}}$

(4-(tert-Butyl)phenyl)methanol $\quad$ (164 $\mathrm{mg}, \quad 1.00 \mathrm{mmol}, \quad 1.00$ equiv), $\mathrm{N}$-allylformamide $(1.0 \mathrm{M}$ in 1,4-dioxane, $100 \mu \mathrm{L}, 0.10 \mathrm{mmol}, 10 \mathrm{~mol} \%)$, mesitylene (138 $\mu \mathrm{L}, 1.00 \mathrm{mmol}, 1.00$ equiv) and $\mathrm{BzCl}(139 \mu \mathrm{L}, 1.20 \mathrm{mmol}$, 1.20 equiv) in 1,4-dioxane $(0.50 \mathrm{~mL})$ were reacted according to general procedure 7 (chapter 2.5.3, page 114).

Table S22. Determination of $k_{\text {start }}$ and $k_{\text {total }}$.

\begin{tabular}{llll} 
Measurement & $\boldsymbol{k}_{\text {start }} / \mathbf{1 0}^{-\mathbf{3}} \mathbf{~} \mathbf{n i n}^{\mathbf{- 1}}$ & $\boldsymbol{k}_{\text {total }} / \mathbf{1 0}^{\mathbf{- 3}} \mathbf{~} \mathbf{n i n}^{\mathbf{- 1}}$ & Induction Period $/ \mathbf{~} \mathbf{i n}$ \\
\hline 1 & 3.33 & 2.32 & 25 \\
2 & 3.62 & 2.08 & 15 \\
$\varnothing$ & 3.48 & 2.20 & -
\end{tabular}
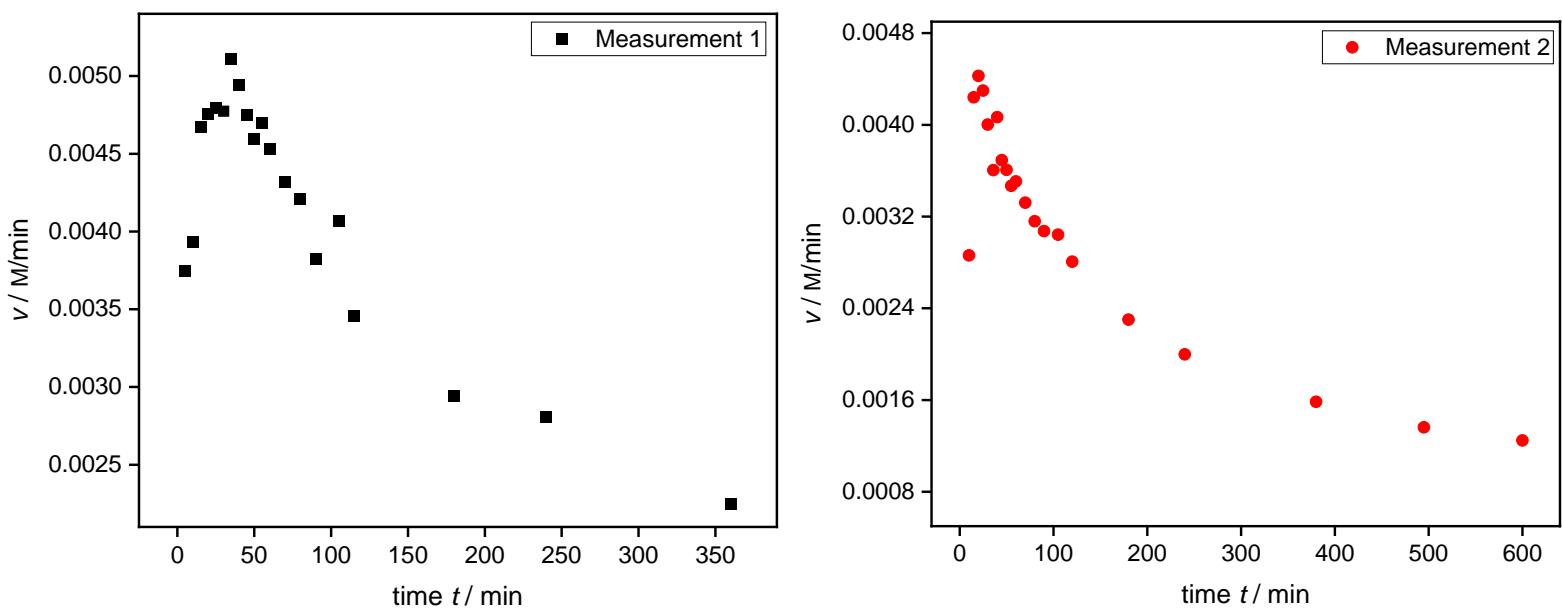

Figure S37. Reaction profiles plotting reaction rate $v$ versus time $t$.
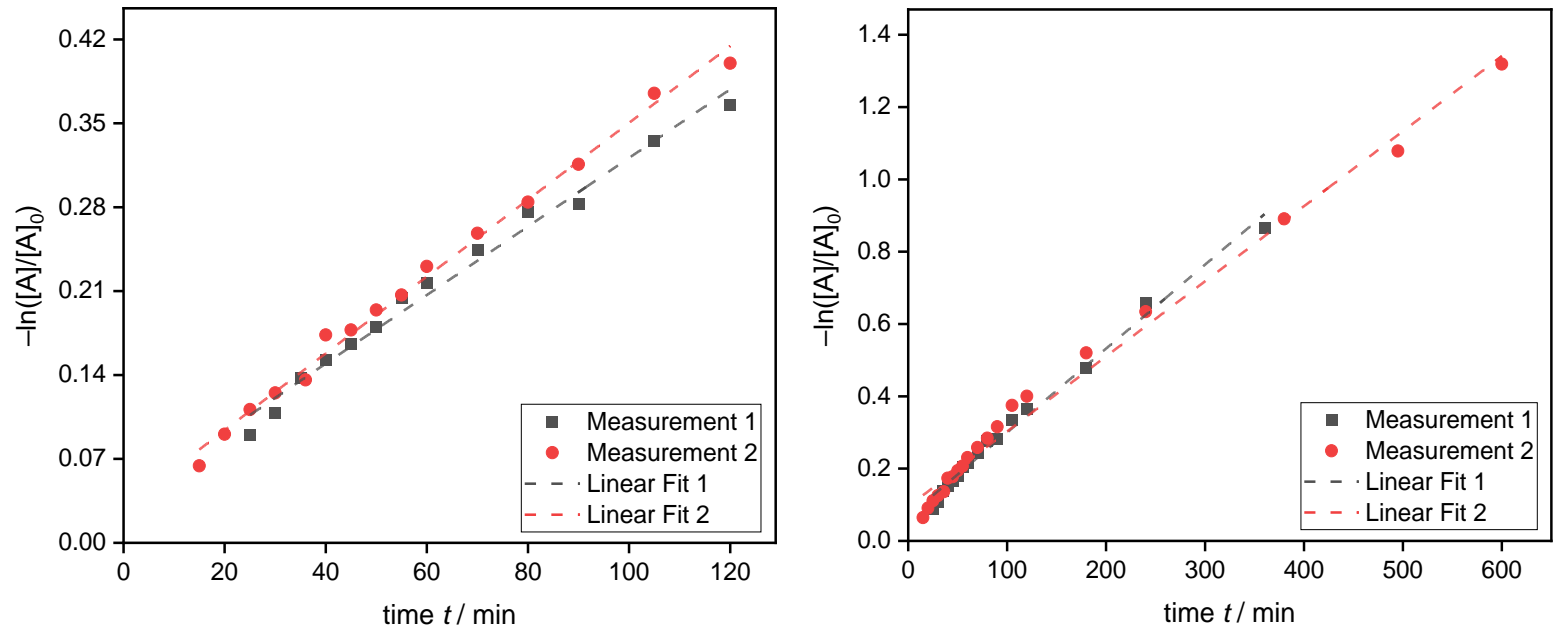

Figure S38. Logarithmic plots to determine $k_{\text {start }}$ (left) and $k_{\text {total }}$ (right). 


\subsubsection{Kinetic Measurements with $\mathrm{N}$-Cyclohexylformamide (cHexF)}<smiles>O=CNC1CCCCC1</smiles>
(4-(tert-Butyl)phenyl)methanol $\quad$ (164 mg, $\quad 1.00 \mathrm{mmol}, \quad 1.00$ equiv), $\mathrm{N}$-cyclohexylformamide (1.0 M in 1,4-dioxane, $100 \mu \mathrm{L}, 0.10 \mathrm{mmol}, 10 \mathrm{~mol} \%$ ), mesitylene (138 $\mu \mathrm{L}, 1.00 \mathrm{mmol}, 1.00$ equiv) and $\mathrm{BzCl}(139 \mu \mathrm{L}, 1.20 \mathrm{mmol}$, 1.20 equiv) in 1,4-dioxane $(0.50 \mathrm{~mL})$ were reacted according to general procedure 7 (chapter 2.5.3, page 114).

Table S23. Determination of $k_{\text {start }}$ and $k_{\text {total }}$.

\begin{tabular}{|c|c|c|c|}
\hline Measurement & $k_{\text {start }} / 10^{-3} \min ^{-1}$ & $k_{\text {total }} / 10^{-3} \min ^{-1}$ & Induction Period / min \\
\hline 1 & 2.99 & 2.99 & 35 \\
\hline 2 & 3.33 & 3.03 & 15 \\
\hline$\varnothing$ & 3.16 & 3.01 & - \\
\hline
\end{tabular}
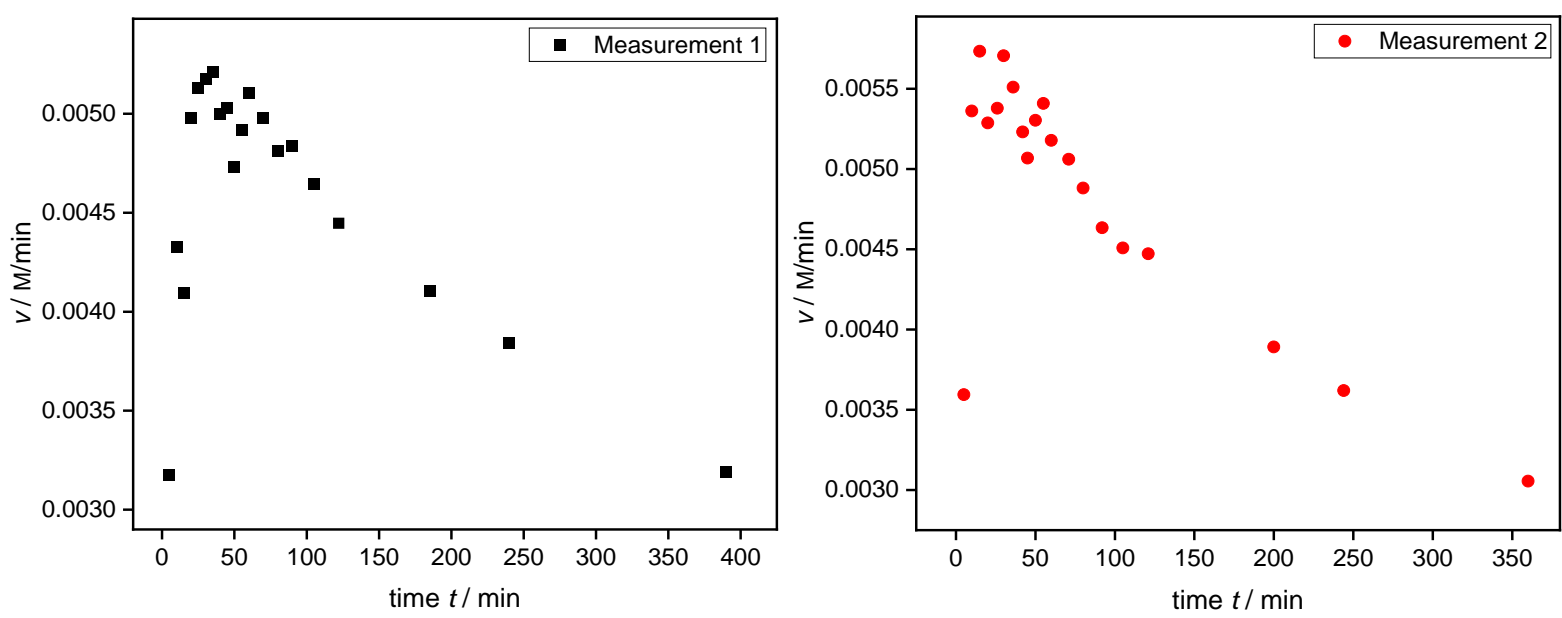

Figure S39. Reaction profiles plotting reaction rate $v$ versus time $t$.
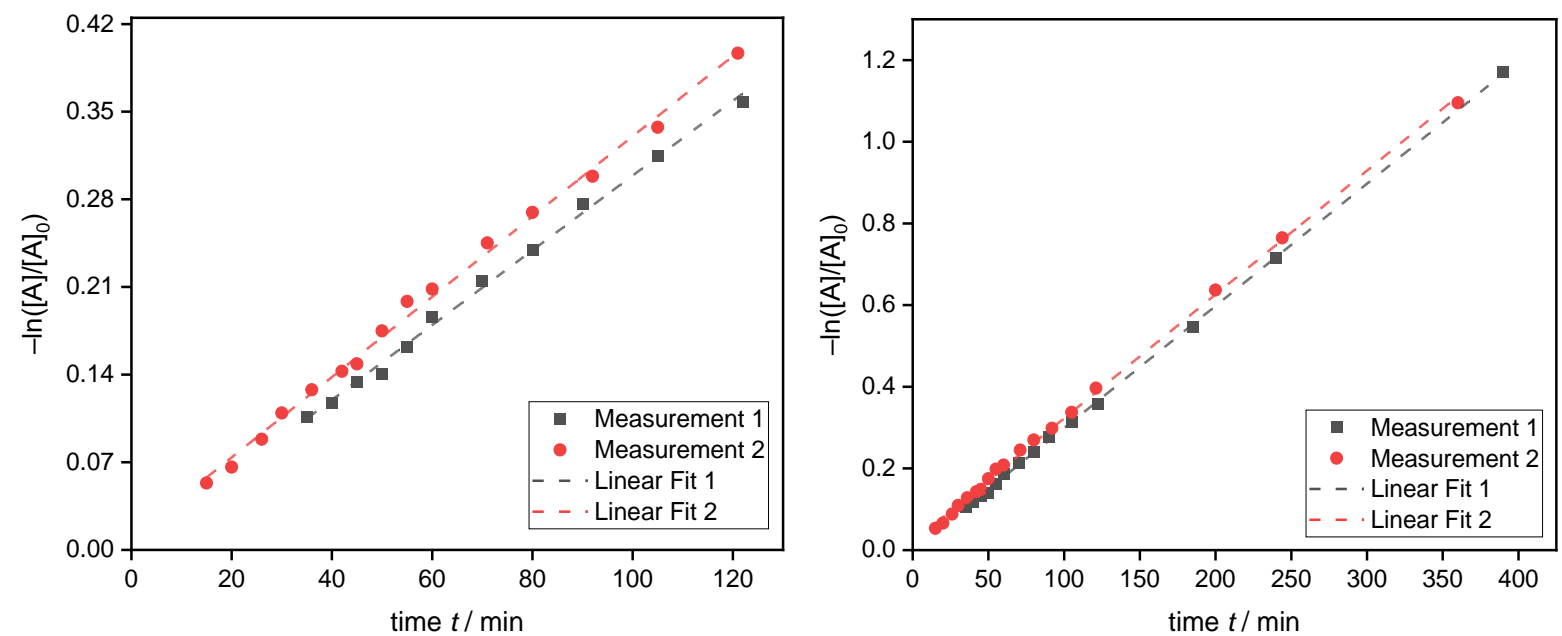

Figure S40. Logarithmic plots to determine $k_{\text {start }}$ (left) and $k_{\text {total }}$ (right). 


\subsubsection{Kinetic Measurements with $\mathrm{N}$-(2,2-Dimethoxyethyl)formamide (DMEF)}<smiles>COC(CNC=O)OC</smiles>

(4-(tert-Butyl)phenyl)methanol (164 mg, 1.00 mmol, 1.00 equiv), $\mathrm{N}-(2,2-$ dimethoxyethyl)formamide $(1.0 \mathrm{M}$ in 1,4-dioxane, $100 \mu \mathrm{L}, 0.10 \mathrm{mmol}$, 10 mol\%), mesitylene (138 $\mu \mathrm{L}, 1.00 \mathrm{mmol}, 1.00$ equiv) and $\mathrm{BzCl}(139 \mu \mathrm{L}$, $1.20 \mathrm{mmol}, 1.20$ equiv) in 1,4-dioxane $(0.50 \mathrm{~mL})$ were reacted according the general procedure 7 (chapter 2.5.3, page 114).

Table S24. Determination of $k_{\text {start }}$ and $k_{\text {total }}$.

\begin{tabular}{llll} 
Measurement & $\boldsymbol{K}_{\text {start }} / \mathbf{1 0}^{\mathbf{- 3}} \mathbf{~ m i n}^{\mathbf{- 1}}$ & $\boldsymbol{k}_{\text {total }} / \mathbf{1 0}^{\mathbf{- 3}} \mathbf{~ m i n}^{-\mathbf{1}}$ & Induction Period / $\mathbf{~ i n ~}$ \\
\hline $\mathbf{1}$ & 2.53 & 1.46 & 31 \\
$\mathbf{2}$ & 2.99 & 2.34 & 45 \\
$\varnothing$ & 2.76 & 1.90 & -
\end{tabular}
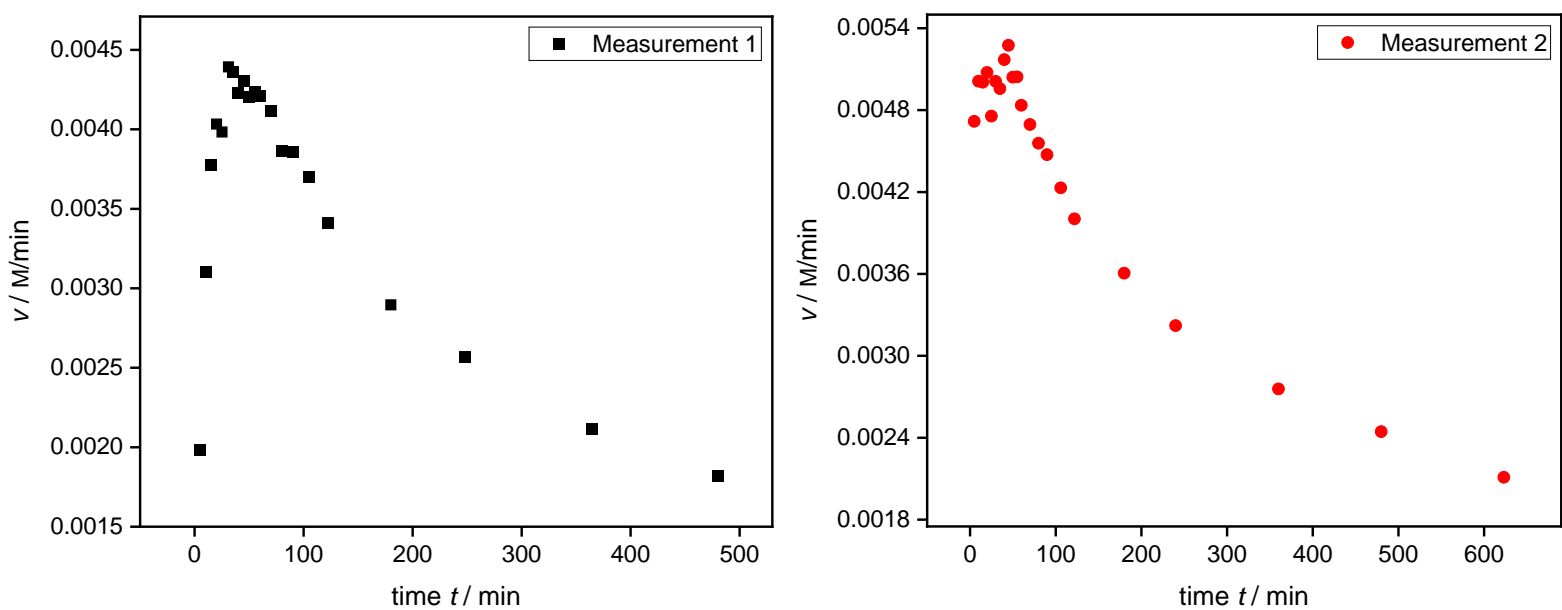

Figure S41. Reaction profiles plotting reaction rate $v$ versus time $t$.
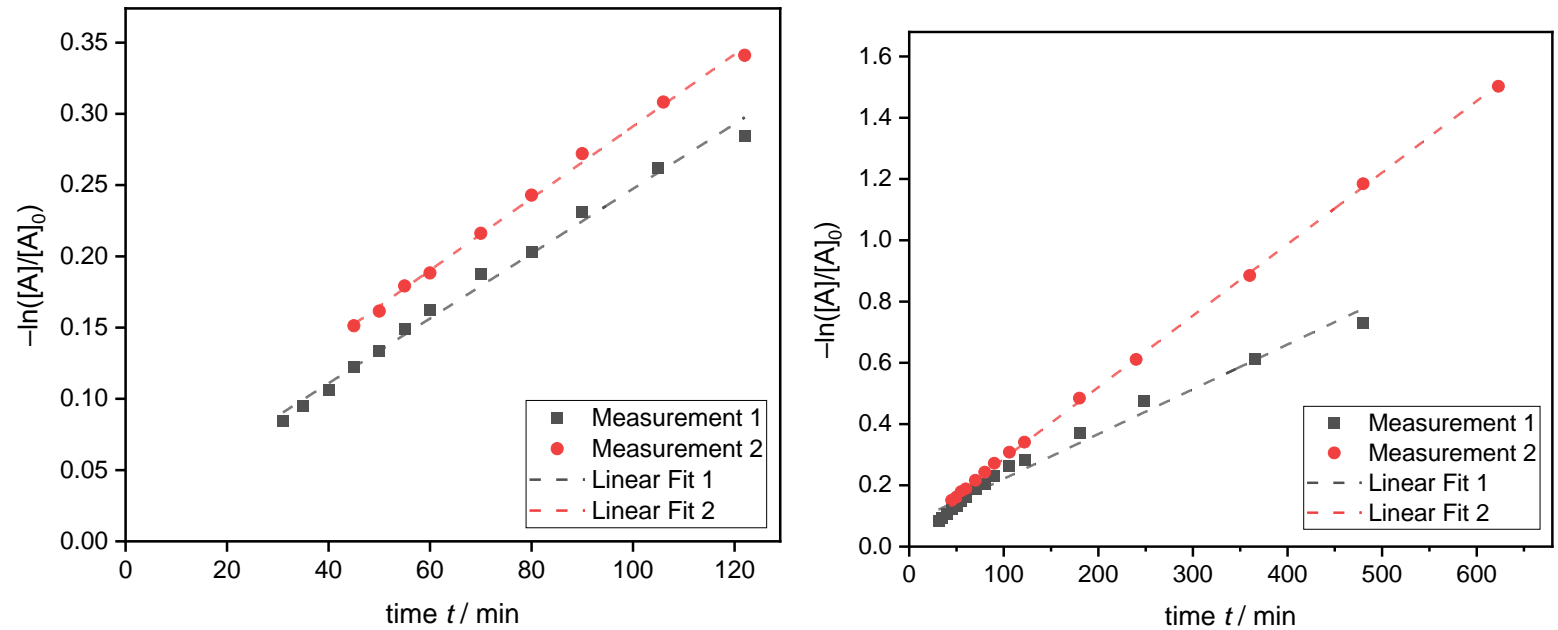

Figure S42. Logarithmic plots to determine $k_{\text {start }}$ (left) and $k_{\text {total }}$ (right). 


\subsubsection{Kinetic Measurements with $\mathbf{N}$-Phenethylformamide (PEF)}

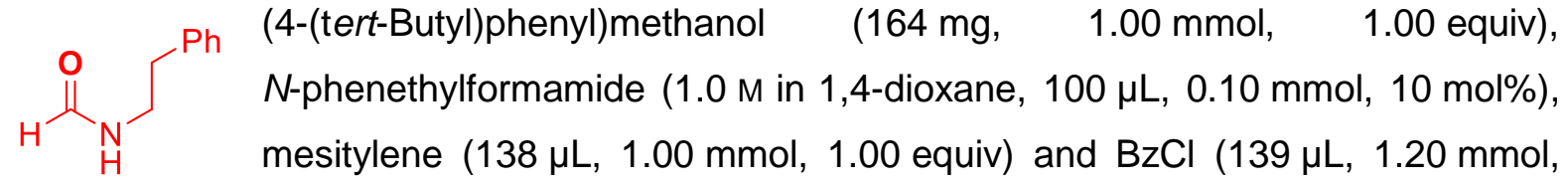
1.20 equiv) in 1,4-dioxane $(0.50 \mathrm{~mL})$ were reacted according to general procedure 7 (chapter 2.5.3, page 114).

Table S25. Determination of $k_{\text {start }}$ and $k_{\text {total. }}$.

\begin{tabular}{llll} 
Measurement & $\boldsymbol{K}_{\text {start }} / \mathbf{1 0}^{\mathbf{- 3}} \mathbf{~ m i n}^{\mathbf{- 1}}$ & $\boldsymbol{K}_{\text {total }} / \mathbf{1 0}^{\mathbf{- 3}} \mathbf{~ m i n}^{\mathbf{- 1}}$ & Induction Period / $\mathbf{~ i n ~}$ \\
\hline 1 & 4.11 & 3.88 & 10 \\
2 & 3.24 & 2.31 & 40 \\
$\varnothing$ & 3.68 & 3.10 & -
\end{tabular}
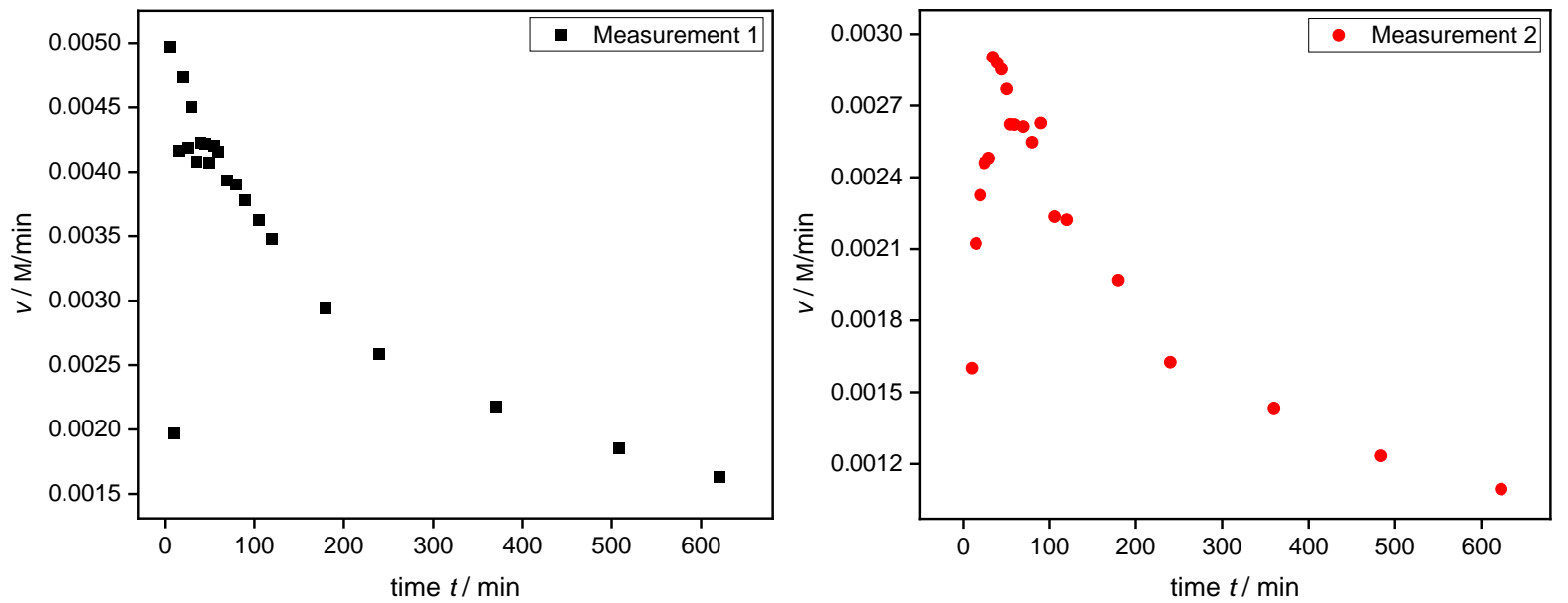

Figure S43. Reaction profiles plotting reaction rate $v$ versus time $t$.
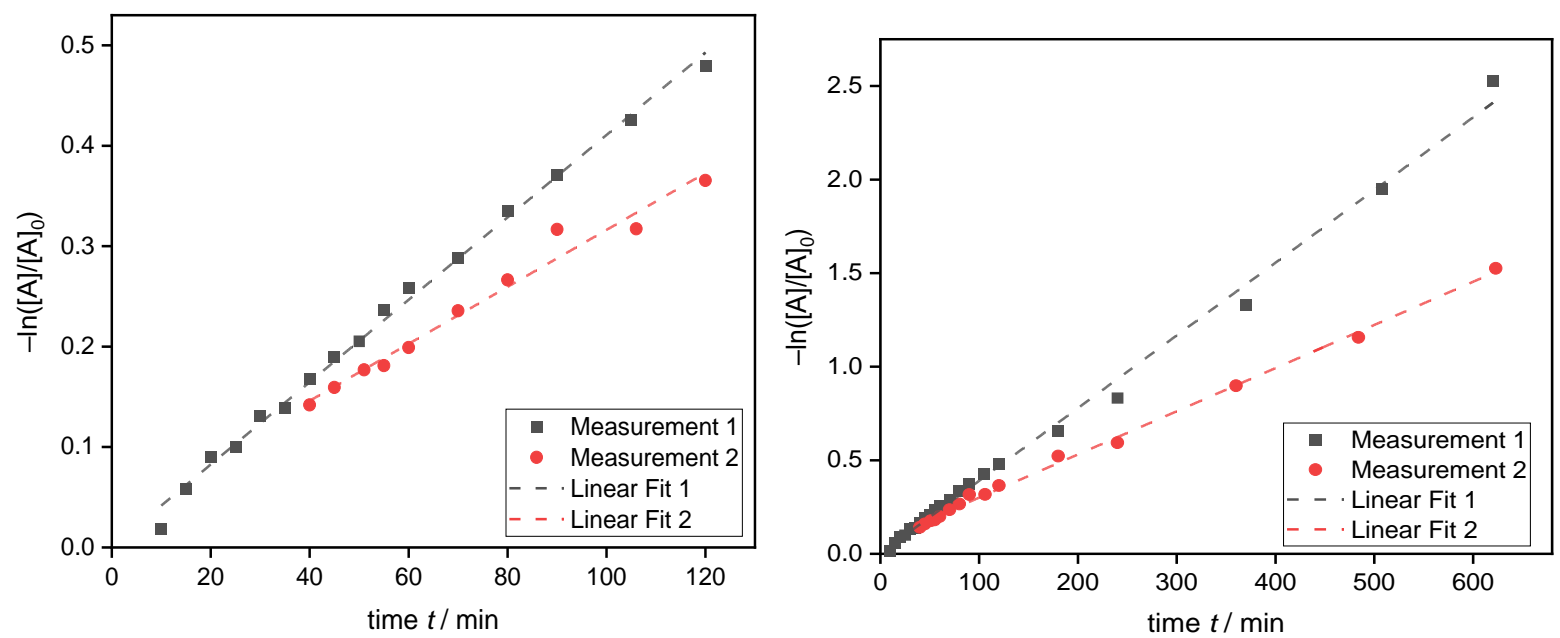

Figure S44. Logarithmic plots to determine $k_{\text {start }}$ (left) and $k_{\text {total }}$ (right). 


\subsubsection{Kinetic Measurements with $N$-Benzyl- $N$-(tert-butyl)formamide (BntBuF)}

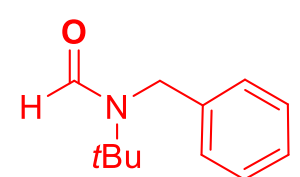

(4-(tert-Butyl)phenyl)methanol (164 mg, $1.00 \mathrm{mmol}, 1.00$ equiv), $N$-benzyl$N$-(tert-butyl)formamide $(1.0 \mathrm{M}$ in 1,4-dioxane, $100 \mu \mathrm{L}, 0.10 \mathrm{mmol}$, 10 mol\%), mesitylene (138 $\mu \mathrm{L}, 1.00 \mathrm{mmol}, 1.00$ equiv) and $\mathrm{BzCl}(139 \mu \mathrm{L}$, $1.20 \mathrm{mmol}, 1.20$ equiv) in 1,4-dioxane $(0.50 \mathrm{~mL})$ were reacted according to general procedure 7 (chapter 2.5.3, page 114)

Table S26. Determination of $k_{\text {start }}$ and $k_{\text {total }}$.

\begin{tabular}{llll} 
Measurement & $\boldsymbol{k}_{\text {start }} / \mathbf{1 0}^{\mathbf{- 3}} \mathbf{~ m i n}^{-\mathbf{1}}$ & $\boldsymbol{k}_{\text {total }} / \mathbf{1 0}^{\mathbf{- 3}} \mathbf{~ m i n}^{-\mathbf{1}}$ & Induction Period / $\mathbf{~ i n ~}$ \\
\hline 1 & 3.98 & 2.22 & - \\
2 & 3.91 & 2.27 & - \\
$\varnothing$ & 3.95 & 4.49 & -
\end{tabular}
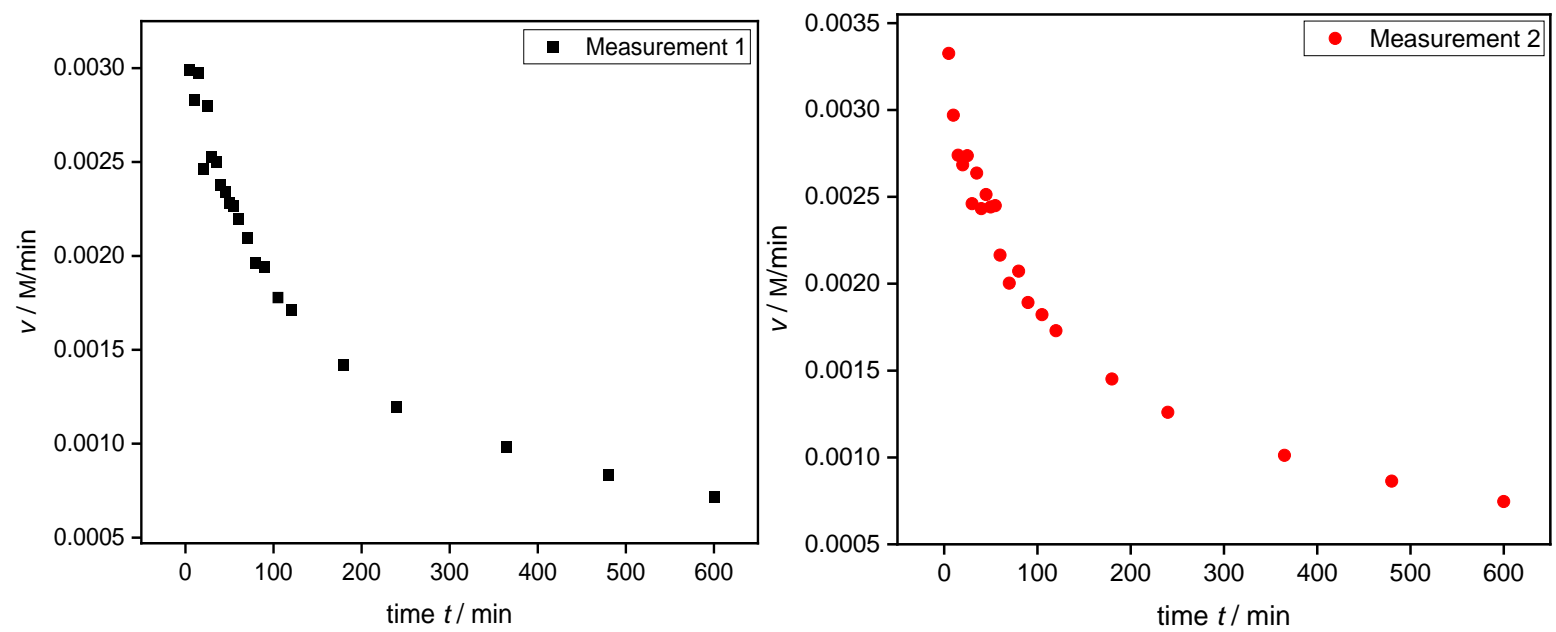

Figure S45. Reaction profiles plotting reaction rate $v$ versus time $t$.
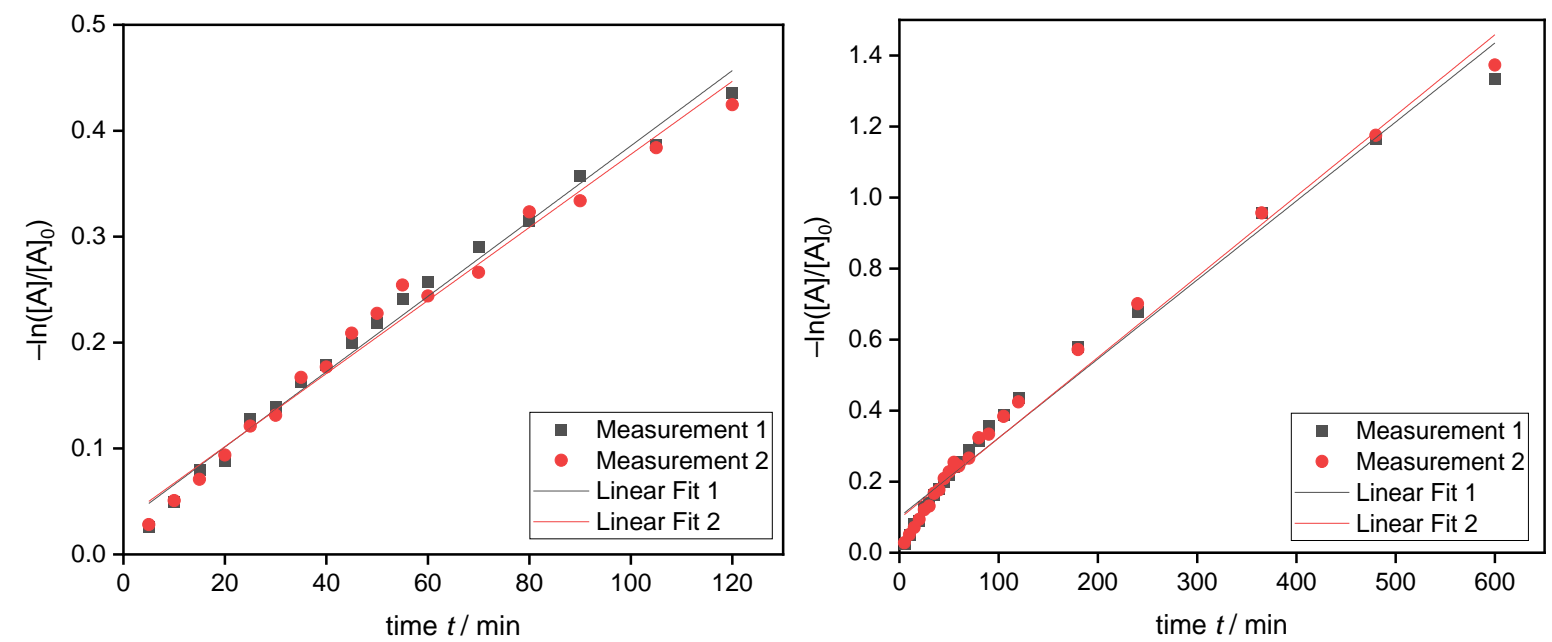

Figure S46. Logarithmic plots to determine $k_{\text {start }}$ (left) and $k_{\text {total }}$ (right). 


\subsubsection{Kinetic Measurements with $\quad \mathrm{N}$-(Naphthalen-1-ylmethyl)formamide (NaphMF)}<smiles>O=CNCc1cccc2ccccc12</smiles>

(4-(tert-Butyl)phenyl)methanol (164 mg, $1.00 \mathrm{mmol}, \quad 1.00$ equiv), $N$-(naphthalen-1-ylmethyl)formamide $(1.0 \mathrm{M}$ in 1,4-dioxane, $100 \mu \mathrm{L}$, $0.10 \mathrm{mmol}, 10 \mathrm{~mol} \%)$, mesitylene (138 $\mu \mathrm{L}, 1.00 \mathrm{mmol}, 1.00$ equiv) and $\mathrm{BzCl}(139 \mu \mathrm{L}, 1.20 \mathrm{mmol}, 1.20$ equiv) in 1,4-dioxane $(0.50 \mathrm{~mL})$ were reacted according to general procedure 7 (chapter 2.5.3, page 114).

Table S27. Determination of $k_{\text {start }}$ and $k_{\text {total. }}$

\begin{tabular}{|c|c|c|c|}
\hline Measurement & $k_{\text {start }} / 10^{-3} \mathrm{~min}^{-1}$ & $k_{\text {total }} / 10^{-3} \mathrm{~min}^{-1}$ & Induction Period / $\mathrm{min}$ \\
\hline 1 & 3.22 & 1.91 & 50 \\
\hline 2 & 3.52 & 2.15 & 50 \\
\hline$\varnothing$ & 3.37 & 2.03 & - \\
\hline
\end{tabular}
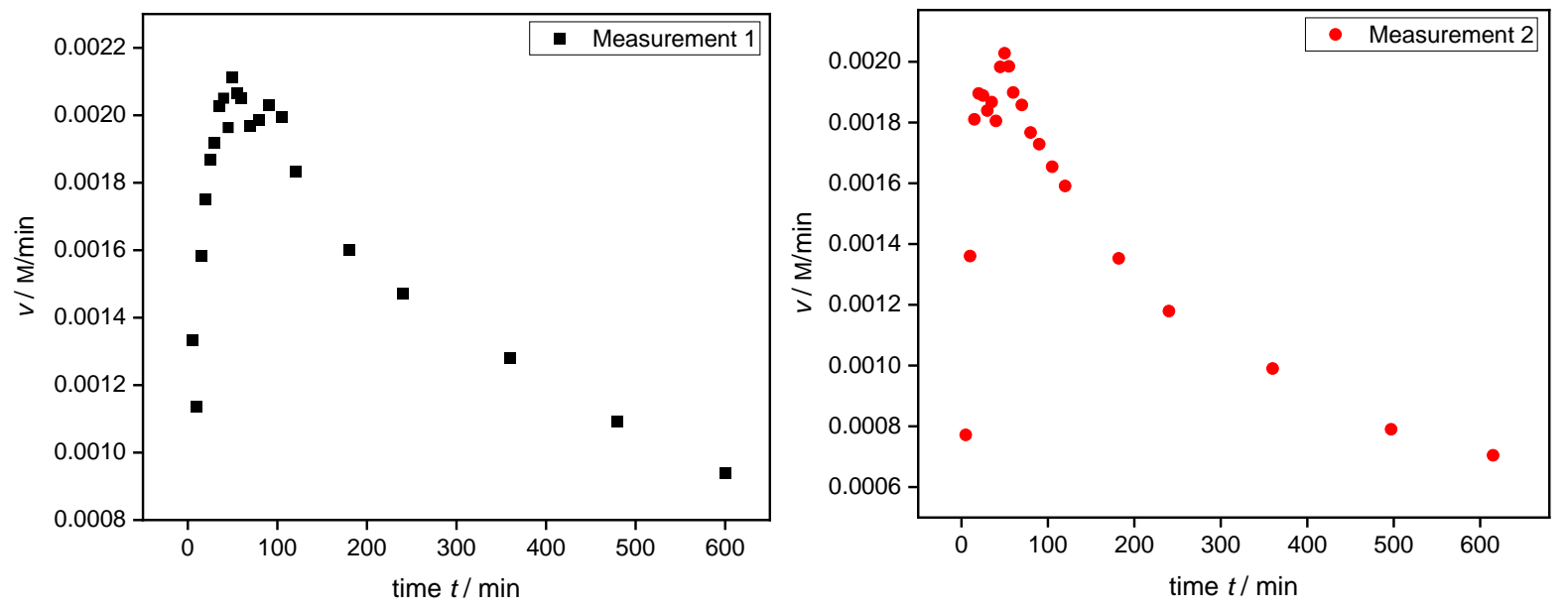

Figure S47. Reaction profiles plotting reaction rate $v$ versus time $t$.
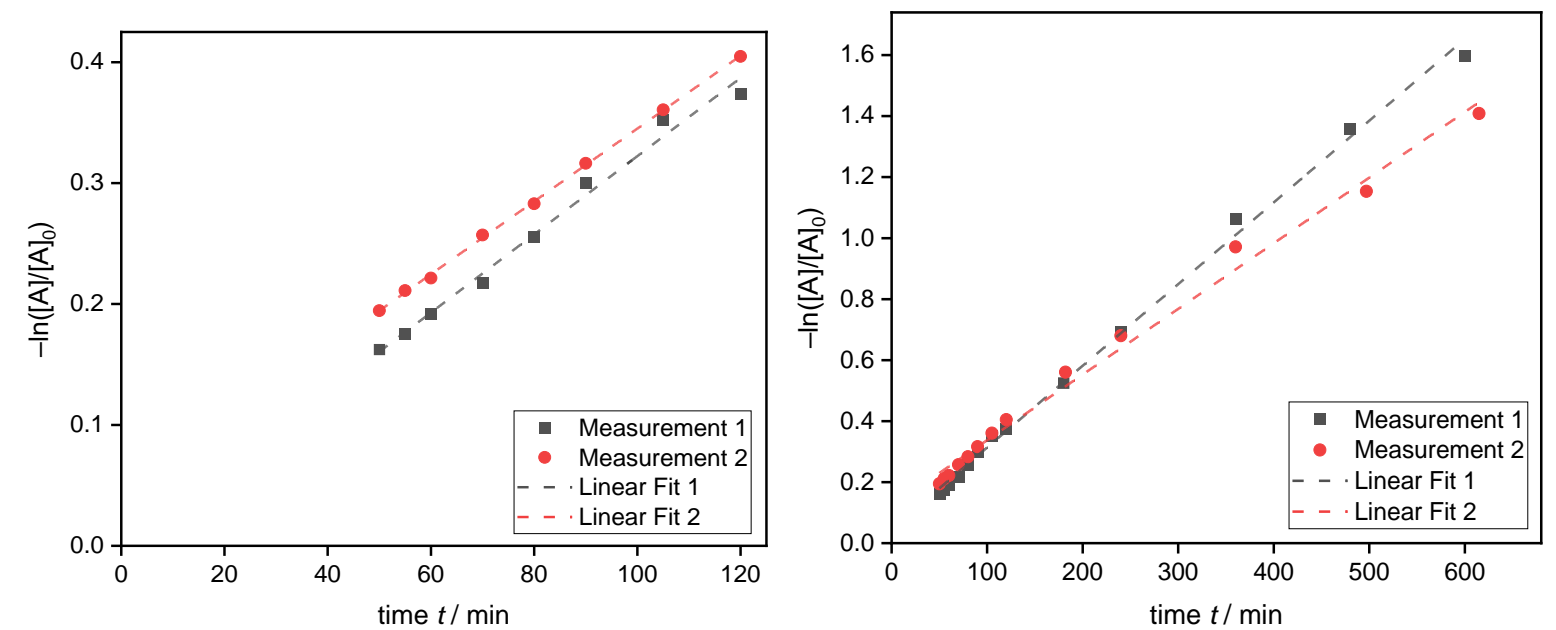

Figure S48. Logarithmic plots to determine $k_{\text {start }}\left(\right.$ left) and $k_{\text {total }}$ (right). 


\subsubsection{Kinetic Measurements with 3,4-Dihydroisoquinoline-2(1H)-carbaldehyde (F/soChin)}

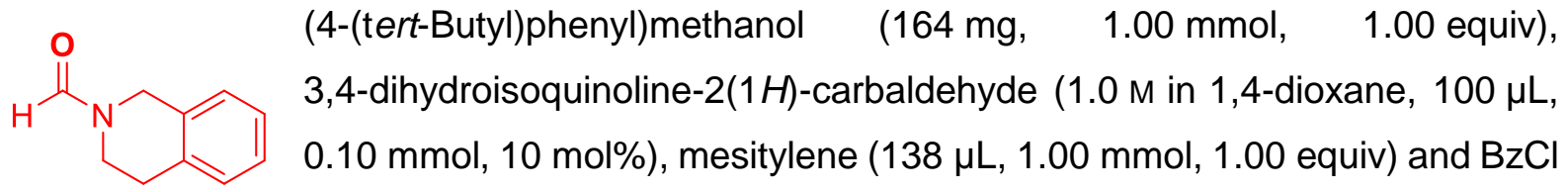

(139 $\mu \mathrm{L}, 1.20 \mathrm{mmol}, 1.20$ equiv) in 1,4-dioxane $(0.50 \mathrm{~mL})$ were reacted according to general procedure 7 (chapter 2.5.3, page 114).

Table S28. Determination of $k_{\text {start }}$ and $k_{\text {total. }}$.

\begin{tabular}{llll} 
Measurement & $\boldsymbol{k}_{\text {start }} / \mathbf{1 0}^{\mathbf{- 3}} \mathbf{~} \mathbf{~ i n}^{\mathbf{- 1}}$ & $\boldsymbol{k}_{\text {total }} / \mathbf{1 0}^{\mathbf{- 3}} \mathbf{~ m i n}^{-\mathbf{1}}$ & Induction Period / $\mathbf{~ i n ~}$ \\
\hline $\mathbf{1}$ & 3.15 & 2.26 & 42 \\
$\mathbf{2}$ & 3.28 & 2.26 & - \\
$\varnothing$ & 3.22 & 2.26 & -
\end{tabular}
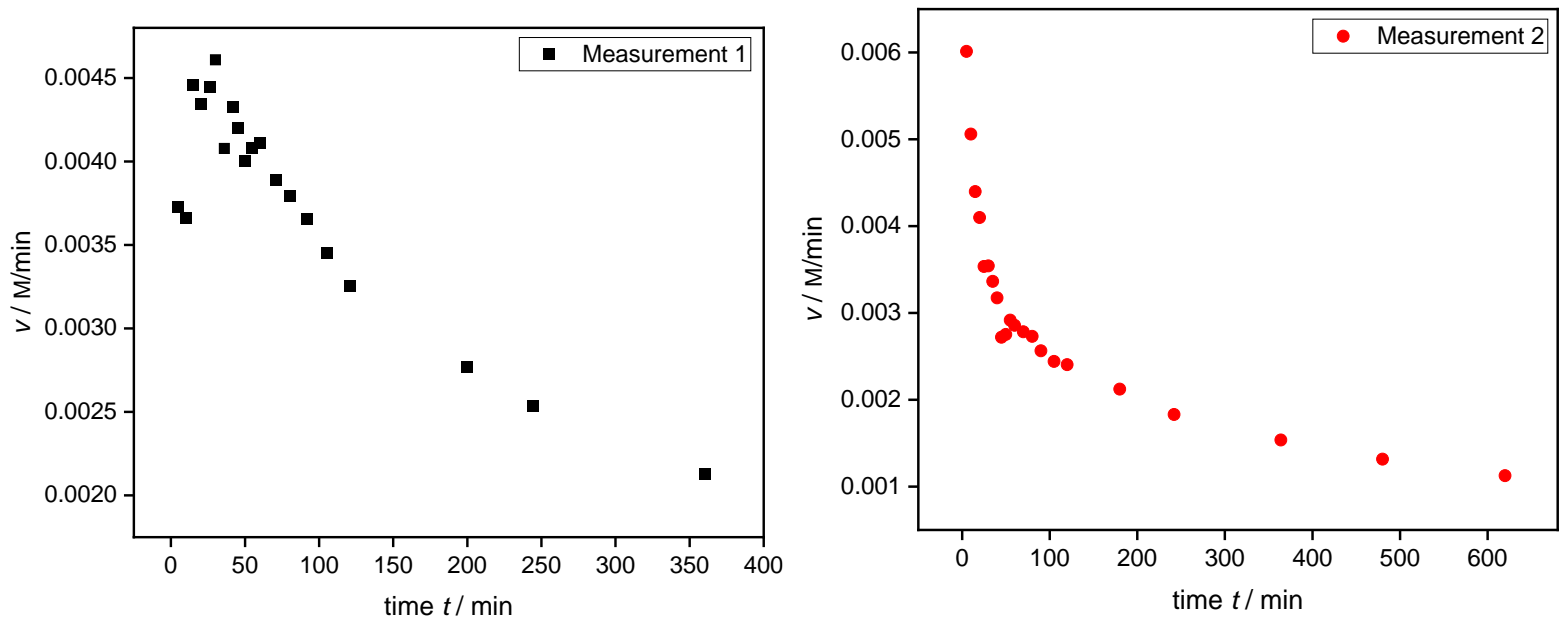

Figure S49. Reaction profiles plotting reaction rate $v$ versus time $t$.
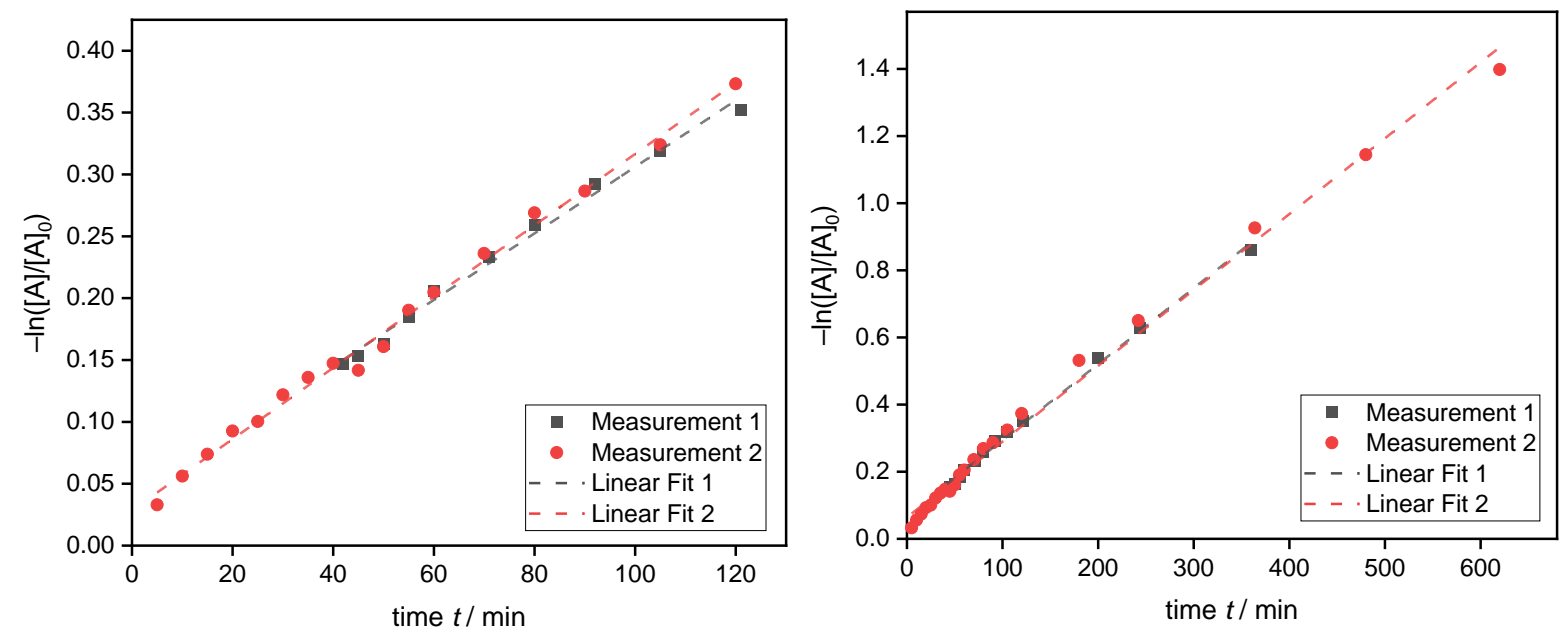

Figure S50. Logarithmic plots to determine $k_{\text {start }}$ (left) and $k_{\text {total }}$ (right). 


\subsection{Quantification of LeWIS-Basicity by Titration of $B\left(\mathrm{C}_{6} \mathrm{~F}_{5}\right)_{3}$ (Figure 3)}

Based on a change in chemical shift of $\mathrm{B}\left(\mathrm{C}_{6} \mathrm{~F}_{5}\right)_{3}$ by coordination of formamides, the Lewis basicity of a broad variety of formamides was quantified. The general principle of this quantification is illustrated in Scheme S3.

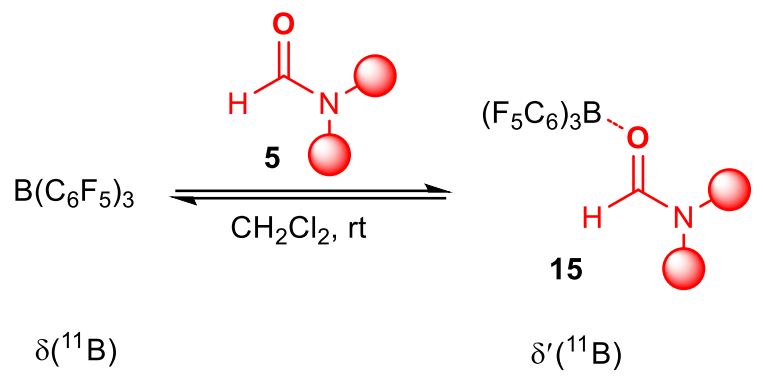

Scheme S3. Principle of quantification of the Lewis basicity of formamides using $B\left(\mathrm{C}_{6} \mathrm{~F}_{5}\right)_{3}$ as NMR probe.

The measurements were performed by adding stepwise 1.00 equiv-15.0 equiv of the corresponding formamide to a solution of $\mathrm{B}\left(\mathrm{C}_{6} \mathrm{~F}_{5}\right)_{3}$ in $\mathrm{CH}_{2} \mathrm{Cl}_{2}$. After each addition, a ${ }^{11} \mathrm{~B}$ NMR spectra was recorded. The maximal shift $\delta^{\prime}\left({ }^{11} \mathrm{~B}\right)$ of the formed complex 15 served as parameter to assess the Lewis basicity. Exemplary, the ${ }^{11} \mathrm{~B} N M R$ spectra of the NMR probe $\mathrm{B}\left(\mathrm{C}_{6} \mathrm{~F}_{5}\right)_{3}$ and the complex formed with N-Benzylformamide (BnF) are shown in Figure S51.
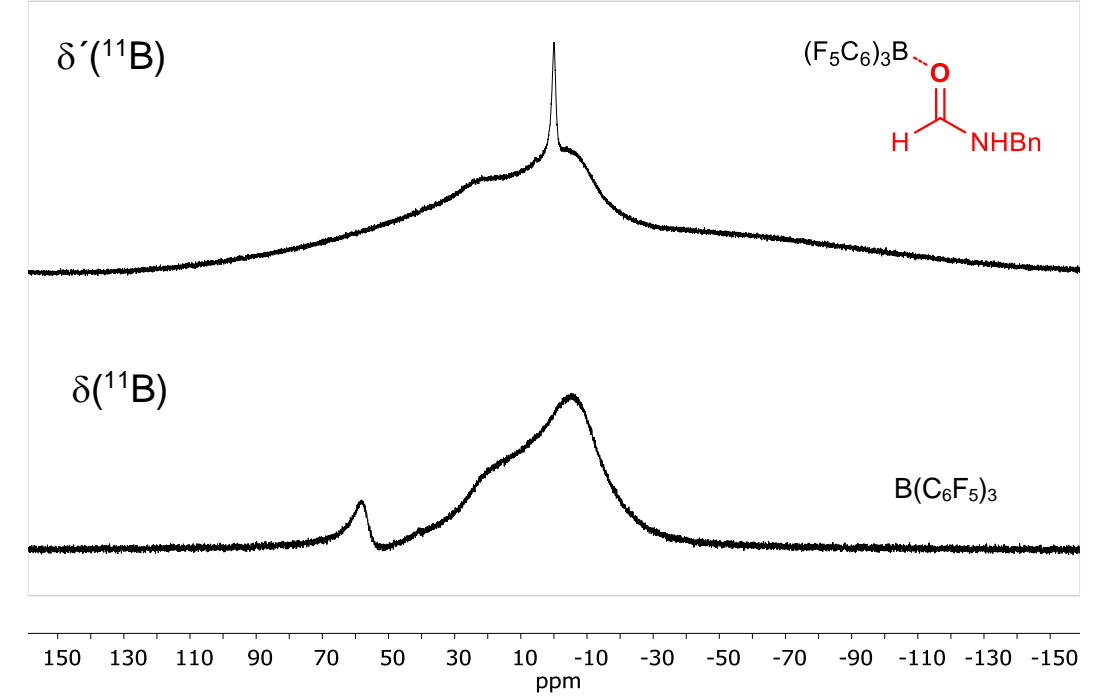

Figure S51. Comparison of the ${ }^{11} \mathrm{~B} N M R$ spectra of $\mathrm{B}\left(\mathrm{C}_{6} \mathrm{~F}_{5}\right)_{3}$ and the complex formed with $N$-Benzylformamide (BnF). 


\subsubsection{General Procedure for the Quantification of Lewis Basicity by Titration of} $B\left(\mathrm{C}_{6} \mathrm{~F}_{5}\right)_{3}$ Monitored by NMR Spectroscopy

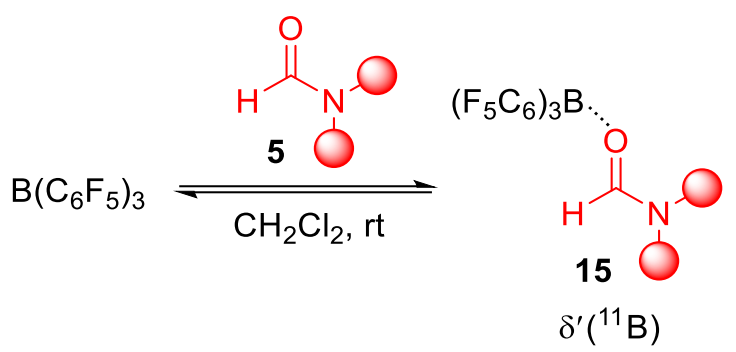

In a glove box, a Schlenk flask is charged with $\mathrm{B}\left(\mathrm{C}_{6} \mathrm{~F}_{5}\right)_{3}(66.6 \mathrm{mg}, 0.13 \mathrm{mmol})$. The flask is transferred out of the glove box and under argon atmosphere a stock solution with freshly distilled $\mathrm{CH}_{2} \mathrm{Cl}_{2}(0.13 \mathrm{M})$ is prepared. An aliquot of the stock solution of the NMR probe $(0.40 \mathrm{~mL}, 52.0 \mu \mathrm{mol}, 1.00$ equiv) is transferred to an NMR tube with a ground joint, which is sealed with a glass stopper. Then, a ${ }^{11} \mathrm{~B}$ NMR spectra is recorded using a glass capillary containing $\mathrm{D}_{2} \mathrm{O}$ for locking. Stepwise another aliquot of the stock solution of formamide in freshly distilled $\mathrm{CH}_{2} \mathrm{Cl}_{2}$ (1.00 equiv, 3.00 equiv, 5.00 equiv, 10.0 equiv, 15.0 equiv) is added to the NMR tube. After each addition of an aliquot of formamide, a ${ }^{11} \mathrm{~B}$ NMR spectra is recorded.

\subsubsection{Titration of $\mathrm{B}\left(\mathrm{C}_{6} \mathrm{~F}_{5}\right)_{3}$ with $\mathrm{N}, \mathrm{N}$-Dimethylformamide (DMF)}

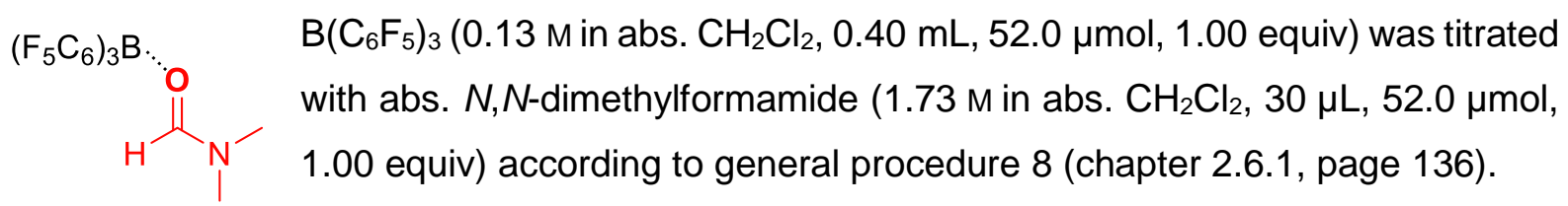

Table S29. ${ }^{11} \mathrm{~B}$ NMR Shifts resulting from the titration of $\mathrm{B}\left(\mathrm{C}_{6} \mathrm{~F}_{5}\right)_{3}$ with $\mathrm{DMF}$.

\begin{tabular}{lll} 
entry & Foramide & $\boldsymbol{\delta}^{\prime}\left({ }^{11} \mathbf{B}\right) / \mathbf{p p m}$ \\
\hline 1 & 1.00 equiv & -0.14 \\
2 & 3.00 equiv & -0.21 \\
3 & 5.00 equiv & -0.27 \\
4 & 10.0 equiv & -0.26 \\
5 & 15.0 equiv & -0.27
\end{tabular}


2.6.2.1 Titration of $\mathbf{B}\left(\mathbf{C}_{6} \mathbf{F}_{5}\right)_{3}$ with $\mathbf{N}$-Formylpyrrolidine (FPyr) $\mathrm{B}\left(\mathrm{C}_{6} \mathrm{~F}_{5}\right)_{3} \quad(0.13 \mathrm{M}$ in abs. $\left(\mathrm{F}_{5} \mathrm{C}_{6}\right)_{3} \mathrm{~B}$

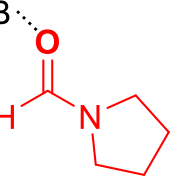

$\mathrm{CH}_{2} \mathrm{Cl}_{2}, \quad 0.40 \mathrm{~mL}, \quad 52.0 \mu \mathrm{mol}, 1.00$ equiv) was titrated with abs. $N$-formylpyrrolidine ( $1.73 \mathrm{M}$ in abs. $\mathrm{CH}_{2} \mathrm{Cl}_{2}, 30 \mu \mathrm{L}, 52.0 \mu \mathrm{mol}, 1.00$ equiv) according to general procedure 8 (chapter 2.6.1, page 136).

Table S30. ${ }^{11} \mathrm{~B}$ NMR Shifts resulting from the titration of $\mathrm{B}\left(\mathrm{C}_{6} \mathrm{~F}_{5}\right)_{3}$ with FPyr.

\begin{tabular}{lll} 
entry & Formamide & $\boldsymbol{\delta}^{\prime}\left({ }^{11} \mathbf{B}\right) / \mathbf{p p m}$ \\
\hline 1 & 1.00 equiv & -0.23 \\
2 & 3.00 equiv & -0.22 \\
3 & 5.00 equiv & -0.23 \\
4 & 10.0 equiv & -0.30 \\
5 & 15.0 equiv & -0.30 \\
\hline
\end{tabular}

\subsubsection{Titration of $\mathrm{B}\left(\mathrm{C}_{6} \mathrm{~F}_{5}\right)_{3}$ with Morpholine-4-carbaldehyde (FMor)}

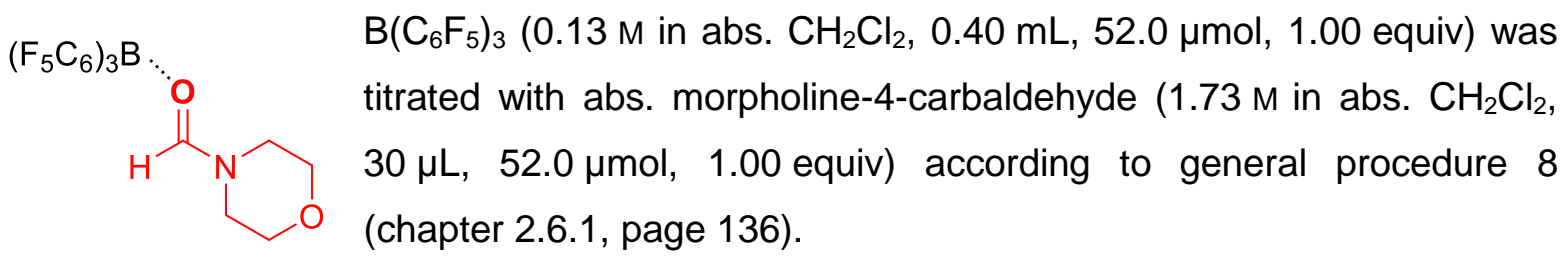

Table S31. ${ }^{11} \mathrm{~B}$ NMR Shifts resulting from the titration of $\mathrm{B}\left(\mathrm{C}_{6} \mathrm{~F}_{5}\right)_{3}$ with FMor.

\begin{tabular}{lll} 
entry & Formamide & $\boldsymbol{\delta}^{\prime}\left({ }^{11} \mathbf{B}\right) / \mathbf{p p m}$ \\
\hline 1 & 1.00 equiv & 0.16 \\
2 & 3.00 equiv & 0.08 \\
3 & 5.00 equiv & 0.06 \\
4 & 10.0 equiv & -0.03 \\
5 & 15.0 equiv & -0.03
\end{tabular}

\subsubsection{Titration of $\mathrm{B}\left(\mathrm{C}_{6} \mathrm{~F}_{5}\right)_{3}$ with Piperazine-1,4-dicarbaldehyde (FPip)}

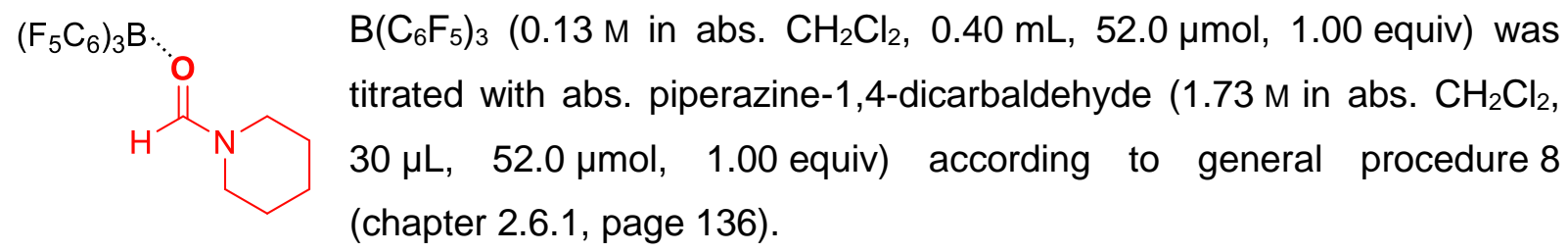


Table S32. ${ }^{11} \mathrm{~B}$ NMR Shifts resulting from the titration of $\mathrm{B}\left(\mathrm{C}_{6} \mathrm{~F}_{5}\right)_{3}$ with FPip.

\begin{tabular}{lll} 
entry & Formamide & $\boldsymbol{\delta}^{\prime}\left({ }^{11} \mathbf{B}\right) / \mathbf{p p m}$ \\
\hline 1 & 1.00 equiv & -0.10 \\
2 & 3.00 equiv & -0.10 \\
3 & 5.00 equiv & -0.20 \\
4 & 10.0 equiv & -0.20 \\
5 & 15.0 equiv & -0.20
\end{tabular}

\subsubsection{Titration of $\mathrm{B}\left(\mathrm{C}_{6} \mathrm{~F}_{5}\right)_{3}$ with $\mathrm{N}$-tert-Butylformamide (tBuF)}

$\left(\mathrm{F}_{5} \mathrm{C}_{6}\right)_{3} \mathrm{~B} \cdot \mathrm{H}_{\mathrm{H}^{-}}$

$\mathrm{B}\left(\mathrm{C}_{6} \mathrm{~F}_{5}\right)_{3}\left(0.13 \mathrm{M}\right.$ in abs. $\mathrm{CH}_{2} \mathrm{Cl}_{2}, 0.40 \mathrm{~mL}, 52.0 \mu \mathrm{mol}, 1.00$ equiv) was titrated with abs. $N$-tert-butylformamide $\left(1.73 \mathrm{M}\right.$ in abs. $\mathrm{CH}_{2} \mathrm{Cl}_{2}, 30 \mu \mathrm{L}$, $52.0 \mu \mathrm{mol}, 1.00$ equiv) according to general procedure 8 (chapter 2.6.1, page 136).

Table S33. ${ }^{11} \mathrm{~B}$ NMR Shifts resulting from the titration of $\mathrm{B}\left(\mathrm{C}_{6} \mathrm{~F}_{5}\right)_{3}$ with $t \mathrm{BuF}$.

\begin{tabular}{ccc} 
entry & Formamide & $\boldsymbol{\delta}^{\prime}\left({ }^{11} \mathbf{B}\right) / \mathbf{p p m}$ \\
\hline 1 & 1.00 equiv & -0.20 \\
2 & 3.00 equiv & -0.20 \\
3 & 5.00 equiv & -0.30 \\
4 & 10.0 equiv & -0.20 \\
5 & 15.0 equiv & -0.20
\end{tabular}

\subsubsection{Titration of $\mathrm{B}\left(\mathrm{C}_{6} \mathrm{~F}_{5}\right)_{3}$ with $\mathrm{N}$-Benzylformamide (BnF)}

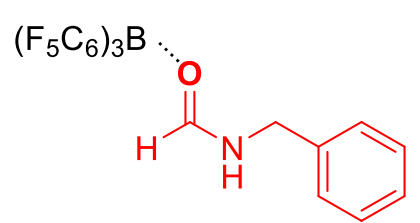

$\mathrm{B}\left(\mathrm{C}_{6} \mathrm{~F}_{5}\right)_{3}\left(0.13 \mathrm{M}\right.$ in abs. $\mathrm{CH}_{2} \mathrm{Cl}_{2}, 0.40 \mathrm{~mL}, 52.0 \mu \mathrm{mol}, 1.00$ equiv $)$ was titrated with abs. $\mathrm{N}$-tert-butylformamide $\left(1.73 \mathrm{M}\right.$ in abs. $\mathrm{CH}_{2} \mathrm{Cl}_{2}$, $30 \mu \mathrm{L}, 52.0 \mu \mathrm{mol}, 1.00$ equiv) according to general procedure 8 (chapter 2.6.1, page 136). 
Table S34. ${ }^{11} \mathrm{~B}$ NMR Shifts resulting from the titration of $\mathrm{B}\left(\mathrm{C}_{6} \mathrm{~F}_{5}\right)_{3}$ with $\mathrm{BnF}$.

\begin{tabular}{lll} 
entry & Formamide & $\boldsymbol{\delta}^{\prime}\left({ }^{11} \mathbf{B}\right) / \mathbf{p p m}$ \\
\hline 1 & 1.00 equiv & 0.72 \\
2 & 3.00 equiv & 0.14 \\
3 & 5.00 equiv & 0.22 \\
4 & 10.0 equiv & -0.10 \\
5 & 15.0 equiv & -0.01 \\
\hline
\end{tabular}

\subsubsection{Titration of $\mathrm{B}\left(\mathrm{C}_{6} \mathrm{~F}_{5}\right)_{3}$ with $N, N$-Di- $n$-butylformamide (DnBuF)}

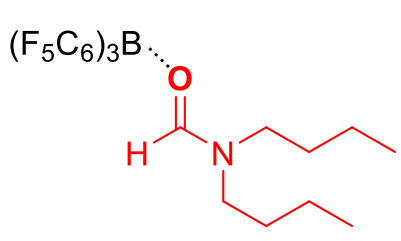

$\mathrm{B}\left(\mathrm{C}_{6} \mathrm{~F}_{5}\right)_{3}\left(0.13 \mathrm{M}\right.$ in abs. $\mathrm{CH}_{2} \mathrm{Cl}_{2}, 0.40 \mathrm{~mL}, 52.0 \mu \mathrm{mol}, 1.00$ equiv $)$ was titrated with abs. $N, N$-di- $n$-butylformamide $(1.73 \mathrm{M}$ in abs. $\mathrm{CH}_{2} \mathrm{Cl}_{2}, \quad 30 \mu \mathrm{L}, 52.0 \mu \mathrm{mol}, 1.00$ equiv) according to general procedure 8 (chapter 2.6.1, page 136).

Table S35. ${ }^{11} \mathrm{~B}$ NMR Shifts resulting from the titration of $\mathrm{B}\left(\mathrm{C}_{6} \mathrm{~F}_{5}\right)_{3}$ with $\mathrm{D} n \mathrm{BuF}$.

\begin{tabular}{lll} 
entry & Formamide & $\boldsymbol{\delta}^{\prime}\left({ }^{\mathbf{1}} \mathbf{B}\right) / \mathbf{p p m}$ \\
\hline $\mathbf{1}$ & 1.00 equiv & 0.00 \\
$\mathbf{2}$ & 3.00 equiv & 0.00 \\
$\mathbf{3}$ & 5.00 equiv & -0.10 \\
$\mathbf{4}$ & 10.0 equiv & 0.00 \\
$\mathbf{5}$ & 15.0 equiv & -0.10
\end{tabular}

\subsubsection{Titration of $\mathrm{B}\left(\mathrm{C}_{6} \mathrm{~F}_{5}\right)_{3}$ with Piperazine-1,4-dicarbaldehyde (DFPiper)}

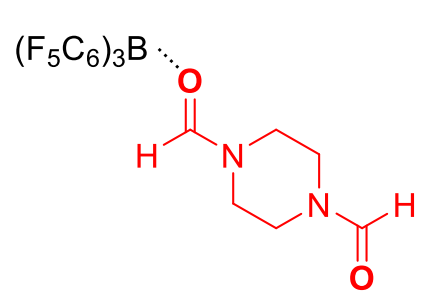

$\mathrm{B}\left(\mathrm{C}_{6} \mathrm{~F}_{5}\right)_{3}\left(0.13 \mathrm{M}\right.$ in abs. $\mathrm{CH}_{2} \mathrm{Cl}_{2}, 0.40 \mathrm{~mL}, 52.0 \mu \mathrm{mol}, 1.00$ equiv $)$ was titrated with abs. piperazine-1,4-dicarbaldehyde (1.73 $\mathrm{M}$ in abs. $\mathrm{CH}_{2} \mathrm{Cl}_{2}, 30 \mu \mathrm{L}, 52.0 \mu \mathrm{mol}, 1.00$ equiv) according to general procedure 8 (chapter 2.6.1, page 136). Indeed, no measurement using 15.0 equiv $B\left(\mathrm{C}_{6} \mathrm{~F}_{5}\right)_{3}$ was performed because the obtained suspension was not measurable with NMR spectroscopy. 
Table S36. ${ }^{11} \mathrm{~B}$ NMR Shifts resulting from the titration of $\mathrm{B}\left(\mathrm{C}_{6} \mathrm{~F}_{5}\right)_{3}$ with DFPiper.

\begin{tabular}{lll} 
entry & Formamide & $\boldsymbol{\delta}^{\prime}\left({ }^{\mathbf{1 1}} \mathbf{B}\right) / \mathbf{p p m}$ \\
\hline 1 & 1.00 equiv & 0.70 \\
2 & 3.00 equiv & 0.40 \\
3 & 5.00 equiv & 0.40 \\
4 & 10.0 equiv & 0.00
\end{tabular}

\subsubsection{Titration of $\mathrm{B}\left(\mathrm{C}_{6} \mathrm{~F}_{5}\right)_{3}$ with $\mathrm{N}$-Methylformamide (MF)}

$\left(\mathrm{F}_{5} \mathrm{C}_{6}\right)_{3} \mathrm{~B} . \quad \mathrm{B}\left(\mathrm{C}_{6} \mathrm{~F}_{5}\right)_{3}\left(0.13 \mathrm{M}\right.$ in abs. $\mathrm{CH}_{2} \mathrm{Cl}_{2}, 0.40 \mathrm{~mL}, 52.0 \mu \mathrm{mol}, 1.00$ equiv) was titrated 이 with abs. $N$-methylformamide $\left(1.73 \mathrm{M}\right.$ in abs. $\mathrm{CH}_{2} \mathrm{Cl}_{2}, 30 \mu \mathrm{L}, 52.0 \mu \mathrm{mol}$, $\mathrm{H}_{\mathrm{H}}^{\prime} 1.00$ equiv) according to general procedure 8 (chapter 2.6.1, page 136).

Table S37. ${ }^{11} \mathrm{~B}$ NMR Shifts resulting from the titration of $\mathrm{B}\left(\mathrm{C}_{6} \mathrm{~F}_{5}\right)_{3}$ with MF.

\begin{tabular}{lll} 
entry & Formamide & $\boldsymbol{\delta}^{\prime}\left({ }^{11} \mathbf{B}\right) / \mathbf{p p m}$ \\
\hline 1 & 1.00 equiv & 0.00 \\
\hline 2 & 3.00 equiv & -0.20 \\
3 & 5.00 equiv & -0.20 \\
4 & 10.0 equiv & -0.20 \\
\hline 5 & 15.0 equiv & -0.20
\end{tabular}

\subsubsection{Titration of $\mathrm{B}\left(\mathrm{C}_{6} \mathrm{~F}_{5}\right)_{3}$ with $\mathrm{N}$-Benzyl-N-methylformamide (BnMF)}<smiles>CN(Cc1ccccc1)C(=O)O[Ga]C(F)(F)C(F)(F)F</smiles>

$\mathrm{B}\left(\mathrm{C}_{6} \mathrm{~F}_{5}\right)_{3}\left(0.13 \mathrm{M}\right.$ in abs. $\mathrm{CH}_{2} \mathrm{Cl}_{2}, 0.40 \mathrm{~mL}, 52.0 \mu \mathrm{mol}, 1.00$ equiv $)$ was titrated with abs. $\mathrm{N}$-benzyl- $\mathrm{N}$-methylformamide (1.73 $\mathrm{M}$ in abs. $\mathrm{CH}_{2} \mathrm{Cl}_{2}, \quad 30 \mu \mathrm{L}, 52.0 \mu \mathrm{mol}, 1.00$ equiv) according to general procedure 8 (chapter 2.6.1, page 136). 
Table S38. ${ }^{11} \mathrm{~B}$ NMR Shifts resulting from the titration of $\mathrm{B}\left(\mathrm{C}_{6} \mathrm{~F}_{5}\right)_{3}$ with $\mathrm{BnMF}$.

\begin{tabular}{lll} 
entry & Formamide & $\boldsymbol{\delta}^{\prime}\left({ }^{11} \mathbf{B}\right) / \mathbf{p p m}$ \\
\hline 1 & 1.00 equiv & 0.20 \\
\hline 2 & 3.00 equiv & 0.20 \\
3 & 5.00 equiv & 0.20 \\
4 & 10.0 equiv & 0.10 \\
\hline 5 & 15.0 equiv & 0.10 \\
\hline
\end{tabular}

\subsubsection{Titration of $\mathrm{B}\left(\mathrm{C}_{6} \mathrm{~F}_{5}\right)_{3}$ with $\mathrm{N}, \mathrm{N}$-Dibenzylformamide (DBnF)}

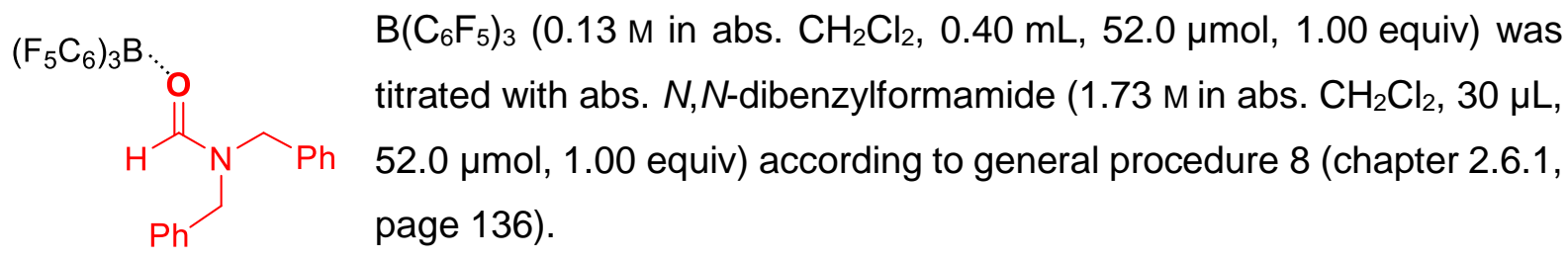

Table S39. ${ }^{11} \mathrm{~B}$ NMR Shifts resulting from the titration of $\mathrm{B}\left(\mathrm{C}_{6} \mathrm{~F}_{5}\right)_{3}$ with $\mathrm{DBF}$.

\begin{tabular}{|lll|} 
entry & Formamide & $\boldsymbol{\delta}^{\prime}\left({ }^{11} \mathbf{B}\right) / \mathbf{p p m}$ \\
\hline 1 & 1.00 equiv & 0.70 \\
\hline 2 & 3.00 equiv & 0.40 \\
\hline 3 & 5.00 equiv & 0.50 \\
4 & 10.0 equiv & 0.24 \\
\hline 5 & 15.0 equiv & 0.24 \\
\hline
\end{tabular}

\subsubsection{Titration of $\mathrm{B}\left(\mathrm{C}_{6} \mathrm{~F}_{5}\right)_{3}$ with $\quad \mathrm{N}$-(4-Methoxyphenyl)- $\mathrm{N}$-methylformamide (pMPMF)}<smiles>COc1ccc(N(C)C(=O)O[Ga]C(F)(F)F)cc1</smiles>

$\mathrm{B}\left(\mathrm{C}_{6} \mathrm{~F}_{5}\right)_{3}\left(0.13 \mathrm{M}\right.$ in abs. $\mathrm{CH}_{2} \mathrm{Cl}_{2}, 0.40 \mathrm{~mL}, 52.0 \mu \mathrm{mol}, 1.00$ equiv $)$ was titrated with abs. $N$-(4-methoxyphenyl)- $N$-methylformamide (1.73 $\mathrm{M}$ in abs. $\mathrm{CH}_{2} \mathrm{Cl}_{2}, 30 \mu \mathrm{L}, 52.0 \mu \mathrm{mol}, 1.00$ equiv) according to general procedure 8 (chapter 2.6.1, page 136). 
Table S40. ${ }^{11} \mathrm{~B}$ NMR Shifts resulting from the titration of $\mathrm{B}\left(\mathrm{C}_{6} \mathrm{~F}_{5}\right)_{3}$ with $p M P M F$.

\begin{tabular}{lll} 
entry & Formamide & $\boldsymbol{\delta}^{\prime}\left({ }^{\mathbf{1}} \mathbf{B}\right) / \mathbf{p p m}$ \\
\hline 1 & 1.00 equiv & 0.58 \\
2 & 3.00 equiv & 0.36 \\
3 & 5.00 equiv & 0.61 \\
4 & 10.0 equiv & 0.26 \\
5 & 15.0 equiv & 0.26
\end{tabular}




\subsection{Linear Free Energy Relationship.}

As shown in Figure S52, the Lewis basicity demonstrated by quantified activation of $B\left(C_{6} F_{5}\right)_{3}$ correlates well with the catalytic activity which is expressed by the logarithm of the initial rate constant $k_{\text {start. }}$

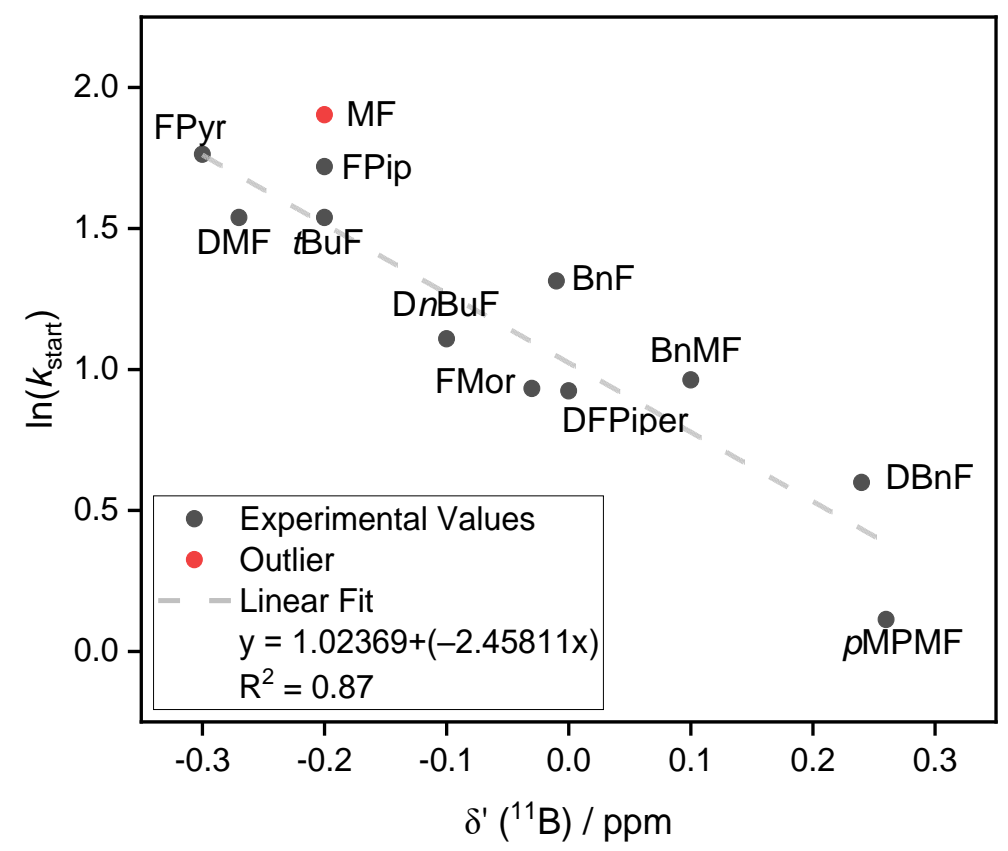

Figure S52. LFER between the Quantified Lewis Basicity and the Catalytic Activity of Formamides. 


\section{Multivariate Linear Regression Analysis (Figure 4)}

\subsection{Extension of the Catalyst Scope}

At the outset of the MLR study, several formamides with diverse structural and electronic properties were subjected to the Lewis base catalyzed nucleophilic substitution. The yield of $\mathbf{3 I}$ as well as the selectivity between $\mathbf{3 I}$ and $\mathbf{1 3}$ I were determined by GC analysis and are summarized and visualized by a color code in Figure S53. Those formamides that catalyze the model rection in adequate yield and good selectivity were used for determination of the initial rate constant $k_{\text {start. }}$ Based on the mean value of two measurements of $k_{\text {start, }}$ we applied MLR to investigate the catalytic activity of the formamides. 


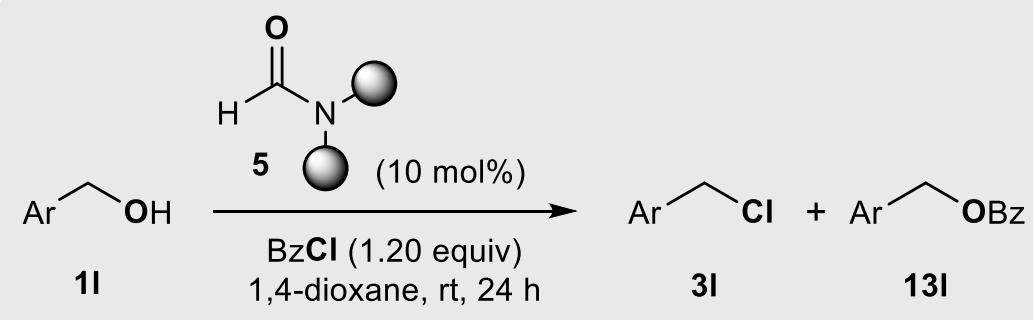

3I:13|

$>90: 10$

75:25-90:10

$<75: 25$

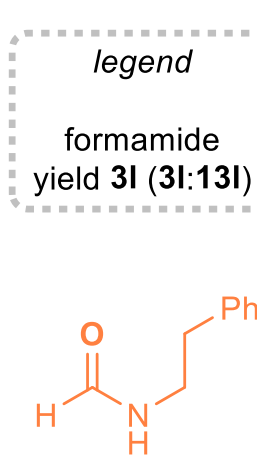

PEF<smiles>C=CCNC=O</smiles>

AlIF $86 \%(91: 9)$<smiles>O=CN1CCCCCC1</smiles>

$\mathrm{FAz}$<smiles>O=CNC1CCC2CC1C2</smiles>

$c \mathrm{HexF}$

$91 \%(90: 10)$<smiles>O=CN(Cc1ccccc1)Cc1ccccc1</smiles>

BntBuF $68 \%(78: 22)$<smiles>COC(CNC=O)OC</smiles>

DMEF

$75 \%(86: 14)$

$96 \%(85: 15)$

$76 \%(80: 20)$<smiles>O=CN1CCc2ccccc2C1</smiles><smiles>O=CN1CCCCCCC1</smiles><smiles>CC1(C)CCCC(C)(C)N1C=O</smiles>

$67 \%(76: 24)$<smiles>[R16][R6]([H])([18F])[Nb]C(=O)NCc1cccc2ccccc12</smiles>

FlsoChin $68 \%(78: 22)$

$68 \%(54: 46)$<smiles>CC(C)N(C=O)C(C)C</smiles>

$34 \%(31: 69)$<smiles>CN(C=O)c1cccc2ccccc12</smiles>

$18 \%(25: 75)$<smiles>COc1ccccc1N(C)C=O</smiles>

$15 \%(25: 75)$<smiles>O=CNc1ccccc1</smiles>

$1 \%(3: 97)$<smiles>COc1ccc(NC=O)cc1</smiles>

$4 \%(4: 96)$<smiles>O=CNc1ccc([N+](=O)[O-])cc1</smiles>

$1 \%(3: 97)$<smiles>O=CNc1ccc(Br)cc1</smiles>

$2 \%(3: 97)$<smiles>O=CNCc1ccccn1</smiles>

0\% (0:100)

Figure S53. Extension of the catalyst scope. 


\subsection{Calculation of the Molecular Descriptors}

All calculations were performed using the Gaussian 09, Revision D.01 software package. ${ }^{[29]}$

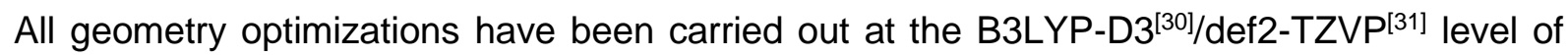
theory. Every optimized structure was confirmed to be a minimum on the potential energy surface by a subsequent frequency analysis (all positive eigenvalues). NBO charges were obtained via a full Natural Bond Orbital analysis, using NBO3. ${ }^{[32]}$

Based on the the energy-minimized structures, various molecular descriptors were calculated. An overview of all calculated parameter is shown in Table S41.

Table S41. Overview of all calculated molecular descriptors.

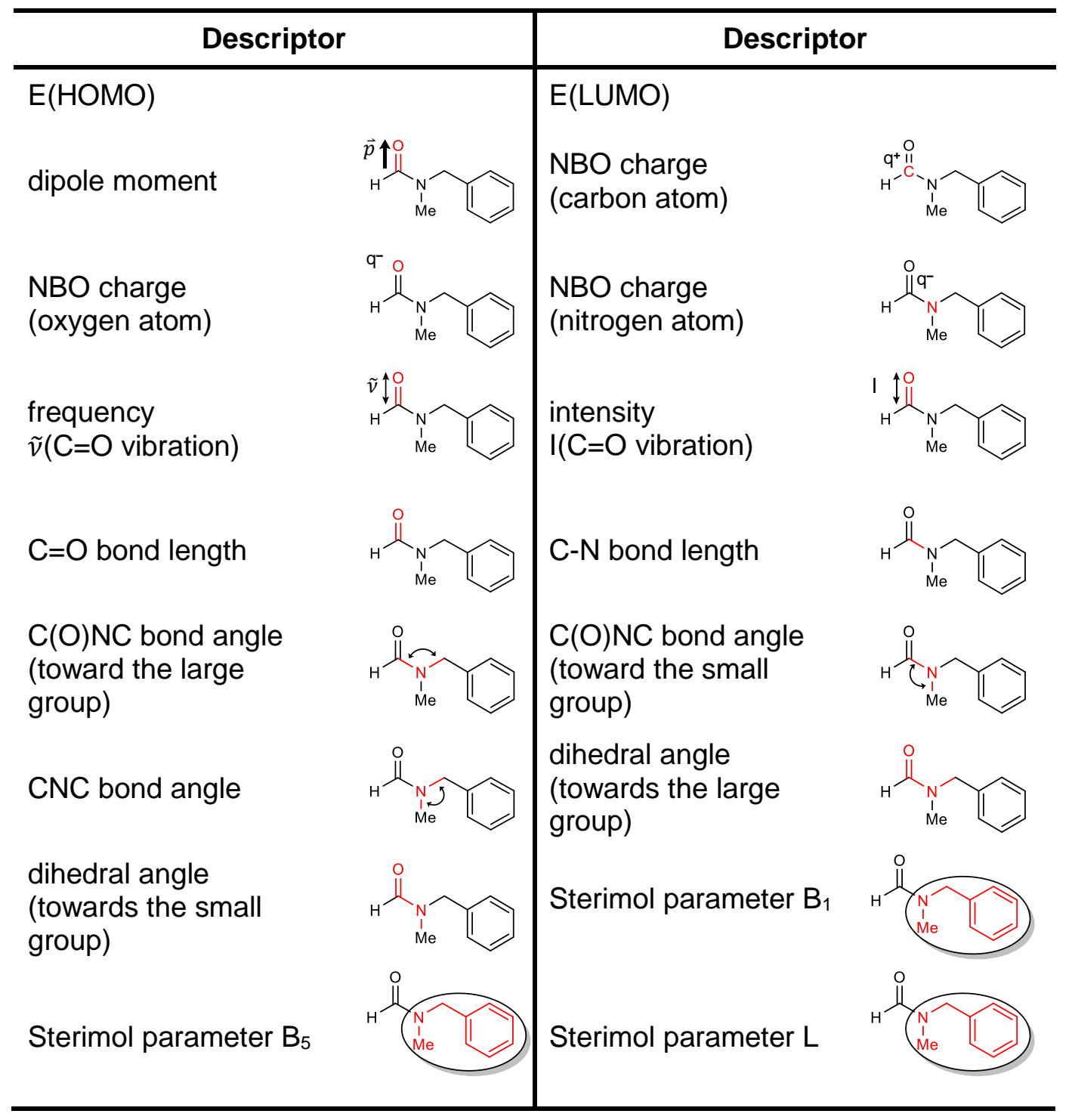

Multivariate linear regression analysis revealed that NBO charge of the oxygen atom at the carbonyl group, dipole moment of the carbonyl group and Sterimol parameter $\mathrm{B}_{5}$ led to an adequate prediction of the initial rate constant $k_{\text {start }}$. 


\section{Formamides}

\section{symmetrical}<smiles>O=CN1CCCC1</smiles>

FPyr<smiles>O=CN1CCCCC1</smiles>

FPip<smiles>CN(C)C=O</smiles>

DMF<smiles>CCCCN(C=O)CCCC</smiles>

DnBuF<smiles>O=CN1CCOCC1</smiles>

FMor<smiles>O=CN1CCN(C(=O)C2CCCCC2)CC1</smiles><smiles>O=CN(Cc1ccccc1)Cc1ccccc1</smiles><smiles>CC(C)(C)C(=O)N1CCCCC(C(C)(C)C)C1</smiles><smiles></smiles>

\section{unsymmetrical}

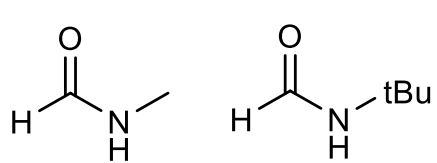

MF<smiles>CN(C=O)Cc1ccccc1</smiles>

BnMF<smiles>COc1ccc(N(C)C=O)cc1</smiles>

pMPMF<smiles>CC(C)(C)N(Cc1ccccc1)C(=O)Cc1ccccc1</smiles>

PEF
BntBuF<smiles>C=CCNC=O</smiles>

AlIF<smiles>O=CNCc1cccc2ccccc12</smiles>

NaphMF<smiles>O=CNC1CCCCC1</smiles>

cHexF<smiles>COC(CNC=O)OC</smiles>

DMEF<smiles>O=CNCc1ccccc1</smiles>

$\mathrm{BnF}$

Scheme S4. Summary of all formamides for model construction. 
Table S42. Relevant molecular descriptors for all symmetrical formamides.

\begin{tabular}{llllll}
\hline entry & formamide & $\begin{array}{l}\text { dipole } \\
\text { moment } / \\
\text { Debye }\end{array}$ & $\begin{array}{l}\text { NBO } \\
\text { charge } \\
\text { (oxygen } \\
\text { atom) }\end{array}$ & $\begin{array}{l}\text { Sterimol } \\
\text { parameter } \\
\text { B }_{5}\end{array}$ & $\begin{array}{l}\text { measured } \\
\boldsymbol{k}_{\text {start }} / \mathbf{1 0}^{-3} \\
\text { min-1[a] }^{-1}\end{array}$ \\
\hline 1 & FPyr & 4.581476 & -0.603 & 3.2741 & 5.83 \\
\hline 2 & FPip & 4.321052 & -0.605 & 3.7282 & 5.58 \\
\hline 3 & DMF & 4.060707 & -0.601 & 3.2844 & 4.66 \\
\hline 4 & DnBuF & 3.946586 & -0.611 & 6.8202 & 3.03 \\
\hline 5 & FMor & 3.043287 & -0.600 & 3.6384 & 2.54 \\
\hline 6 & DFPiper & 3.200700 & -0.594 & 4.2801 & 2.52 \\
\hline 7 & DBnF & 3.902455 & -0.601 & 6.8818 & 1.82 \\
\hline 8 & FAz & 4.325258 & -0.605 & 4.5409 & 4.20 \\
\hline 9 & FlsoChin & 3.857692 & -0.602 & 5.4639 & 3.21 \\
\hline
\end{tabular}

[a] The mean value of two measurements of $k_{\text {start }}$ is depicted. 
Table S43. Relevant molecular descriptors for all unsymmetrical formamides.

\begin{tabular}{|c|c|c|c|c|c|}
\hline entry & formamide & $\begin{array}{l}\text { dipole } \\
\text { moment / } \\
\text { Debye }\end{array}$ & $\begin{array}{l}\text { NBO charge } \\
\text { (oxygen atom) }\end{array}$ & $\begin{array}{l}\text { Sterimol } \\
\text { parameter } B_{5}\end{array}$ & $\begin{array}{l}\text { measured } k_{\text {start }} / \\
10^{-3} \mathrm{~min}^{-1}\end{array}$ \\
\hline 1 & $E-M F$ & 4.236315 & -0.590 & 3.2678 & 6.71 \\
\hline 2 & $Z-\mathrm{MF}$ & 3.992725 & -0.599 & 3.2605 & 6.71 \\
\hline 3 & $E-t B u F$ & 4.553916 & -0.596 & 4.3524 & 4.66 \\
\hline 4 & $Z$-tBuF & 3.813459 & -0.608 & 4.3677 & 4.66 \\
\hline 5 & $E-B n F$ & 4.214631 & -0.588 & 6.1829 & 3.72 \\
\hline 6 & $Z-B n F$ & 3.654161 & -0.604 & 6.8575 & 3.72 \\
\hline 7 & $E-B n M F$ & 4.096167 & -0.600 & 6.2195 & 2.62 \\
\hline 8 & Z-BnMF & 3.81455 & -0.609 & 6.7499 & 2.62 \\
\hline 9 & E-pMPMF & 4.961261 & -0.593 & 7.2838 & 1.12 \\
\hline 10 & Z-pMPMF & 4.701277 & -0.581 & 6.9889 & 1.12 \\
\hline 11 & E-PEF & 4.322410 & -0.590 & 7.5217 & 3.68 \\
\hline 12 & Z-PEF & 3.701784 & -0.601 & 7.8479 & 3.68 \\
\hline 13 & $E-B n t B u F$ & 4.100704 & -0.615 & 6.9609 & 3.95 \\
\hline 14 & Z-BntBuF & 3.859520 & -0.609 & 6.918 & 3.95 \\
\hline 15 & E-AllF & 4.155724 & -0.587 & 3.7611 & 3.48 \\
\hline 16 & Z-AllF & 3.787948 & -0.597 & 3.7107 & 3.48 \\
\hline 17 & E-NaphMF & 4.333247 & -0.587 & 7.1171 & 3.37 \\
\hline 18 & Z-NaphMF & 3.702722 & -0.592 & 7.1064 & 3.37 \\
\hline 19 & $E-c H e x F$ & 4.775163 & -0.596 & 4.8273 & 3.17 \\
\hline 20 & $Z$-cHexF & 3.937466 & -0.604 & 5.4082 & 3.17 \\
\hline 21 & E-DMEF & 4.86563 & -0.587 & 5.2308 & 2.76 \\
\hline 22 & Z-DMEF & 2.850840 & -0.604 & 6.2255 & 2.76 \\
\hline
\end{tabular}

[a] The mean value of two measurements of $k_{\text {start }}$ is depicted. 


\subsection{Model Construction for Symmetrical Formamides}

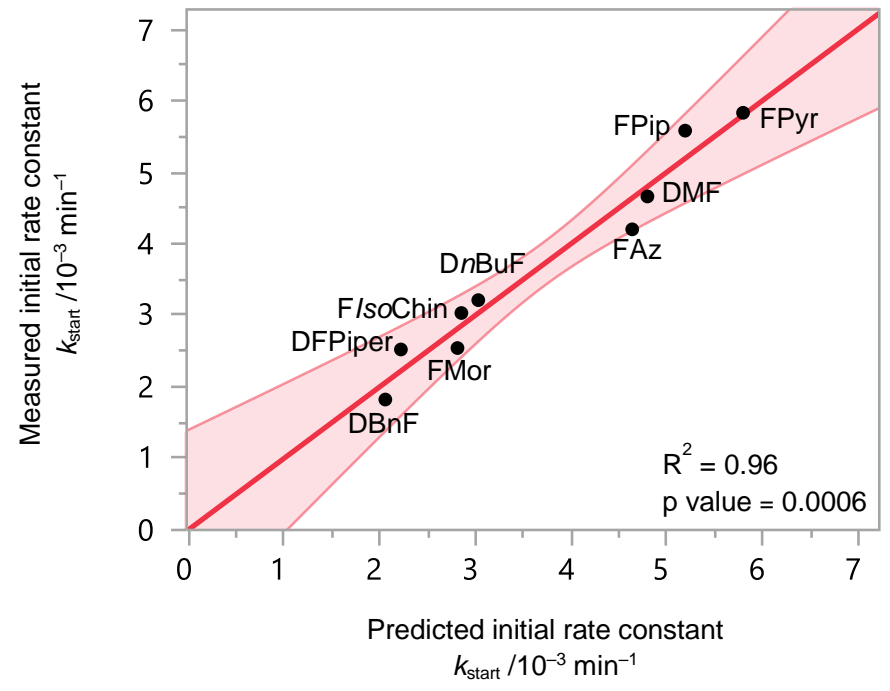

- symmetrical formamides

$$
\begin{aligned}
k_{\text {start }}=-54.819 & +1.121 \cdot\left(\frac{(\text { dipole moment }-3.951}{0.750}\right)+(-0.811) \cdot\left(\frac{\left(B_{5}-2.821\right)}{0.957}\right) \\
& +(-100.156) \cdot N B O(O)
\end{aligned}
$$

Figure S54. Prediction model for $k_{\text {start. }}$

\begin{tabular}{|c|c|c|c|}
\hline Source & Log Worth & & P Value \\
\hline $\begin{array}{l}\text { Sterimol parameter } B_{5}(1.8634 \text {, } \\
3.7777)\end{array}$ & 2.938 & 1 & 0.00115 \\
\hline dipole moment $(3.2007,4.7013)$ & 2.392 & -十： & 0.00405 \\
\hline NBO charge (oxygen atom) & 0.847 & $\square ! \quad ! \quad \vdots$ & 0.14221 \\
\hline
\end{tabular}

Table S44. Effect summary. 


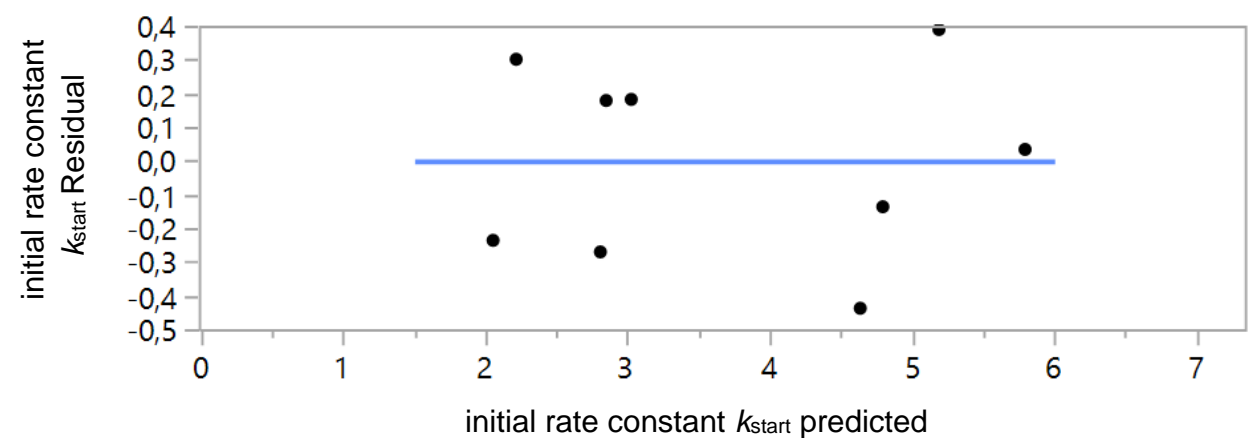

Figure S55. Residual by predicted plot.

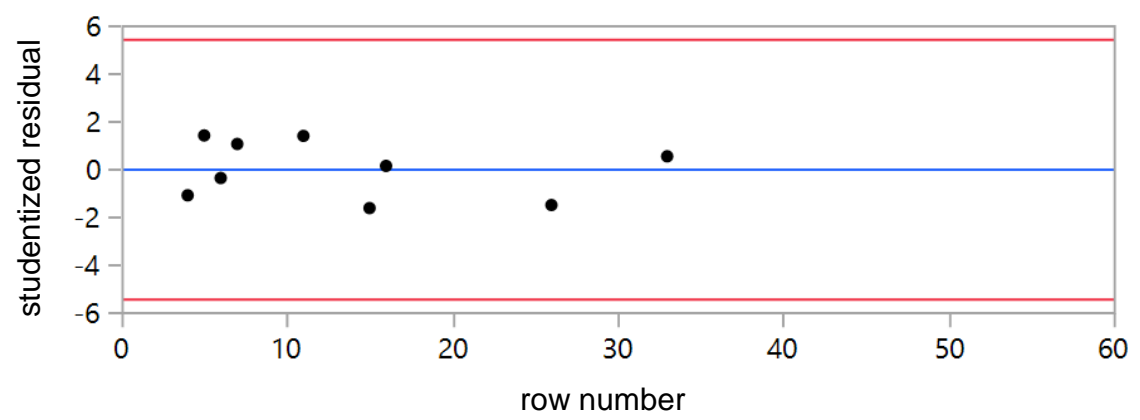

Figure S56. Studentized Residuals.

Table S45. Parameter Estimates.

$\begin{array}{lcrrr}\text { Term } & \text { Estimate } & \text { Std. Error } & \text { t Ratio } & \text { Prob. }>|\mathbf{t}| \\ \text { intercept } & -35.93972 & 23.45222 & -1.53 & 0.1860 \\ \begin{array}{l}\text { dipole moment } \\ \text { (3.2007, 4.7013) }\end{array} & 1.23443 & 0.246154 & 5.01 & 0.0041^{*} \\ \begin{array}{l}\text { Sterimol parameter B } \\ \text { (1.8634, 3.7777) }\end{array} & -0.659817 & 0.099105 & -6.66 & 0.0012^{*} \\ \text { NBO charge (oxygen atom) } & -68.01303 & 39.07168 & -1.74 & 0.1422\end{array}$

Table S46. Effect Tests.

\begin{tabular}{|c|c|c|c|c|c|}
\hline Source & Nparm & DF & Sum of Squares & F Ratio & Prob. $>$ F \\
\hline $\begin{array}{l}\text { dipole moment (3.2007, } \\
4.7013)\end{array}$ & 1 & 1 & 3.2551585 & 25.1489 & $0.0041^{*}$ \\
\hline $\begin{array}{l}\text { Sterimol parameter } B_{5} \\
(1.8634,3.7777)\end{array}$ & 1 & 1 & 5.7373400 & 44.3258 & $0.0012^{*}$ \\
\hline NBO charge (oxygen atom) & 1 & 1 & 0.3922059 & 3.0301 & 0.1422 \\
\hline
\end{tabular}




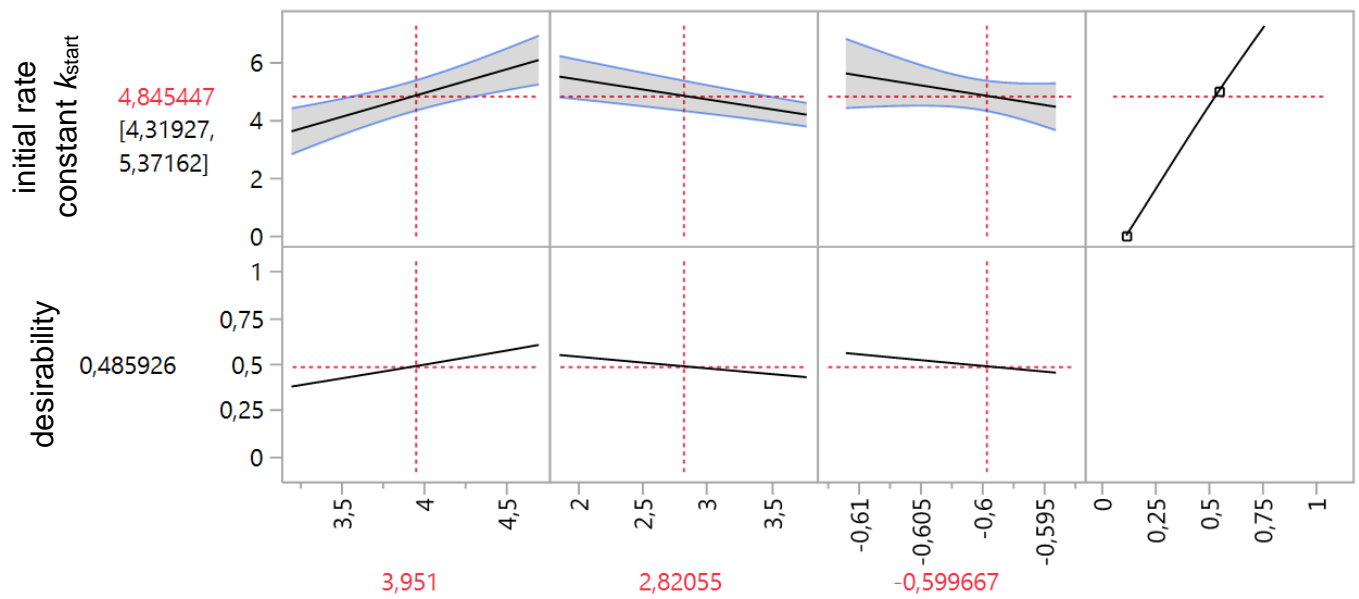

$\begin{array}{llll}\text { dipole moment } & \mathrm{B}_{5} & \mathrm{NBO} & \text { desirability }\end{array}$

Figure S57. Prediction profiler. 


\subsection{Addition of E-configured Formamides to the Model}

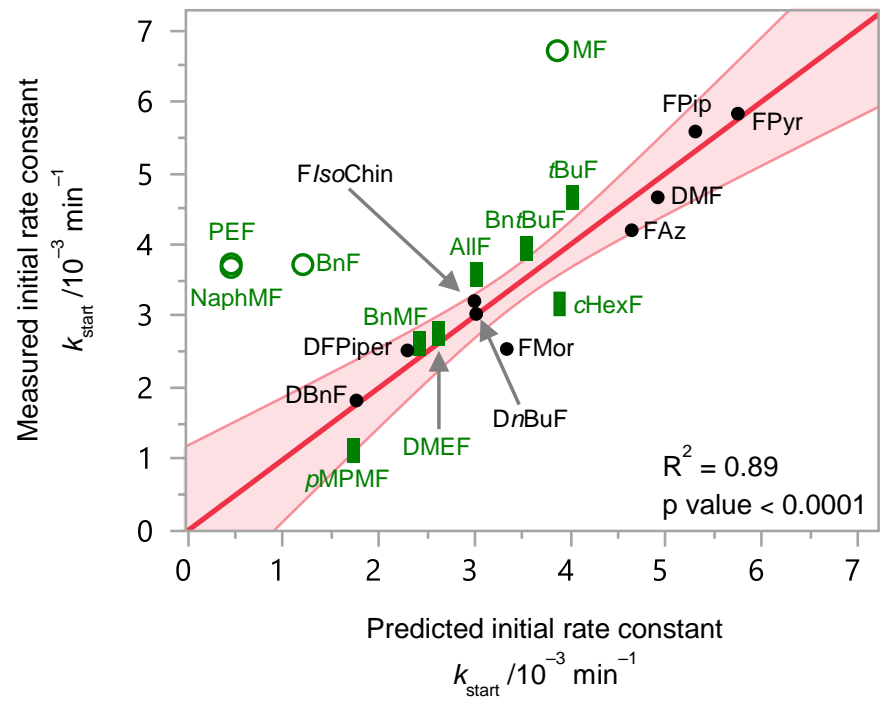

- symmetrical formamides E-configurated formamides o outlier

Table S47. Effect summary.

\section{Source}

Sterimol parameter $\mathrm{B}_{5}(1.8634$, 3.7777)

NBO charge (oxygen atom)

dipole moment $(3.2007,4.7013)$

\section{Log Worth}

5.749

4.447

3.202

\section{P Value}

0.00000

0.00004

0.00063

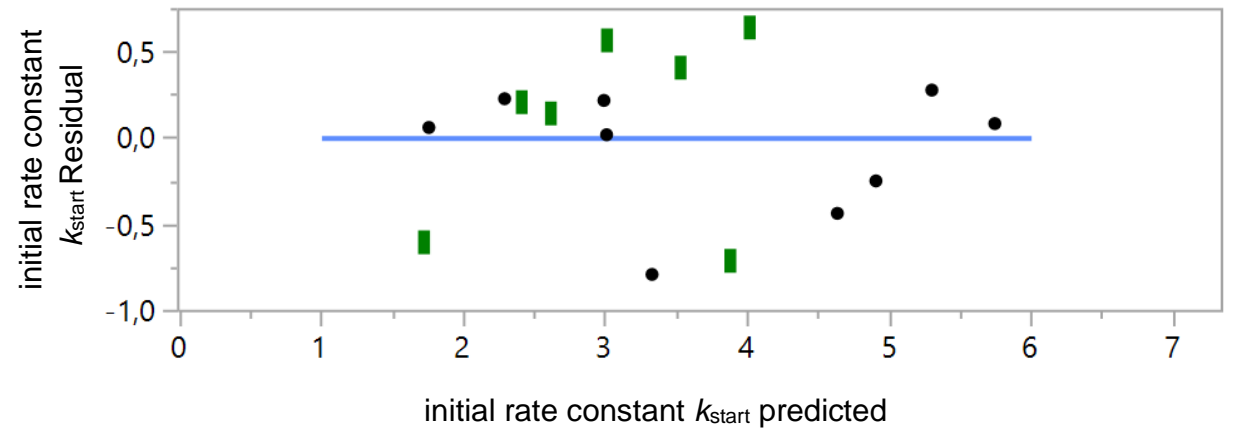

Figure S58. Residual by predicted plot. 


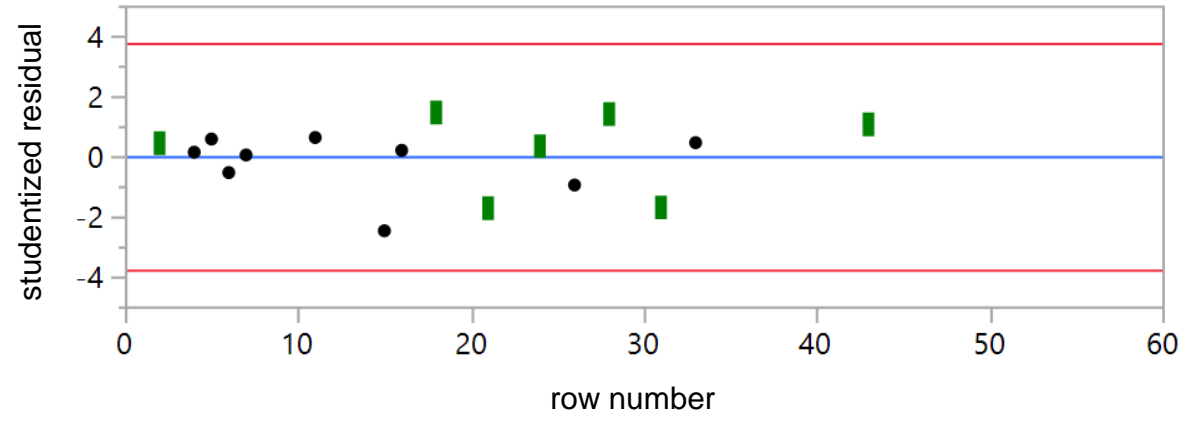

Figure S59. Studentized Residuals.

Table S48. Parameter Estimates.

$\begin{array}{lcccc}\text { Term } & \text { Estimate } & \text { Std. Error } & \text { t Ratio } & \text { Prob. }>|\mathbf{t}| \\ \text { intercept } & -63.977 & 10.79061 & -5.93 & <0.0001^{*} \\ \begin{array}{l}\text { dipole moment } \\ \text { (3.2007, 4.7013) }\end{array} & 0.8632978 & 0.188341 & 4.58 & 0.0006^{*} \\ \begin{array}{l}\text { Sterimol parameter B } \\ \text { (1.8634, 3.7777) }\end{array} & -0.789569 & 0.091825 & -8.60 & <0.0001^{*} \\ \text { NBO charge (oxygen atom) } & -115.0506 & 18.07018 & -6.37 & <0.0001^{*}\end{array}$

Table S49. Effect Tests.

\begin{tabular}{|c|c|c|c|c|c|}
\hline Source & Nparm & DF & $\begin{array}{l}\text { Sum of } \\
\text { Squares }\end{array}$ & F Ratio & Prob. > F \\
\hline $\begin{array}{l}\text { dipole moment }(3.2007 \text {, } \\
4.7013)\end{array}$ & 1 & 1 & 5.074874 & 21.0103 & $0.0006^{*}$ \\
\hline $\begin{array}{l}\text { Sterimol parameter } B_{5} \\
(1.8634,3.7777)\end{array}$ & 1 & 1 & 17.858739 & 73.9364 & $<0.0001^{*}$ \\
\hline NBO charge (oxygen atom) & 1 & 1 & 9.791418 & 40.5371 & $<0.0001^{*}$ \\
\hline
\end{tabular}

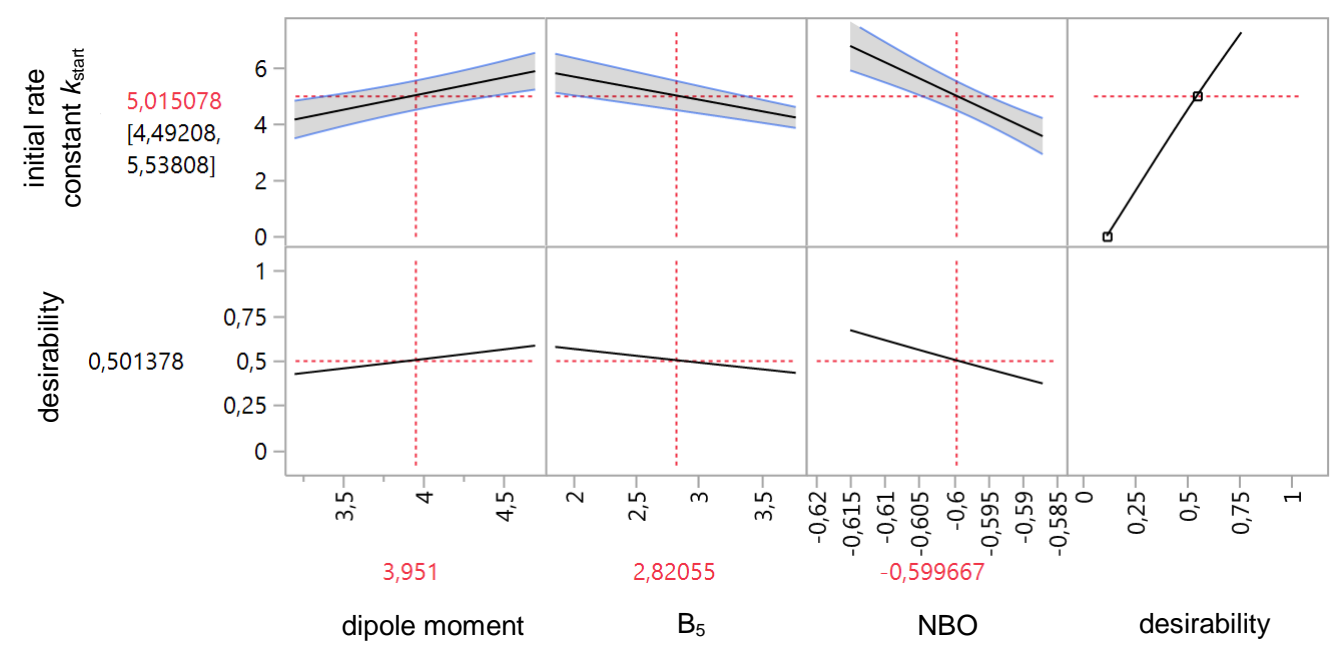

Figure S 60. Prediction Profiler. 


\subsection{Addition of Z-configured Formamides to the Model}

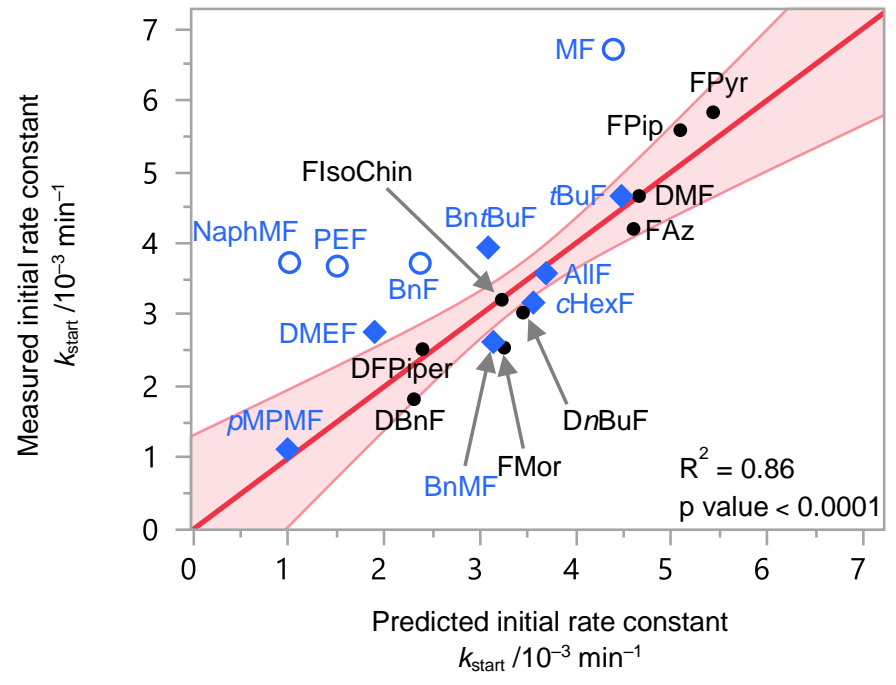

- symmetrical formamides Z-configurated formamides o outlier

Table S50. Effect summary.

\begin{tabular}{|c|c|c|c|}
\hline Source & Log Worth & & P Value \\
\hline Sterimol parameter $\mathrm{B}_{5}(1.8634,3.7777)$ & 4.421 & 1 & 0.00004 \\
\hline NBO charge (oxygen atom) & 3.803 & 1 & 0.00016 \\
\hline dipole moment $(3.2007,4.7013)$ & 2.644 & 1 & 0.00227 \\
\hline
\end{tabular}

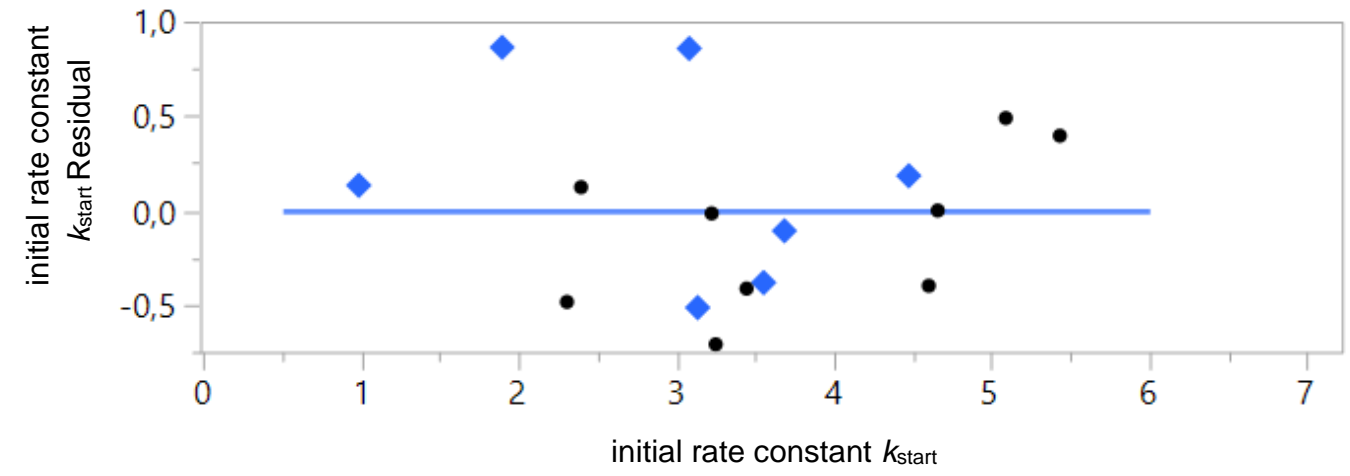

Figure S61. Residual by predicted plot. 


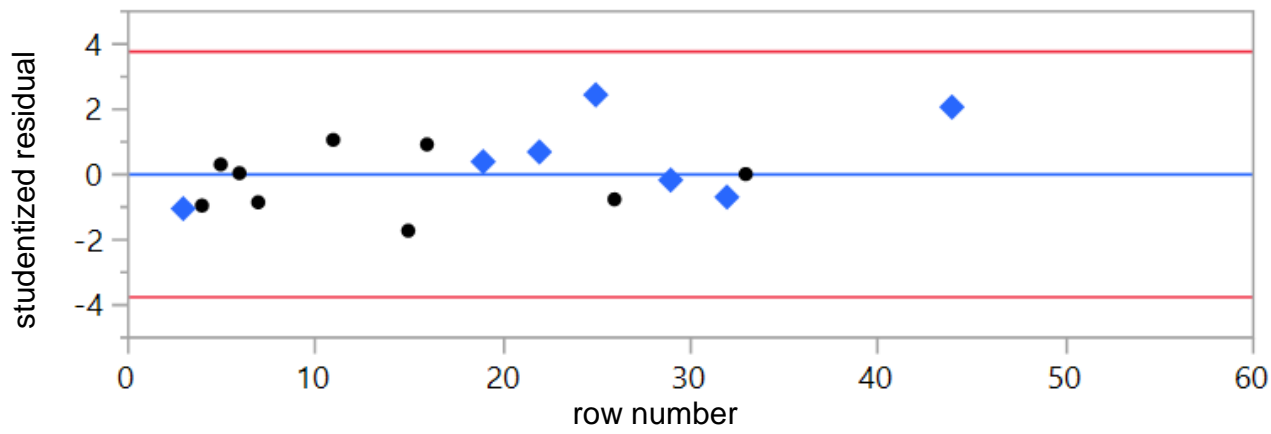

Figure S62. Studentized Residuals.

Table S51. Parameter Estimates.

$\begin{array}{lcccc}\text { Term } & \text { Estimate } & \text { Std. Error } & \text { t Ratio } & \text { Prob. }>|\mathbf{t}| \\ \begin{array}{l}\text { intercept } \\ \text { dipole moment }\end{array} & -58.58329 & 11.72927 & -4.99 & 0.0003^{*} \\ \begin{array}{l}\text { (3.2007, 4.7013) } \\ \text { Sterimol parameter B }\end{array} & 0.8027433 & 0.20801 & 3.86 & 0.0023^{*} \\ \begin{array}{l}(1.8634,3.7777) \\ \text { NBO charge (oxygen atom) }\end{array} & -0.580864 & 0.091809 & -6.33 & <0.0001^{*} \\ & -105.503 & 19.49772 & -5.41 & 0.0002^{*}\end{array}$

Table S52. Effect Tests.

\begin{tabular}{|c|c|c|c|c|c|}
\hline Source & Nparm & DF & Sum of Squares & F Ratio & Prob. $>$ F \\
\hline $\begin{array}{l}\text { dipole moment (3.2007, } \\
4.7013)\end{array}$ & 1 & 1 & 4.278613 & 14.8932 & $0.0023^{*}$ \\
\hline $\begin{array}{l}\text { Sterimol parameter } \mathrm{B}_{5} \\
(1.8634,3.7777)\end{array}$ & 1 & 1 & 11.499853 & 40.0291 & $<0.0001^{*}$ \\
\hline NBO charge (oxygen atom) & 1 & 1 & 8.411582 & 29.2794 & $0.0002^{*}$ \\
\hline
\end{tabular}




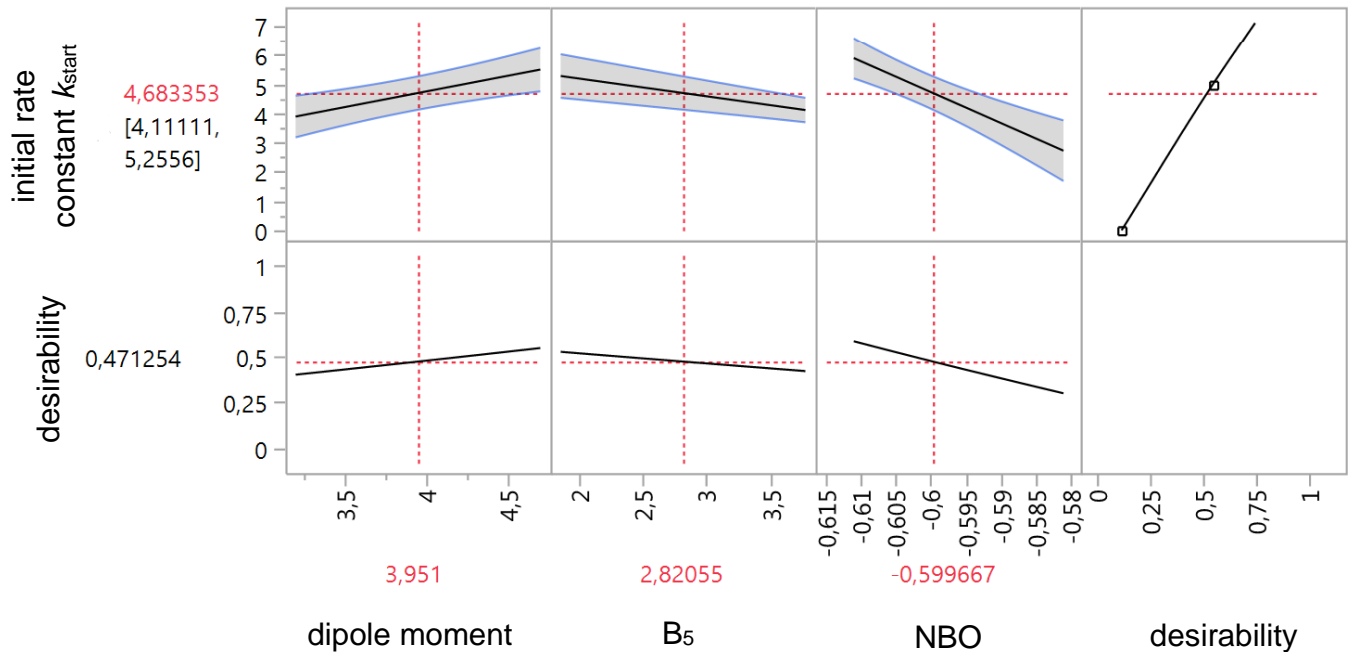

Figure S63. Prediction Profiler. 


\section{References}

(1) Collins, K. D.; Glorius, F. A robustness screen for the rapid assessment if chemical reactions. Nature Chem. 2013, 5, 597-601.

(2) Collins, K. D.; Glorius, F. Employing a robustness screen: rapid assessment of rhodium(III)-catalysed C-H activation reactions. Tetrahedron 2013, 69, 7817-7825.

(3) Gensch, T.; Teders, M.; Glorius, F. Approach to Comparing the Functional Group Tolerance of Reactions. J. Org. Chem. 2017, 82, 9154-9159.

(4) Huy, P. H.; Filbrich, I. A General Catalytic Method for Highly Cost- and Atom-Efficient Nucleophilic Substitutions. Chem. Eur. J. 2018, 24, 7410-7416.

(5) Huy, P. H.; Motsch, S.; Kappler, S. M. Formamides as Lewis Base Catalysts in SN Reactions-Efficient Transformation of Alcohols into Chlorides, Amines, and Ethers. Angew. Chem. Int. Ed. 2016, 55, 10145-10149.

(6) Pitzer, L.; Schäfers, F.; Glorius, F. Rapid Assessment of the Reaction-Condition-Based Sensitivity of Chemical Transformations. Angew. Chem. Int. Ed. 2019, 58, 8572-8576.

(7) Fulmer, G. R.; Miller, A. J. M.; Sherdan, N. H.; Gottlieb, H. E.; Nudelman, A.; Stoltz, B. M.; Bercaw, J. E.; Goldberg, K. I. NMR Chemical Shifts of Trace Impurities: Common Laboratory Solvents, Organics, and Gases in Deuterated Solvents Relevant to the Organometallic Chemist. Organometallics 2010, 29, 2176-2179.

(8) Kazmierczak, P.; Skulski, L.; Kraszkiewicz, L. Syntheses of (Diacetoxyiodo)arenes or lodylarenes from lodoarenes, with Sodium Periodate as the Oxidant. Molecules 2001, 6, 881-891.

(9) Behrends, I.; Bähr, S.; Czekelius, C. Perfluoroalkylation of Alkenes by Frustrated Lewis Pairs. Chem. Eur. J. 2016, 22, 17177-17181.

(10) (a) Verloop, A. IUPAC Pesticide Chemistry, Pergamon, Oxford, 1983. (b) Verloop, A.; Hoogenstraaten, W.; Tipker, J. Sterimol. Drug Design - Medicinal Chemistry: A Series of Monographs, Academic Press, Amsterdam, 1976. (c) Verloop, A.; Tipker, J. Biological Activity and Chemical Structure, Elsevir, New York, 1977. (d) Verloop, A.; Tipker, J. QSAR in Drug Dosing and Toxicology, Elsevir, New York, 1987.

(11) Vanjari, R.; Allam, B. K.; Singh, K. N. Hypervalent iodine catalyzed transamidation of carboxamides with amines. RSC Adv. 2013, 3, 1691-1694.

(12) Chapman, R. S. L.; Lawrence, R.; Williams, J. M. J.; Bull, S. D. Formyloxyacetoxyphenylmethane as an $\mathrm{N}$-Formylating Reagent for Amines, Amino Acids, and Peptides. Org. Lett. 2017, 19, 4908-4911.

(13) Ortega, N.; Richter, C.; Glorius, F. N-Formylation of Amines by Methanol Activation. Org. Lett. 2013, 15, 1776-1779. 
(14) El Dine, T. M.; Evans, D.; Rouden, J.; Blanchet, J. Formamide Synthesis through Borinic Acid Catalysed Transamidation under Mild Conditions. Chem. Eur. J. 2016, 22, 5894-5898.

(15) Ma, J.; Zhang, F.; Zhang, J.; Gong, H. Cobalt(II)-Catalyzed N-Acylation of Amines through a Transamidation Reaction. Eur. J. Org. Chem. 2018, 35, 4940-4948.

(16) Klapars, A.; Huang, X.; Buchwald, S. L. A General and Efficient Copper Catalyst for the Amidation of Aryl Halides. J. Am. Chem. Soc. 2002, 124, 7421-7428.

(17) Netz, N.; Opatz, T. A Modular Formal Total Synthesis of ( \pm )-Cycloclavine. J. Org. Chem. 2016, 81, 1723-1730.

(18) Tan, Z.; Li, Z.; Ma. Y.; Qin, J.; Yu, C. Potassium tert-Butoxide Prompted Highly Efficient Transamidation and Its Coordination Radical Mechanism. Eur. J. Org. Chem. 2019, 45384545.

(19) Zhang, B.; Du, G.; Hang, W.; Wang, S.; Xi, C. Lewis Base Promoted Reduction of $\mathrm{CO}_{2}$ with $\mathrm{BH}_{3} \mathrm{NH}_{3}$ into Boryl Formates: $\mathrm{CO}_{2}$ as a Carbon Source in Organic Synthesis Under Mild Conditions. Eur. J. Org. Chem. 2018, 14, 1739-1743.

(20) Motsch, S.; Schütz, C.; Huy, P. H. Systematic Evaluation of Sulfoxides as Catalysts in Nucleophilic Substitutions of Alcohols. Eur. J. Org. Chem. 2018, 4541-4547.

(21) Neochoritis, C. G.; Stotani, S.; Mishra, B.; Dömling, A. Efficient Isocyanide-less Isocyanide-Based Multicomponent Reactions. Org. Lett. 2015, 17, 2002-2005.

(22) Yin, J.; Zhang, J.; Cai, C.; Deng, G.-J.; Gong, H. Catalyst-Free Transamidation of Aromatic Amines with Formamide Derivatives and Tertiary Amides with Aliphatic Amines.

Org. Lett. 2019, 21, 387-392.

(23) Chen, X.; Fan. H.; Zhang, S.; Yu, C.; Wang, W. Facile Installation of 2-Reverse Prenyl Functionality into Indoles by a Tandem N-Alkylation-Aza-Cope Rearrangement Reaction and Its Application in Synthesis. Chem. Eur. J. 2016, 22, 716-723.

(24) (a) Burés, J. A simple graphical method to determine the order in catalyst. Angew. Chem. Int. Ed. 2016, 55, 2028-2031. (b) Burés, J. A Simple Graphical Method to Determine the Order in Catalyst. Angew. Chem. Int. Ed. 2016, 55, 16084-16087.

(25) Li, J.; Liu, T., Zheng, M.; Sun, M.; Zhang, D.; Zhang, H.; Sun, P.; Xue, S.; Zhang, B. Dibutylaminophenyl- and/or Pyridinyl-Capped 2,6,9,10-Tetravinylanthracene Cruciforms: Synthesis and Aggregation-Enhanced One- and Two-Photon Excited Fluorescence. J. Phys. Chem. C 2013, 117, 8404-8410.

(26) Tanaka, H.; Chou, J.; Mine, M.; Kuroboshi, M. The Oxidation of Alcohols in N-OxylImmobilized Silica Gel/Aqueous $\mathrm{NaOCI}$ Disperse Systems. A Prominent Access to a Column-Flow System. Bull. Chem. Soc. Jpn. 2004, 77, 1745-1755.

(27) Champagne, P. A.; Benhassine, Y.; Desroches, J.; Paquin, J.-F. Friedel-Crafts Reaction of Benzyl Fluorides: Selective Activation of $\mathrm{C}-\mathrm{F}$ Bonds as Enabled by Hydrogen Bonding. Angew. Chem. Int. Ed. 2014, 53, 13835-13839. 
(28) Shaikh, N. S.; Junge, K.; Beller, M. A convenient and general iron-catalyzed hydrosilylation of aldehydes. Org. Lett. 2007, 9, 5429-5432.

(29) Gaussian 09, Revision D.01, Frisch, M. J.; Trucks, G. W.; Schlegel, H. B.; Scuseria, G. E.; Robb, M. A.; Cheeseman, J. R.; Scalmani, D.; Barone, V.; Petersson, G. A.; Nakatsuji, H.; Li, X.; Caricato, M.; Marenich, A.; Bloino, J.; Janesko, B. G.; Gomperts, R.; Mennucci, B.; Hratchian, H. P.; Ortiz, J. V.; Izmaylov, A. F.; Sonnenberg, J. L.; Williams-Young, D.; Ding, F.; Lipparini, F.; Egidi, F.; Goings, J.; Peng, B.; Petrone, A.; Henderson, T.; Ranasinghe, D.; Zakrzewski, V. G.; Gao, J.; Rega, N.; Zheng, G.; Liang, W.; Hada, M; Ehara, M.; Toyota, K.; Fukuda, R.; Hasegawa, J.; Ishida, M.; Nakajima, T.; Honda, Y.; Kitao, O.; Nakai, H.; Vreven, T.; Throssell, K.; Montgomery, J. A.; Peralta, Jr. J. E.; Ogliaro, F.; Bearpark, M.; Heyd, J. J.; Brothers, E.; Kudin, K. N.; Staroverov, V. N.; Keith, T.; Kobayashi, R.; Normand, J.;

Raghavachari, K.; Rendell, A.; Burant, J. C.; lyengar, S. S.; Tomasi, J.; Cossi, M.; Millam, J. M.; Klene, M.; Adamo, C.; Cammi, R.; Ochterski, J. W.; Martin, R. L.; Morokuma, K.; Farkas, V; Foresman, J. B.; Fox, D. J. Gaussian, Inc., Wallingford CT, 2016.

(30) (a) Becke, A. D. Density-functional thermochemistry. III. The role of exact exchange. J. Chem. Phys. 1993, 98, 5648-5652. (b) Lee, W.; Yang, R. G. Development of the ColleSalvetti correlation-energy formula into a functional of the electron density. Phys. Rev. $B$ 1988, 37, 785-789. (c) Vosko, S. H.; Wilk, L.; Nusair, M. Accurate spin-dependent electron liquid correlation energies for local spin density calculations: a critical analysis. Can. J. Phys. 1980, 58, 1200-1211. (d) Stephens, P. J.; Devlin, F. J.; Chabalowski, C. F.; Frisch, M. J. Ab initio calculation of vibrational absorption and circular dichroism spectra using density functional force fields. J. Phys. Chem. 1994, 11623-11627. (e) Grimme, S.; Antony, J.;

Ehrlich, S.; Krieg, H. A consistent and accurate ab initio parametrization of density functional dispersion correction (DFT-D) for the 94 elements H-Pu. J. Chem. Phys. 2010, 132, 154104. (32) (a) Foster, J. P.; Weinhold, F. Natural hybrid orbitals. J. Am. Chem. Soc. 1980, 102, 7211-7218. (b) A. E. Reed, F. Weinhold, Natural bond orbital analysis of near-Hartree-Fock water dimer. J. Chem. Phys. 1983, 78, 4066-4073. (c) Reed, A. E.; Weinstock, R. B.; Weinhold, F. Natural-population analysis. J. Chem. Phys. 1985, 83, 735-746. (d) Reed, A. E.; Weinhold, F. Natural Localized Molecular Orbitals. J. Chem. Phys. 1985, 83, 1736-1740. (e) Carpenter, J. E. Extension of Lewis structure concepts to open-shell and excited-state molecular species, Ph.D. thesis, University of Wisconsin, Madison, WI, 1987. (f) Carpenter, J. E.; Weinhold, F. Analysis of the geometry of the hydroxymethyl radical by the different hybrids for different spins natural bond orbital procedure. J. Mol. Struct. (Theochem) 1988, 139, 41-62. (g) Reed, A. E. Intermolecular interactions from a natural bond orbital, donoracceptor viewpoint. Chem. Rev. 1988, 88, 899-926. (h) Weinhold, F.; Carpenter, J. E. The Structure of Small Molecules and Ions, Ed. R. Naaman and Z. Vager, Plenum, 1988, 227236. 